\title{
Water maser variability over 20 years in a large sample of star-forming regions: the complete database ${ }^{\star}$
}

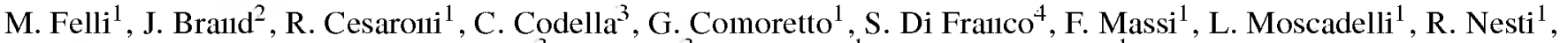 \\ L. Olmi ${ }^{3}$, F. Palagi ${ }^{3}$, D. Panella ${ }^{1}$, and R. Valdettaro ${ }^{1}$ \\ 1 INAF - Osservatorio Astrofisico di Arcetri. Largo E. Fermi 5, 50125 Firenze. Italy \\ e-mail: cesa@arcetri.astro.it \\ INAF - Istituto di Radioastronomia, via Gobetti 101, 40129 Bologna, Italy \\ INAF - Istituto di Radioastronomia, Sez. di Firenze, Largo E. Fermi 5, 50125 Firenze, Italy \\ + Dipartimento di Astronomia. Università degli Studi di Firenze, Largo E. Fermi 2. 50125 Firenze. Italy
}

Received 7 May 2007 / Accepted 3 July 2007

\begin{abstract}
Context. Water vapor' emission at $22 \mathrm{GHz}$ from maser's associated with sta1-forming r'egions is highly variable. Aims. We present a database of up to 20 years of monitoring of a sample of 43 masers within star-forming regions. The sample covers a large range of luminosities of the associated IRAS source and is representative of the entire population of $\mathrm{H}_{2} \mathrm{O}$ maser's of this type. The database forms a good starting point for any further study of $\mathrm{H}_{2} \mathrm{O}$ maser variability.

Methods. The observations were obtained with the Medicina 32-m radiotelescope, at a rate of 4-5 observations per year.

Results. To provide a database that can be easily accessed through the web, we give for each source: plots of the calibrated spectra, the velocity-time-flux density plot, the light curve of the integrated flux, the lower and upper envelopes of the maser emission. the mean spectrum. and the rate of the maser occurrence as a function of velocity. Figures for just one source are given in the text for representative purposes. Figures for all the sources are given in electronic form the appendix. A discussion of the main properties of the $\mathrm{H}_{2} \mathrm{O}$ variability in our sample will be presented in a forthcoming paper.
\end{abstract}

Key words. masers - radio lines: ISM - ISM: molecules

\section{Introduction}

Since the discovery of $22 \mathrm{GHz} \mathrm{H_{2 }} \mathrm{O}$ maser emission associated with young stellar objects (YSOs) within star-forming regions (SFRs), variability of maser emission is well-known. Changes as large as several orders of magnitude in the maser emission have been observed (e.g., Little et al. 1977; Liljeström et al. 1989; Claussen et al. 1996; Comoretto et al. 1990; Wouterloot et al. 1995). At the same time, velocity drifts of individual components of up to a few $\mathrm{km} \mathrm{s}^{-1}$ per year have also been reported (e.g., Brand et al. 2003). The variability can be slow or burst-like and covers all ranges of timescales, from hours-days to monthsyears. In the present study we are mainly concerned with the latter and, for the first time, deal with a large sample of sources (43) and with a time-span of up to 20 years, with $4-5$ spectra per year.

Due to the large amount of telescope time required to follow the evolution of $\mathrm{H}_{2} \mathrm{O}$ maser emission, inevitably the variability is more easily monitored through single dish observations. In fact, besides our campaign only the Pushchino 22-1n single-dish maser patrol covers a comparably long period (e.g., Rudnitskij et al. 2007). Furuya et al. (2003) carried out a multiepoch $22 \mathrm{GHz} \mathrm{H}_{2} \mathrm{O}$ maser survey towards 173 low-mass YSOs (Class 0 to Class III sources) using the Nobeyama $45 \mathrm{~m}$ telescope. This was the first complete water maser survey towards Class 0 sources in the northern sky. However, their observations extend non-uniformly over a period of only three years.

* Based on observations with the Medicina radiotelescope operated by INAF - Istituto di Radioastronomia.
It is well-known that the variability depends strongly on the luminosity of the SFR (usually derived from the associated IRAS source). For instance, there is a minimum luminosity ( $\left.\sim 25 L_{\odot}\right)$ below which $\mathrm{H}_{2} \mathrm{O}$ maser emission may be present only for about one third of the entire duration of the maser activity (Persi et al. 1994; Claussen et al. 1996). The results obtained so far suggest that the lower the SFR luminosity, the higher the observed degree of variability of the $\mathrm{H}_{2} \mathrm{O}$ maser emission. Higher luminosity sources may show more steady components. Consequently, a large sample of sources is needed to better understand the dependence of the variability on other parameters of the SFRs, in particular on their luminosities which cover a range of several orders of magnitude, and on their (molecular) environment.

The use of a single dish instrument is of course a limitation because interferometric observations clearly show that $\mathrm{H}_{2} \mathrm{O}$ masers within a SFR often consist of many spatially separated, unresolved components, generally clustered in groups (usually with different velocities), which in most cases cannot be separated in single dish observations (e.g., Forster \& Caswell 1989, 1999; Tofani et al. 1995). However, Felli et al. (2006) reported a case in which single dish observations were able to separate the evolution of spatially distinct components. Nevertheless, frequent single dish observations have the advantage of being able to follow potential velocity drifts of individual velocity components, which can be easily identified in the spectra, and thus give a better view of the dynamics (e.g., accelerations) occurring in the circumstellar environment of the YSO.

In two earlier papers (Valdettaro et al. 2002; Brand et al. 2003), we set the basis for a systematic study of the $\mathrm{H}_{2} \mathrm{O}$ vari- 
ability in SFRs with the Medicina 32-m radiotelescope using a sample of 14 sources which covered a wide range of luminosities and had been observed for more than 10 years. Here we report on a larger sample of 43 SFRs (including the former 14 sources) and increase the time coverage up to 20 years. The database is presented in the form of plots of the calibrated spectra and additional plots of derived quantities, in a form that can be easily accessed through the web. In a forthcoming paper, we will analyze the properties of $\mathrm{H}_{2} \mathrm{O}$ variability of our sample.

\section{The sample}

A large sample of sources selected from the Arcetri Atlas (Cesaroni et al. 1988; Comoretto et al. 1990; Palagi et al. 1993; Brand et al. 1994; Valdettaro et al. 2001) has been monitored regularly since the beginning of the operating period of the Arcetri digital autocorrelator at the Medicina 32-m radiotelescope (1987), at a rate of 4-5 observations per year. Our goal was to study the dependence of the variability of $\mathrm{H}_{2} \mathrm{O}$ masers in SFRs on the luminosity of the associated YSO, assumed to remain constant during our patrol. It should be pointed out that the FIR luminosity is derived from IRAS observations and, consequently, is representative of the entire luminosity of the SFR and not necessarily of the YSO that powers the $\mathrm{H}_{2} \mathrm{O}$ maser. In fact a SFR may have a very complex structure, with several YSOs in various evolutionary stages and with different luminosities present within the same SFR, as for instance in W75-N (Hunter et al. 1994). Variability might also depend on other characteristics of the SFR such as the presence of molecular outflows (Felli et al. 1992), its IRAS colours and the presence or absence of an UC HII region (Palla et al. 1991, 1993; Codella et al. 1994, 1996; Codella \& Felli 1995; Codella \& Palla 1995).

The present sample of $43 \mathrm{H}_{2} \mathrm{O}$ masers was chosen out of the several hundred known $\mathrm{H}_{2} \mathrm{O}$ masers of SFR type (e.g., Comoretto et al. 1990) using the following criteria:

(1) the maser sources were to be of the SFR-type, i.e. associated with a SFR, to be distinguished from those associated with late-type stars. The SFR classification of Palagi et al. (1993) and Valdettaro et al. (2001) is based on IRAS colours. The location of the sources of our sample in an IRAS colour-colour plot is shown in Fig. 1 and compared with that of a much larger sample of SFRs with $\mathrm{H}_{2} \mathrm{O}$ maser emission (a merger of Comoretto et al. 1990; Brand et al. 1994; and Valdettaro et al. 2001). The dashed box defines the region occupied by sources with colours characteristic of ultracompact HII regions (Wood \& Churchwell 1989). To double-check the true nature of the sources exciting the $\mathrm{H}_{2} \mathrm{O}$ masers, other morphological indicators of the SFRs were also searched for in the literature, e.g. association with dense molecular cores (e.g. Cesaroni et al. 1999), presence of UC HII regions, presence of molecular outflows and other types of masers. All investigations further support the SFR identification ${ }^{l}$.

(2) the luminosities of the associated IRAS source was to cover as large a range as possible;

\footnotetext{
1 We note that in SIMBAD. AFGL2789 is identified witl1 "V645 Cyg. pulsating variable star". However, as stated by Clarke et al. (2006), V645 Cyg is a "relatively unembedded young massive star, witll a liigl1velocity wind and an associated optical and molecular outflow", and it "may represent a relatively rare class of transition objects between a genuine massive YSO and a normal young Oe-type star in a weak HII region". Its presence in our sample is tlerefore justified.
}

(3) the sample was to be sufficiently large to be representative of the entire population of $\mathrm{H}_{2} \mathrm{O}$ masers within SFRs;

(4) the sources were to be suitably distributed in right ascension, to avoid scheduling problems due to clustering of sources in the inner region of the Galactic plane in the large sample;

(5) the peak flux density was to be greater than a few Jy so that the source could be easily observed with a 5 min on-source integration time with the Medicina 32-m radio telescope;

(6) the number of selected sources was not to be too large to allow observations in a $<2-3$ day session;

(7) finally, in order to prevent confusion in the single beam from nearby, but unrelated, maser sources, we selected sources from the Arcetri Atlas for which no other masers had been reported within a circle of several arcminutes with single dish observations ${ }^{2}$.

The main source parameters are given in Table 1, which lists:

(1) a running number;

(2) the source name;

(3) the associated IRAS source, when available;

(4) J2000.0 right ascension;

(5) J2000.0 declination;

(6) $V_{\mathrm{cl}}$, the velocity of the associated molecular cloud relative to the LSR, derived from the literature using tracers sensitive to high molecular density, such as $\mathrm{NH}_{3}$ and $\mathrm{CS}$ (in $\mathrm{k} \mathrm{n} \mathrm{s}^{-1}$ );

(7) $d$, the distance taken from the literature (in $\mathrm{kpc}$ );

(8) $L_{\mathrm{IR}}$, the integrated FIR luminosity (in $L_{\odot}$ ), usually derived from IRAS data or, otherwise, taken from the literature;

(9) the date of the first observation;

(10) references for the distance.

The IRAS luminosity was obtained by adopting the distance from Col. 7, assuming an emissivity proportional to the frequency, correcting the observed IRAS flux densities for the derived colour temperatures between two adjacent bands, and extrapolating the fluxes to 6 and $400 \mu \mathrm{m}$ (Wouterloot et al. 1995).

The sample covers a range of IR luminosities from $20 L_{\odot}$ to $1.5 \times 10^{6} L_{\odot}$. These values bracket the entire luminosity interval of the regions where $\mathrm{H}_{2} \mathrm{O}$ masers of the SFR-type are found (Palagi et al. 1993). In the histogram of Fig. 2 the distribution of the IR luminosities of the sources in our sample is compared with that of a much larger sample of SFR with $\mathrm{H}_{2} \mathrm{O}$ maser emission (Palagi et al. 1993). Both histograms are normalized to their respective total number of sources. The small decrease of sources in our sample at large luminosities is probably due to the clustering of bright masers in the inner region

2 A possible exception miglit be OMC 2 and KL IRC2 wlicli are separated by 13:3. During the mega burst of KL IRC2 in January 1999. in which the flux density increased by at least a factor $10^{3}$ and reaclied $\sim 2.6 \times 10^{7} \mathrm{Jy}$, we observed a similar burst in OMC 2 at the same velocity (around $8 \mathrm{~km} \mathrm{~s}^{-1}$ ) and witli a flux density of $\sim 3.6 \times 10^{+} \mathrm{Jy}$. Beam sliape mapping indicates that at this distance from the beam axis the attenuation slould be about $10^{-3}$. Consequently, a side-lobe effect of the observed intensity is expected. However, apart for tlie period of the mega-burst. all the remaining data for OMC 2 slould be unaffected by the presence of the close-by source KL IRC2, at least outside the velocity range close to the KL IRC2 peak emission. approximately for velocities less than $6 \mathrm{~km} \mathrm{~s}^{-1}$ or greater thian $10 \mathrm{~km} \mathrm{~s}^{-1}$. In fact. 1) wlien the flux of KL IRC 2 is at a level of $2-3 \times 10^{3}$ Jy tliere are OMC 2 spectra witlı no signal above the noise level of $1-2 \mathrm{Jy}, 2$ ) there are features observed in OMC 2 outside the above-mentioned velocity range wlicl1 do not matcli witll any emission in KL IRC 2 . In conclusion, the data of OMC 2 slould be used witli the caveat of possible KL IRC2 side lobe effects in the range of velocities witl1 strong KL IRC2 emission. 


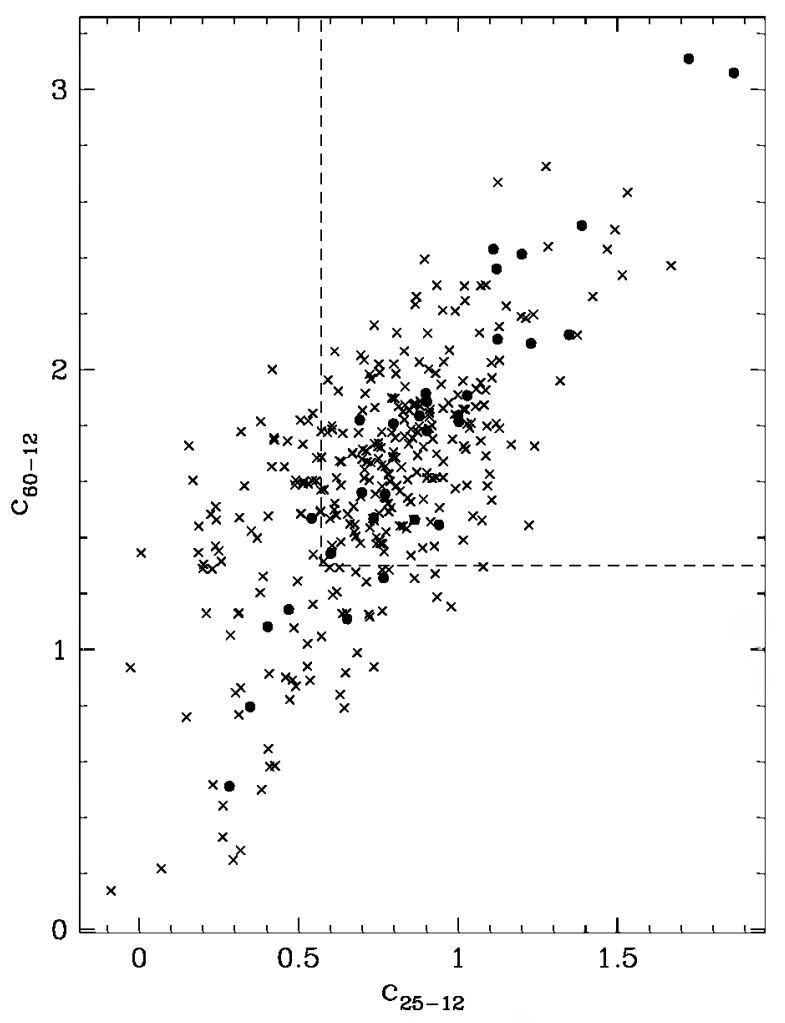

Fig. 1. Colour-colour $\left(C_{a-b}=\log \left(F_{a l} / F_{b}\right), F_{a}=\right.$ IRAS flux in band $a \mu \mathrm{m}$ ) diagram of the IRAS counterparts of the sources listed in Table 1 (•), compared to that of a larger sample of $\mathrm{H}_{2} \mathrm{O}$ masers of SFR type (a merger of Comoretto et al. 1990: Brand et al. 1994: and Valdettaro et al. 2001) ( $x$ ). The dashed box defines the location of Ultracompact Hпr regions (Wood \& Churchwell 1989).

of the Galactic plane which is less sampled according to criterion (4). The slightly larger percentage of sources in our sample at low luminosities is due to $\mathrm{H}_{2} \mathrm{O}$ masers added after the publication of the Palagi et al. (1993) sample.

Similarly, in Fig. 3 the distribution of $\mathrm{H}_{2} \mathrm{O}$ maser integrated fluxes of our sources in the last observing run (February 2007) is compared with that of a much larger sample of SFR (a merger of Comoretto et al. 1990; Brand et al. 1994; and Valdettaro et al. 2001). Both histograms are normalized to their respective total number of sources.

Figure 3 shows that in Feb. 2007 there were percentage-wise, more sources with high integrated flux densities in our present sample than there are in the comparison catalogue. Likewise there is a smaller percentage of sources with low integrated flux densities. For a large range in $L_{\mathrm{IR}}$ the general trend is for the average integrated flux density to increase with increasing $L_{\mathrm{IR}}$ (e.g., Brand et al. 2003; this trend will be further investigated in a forthcoming paper) and the difference between the distributions shown in Fig. 3 reflects the fact that we have biased our present sample towards sources of high $L_{\mathrm{IR}}$, in order to have a good chance of yielding a maser detection in a reasonable amount of integration time. On the other hand, sources of lower $L_{\mathrm{IR}}$ usually have a lower flux density, and do not have a $100 \%$ detection rate (e.g., Brand et al. 2007). This, in combination with our present sample's bias towards sources with higher $L_{\mathrm{IR}}$ can be seen as the main cause of the difference between the histograms in Fig. 3 for the lower integrated flux density bins. Furthermore, on the low flux density side, the difference between the histograms is en- hanced by the way sources from the comparison catalogue were counted (if there was more than one observation, we always selected the one in which the source was detected). Note that although in Fig. 3 we show the distribution of integrated flux densities for the observing session of Feb. 2007, any other session would have given the same statistical result. In summary and within the limitations inherent in the comparison, we confirm that our sample is fairly representative of the entire population of SFRs.

Ideally, from the observed maser variability, we would like to infer the properties of the exciting YSO and of its surroundings. However, while for $\mathrm{H}_{2} \mathrm{O}$ masers from late-type stars the association is unambiguous (i.e. there is only one star pumping the maser), for SFR-type masers, several distinct YSOs may simultaneously be present within our beam. This ambiguity can only be solved with high-resolution interferometric observations, but even if in many cases these are available, they cover at most a few epochs and there is no guarantee that the spatial/velocity situation of the maser spots remains the same over long time intervals, given the large variability observed.

\section{Observations}

The present study is based on observations carried out with the Medicina 32-1m radiotelescope ${ }^{3}$ (HPBW $\sim 1$ '.9 at $22 \mathrm{GHz}$ ). This is primarily set up to be used for VLBI measurements, and therefore the front-end is optimized for such work.

The monitoring program is ongoing but the time interval considered in this work spans 20 years, from March 1987 to February 2007, with shorter time coverage for a few sources which were added to the list at a later date. During this period, various parts of the whole system (antenna, receiver, altocorrelator) were improved, increasing the sensitivity and spectral resolution of the observations. A detailed description of the system and its improvements can be found in Comoretto et al. (1990) and in Brand et al. (1994), respectively. In 1989 realigning of the antenna surface resulted in an improvement of the efficiency at $22 \mathrm{GHz}$ from 17 to $38 \%$. The original backend was a 512-channel digital autocorrelator; in 1991 the number of channels was increased to 1024. In the same year a HEMT amplifier replaced the GaAs FET front-end, reducing the system temperature. In 1991 an active sub-reflector control increased the gain at low and high elevations.

The available bandwidth varied between $3.125 \mathrm{MHz}$ and $25 \mathrm{MHz}$ (at $22 \mathrm{GHz}$ this corresponds to a velocity resolution between $0.041 \mathrm{~km} \mathrm{~s}^{-1}$ and $0.658 \mathrm{~km} \mathrm{~s}^{-1}$ ). In February 1997, the VLA-1 chip correlator was replaced by a new one based on the NFRA correlator chips and boards (Bos 1991). The new autocorrelator has 2048 delay channels and $160 \mathrm{MHz}$ maximum bandwidth in its standard configuration. In order to maintain compatibility with previous observations, we have continued to use only 1024 channels and a $10 \mathrm{MHz}$ bandwidth, giving a velocity resolution of $0.132 \mathrm{~km} \mathrm{~s}^{-1}$. Finally, owing to extensive maintenance works on the radiotelescope, there is a gap in the observations from April 1996 to February 1997.

The telescope pointing model is typically updated a few times per year, and is quickly checked every few weeks by observing strong maser sources (e.g., W3 OH, Orion-KL, W49 N, Sgr B2 and W51). The pointing accuracy was always better than $25^{\prime \prime}$; from about mid-2004 the rms residuals from the pointing model were about $8^{\prime \prime}-10^{\prime \prime}$.

\footnotetext{
3 The Medicina $32-\mathrm{m}$ VLBI radiotelescope is operated by INAFIstituto di Radioastronomia.
} 
Table 1. The $\mathrm{H}_{2} \mathrm{O}$ maser sample.

\begin{tabular}{|c|c|c|c|c|c|c|c|c|c|}
\hline 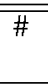 & Name & IRAS & $\alpha \overline{\alpha(\mathrm{J} 2000)}$ & $\overline{\bar{\delta}(\mathrm{J} 2000)}$ & $\begin{array}{c}V_{\mathrm{cl}} \\
\left(\mathrm{km} \mathrm{s}^{-1}\right)\end{array}$ & $\begin{array}{c}d \\
(\mathrm{kpc})\end{array}$ & $\begin{array}{c}L_{\mathrm{IR}} \\
\left(L_{\mathrm{g}}\right)\end{array}$ & Fir'st obs. & Ref. \\
\hline 1 & NGC 281 & $00494+5617$ & $00: 52: 24.7$ & $+56: 33: 50$ & -30.8 & 2.2 & $7.9 \times 10^{3}$ & 01-Apr:-1987 & 1 \\
\hline 2 & W3 OH & $02232+6138$ & $02: 27: 04.7$ & $+61: 52: 26$ & -46.8 & 2.04 & $6.6 \times 10^{+}$ & 27-Mar.-1987 & 2 \\
\hline 3 & RNO 15-FIR & $03245+3002$ & $03: 27: 38.1$ & $+30: 12: 59$ & 4.7 & 0.35 & $2.0 \times 10^{1}$ & 30-Mar.- 1987 & 3 \\
\hline 4 & AFGL 5142 & $05274+3345$ & $05: 30: 48.0$ & $+33: 47: 54$ & -4.1 & 1.8 & $5.3 \times 10^{3}$ & 14-Sep.-1995 & 4 \\
\hline 5 & Ori A-west & $05302-0537$ & $05: 32: 41.6$ & $-05: 35: 47$ & 8.9 & 0.5 & $4.0 \times 10^{1}$ & 13-Feb.- 1990 & 5.6 \\
\hline 6 & KL IRC2 & & $05: 35: 14.5$ & $-05: 22: 30$ & 9.0 & 0.45 & $8.0 \times 10^{4}$ & 30-Mar.- 1987 & 6 \\
\hline 7 & OMC 2 & $05329-0512$ & $05: 35: 27.6$ & $-05: 09: 35$ & 11.1 & 0.45 & $2.0 \times 10^{2}$ & 26-Mar.- 1987 & 6 \\
\hline 8 & Sh $2-231$ & $05358+3543$ & $05: 39: 12.9$ & $+35: 45: 51$ & -17.6 & 2.00 & $6.4 \times 10^{3}$ & 23-Mar.- 1987 & 7 \\
\hline 9 & HHL 26 & $05373+2349$ & $05: 40: 24.1$ & $+23: 50: 55$ & 2.0 & 2.4 & $1.8 \times 10^{3}$ & 09-Jul-1988 & 8 \\
\hline 10 & Sh $2-235$ & $05375+3540$ & $05: 40: 53.3$ & $+35: 41: 49$ & -57.0 & 1.8 & $1.1 \times 10^{4}$ & 31-Mar.- 1987 & 9,34 \\
\hline 11 & NGC 2071 & $05445+0020$ & $05: 47: 05.4$ & $+00: 21: 43$ & 9.5 & 0.39 & $4.1 \times 10^{2}$ & 20-Mar.- 1987 & 10 \\
\hline 12 & HH 397A & $05553+1631$ & $05: 58: 13.9$ & $+16: 32: 00$ & 5.7 & 2.0 & $3.0 \times 10^{3}$ & 20-Mar.- 1989 & 11 \\
\hline 13 & Mon R2 IRS3 & $06053-0622$ & $06: 07: 48.0$ & $-06: 22: 57$ & 10.5 & 0.8 & $3.2 \times 10^{4}$ & 03-May- 1988 & 12 \\
\hline 14 & Sh $2-252$ & $06055+2039$ & $06: 08: 35.5$ & $+20: 39: 13$ & 8.9 & 2.0 & $4.0 \times 10^{3}$ & 01-Apr:-1987 & 13 \\
\hline 15 & AFGL 5180 & $06058+2138$ & $06: 08: 53.3$ & $+21: 38: 12$ & 3.3 & 1.5 & $5.0 \times 10^{3}$ & 19-Dec.-1998 & 4 \\
\hline 16 & GGD $12-15$ & $06084-0611$ & $06: 10: 52.2$ & $-06: 11: 32$ & 11.4 & 1.0 & $7.9 \times 10^{3}$ & 17-Jun.-1987 & 14 \\
\hline 17 & Sh $2-255 / 7$ & $06099+1800$ & $06: 12: 53.6$ & $+17: 59: 27$ & 7.0 & 2.5 & $4.7 \times 10^{4}$ & 22-Mar.-1987 & 15 \\
\hline 18 & Sh $2-269$ & $06117+1350$ & $06: 14: 36.5$ & $+13: 49: 35$ & 18.2 & 5.3 & $1.2 \times 10^{5}$ & 22-Mar.- 1987 & 16 \\
\hline 19 & NGC 2264 & $06384+0932$ & $06: 41: 10.1$ & $+09: 29: 22$ & 9.0 & 0.8 & $2.1 \times 10^{3}$ & 24-Oct.-1992 & 17 \\
\hline 20 & $\mathrm{G} 31.41+0.31$ & $18449-0115$ & $18: 47: 34.7$ & $-01: 12: 46$ & 98.0 & 7.9 & $2.6 \times 10^{5}$ & 22-Mar.- 1987 & 18 \\
\hline 21 & W43 Main3 & & $18: 47: 47.0$ & $-01: 54: 35$ & 98.7 & 6.54 & $1.5 \times 10^{6}$ & 31-Mar.- 1987 & 19 \\
\hline 22 & G32.74-0.08 & $18487-0015$ & $18: 51: 21.9$ & $-00: 12: 09$ & 38.2 & 2.6 & $5.3 \times 10^{3}$ & 12-Jun.-1987 & 20 \\
\hline 23 & $\mathrm{G} 34.26+0.15$ & $18507+0110$ & $18: 53: 18.8$ & $+01: 14: 56$ & 57.8 & 3.9 & $7.5 \times 10^{5}$ & 22-Mar.- 1987 & 20,18 \\
\hline 24 & $\mathrm{G} 35.20-0.74$ & $18556+0136$ & $18: 58: 12.6$ & $+01: 40: 37$ & 34.0 & 1.8 & $1.4 \times 10^{4}$ & 01-Apr:- 1987 & 20 \\
\hline 25 & $\mathrm{OH} 43.8-0.1$ & $19095+0930$ & $19: 11: 54.2$ & $+09: 35: 55$ & 41.0 & 2.8 & $2.7 \times 10^{4}$ & 22-Mar.- 1987 & 21 \\
\hline 26 & $\mathrm{G} 45.07+0.13$ & $19110+1045$ & $19: 13: 22.0$ & $+10: 50: 52$ & 58.5 & 6.0 & $4.4 \times 10^{5}$ & 01-Apl:- 1987 & 18 \\
\hline 27 & $\mathrm{G} 59.78+0.06$ & $19410+2336$ & $19: 43: 11.5$ & $+23: 43: 54$ & 22.3 & 2.2 & $1.5 \times 10^{4}$ & 01-Apr.-1987 & 22 \\
\hline 28 & ON 1 & $20081+3122$ & $20: 10: 09.1$ & $+31: 31: 37$ & 13.0 & 3.0 & $2.7 \times 10^{4}$ & 23-Mar.- 1987 & 7 \\
\hline 29 & IRAS $20126+4104$ & $20126+4104$ & $20: 14: 26.0$ & $+41: 13: 33$ & -3.6 & 1.7 & $1.0 \times 10^{4}$ & 21-Mar.-1989 & 23 \\
\hline 30 & AFGL 2591 & $20275+4001$ & $20: 29: 24.9$ & $+40: 11: 20$ & -5.7 & 1.0 & $2.0 \times 10^{4}$ & 31-Mar-- 1987 & 24 \\
\hline 31 & W75-N & & $20: 38: 36.4$ & $+42: 37: 35$ & 10.0 & 2.0 & $1.4 \times 10^{5}$ & 22-Mar.-1987 & 25 \\
\hline 32 & $\mathrm{Sh} 2-128\left(\mathrm{H}_{2} \mathrm{O}\right)$ & $21306+5540$ & $21: 32: 11.4$ & $+55: 53: 55$ & -71.0 & 6.5 & $8.9 \times 10^{+}$ & 26-Mar.- 1987 & 20 \\
\hline 33 & AFGL 2789 & $21381+5000$ & $21: 39: 58.2$ & $+50: 14: 22$ & -43.9 & 5.7 & $4.5 \times 10^{4}$ & 29-Jan.-1989 & 26 \\
\hline 34 & IC $1396 n$ & $21391+5802$ & $21: 40: 41.9$ & $+58: 16: 12$ & 0.0 & 0.62 & $3.2 \times 10^{2}$ & 21-Mar.- 1989 & 27 \\
\hline 35 & NGC 7129 FIRS2 & & $21: 43: 00.2$ & $+66: 03: 26$ & -10.1 & 1.0 & $4.3 \times 10^{2}$ & 26-Oct.-1991 & 20 \\
\hline 36 & Sh 2-140 IRS 1 & $22176+6303$ & $22: 19: 18.3$ & $+63: 18: 47$ & -7.1 & 0.9 & $2.6 \times 10^{4}$ & 30-Mar.- 1987 & 7 \\
\hline 37 & L1204-G & $22198+6336$ & $22: 21: 26.7$ & $+63: 51: 38$ & -10.8 & 0.9 & $5.9 \times 10^{2}$ & 04-Dec.- 1989 & 28.7 \\
\hline 38 & IRAS $22506+5944$ & $22506+5944$ & $22: 52: 36.9$ & $+60: 00: 48$ & -51.5 & 5.7 & $2.2 \times 10^{4}$ & 12-Jun.-1987 & 29 \\
\hline 39 & Cepheus A & $22543+6145$ & $22: 56: 18.1$ & $+62: 01: 46$ & -10.7 & 0.73 & $2.5 \times 10^{4}$ & 11-Feb.- 1987 & 30 \\
\hline 40 & $\mathrm{WB} 89-234 \mathrm{H}_{2} \mathrm{O}$ & $23004+5642$ & $23: 02: 31.8$ & $+56: 57: 44$ & -53.5 & 5.6 & $9.6 \times 10^{3}$ & 17-Sep.-1995 & 31 \\
\hline 41 & Sh $2-158$ & $23116+6111$ & $23: 13: 44.7$ & $+61: 28: 10$ & -56.9 & 2.5 & $2.5 \times 10^{5}$ & 12-Jun.-1987 & 32 \\
\hline 42 & IRAS $23139+5939$ & $23139+5939$ & $23: 16: 10.3$ & $+59: 55: 29$ & -44.0 & 3.5 & $1.0 \times 10^{+}$ & 12-Jun.-1987 & 33 \\
\hline 43 & IRAS $23151+5912$ & $23151+5912$ & $23: 17: 20.8$ & $+59: 28: 47$ & -54.7 & 3.5 & $3.9 \times 10^{-}$ & 12-Jun.-1987 & 33 \\
\hline
\end{tabular}

Distances from: (1) Georgelin (1975): (2) Hachisuka et al. (2006): (3) Herbig \& Jones (1983): (4) Snell et al. (1988): (5) adopted distance by Fukui et al. (1986). Meehan et al. (1998) quote $d=0.45 \mathrm{kpc}$ : (6) adopted distance. for a recent review of distance determinations to the Orion region see Jeffries (2007); (7) Palagi et al. (1993); (8) Molinari et al. (2002); (9) Evans \& Blair (1981); (10) Anthony-Twarog (1982): (11) Shepherd \& Churchwell (1996): (12) Racine (1968); (13) Lada \& Wooden (1979): (14) Racine \& van den Bergh (1970): (15) Hunter \& Massey (1990); (16) M. Honma, private communication; (17) Walker (1956); (18) Churchwell et al. (1990); (19) kinematic distance, using the rotation curve of Brand \& Blitz (1993): (20) Valdettaro et al. (2002); (21) Honma et al. (2005); (22) Y. Xu, private communication; (23) Wilking et al. (1989); (24) van der Tak et al. (1999): (25) Hunter et al. (1994): (26) Clarke et al. (2006): (27) de Zeeuw et al. (1999): (28) Crampton \& Fisher (1974): (29) Molinari et al. (1996): (30) Blaauw et al. (1959): (31) Brand \& Wouterloot (1998); (32) L. Moscadelli, private communication: (33) Wouterloot \& Walmsley (1986): (34) Armandroff \& Herbst (1981).

Observations were taken in position-switching mode, with both ON and OFF scans of 5 min duration. The OFF position was taken $1.25^{\circ} \mathrm{E}$ of the source position to rescan the same path as the ON scan. Typically, one ON/OFF pair was taken, though for weak sources this was repeated several times. The typical $1 \sigma$ noise-level in the spectra is $\sim 1.5 \mathrm{Jy}$.

\subsection{Calibration}

The calibration of the intensities and the velocities is one of the main concerns for variability studies.

The antenna gain as a function of elevation is determined by daily observations of the continuum source DR 21 (for which we assume a flux density of $16.4 \mathrm{Jy}$ after scaling the value of $17.04 \mathrm{Jy}$ given by Ott et al. 1994 for the ratio of the source size 


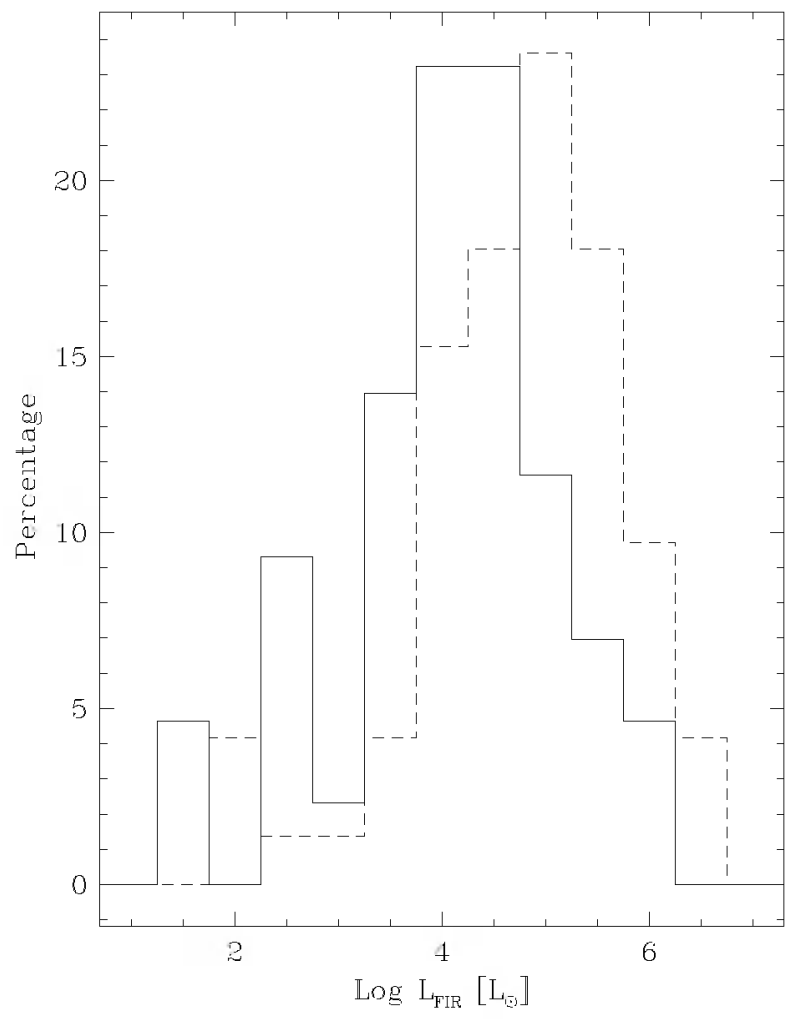

Fig. 2. The distribution of IR luminosity of the SFRs in our sample (solid line) and that of a larger sample of SFRs (Palagi et al. 1993) (dashed line). Both histograms are normalized to the total number of sources.

to the Medicina beam ${ }^{4}$ ) at a range of elevations. Antenna temperatures were derived from total power measurements in position switching mode. Integration time in each position is $10 \mathrm{~s}$ with $400 \mathrm{MHz}$ bandwidth. The zenith system temperature is about $120 \mathrm{~K}$ in clear weather conditions.

The daily gain curve was determined by fitting a polynomial curve to the data, which was then used to convert antenna temperature to flux density for all spectra taken that day. From the dispersion of the single measurements around the curve we find the typical calibration uncertainty to be $19 \%$. On the few days for which no separate gain curve was measured, we applied that which was closest in time, and estimated a corresponding calibration uncertainty of $7 \%$. The average overall calibration uncertainty is therefore estimated to be $\sim 20 \%$.

To reduce the effects of atmospheric attenuation variations, observations were obtained in good weather conditions and at elevations $\geq 30^{\circ}$, or around source meridian transit for low declination sources. For several sources we compared our intensities with those available in the literature and close in time with our observations, virtually always finding very good agreement within the above-quoted uncertainty (e.g., Valdettaro et al. 2002). A good agreement was also found comparing observations taken at Effelsberg in a program to study $\mathrm{H}_{2} \mathrm{O}$ masers associated with late-type stars which was run jointly by the Medicina and Effelsberg radiotelescopes.

As for the calibration of the velocity scale and its stability over time, checks were made with quasi simultaneous Effelsberg observations showing a good agreement. However, it is difficult

\footnotetext{
${ }^{4}$ In previous works and up to April 2003 the flux density of $18.8 \mathrm{Jy}$ was adopted for DR 21, following Dent (1972). In this paper, all data were recalibrated using the value given by Ott et al. (1994).
}

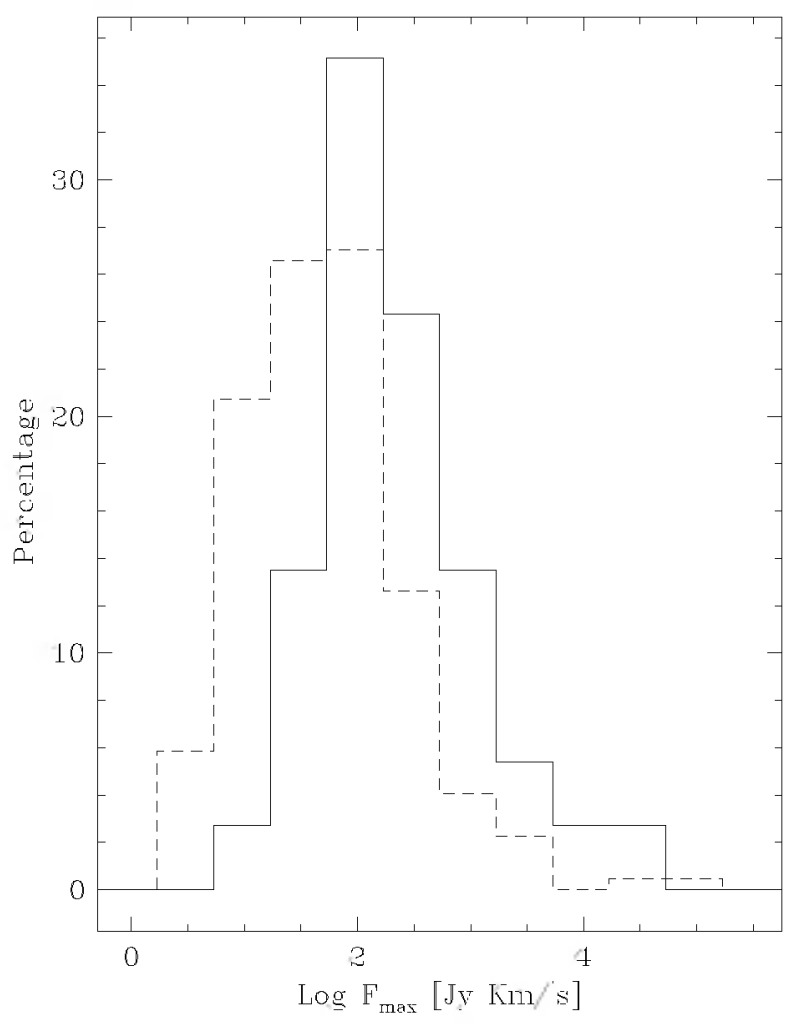

Fig. 3. The distribution of the integrated fluxes of the $\mathrm{H}_{2} \mathrm{O}$ masers in our sample as measured in the Feb. 2007 observing session (solid line). and that of a larger sample of SFRs (a merger of Comoretto et al. 1990: Brand et al. 1994. and Valdettaro et al. 2001) (dashed line).

to quote a definite uncertainty considering the variable nature of the sources and the not simultaneous observations. To make sure that the velocity changes that will be reported are real effects and not due to instrumental changes (e.g., local oscillator drifts in our system) and to evaluate the uncertainty, we performed internal consistency checks within the sources of our sample. The source G32.74-0.08 is characterized by a single, narrow and intense velocity component. The observed velocity of the peak of the emission displays a maximum deviation from a linear fit over the entire period of $<0.1 \mathrm{~km} \mathrm{~s}^{-1}$, a value that will be used as maximum possible uncertainty on the velocity throughout our patrol.

\section{The database}

For the purposes of the present study, the standard reduction procedures were applied to the observed spectra before including them in the database:

(a) the edges of the available band were deleted to limit the spectra to the flatter part of the band. This means that the velocity coverage shown may be smaller (generally by $20-30 \%$ ) than the nominal bandwidth of the autocorrelator;

(b) the spectra taken within 10 days were averaged, unless a significant difference was found;

(c) a polynomial fit to the channels free of line emission was removed from the spectra;

(d) rebinning to $0.3 \mathrm{~km} \mathrm{~s}^{-1}$ was applied. 
The whole set of Medicina 32-m observations is archived in CLASS $^{5}$ format. The raw data can be accessed through contact with the authors.

The amount of information present in the database is so large that a concise way of presentation is essential for a rapid visual appreciation of the properties of maser variability. Below we describe our selection. All plots and figures presented can be found in the appendix.

\subsection{The spectra}

The most obvious presentation is a time sequence of spectra. These are arranged in a compact form as page-plots in which each page contains 18 spectra, with time running from top to bottom and from left to right. In each spectrum, the date of observation is reported in the top left corner, and the number of days elapsed since the first observation of the source is shown in the top right corner. The velocity scale is the same for all spectra of each source and is bracketed by the minimum and maximum velocities at which emission has been detected (above the $5 \sigma$ level) throughout the monitoring period. Given the large range of intensities present during our patrol, we present the spectra using an autoscaled flux density scale. These plots are convenient to view the full details of each spectrum except for those with bursts, although they have the disadvantage that the flux density scale changes from spectrum to spectrum and the visual impression of the variability is lost.

As an example we show in Fig. 4 the first page of the spectra for the source W75-N.

The following two additional presentations of the spectra can be downloaded from our WEB pages ${ }^{6}$.

(1) Fixed-linear flux density scale, where the maximum flux density is determined by the maximum value observed during our monitoring. These plots are convenient for a quick estimate of the variability, but compress the spectra too much when the source presents bursts with intensities orders of magnitude larger than the "quiescent" value.

(2) Same as in (1) but with logarithmic flux density scale. The minimum flux density is equal to the $5 \sigma$ level in each spectrum.

\subsection{The velocity-time-flux density plots}

A more concise visualization of the time variation of the maser emission can be obtained by showing the flux density versus velocity and time in the same diagram.

Since the observed dates are not distributed evenly in time, some kind of interpolation between two adjacent observations must be used. As no assumption can be made on the evolution of the $\mathrm{H}_{2} \mathrm{O}$ maser components, we chose a linear interpolation. This procedure produces an apparent increase in the lifetime of a feature when long time-intervals between two consecutive observations occur.

Two versions of these plots are presented:

(1) full plots, with velocity coverage extending to the maximum velocity covered during the observations;

${ }^{5}$ CLASS is part of the GILDAS software developed at IRAM and Observatoire de Grenoble (http://WWW.iram. fr/IRAMFR/GILDAS)

${ }^{6}$ http://www.arcetri.astro.it/ starform/

water_maser_v2.html.

http://www.ira.inaf.it/papers/masers/

water_maser_v2.html
(2) zoomed plots, with velocity coverage limited to the part of the spectrum where emission has been detected (above the $5 \sigma$ level) at least once during the patrol.

In these plots, only emission above the $5 \sigma$ level is shown. In both plots the vertical solid line indicates the velocity of the thermal molecular gas (either $\mathrm{CO}, \mathrm{CS}$ or $\mathrm{NH}_{3}$ ). In the zoomed plots the additional dashed line represents the mean velocity derived from the histogram of the rate-of-occurrence (see Sect. 4.5). The timescale on the left is in days starting from the first observation. To convert this scale to real dates one should use the date given in the first spectrum of the figure with the time sequence of spectra, e.g., Fig. 4 for W75-N or the date listed in Table 1. These plots are particularly useful to find possible velocity drifts and to separate steady components from bursts of short duration.

As an example of the velocity-time-flux density full and zoomed plots, in Figs. 5-6 we show those of the source W75-N.

\subsection{The light curve of the maser emission}

A plot of the peak flux density as a function of time is often used to study the temporal behaviour of the maser emission. However, the peak flux density is meaningful only if one narrow component is present throughout the entire observing period. It has less physical meaning for complex spectra with multiple components of comparable flux densities varying in an uncorrelated fashion. In this case, the integrated flux density can only be used with the understanding that this represents the contribution of all components and may be representative of the energy input from the YSOs. However, since each velocity component may vary independently, the relative contribution of each to the light curve is lost in this plot.

An example of the light curve of the integrated flux density for the source W75-N is shown in Fig. 7.

\subsection{The upper and lower envelopes and the mean spectrum}

Another meaningful description of the degree of maser variability is given by the comparison between the upper and lower envelopes and the mean spectra of the sources over the whole period of observation.

The upper envelope corresponds to the maximum flux density ever reached at each velocity and is obtained by finding the maximum flux density in each velocity bin. Its integral and the derived luminosity, $L_{\mathrm{H}_{2} \mathrm{O}}$ (up), represent the maximum emission that could be produced by the source if all the velocity components were to emit at their maximum level and at the same time.

The mean spectrum represents the mean flux density at each velocity, and is obtained by computing the arithmetic mean of the flux densities in each velocity bin, assigning the same weight to the individual spectra. In this average the flux density is arbitrarily set to zero if it is below the $5 \sigma$ noise level in the spectrum in question. Clearly, the steadier components dominate this spectrum.

Finally, the lower envelope identifies the components that are always active and is obtained by finding the minimum flux density in each velocity bin, again setting it to zero if it is below the $5 \sigma$ noise level in each spectrum. When the lower envelope is zero over the whole velocity interval, it indicates that no velocity component is constantly present above a $5 \sigma$ level during the monitoring period. This does not necessarily imply that the maser is quiescent at all velocities at some epoch, since the emission may occur at different velocities at different times. 


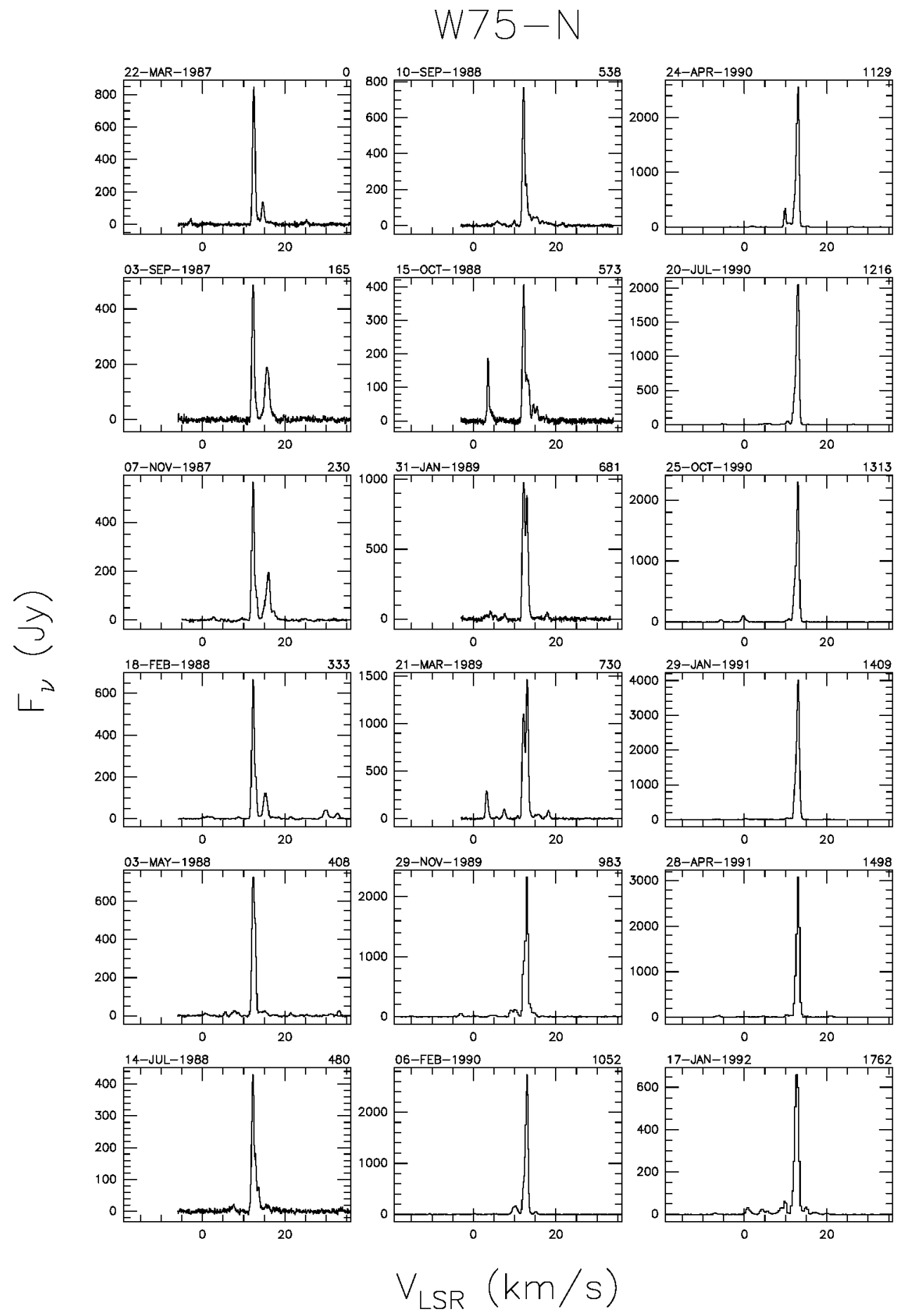

Fig. 4. The first 18 spectra of the source W75-N with an autoscaled flux density scale. Time runs from top to bottom and from left to right. In each spectrum the actual date of observation is given above the top left corner. and the number of days elapsed since the first observation of the source above the top right corner. The velocity scale is the same for all spectra.

An example of the upper and lower envelopes, and mean spectrum of the source W75-N is shown in Fig. 8.

\subsection{The rate-of-occurrence plots}

To identify the velocity components which are more frequently present in the spectra (independently of their flux density), we plot the rate-of-occurrence of maser emission above the $5 \sigma$ noise level as a function of velocity. To produce this plot we have set a counter for each channel that increases by one unit every time the flux density in the channel is greater than the $5 \sigma$ noise level of the spectrum.

An example of the rate-of-occurrence histogram of the source W75-N is shown in Fig. 9.

Two histograms can be produced:

(1) the percentage of detections with respect to the total number of observations (dotted line histogram, scale to the right);

(2) the number of detections (solid line histogram, scale to the left). 


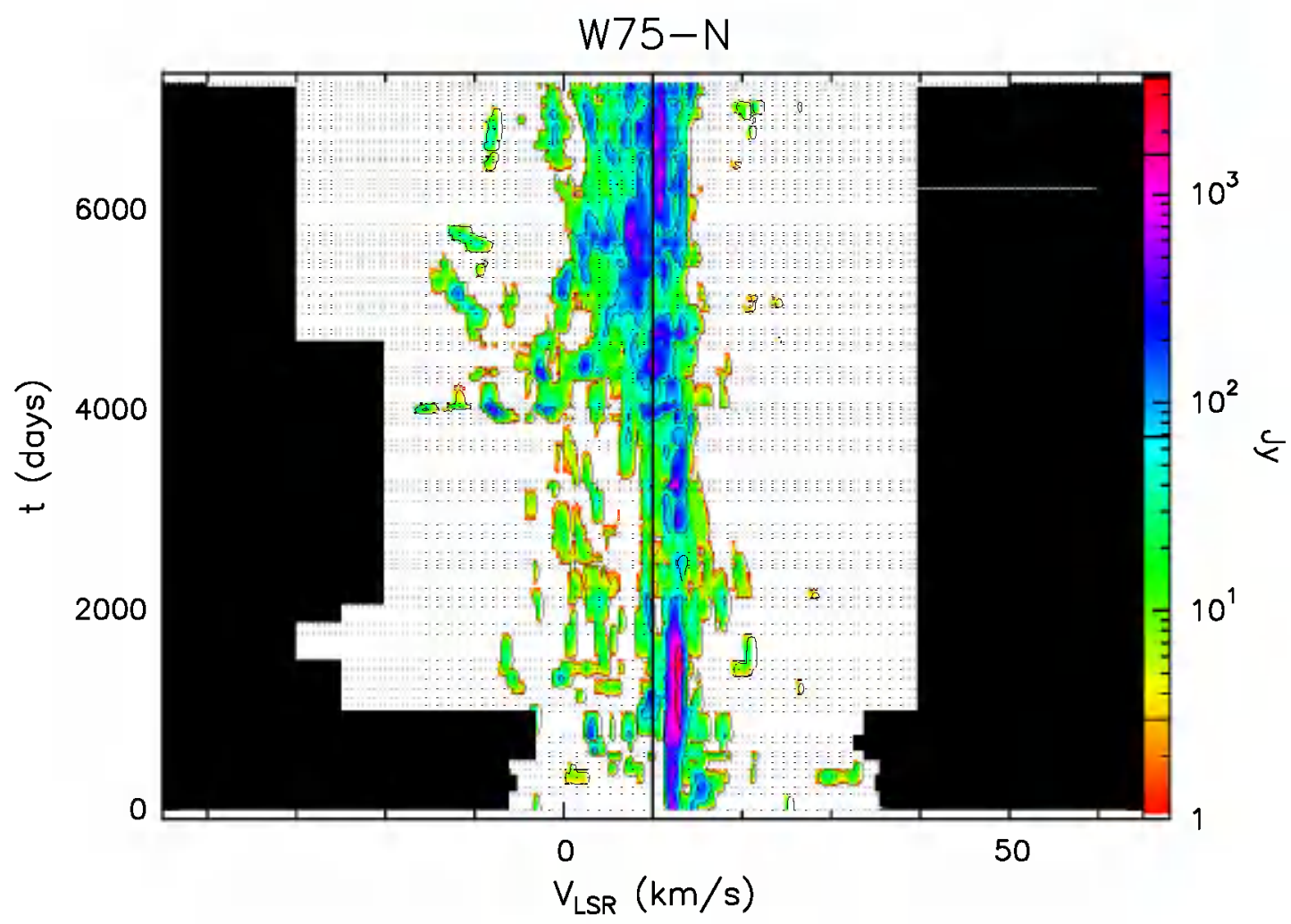

Fig. 5. The velocity-time-flux density full plot of the source W75-N. The vertical solid line indicates the velocity of the associated thermal molecular gas. The flux density scale is shown by the bar on the right. In this bar the three lines give the flux density of the drawn contours. The area in black indicates that the corresponding velocity range was not observed.

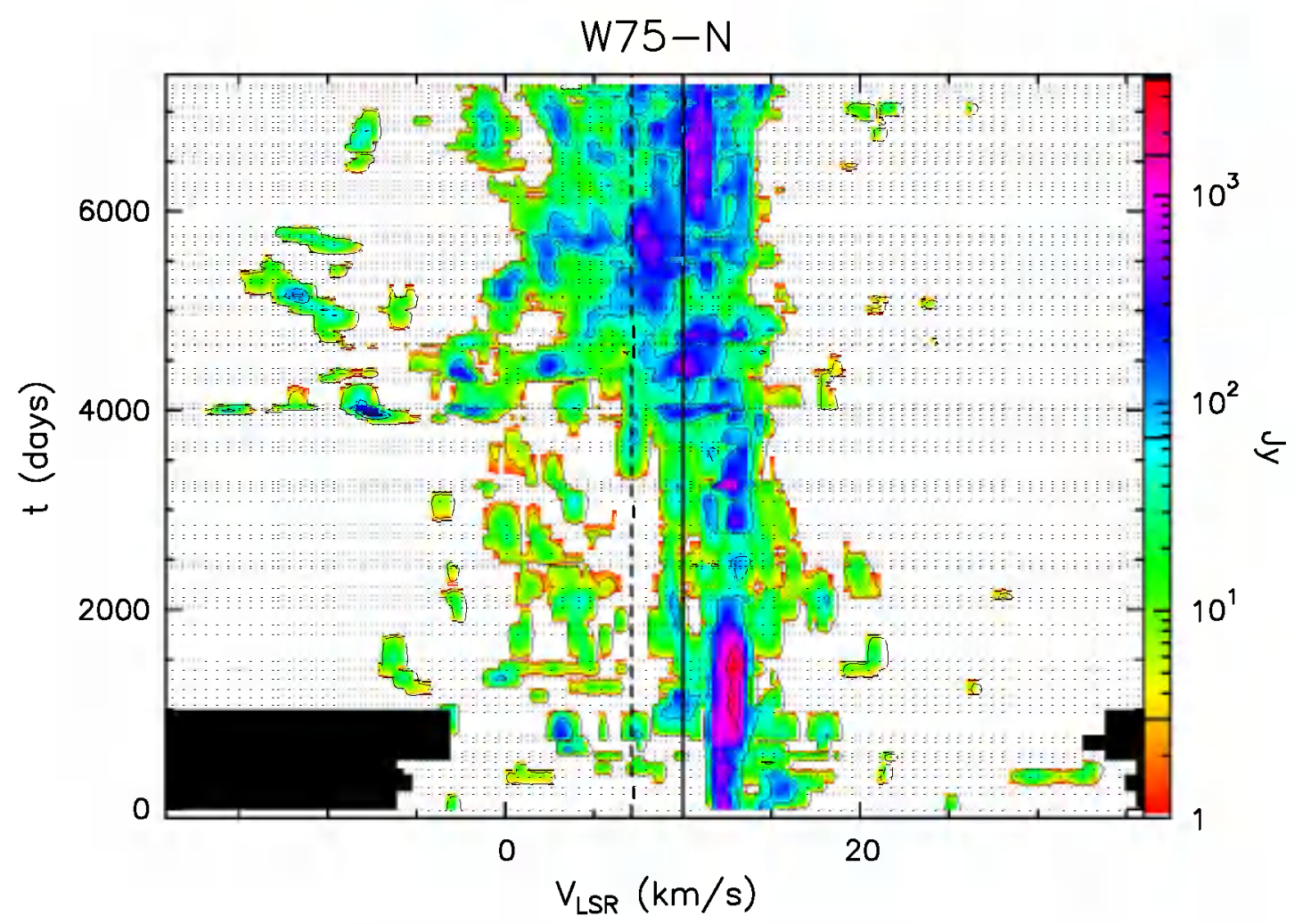

Fig. 6. The velocity-time-flux density zoomed plot of the source W75-N. The vertical solid line indicates the velocity of the associated thermal molecular gas. The vertical dashed line marks the mean velocity derived from the histogram of the rate-of-occurrence. The flux density scale is shown by the bar on the right. In this bar the three lines give the flux density of the drawn contours. The area in black indicates that the corresponding velocity range was not observed. 


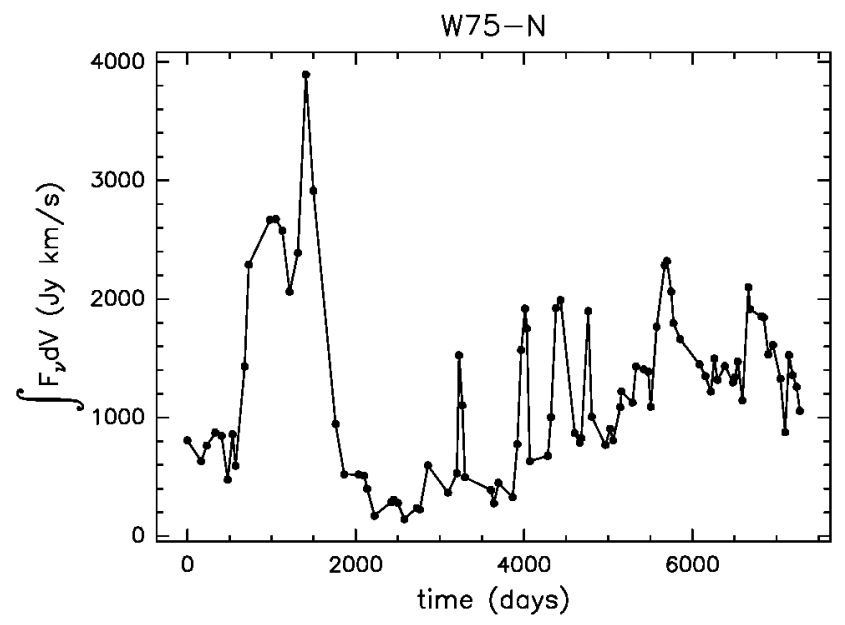

Fig. 7. The integral of the flux density over the observed velocity range as a function of time for the source W75-N.

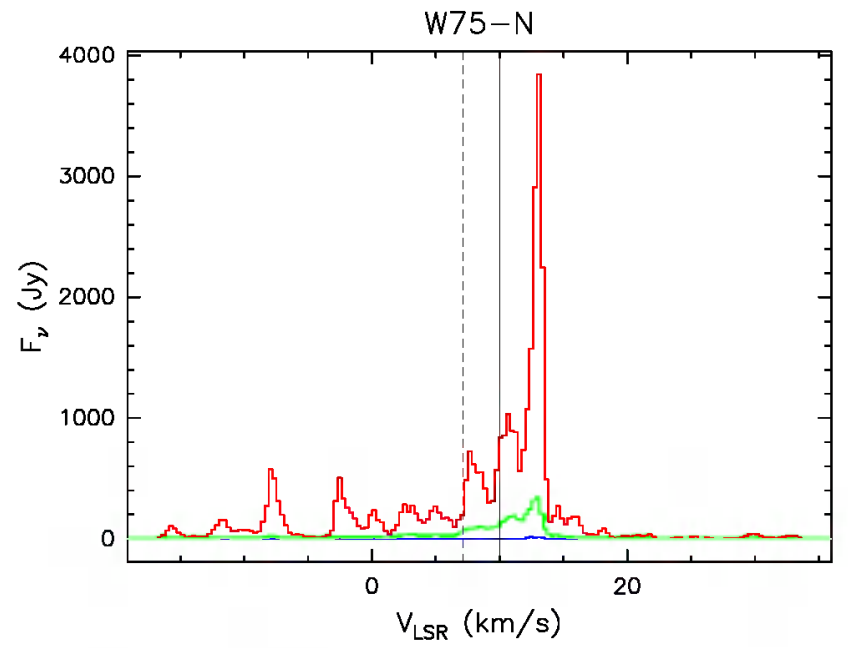

Fig. 8. The upper (red) and lower (blue) envelopes and the mean spectrum (green) of the source W75-N measured during our monitoring. The vertical solid line marks the velocity of the associated thermal molecular gas. The vertical dashed line marks the mean velocity derived from the histogram of the rate-of-occurrence.

The two histograms may differ slightly in velocity ranges where the time coverage is not equal for all channels, produced by using different bandwidths during our monitoring. From this histogram we can derive the mean velocity which is shown in Figs. 6 and 8 .

\section{Conclusions}

We present the observational results of a systematic study extending for almost 20 years of the $\mathrm{H}_{2} \mathrm{O}$ maser variability in 43 SFRs with luminosities of the associated IR sources between 20 and $1.5 \times 10^{6} L_{\odot}$. This database provides the backbone for the discussion of the main long-term properties of maser emission that will be presented in a forthcoming paper. We have identified several ways to describe graphically the main aspects of the $\mathrm{H}_{2} \mathrm{O}$ maser emission. These include:

(a) the spectra in a compressed form, with an autoscaled flux density scales (the same sets of plots, but with fixed linear and logarithmic flux density scales, are also available from our WEB pages);

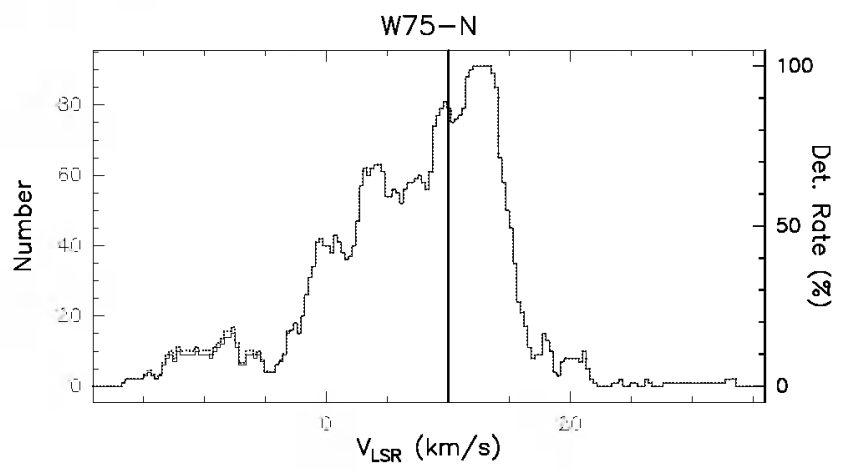

Fig. 9. The rate-of-occurrence plot for the source W75-N. The scale to the right refers to the dotted histogram, the scale to the left to the solid line histogram. The vertical solid line marks the velocity of the associated thermal molecular gas.

(b) the velocity-time-flux density plots, which conveniently describe the morphology of the variability of the maser emission. These diagrams are particularly useful for recognizing the presence of possible velocity drifts and separating steady components from bursts of short duration;

(c) the velocity-integrated flux density as a function of time. This light curve describes the overall emission of the maser components associated with a SFR;

(d) the mean spectrum;

(e) the upper and lower envelopes of the maser emission. The upper envelope represents the maximum emission that the SFR could produce if all the velocity components were present simultaneously and emitting at their maximum rate. Similarly, the lower envelope pinpoints the steady components and their lowest level (but $>5 \sigma$ ) of emission;

(f) the number of times that the maser emission is well above the noise $(>5 \sigma)$ as a function of velocity is directly gauged by the histogram of the rate-of-occurrence.

Acknowledgements. This long tern project could not have been carried out without the help and dedication of the technical staff of the Radio Group of the Arcetri Observatory and the constant assistance of the technical staff of the Medicina 32-nl Radiotelescope. This research has made use of the SIMBAD database, operated at CDS, Strasbourg. France.

\section{Appendix A: Figures}

In this appendix we give all the figures for the sources in our sample. They are organized in the same order as that of Table 1, i.e. in order of increasing source right ascension, and for each source in the same order as they are presented in the text, i.e. figures of all spectra (see Fig. 4), the two velocity-time-flux density plots (see Figs. 5-6), the light curve of the integrated flux (see Fig. 7), the upper and lower envelopes together with the mean spectrum (see Fig. 8) and the rate-of-occurrence (see Fig. 9). For instance, Figure

(A1.a) corresponds to the spectra of NGC 281 in an autoscaled intensity scale;

(Al.b) corresponds to the velocity-time-intensity full plot of NGC 281;

(A1.c) corresponds to the velocity-time-intensity zoomed plot of NGC 281;

(A1.d) corresponds to the light curve of NGC 281;

(A1.e) corresponds to the upper and lower envelopes and the mean spectrum of NGC 281 ;

(A1.f) corresponds to the rate-of-occurrence for NGC 281; 
(A2.a) corresponds to the spectra of $\mathrm{W} 3 \mathrm{OH}$ in an autoscaled intensity scale, etc.

\section{References}

Anthony-Twarog, B. J. 1982, AJ, 87, 1213

Armandroff. T. E.. \& Herbst. W. 1981, AJ, 86, 1923

Blaauw, A., Hiltner, W. A., \& Johnson. H. L. 1959, ApJ, 130, 69

Bos, A. 1991. IEEE Trans. on Instrumentation and Measurements, 40. 591

Brand, J., \& Blitz, L. 1993, A\& A, 275, 67

Brand, J., \& Wouterloot, J. G. A. 1998, A\& A, 337, 539

Brand, J., Cesaroni, R., Caselli, P., et al. 1994, A\&AS, 103, 541

Brand. J.. Cesaroni. R.. Comoretto. G.. et al. 2003. A\&A. 407. 573

Brand. J., Felli. M.. Cesaroni. R., et al. 2007. in Astrophy sical Masers and Their

Environments, ed. J. Chapman, \& W. A. Baan, Proc. IAU Symp., 242. in Press

Cesaroni, R., Palagi, F., Felli. M., et al. 1988, A\&AS, 76, 445

Cesaroni. R.. Felli. M.. \& Walmsley. C. M. 1999. A\&AS, 136, 333

Churchwell, E., Walmsley, C. M., \& Cesaroni. R. 1990, A\&AS, 83, 119

Clarke, A. J., Lumsden, S. L., Oudmaijer, R. D., et al. 2006, A\&A. 457, 183

Claussen, M. J., Wilking. B. A., Benson, P. J., et al. 1996, ApJS, 106, 111

Codella, C., \& Felli, M. 1995, A\&A, 302, 521

Codella. C.. \& Palla. F. 1995. A\&A. 302.528

Codella. C. Felli, M.. Natale. V., Palagi, F.. \& Palla. F. 1994. A\&A. 291, 261

Codella, C., Felli, M., \& Natale, V. 1996, A\&A, 311, 971

Comoretto, G., Palagi, F., Cesaroni, R., et al. 1990, A\& AS, 84, 179

Crampton, D., \& Fisher, W. A. 1974, Pub. Dom. Astrophys. Obs., 14, 283

Dent. W. A. 1972. ApJ. 177.93

de Zeeuw. P. T., Hoogerwerf, R., de Bruijne, J. H. J., Brown, A. G. A., \& Blaauw, A. $1999, \mathrm{AJ}_{,} 117,354$

Evans, N. J., \& Blair, G. N. 1981, ApJ, 246. 394

Felli, M., Palagi, F., \& Tofani, G. 1992, A\&A. 255, 293

Felli. M., Massi. F., Robberto, M., \& Cesaroni, R. 2006, A\&A, 453, 911

Forster. J. R.. \& Caswell. J. L. 1989, A\& A, 213, 339

Forster, J. R., \& Caswell, J. L. 1999, A\&AS, 137, 43

Fukui, Y., Sugitani, K., Takaba, H., et al. 1986, ApJ, 311, L85

Furuya, R. S., Kitamura, Y., Wootten, A., Claussen, M. J., \& Kawabe, R. 2003, ApJS, 144, 71 (Erratum: 2007, ApJS. 171, 349)

Georgelin. Y. 1975. Thèse de Doctorat. Université de Provence

Hachisuka, K., Brunthaler, A., Menten, K. M., et al. 2006, ApJ, 645, 337
Herbig, G. H.., \& Jones, B. F. 1983, AJ, 88, 1040

Honma. M., Bushimata. T., Choi, Y. K.. et al. 2005, PASJ, 57. 595

Hunter. D. A.. \& Massey. P. 1990. AJ. 99, 846

Hunter. T. R.. Taylor. G. B.. Felli. M.. \& Tofani. G. 1994. A\&A. 284. 215

Jeffries, R. D. 2007, MNRAS, 376, 1109

Lada, C. J., \& Wooden, D. 1979, ApJ, 232, 158

Liljeström. T., Mattila, K., Toriseva, M., \& Anttila. R. 1989. A\&AS, 79, 19

Little. L. T.. White. G. J.. \& Riley. P. W. 1977. MNRAS. 180. 639

Meehan, L. S. G., Wilking, B. A., Claussen, M. J., Mundy, L. G., \& Wootten, A. 1998, AJ, 115, 1599

Molinari, S., Brand, J., Cesaroni, R., \& Palla, F. 1996, A\&A, 308, 573

Molinari. S., Testi, L., Rodríguez, L. F., \& Zhang, Q. 2002. ApJ, 570, 758

Ott. M.. Witzel. A.. Quirrenbach. A.. et al. 1994. A\&A. 284. 331

Palagi, F., Cesaroni, R., Comoretto, G., Felli, M., \& Natale, V. 1993, A\&AS, 101,153

Palla, F., Brand, J., Comoretto, G., Felli, M., \& Cesaroni, R. 1991, A\& A, 246, 249

Palla. F.. Cesaroni. R.. Brand. J.. et al. 1993. A\&A. 280. 599

Persi. P.. Palagi. F.. \& Felli. M. 1994. A\&A. 291. 577

Racine, R. 1968, AJ, 73, 233

Racine, R., \& van den Bergh, S. 1970, Reflection Nebulae and Spiral Structure, in The Spiral Structure of our Galaxy, ed. W. Becker, \& G. I. Kontopoulos (Dordrecht: Reidel). IAU Symp.. 38. 219

Rudnitskij. G. M., Paschenko. M. I.. Lekht. E. E., et al. 2007, in Astrophysical Masers and Their Environments, ed. J. Chapman, \& W. A. Baan, Proc. IAU Symp., 242, in Press

Shepherd, D. S., \& Churchwell, E. 1996, ApJ, 472, 225

Snell. R. L.. Huang. Y.-L.. Dickman. R. L.. \& Claussen. M. J. 1988. ApJ. 325. 853

Tofani, G., Felli, M., Taylor, G. B., \& Hunter, T. R. 1995, A\&AS, 112, 299

Valdettaro, R., Palla. F., Brand, J., et al. 2001, A\&A, 368, 845

Valdettaro, R., Palla. F., Brand. J., et al. 2002. A\&A, 383, 244

van der Tak. F. F. S., van Dishoeck, E. F., Evans. N. J., Bakker. E. J., \& Blake, G. A. 1999, ApJ, 522, 991

Walker, M. F. 1956, ApJS, 2, 365

Wilking, B. A., Blackwell, J. H., Mundy, L. G., \& Howe, J. E. 1989, ApJ, 345, 257

Wood. D. O. S.. \& Churchwell. E. 1989. ApJ. 340. 265

Wouterloot, J. G. A., \& Walmsley, C. M. 1986, A\&A, 168, 237

Wouterloot, J. G. A., Fiegle, K., Brand. J., \& Winewisser, G. 1995, A\&A, 301, 236 (Erratum: 1997, A\&A, 319, 360) 


\section{NGC 281}
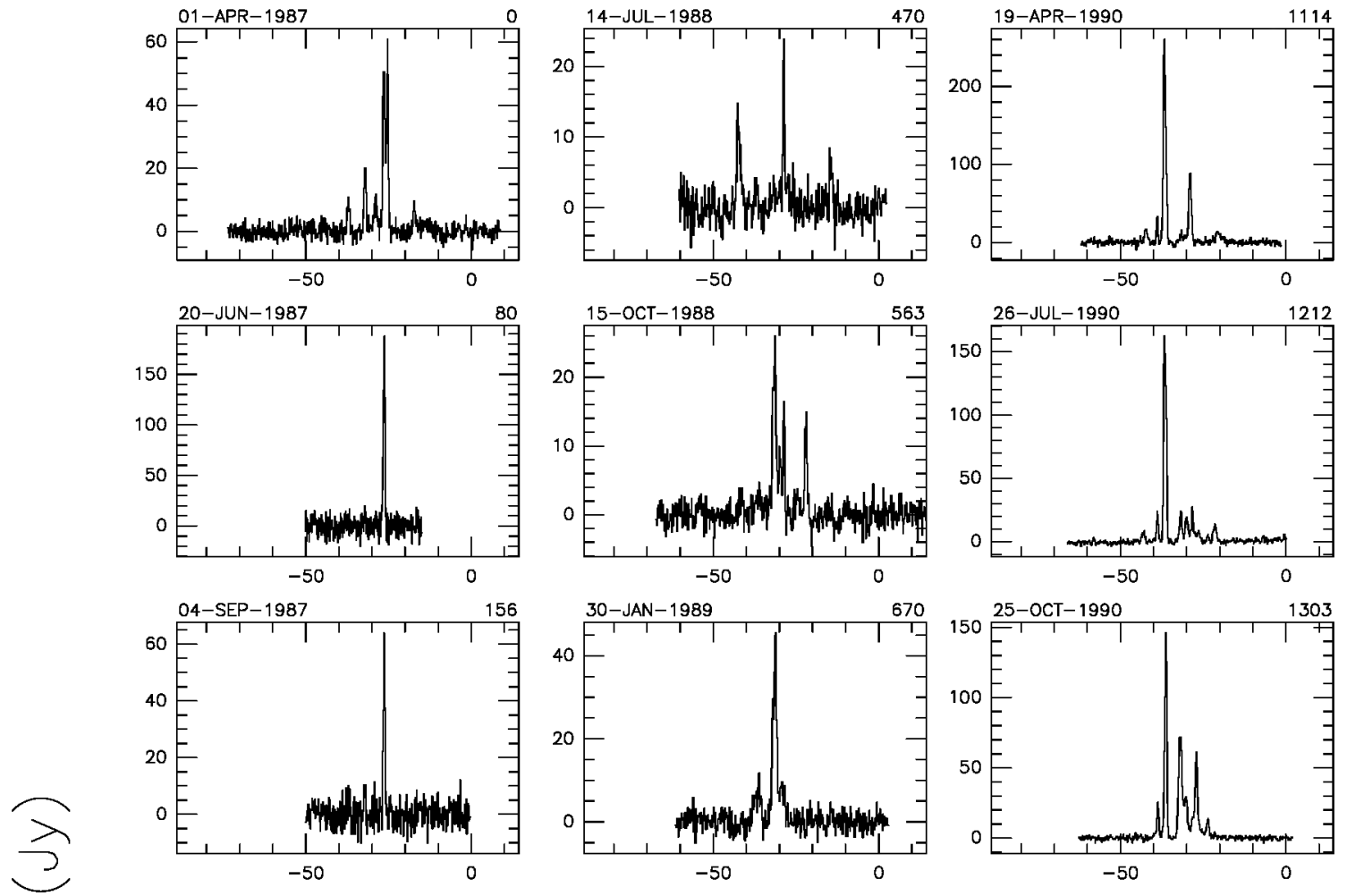

$L^{2}$
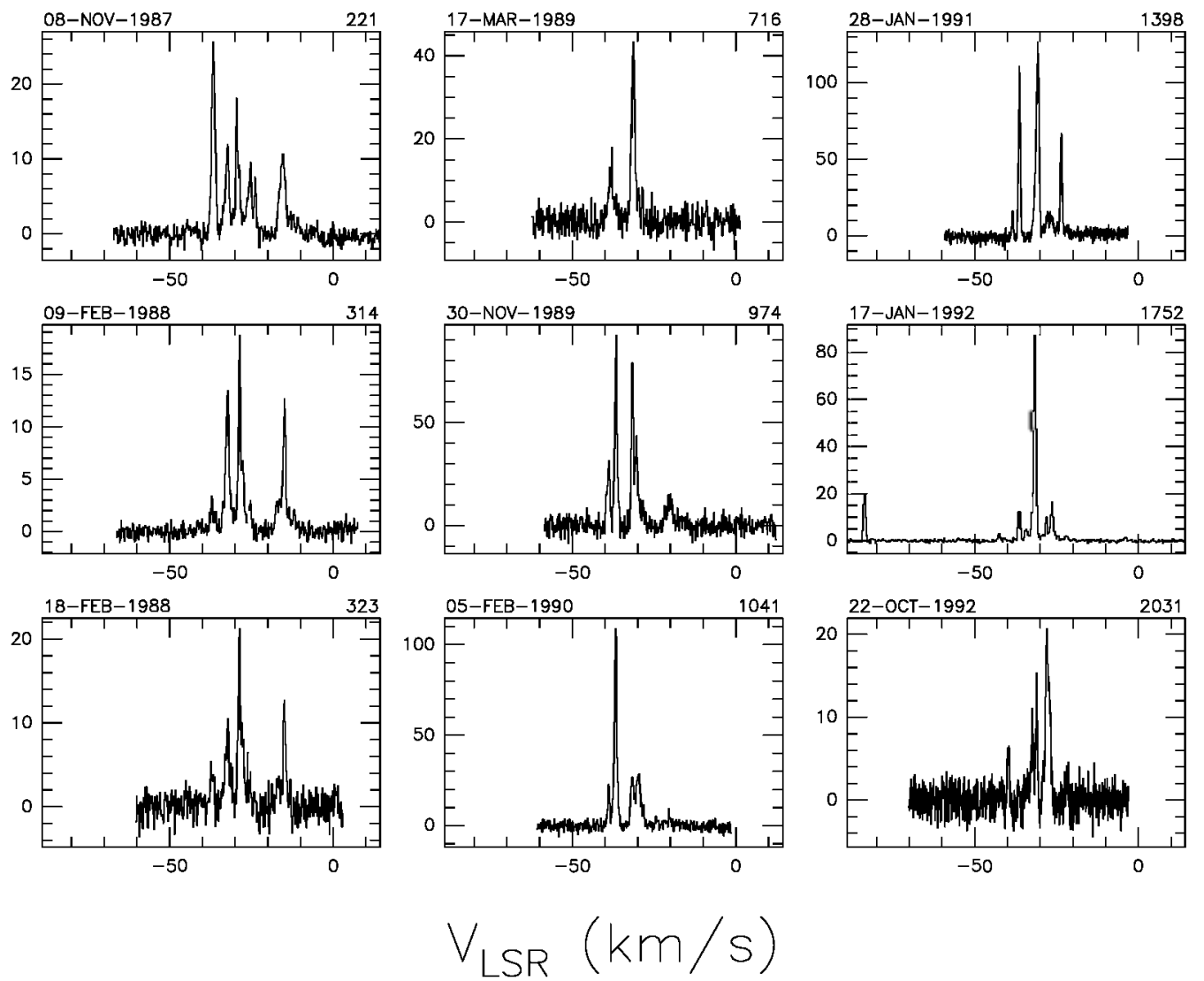

Fig. A.1. a Spectra of source NGC 281 with autoscaled flux density scale. The date of observation is shown above the top left corner of each spectrum and the number of days elapsed since the first observation is given above the top right corner. The velocity scale is the same for all spectra. 


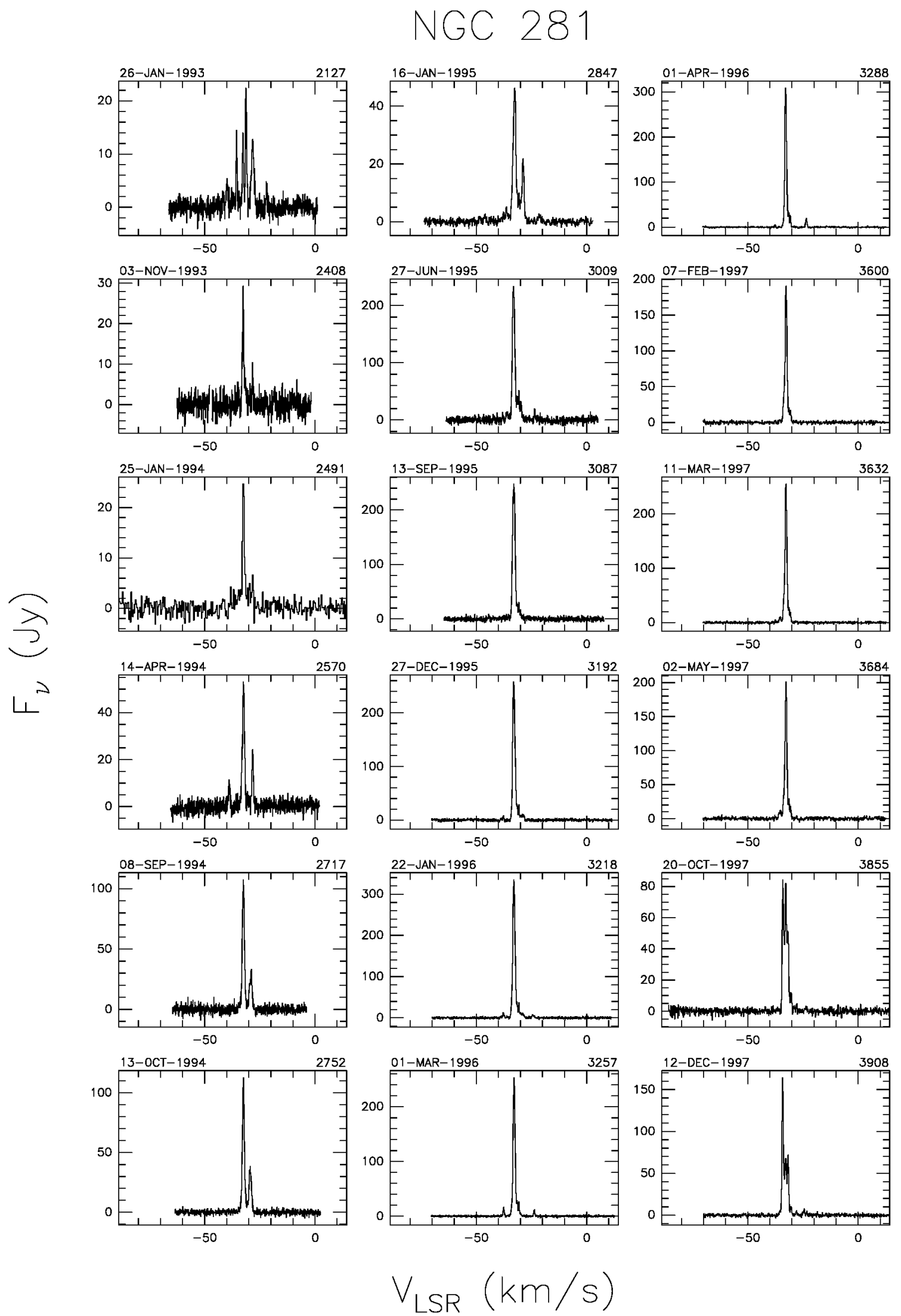

Fig. A.1. a continued. 


\section{NGC 281}
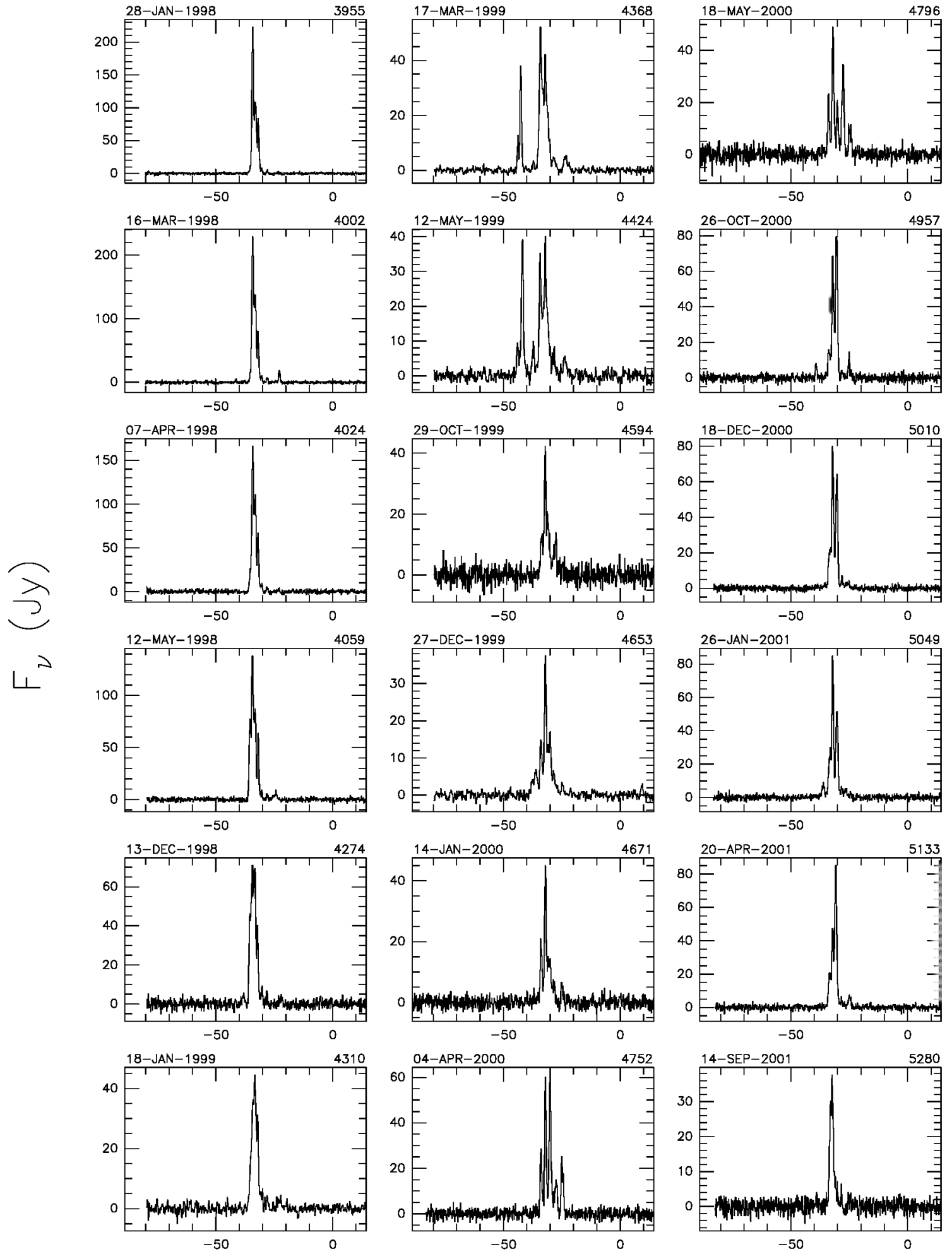

$$
V_{\text {LSR }}(\mathrm{km} / \mathrm{s})
$$

Fig. A.1. a continued. 


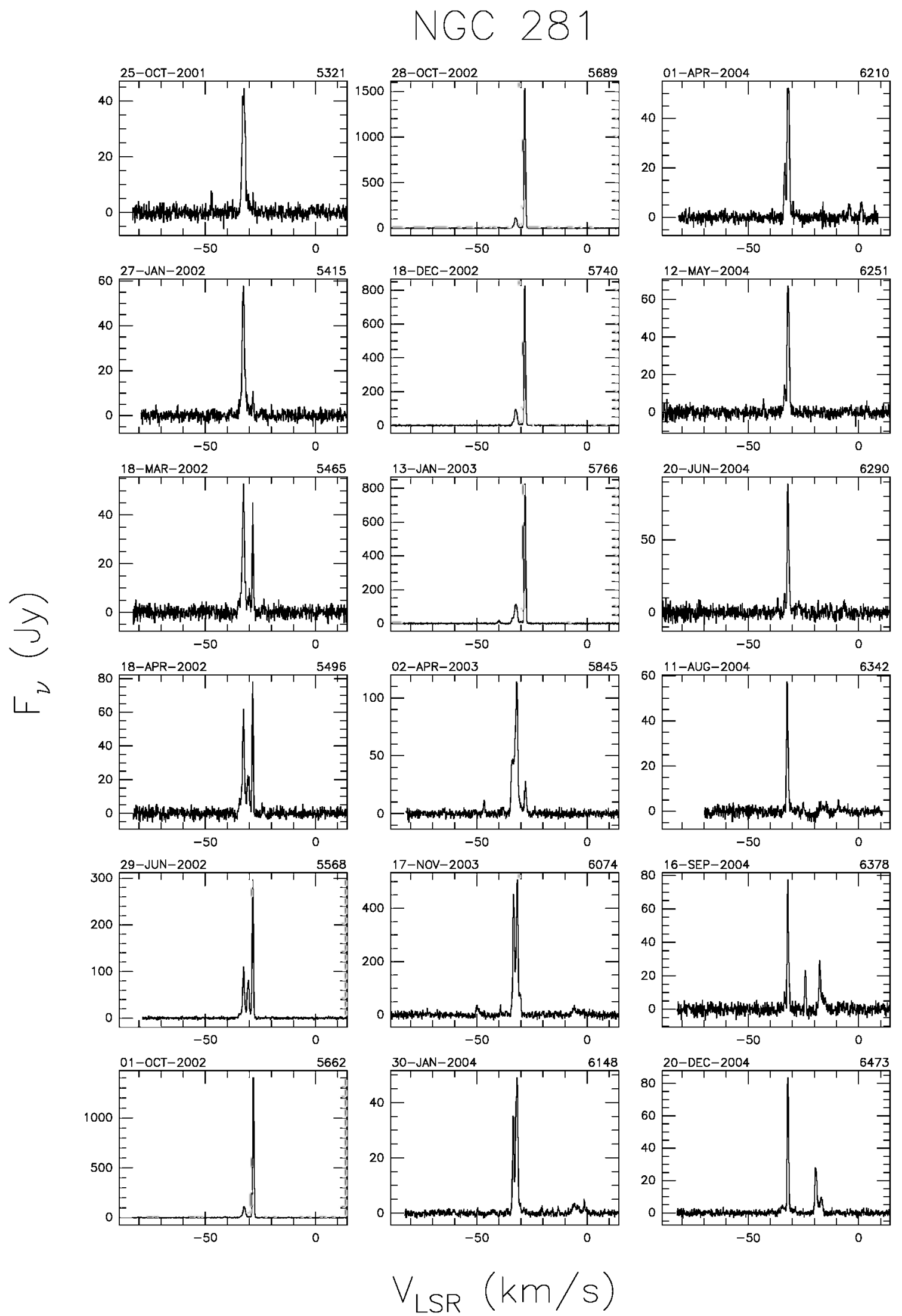

Fig. A.1. a continued. 


\section{NGC 281}
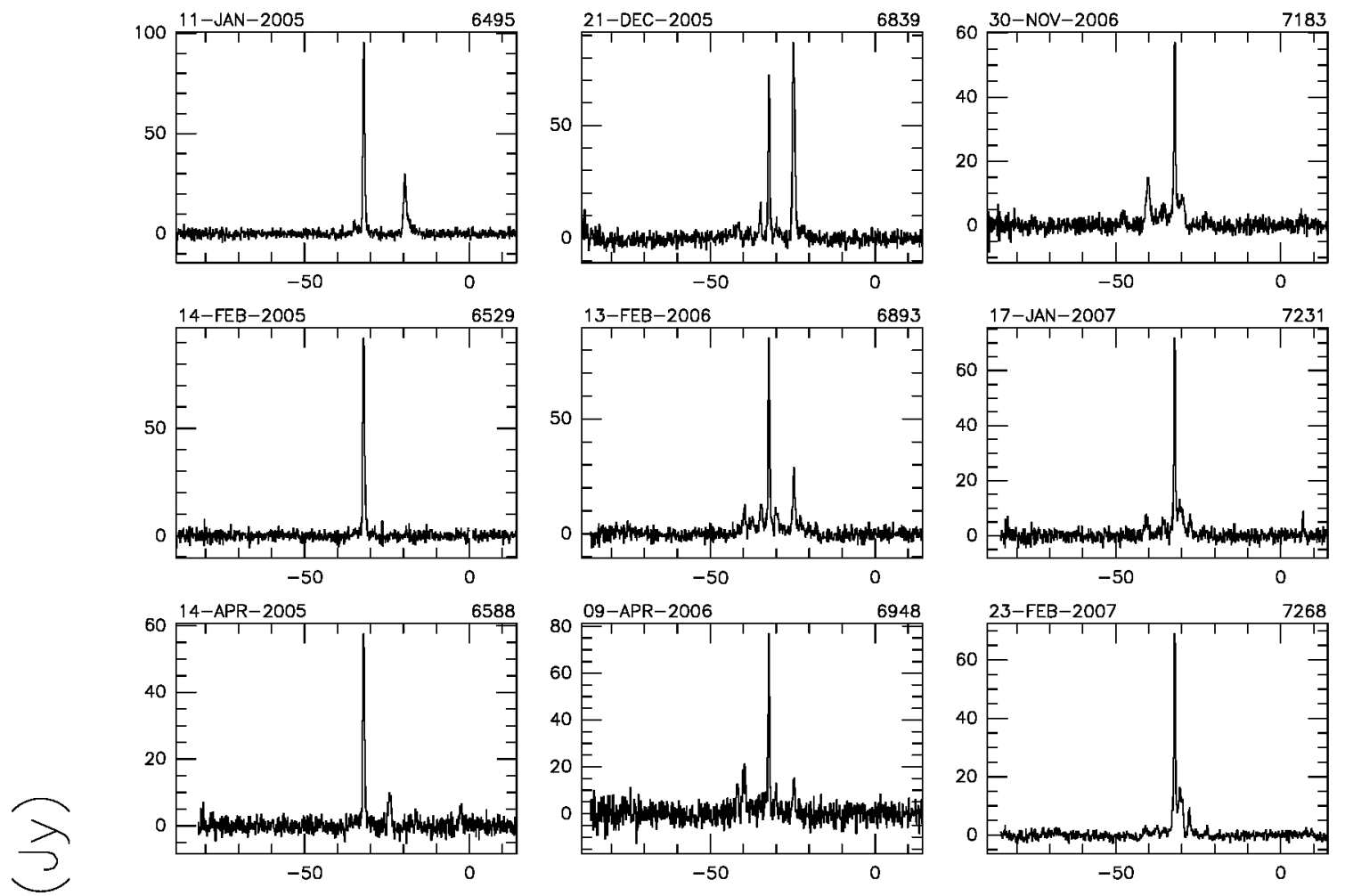

\llcorner
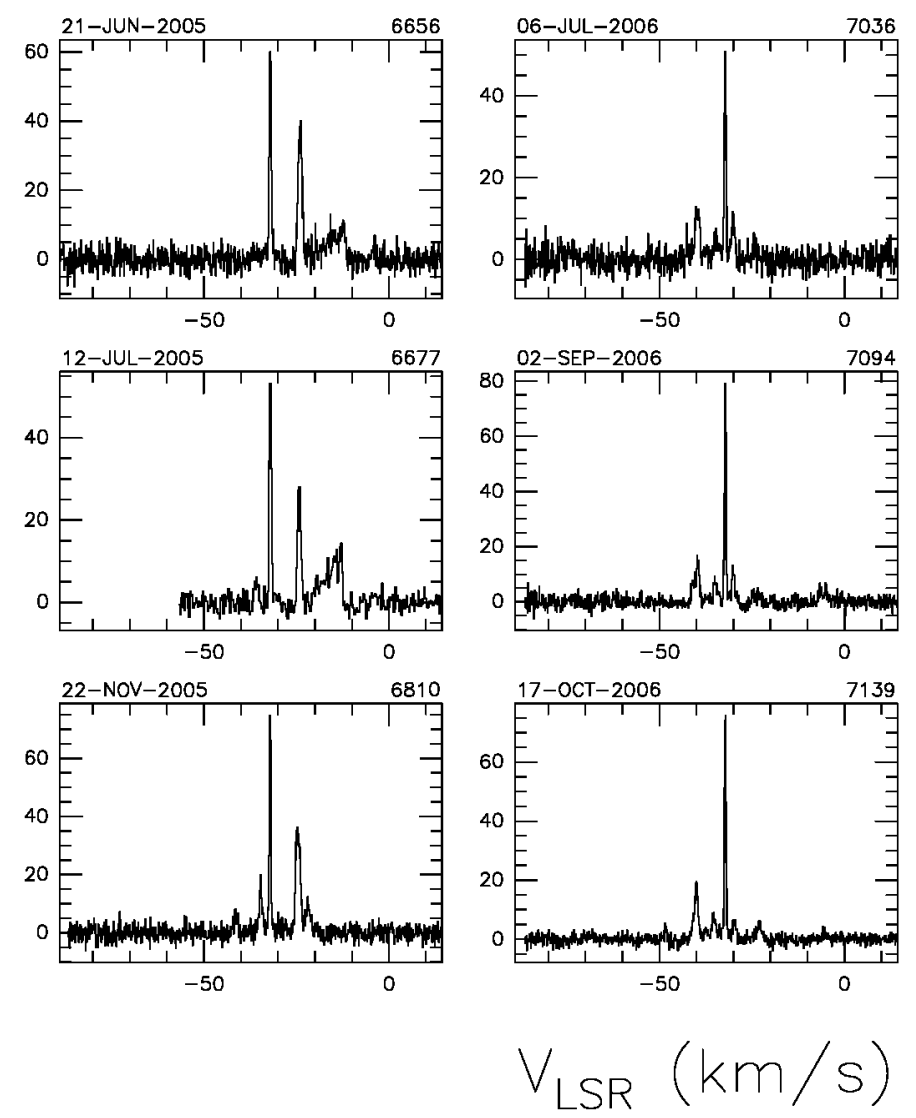

Fig. A.1. a continued. 


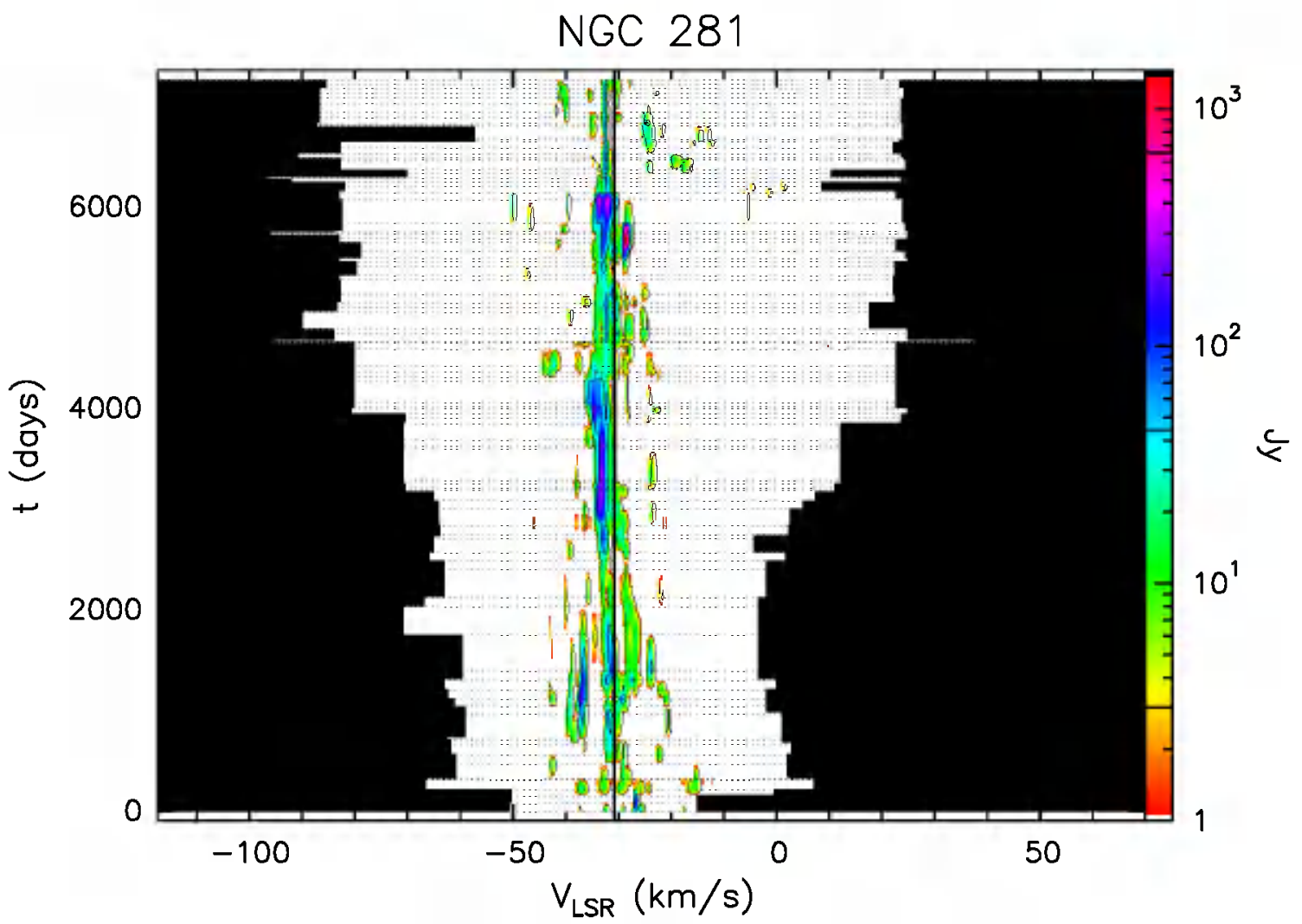

Fig. A.1. b Velocity-time-flux density full plot for source NGC 281. The vertical solid line indicates the velocity of the associated thermal molecular gas. The flux density scale is shown by the bar on the right. In this bar the three lines give the flux density of the drawn contours.

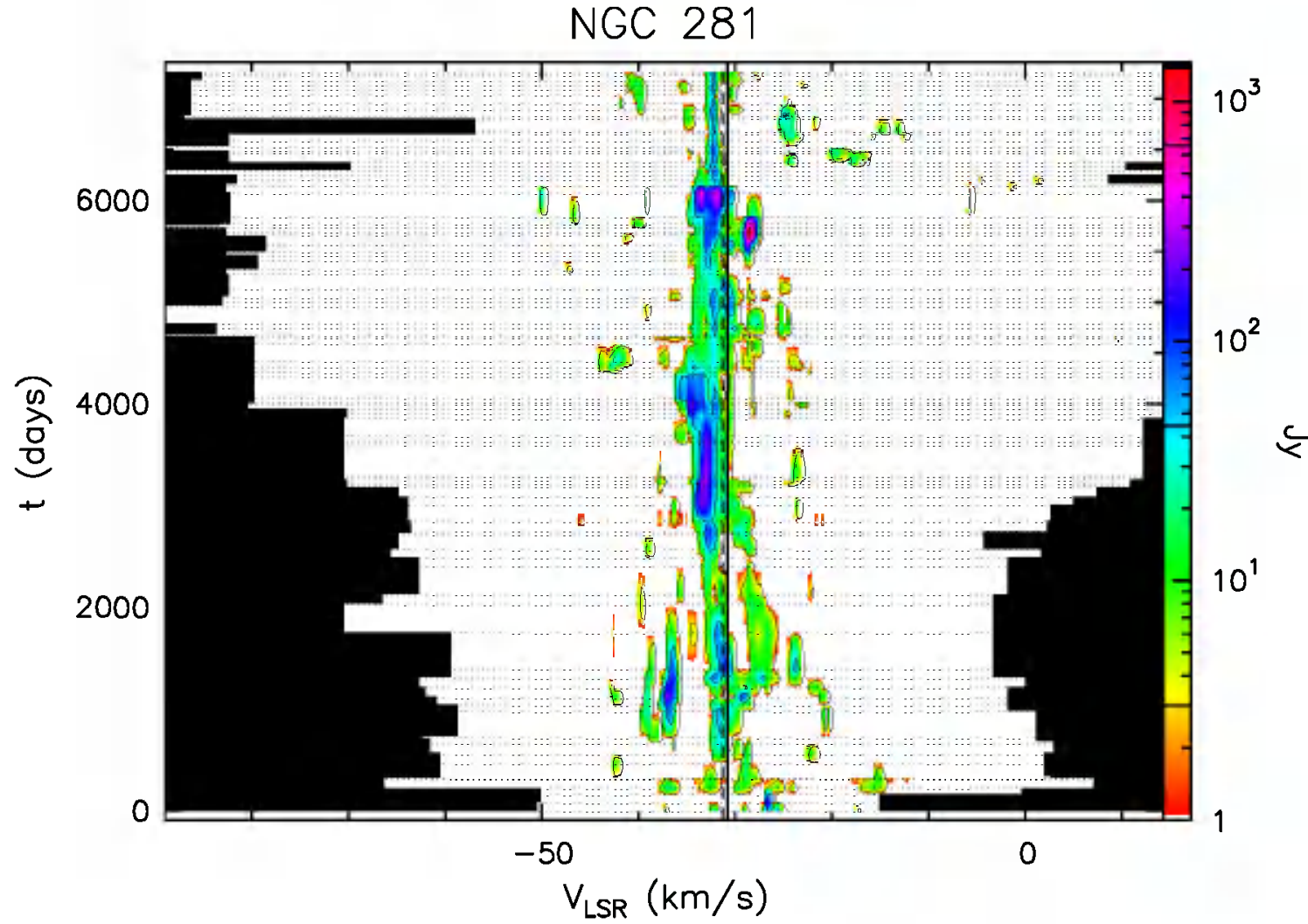

Fig. A.1. c Same as previous figure, but "zoomed" to velocity range over which emission has been detected. 


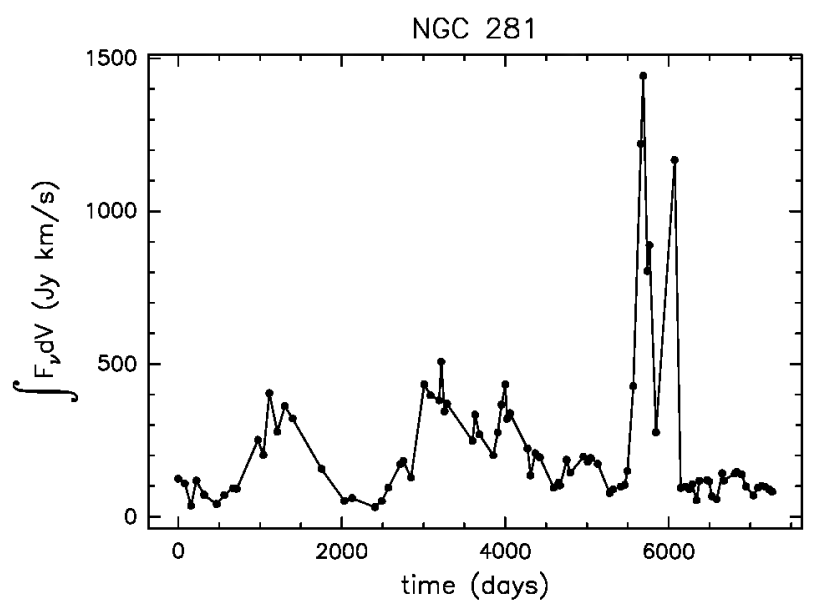

Fig. A.1. d Integral of the flux density over the observed velocity range as a function of time for source NGC 281 .

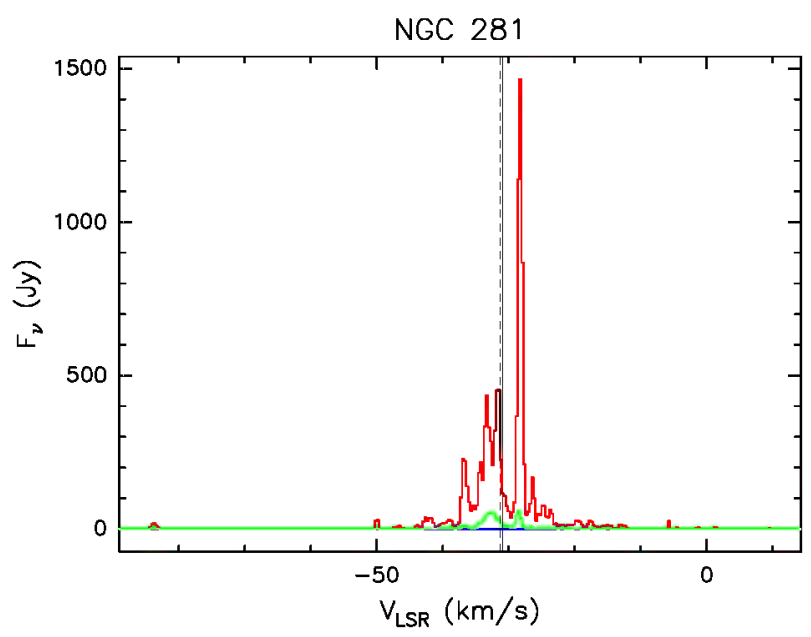

Fig. A.1. e Upper (red) and lower (blue) envelopes and mean spectrum (green) of source NGC 281 measured during our monitoring. The vertical solid line marks the velocity of the associated thermal molecular gas. The vertical dashed line marks the mean velocity derived from the histogram of the rate-of-occurrence.

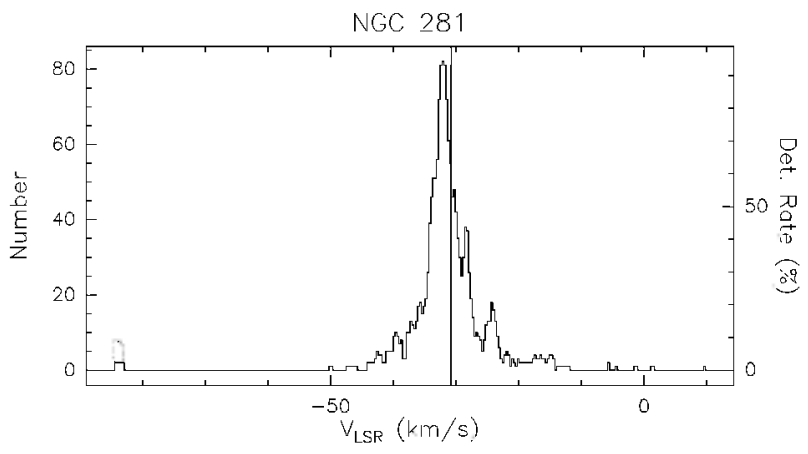

Fig. A.1.f Rate-of-occurrence plot for source NGC 281. The scale to the right refer's to the dotted histogram, the scale to the left to the solid line histogram. The vertical solid line marks the velocity of the associated thermal molecular gas. 


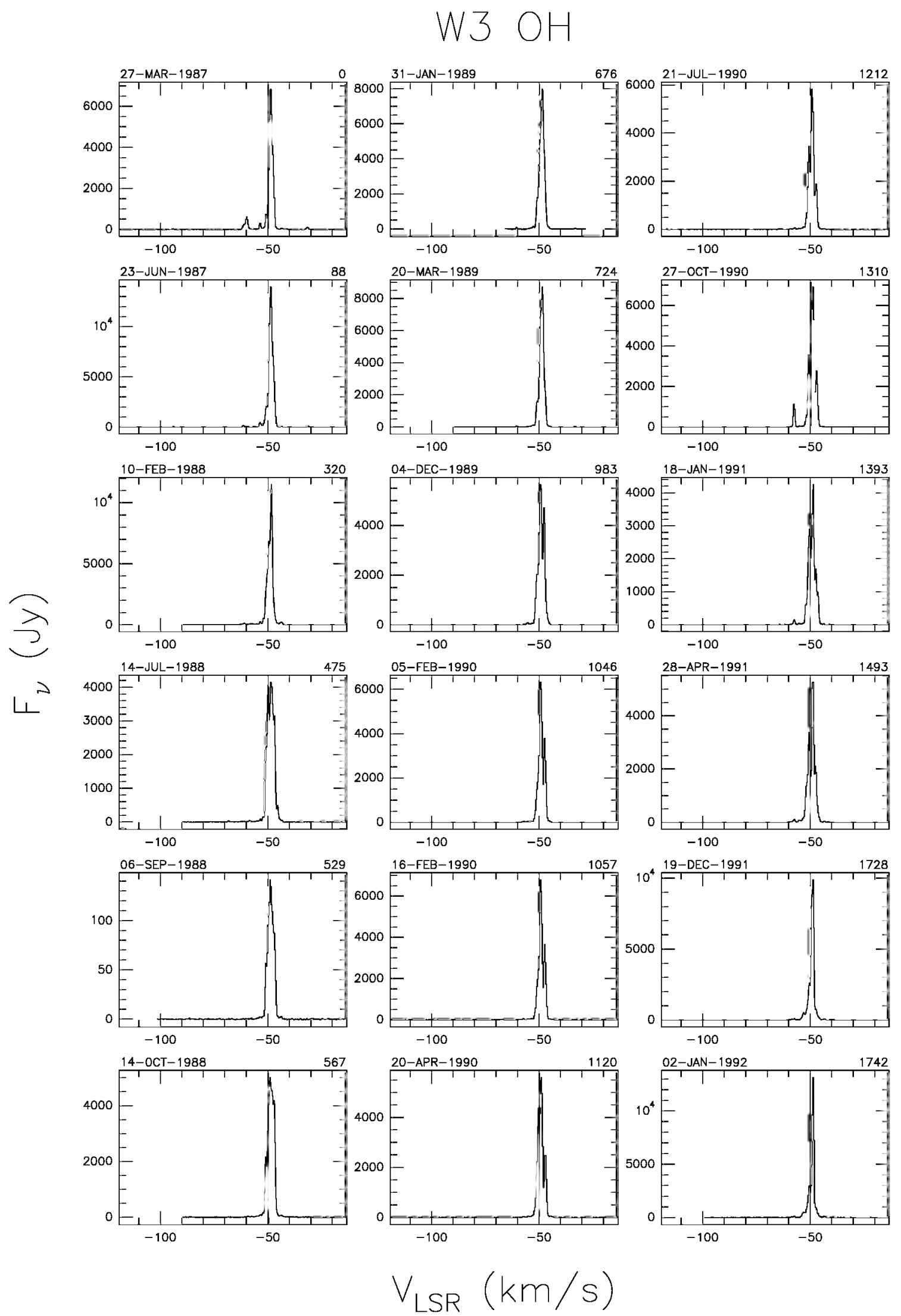

Fig. A.2. a Spectra of source W3 OH with autoscaled flux density scale. The date of observation is shown above the top left corner of each spectrum and the number of days elapsed since the first observation is given above the top right corner. The velocity scale is the same for all spectra. 


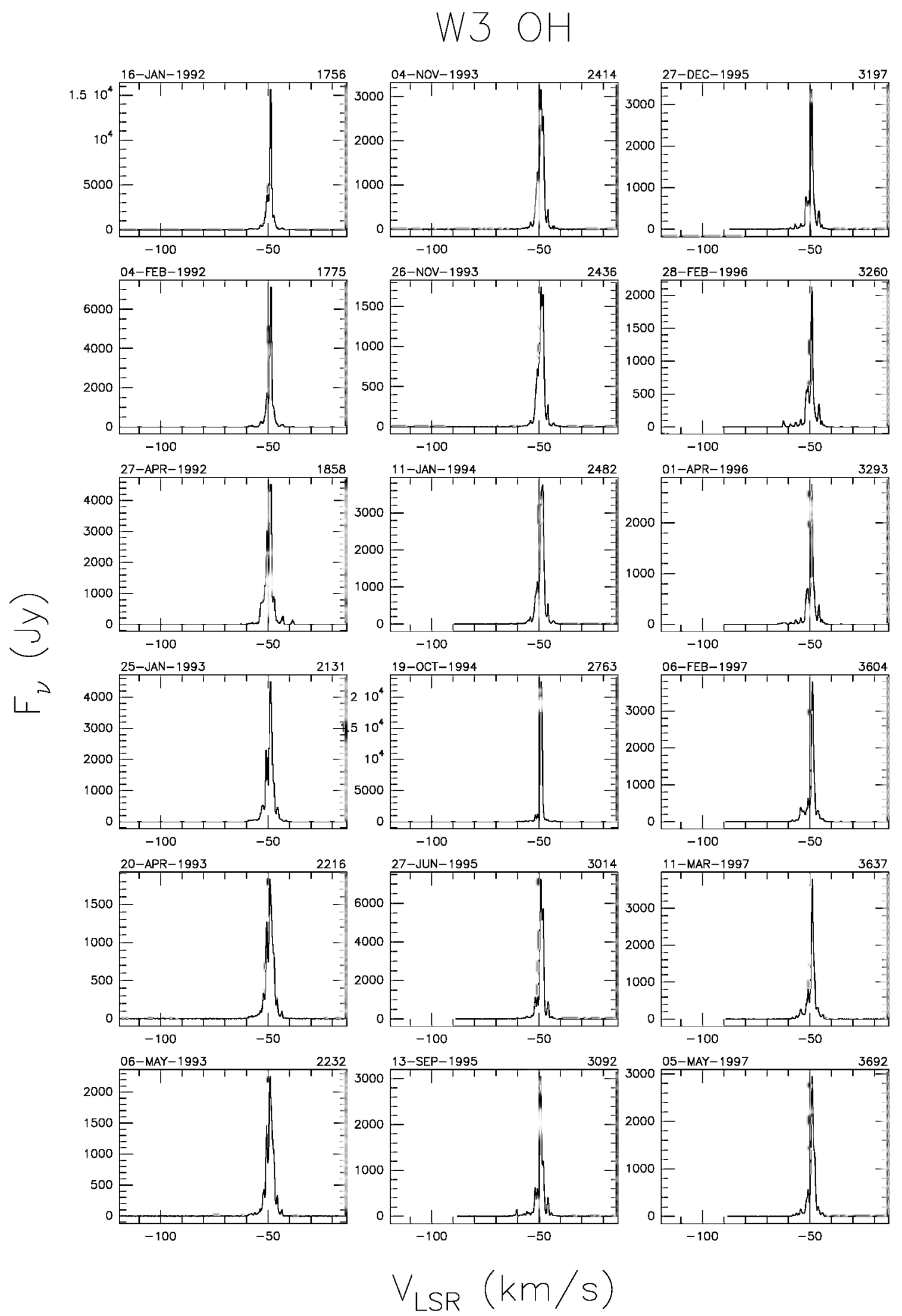

Fig. A.2. a continued. 


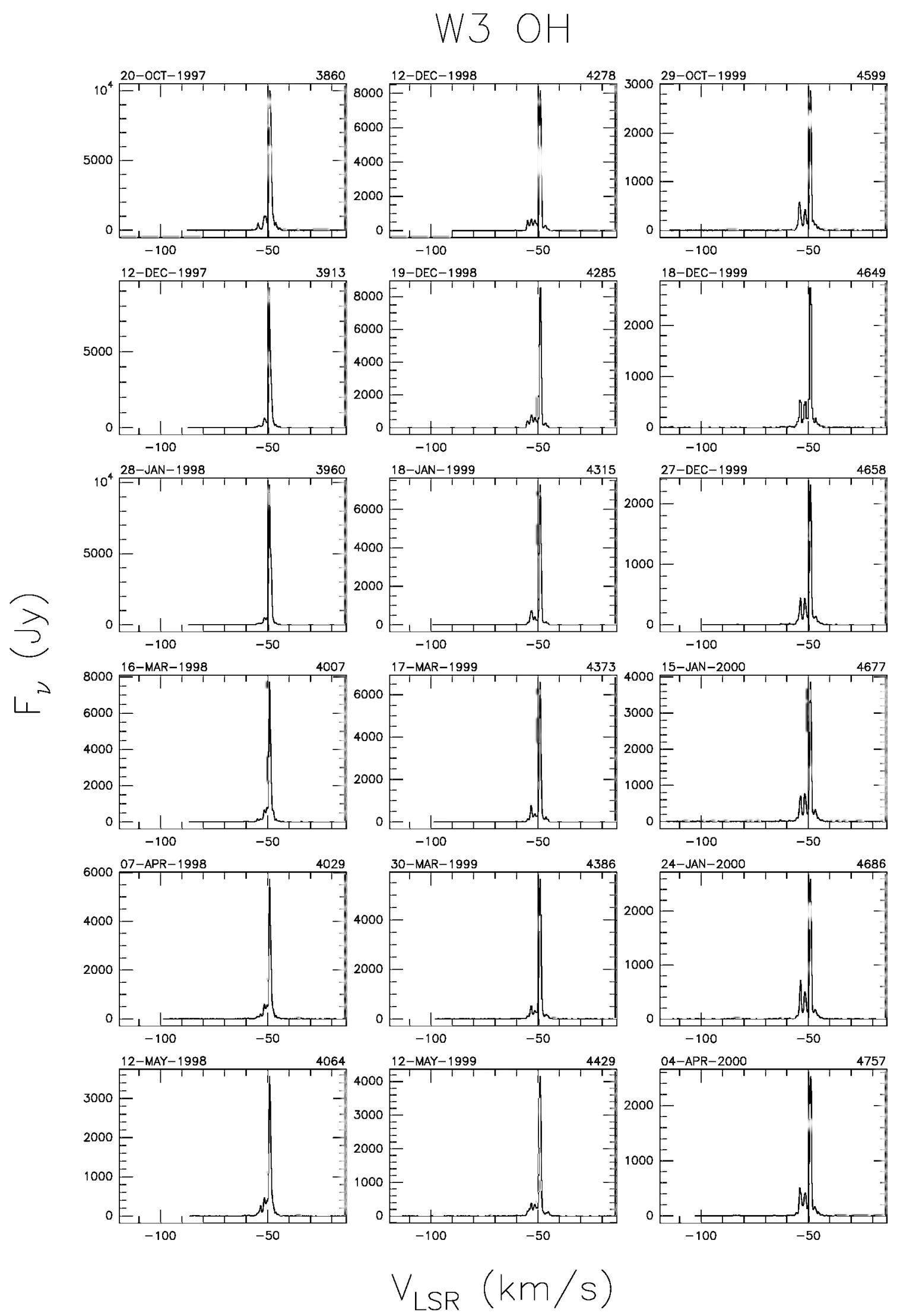

Fig. A.2. a continued. 


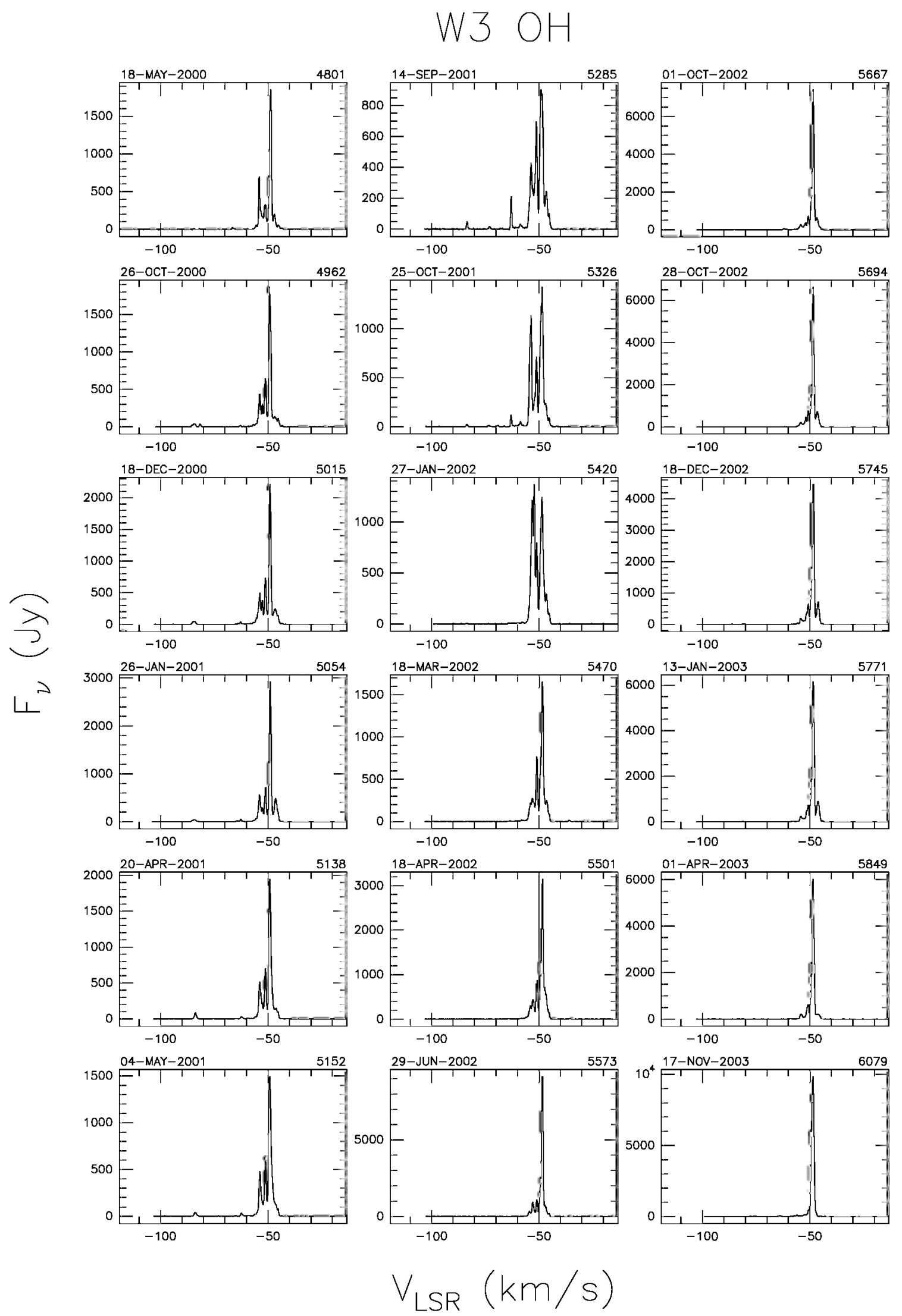

Fig. A.2. a continued. 


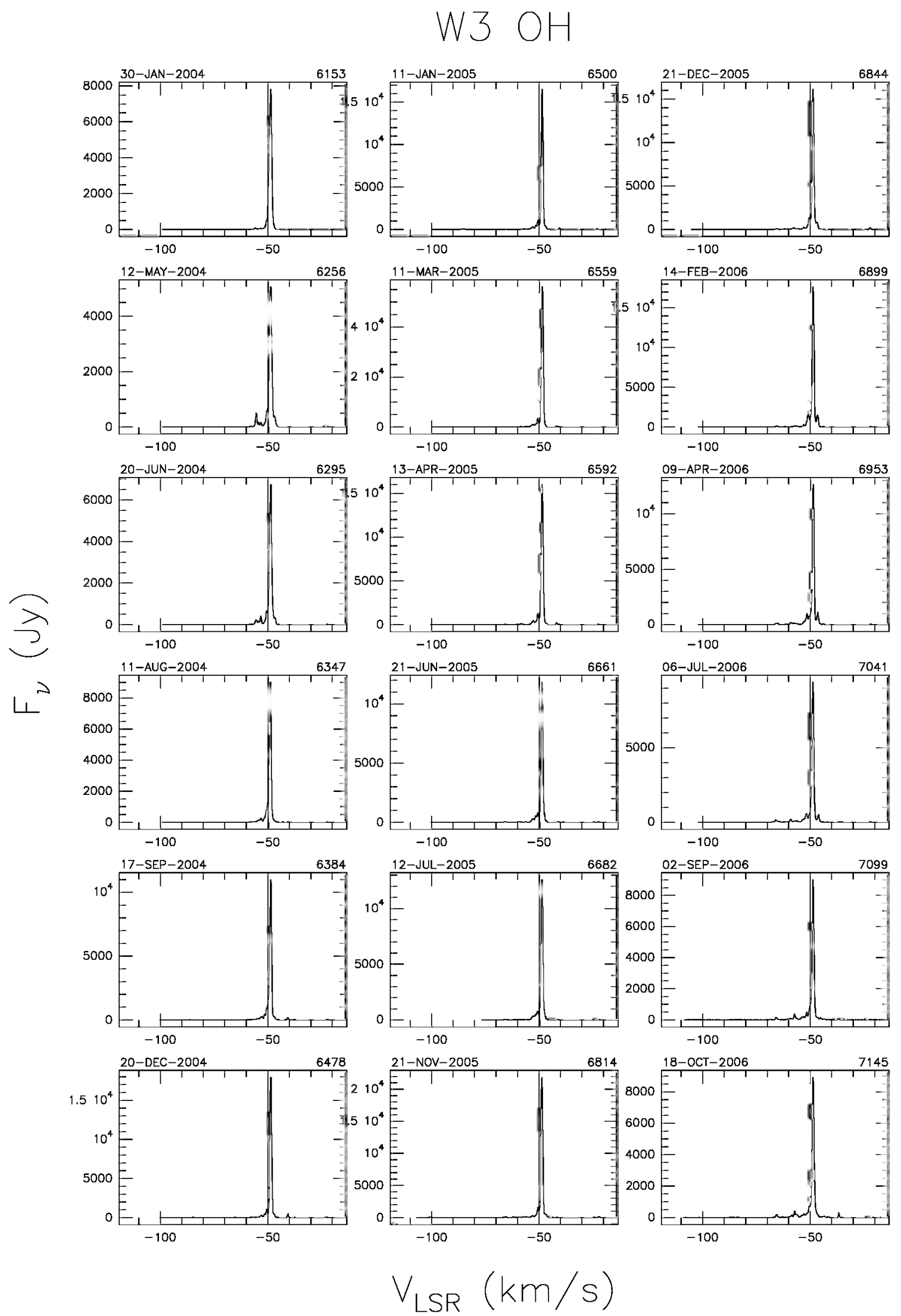

Fig. A.2. a continued. 

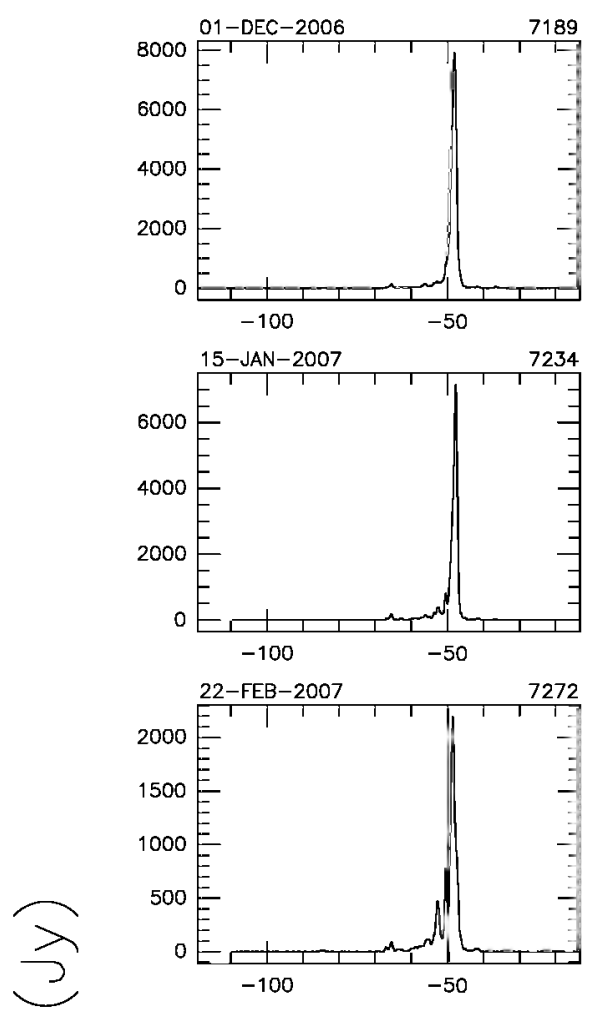

$L^{2}$

$$
V_{\text {LSR }}(\mathrm{km} / \mathrm{s})
$$

Fig. A.2. a continued. 


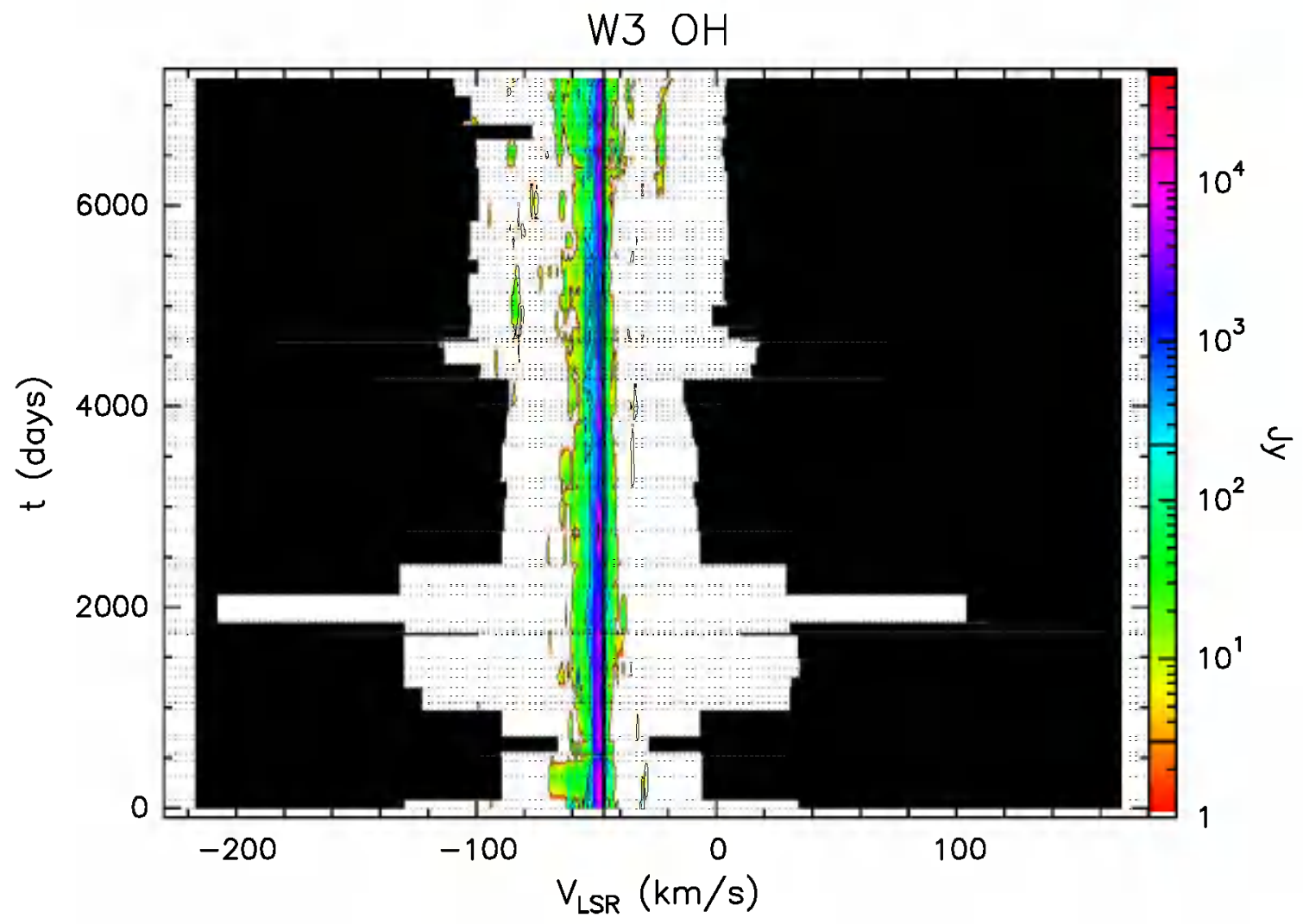

Fig. A.2. b Velocity-time-flux density full plot for source $\mathrm{W} 3 \mathrm{OH}$. The vertical solid line indicates the velocity of the associated thermal molecular gas. The flux density scale is shown by the bar on the right. In this bar the three lines give the flux density of the drawn contours.

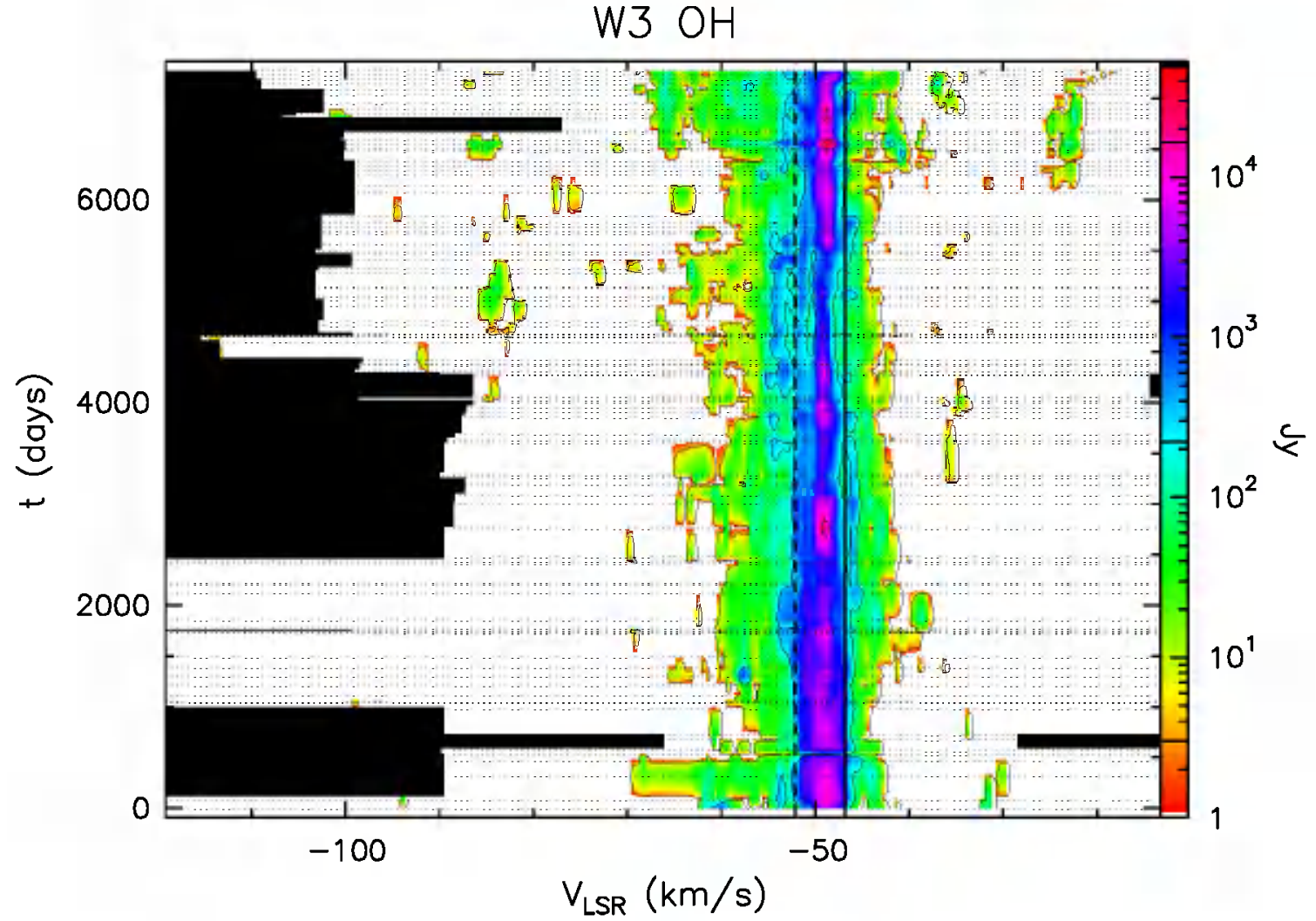

Fig. A.2. c Same as previous figure. but "zoomed" to velocity range over which emission has been detected. 


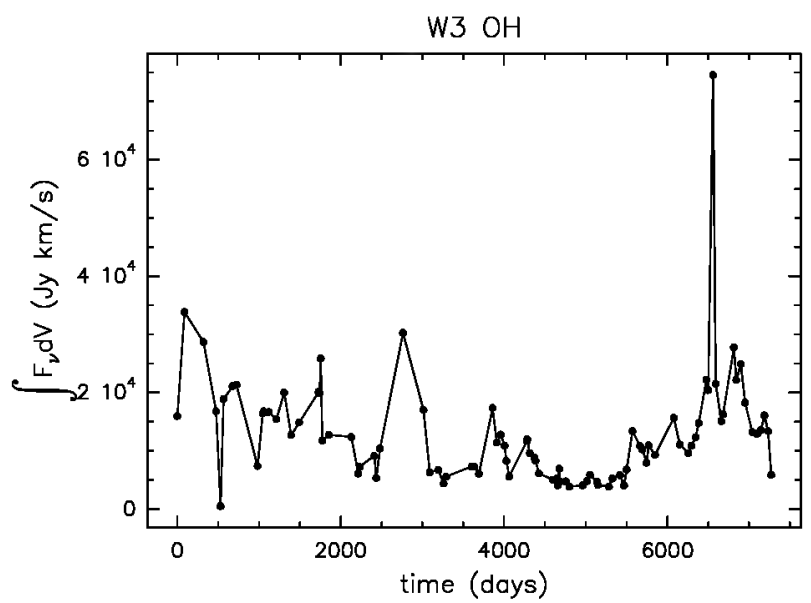

Fig. A.2. d Integral of the flux density over the observed velocity range as a function of time for source $\mathrm{W} 3 \mathrm{OH}$.

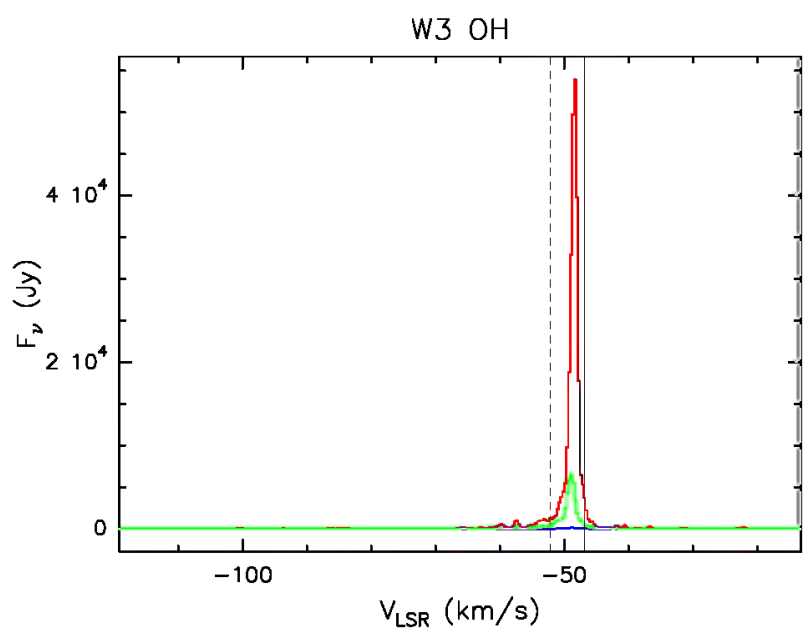

Fig. A.2. e Upper (red) and lower (blue) envelopes and mean spectrum (green) of source W3 OH measured during our monitoring. The vertical solid line marks the velocity of the associated thermal molecular gas. The vertical dashed line marks the mean velocity derived from the histogram of the rate-of-occurrence.

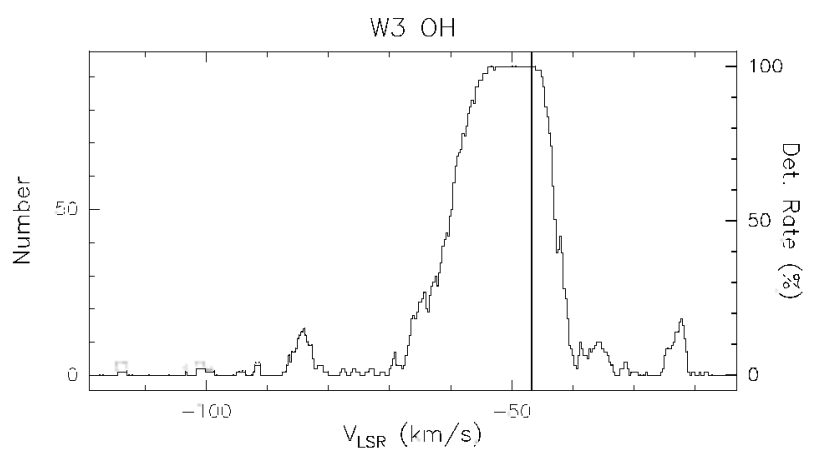

Fig. A.2. f Rate-of-occurrence plot for sonrce W3 OH. The scale to the right refers to the dotted histogram, the scale to the left to the solid line histogram. The vertical solid line marks the velocity of the associated thermal molecular gas. 


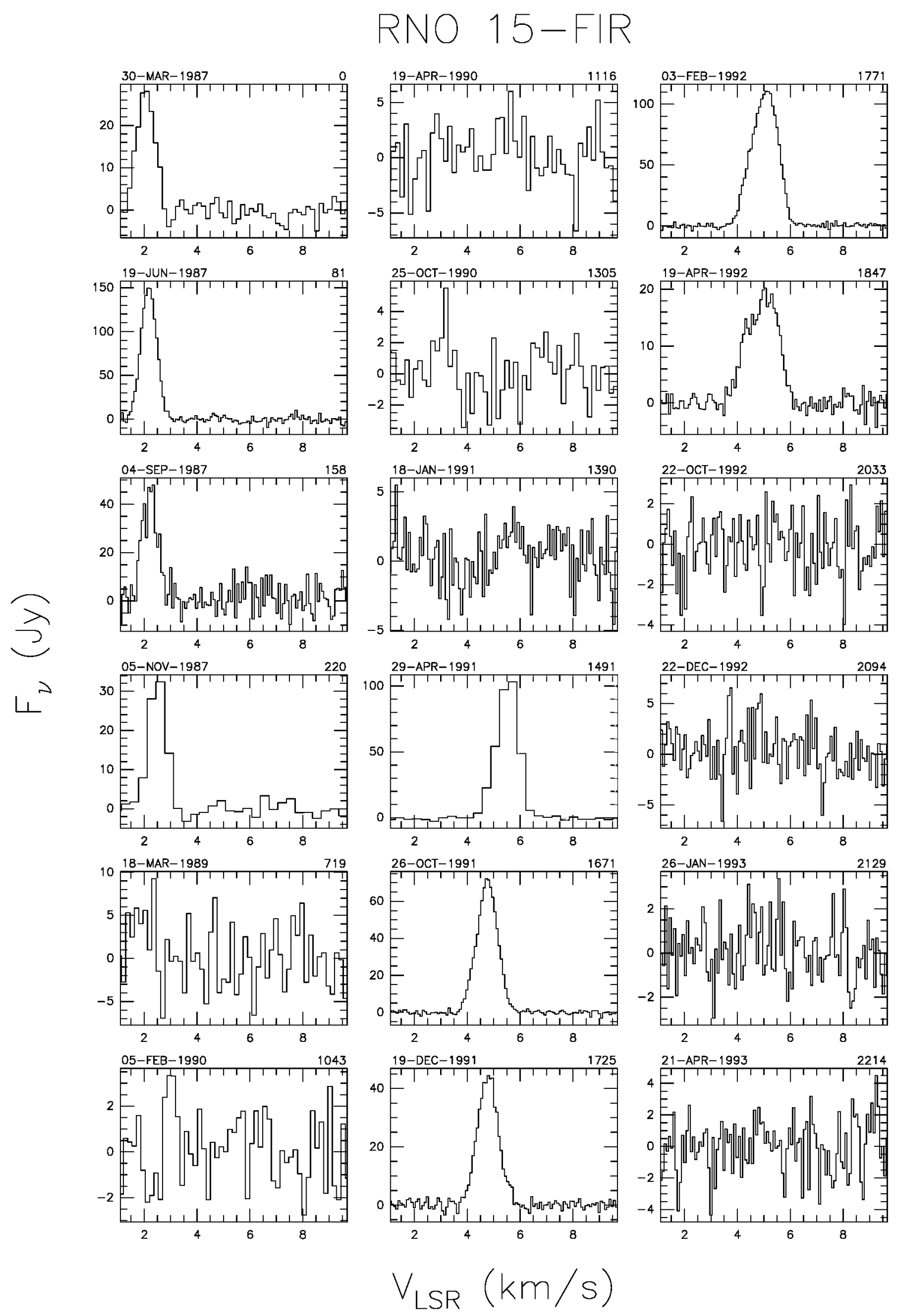

Fig. A.3. a Spectra of source RNO 15-FIR with autoscaled flux density scale. The date of observation is shown above the top left corner of each spectrum and the number of days elapsed since the first observation is given above the top right corner. The velocity scale is the same for all spectra. 


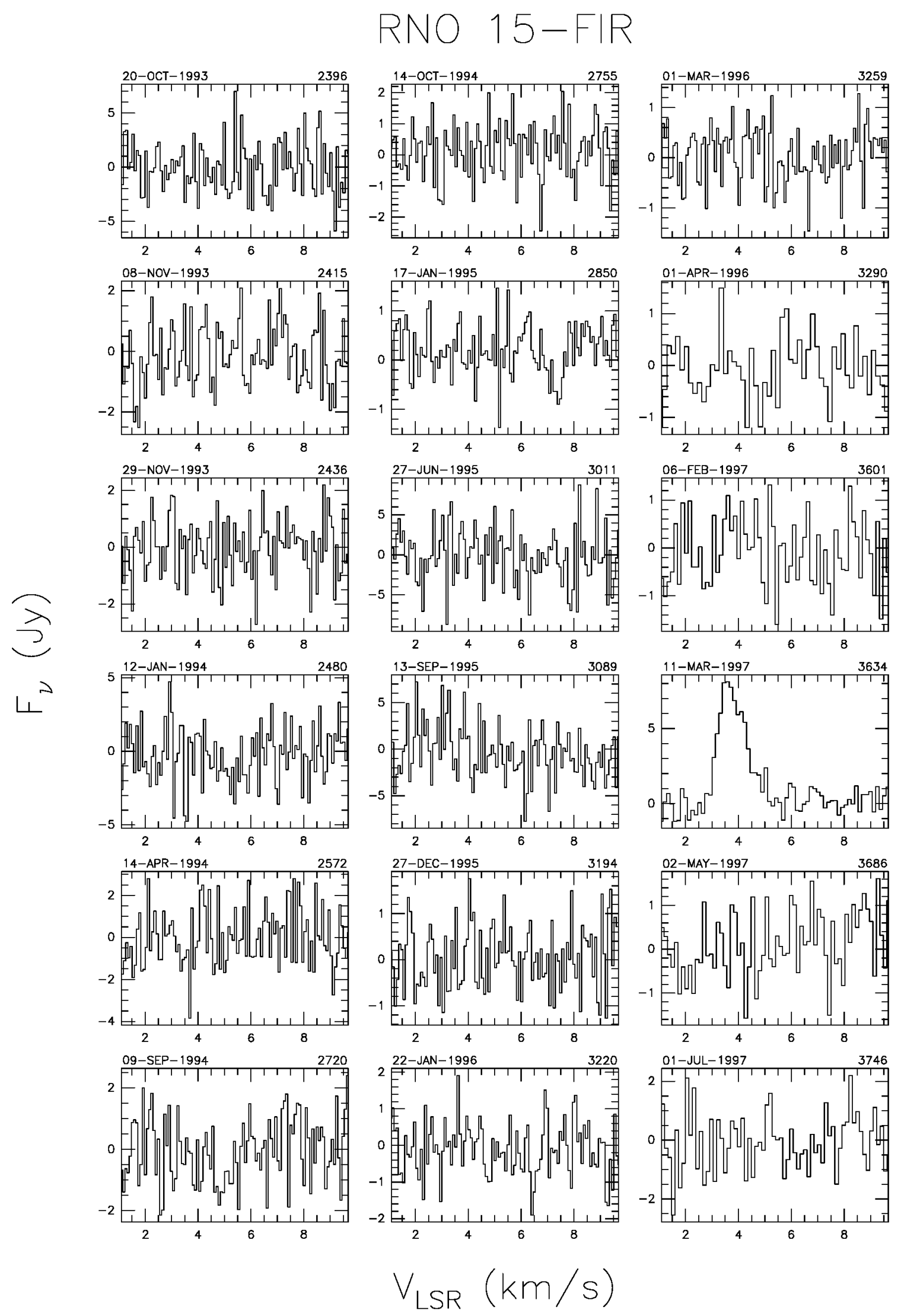

Fig. A.3. a continued. 


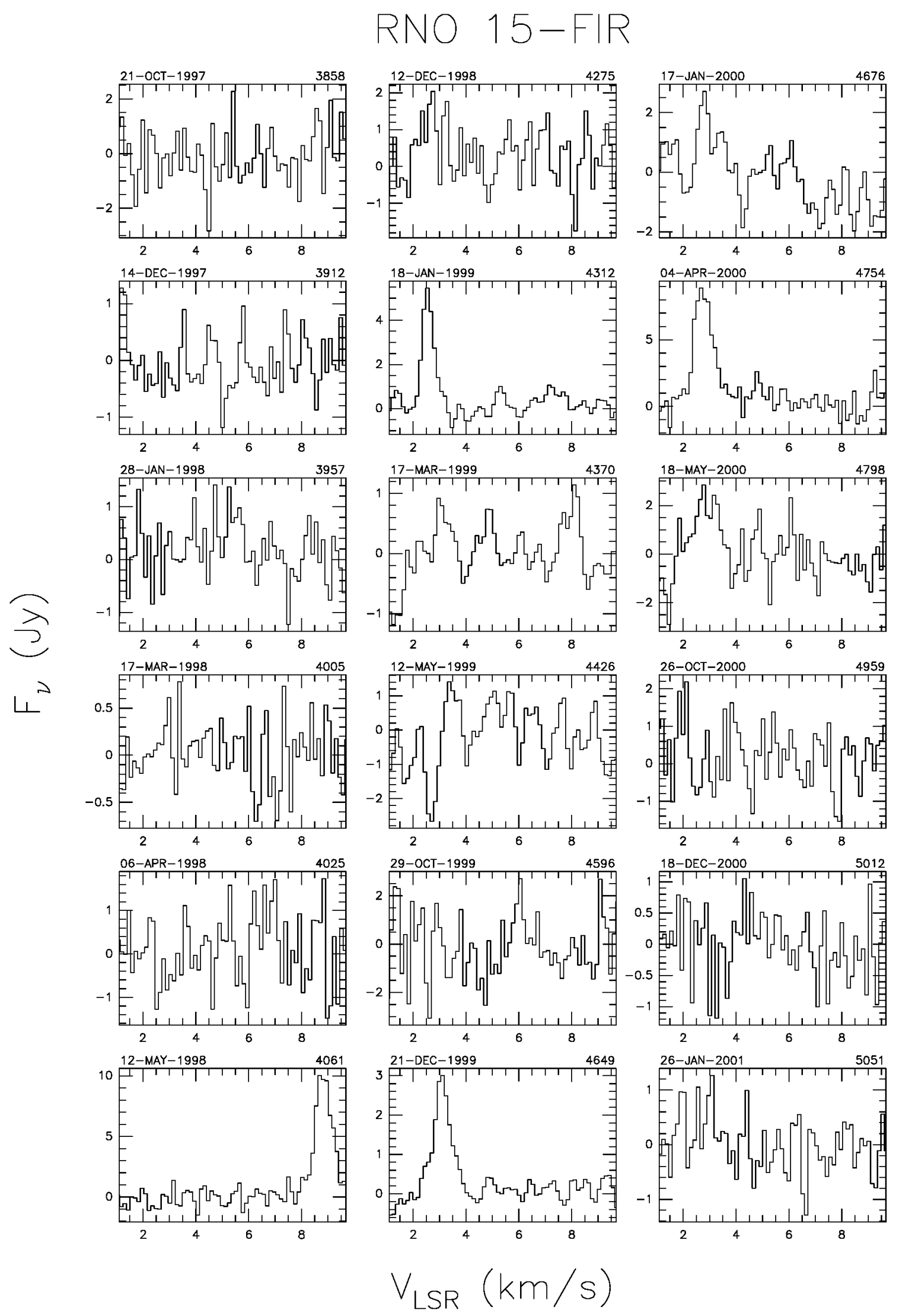

Fig. A.3. a continued. 
RNO 15-FIR
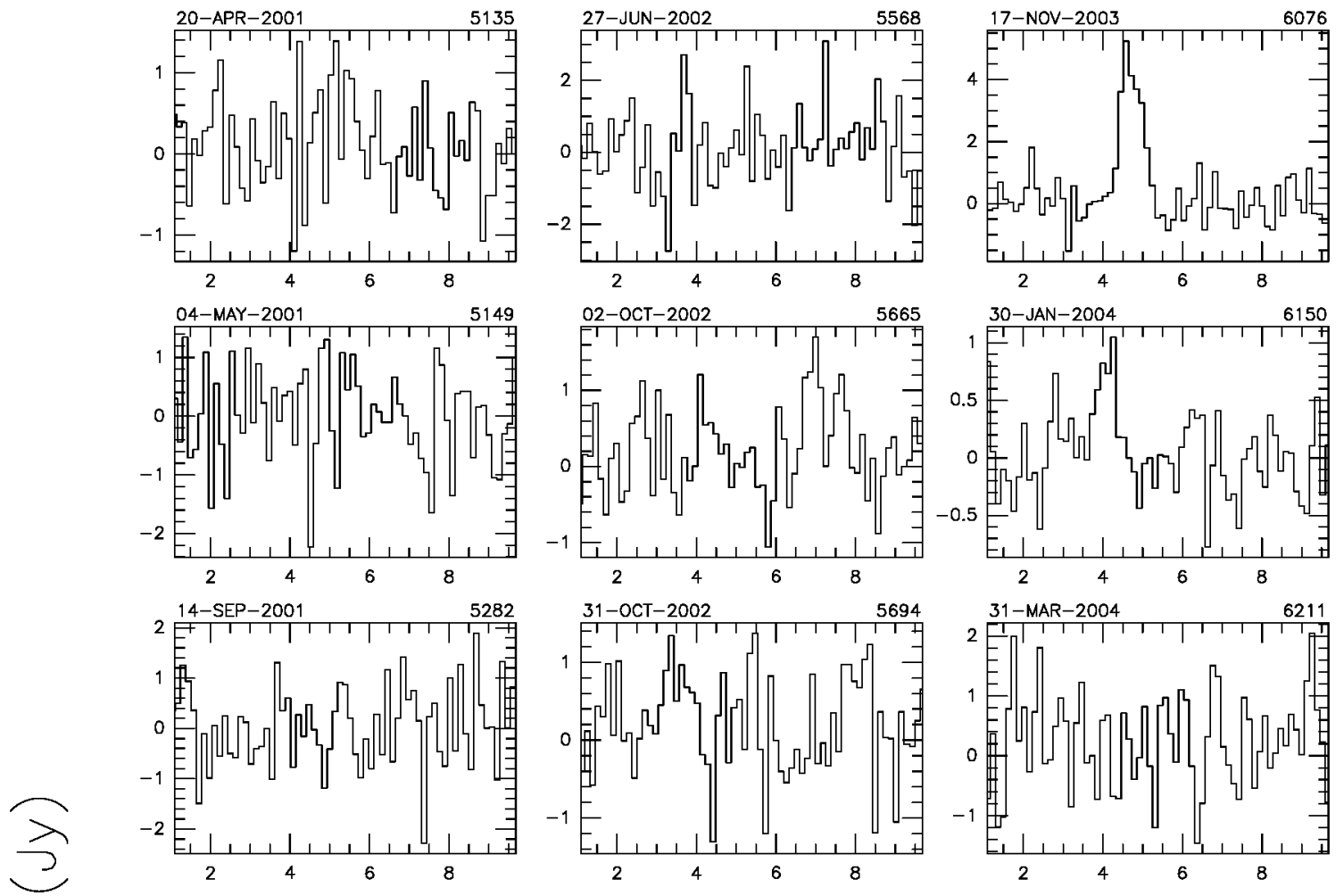

$L^{2}$
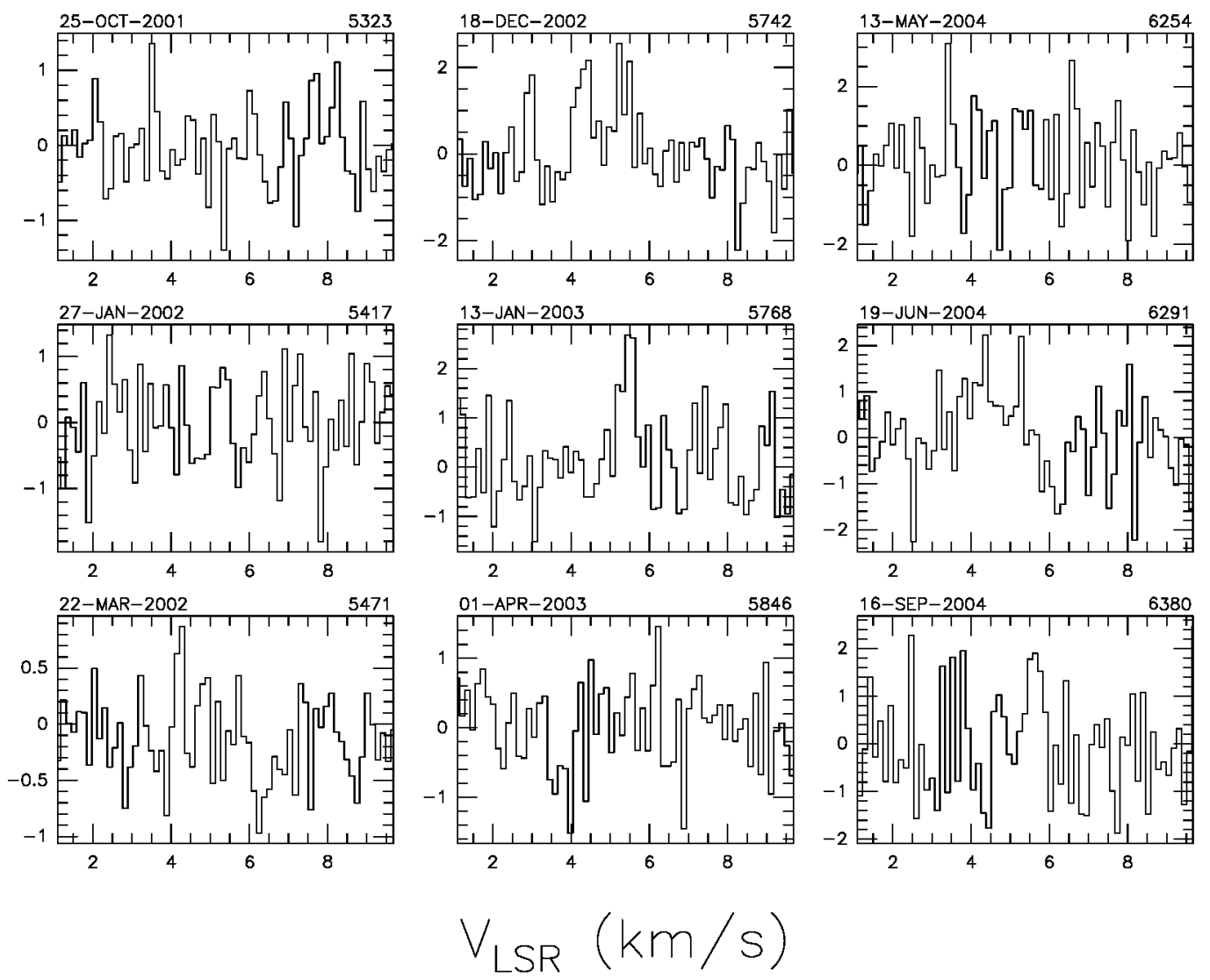

Fig. A.3. a continued. 
RNO 15-FIR
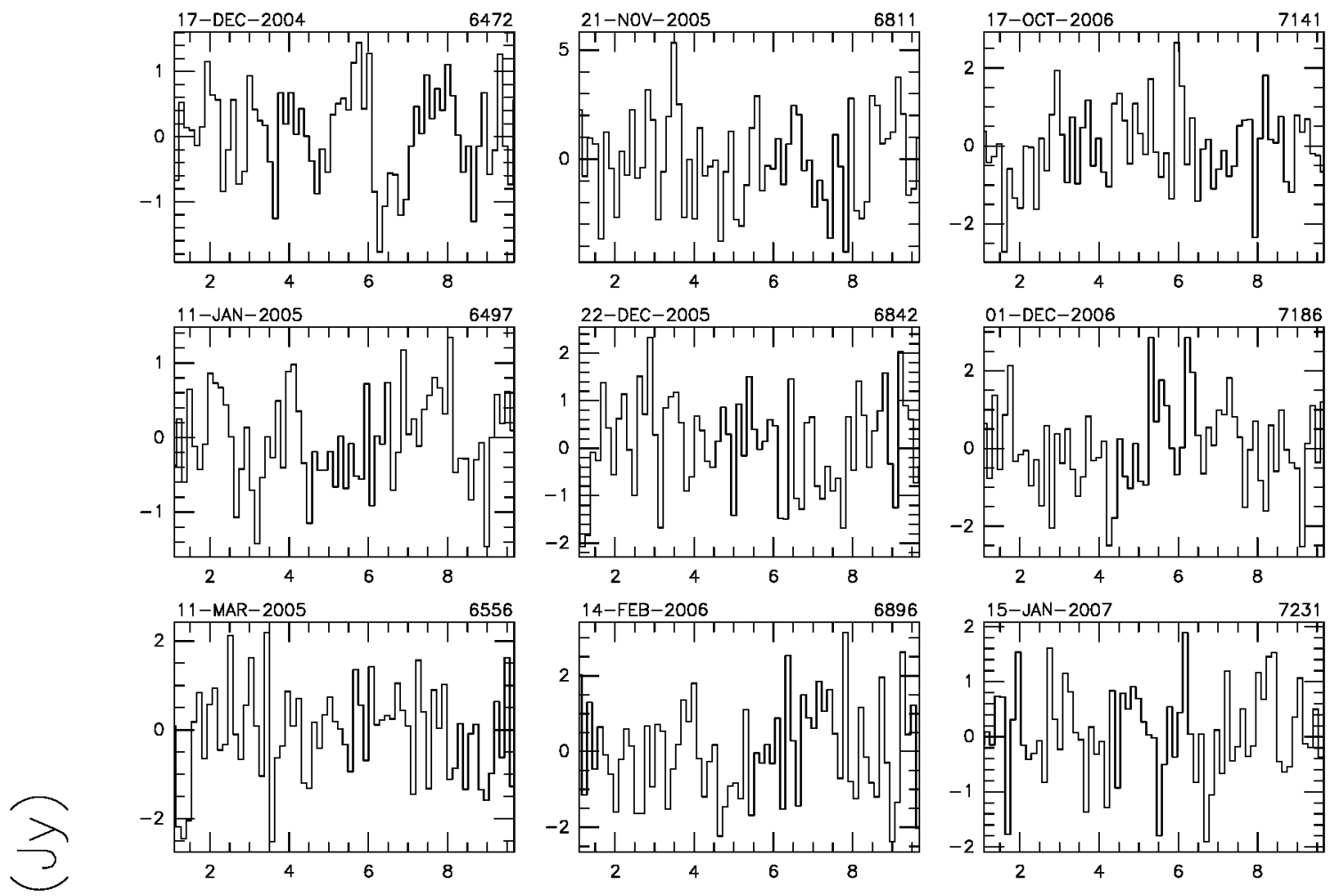

$L^{\curvearrowright}$
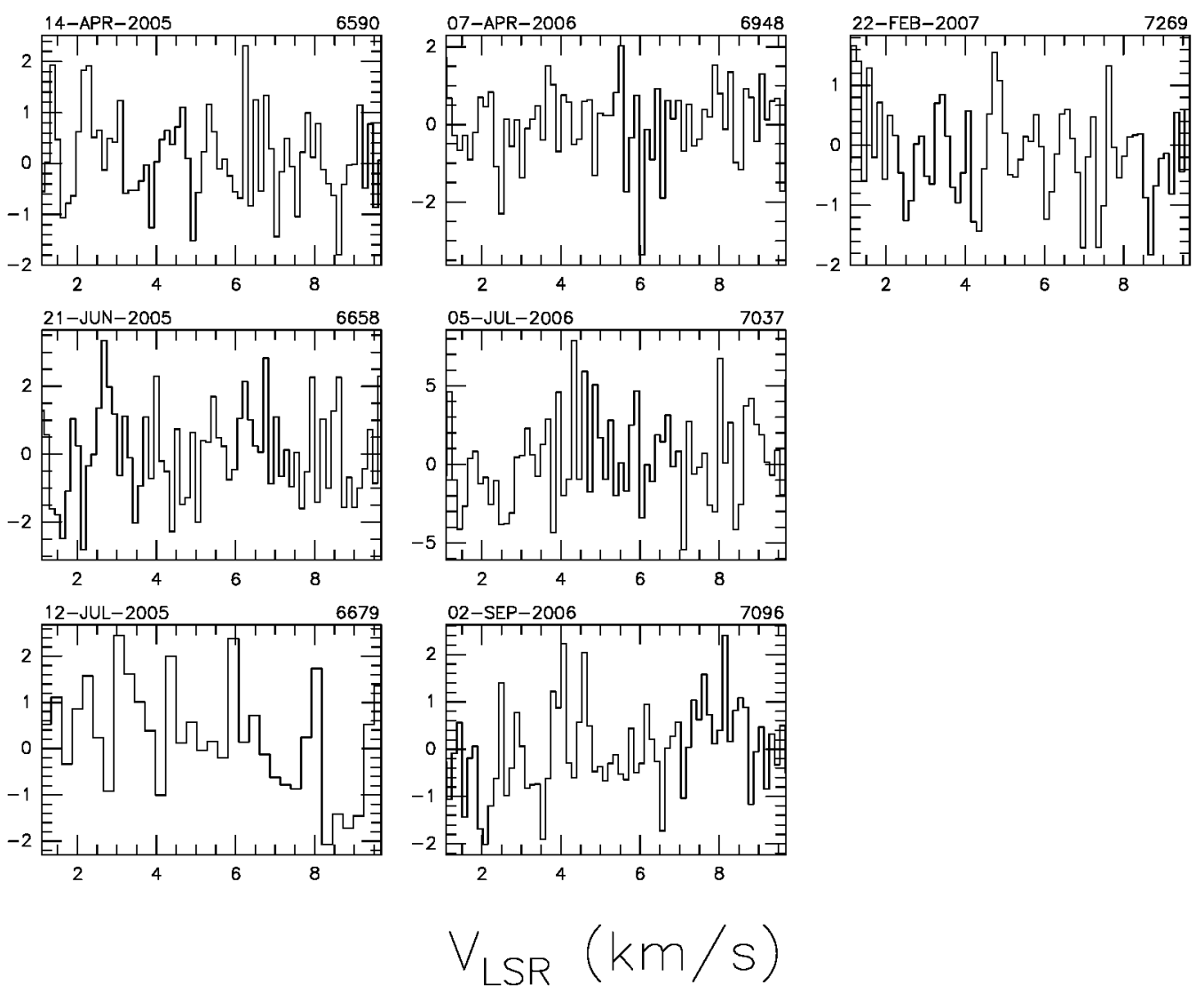

Fig. A.3. a continued. 


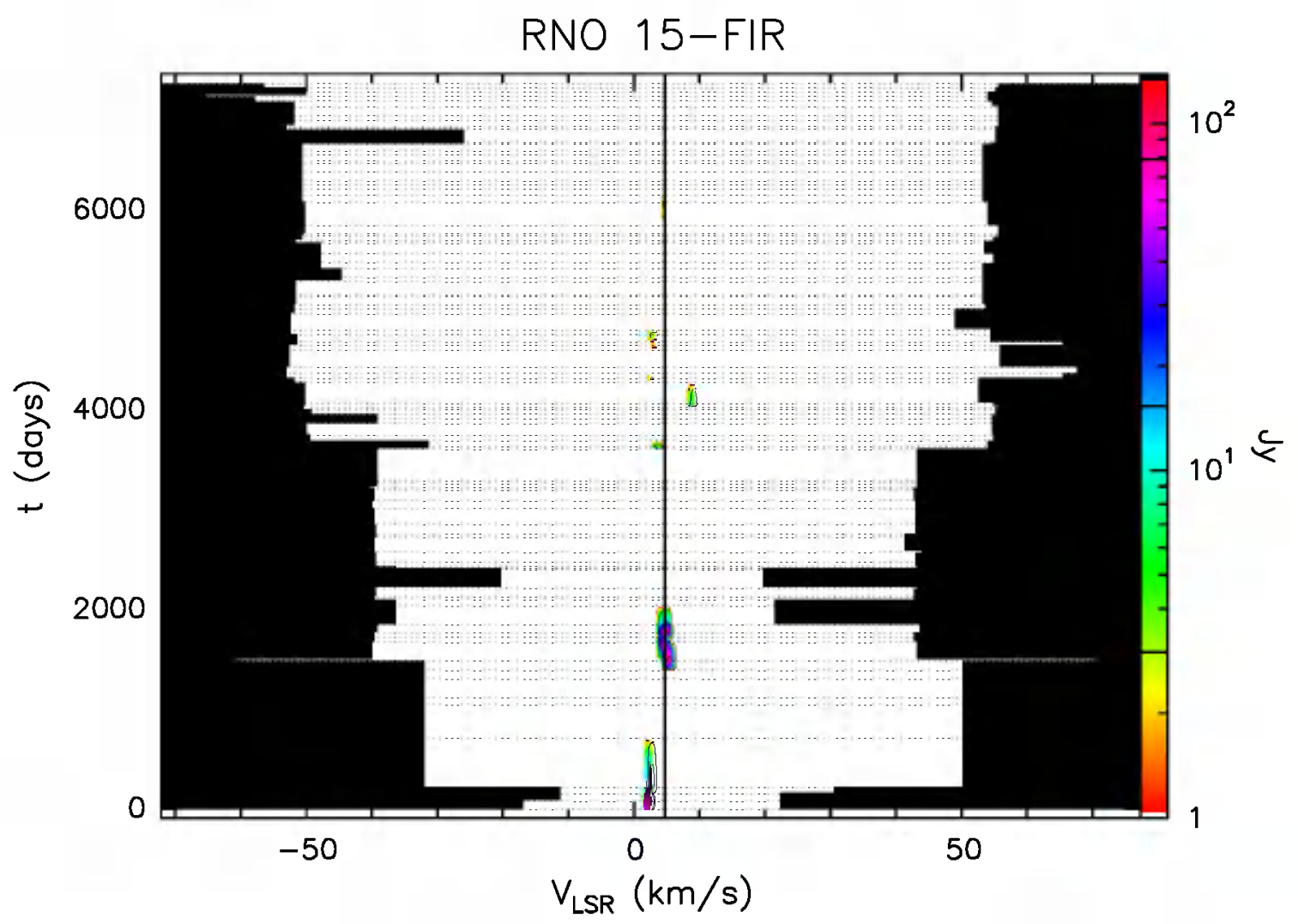

Fig. A.3. b Velocity-time-flux density full plot for source RNO 15-FIR. The vertical solid line indicates the velocity of the associated thermal molecular gas. The flux density scale is shown by the bar on the right. In this bar the three lines give the flux density of the drawn contours.

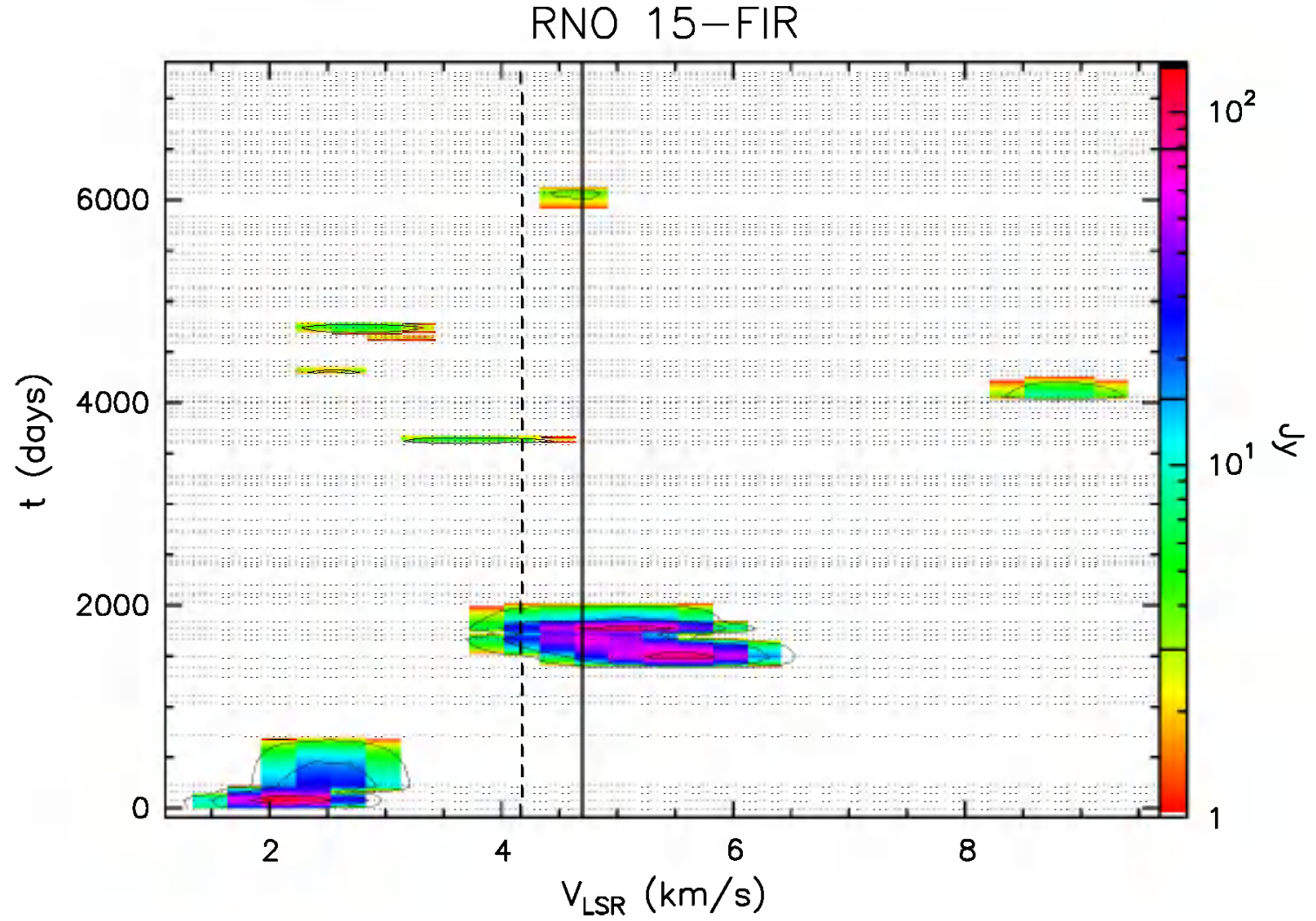

Fig. A.3. c Same as previous figure, but "zoomed" to velocity range over which emission has been detected. 


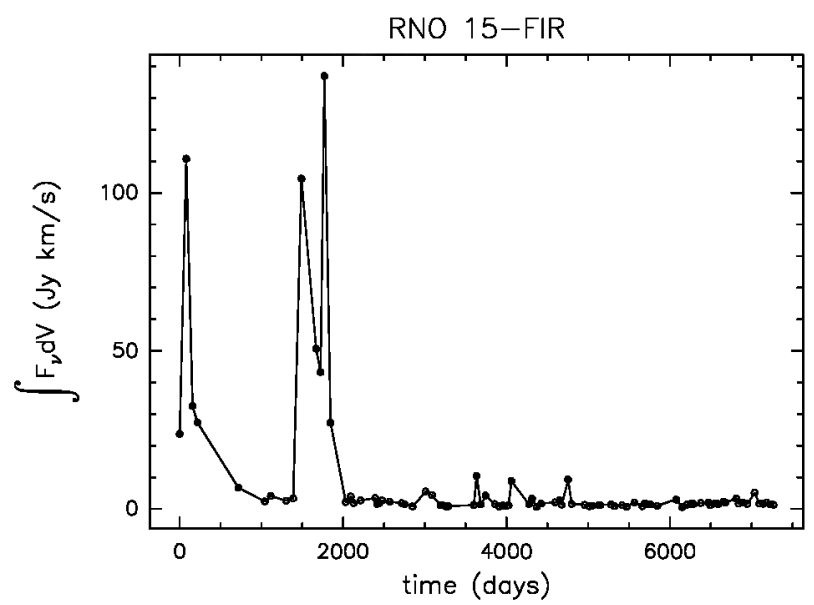

Fig. A.3. d Integral of the flux density over the observed velocity range as a function of time for source RNO 15-FIR.

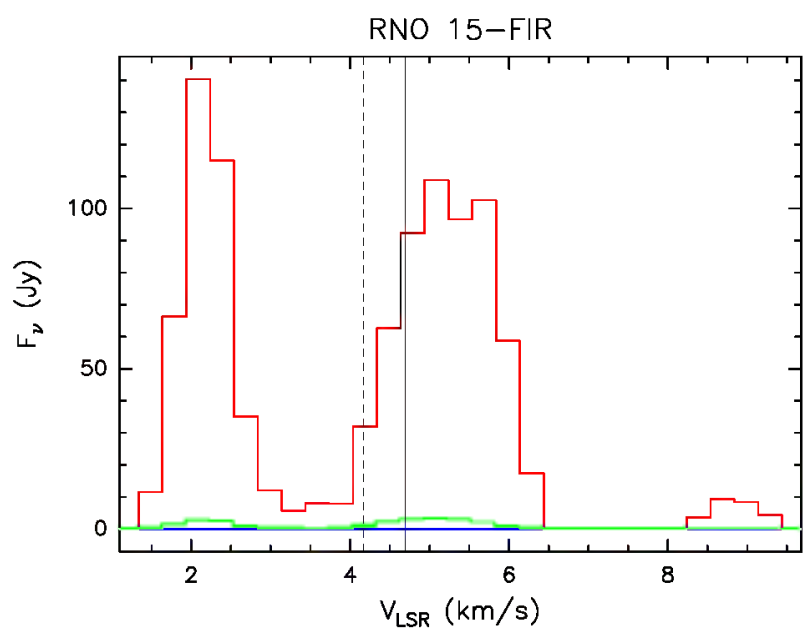

Fig. A.3. e Upper (red) and lower (blue) envelopes and mean spectrum (green) of source RNO 15-FIR measured during our monitoring. The vertical solid line marks the velocity of the associated thermal molectllar gas. The vertical dashed line marks the mean velocity derived from the histogram of the rate-of-occurrence.

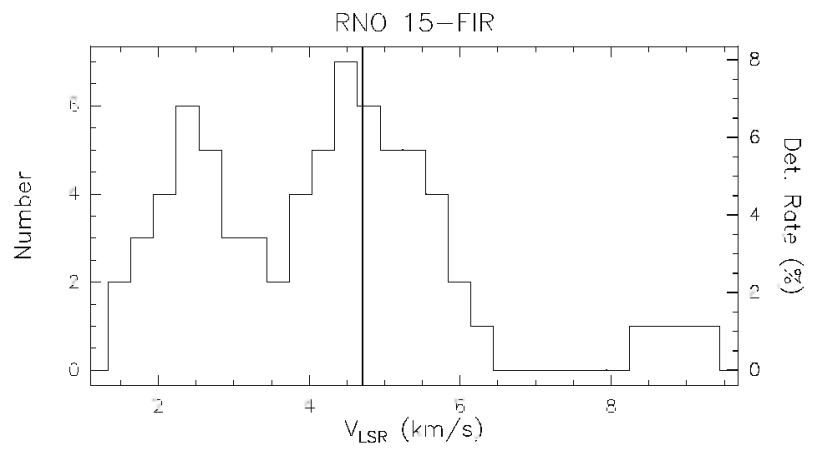

Fig. A.3. f Rate-of-occurrence plot for source RNO 15-FIR. The scale to the right refers to the dotted histogram, the scale to the left to the solid line histogram. The vertical solid line marks the velocity of the associated thermal molecular gas. 

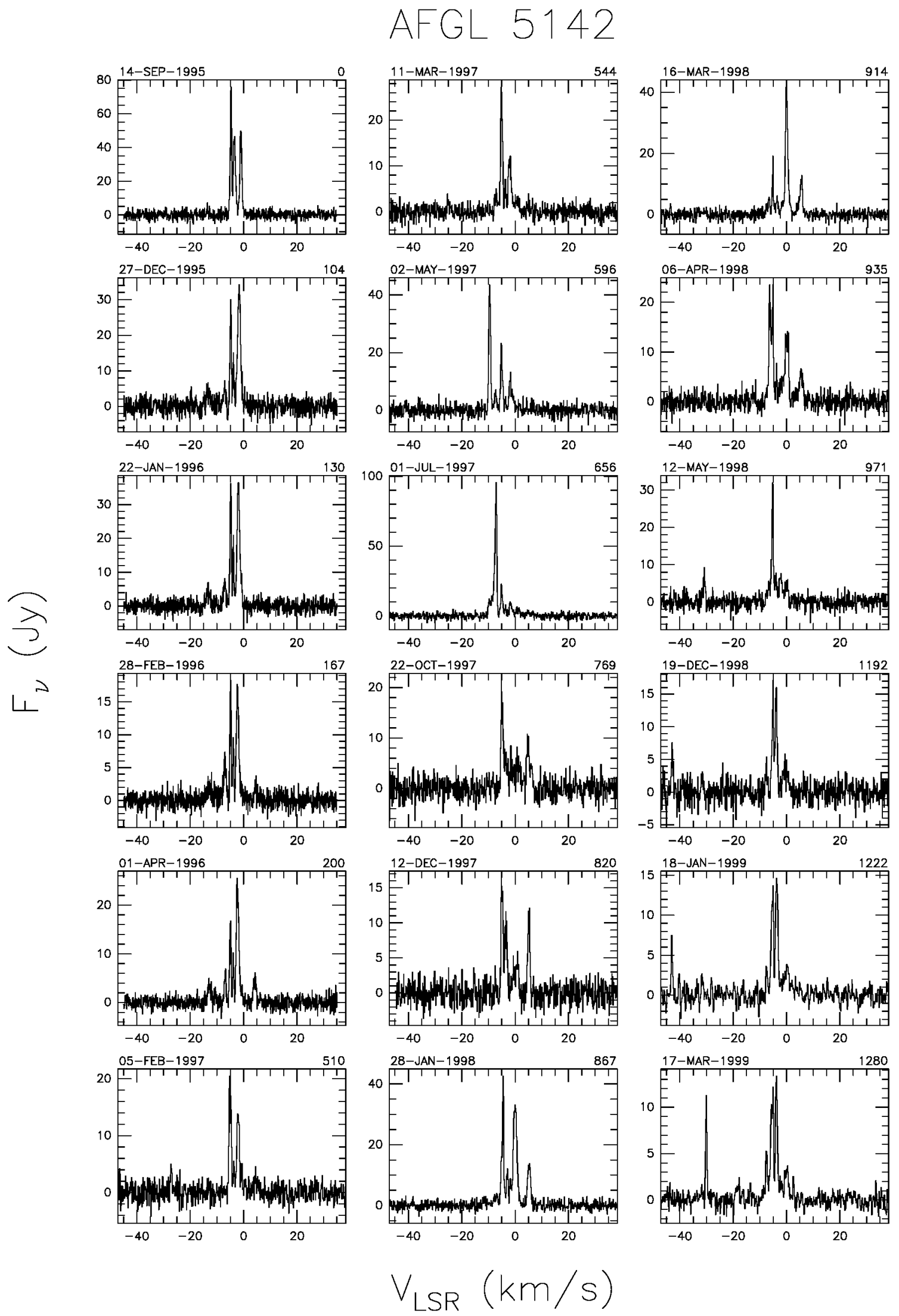

Fig. A.4. a Spectra of source AFGL 5142 with autoscaled flux density scale. The date of observation is shown above the top left corner of each spectrum and the number of days elapsed since the first observation is given above the top right corner. The velocity scale is the same for all spectra. 
AFGL 5142
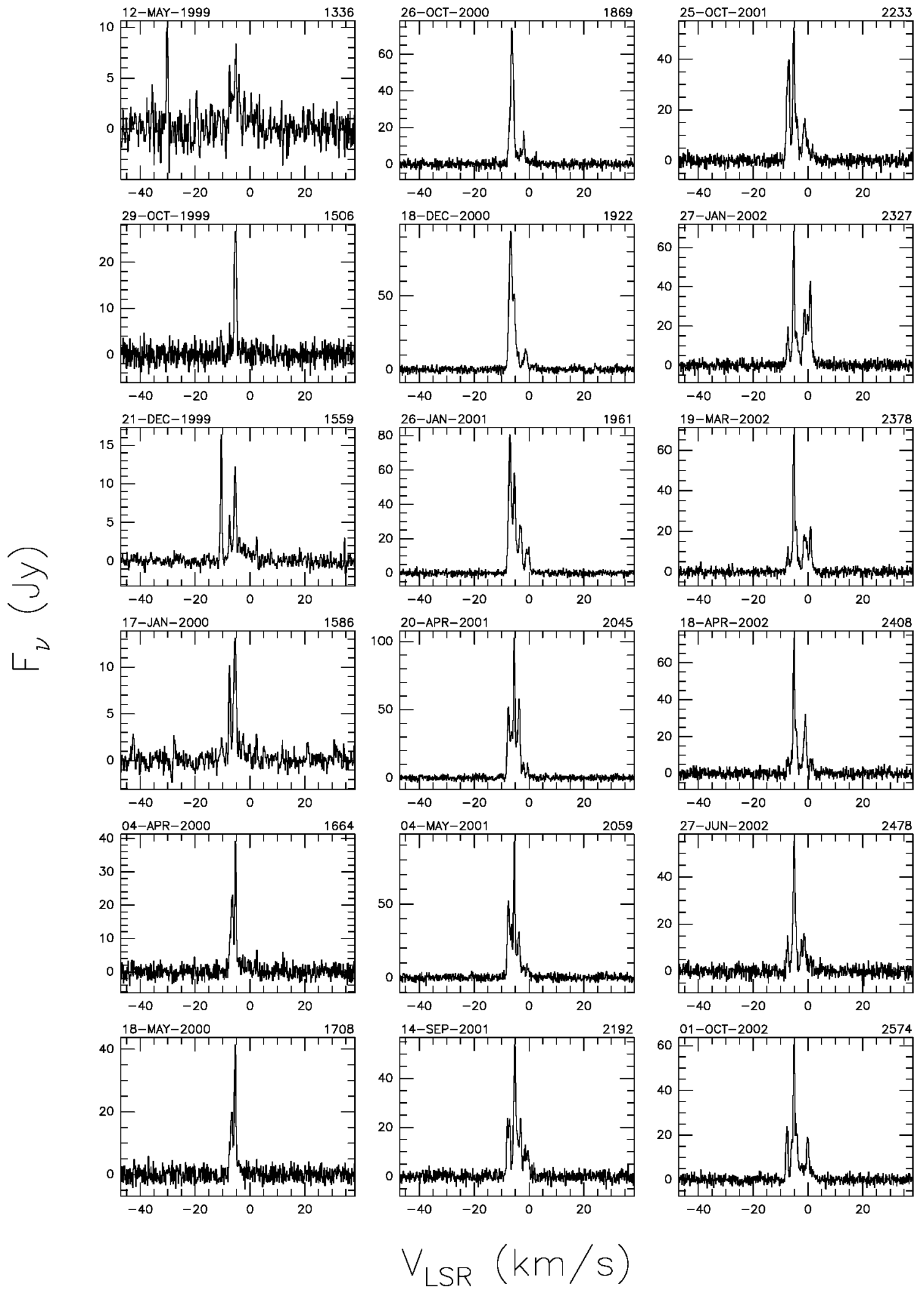

Fig. A.4. a continued. 
AFGL 5142
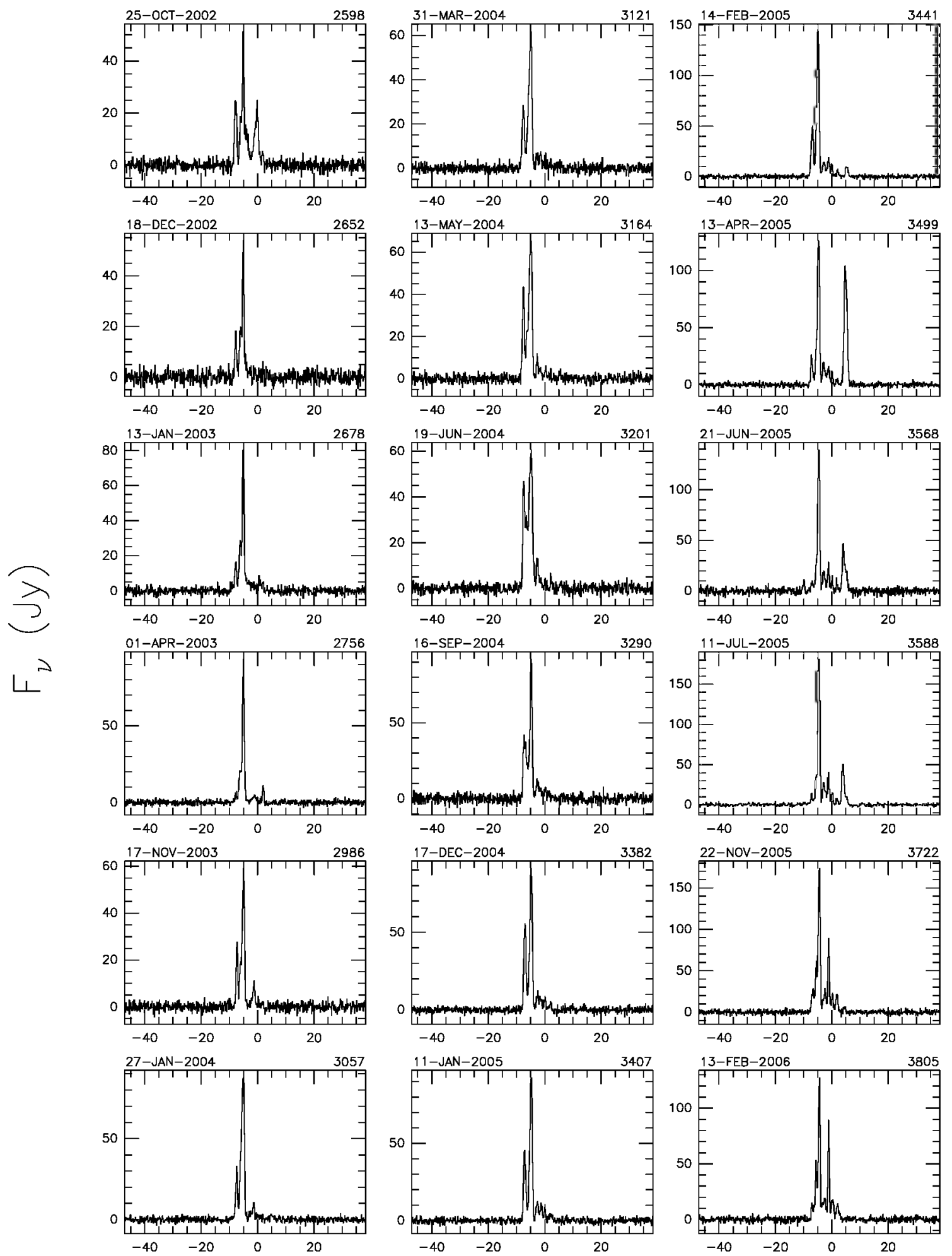

$$
V_{\text {LSR }}(\mathrm{km} / \mathrm{s})
$$

Fig. A.4. a continued. 


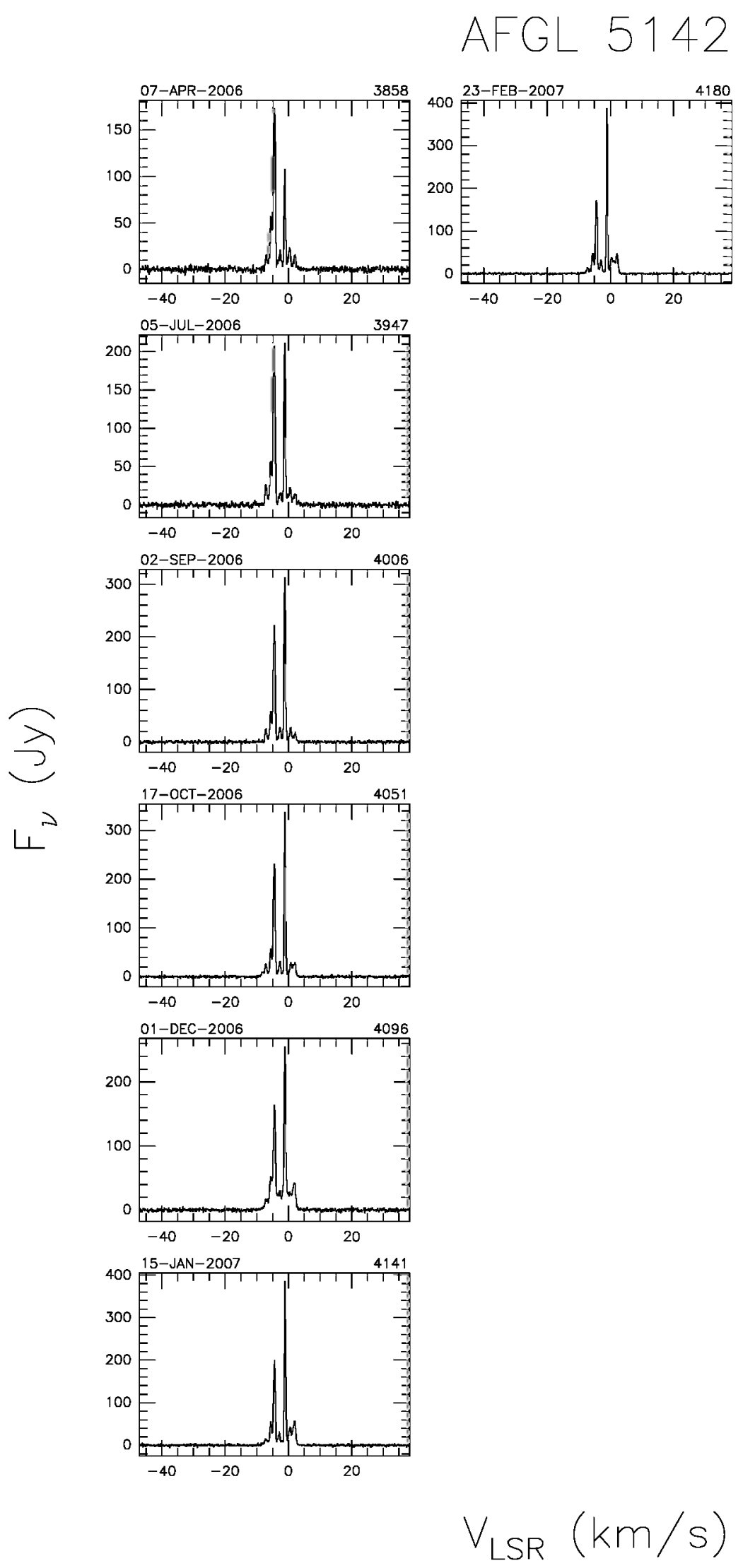

Fig. A.4. a continued. 


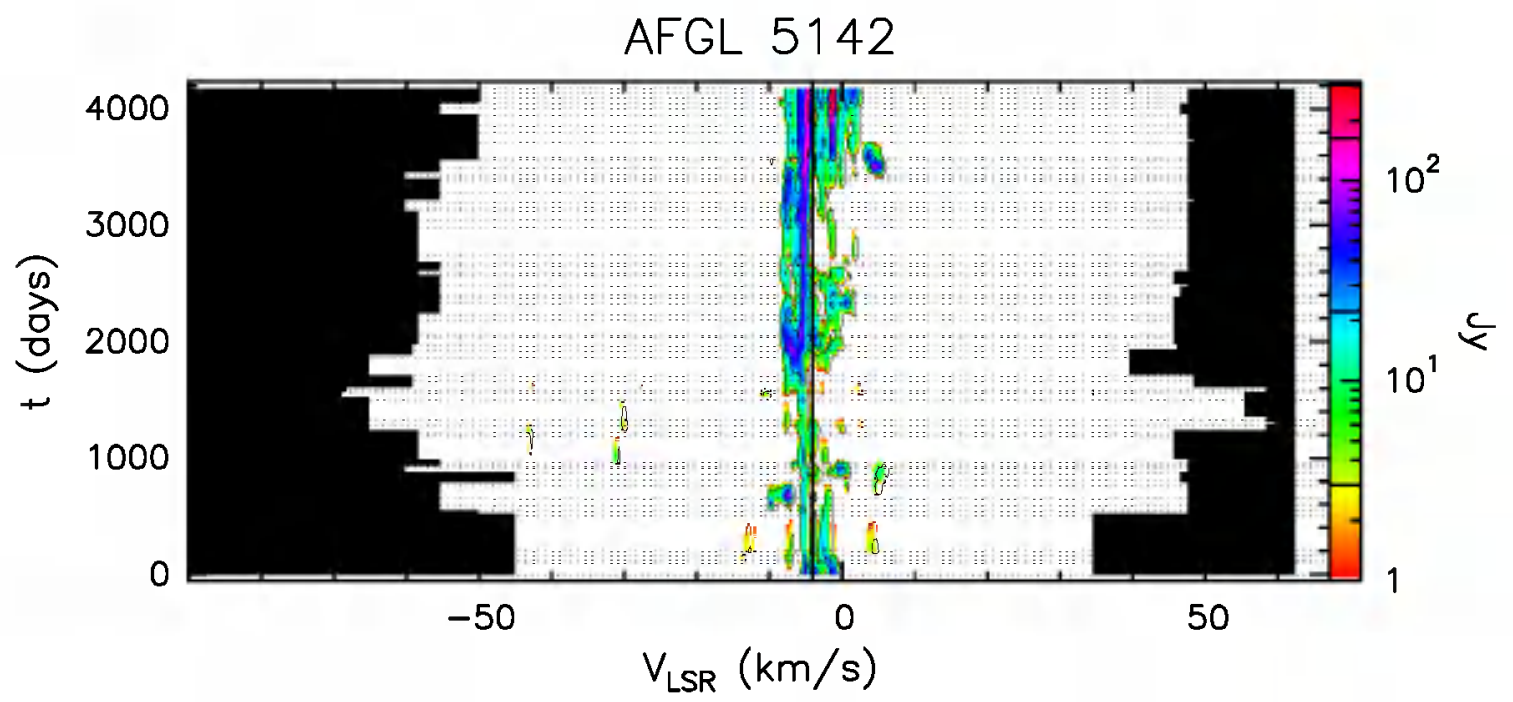

Fig. A.4. b Velocity-time-flux density full plot for source AFGL 5142. The vertical solid line indicates the velocity of the associated theimal molecular gas. The flux density scale is shown by the bar on the right. In this bar the three lines give the flux density of the drawn contours.

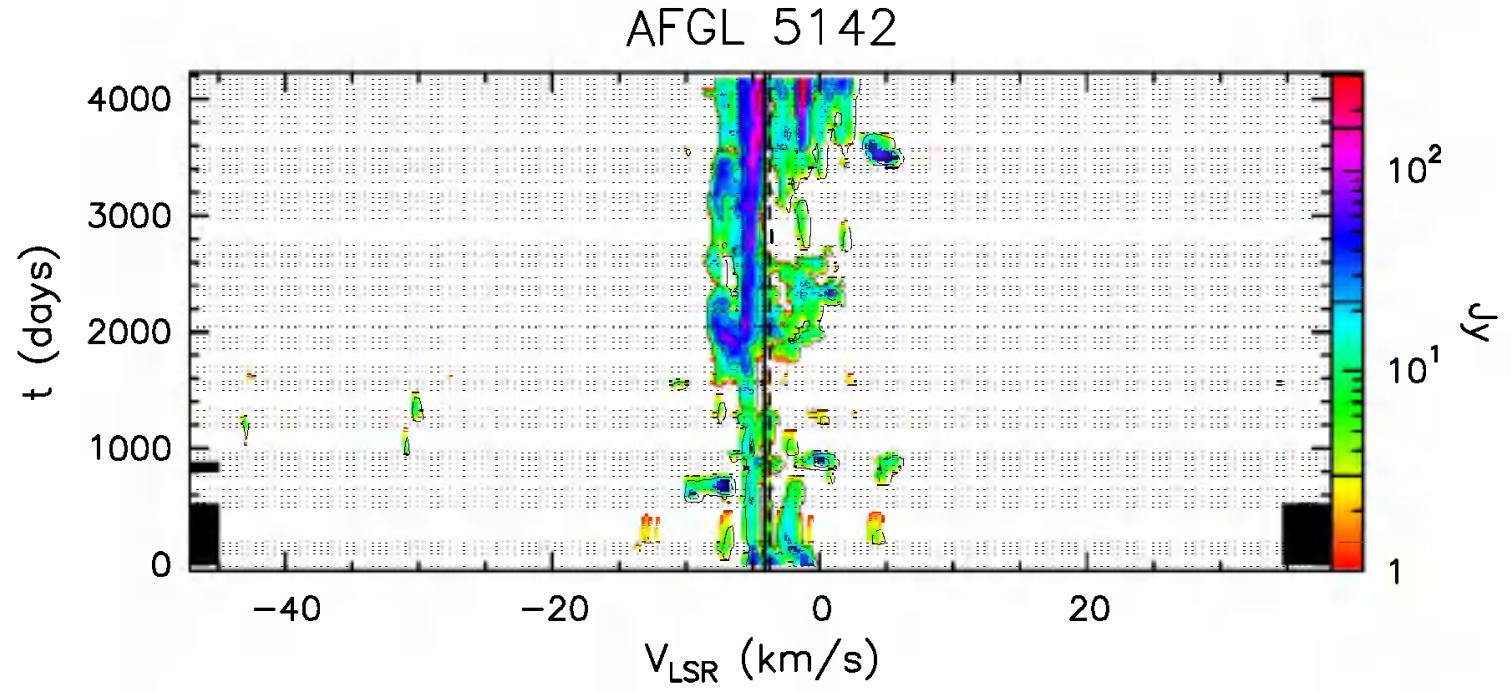

Fig. A.4. c Same as previous figure, but "zoomed" to velocity range over which emission has been detected. 


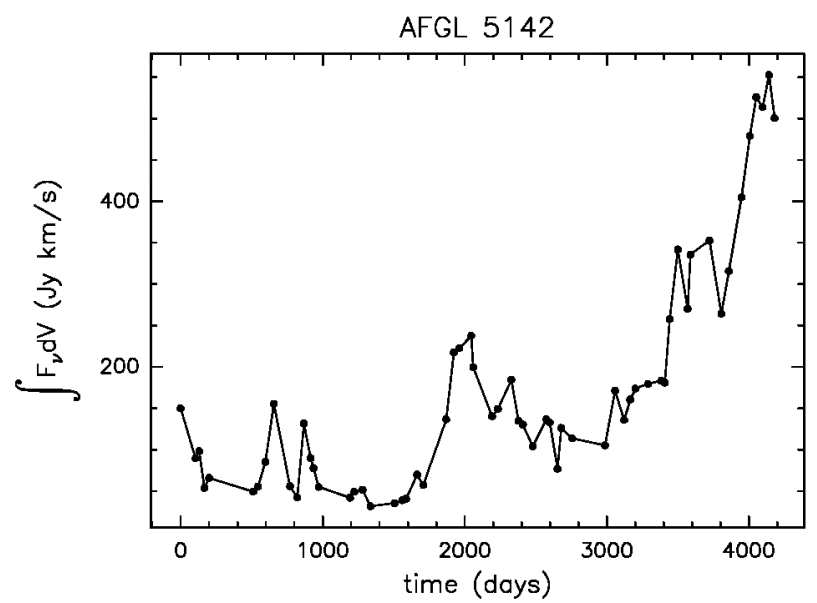

Fig. A.4. d Integral of the flux density over the observed velocity range as a function of time for source AFGL 5142.

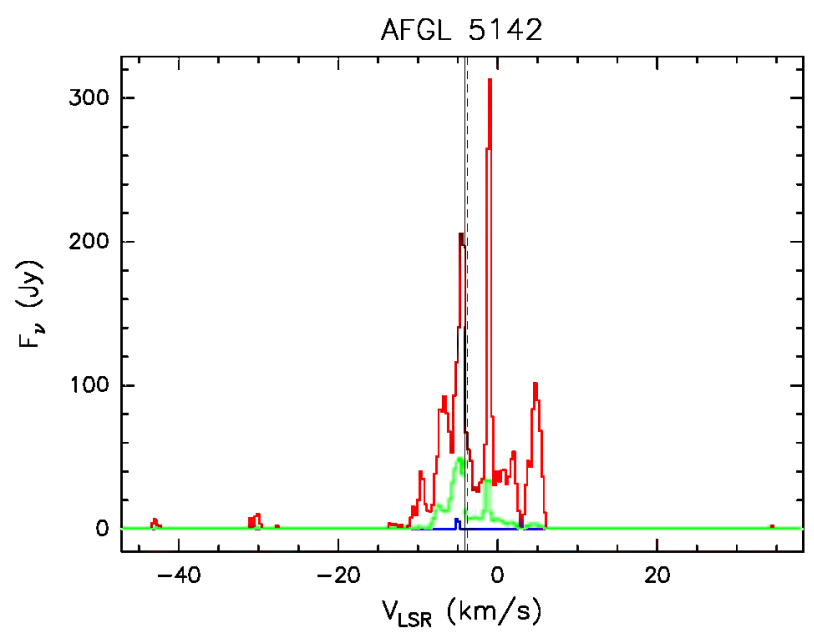

Fig. A.4. e Upper (red) and lower (blue) envelopes and mean spectrum (green) of source AFGL 5142 measured during our monitoring. The vertical solid line marks the velocity of the associated thermal molectllar gas. The vertical dashed line marks the mean velocity derived from the histogram of the rate-of-occurrence.

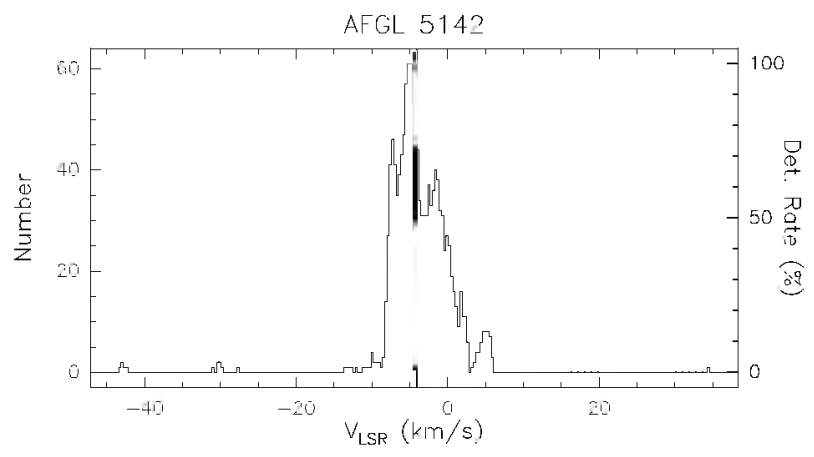

Fig. A.4. f Rate-of-occurrence plot for source AFGL 5142. The scale to the right refers to the dotted histogram, the scale to the left to the solid line histogram. The vertical solid line marks the velocity of the associated thermal molecular gas. 


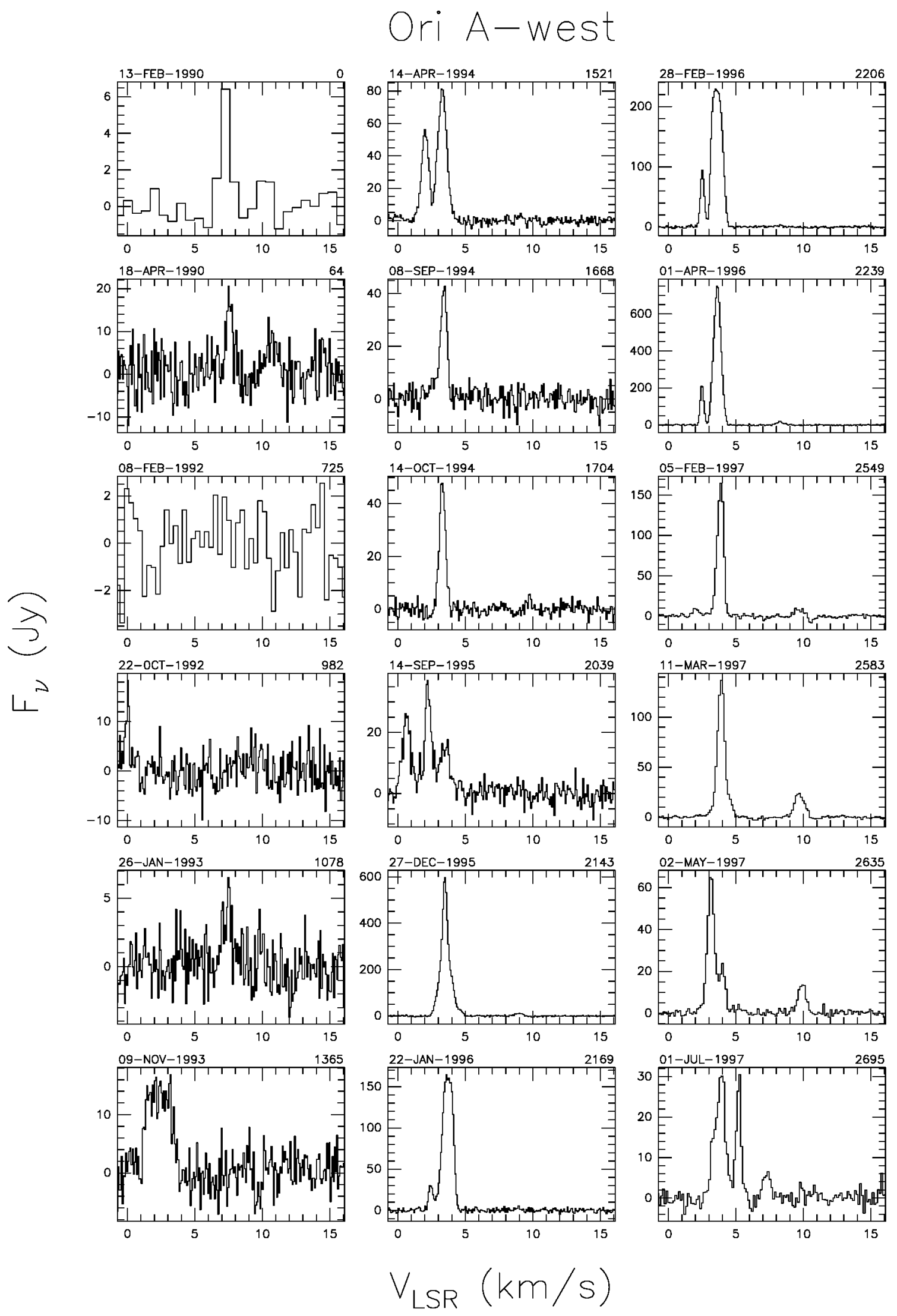

Fig. A.5. a Spectra of source Ori A-west with autoscaled flux density scale. The date of observation is shown above the top left corner of each spectrum and the number of days elapsed since the first observation is given above the top right corner. The velocity scale is the same for all spectra. 


\section{Ori A-west}
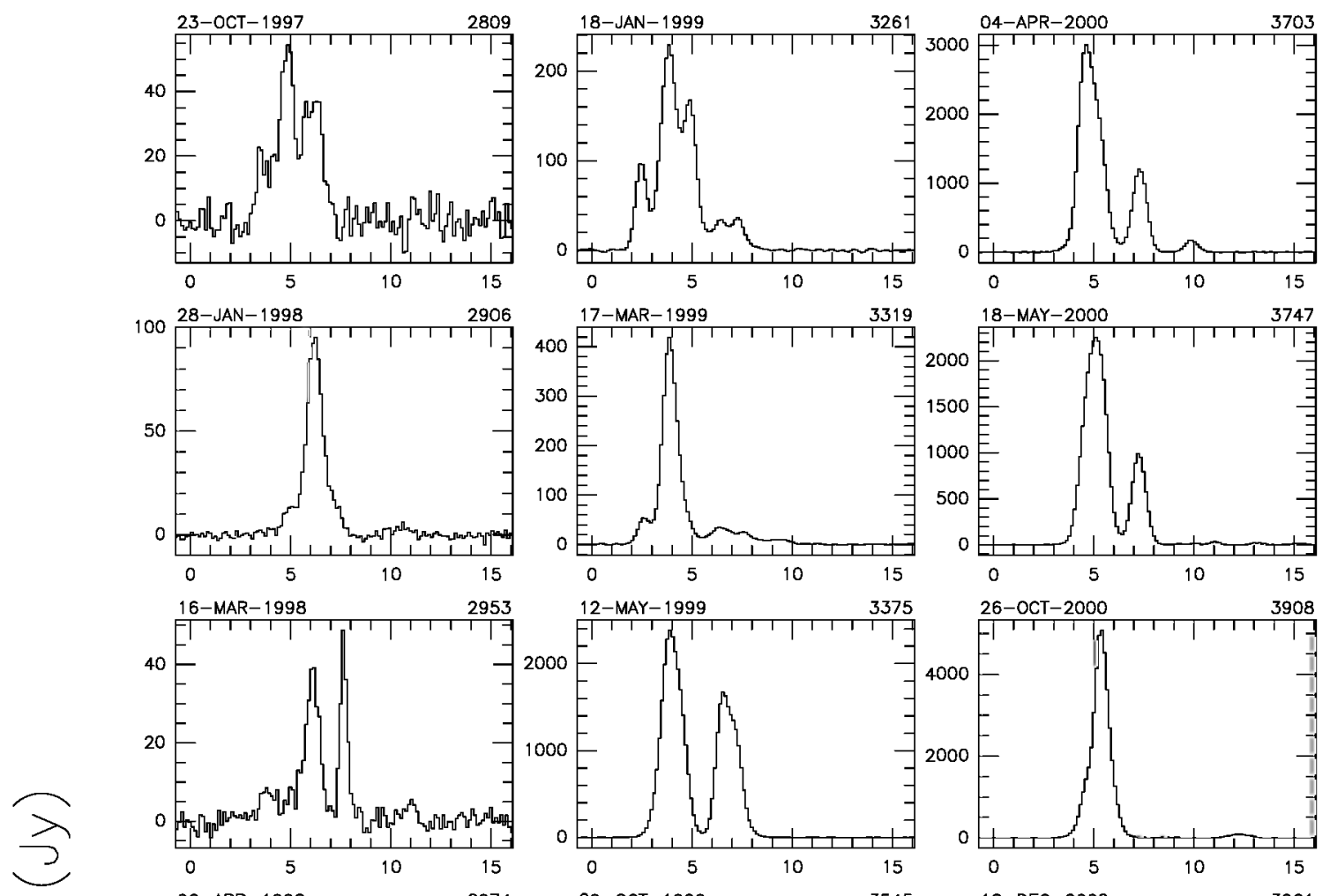

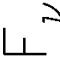
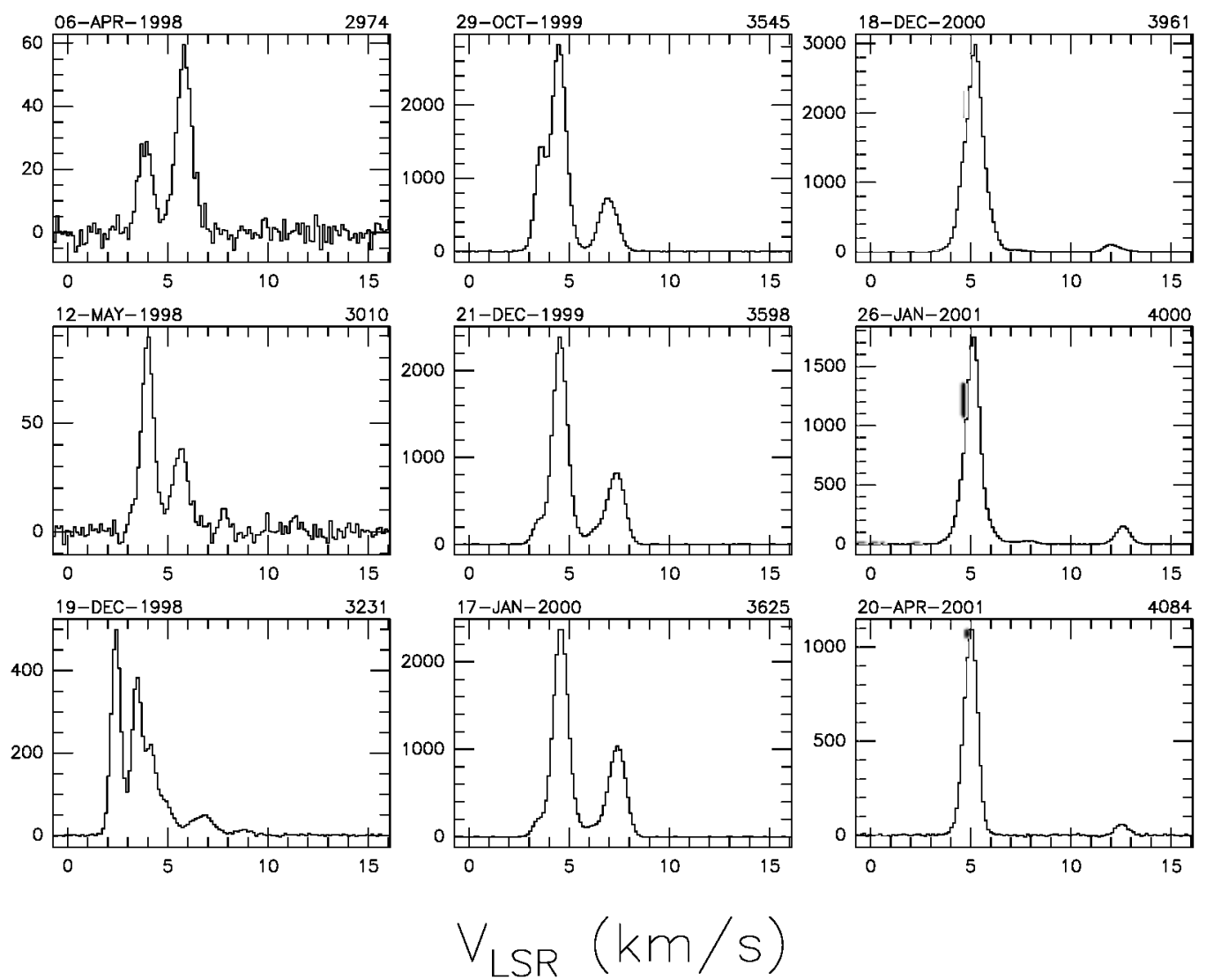

Fig. A.5. a continued. 


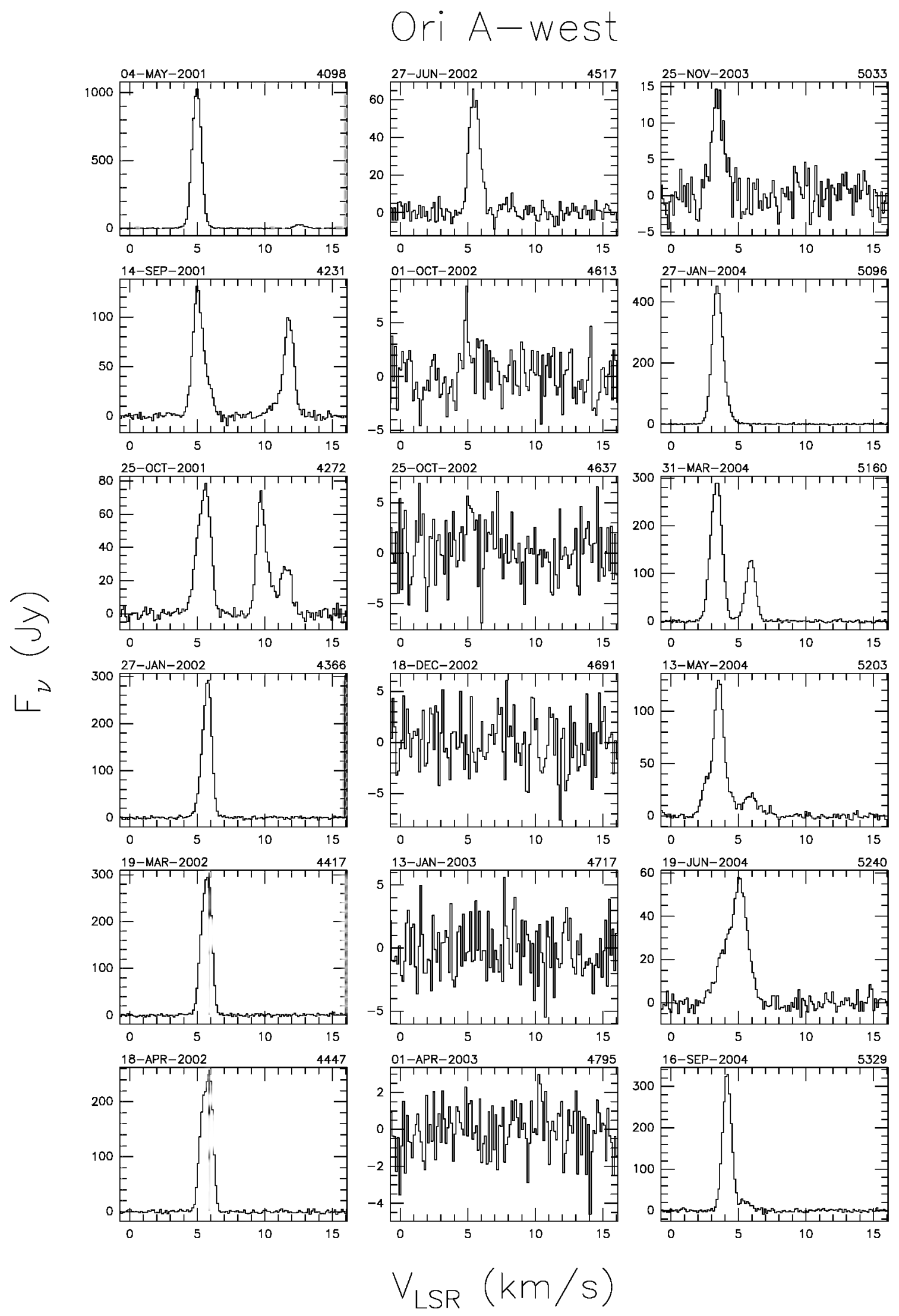

Fig. A.5. a continued. 


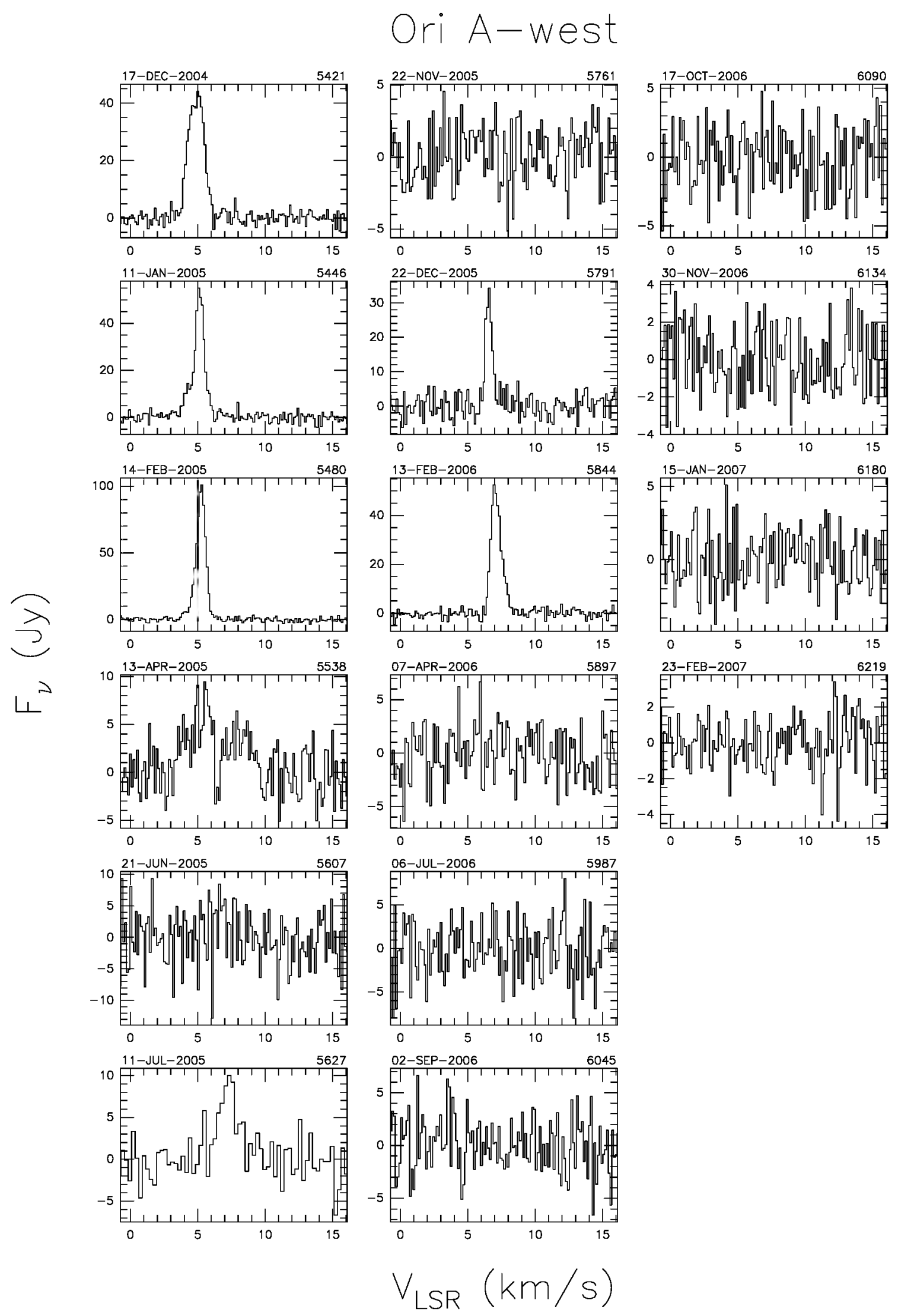

Fig. A.5. a continued. 


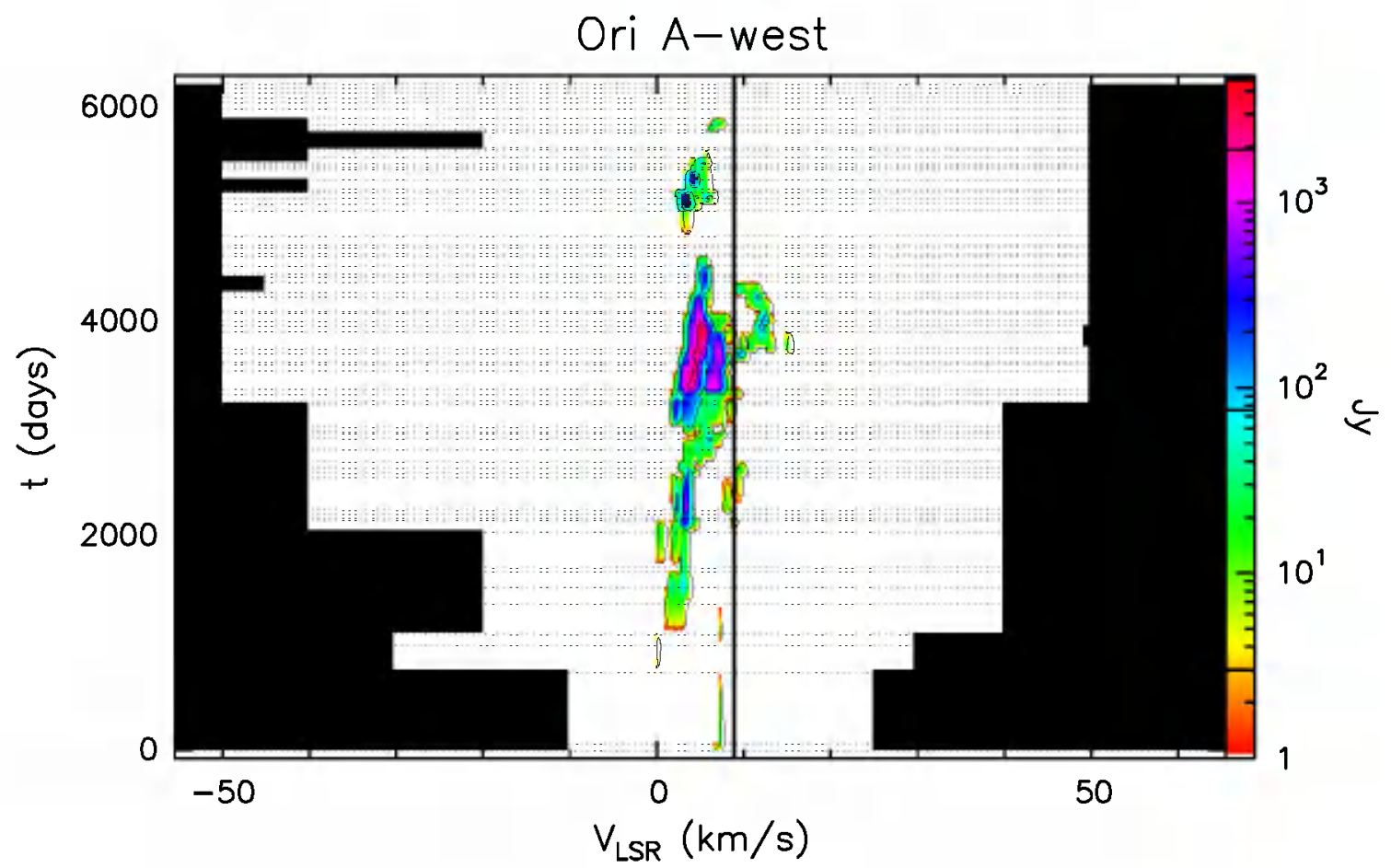

Fig. A.5. b Velocity-time-flux density full plot for source Ori A-west. The vertical solid line indicates the velocity of the associated thermal molecular gas. The flux density scale is shown by the bar on the right. In this bar the three lines give the flux density of the drawn contours.

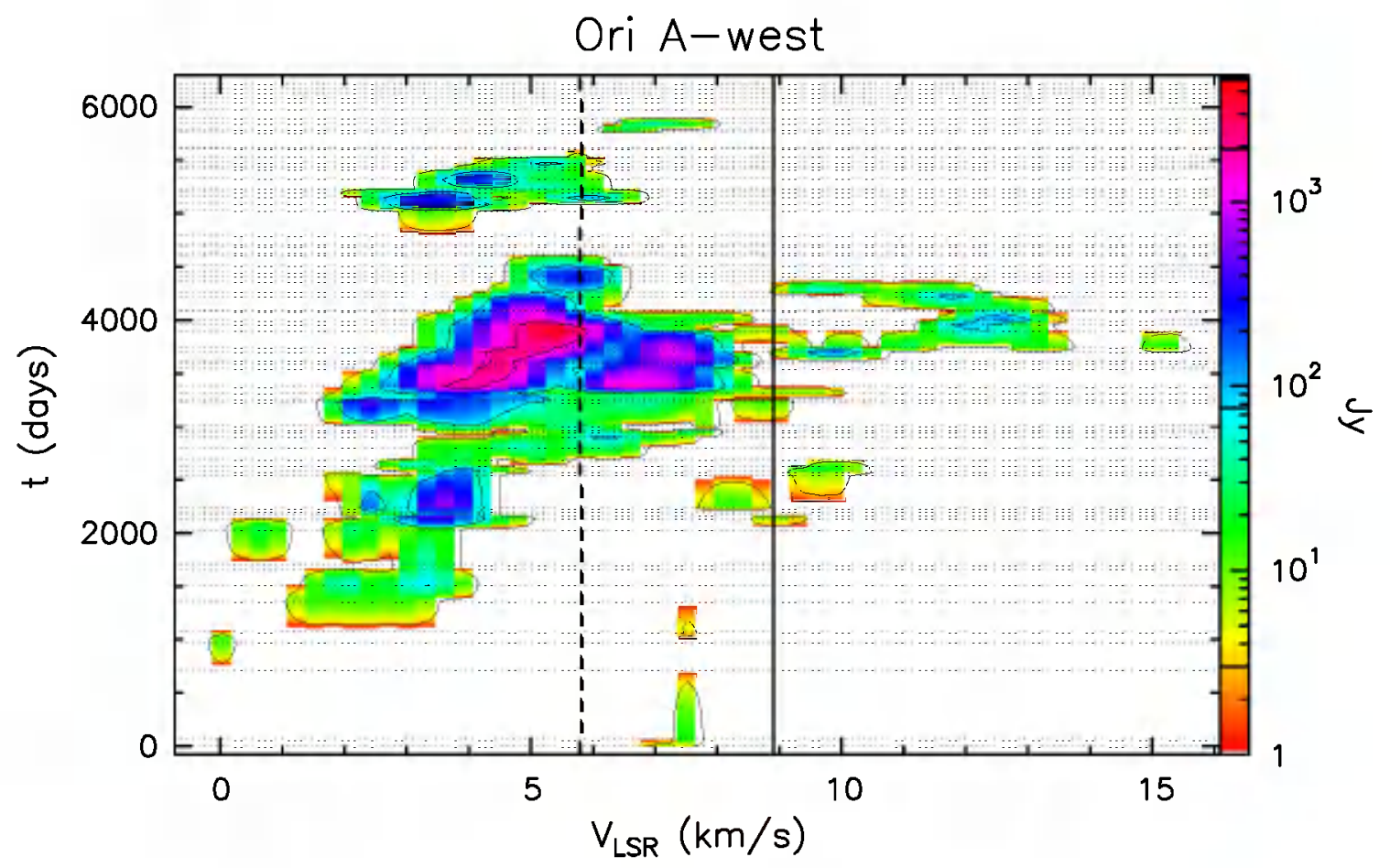

Fig. A.5. c Same as previous figure, but "zoomed" to velocity range over which emission has been detected. 


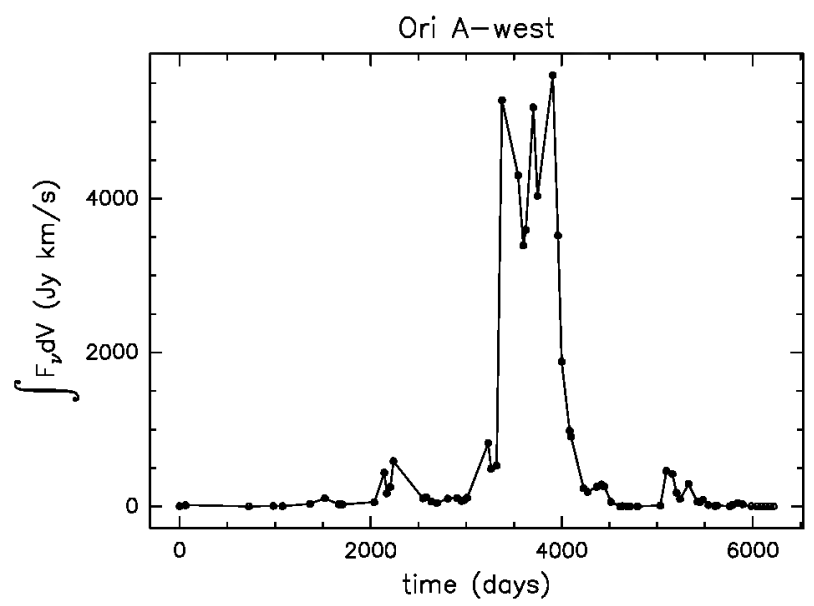

Fig. A.5. d Integral of the flux density over the observed velocity range as a function of time for source Ori A-west.

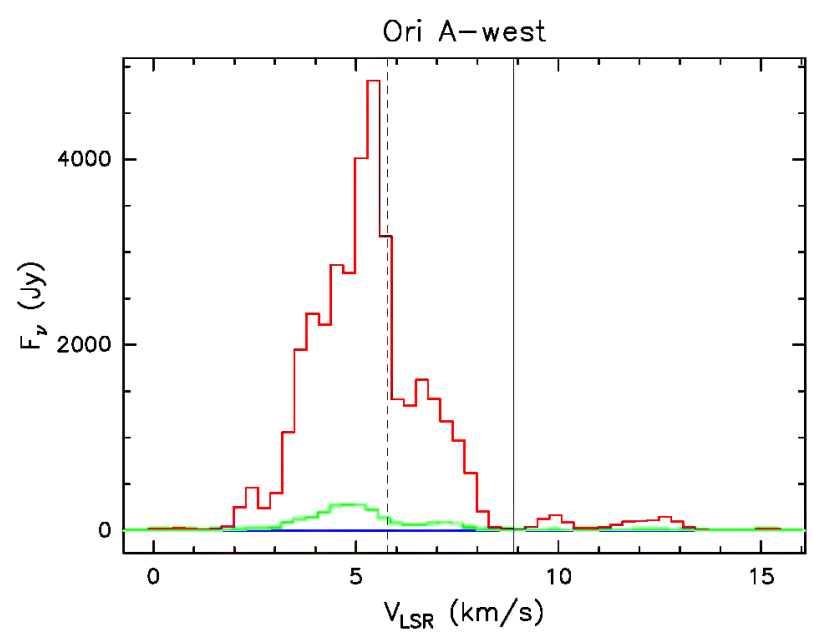

Fig. A.5. e Upper (red) and lower (blue) envelopes and mean spectrum (green) of source Ori A-west measured during our monitoring. The vertical solid line marks the velocity of the associated thermal molecular gas. The vertical dashed line marks the mean velocity derived from the histogram of the rate-of-occurrence.

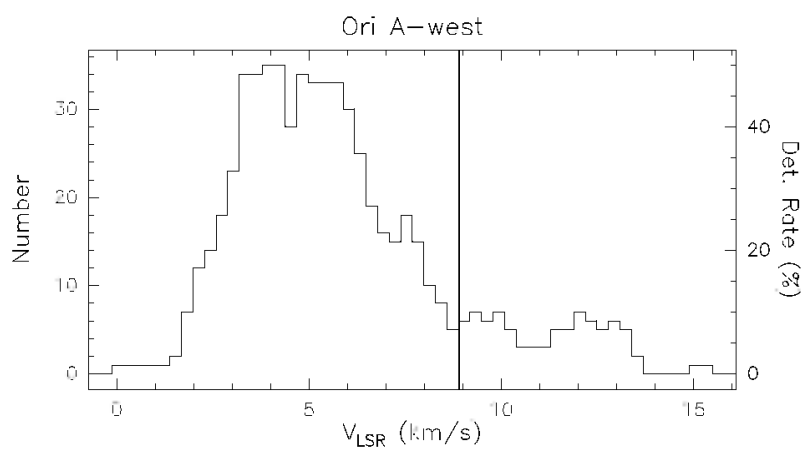

Fig. A.5. f Rate-of-occurrence plot for source Ori A-west. The scale to the right refer's to the dotted histogram, the scale to the left to the solid line histogram. The vertical solid line marks the velocity of the associated thermal molecular gas. 

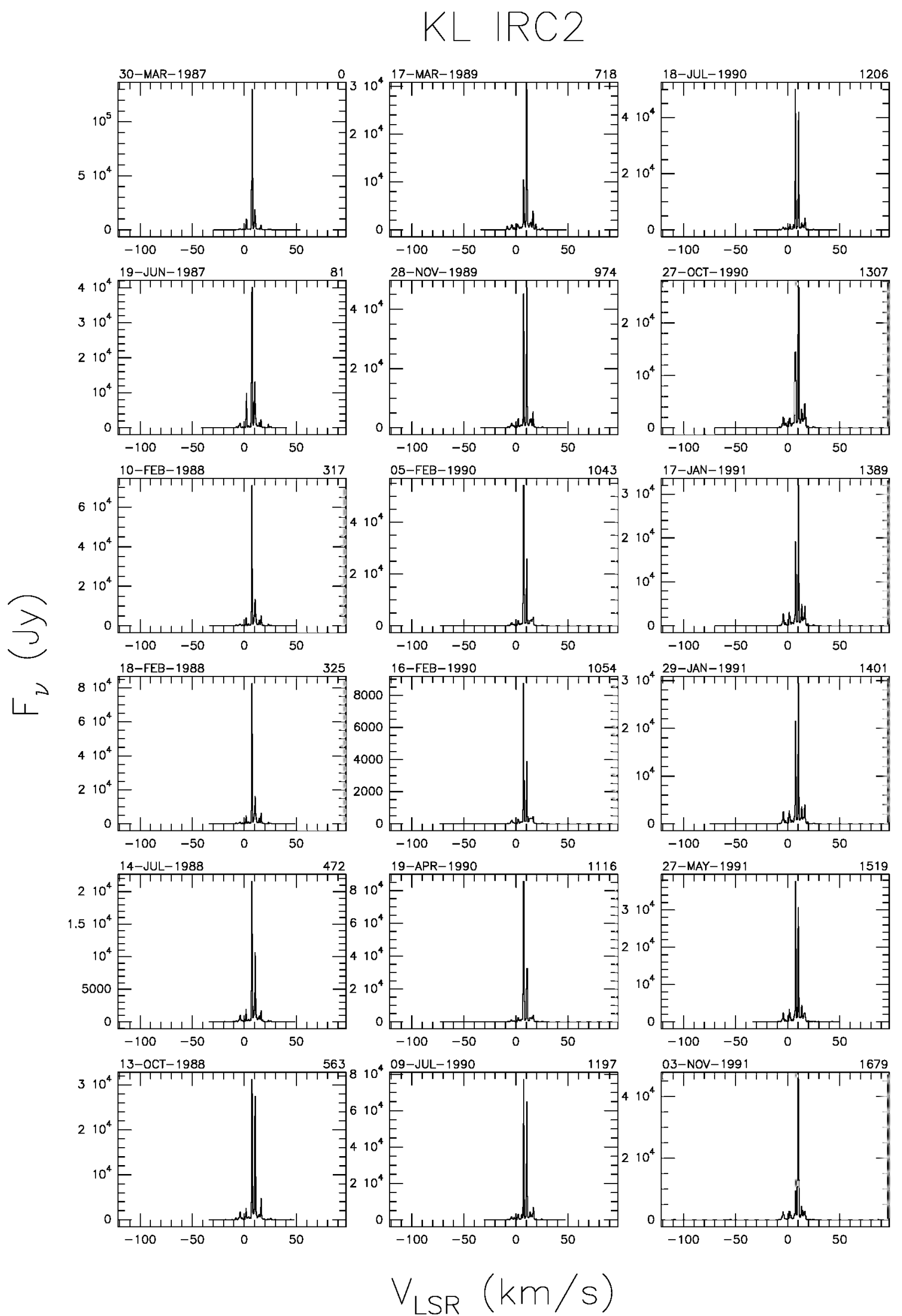

Fig. A.6. a Spectia of source KL IRC2 with antoscaled flux density scale. The date of observation is shown above the top left corner of each spectrum and the number of days elapsed since the first observation is given above the top right corner. The velocity scale is the same for all spectra. 


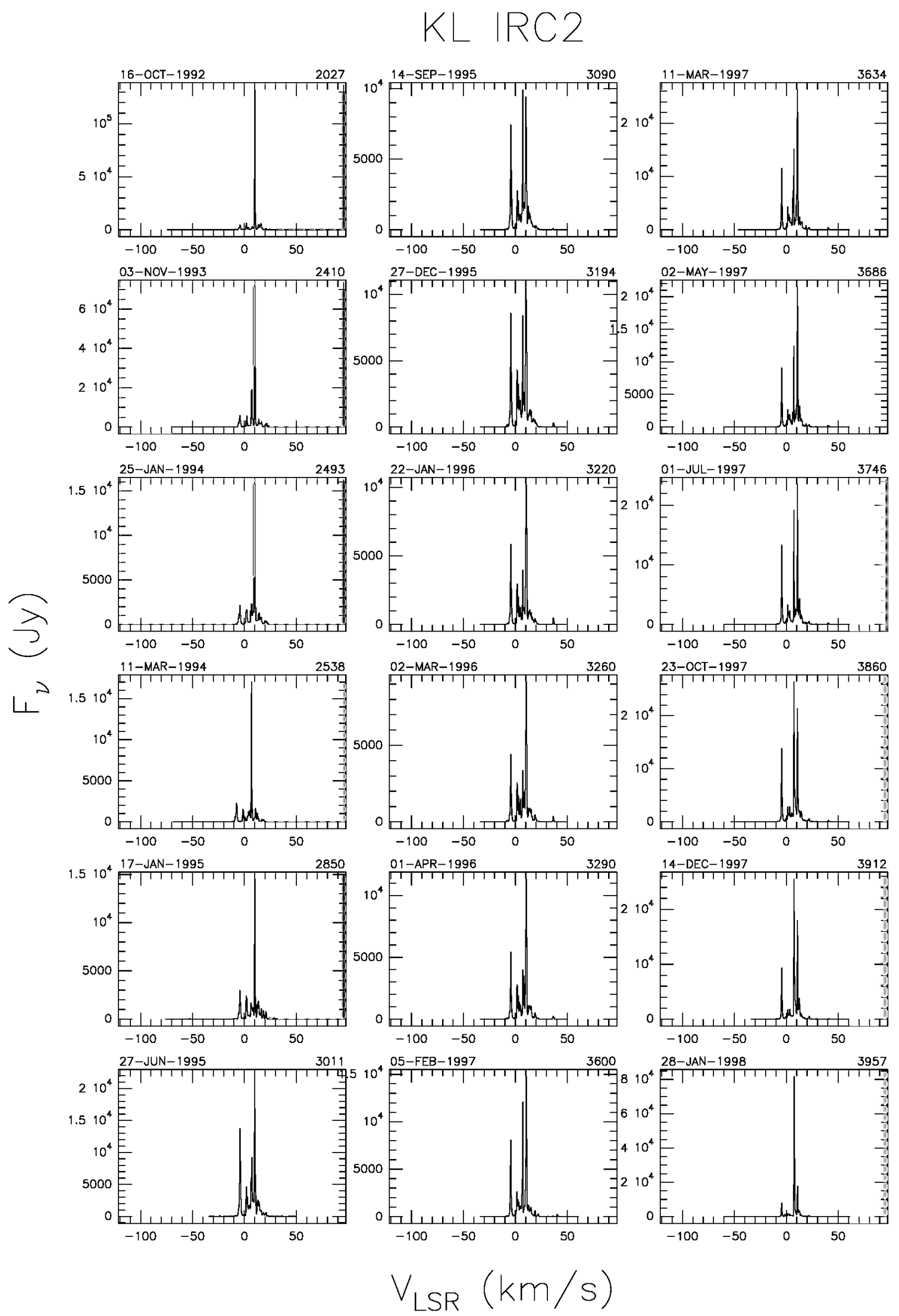

Fig. A.6. a continued. 


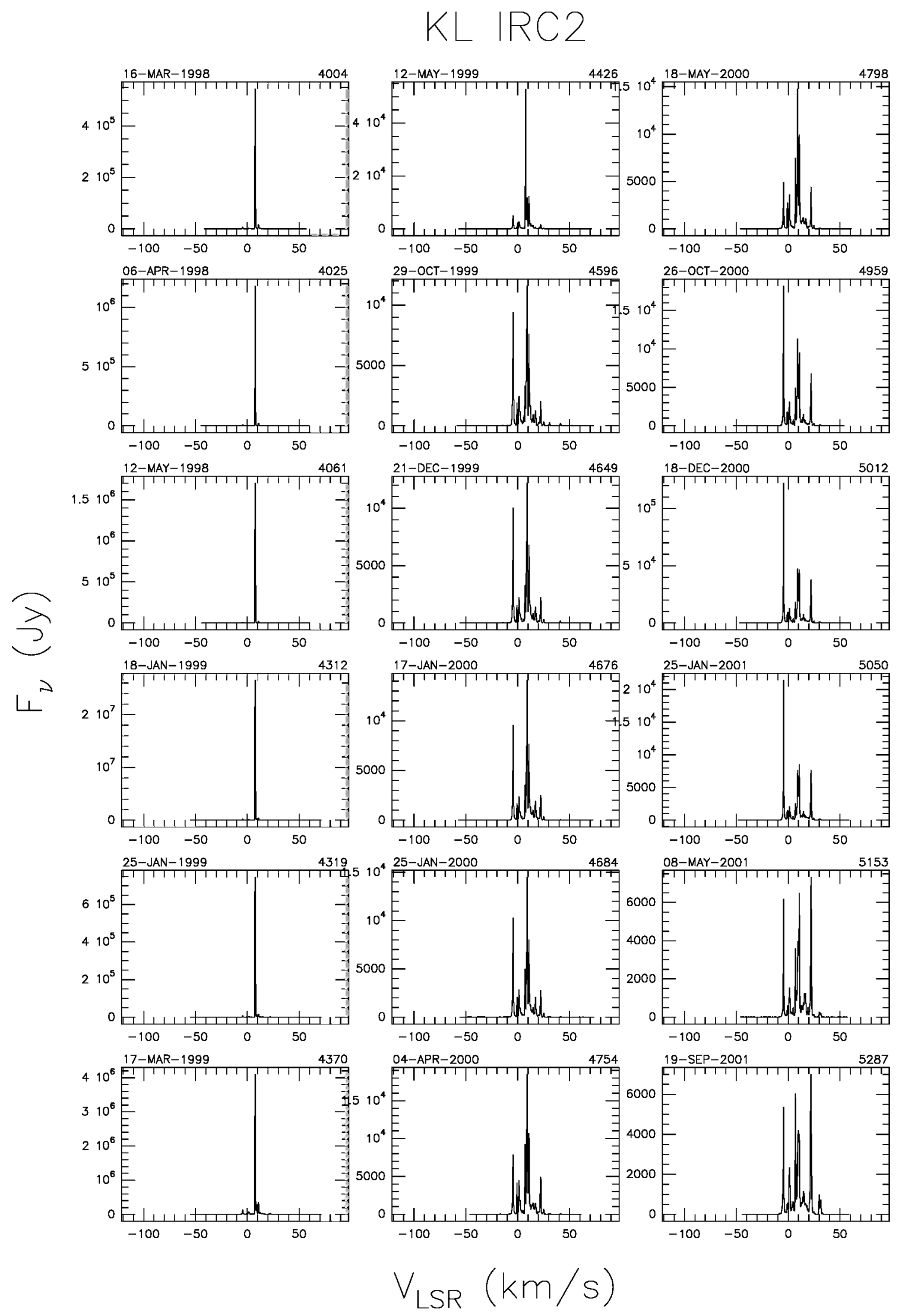

Fig. A.6. a continued. 


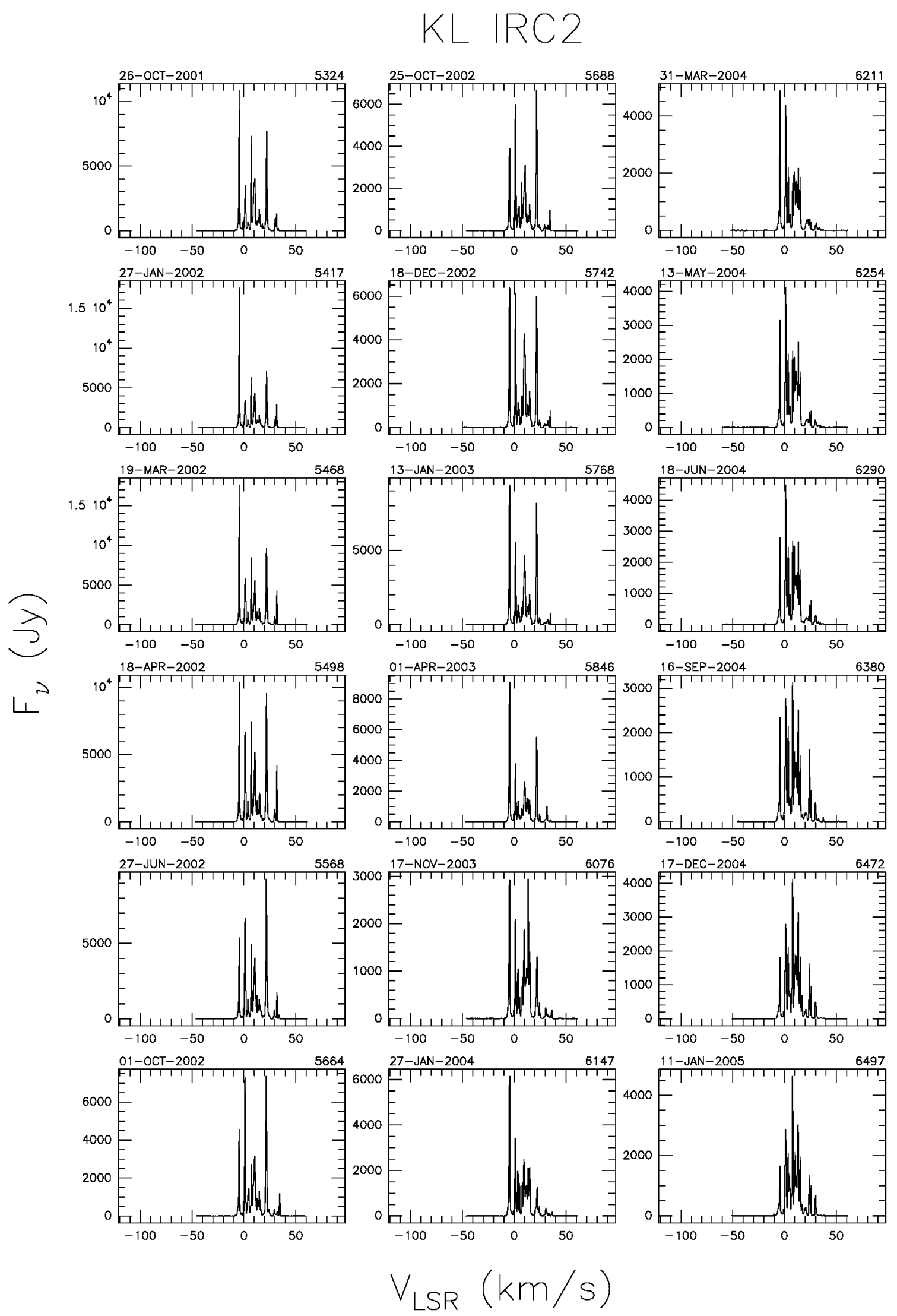

Fig. A.6. a continued. 

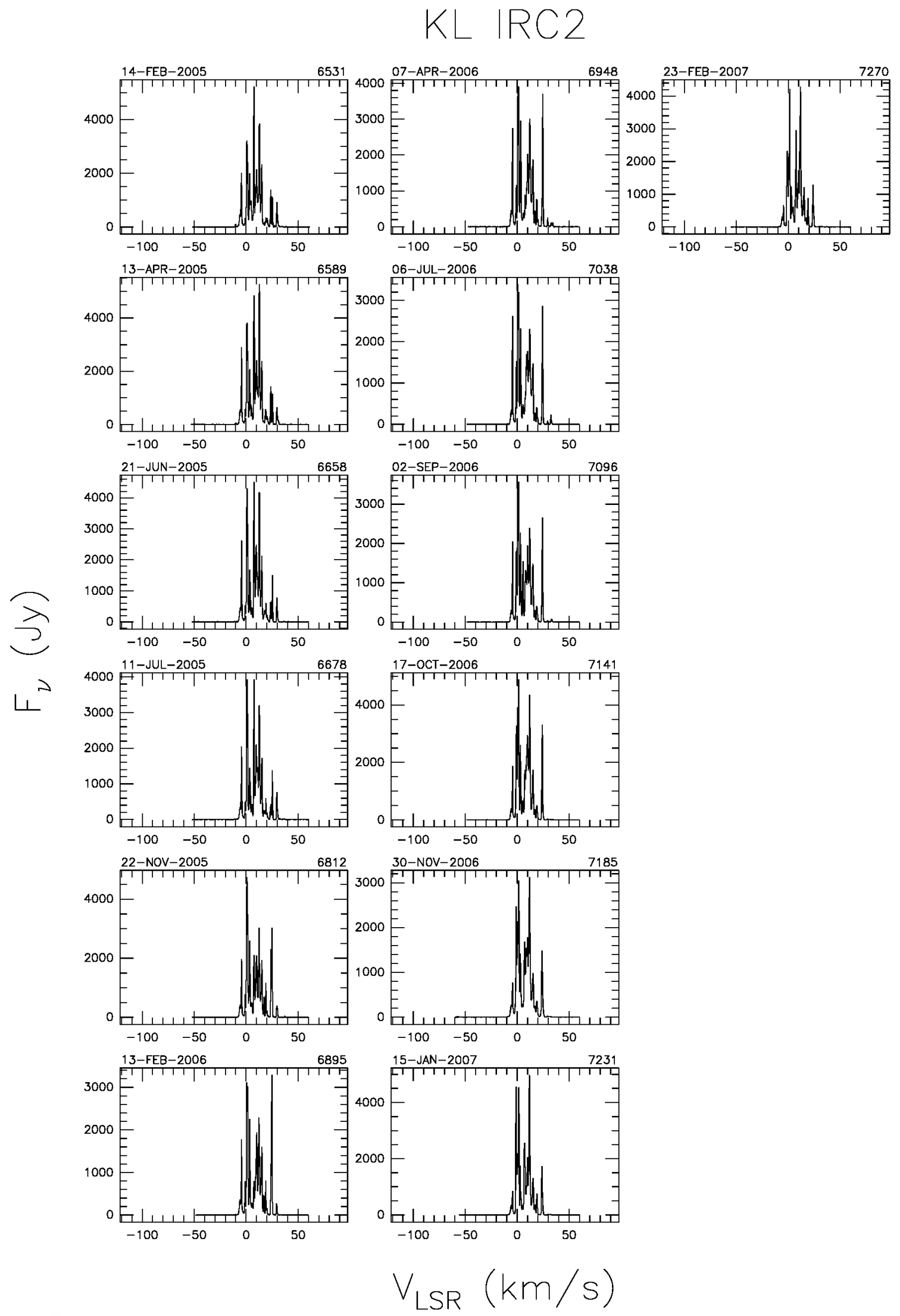

Fig. A.6. a continued. 


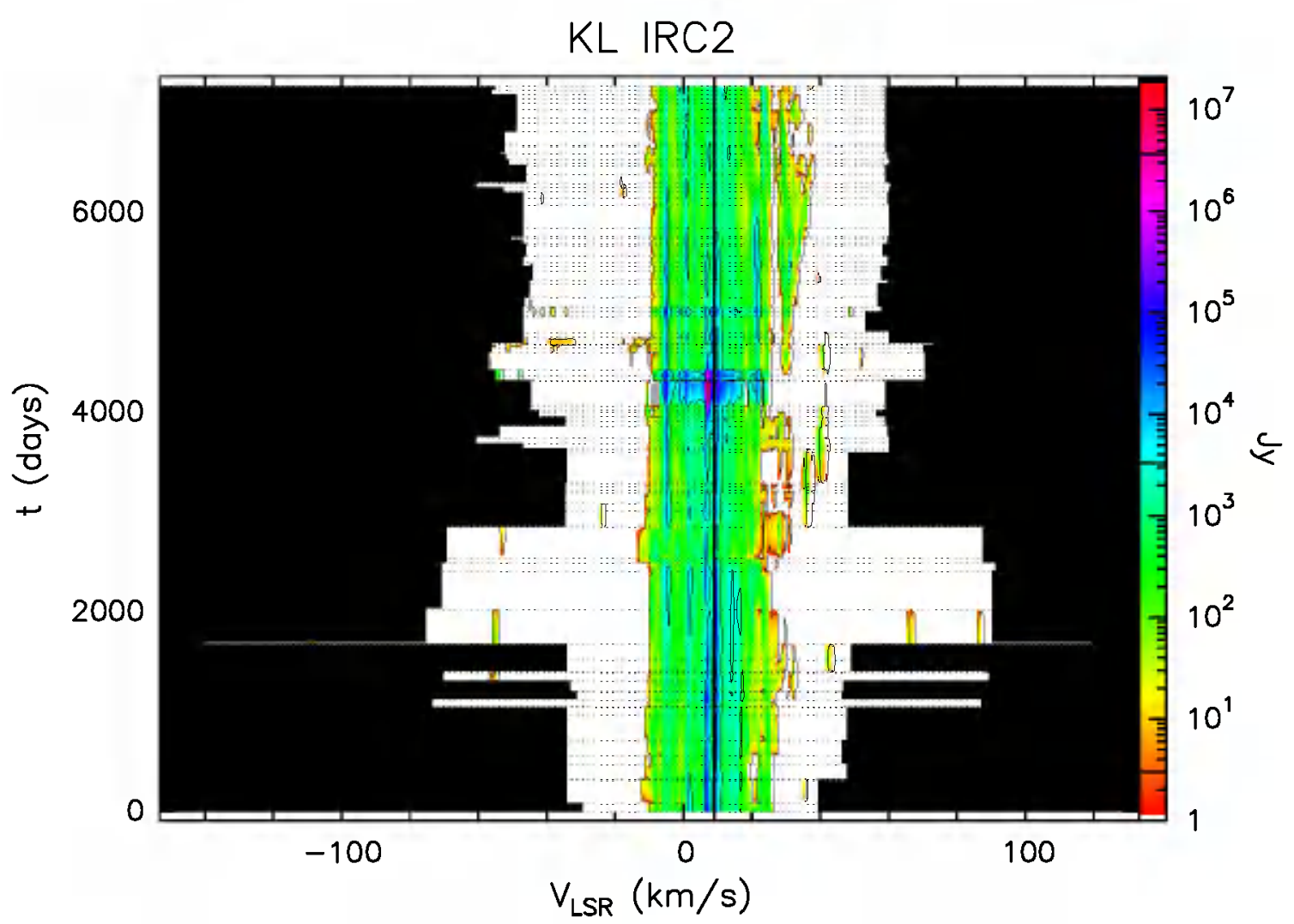

Fig. A.6. b Velocity-time-flux density full plot for source KL IRC2. The vertical solid line indicates the velocity of the associated thermal molecular gas. The flux density scale is shown by the bar on the right. In this bar the three lines give the flux density of the drawn contours.

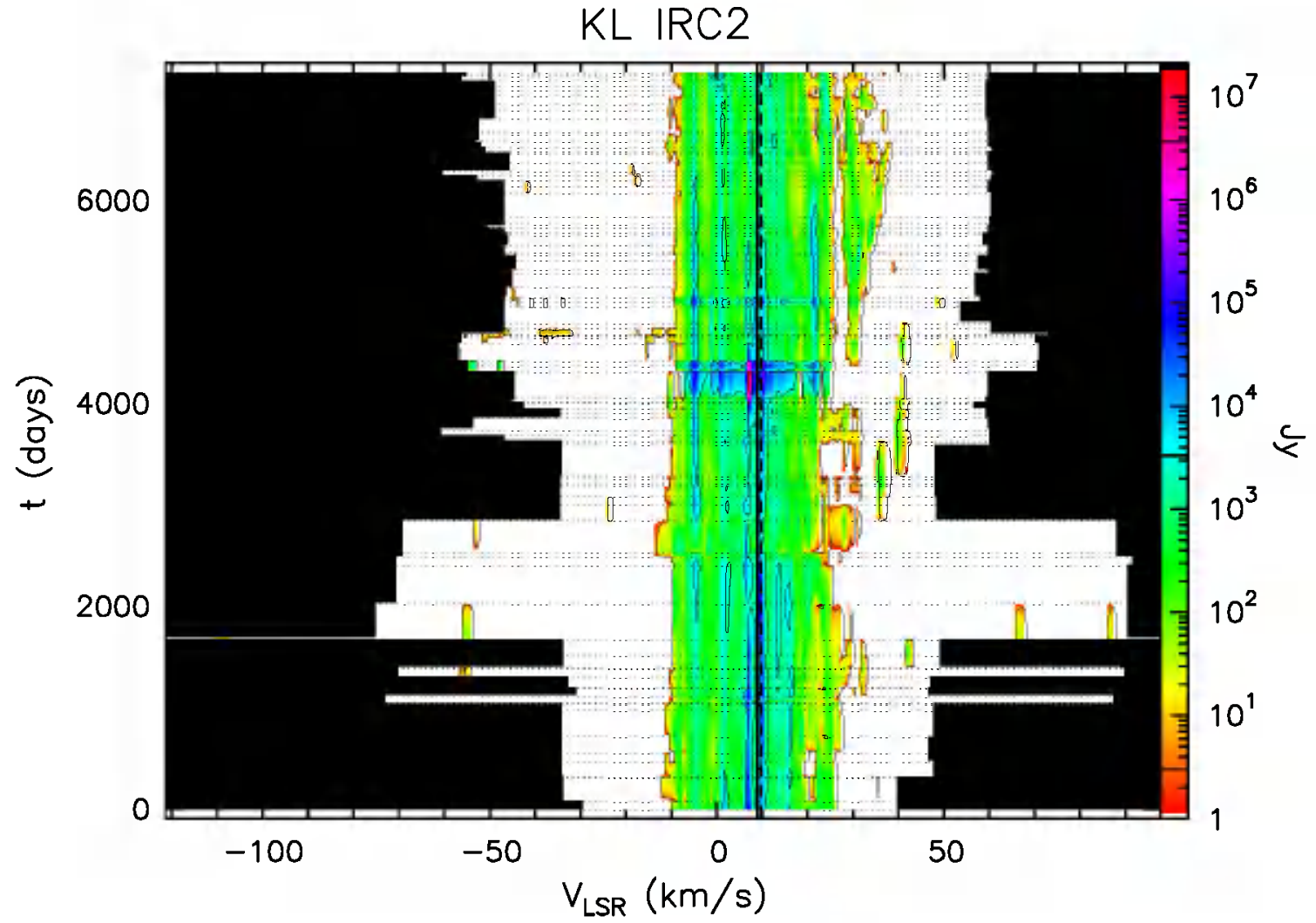

Fig. A.6. c Same as previous figure, but "zoomed" to velocity range over which emission has been detected. 


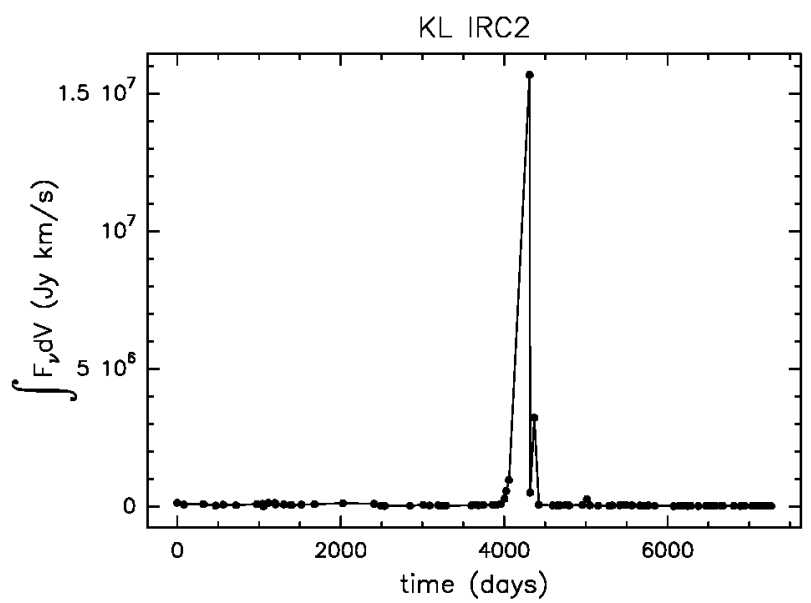

Fig. A.6. d Integral of the flux density over the observed velocity range as a function of time for source KL IRC2.

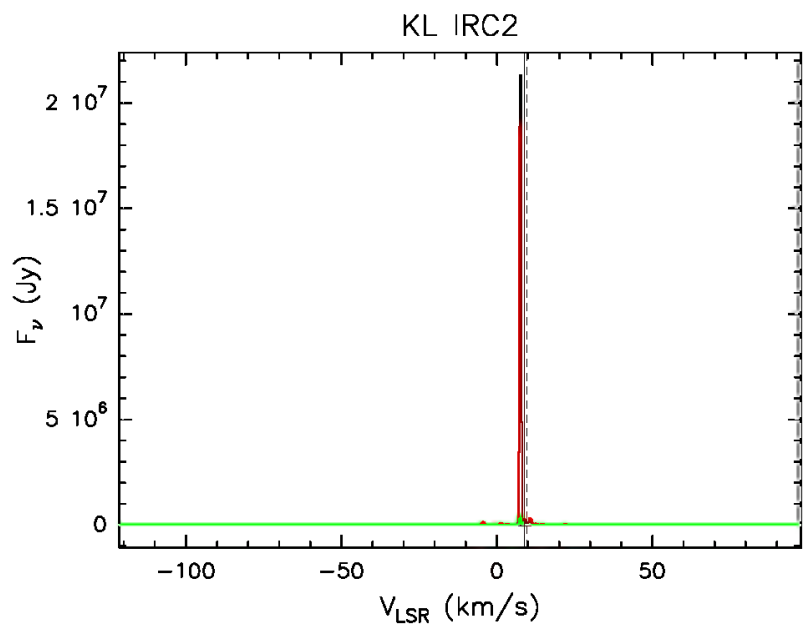

Fig. A.6. e Upper (red) and lower (blue) envelopes and mean spectrum (green) of source KL IRC2 measured during our monitoring. The vertical solid line marks the velocity of the associated thermal molecular gas. The vertical dashed line marks the mean velocity derived from the histogram of the rate-of-occurrence.

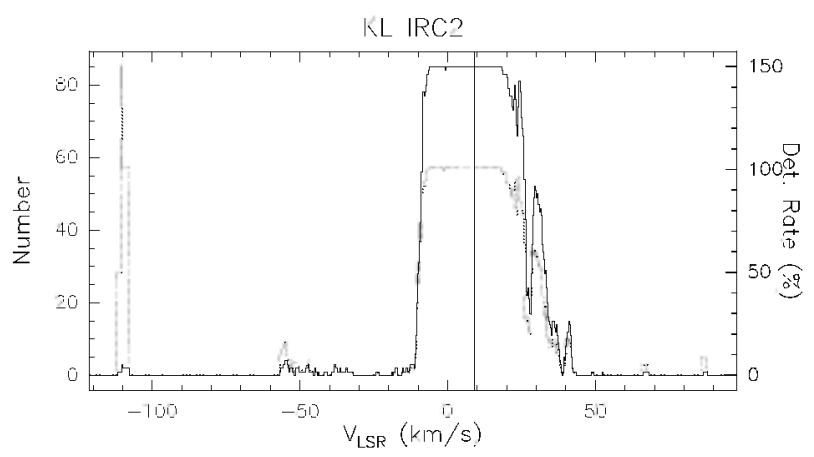

Fig. A.6. f Rate-of-occurrence plot for source KL IRC2. The scale to the right refers to the dotted histogram. the scale to the left to the solid line histogram. The vertical solid line marks the velocity of the associated thermal molecular gas. 


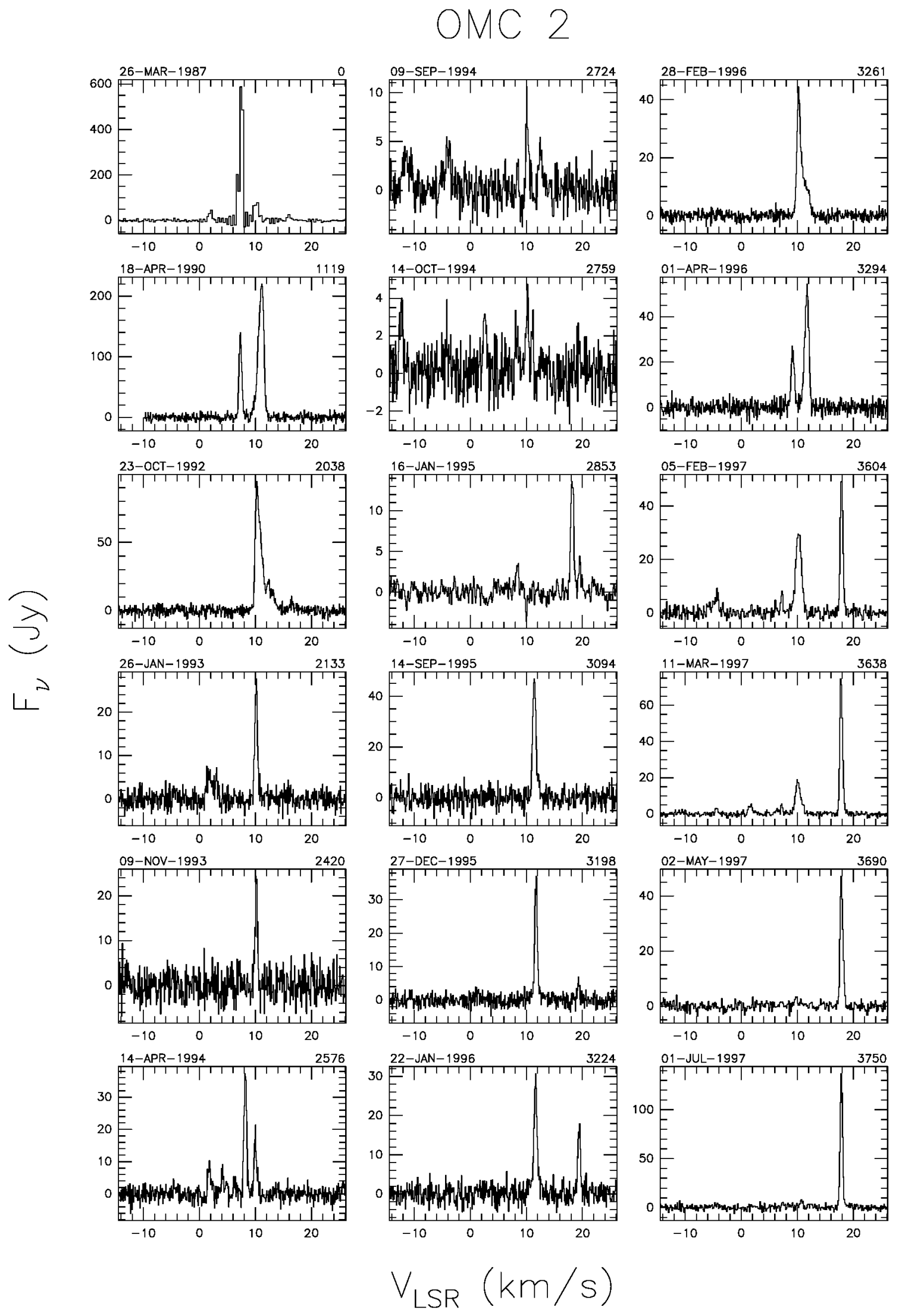

Fig. A.7. a Spectra of source OMC 2 with autoscaled flux density scale. The date of observation is shown above the top left corner of each spectrum and the number of days elapsed since the first observation is given above the top right corner. The velocity scale is the same for all spectra. 
OMC 2
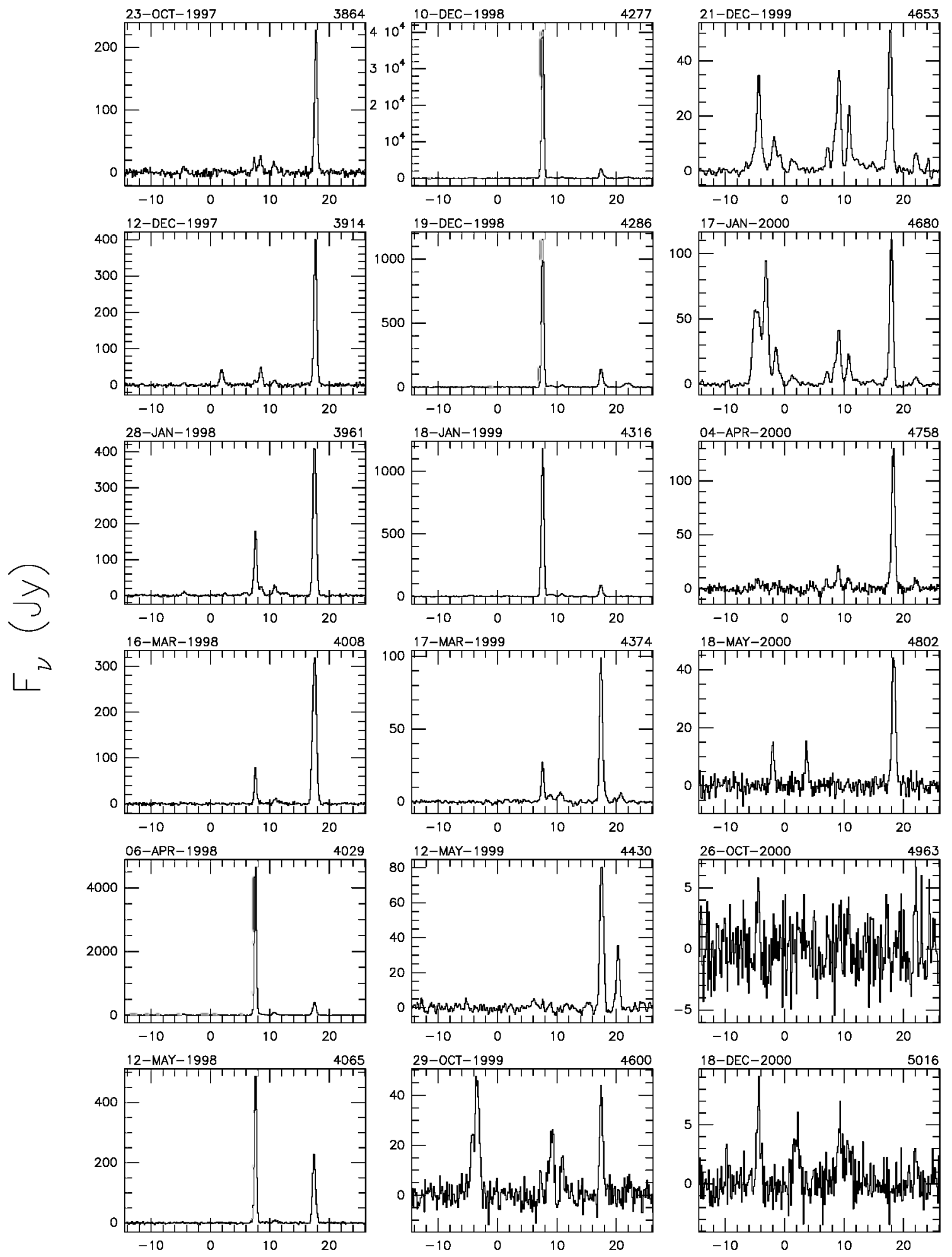

$$
V_{\text {LSR }}(\mathrm{km} / \mathrm{s})
$$

Fig. A.7. a continued. 
OMC 2
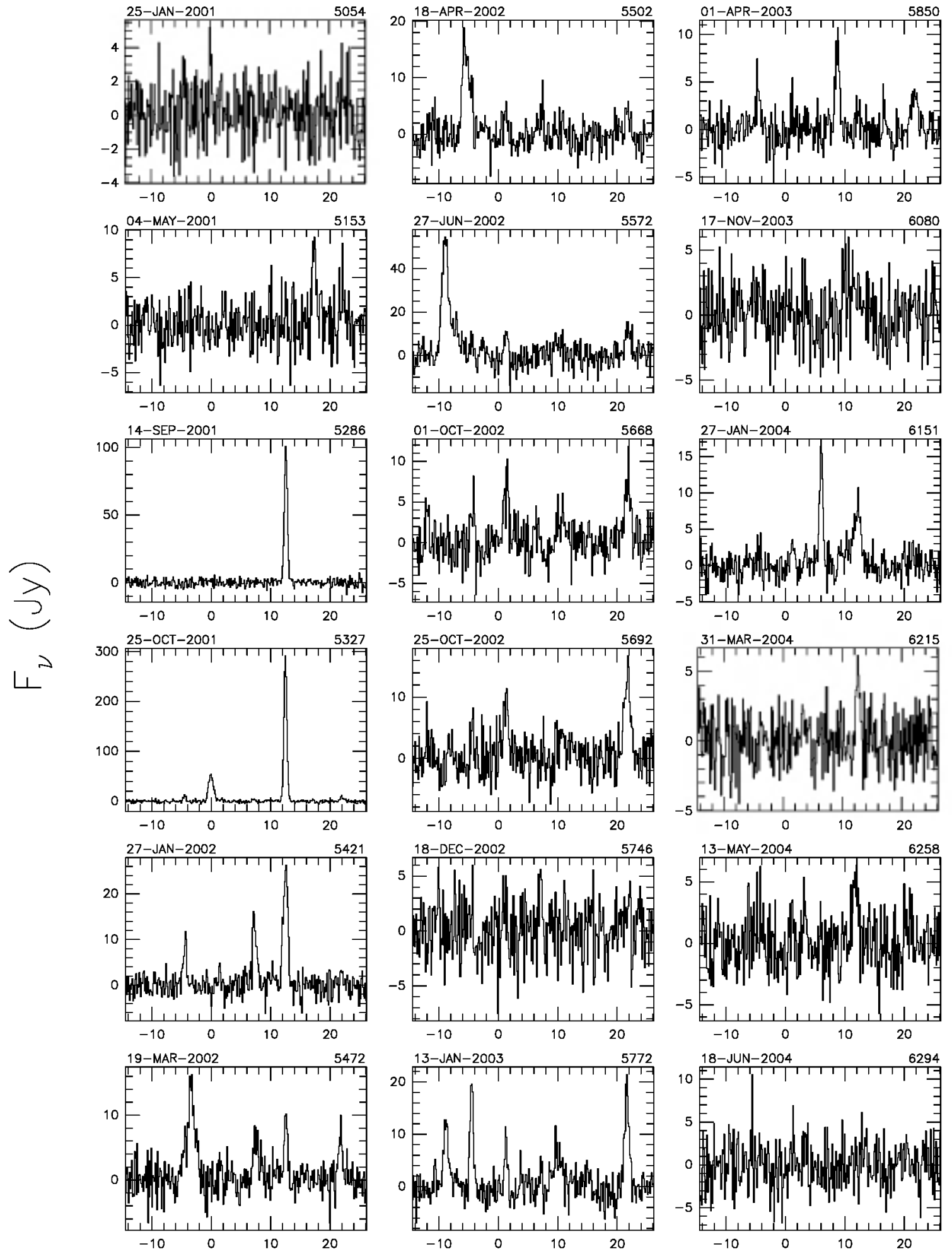

$$
V_{\text {LSR }}(\mathrm{km} / \mathrm{s})
$$

Fig. A.7. a continued. 
OMC 2
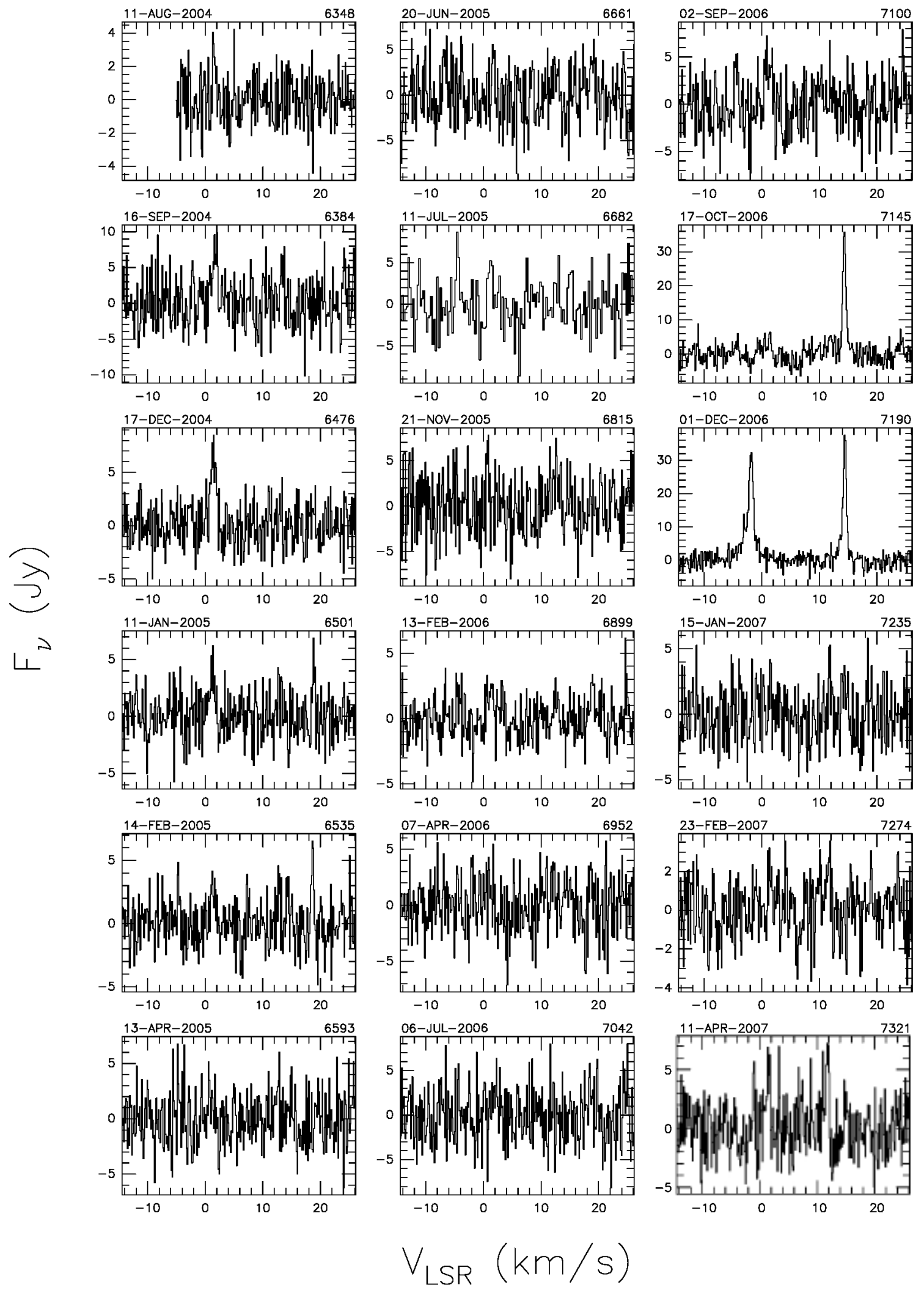

Fig. A.7. a continued. 


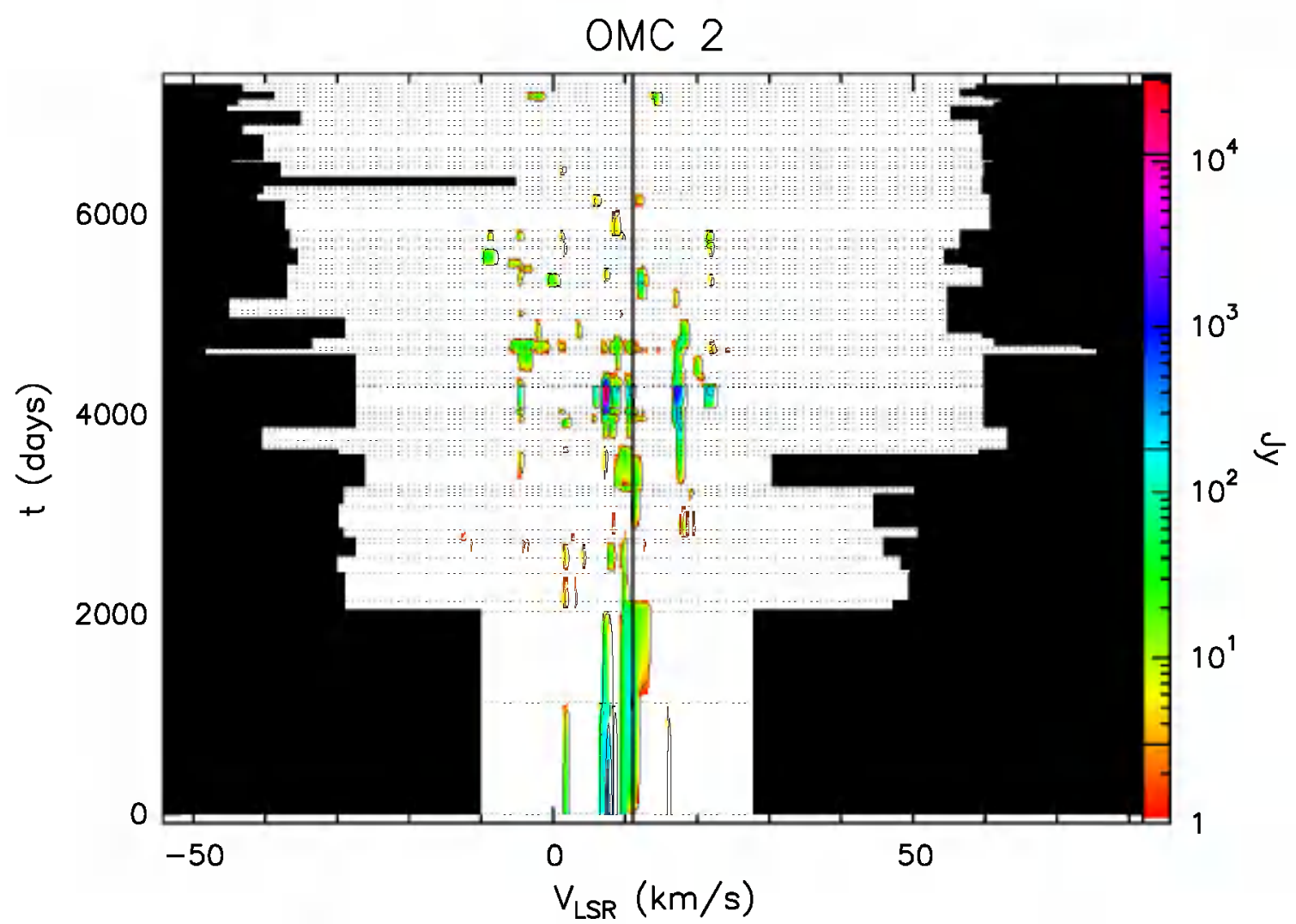

Fig. A.7. b Velocity-time-flux density full plot for source OMC 2. The vertical solid line indicates the velocity of the associated thermal molecular gas. The flux density scale is shown by the bar on the right. In this bar the three lines give the flux density of the drawn contours.

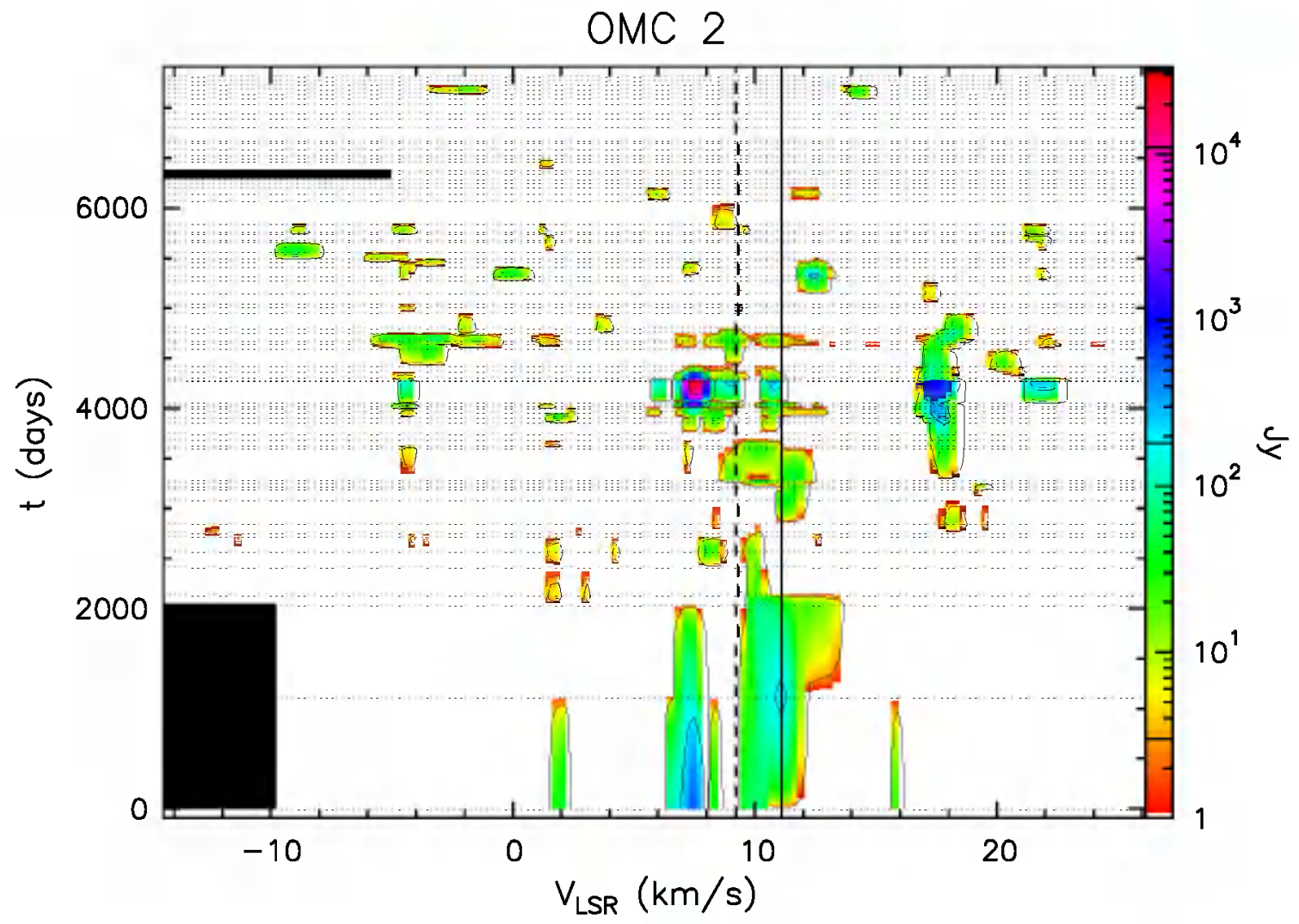

Fig. A.7. c Same as previous figure, but "zoomed" to velocity range over which emission has been detected. 


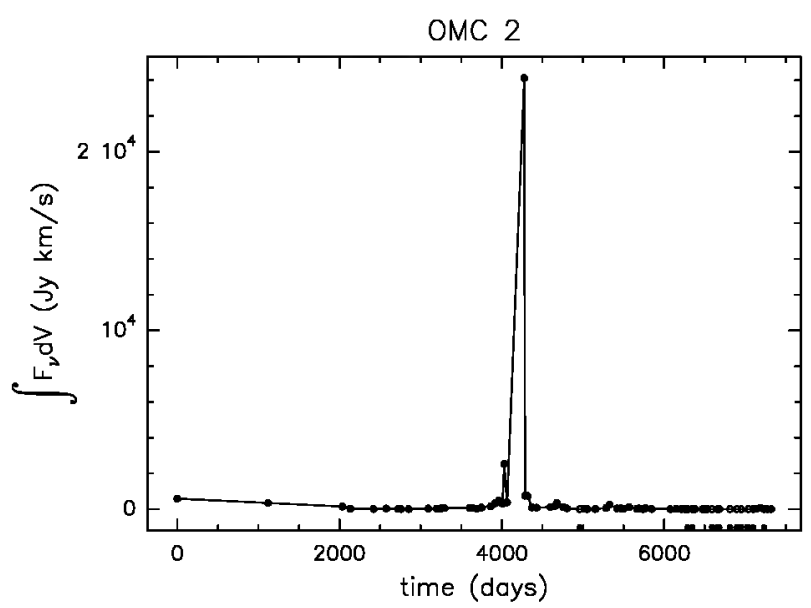

Fig. A.7. d Integial of the flux density over the observed velocity range as a function of time for source OMC 2 .

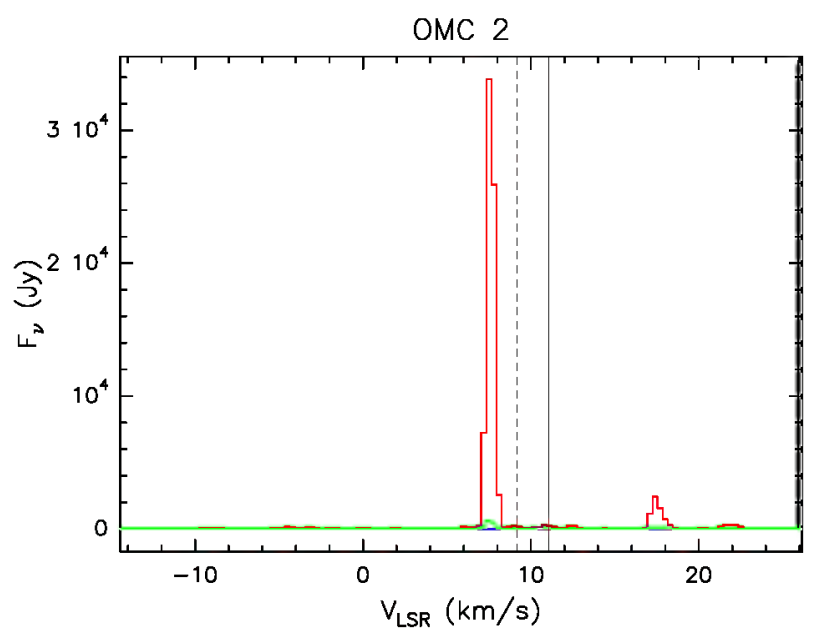

Fig. A.7. e Upper (red) and lower (blue) envelopes and mean spectrum (green) of source OMC 2 measured during our monitoring. The vertical solid line marks the velocity of the associated thermal molecular gas. The vertical dashed line marks the mean velocity derived from the histogram of the rate-of-occurrence.

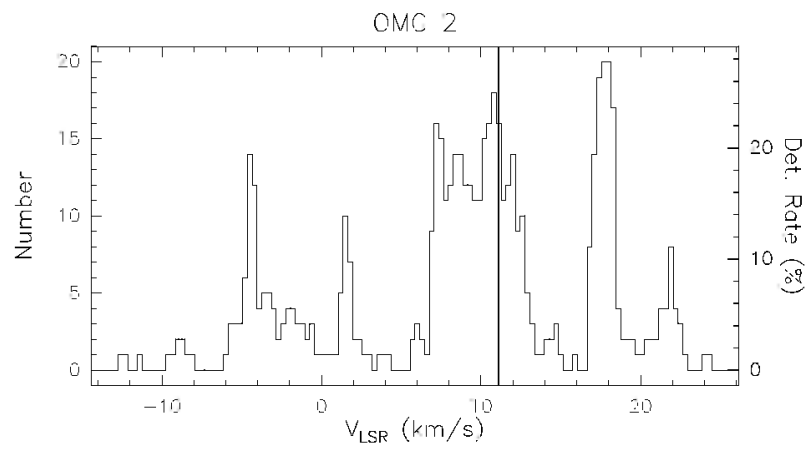

Fig. A.7. f Rate-of-occurrence plot for source OMC 2. The scale to the right refers to the dotted histogram, the scale to the left to the solid line histogram. The vertical solid line marks the velocity of the associated thermal molecular gas. 
Sh 2-231
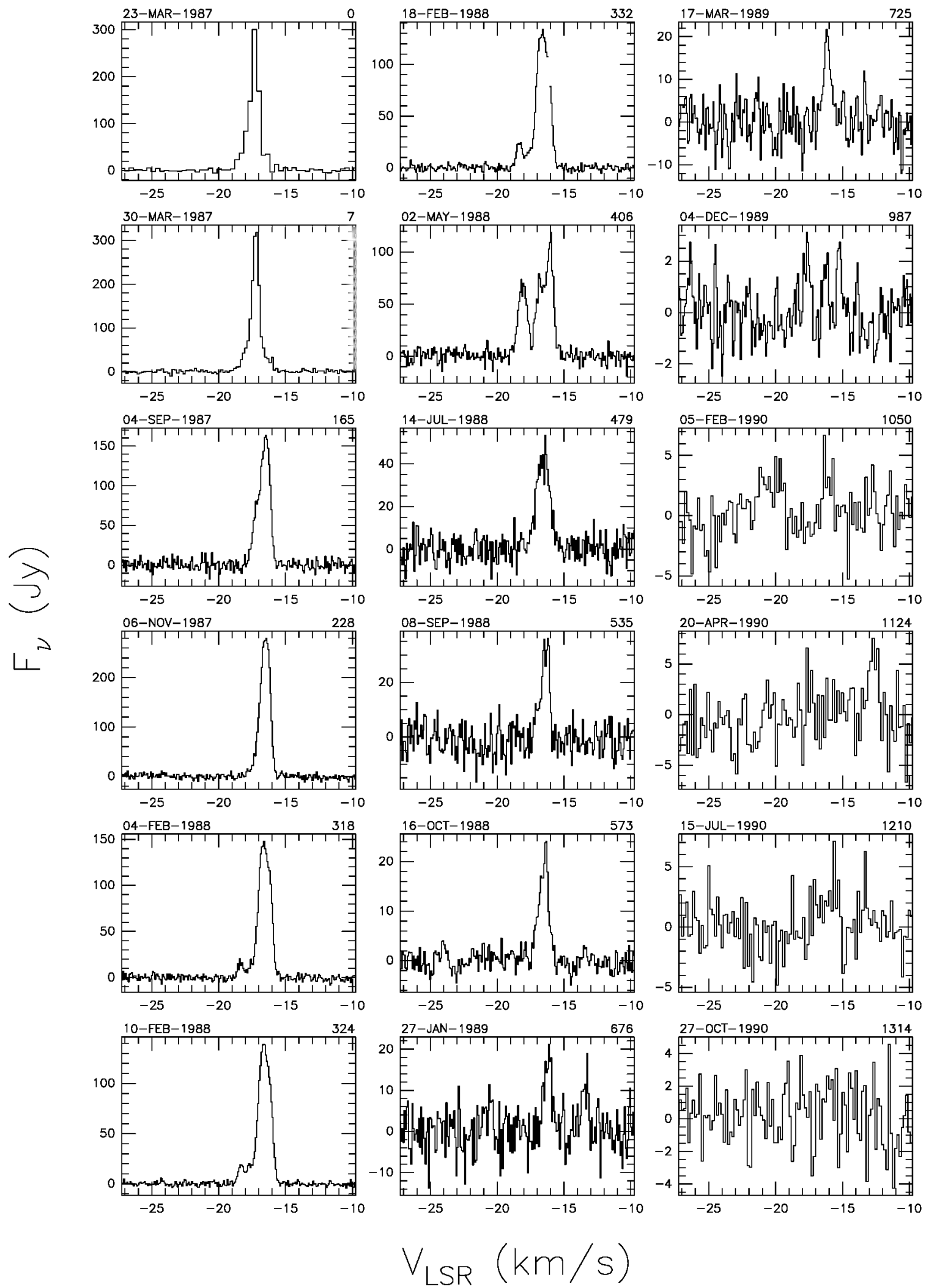

Fig. A.8. a Spectra of source Sh 2-231 with autoscaled flux density scale. The date of observation is shown above the top left corner of each spectrum and the number of days elapsed since the first observation is given above the top right corner. The velocity scale is the same for all spectra. 
Sh 2-231
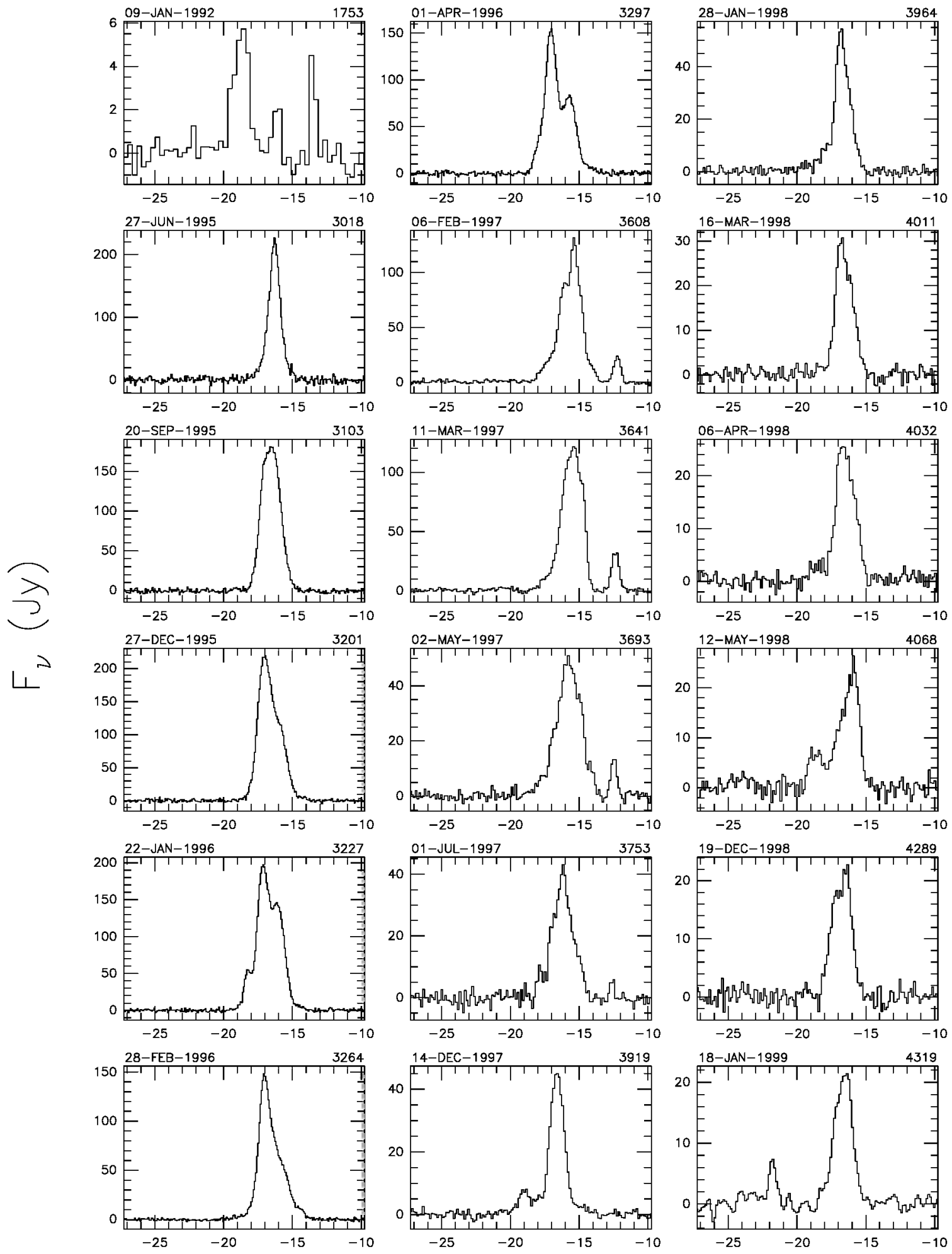

$$
V_{\text {LSR }}(\mathrm{km} / \mathrm{s})
$$

Fig. A.8. a continued. 
Sh 2-231
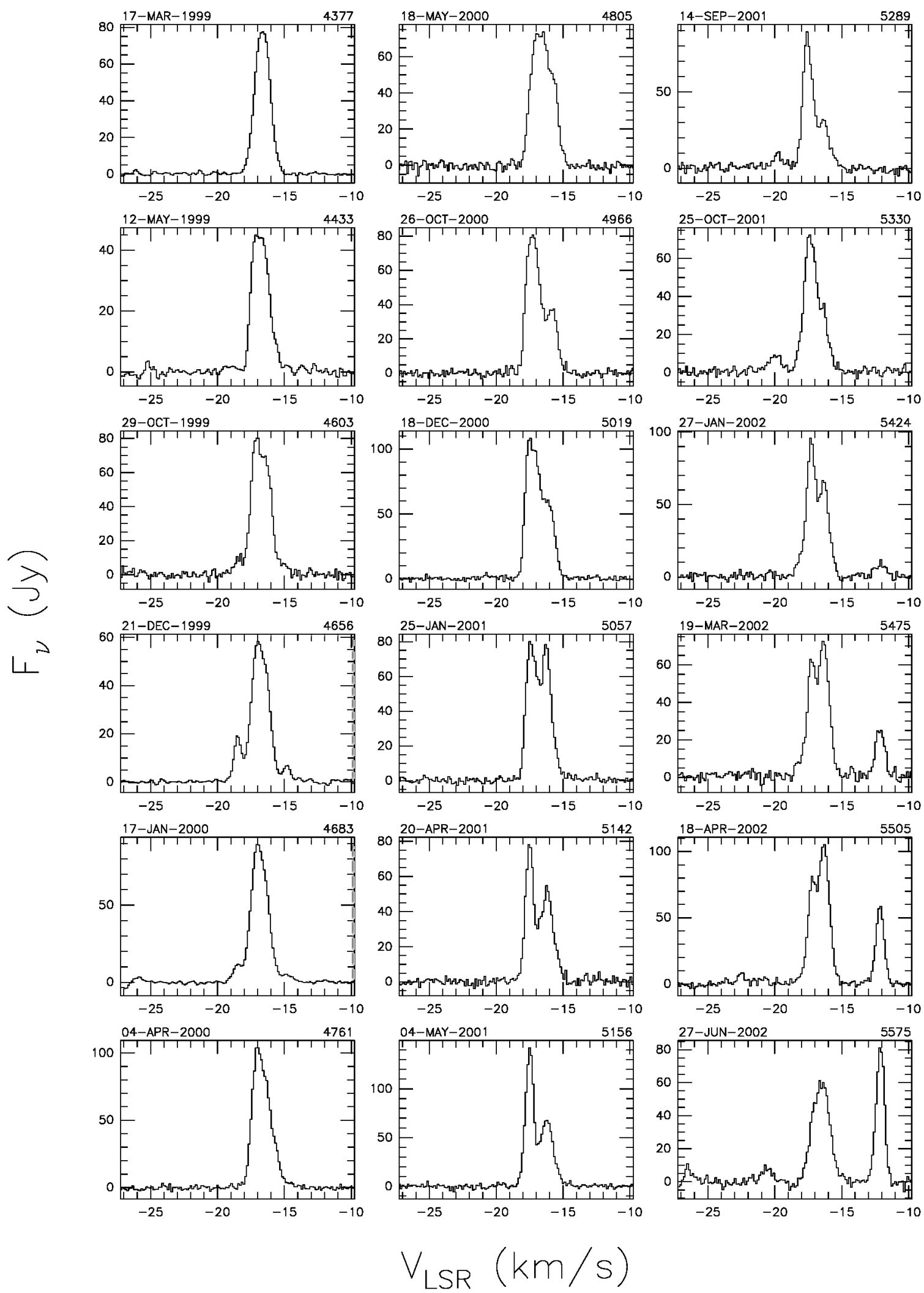

Fig. A.8. a continued. 
Sh 2-231
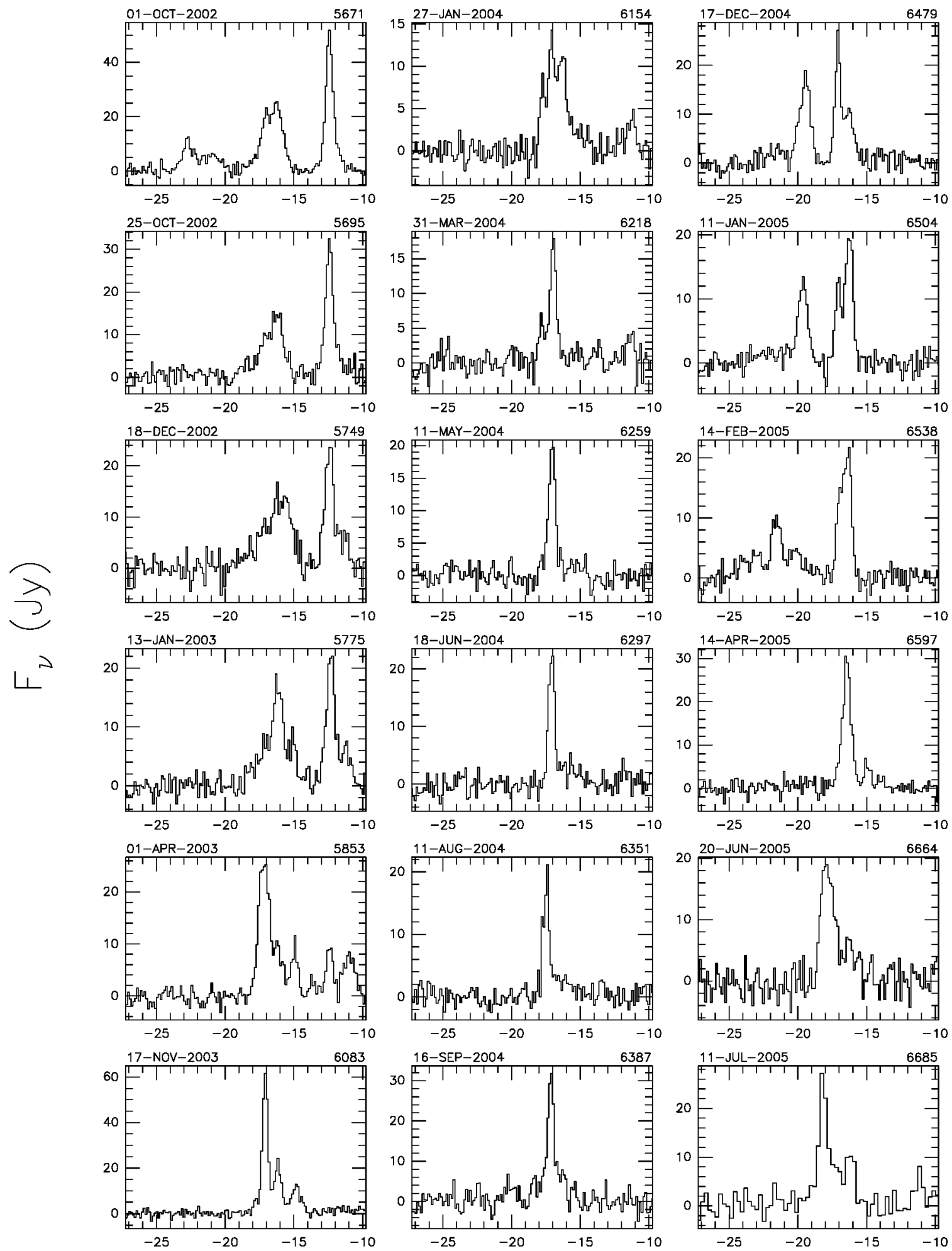

$$
V_{\text {LSR }}(\mathrm{km} / \mathrm{s})
$$

Fig. A.8. a continued. 

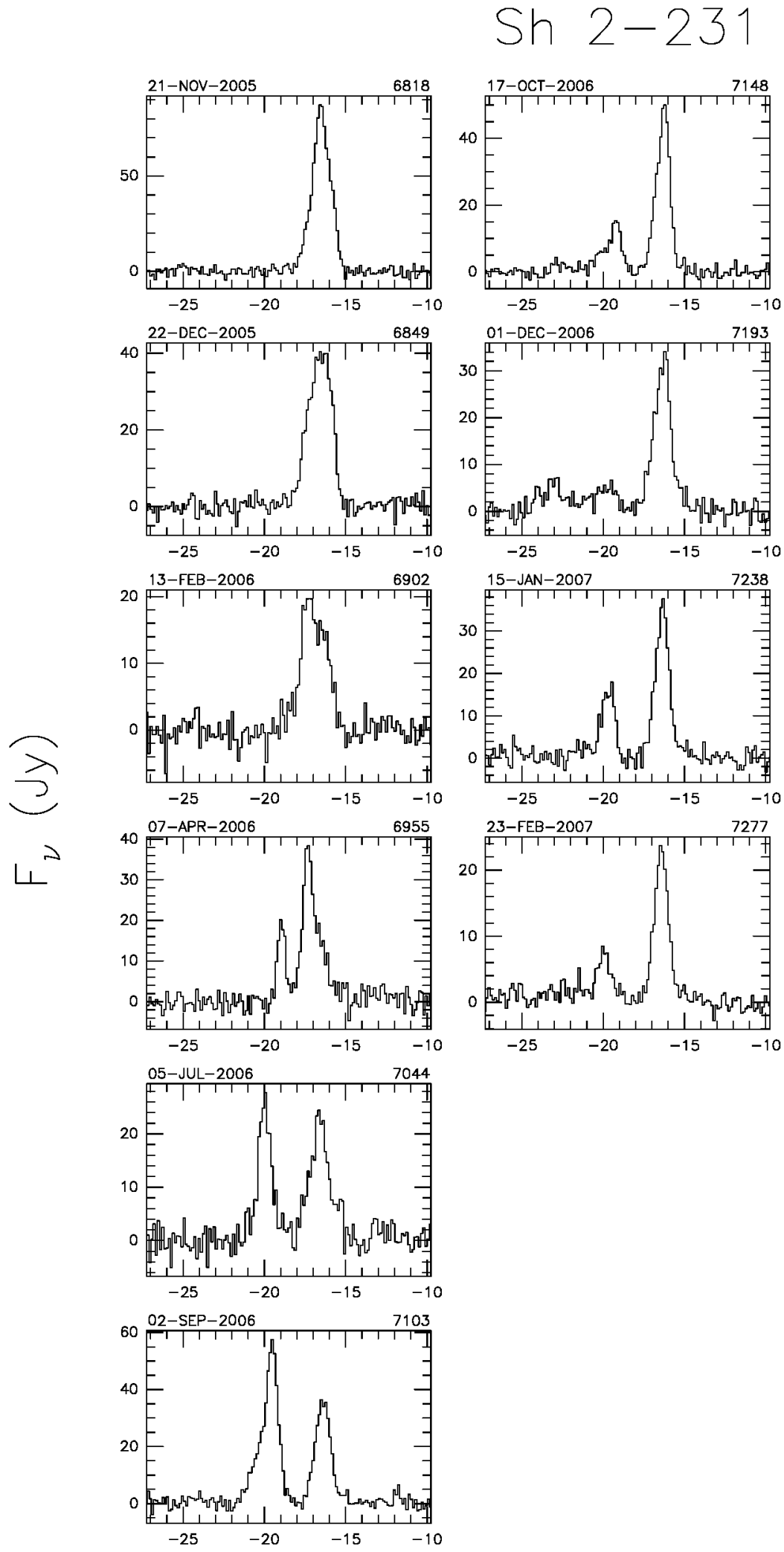

$$
V_{\text {LSR }}(\mathrm{km} / \mathrm{s})
$$

Fig. A.8. a continued. 


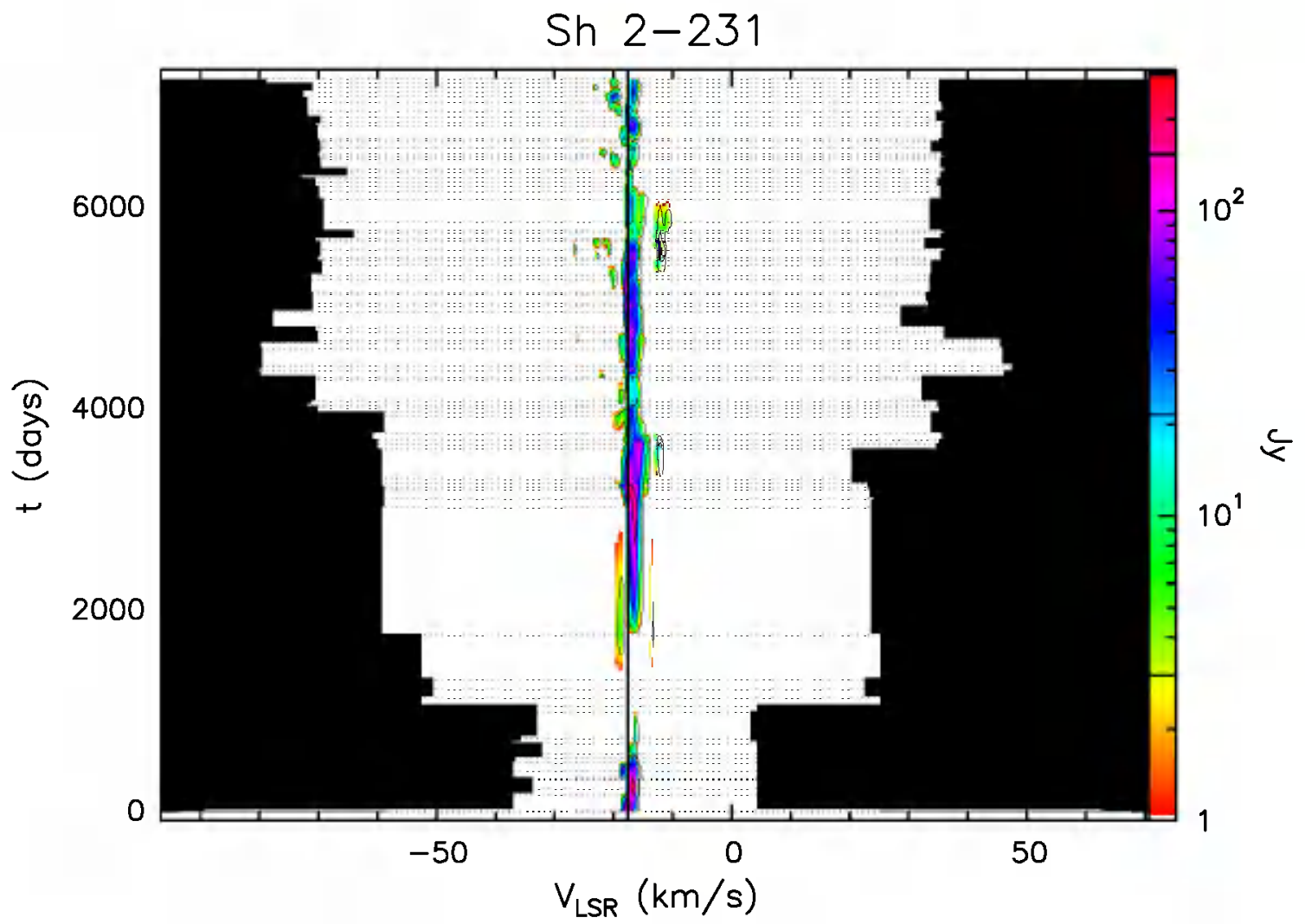

Fig. A.8. b Velocity-time-flux density full plot for source Sh 2-231. The vertical solid line indicates the velocity of the associated thermal molecular gas. The flux density scale is shown by the bar on the right. In this bar the three lines give the flux density of the drawn contours.

Sh $2-231$

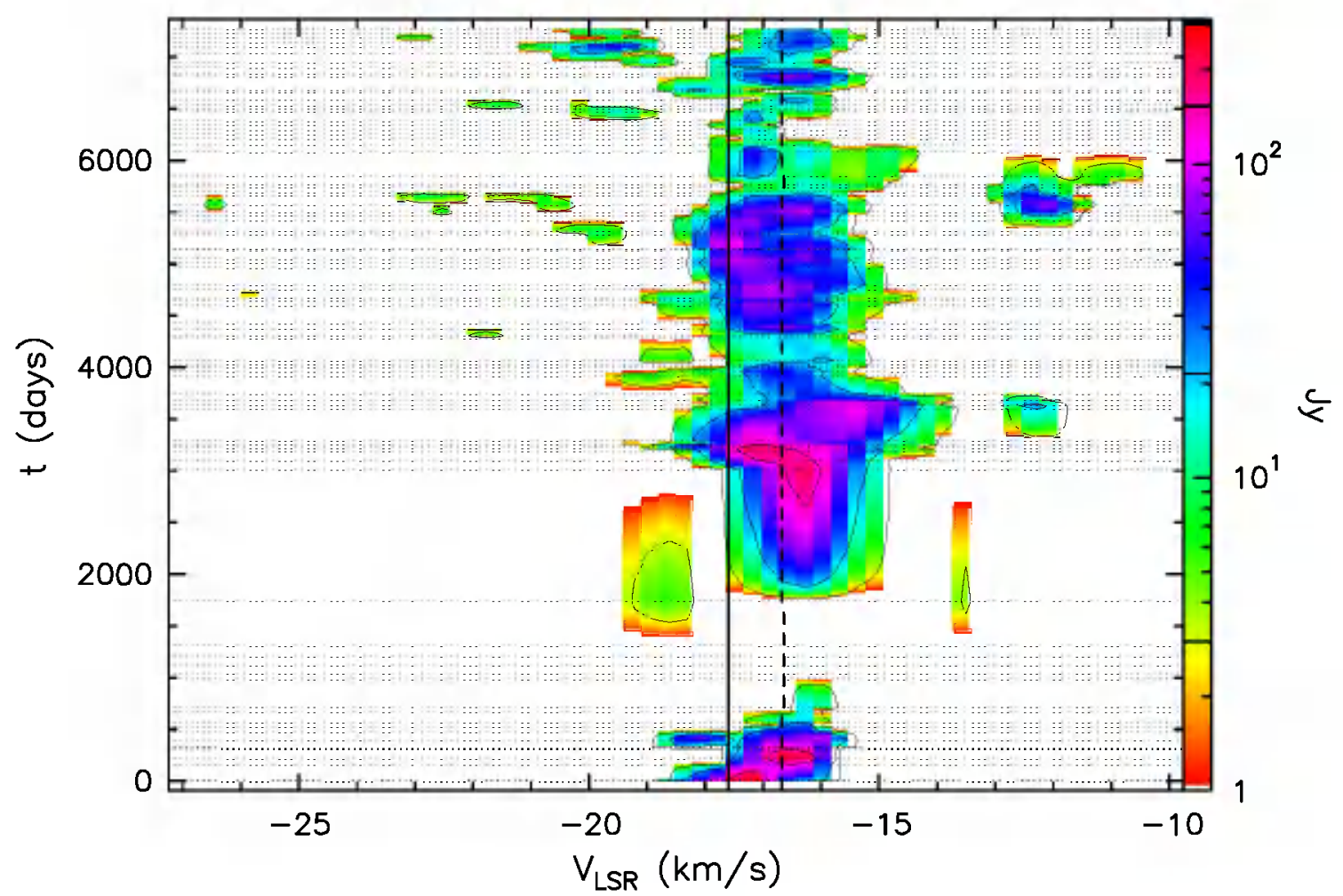

Fig. A.8. c Same as previous figure, but "zoomed" to velocity range over which emission has been detected. 


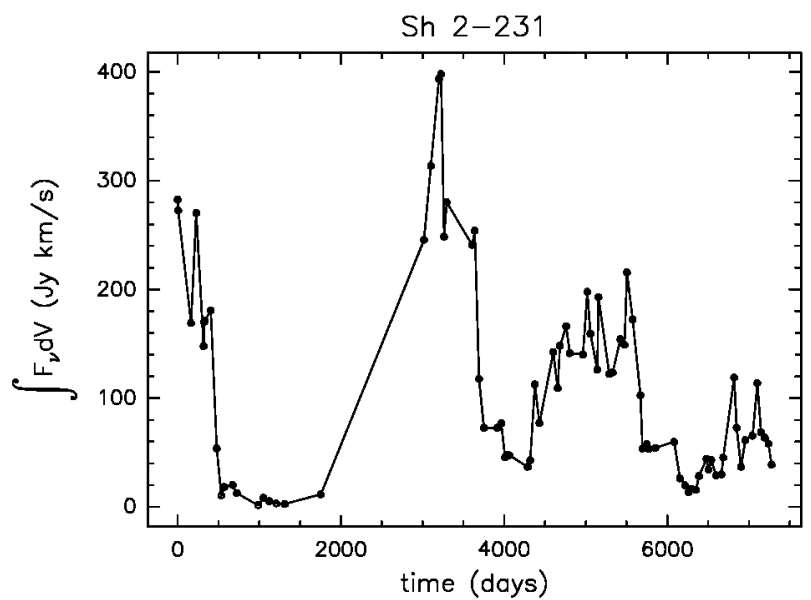

Fig. A.8. $d$ Integral of the flux density over the observed velocity range as a function of time for source Sh 2-231.

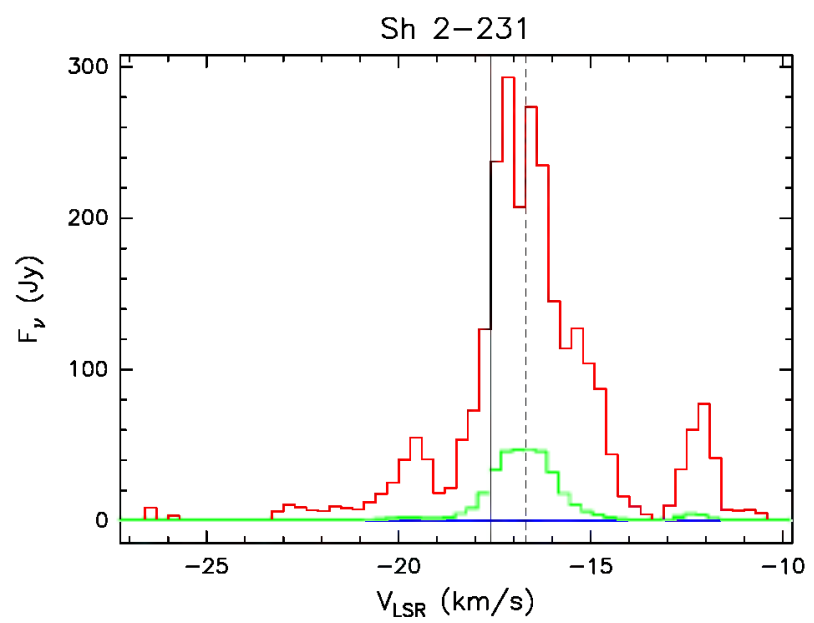

Fig. A.8. e Upper (red) and lower (blue) envelopes and mean spectrum (green) of source Sh 2-231 measured during our monitoring. The vertical solid line marks the velocity of the associated thermal molecular gas. The vertical dashed line marks the mean velocity derived from the histogram of the rate-of-occurrence.

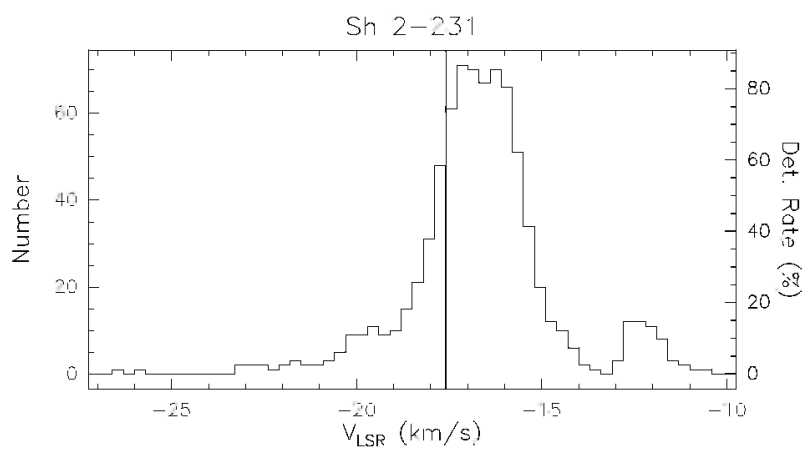

Fig. A.8. $f$ Rate-of-occurrence plot for source Sh 2-231. The scale to the right refers to the dotted histogram. the scale to the left to the solid line histogram. The vertical solid line marks the velocity of the associated thermal molecular gas. 


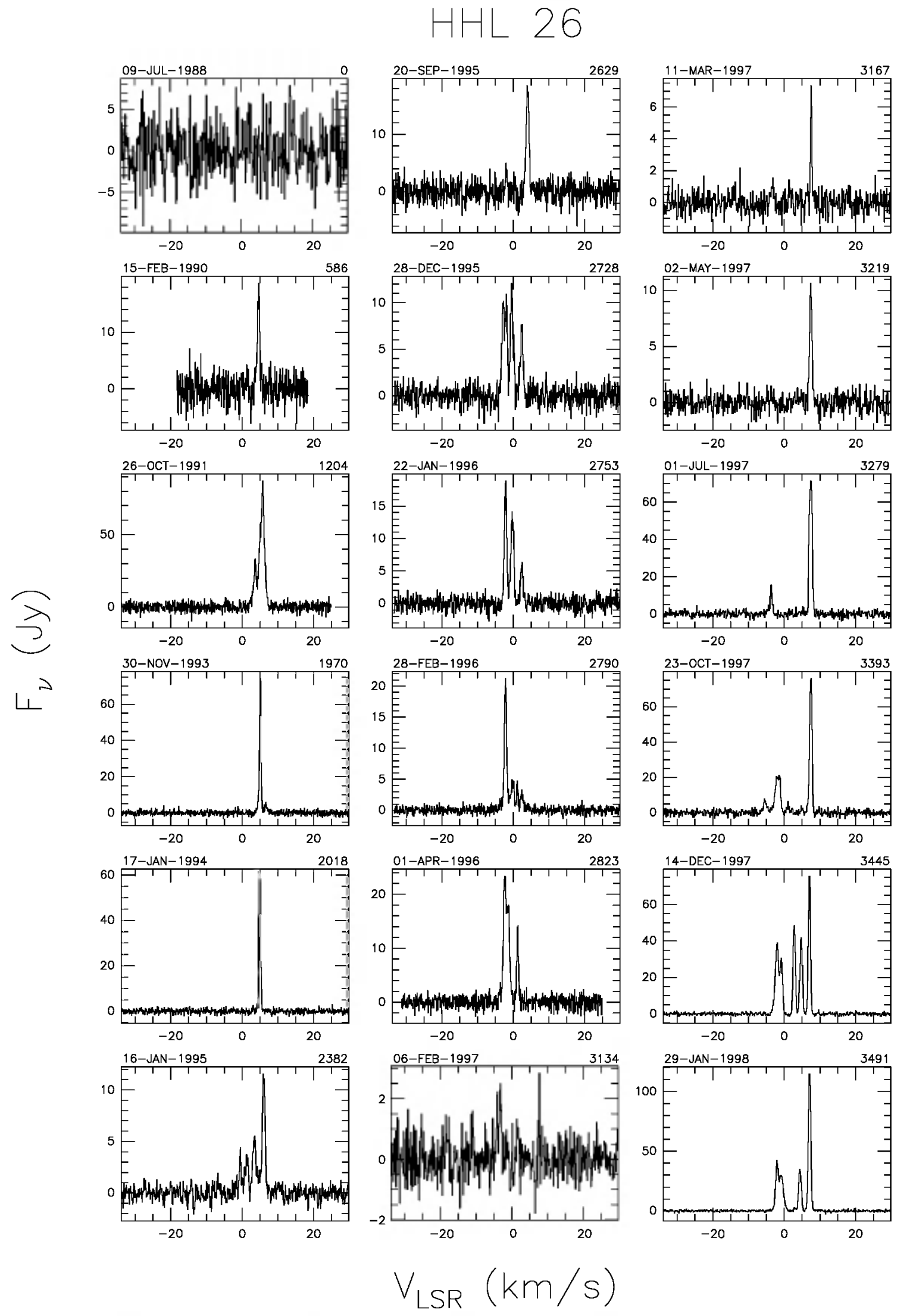

Fig. A.9. a Spectra of source HHL 26 with autoscaled flux density scale. The date of observation is shown above the top left corner of each spectrum and the number of days elapsed since the first observation is given above the top right corner. The velocity scale is the same for all spectia. 


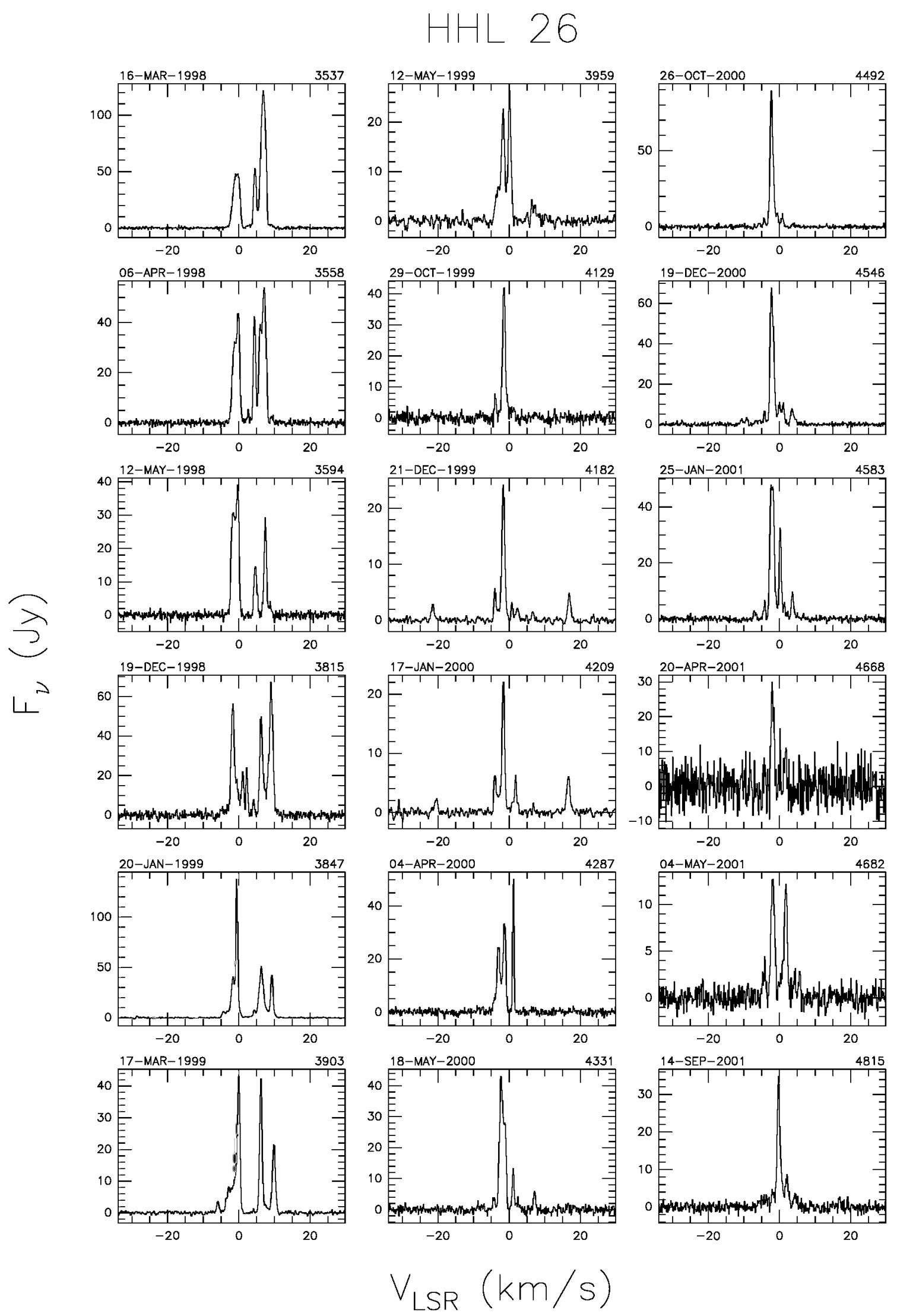

Fig. A.9. a continued. 


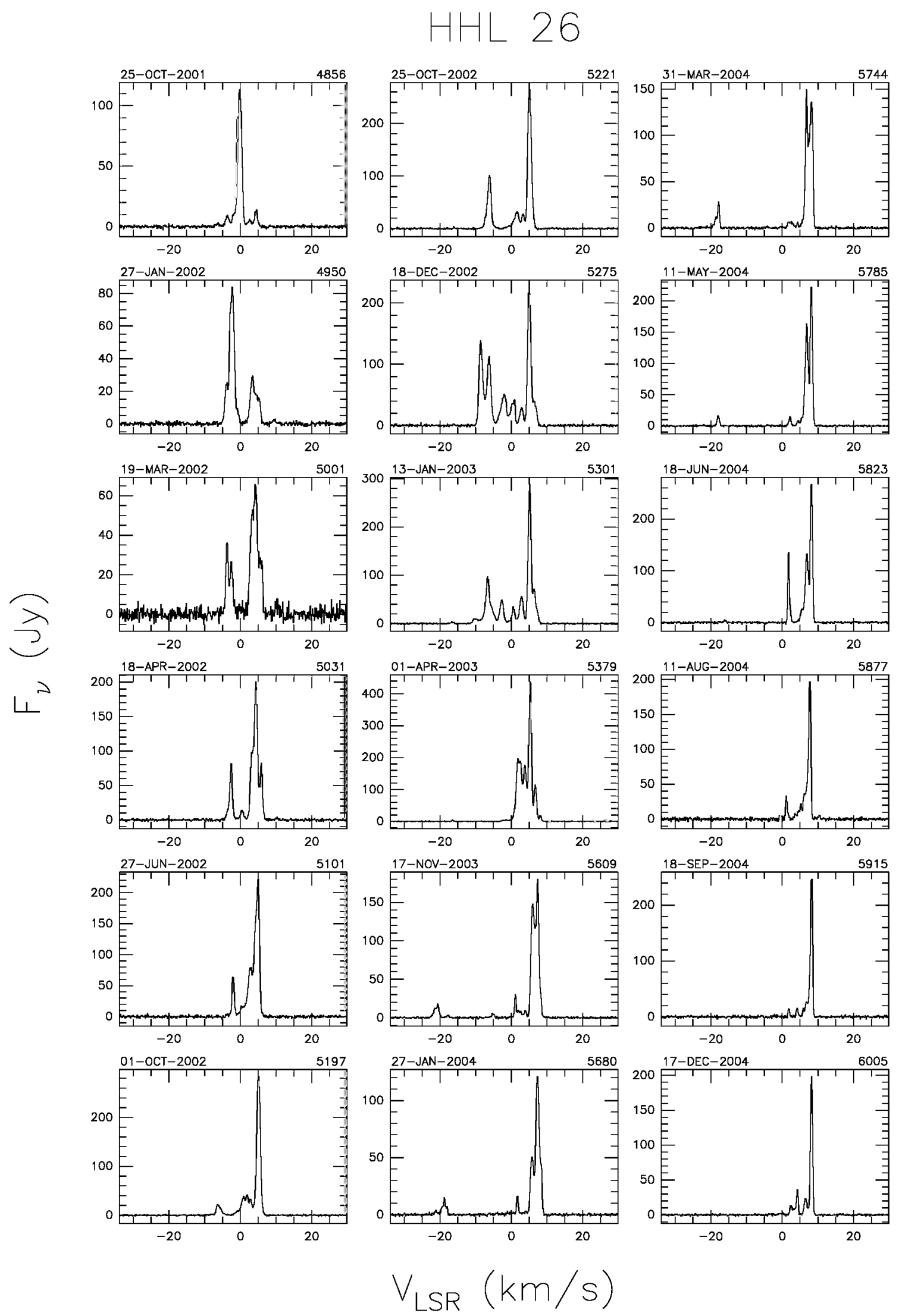

Fig. A.9. a continued. 


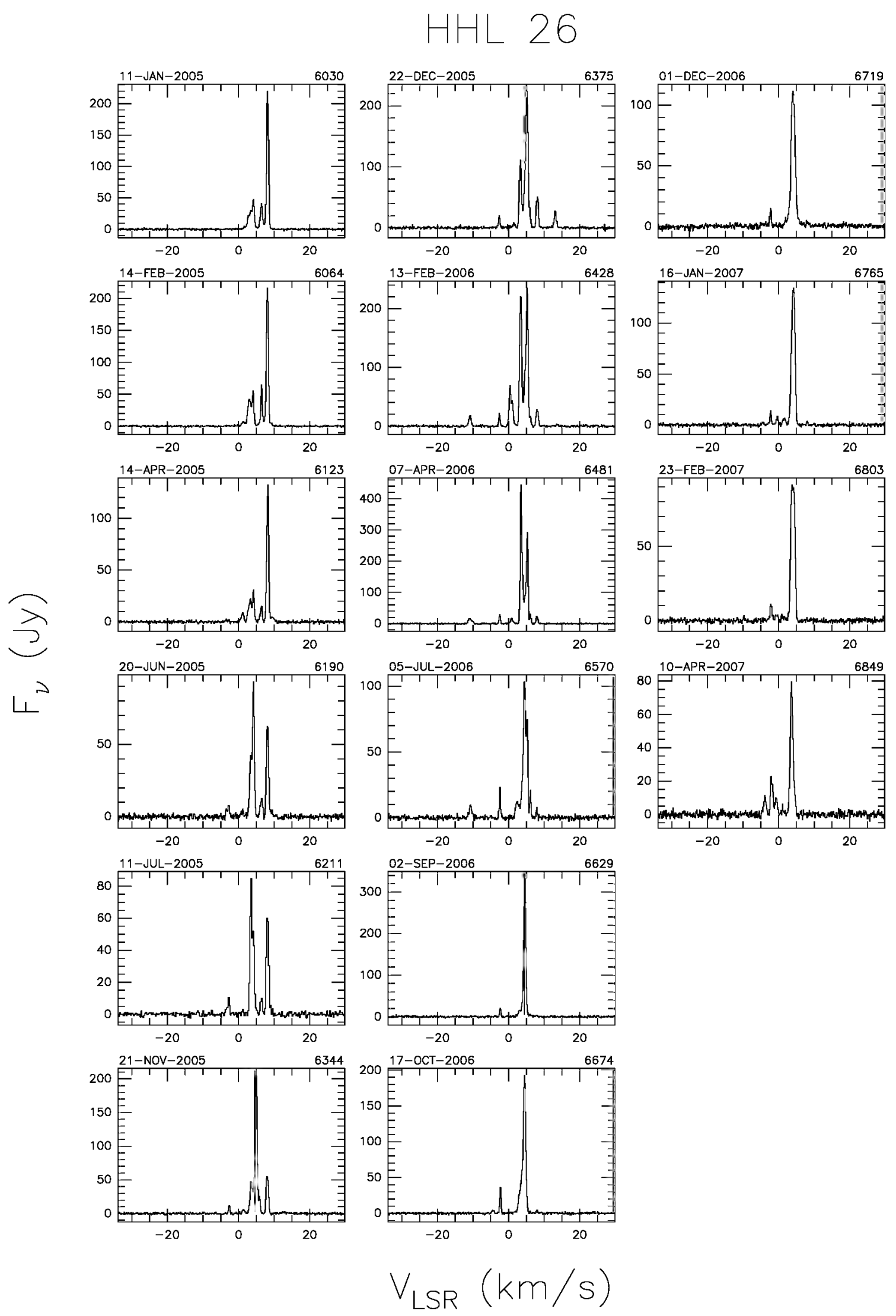

Fig. A.9. a continued. 


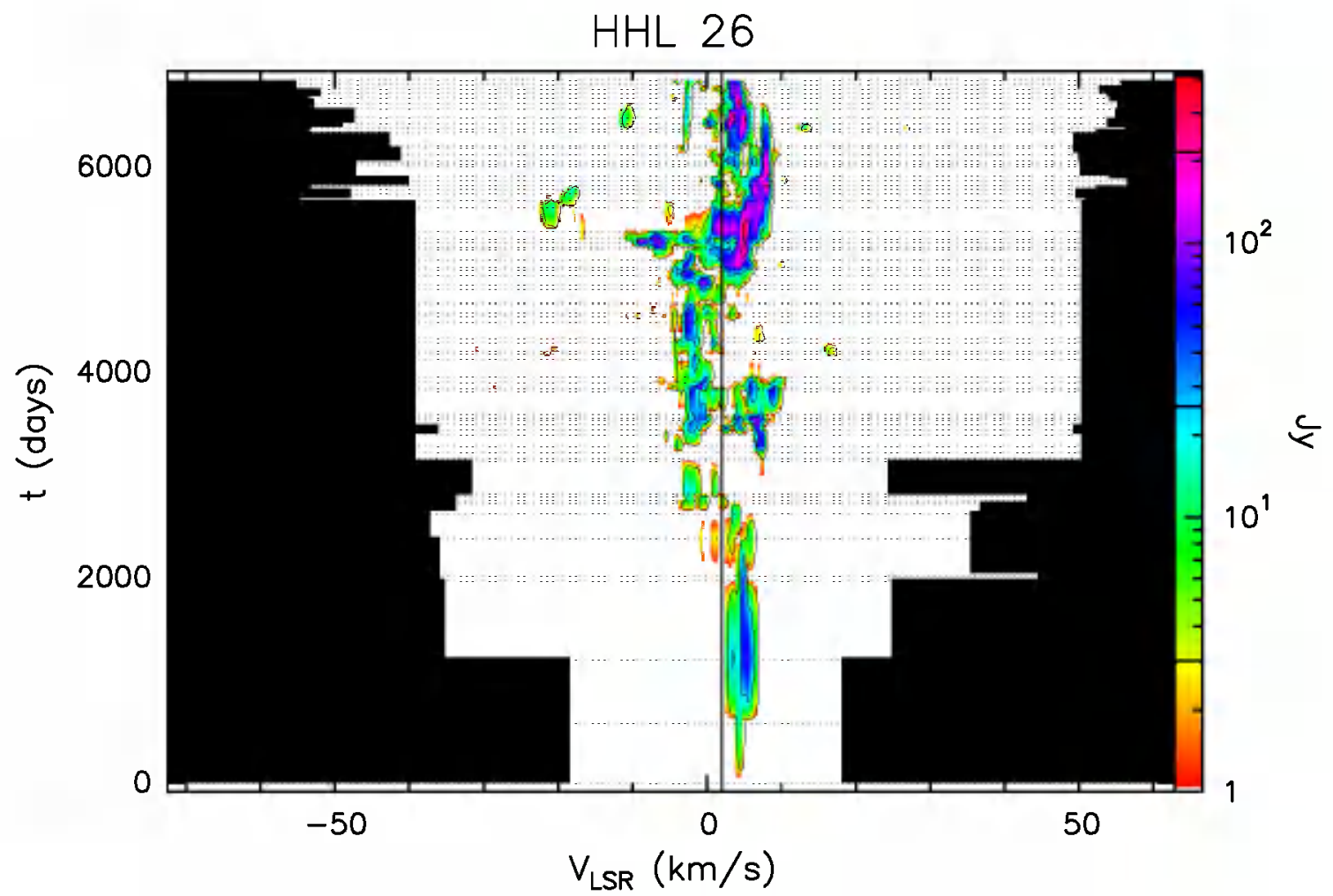

Fig. A.9. b Velocity-time-flux density full plot for source HHL 26. The vertical solid line indicates the velocity of the associated thermal molecular gas. The flux density scale is shown by the bar on the right. In this bar the three lines give the flux density of the drawn contours.

HHL 26

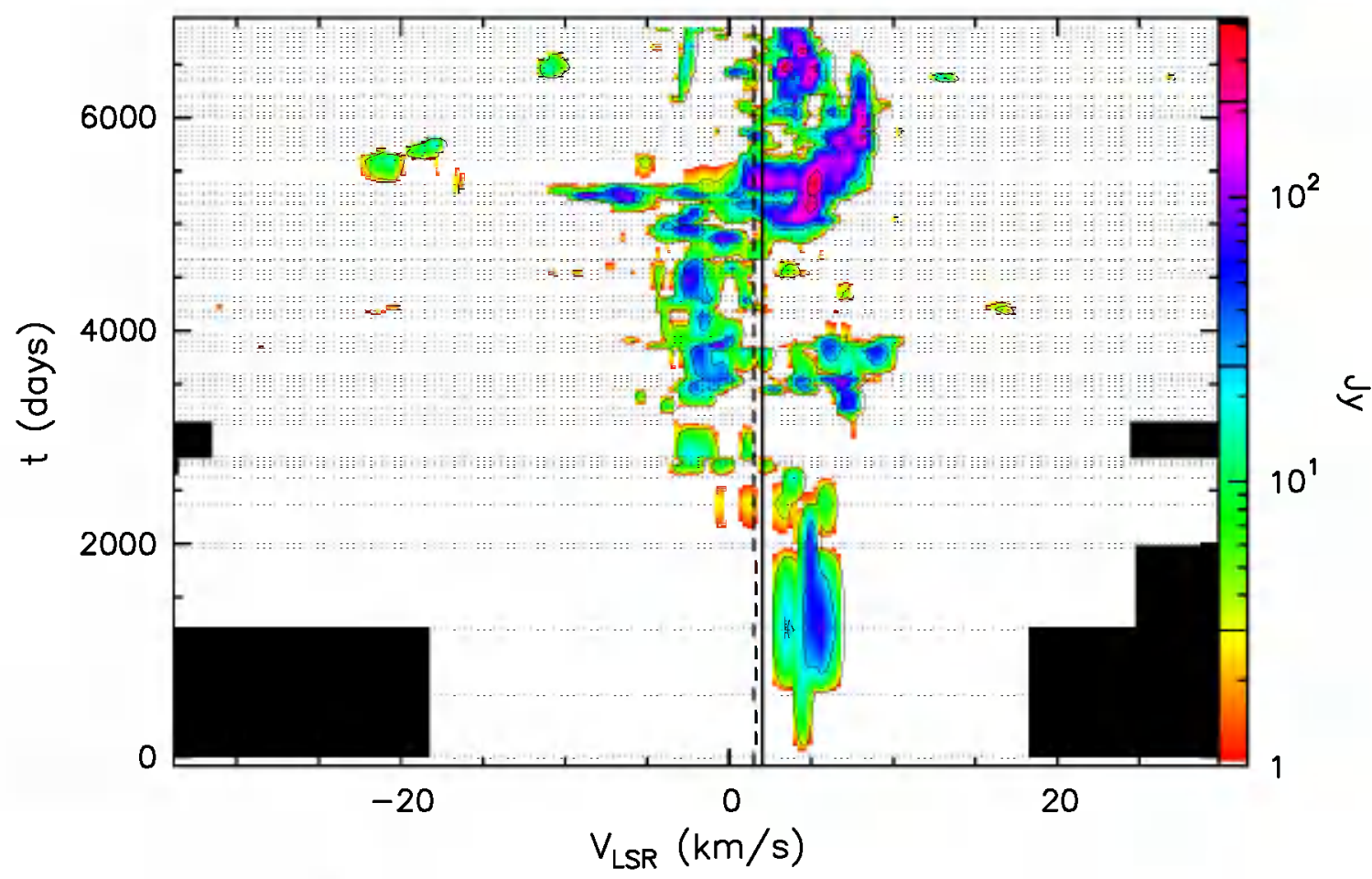

Fig. A.9. c Same as previous figure, but "zoomed" to velocity range over which emission has been detected. 


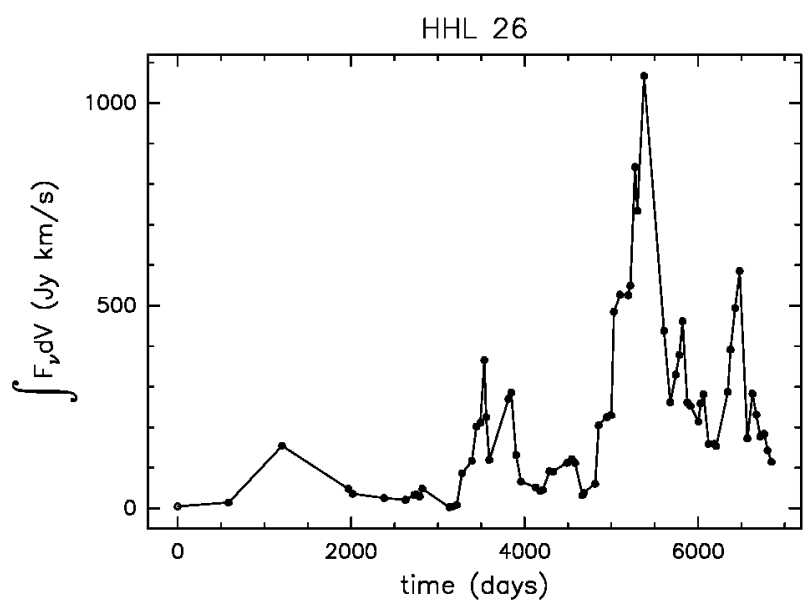

Fig. A.9. $d$ Integral of the flux density over the observed velocity range as a function of time for source HHL 26.

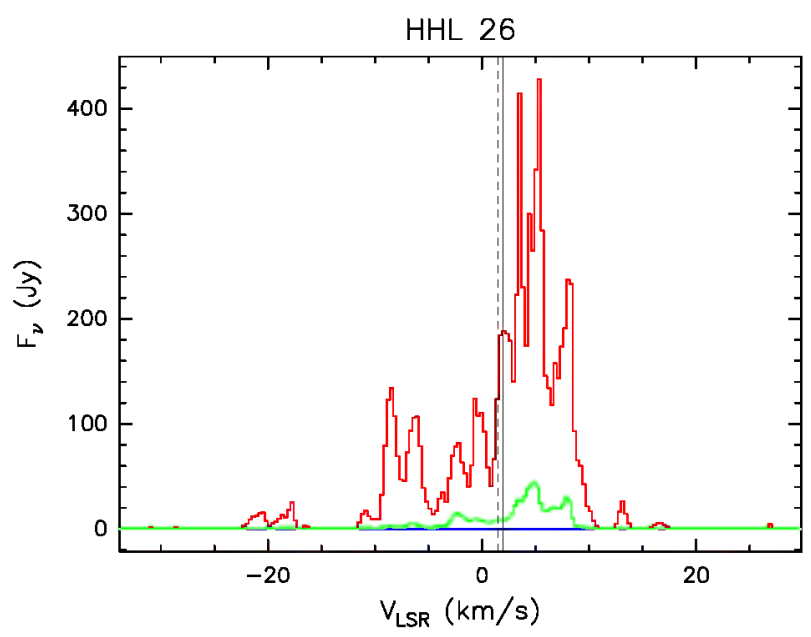

Fig. A.9. e Upper (red) and lower (blue) envelopes and mean spectrum (green) of source HHL 26 measured during our monitoring. The vertical solid line marks the velocity of the associated thermal molecular gas. The vertical dashed line marks the mean velocity derived from the histogram of the rate-of-occurrence.

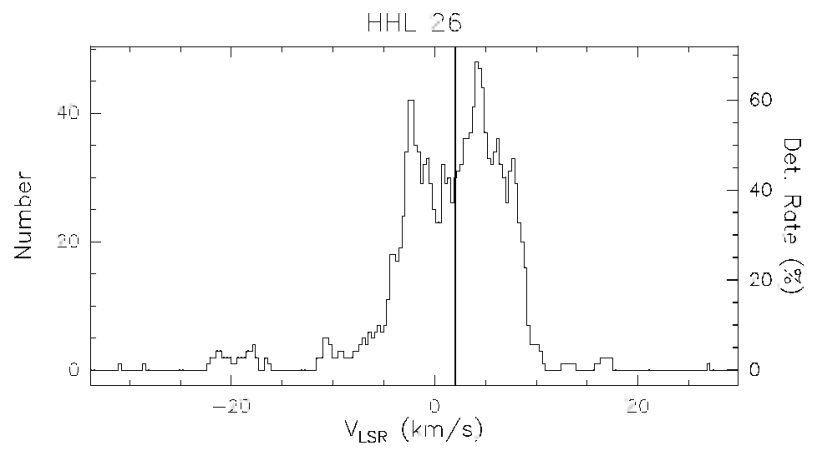

Fig. A.9. f Rate-of-occurrence plot for source HHL 26. The scale to the right refers to the dotted histogram, the scale to the left to the solid line histogram. The vertical solid line marks the velocity of the associated thermal molecular gas. 
Sh 2-235
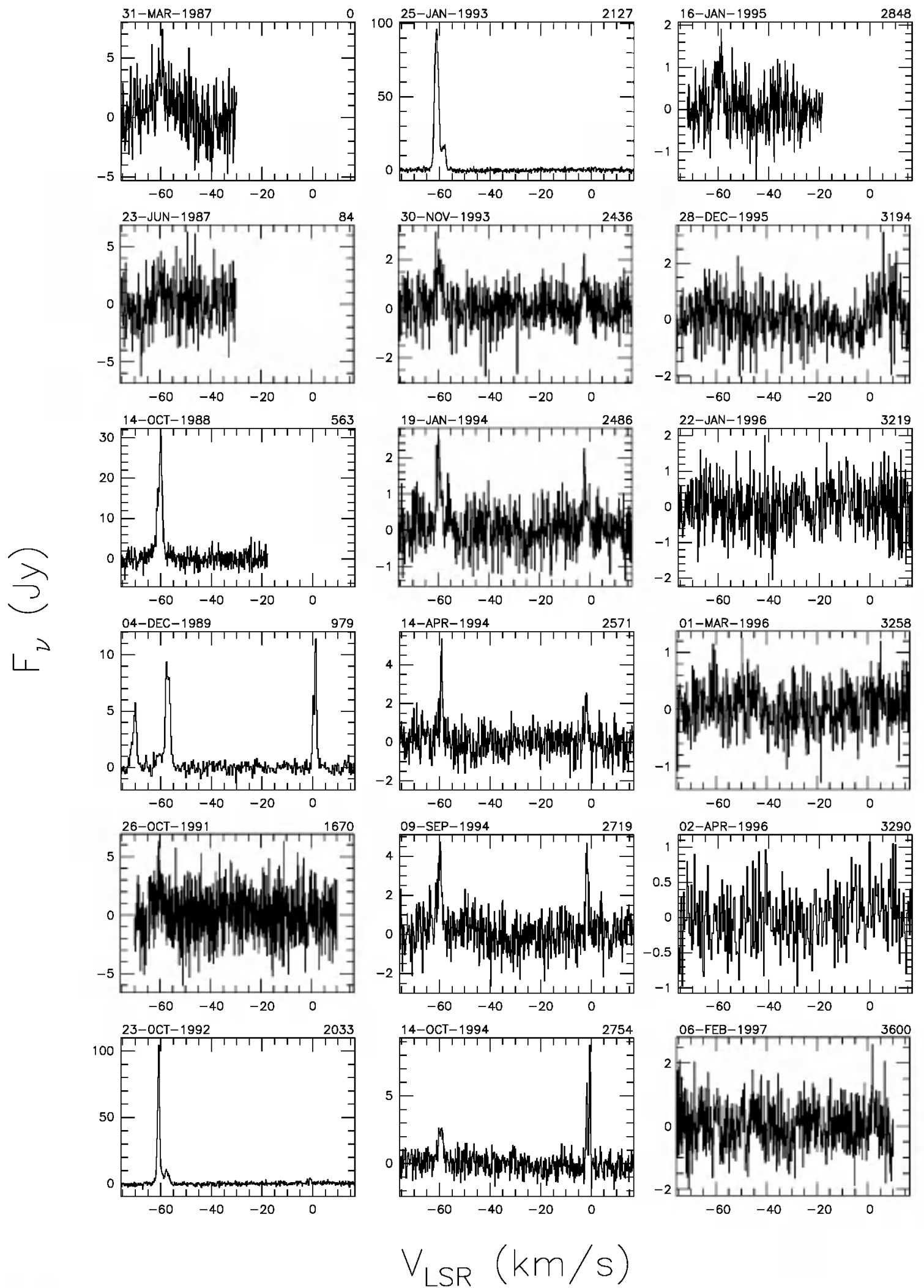

Fig. A.10. a Spectra of source Sh 2-235 with autoscaled flux density scale. The date of observation is shown above the top left corner of each spectrum and the number of days elapsed since the first observation is given above the top right corner. The velocity scale is the same for all spectia. 
Sh 2-235
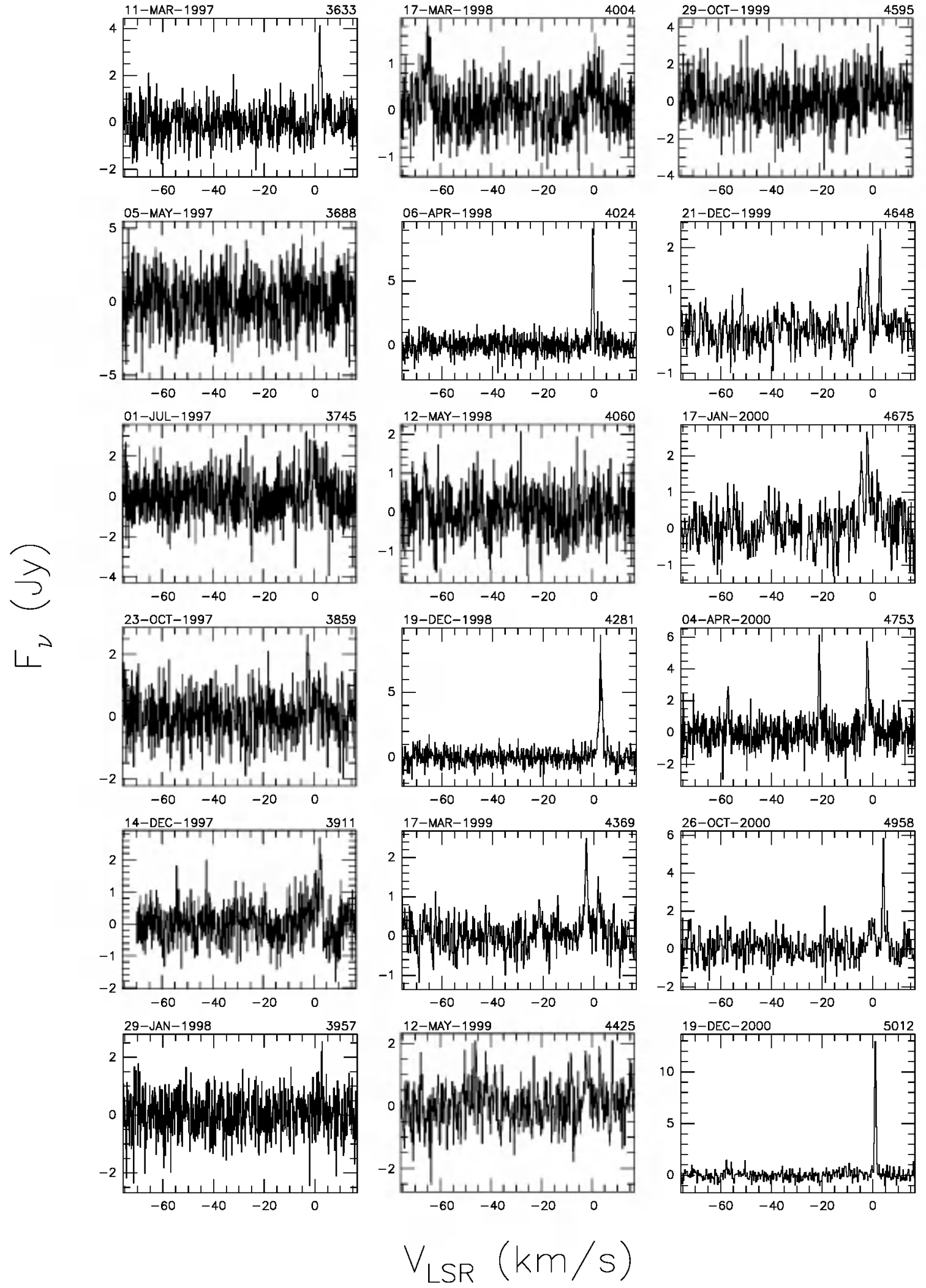

Fig. A.10. a continued. 
Sh 2-235
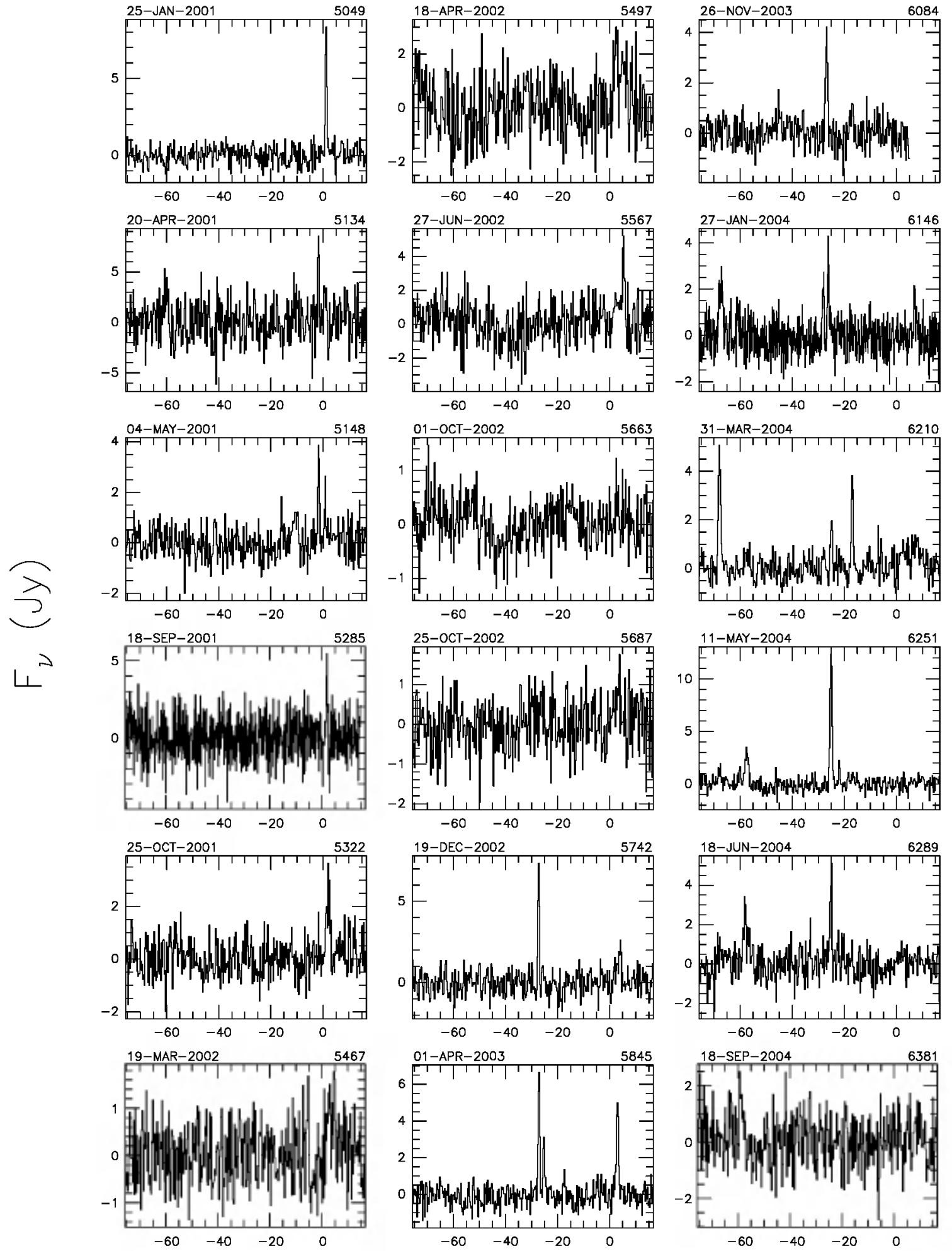

$$
V_{\text {LSR }}(\mathrm{km} / \mathrm{s})
$$

Fig. A.10. a continued. 
Sh 2-235
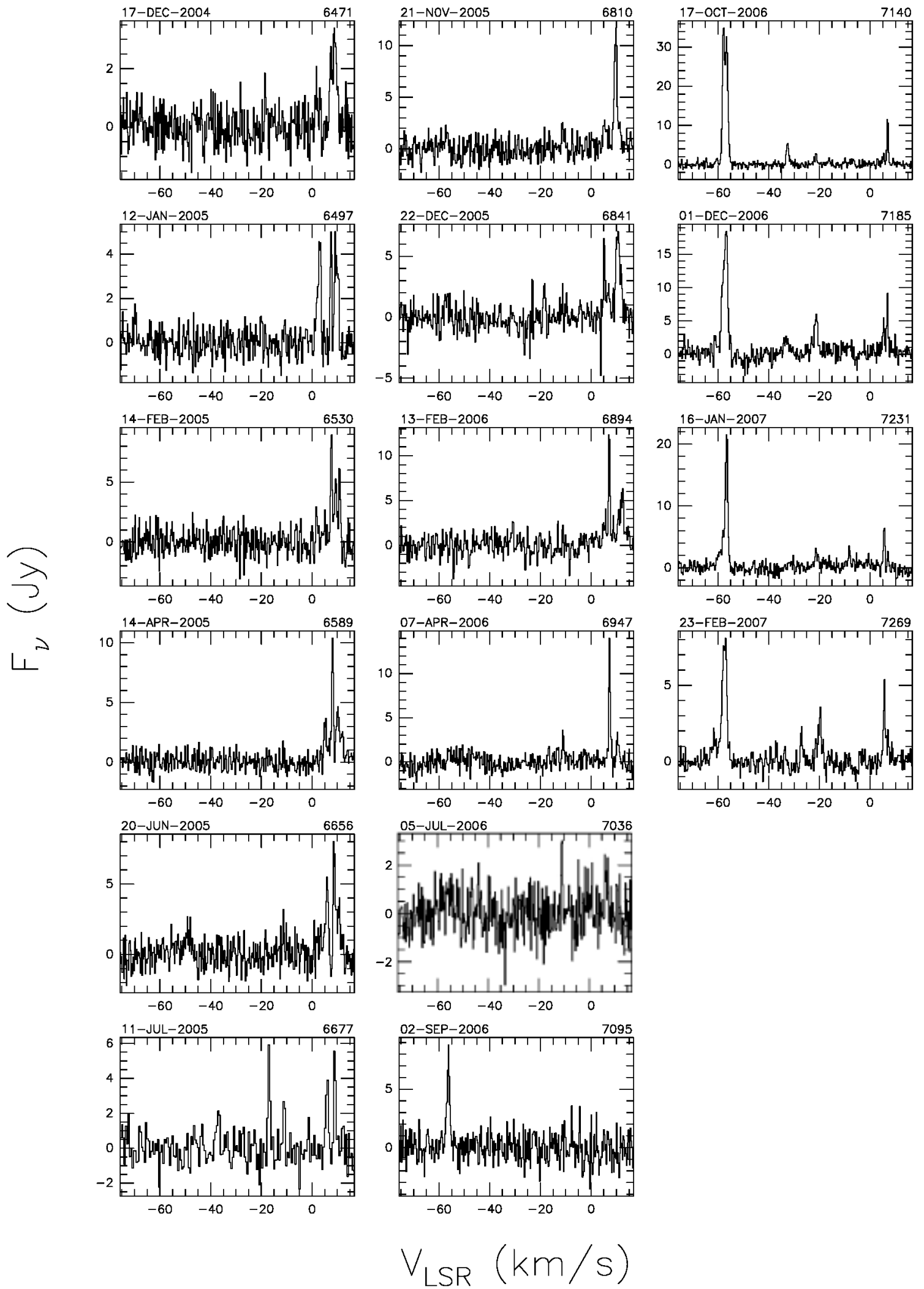

Fig. A.10. a continued. 


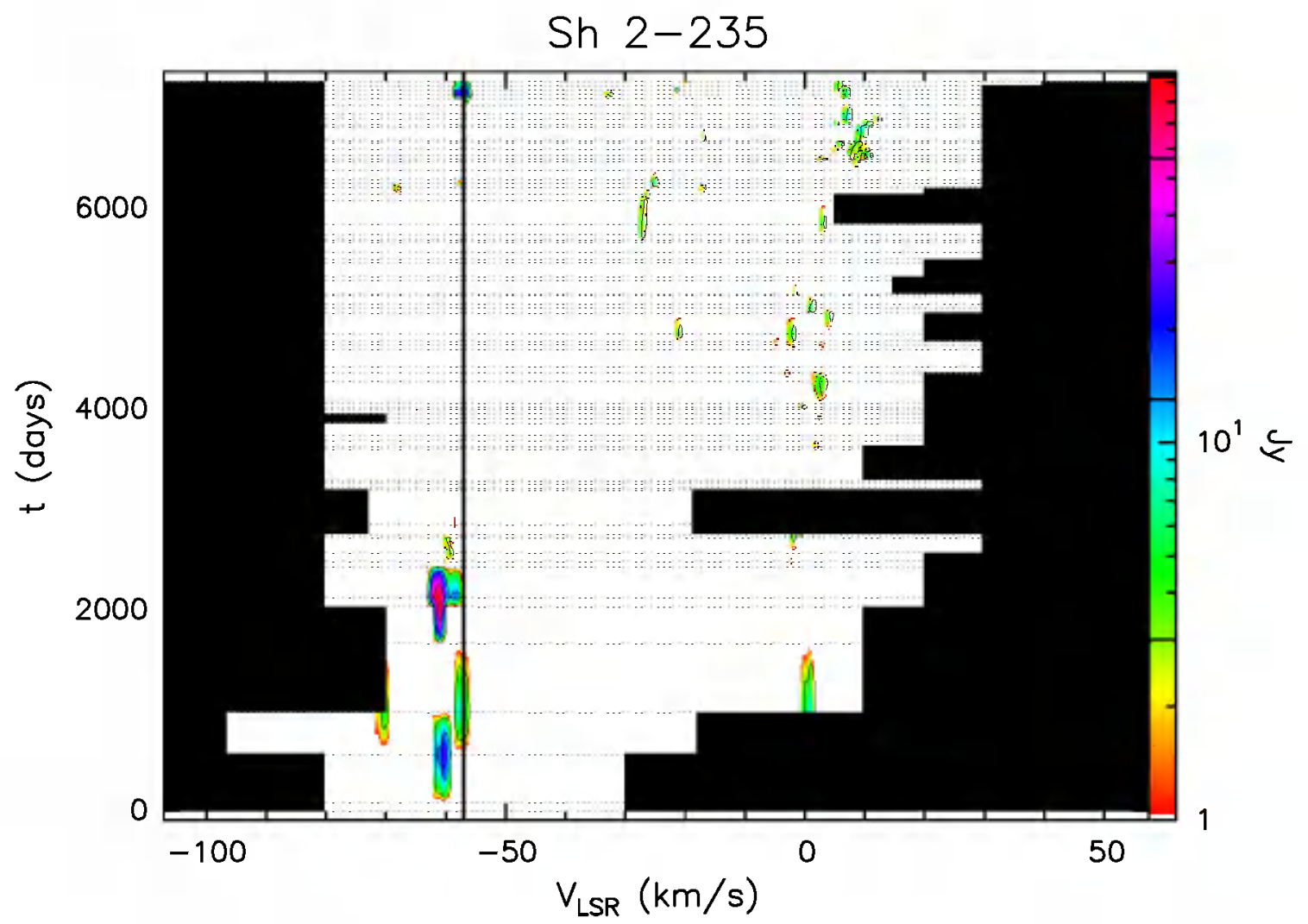

Fig. A.10. b Velocity-time-flux density full plot for source Sh 2-235. The vertical solid line indicates the velocity of the associated thermal molecular gas. The flux density scale is shown by the bar on the right. In this bar the three lines give the flux density of the drawn contours.

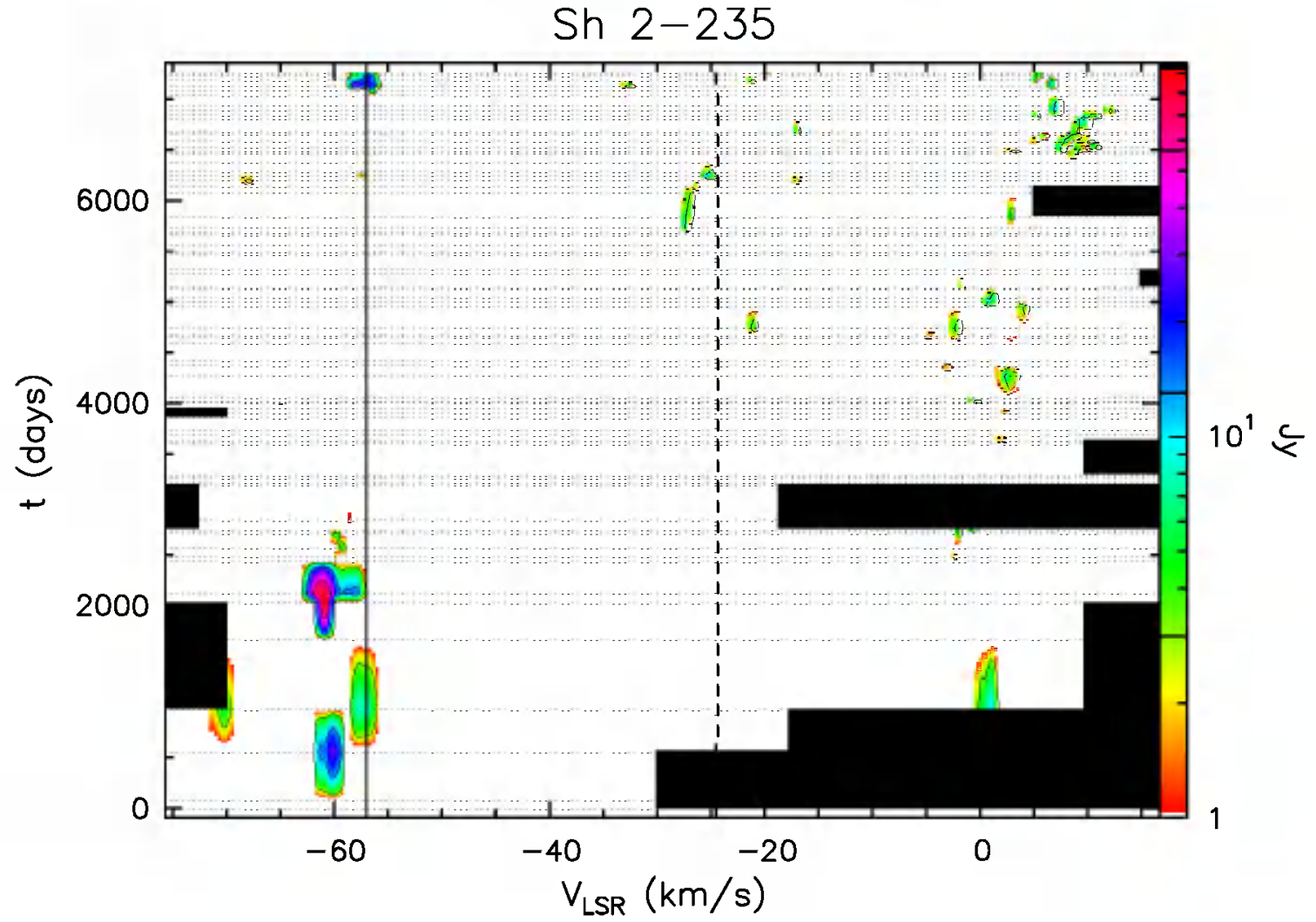

Fig. A.10. c Same as previous figure. but "zoomed" to velocity range over which emission has been detected. 


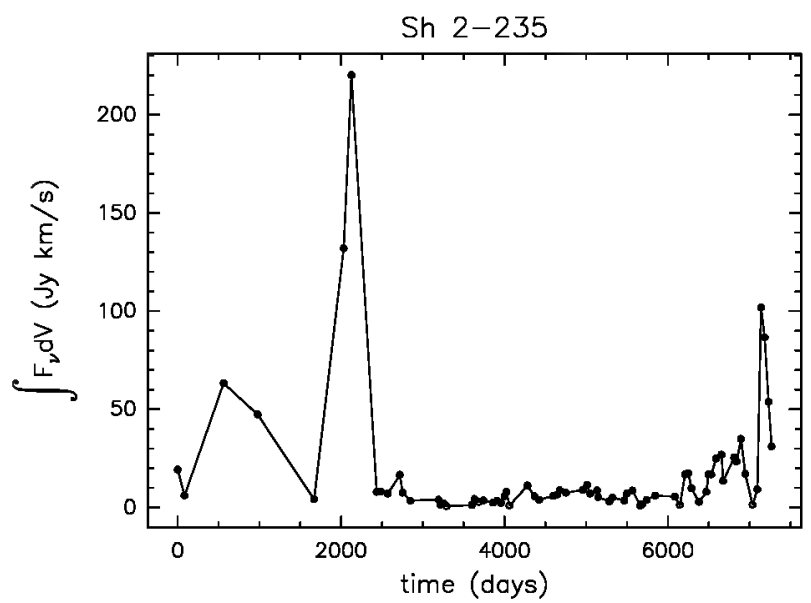

Fig. A.10. d Integral of the flux density over the observed velocity range as a function of time for source Sh 2-235.

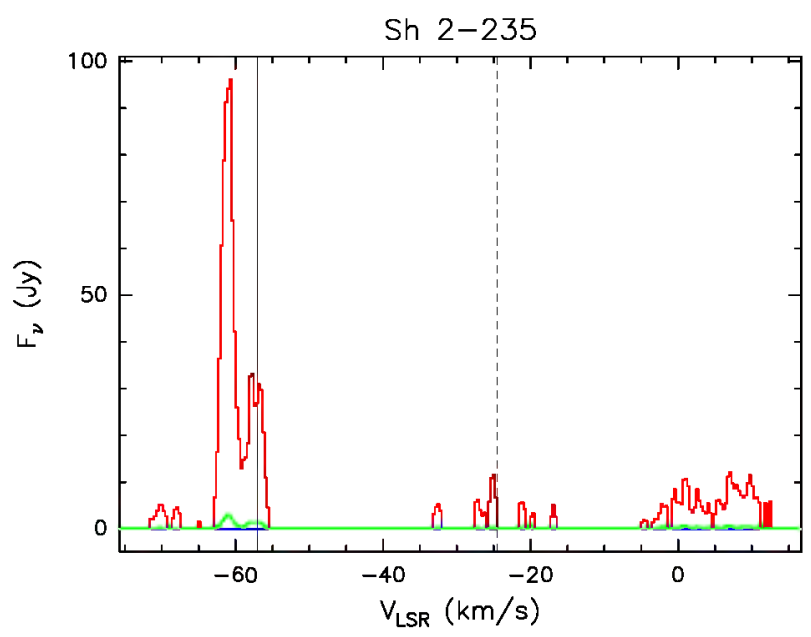

Fig. A.10. e Upper (red) and lower (blue) envelopes and mean spectrum (green) of source Sh 2-235 measured during our monitoring. The vertical solid line marks the velocity of the associated thermal molecular gas. The vertical dashed line marks the mean velocity derived from the histogram of the rate-of-occurrence.

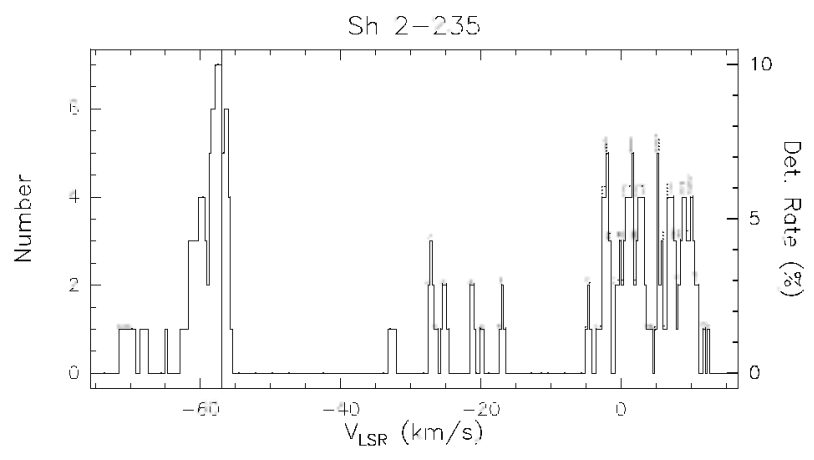

Fig. A.10. f Rate-of-occurrence plot for source Sh 2-235. The scale to the right refers to the dotted histogram, the scale to the left to the solid line histogram. The vertical solid line marks the velocity of the associated thermal molecular gas. 
NGC 2071
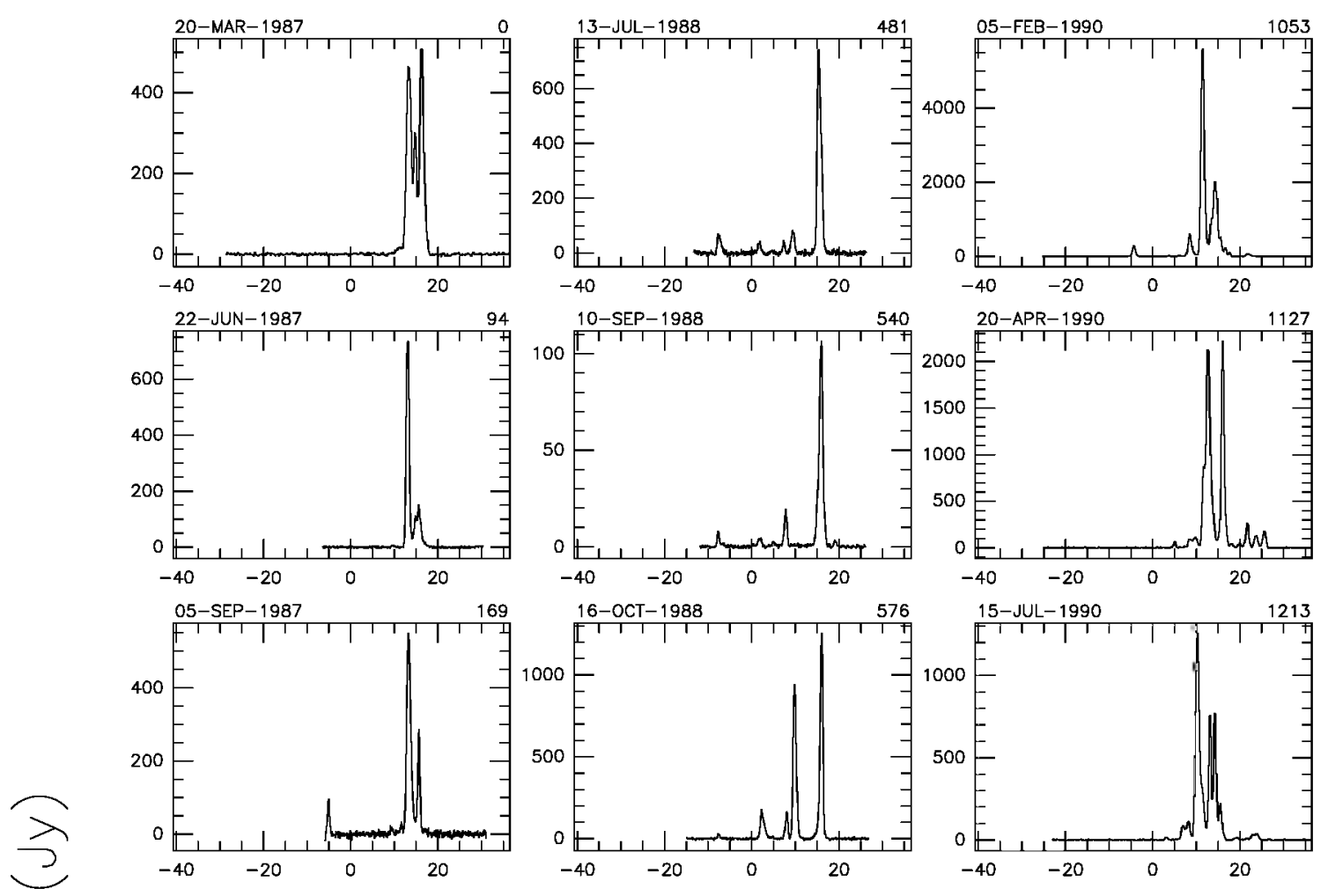

$\left\llcorner^{\curvearrowright}\right.$
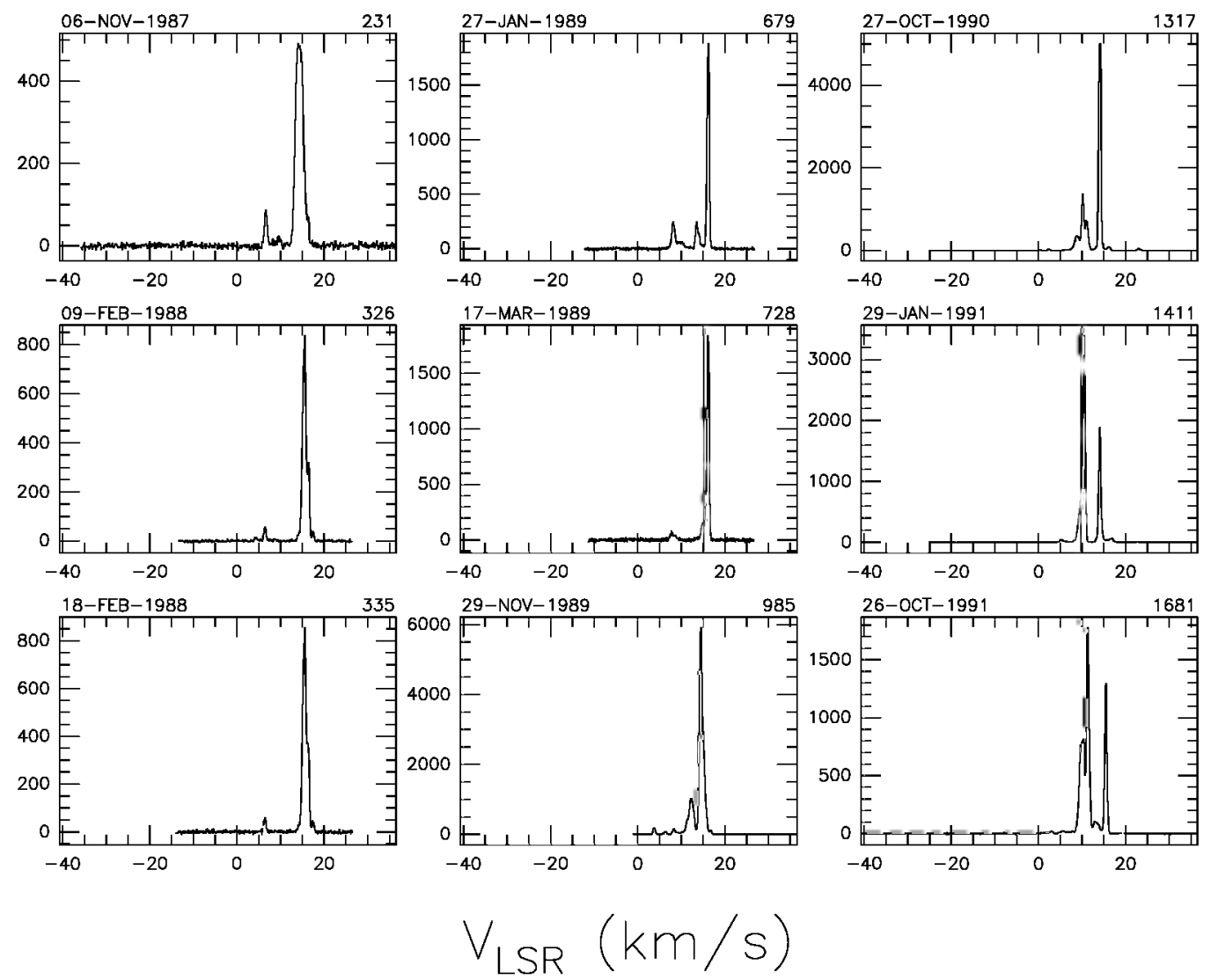

Fig. A.11. a Spectra of source NGC 2071 with autoscaled flux density scale. The date of observation is shown above the top left corner of each spectrum and the number of days elapsed since the first observation is given above the top right corner. The velocity scale is the same for all spectra. 


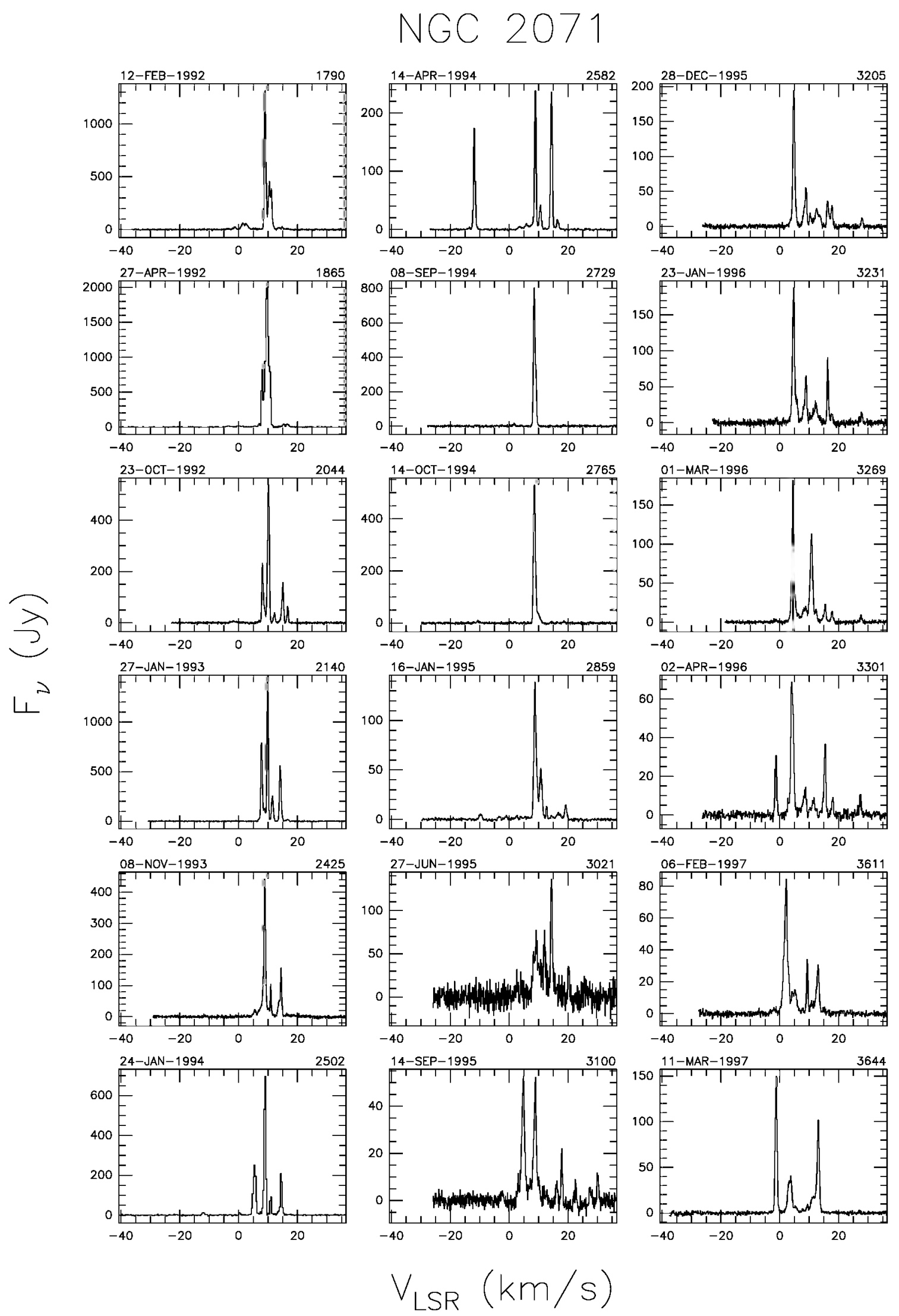

Fig. A.11. a continued. 


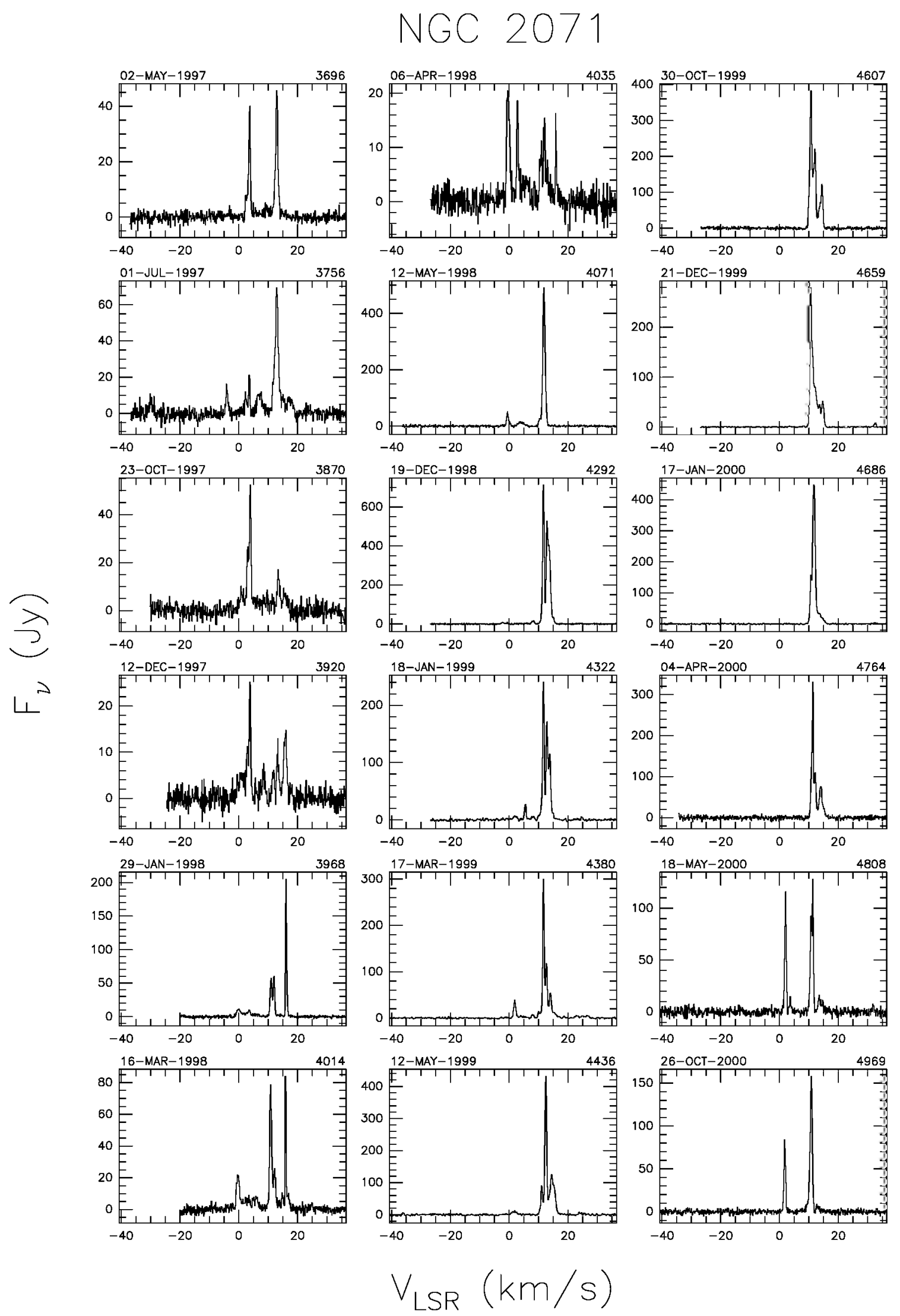

Fig. A.11. a continued. 


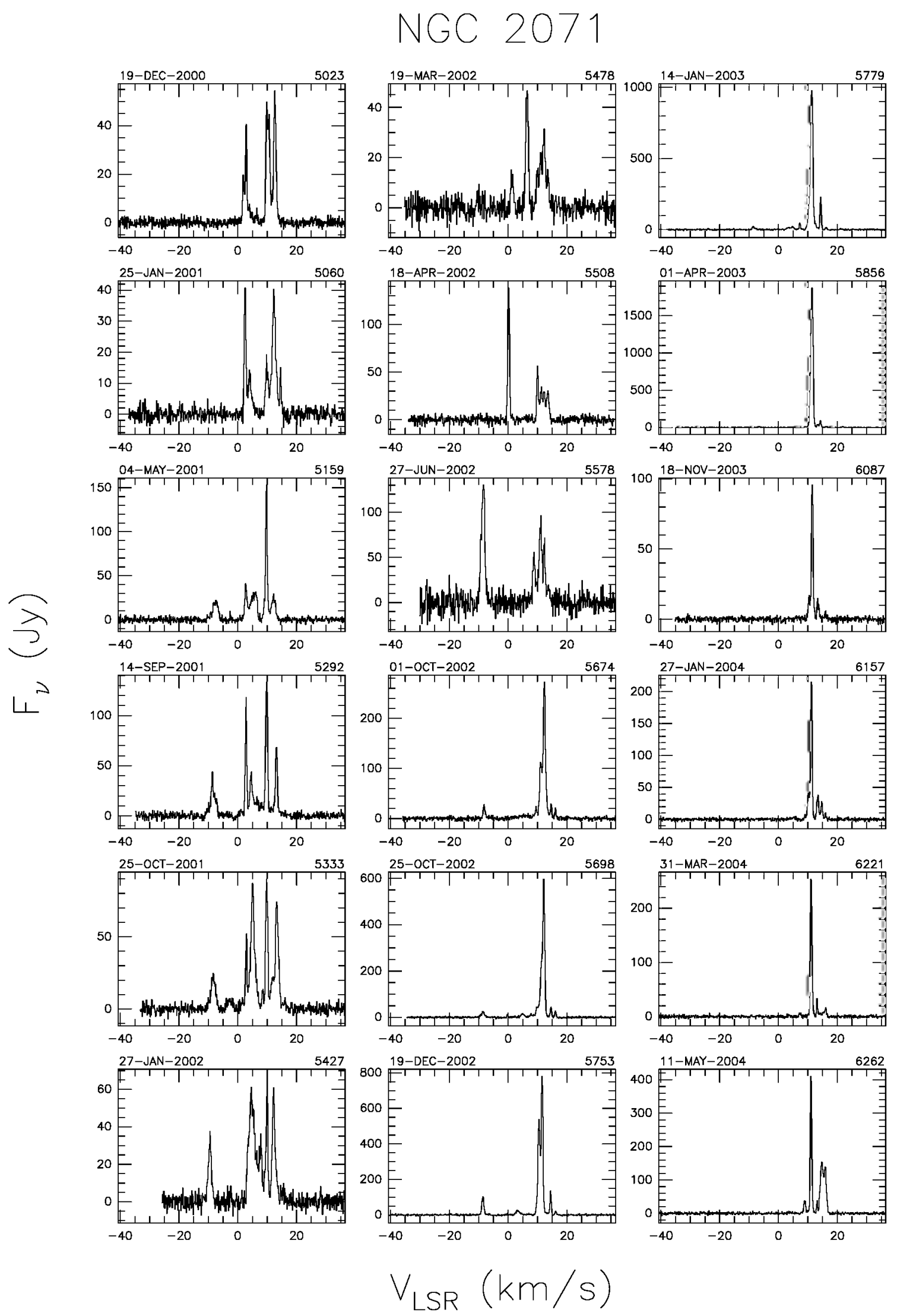

Fig. A.11. a continued. 

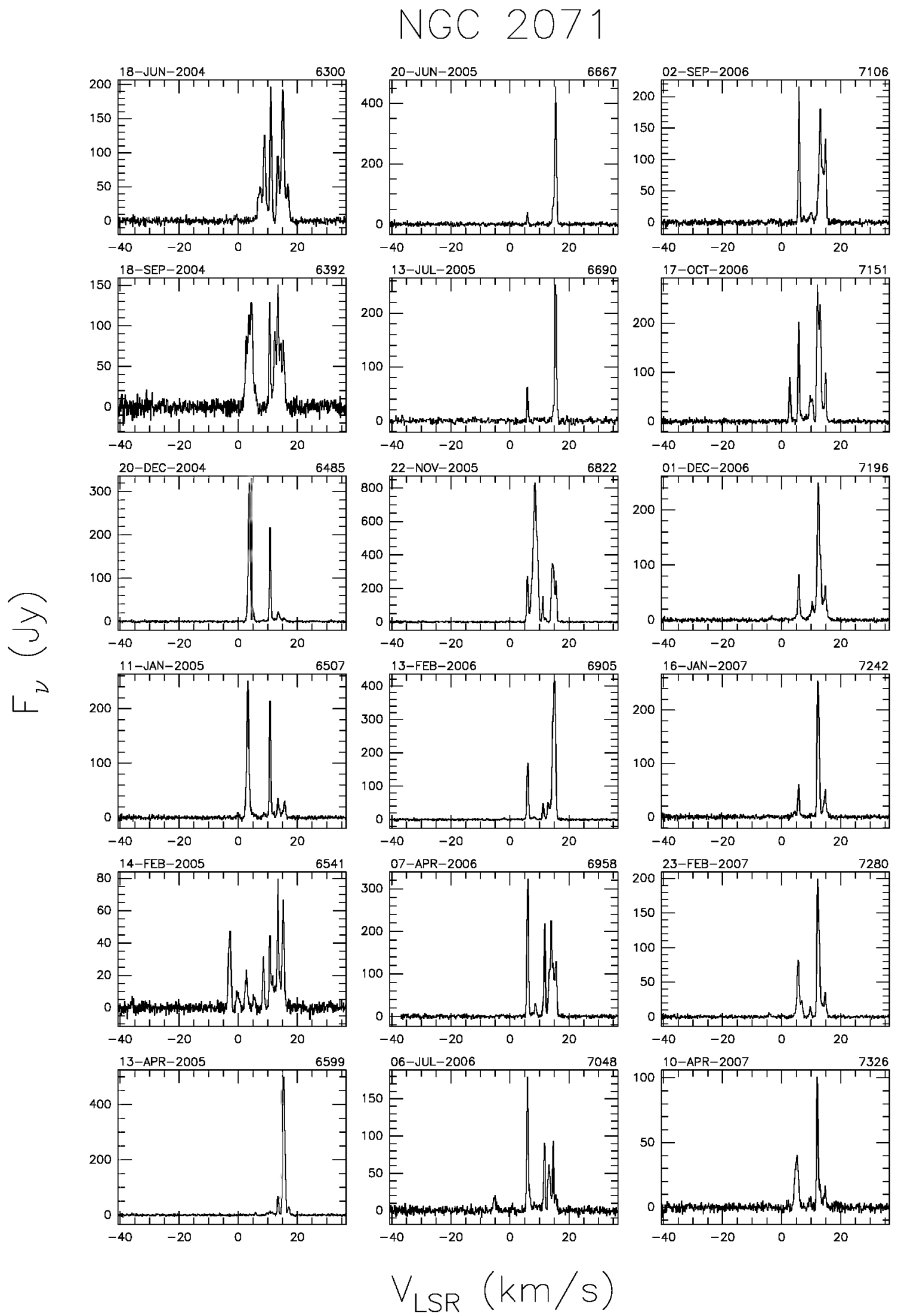

Fig. A.11. a continued. 


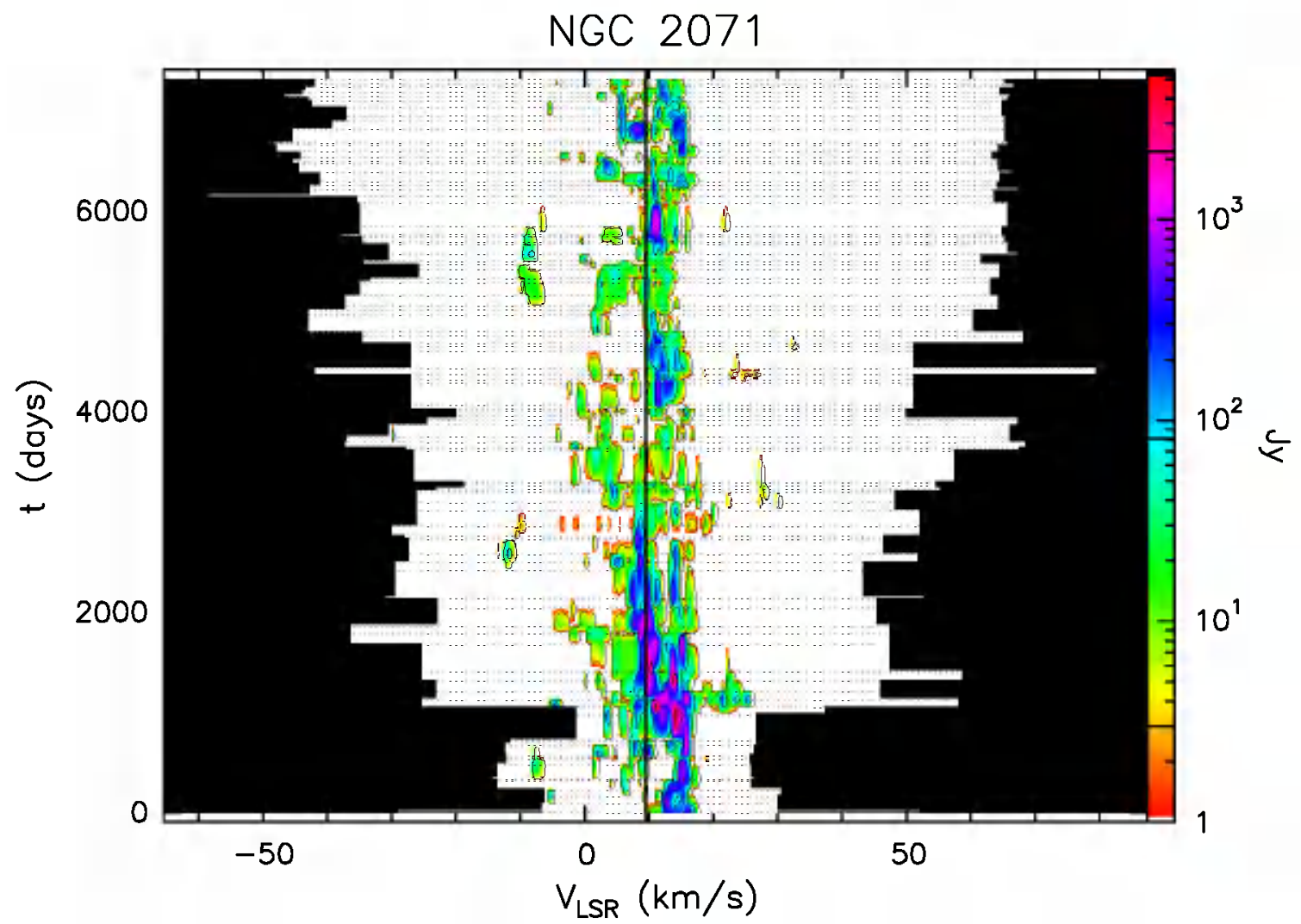

Fig. A.11. b Velocity-time-flux density full plot for source NGC 2071. The vertical solid line indicates the velocity of the associated thermal molecular gas. The flux density scale is shown by the bar on the right. In this bar the three lines give the flux density of the drawn contours.

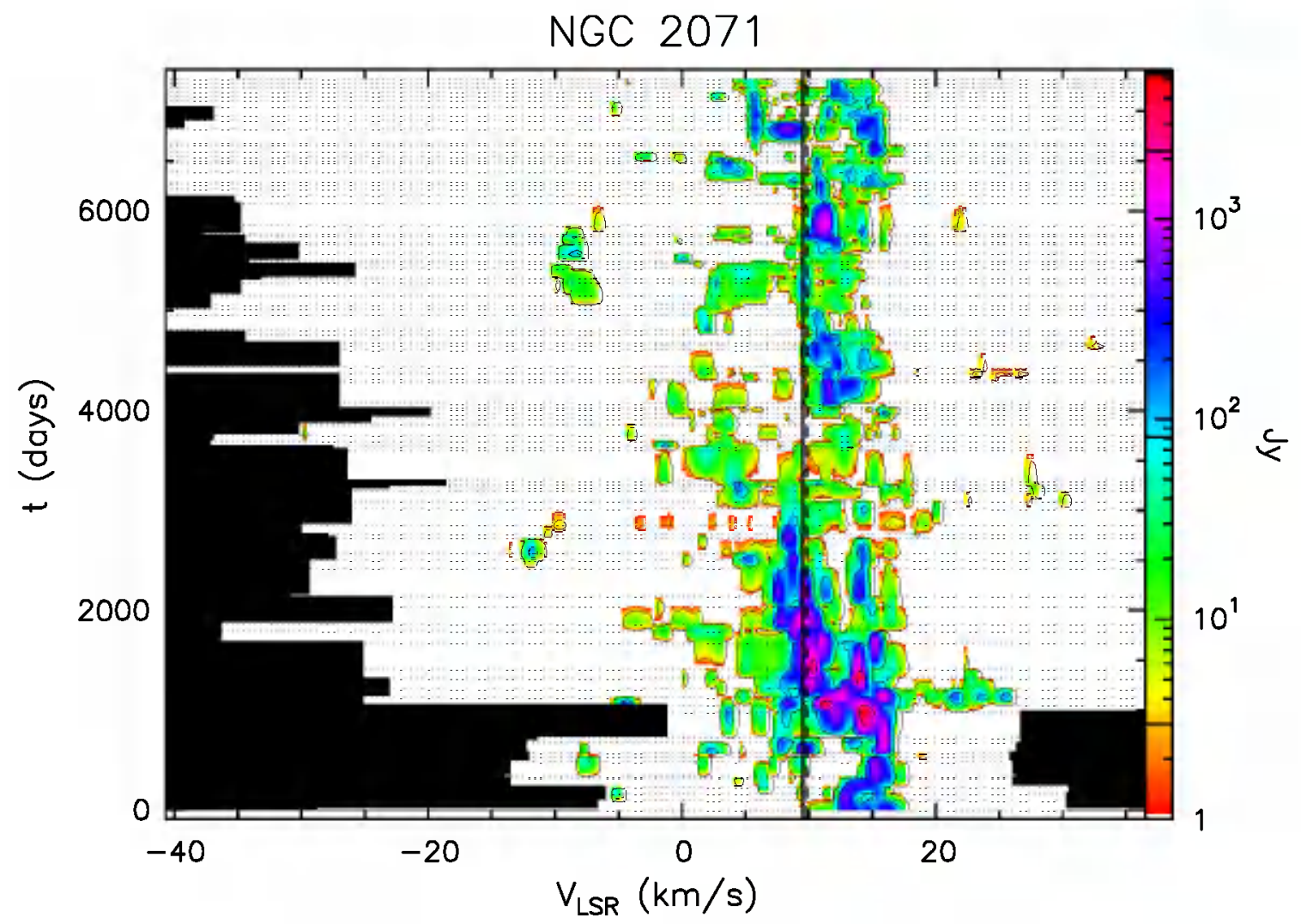

Fig. A.11. c Same as previous figure, but "zoomed" to velocity range over which emission has been detected. 


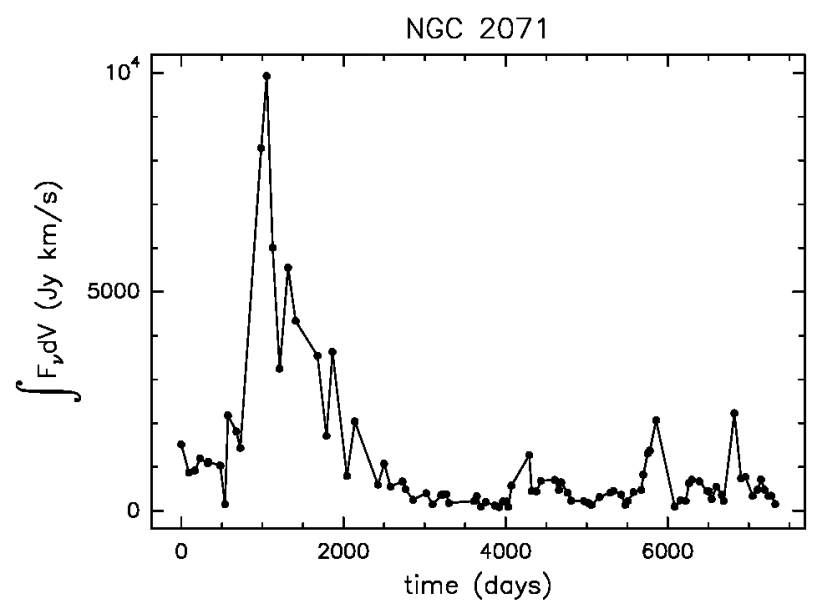

Fig. A.11. d Integral of the flux density over the observed velocity range as a function of time for source NGC 2071.

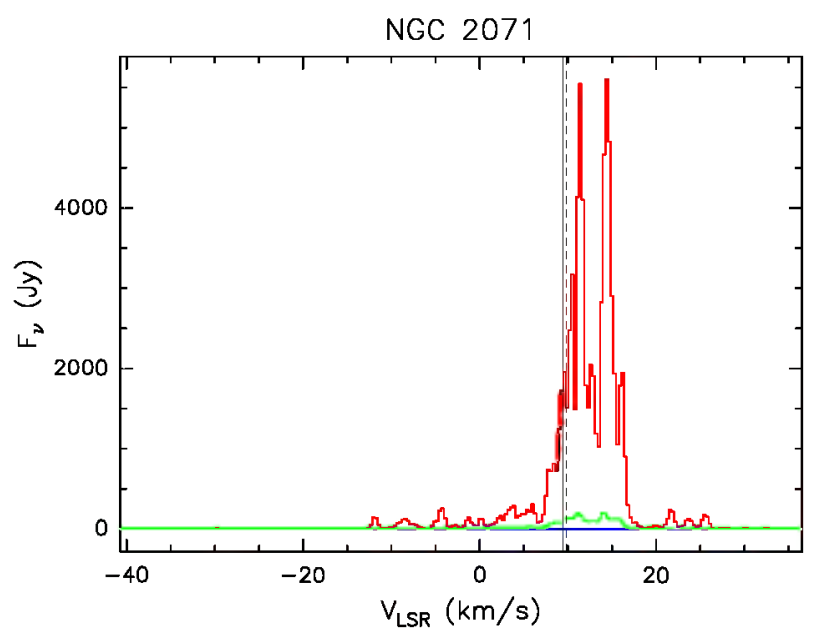

Fig. A.11. e Upper (red) and lower (blue) envelopes and mean spectrum (green) of source NGC 2071 measured during our monitoring. The vertical solid line marks the velocity of the associated thermal molecular gas. The vertical dashed line marks the mean velocity derived from the histogram of the rate-of-occurrence.

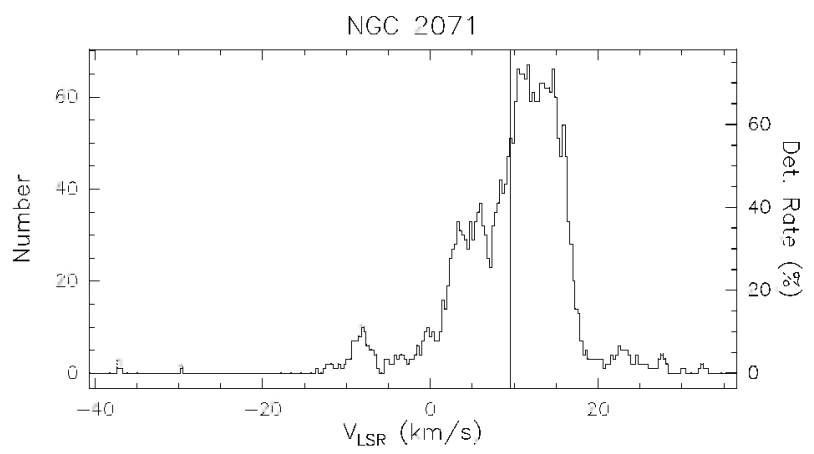

Fig. A.11. f Rate-of-occurrence plot for source NGC 2071. The scale to the right refers to the dotted histogram, the scale to the left to the solid line histogram. The vertical solid line marks the velocity of the associated thermal molecular gas. 


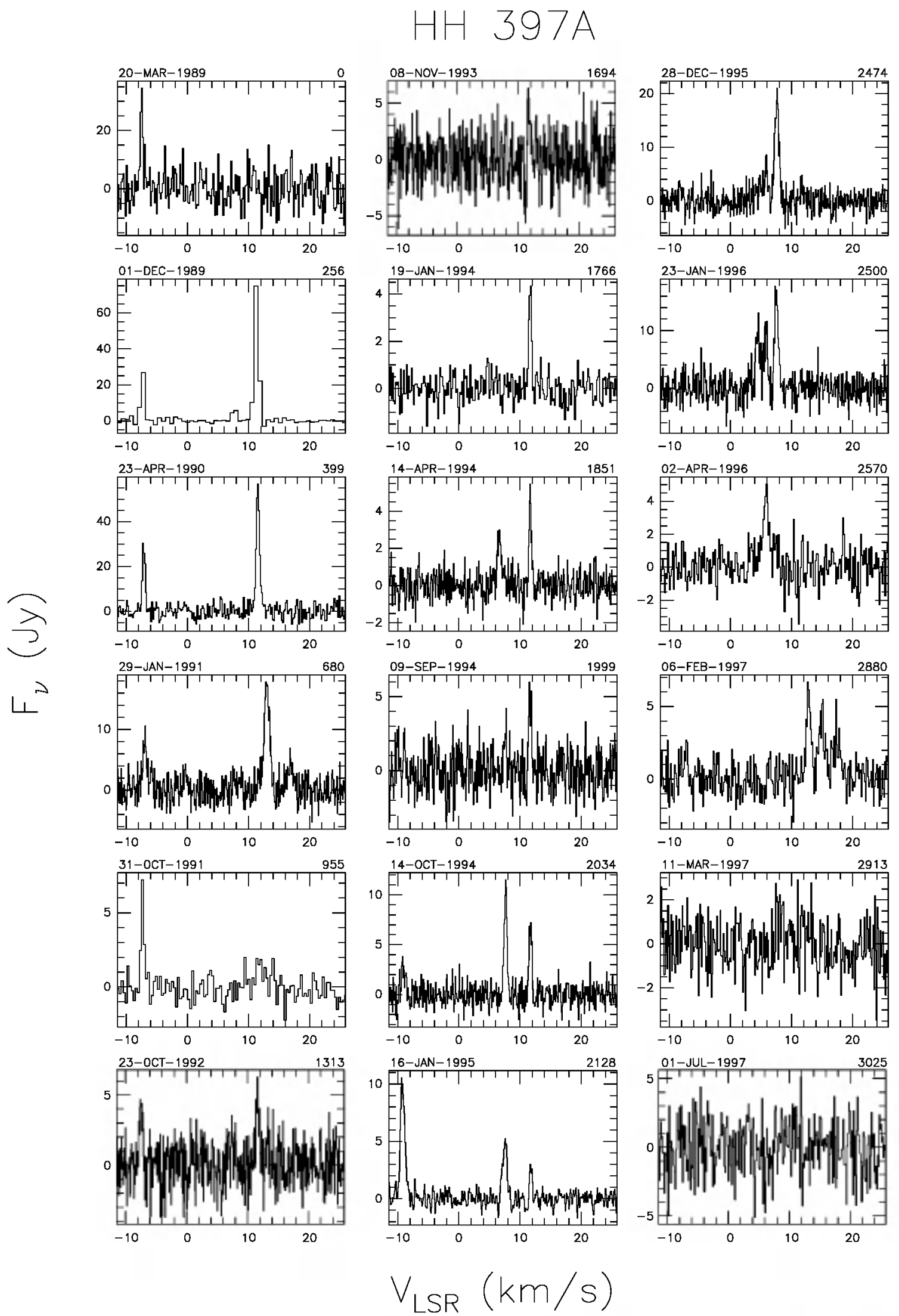

Fig. A.12. a Spectra of source HH 397A with autoscaled flux density scale. The date of observation is shown above the top left corner of each spectrum and the number of days elapsed since the first observation is given above the top right corner. The velocity scale is the same for all spectra. 


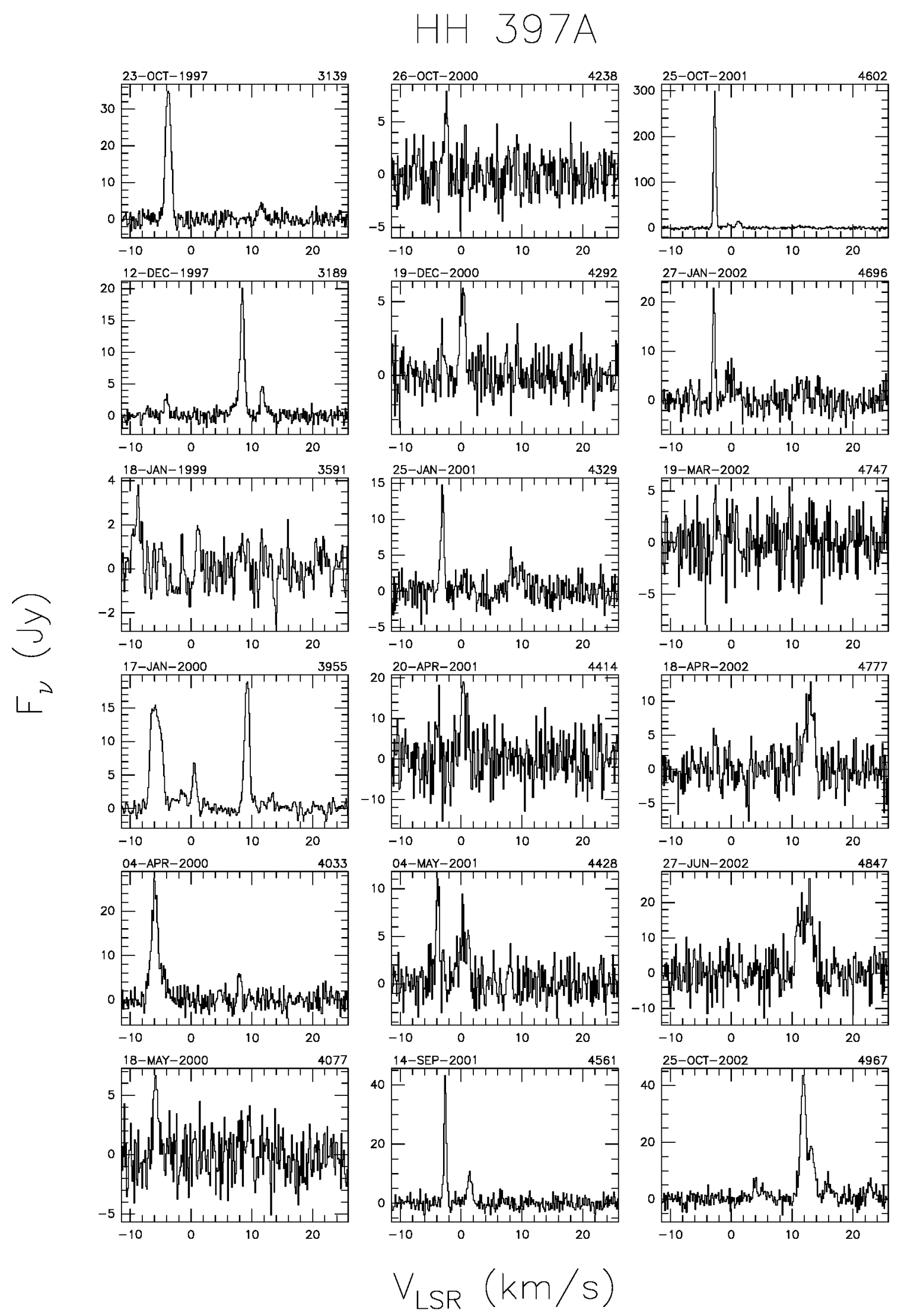

Fig. A.12. a continued. 


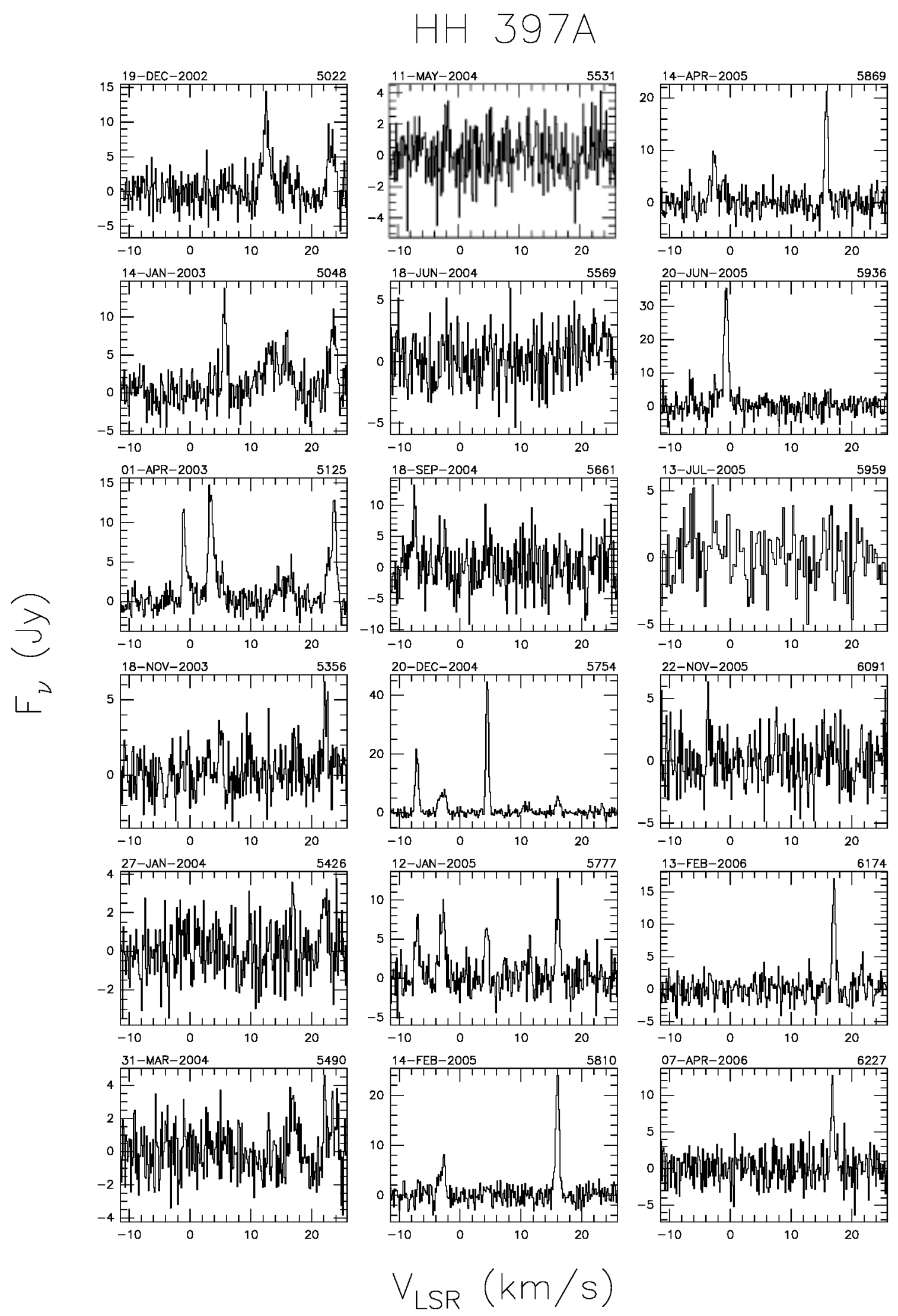

Fig. A.12. a continued. 


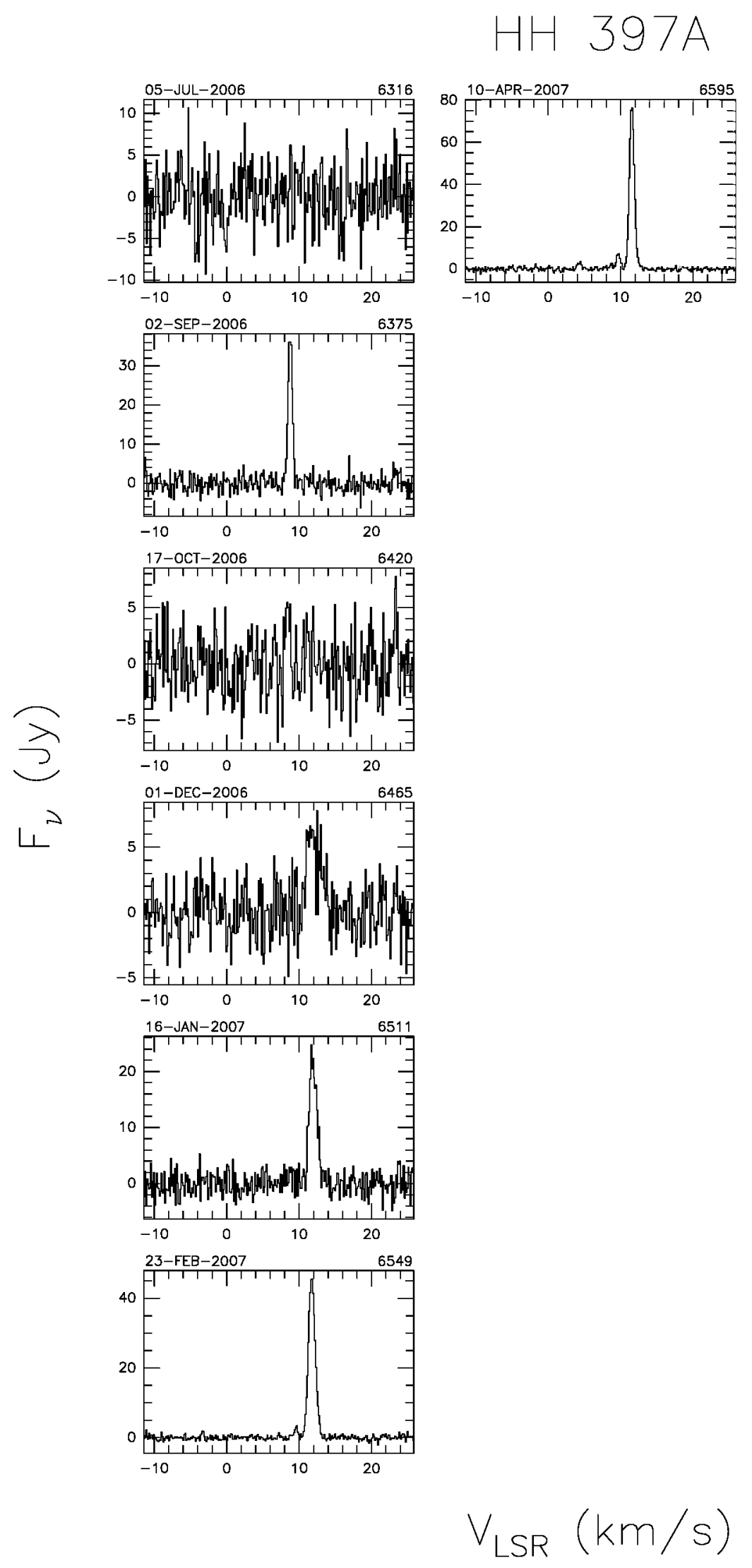

Fig. A.12. a continued. 


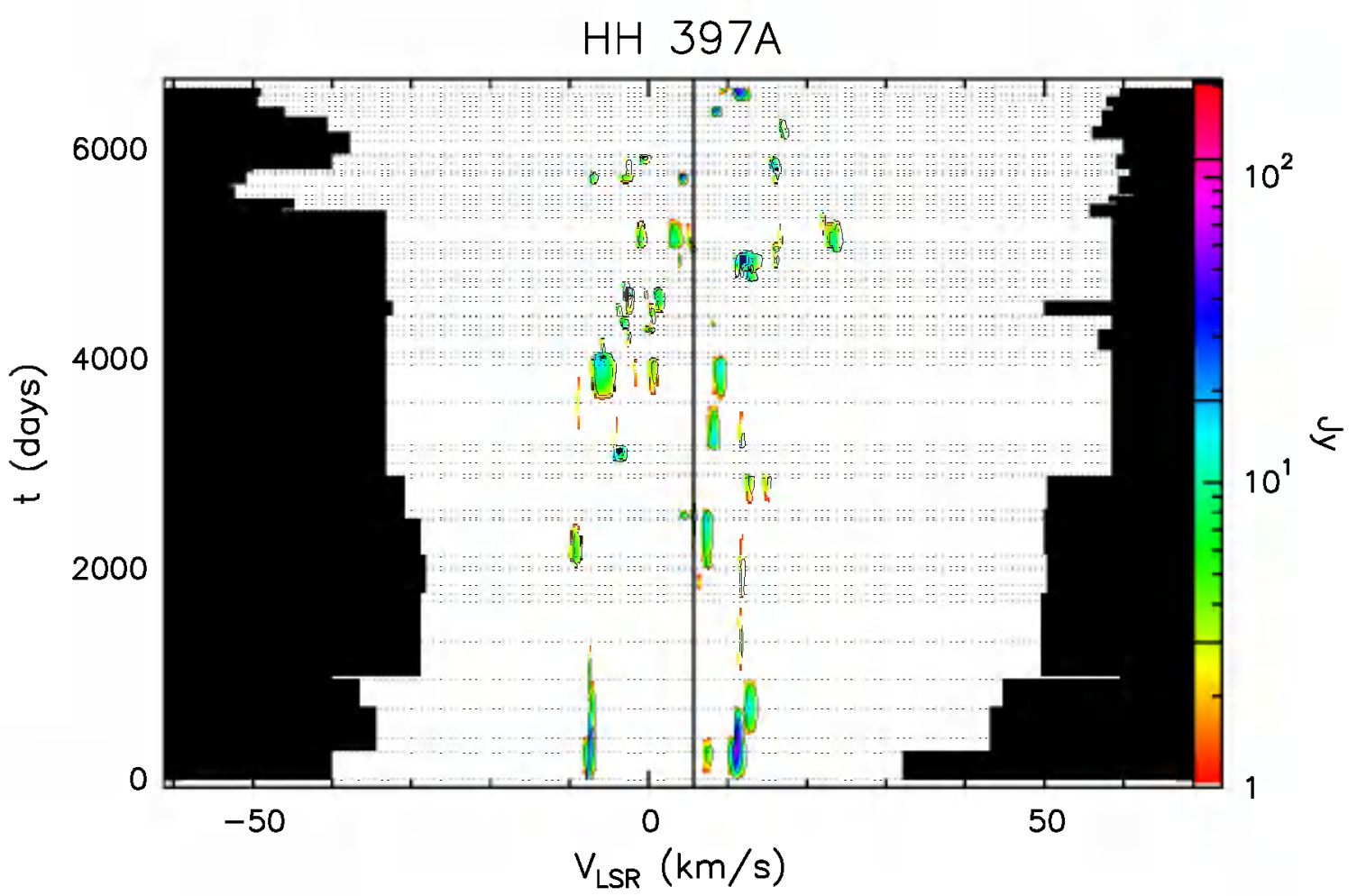

Fig. A.12. b Velocity-time-flux density full plot for source HH 397A. The vertical solid line indicates the velocity of the associated thermal molecular gas. The flux density scale is shown by the bar on the right. In this bar the three lines give the flux density of the drawn contours.

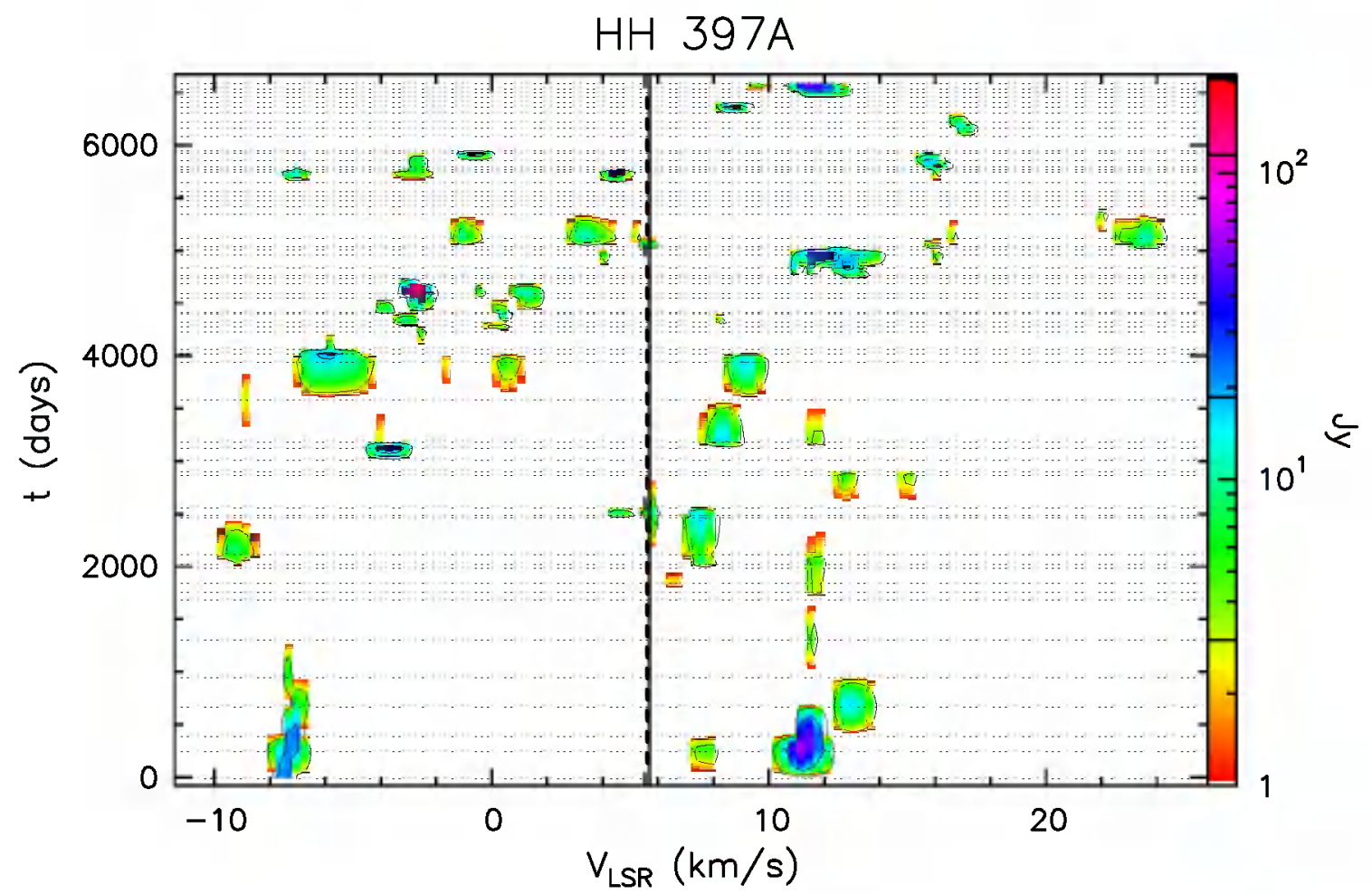

Fig. A.12. c Same as previous figure, but "zoomed" to velocity range over which emission has been detected. 


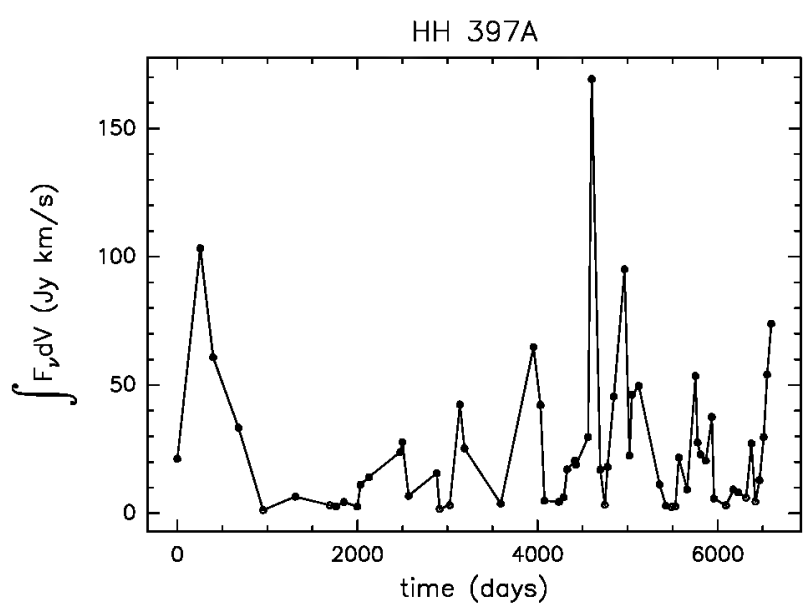

Fig. A.12. d Integral of the flux density over the observed velocity range as a function of time for source HH 397A.

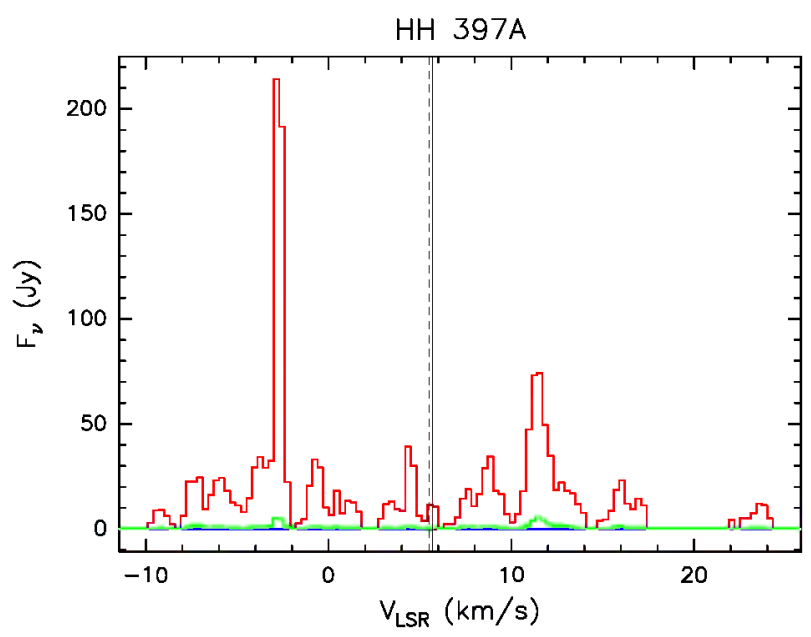

Fig. A.12. e Upper (red) and lower (blue) envelopes and mean spectrum (green) of source HH 397A measured during our monitoring. The vertical solid line marks the velocity of the associated thermal molecular gas. The vertical dashed line marks the mean velocity derived from the histogram of the rate-of-occurrence.

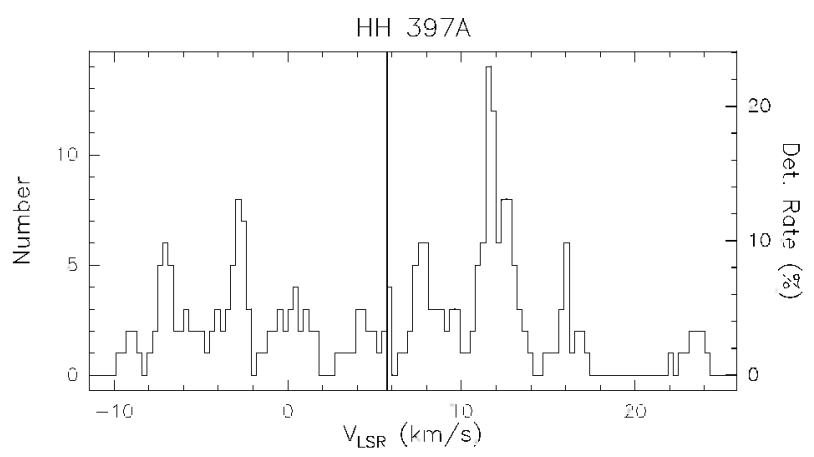

Fig. A.12. f Rate-of-occurrence plot for source HH 397A. The scale to the right refers to the dotted histogram. the scale to the left to the solid line histogram. The vertical solid line marks the velocity of the associated thermal molecular gas. 


\section{Mon R2 IRS3}
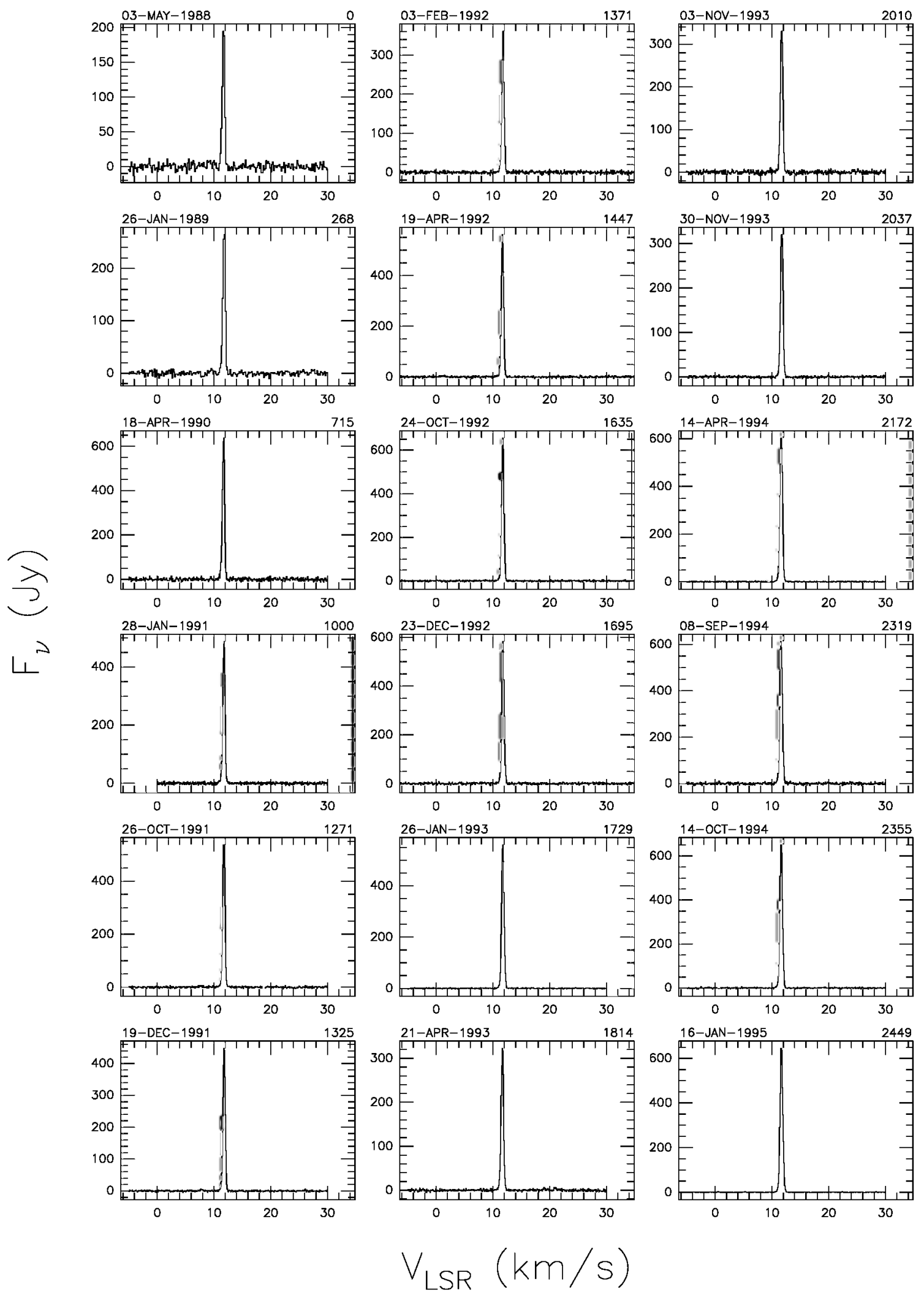

Fig. A.13. a Spectra of source Mon R2 IRS3 with autoscaled flux density scale. The date of observation is shown above the top left corner of each spectrum and the number of days elapsed since the first observation is given above the top right corner. The velocity scale is the same for all spectra. 


\section{Mon R2 IRS3}
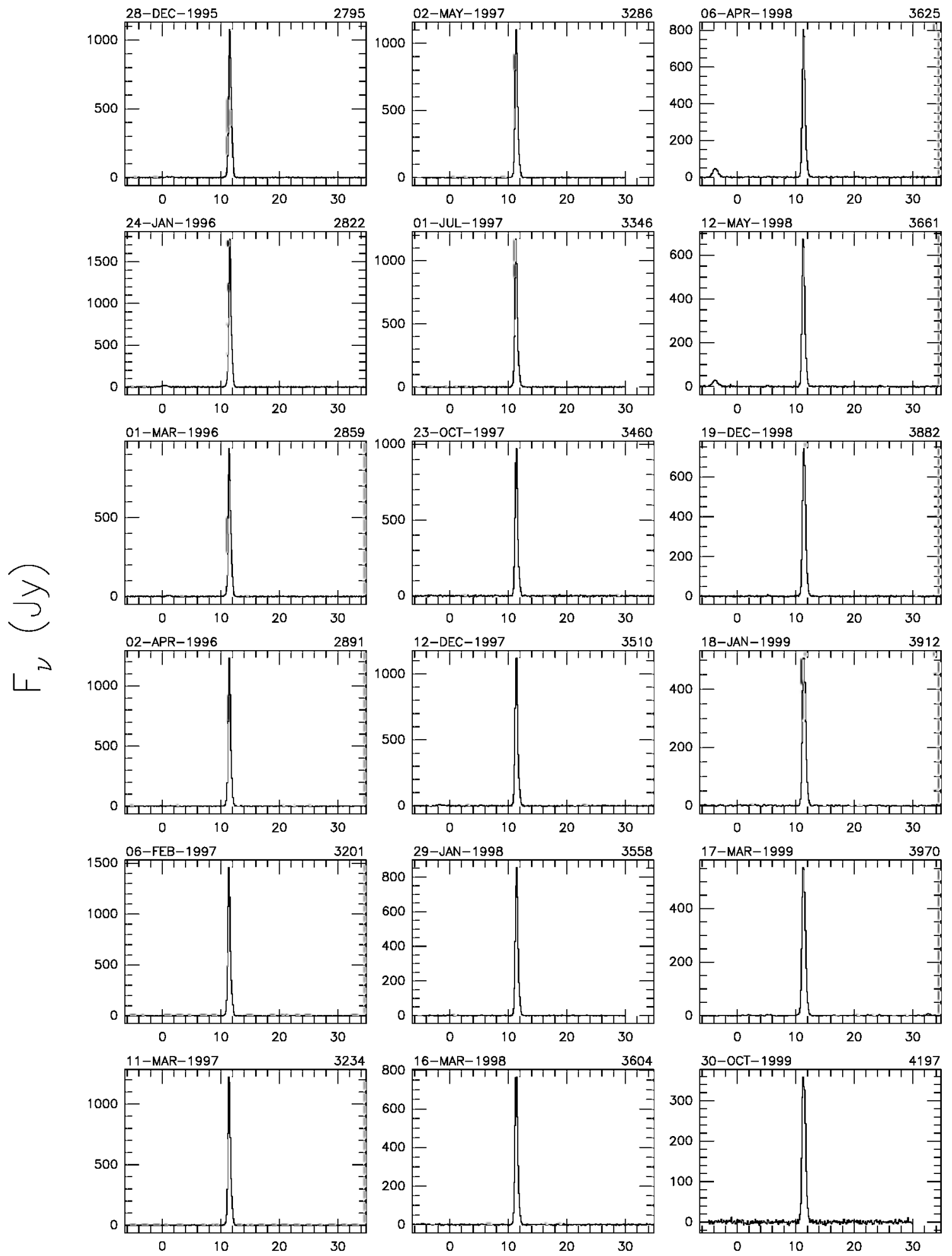

$$
V_{\text {LSR }}(\mathrm{km} / \mathrm{s})
$$

Fig. A.13. a continued. 


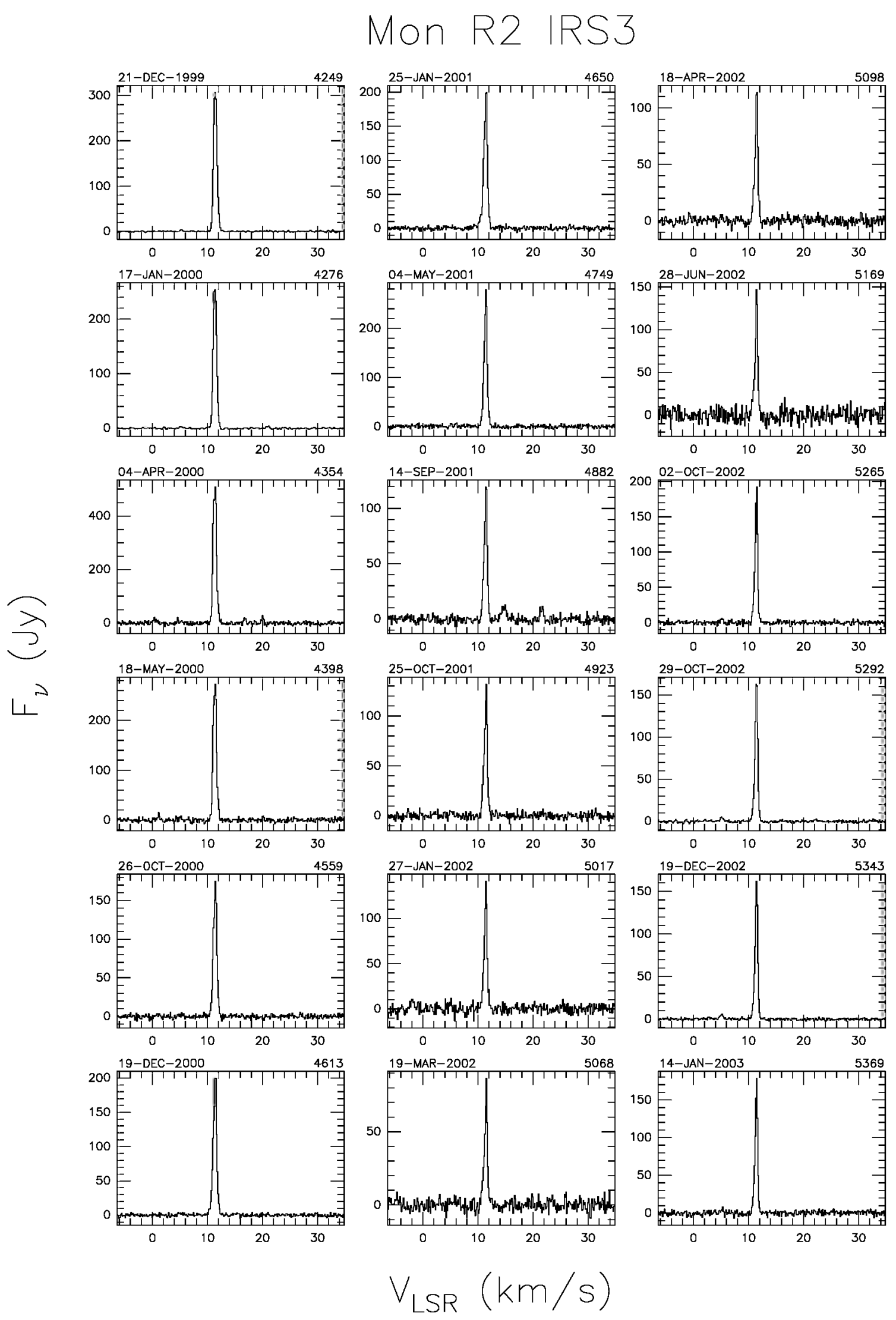

Fig. A.13. a continued. 


\section{Mon R2 IRS3}
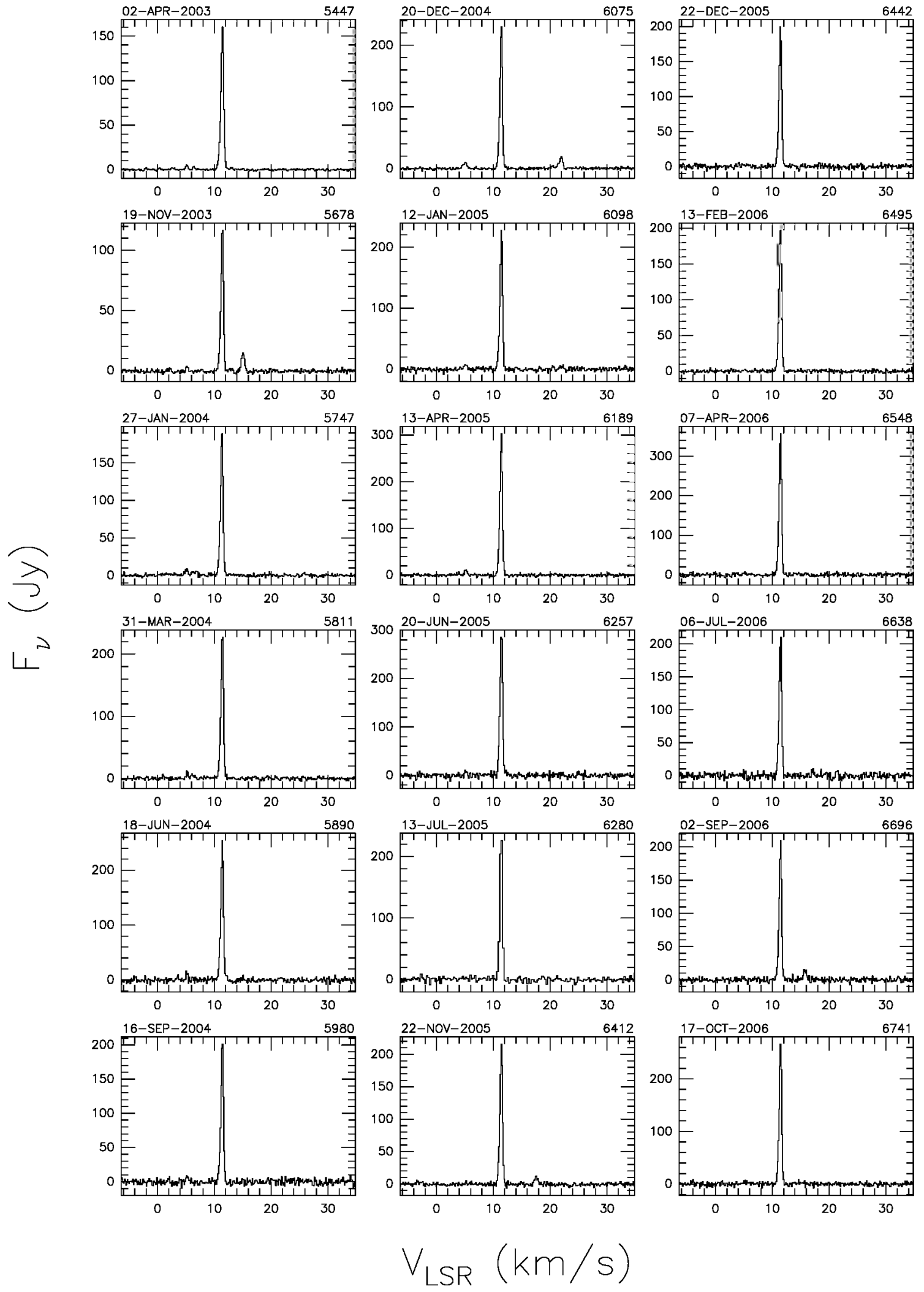

Fig. A.13. a continued. 
Mon R2 IRS3
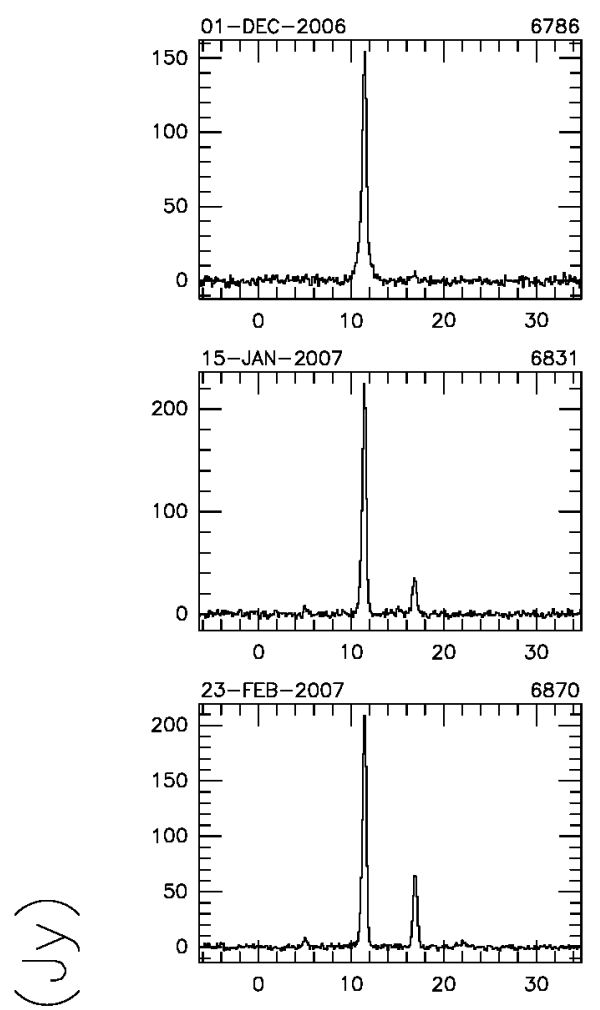

$\lambda$

$V_{\text {LSR }}(\mathrm{km} / \mathrm{s})$

Fig. A.13. a continued. 


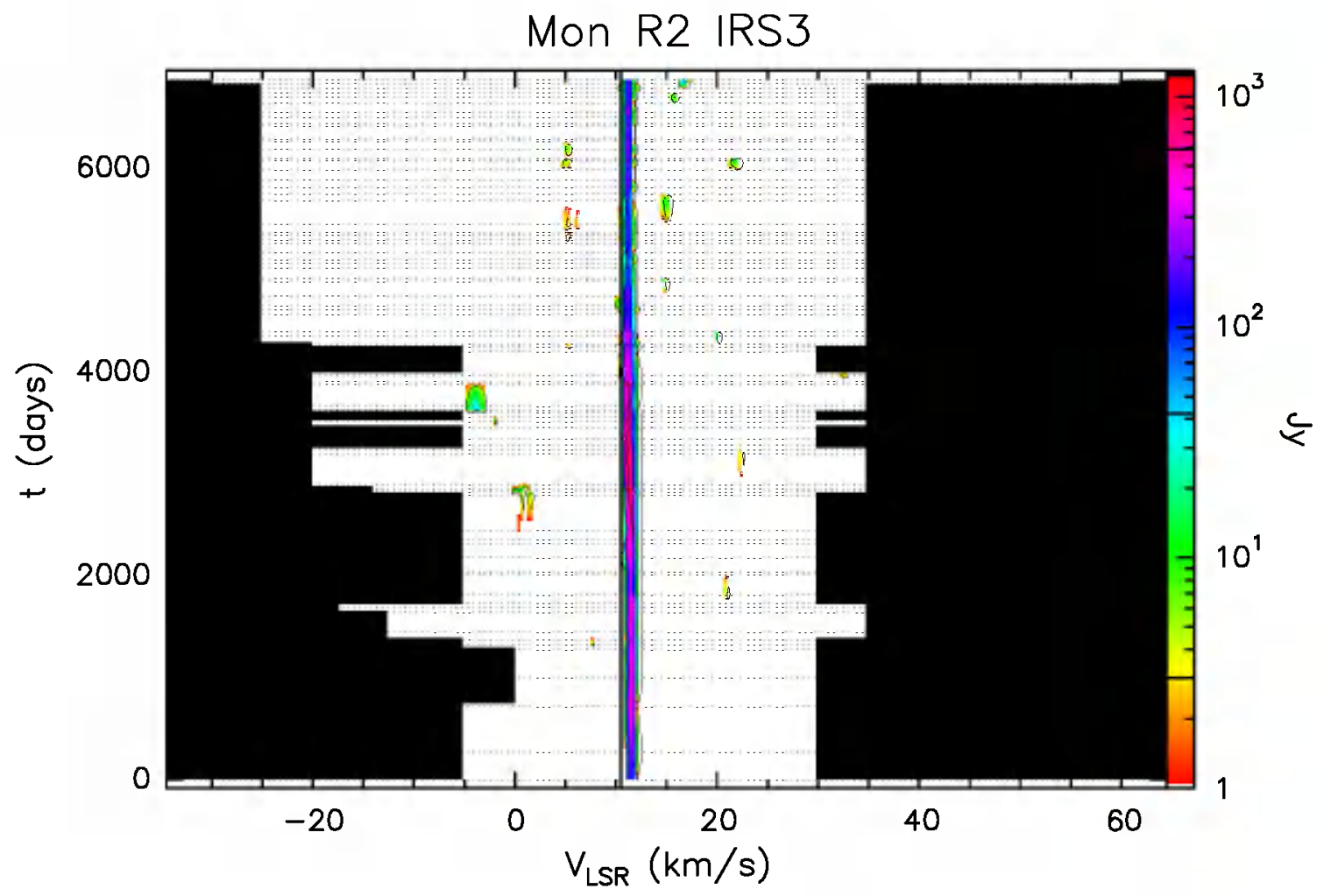

Fig. A.13. b Velocity-time-flux density full plot for source Mon R2 IRS3. The vertical solid line indicates the velocity of the associated thermal molecular gas. The flux density scale is shown by the bar on the right. In this bar the three lines give the flux density of the drawn contours.

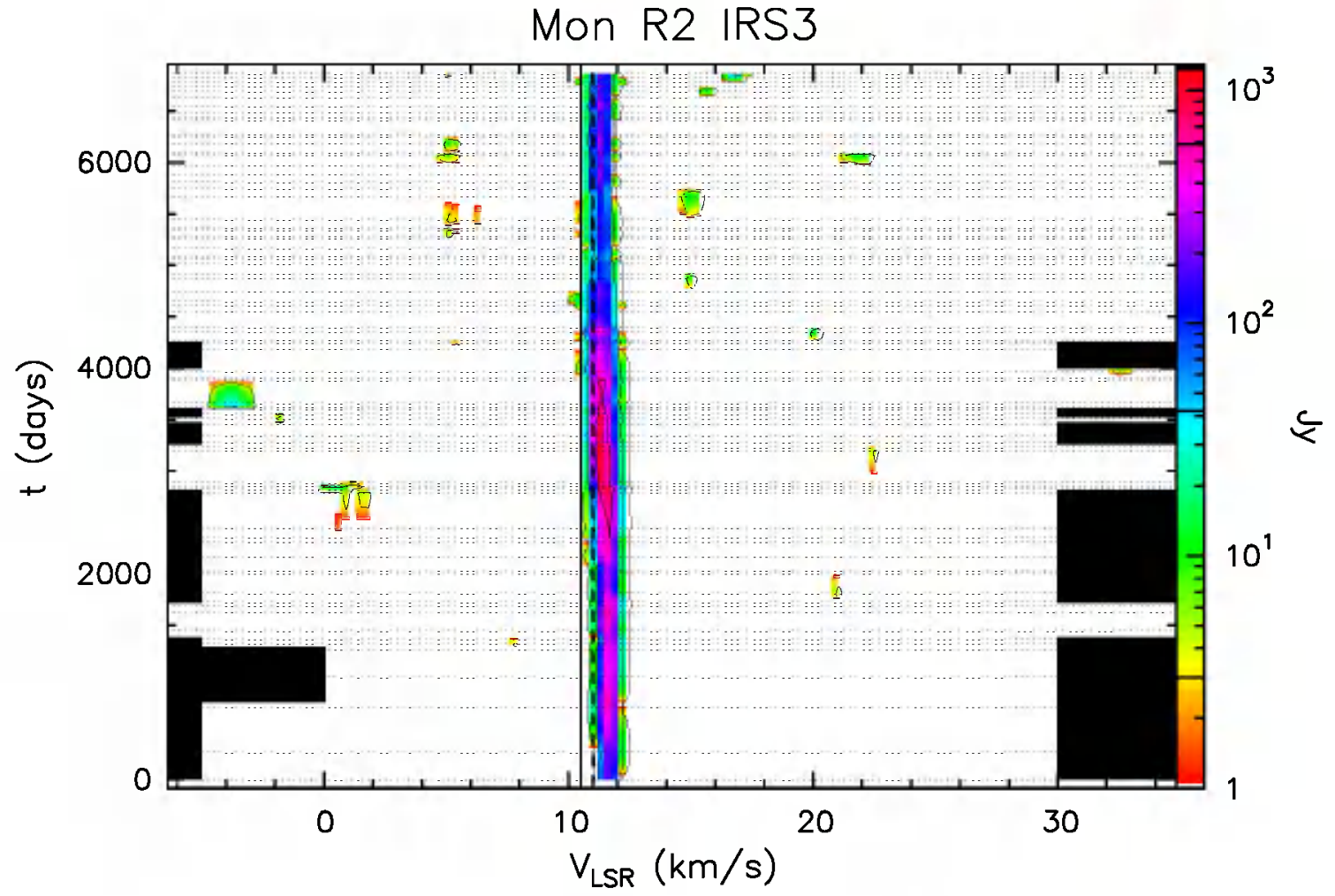

Fig. A.13. c Same as previous figure, but "zoomed" to velocity range over which emission has been detected. 


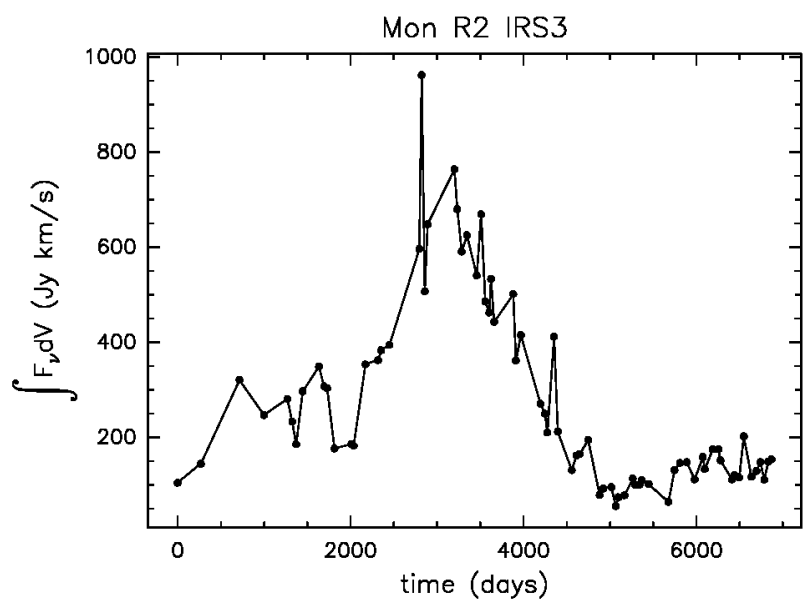

Fig. A.13. d Integral of the flux density over the observed velocity range as a function of time for source Mon R2 IRS3.

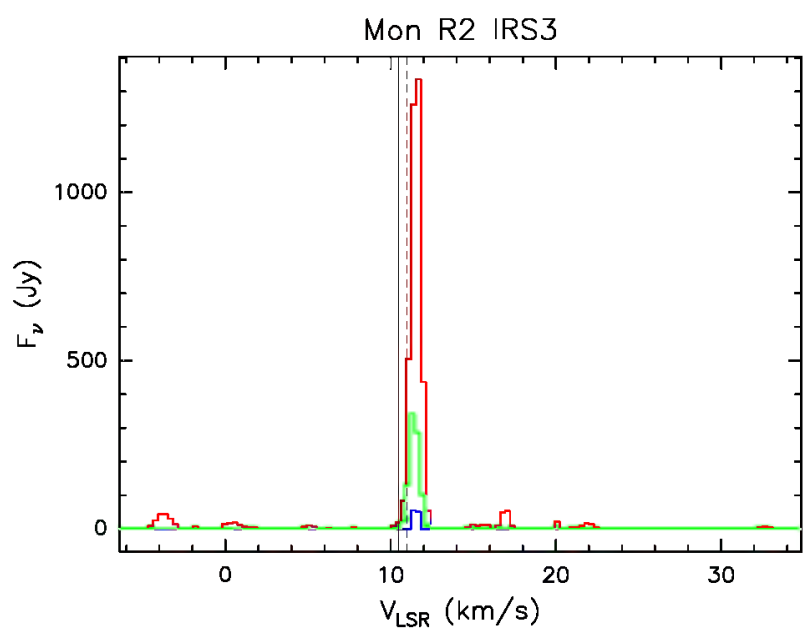

Fig. A.13. e Upper (red) and lower (blue) envelopes and mean spectrum (green) of source Mon R2 IRS3 measured during our monitoring. The vertical solid line marks the velocity of the associated thermal molectllar gas. The vertical dashed line marks the mean velocity derived from the histogram of the rate-of-occurrence.

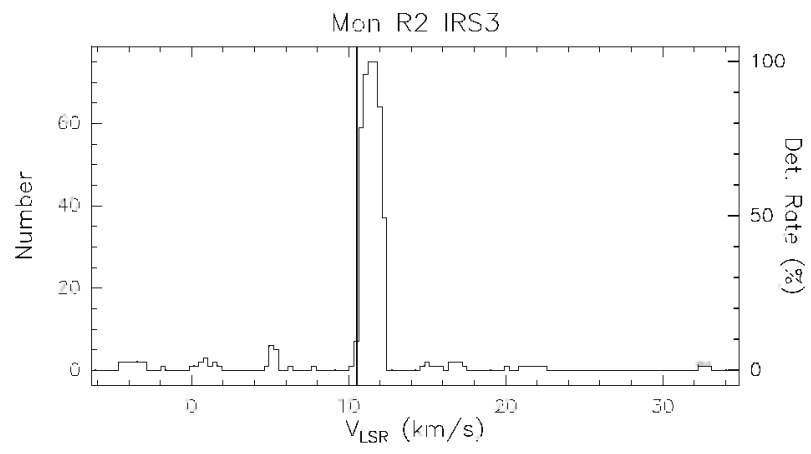

Fig. A.13. f Rate-of-occurrence plot for source Mon R2 IRS3. The scale to the right refers to the dotted histogram, the scale to the left to the solid line histogram. The vertical solid line marks the velocity of the associated thermal molecular gas. 


\section{Sh $2-252$}
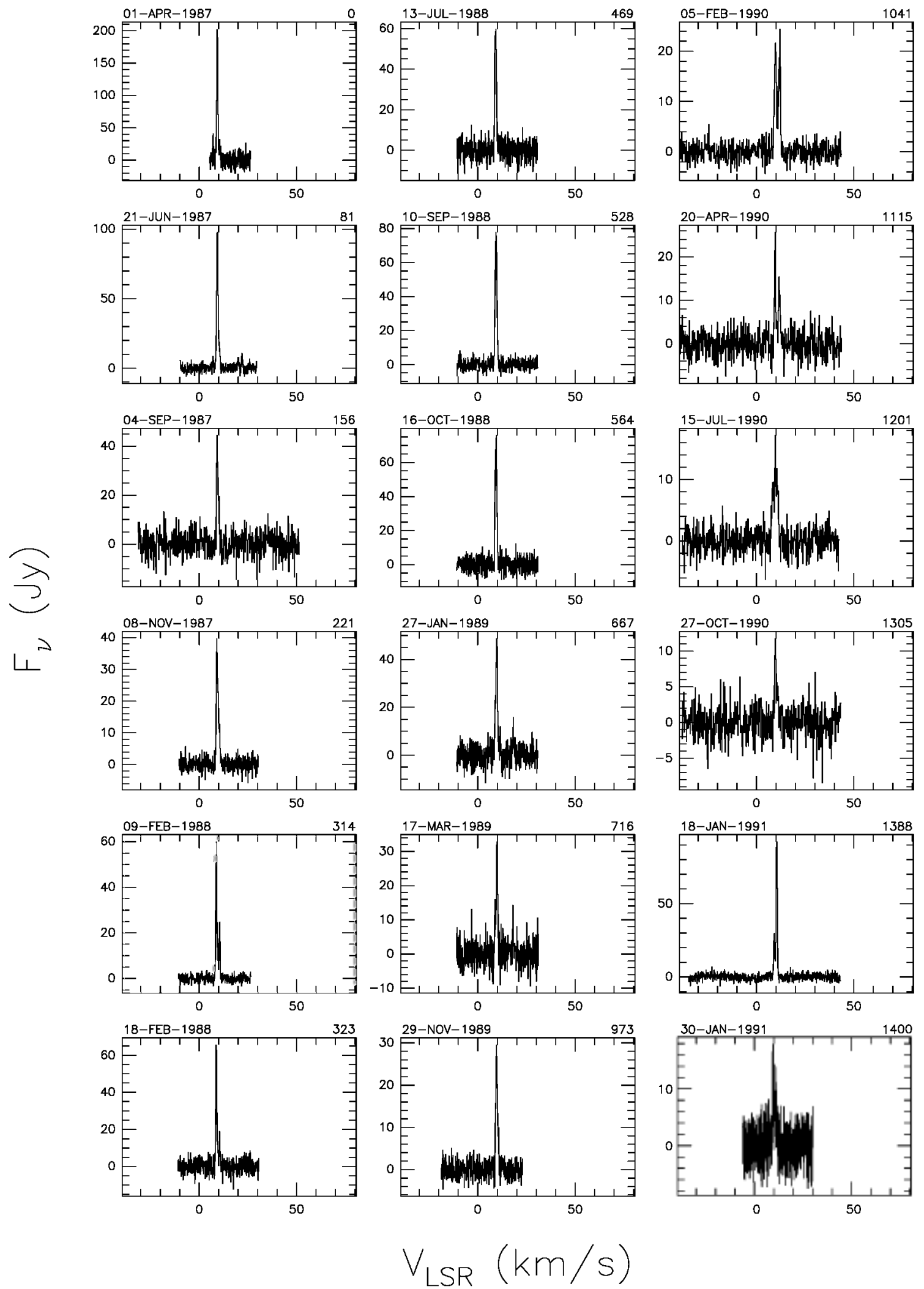

Fig. A.14. a Spectra of source Sh 2-252 with autoscaled flux density scale. The date of observation is shown above the top left corner of each spectrum and the number of days elapsed since the first observation is given above the top right corner. The velocity scale is the same for all spectra. 


$$
\text { Sh 2-252 }
$$
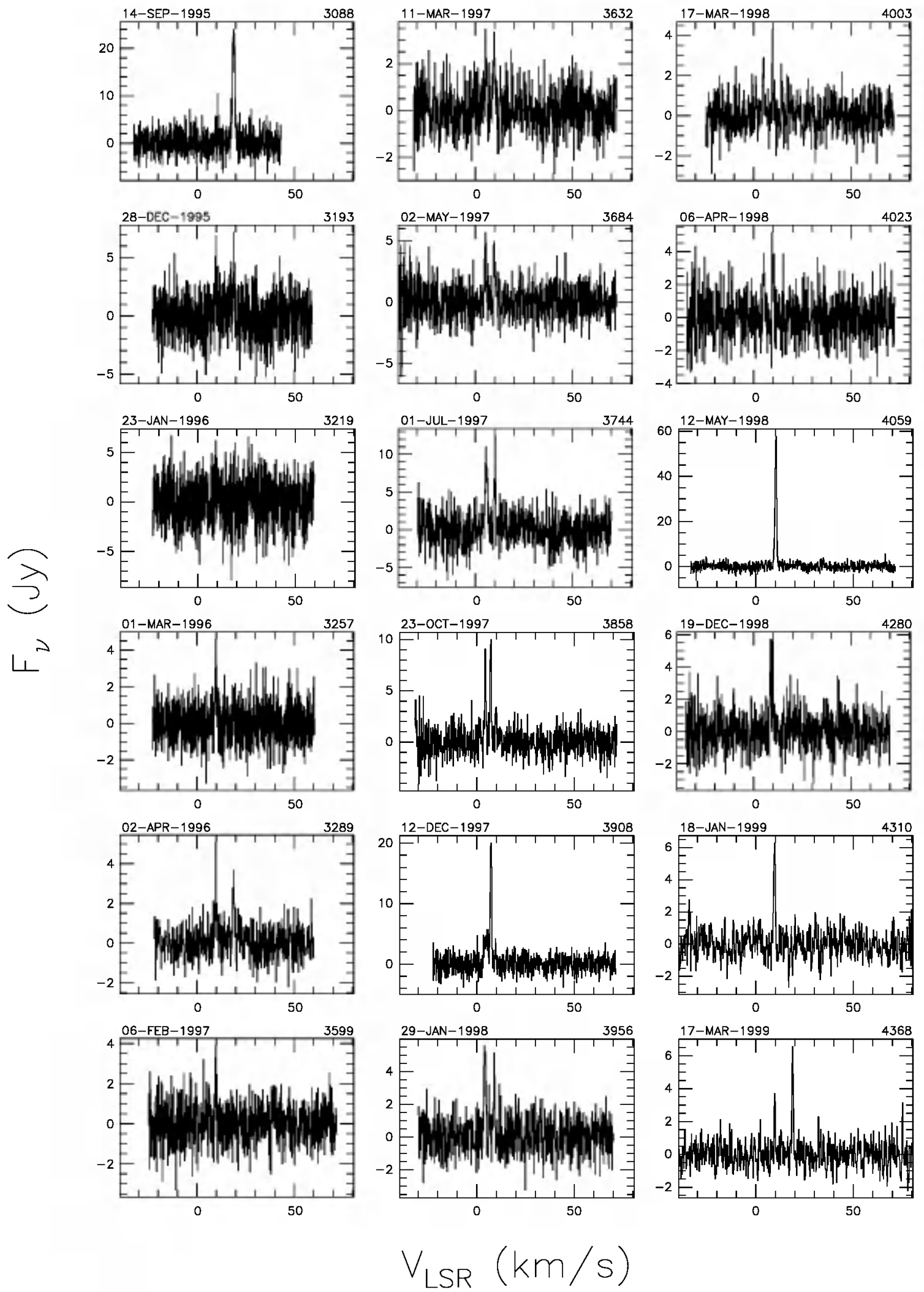

Fig. A.14. a continued. 
Sh 2-252
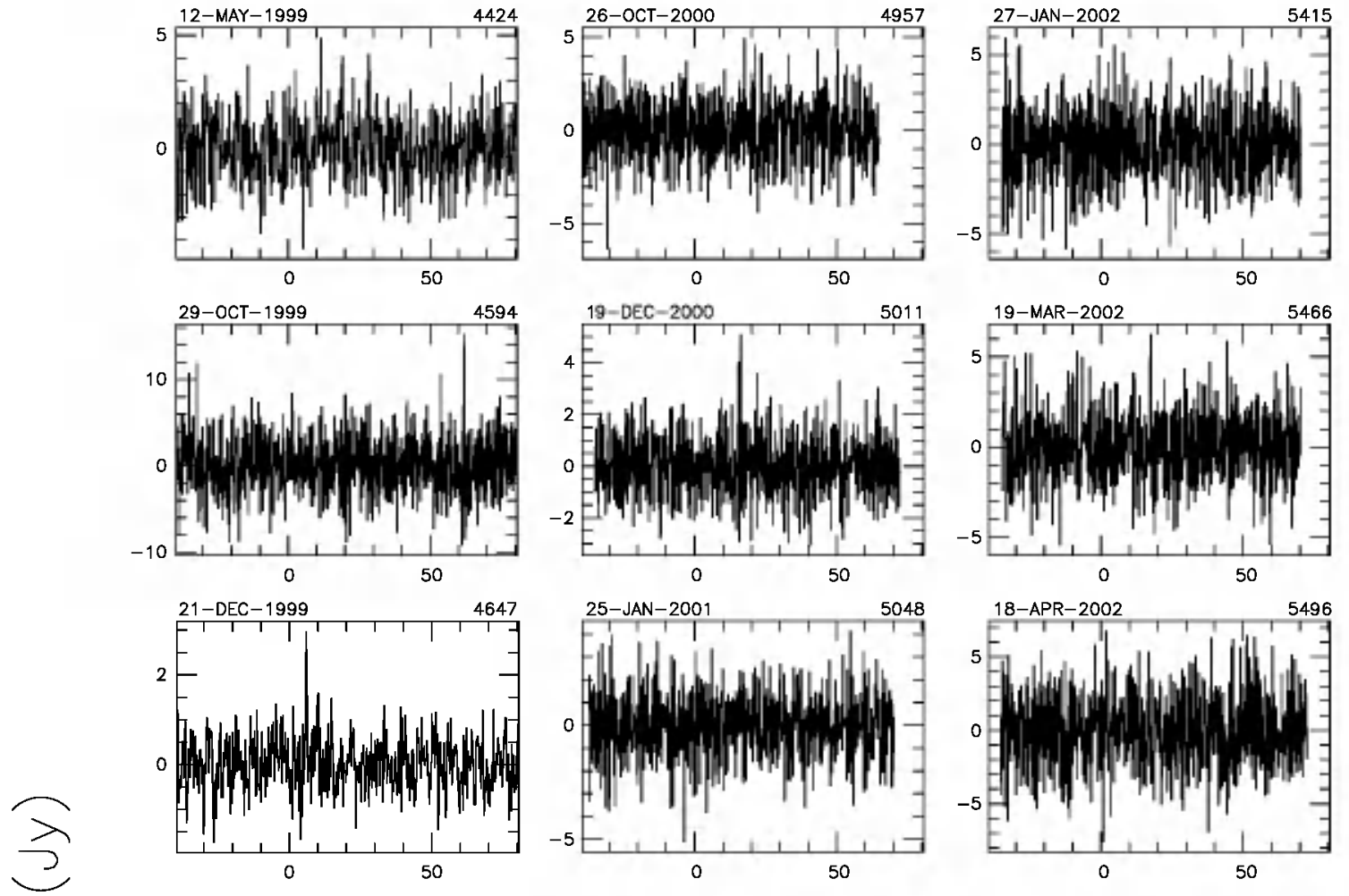

L
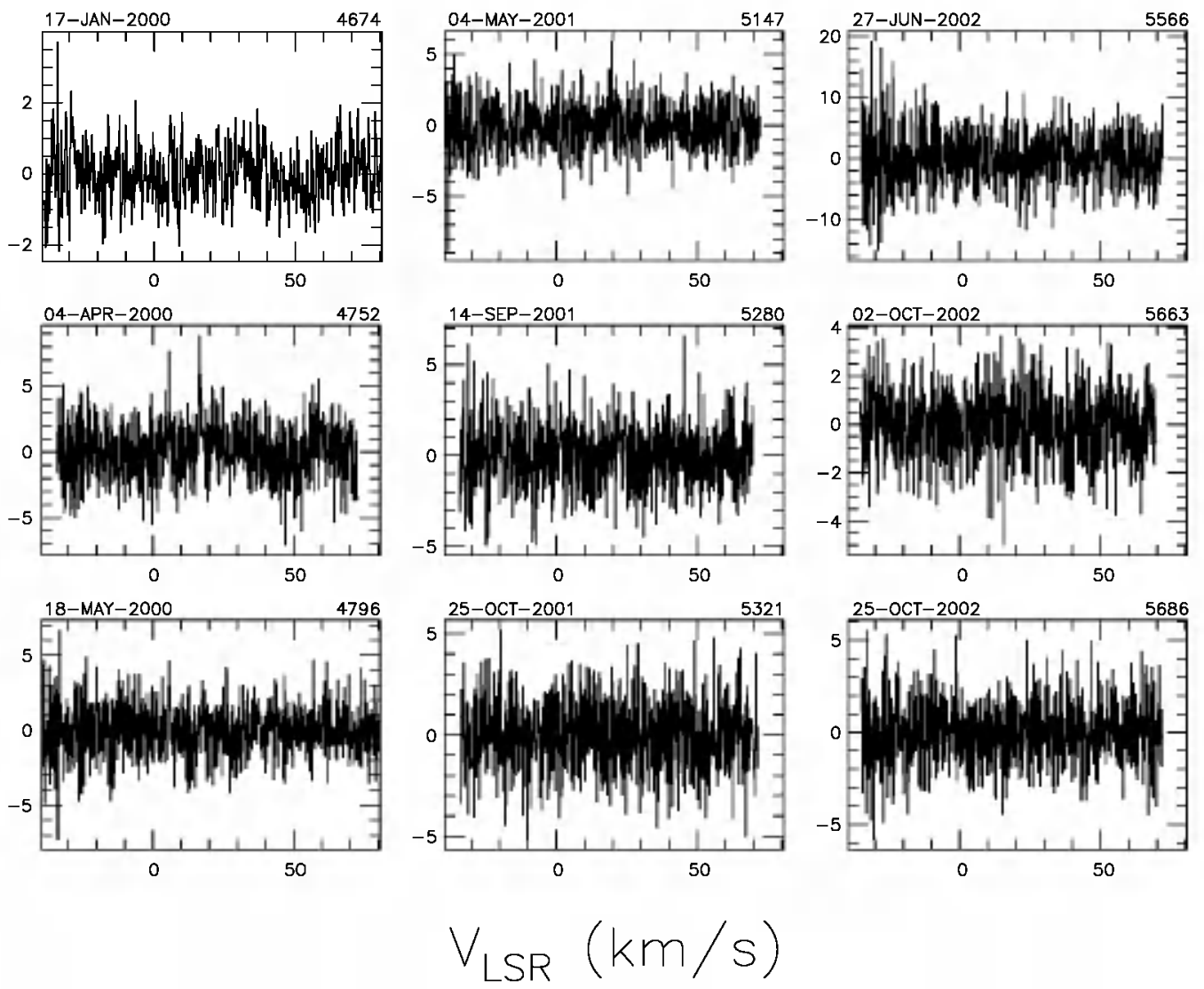

Fig. A.14. a continued. 


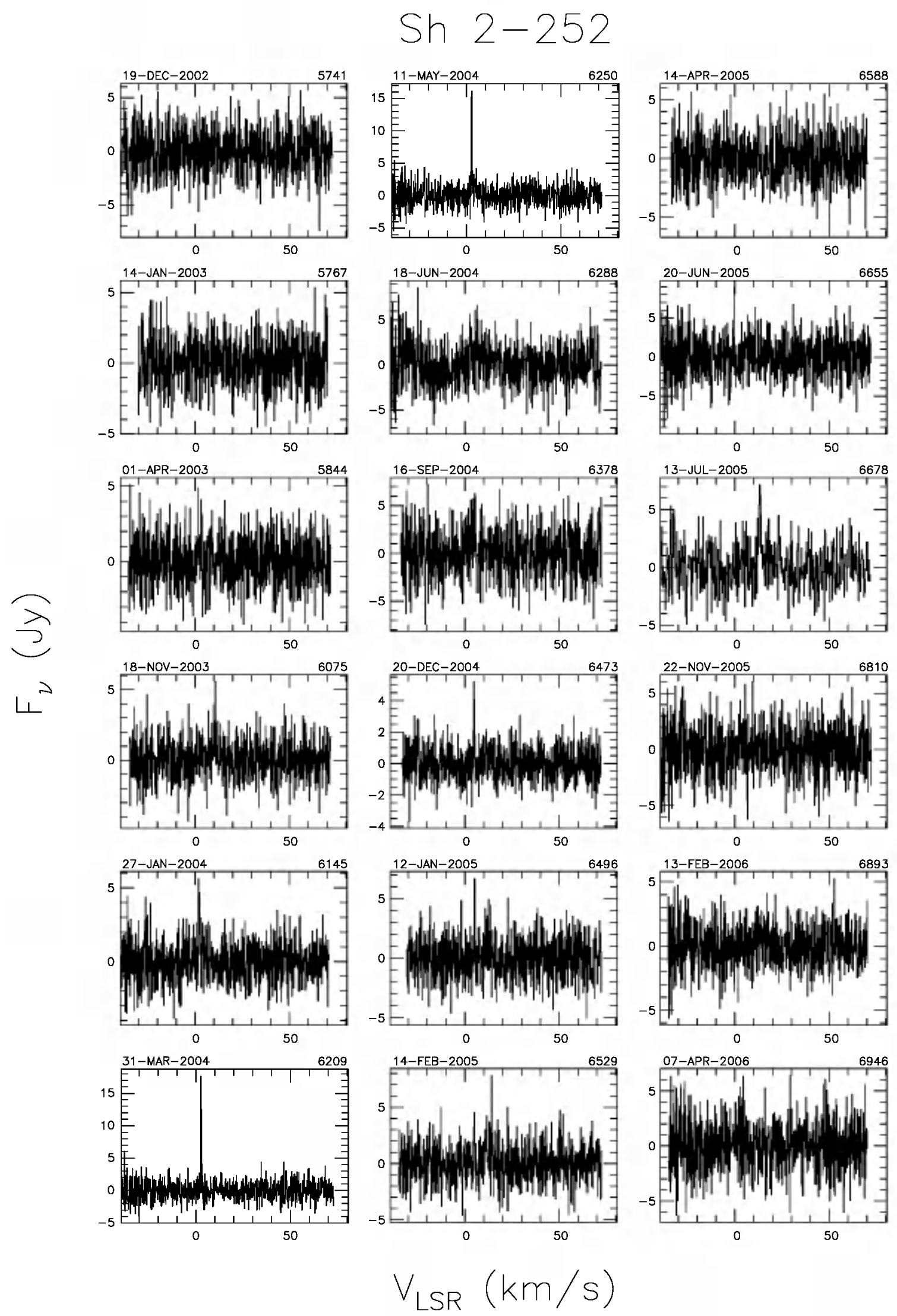

Fig. A.14. a continued. 
Sh $2-252$
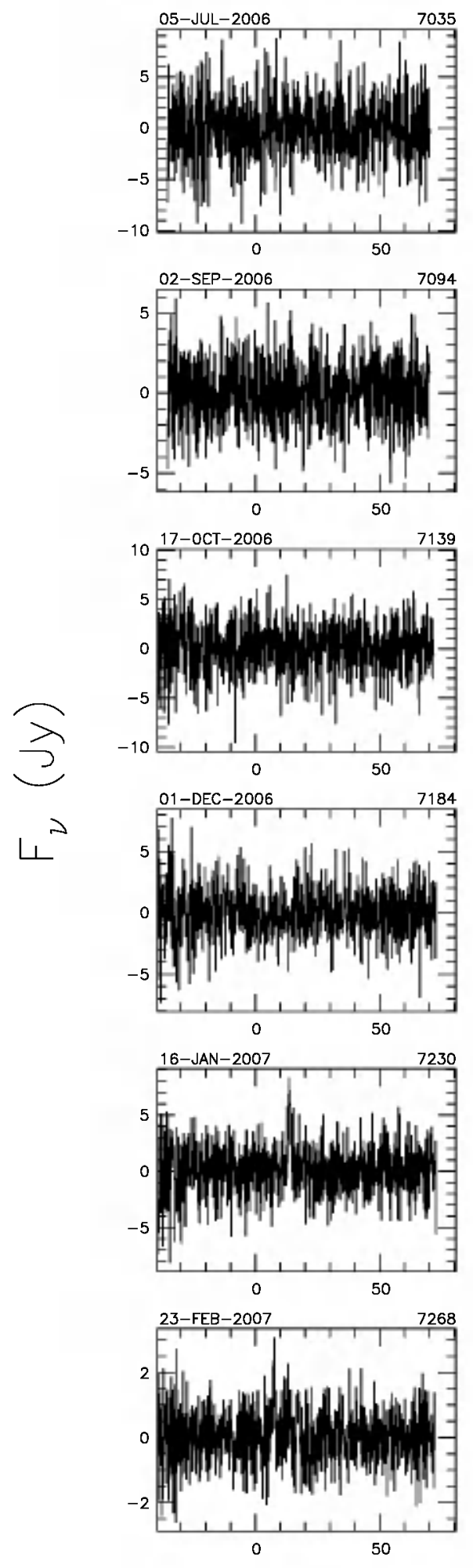

$$
V_{\text {LSR }}(k m / s)
$$

Fig. A.14. a continued. 


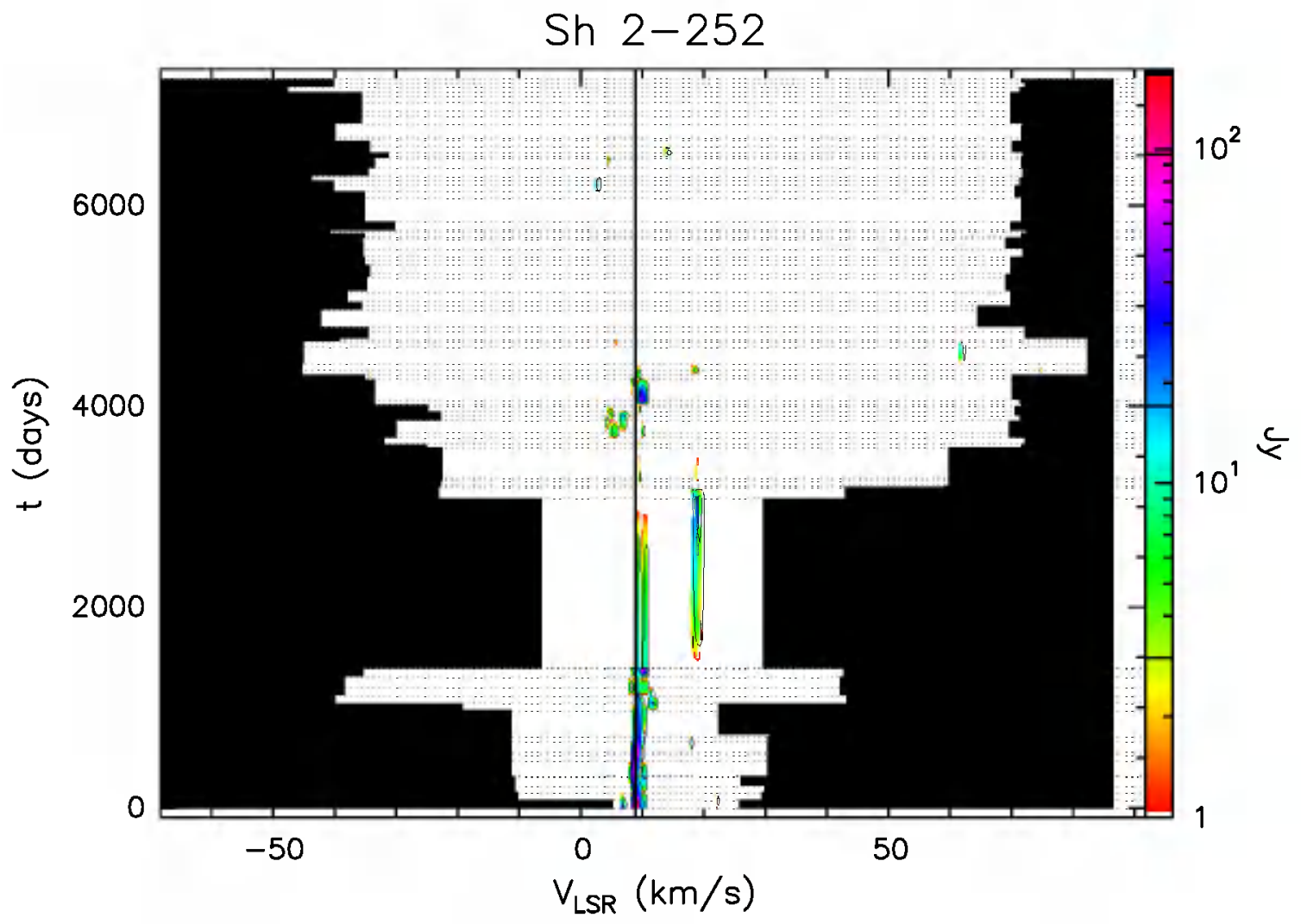

Fig. A.14. b Velocity-time-flux density full plot for source Sh 2-252. The vertical solid line indicates the velocity of the associated thermal molecular gas. The flux density scale is shown by the bar on the right. In this bar the three lines give the flux density of the diawn contours.

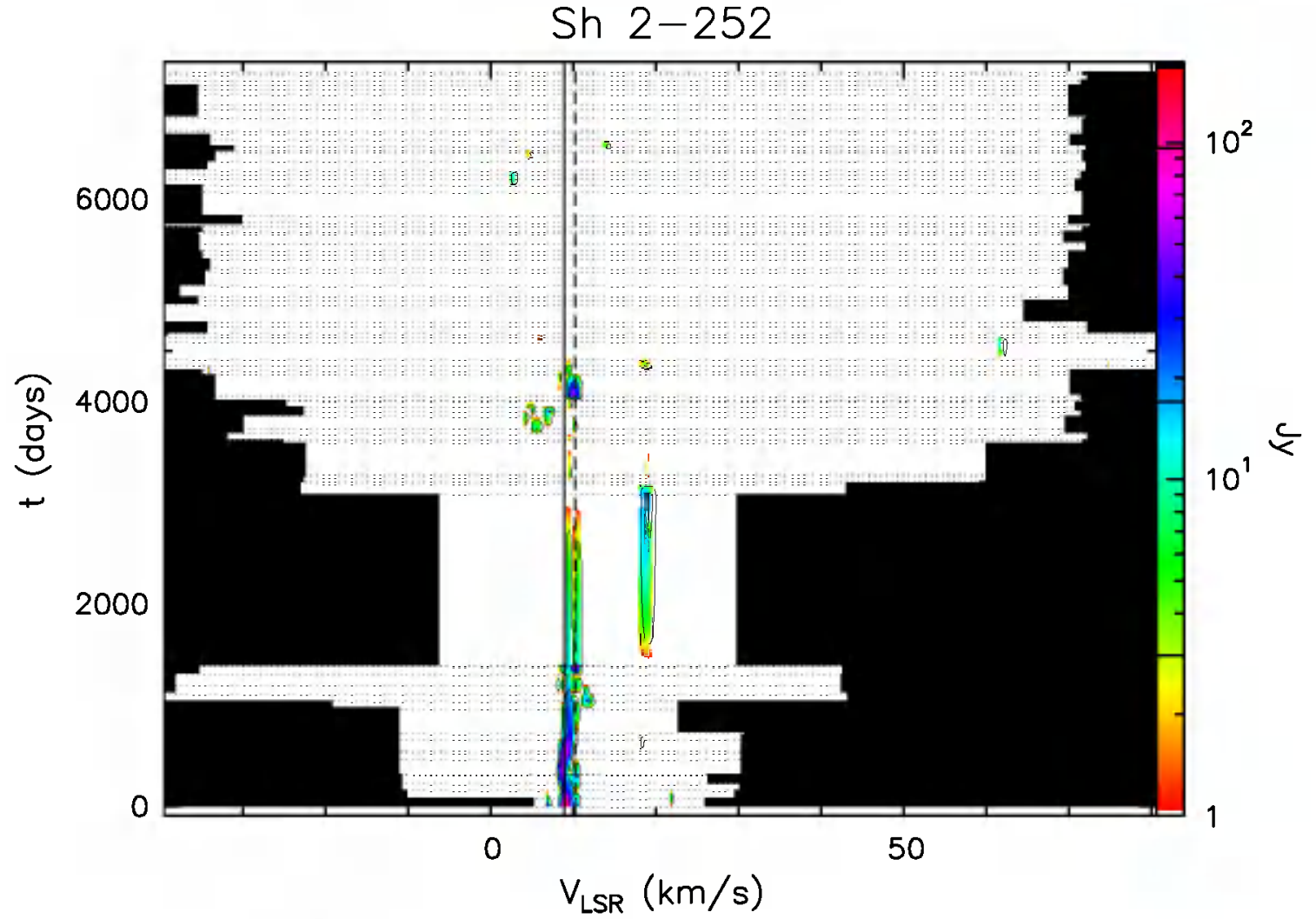

Fig. A.14. c Same as previous figure, but "zoomed" to velocity range over which emission has been detected. 


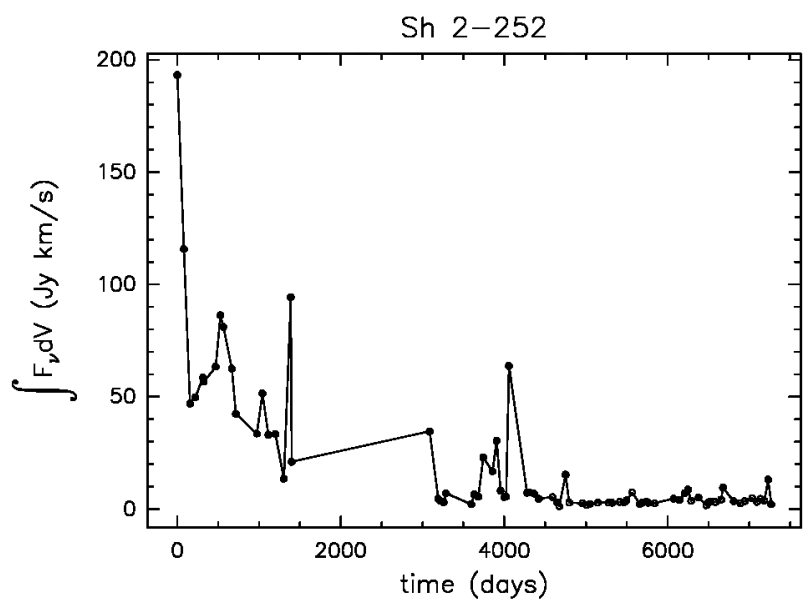

Fig. A.14. d Integral of the flux density over the observed velocity range as a function of time for source Sh 2-252.

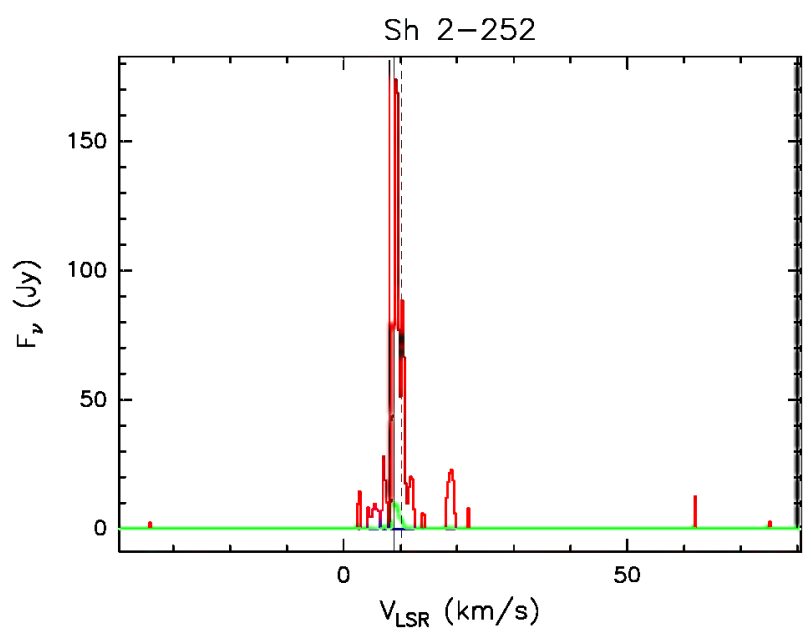

Fig. A.14. e Upper (red) and lower (blue) envelopes and mean spectrum (green) of source Sh 2-252 measured during our monitoring. The vertical solid line marks the velocity of the associated thermal molecular gas. The vertical dashed line marks the mean velocity derived from the histogram of the rate-of-occurrence.

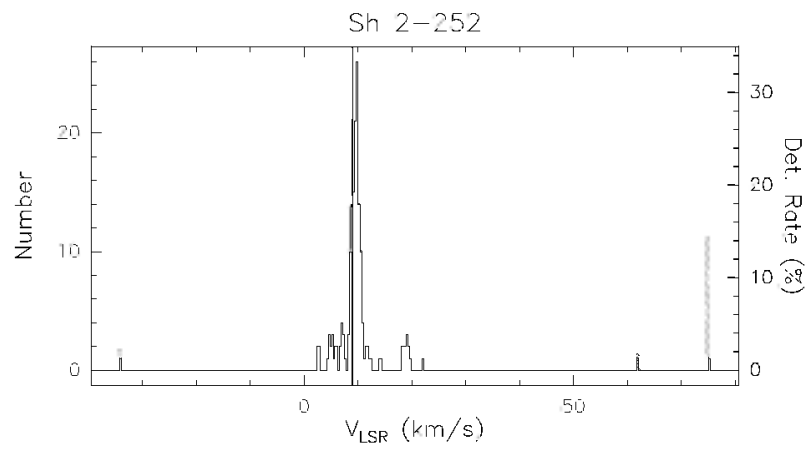

Fig. A.14. f Rate-of-occurrence plot for source Sh 2-252. The scale to the right refers to the dotted histogram, the scale to the left to the solid line histogram. The vertical solid line marks the velocity of the associated thermal molecular gas. 


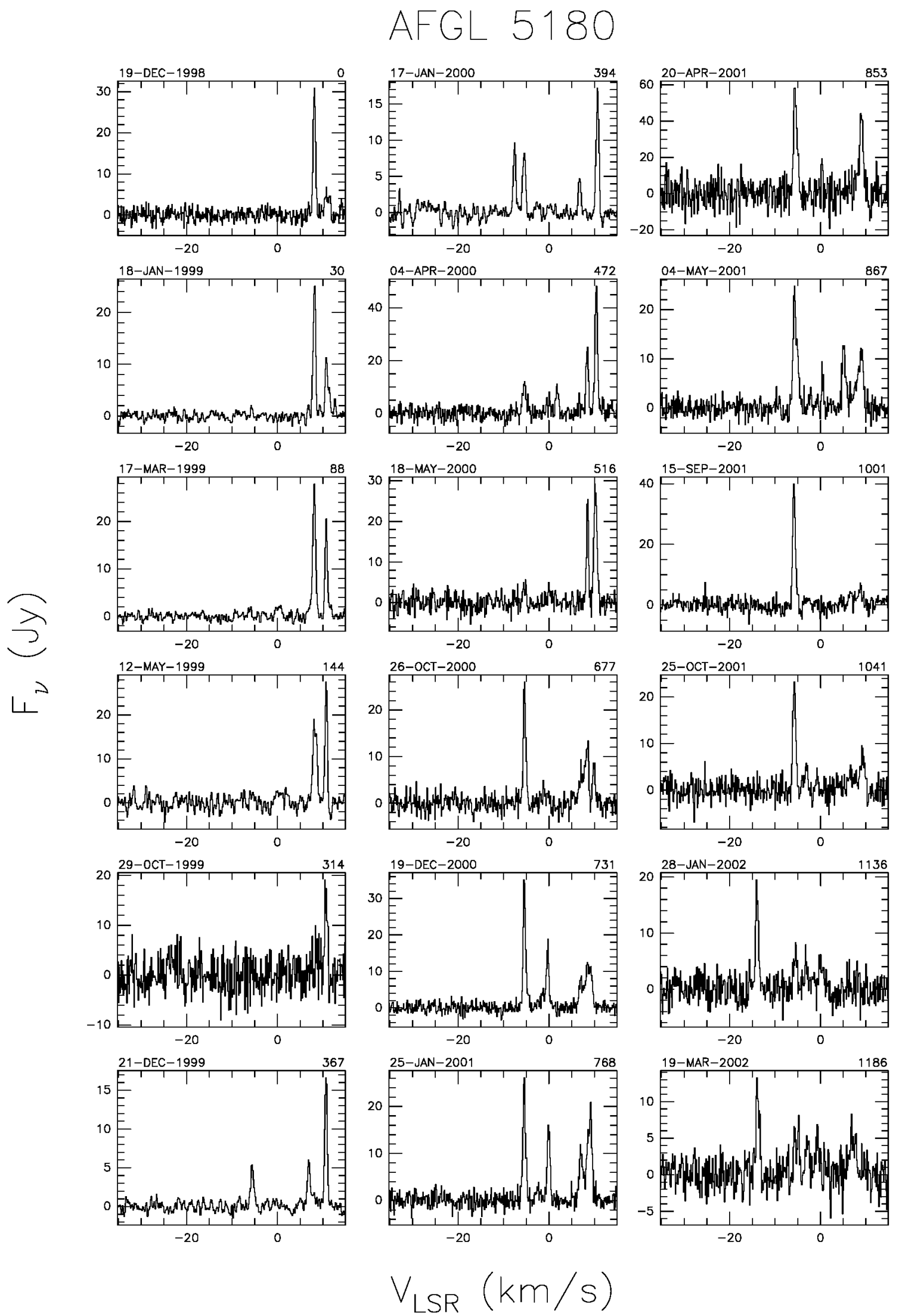

Fig. A.15. a Spectra of source AFGL 5180 with autoscaled flux density scale. The date of observation is shown above the top left corner of each spectrum and the number of days elapsed since the first observation is given above the top right corner. The velocity scale is the same for all spectra. 

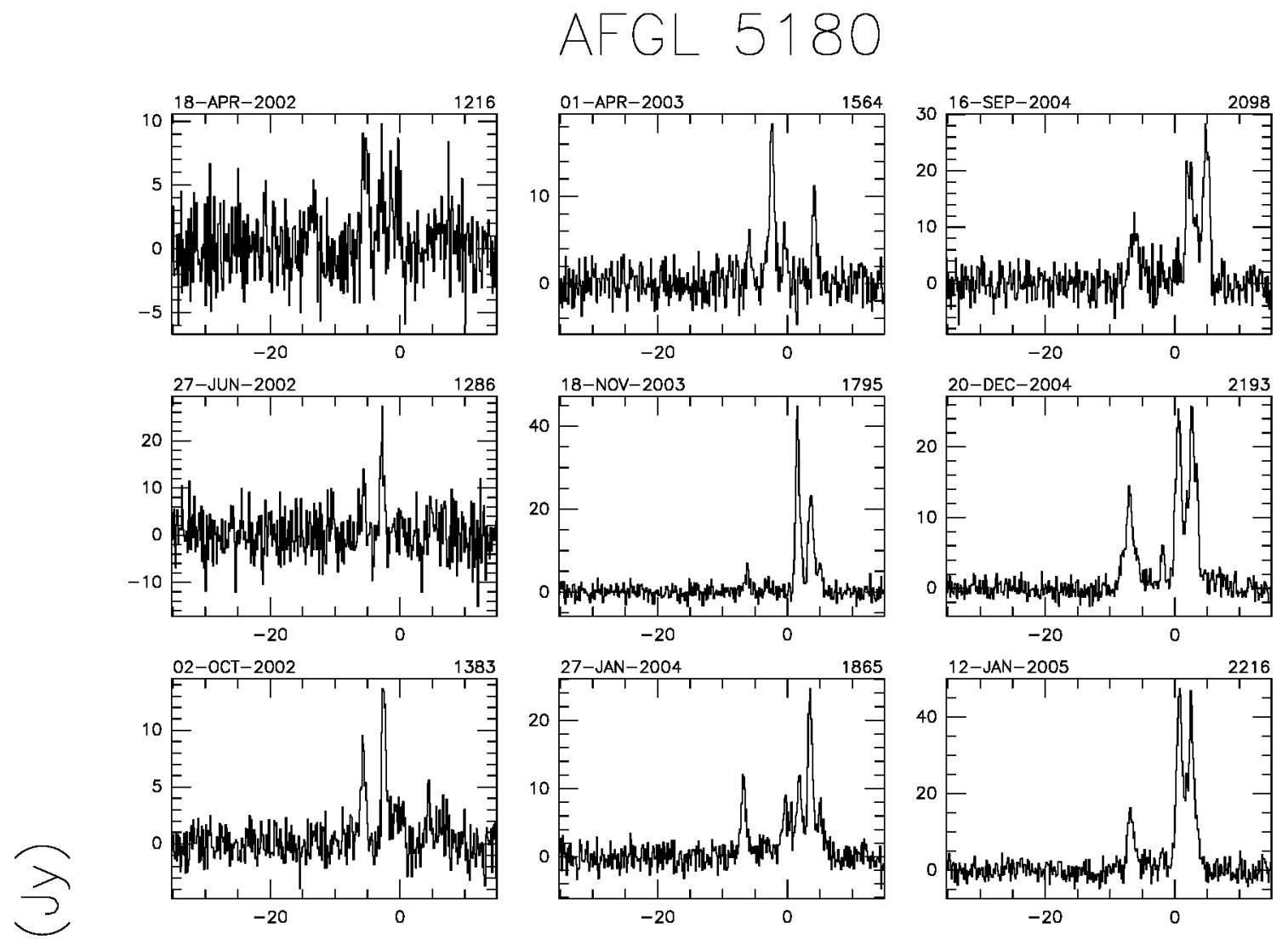

$L^{2}$
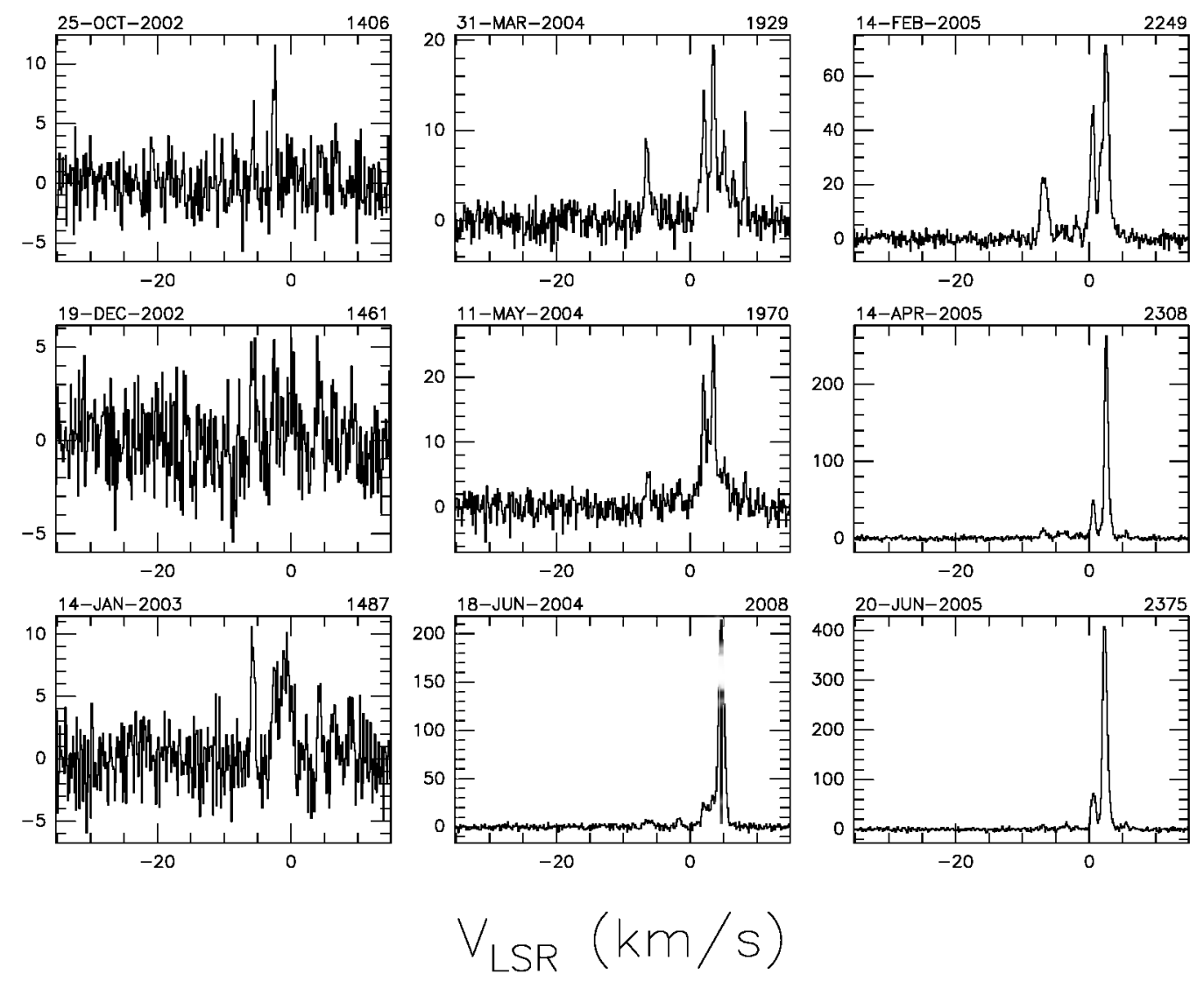

Fig. A.15. a continued. 


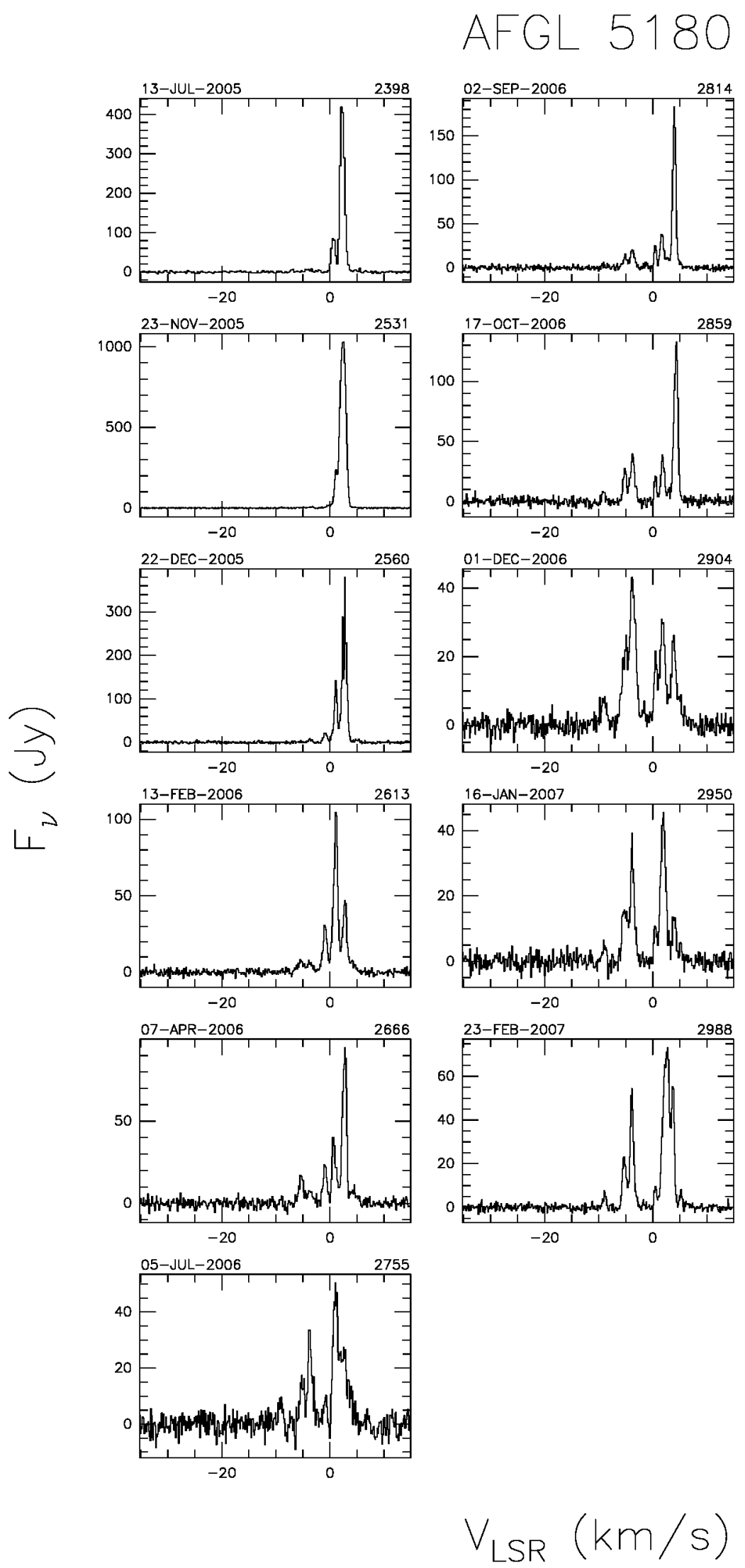

Fig. A.15. a continued. 


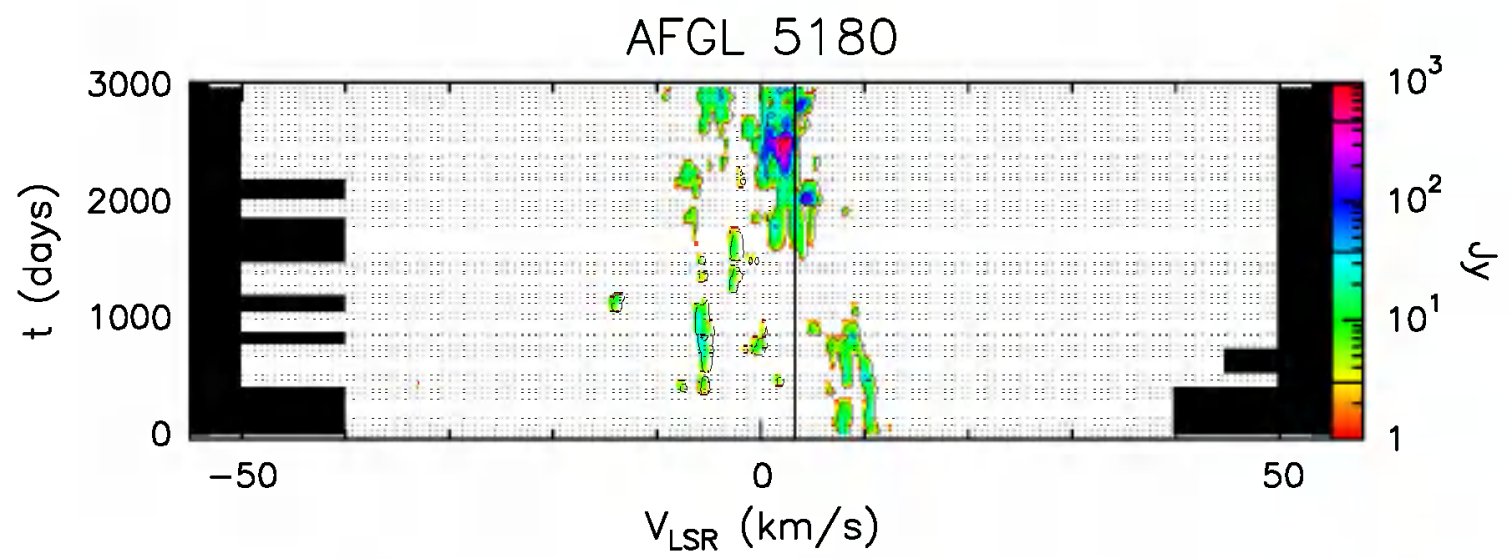

Fig. A.15. b Velocity-time-flux density full plot for source AFGL 5180. The vertical solid line indicates the velocity of the associated thermal molecular gas. The flux density scale is shown by the bar on the right. In this bar the three lines give the flux density of the drawn contours.

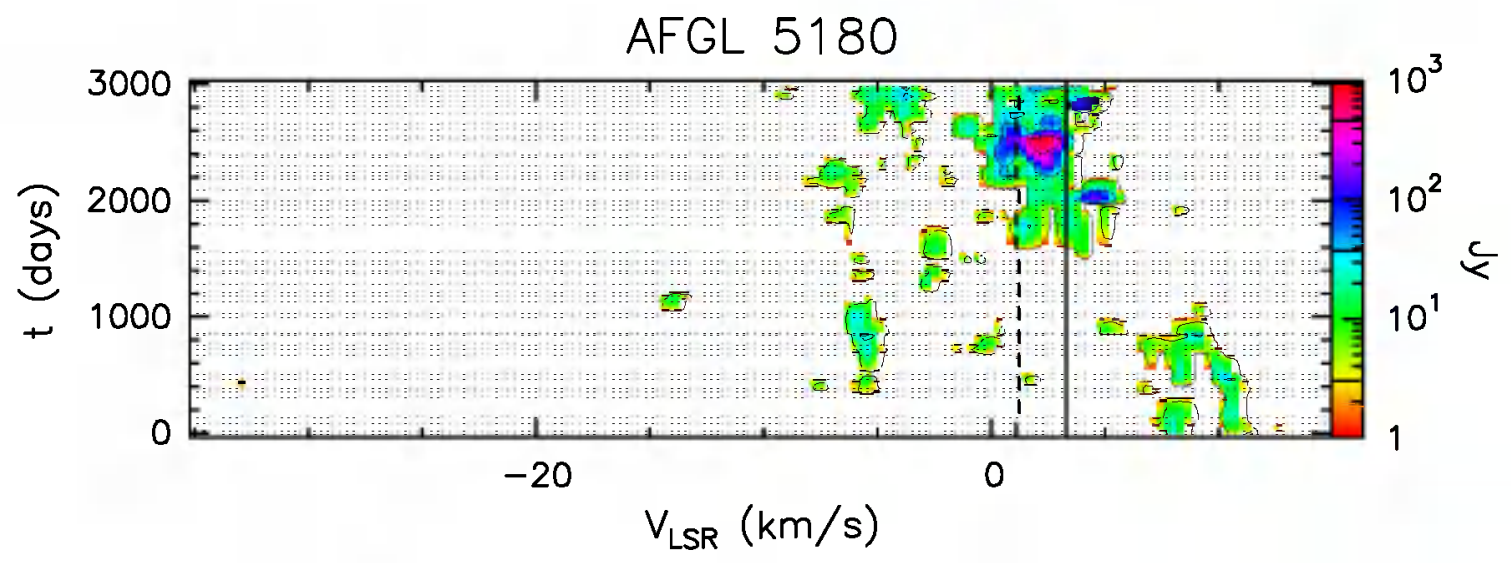

Fig. A.15. c Same as previous figure, but "zoomed" to velocity range over which emission has been detected. 


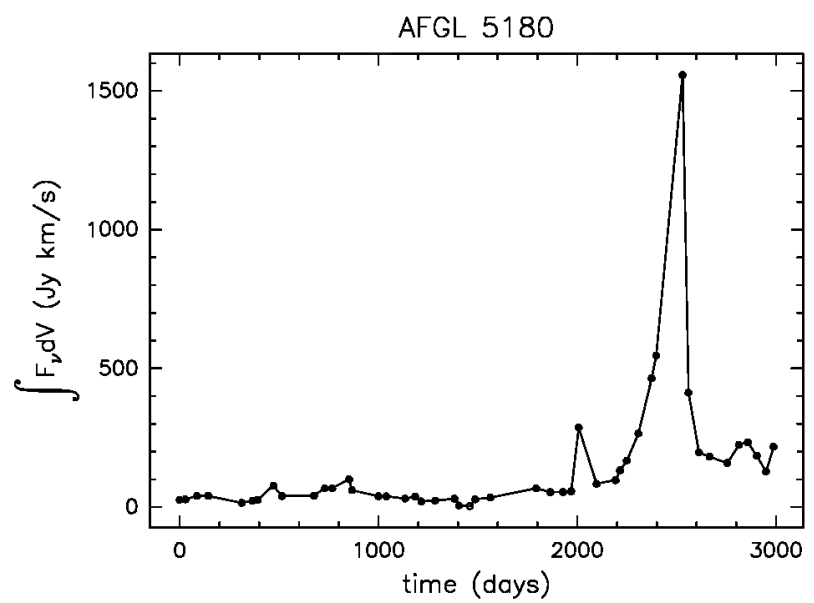

Fig. A.15. d Integral of the flux density over the observed velocity range as a function of time for source AFGL 5180.

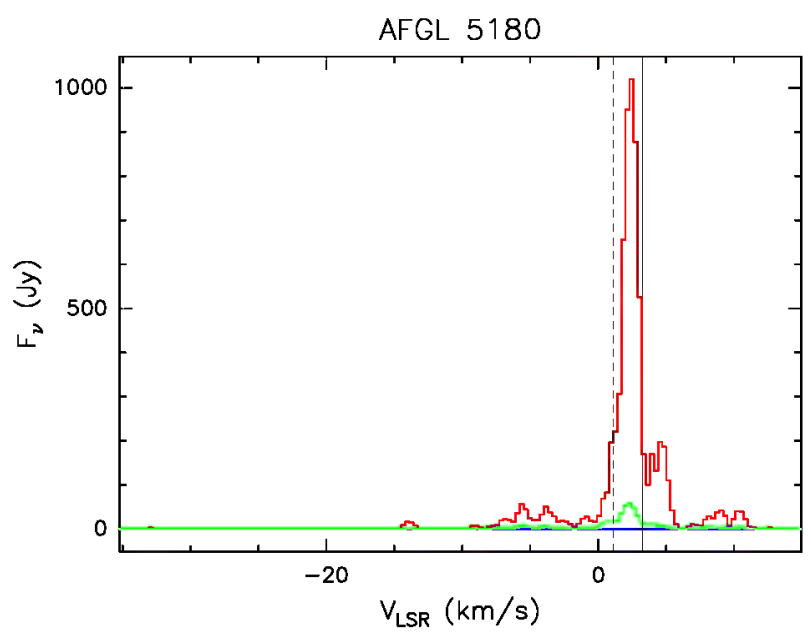

Fig. A.15. e Upper (red) and lower (blue) envelopes and mean spectrum (green) of source AFGL 5180 measured during our monitoring. The vertical solid line marks the velocity of the associated thermal molectllar gas. The vertical dashed line marks the mean velocity derived from the histogram of the rate-of-occurrence.

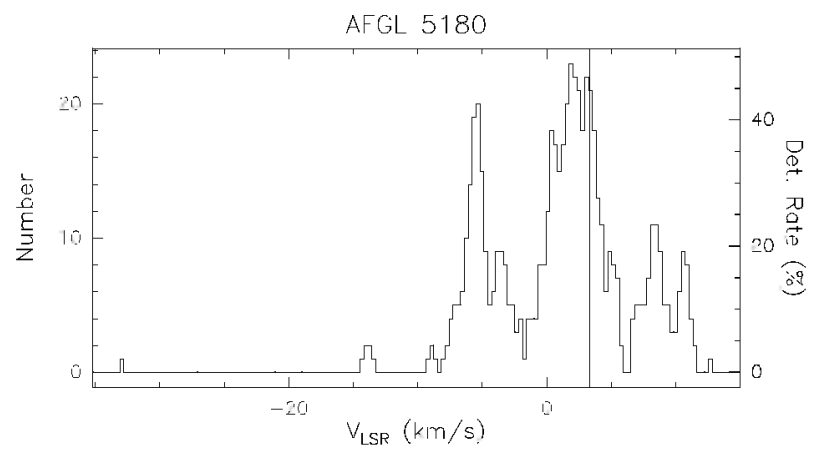

Fig. A.15. f Rate-of-occurrence plot for source AFGL 5180. The scale to the right refers to the dotted histogram, the scale to the left to the solid line histogram. The vertical solid line marks the velocity of the associated thermal molecular gas. 


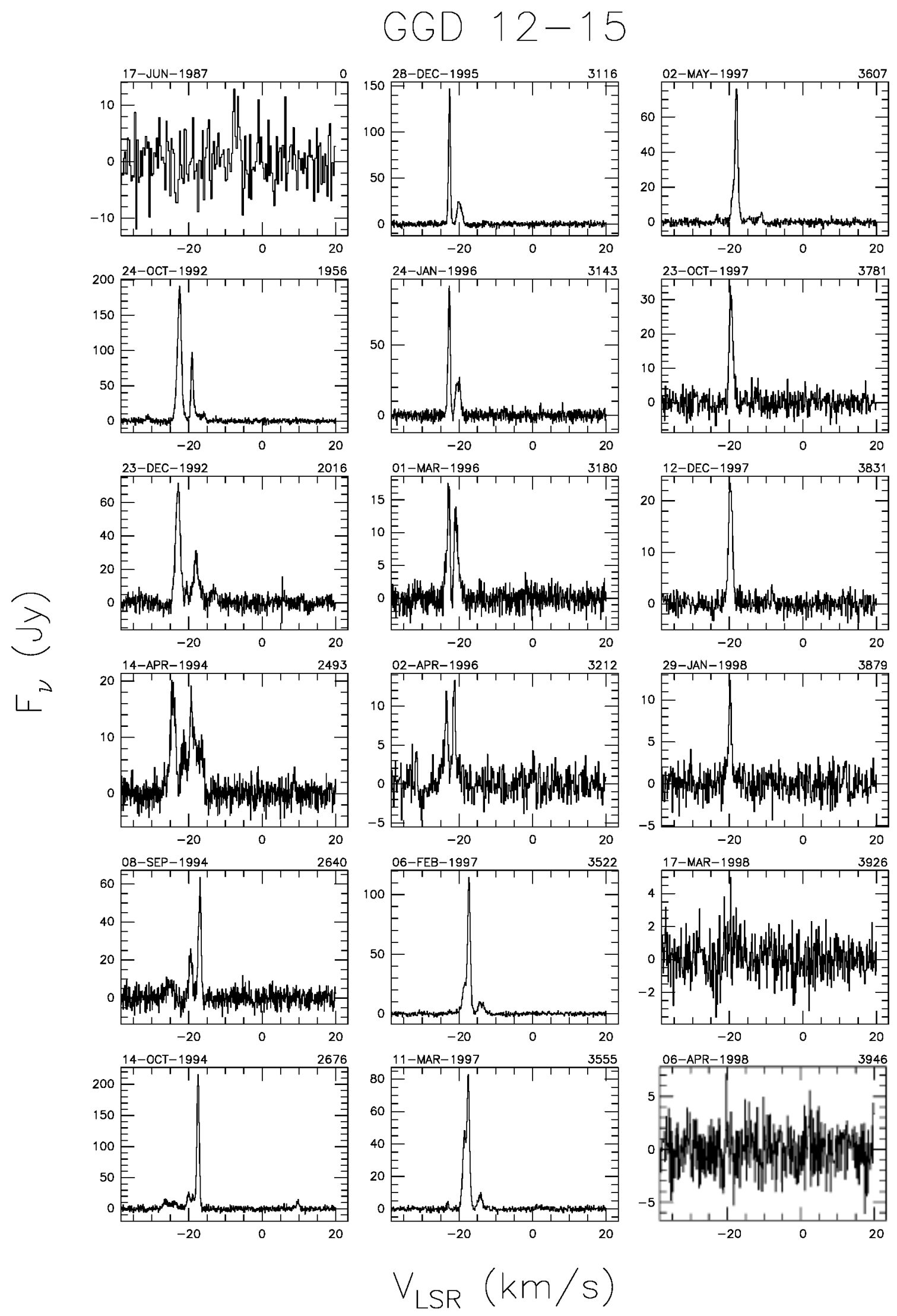

Fig. A.16. a Spectra of source GGD 12-15 with autoscaled flux density scale. The date of observation is shown above the top left corner of each spectrum and the number of days elapsed since the first observation is given above the top right corner. The velocity scale is the same for all spectia. 


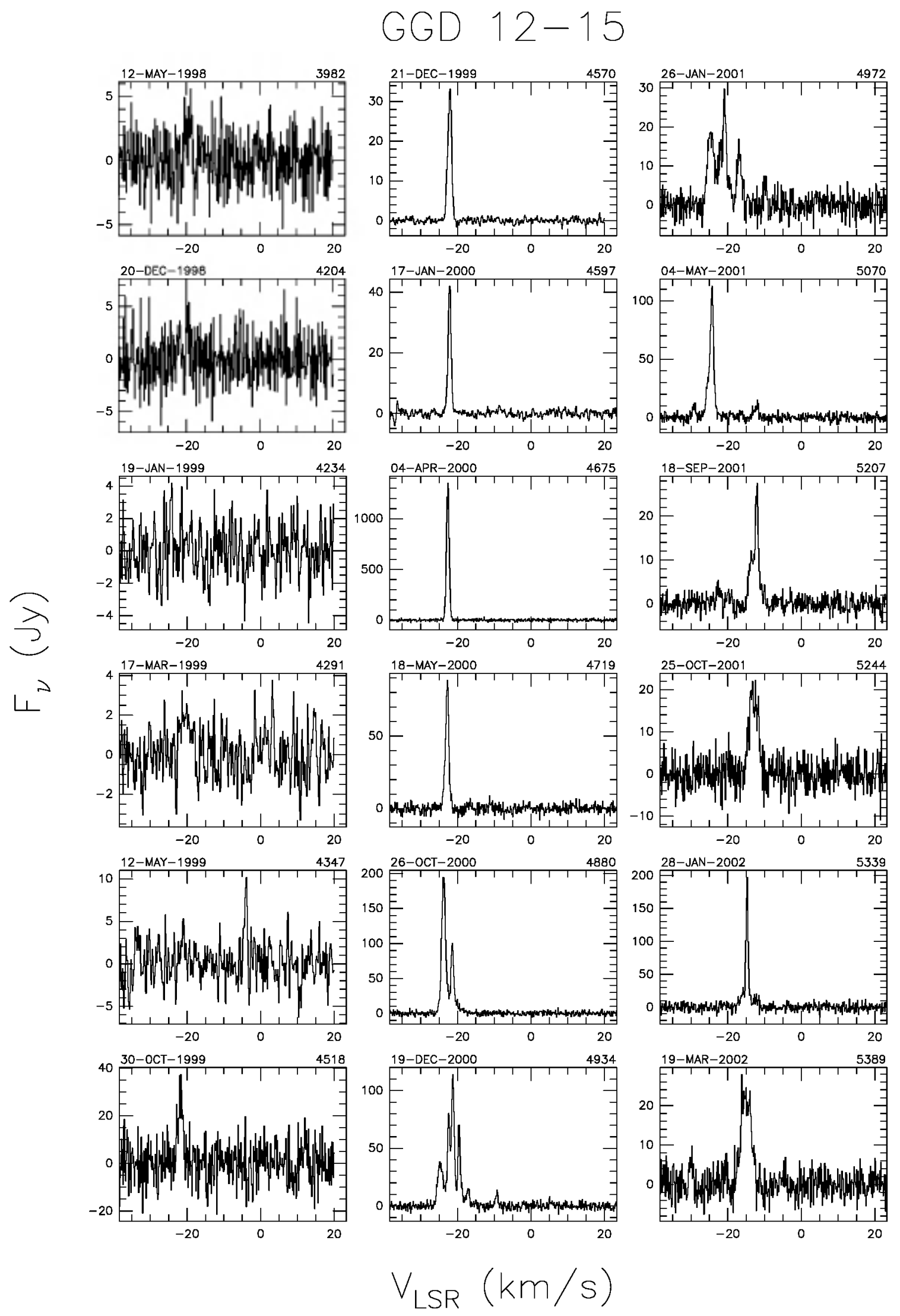

Fig. A.16. a continued. 

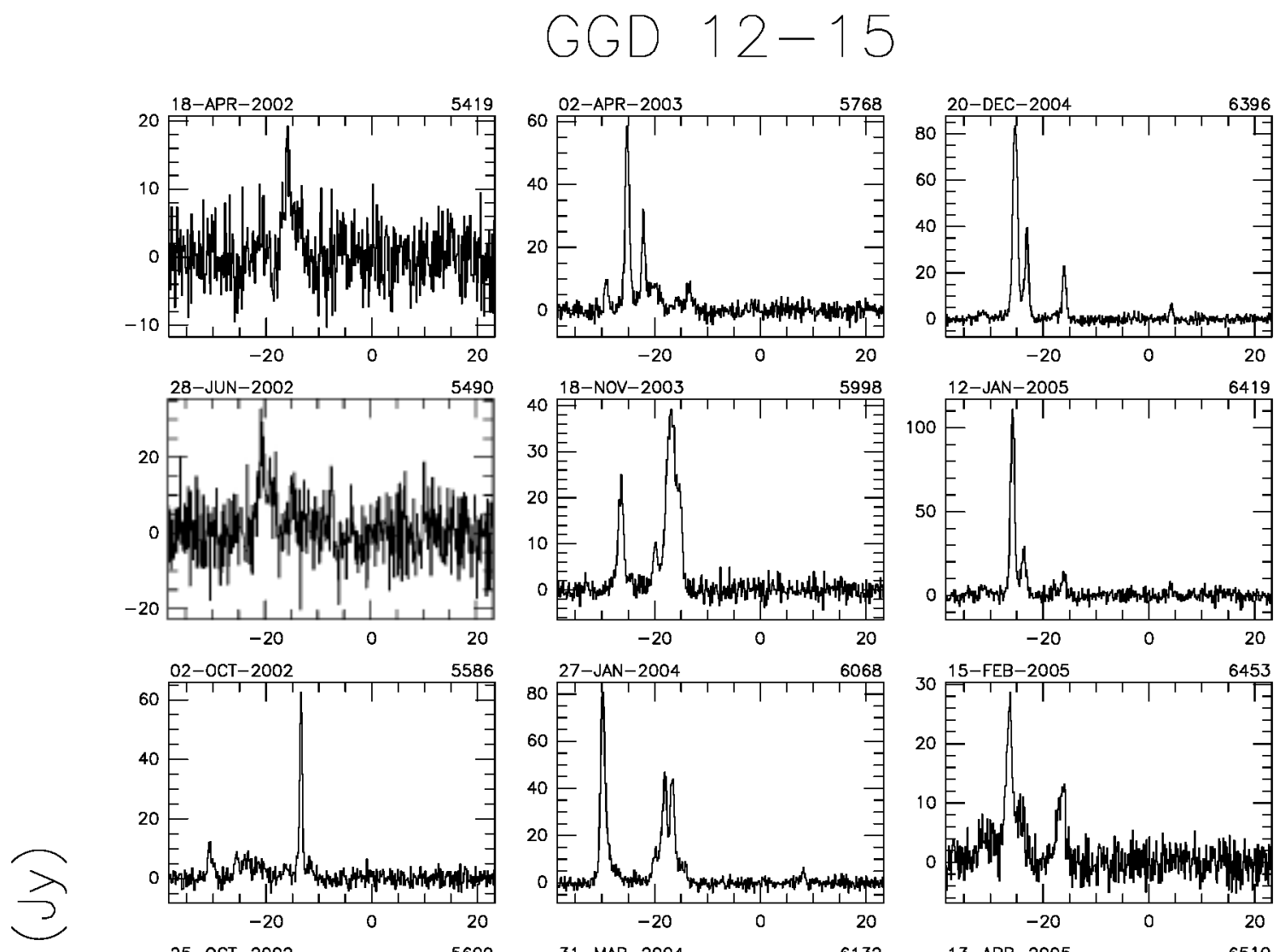

$L^{2}$
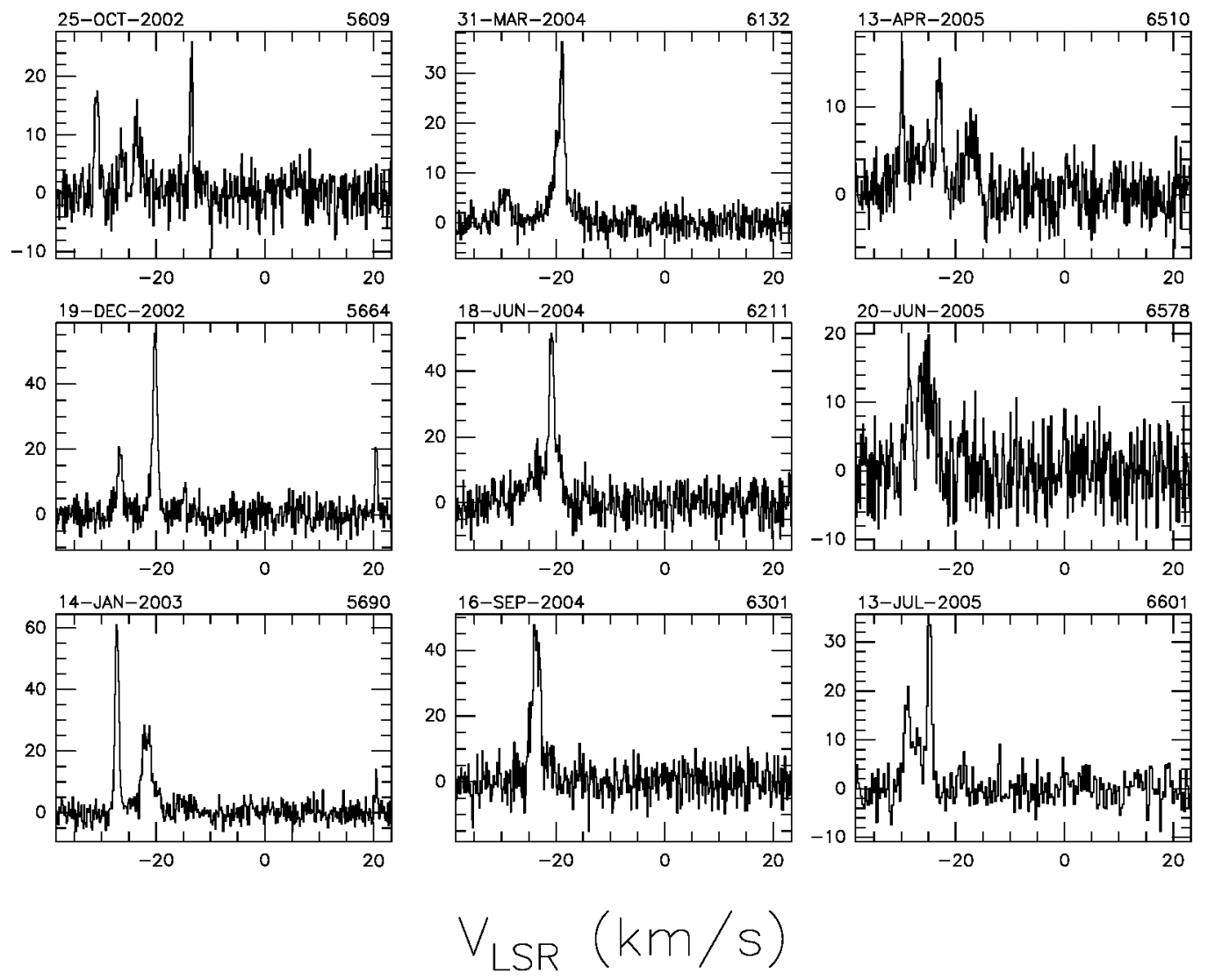

Fig. A.16. a continued. 


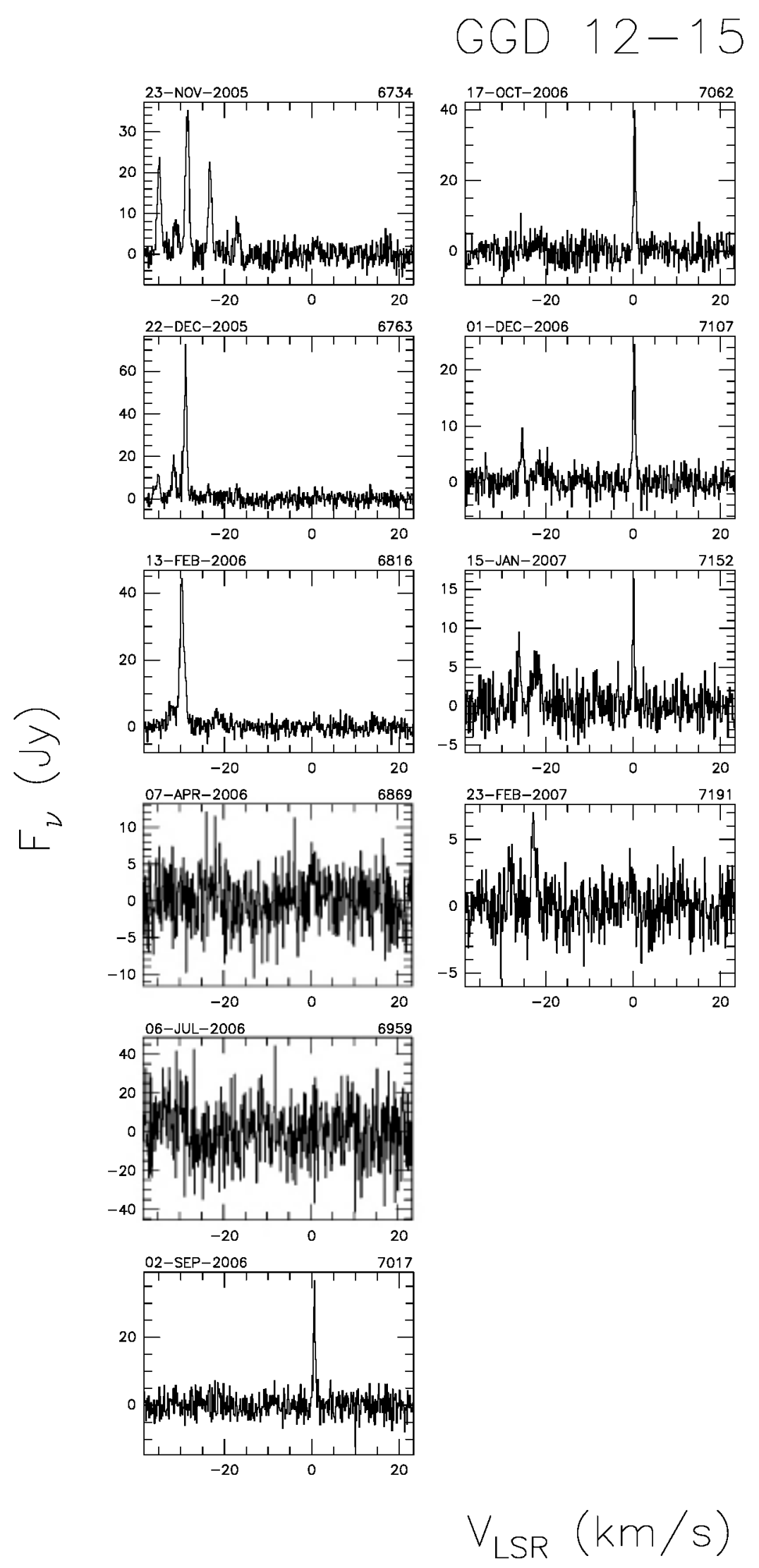

Fig. A.16. a continued. 


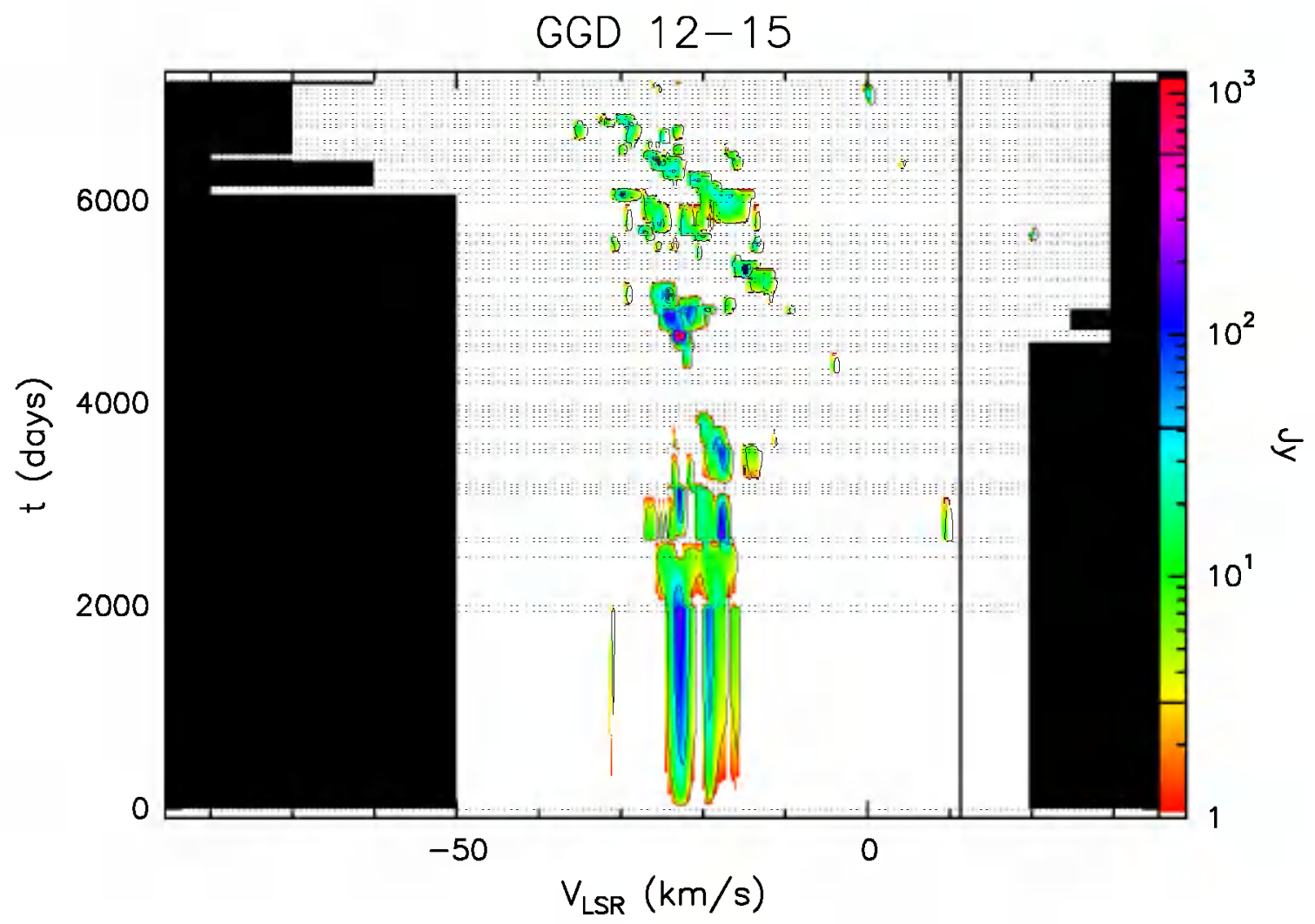

Fig. A.16. b Velocity-time-flux density full plot for source GGD 12-15. The vertical solid line indicates the velocity of the associated thermal molecular gas. The flux density scale is shown by the bar on the right. In this bar the three lines give the flux density of the drawn contours.

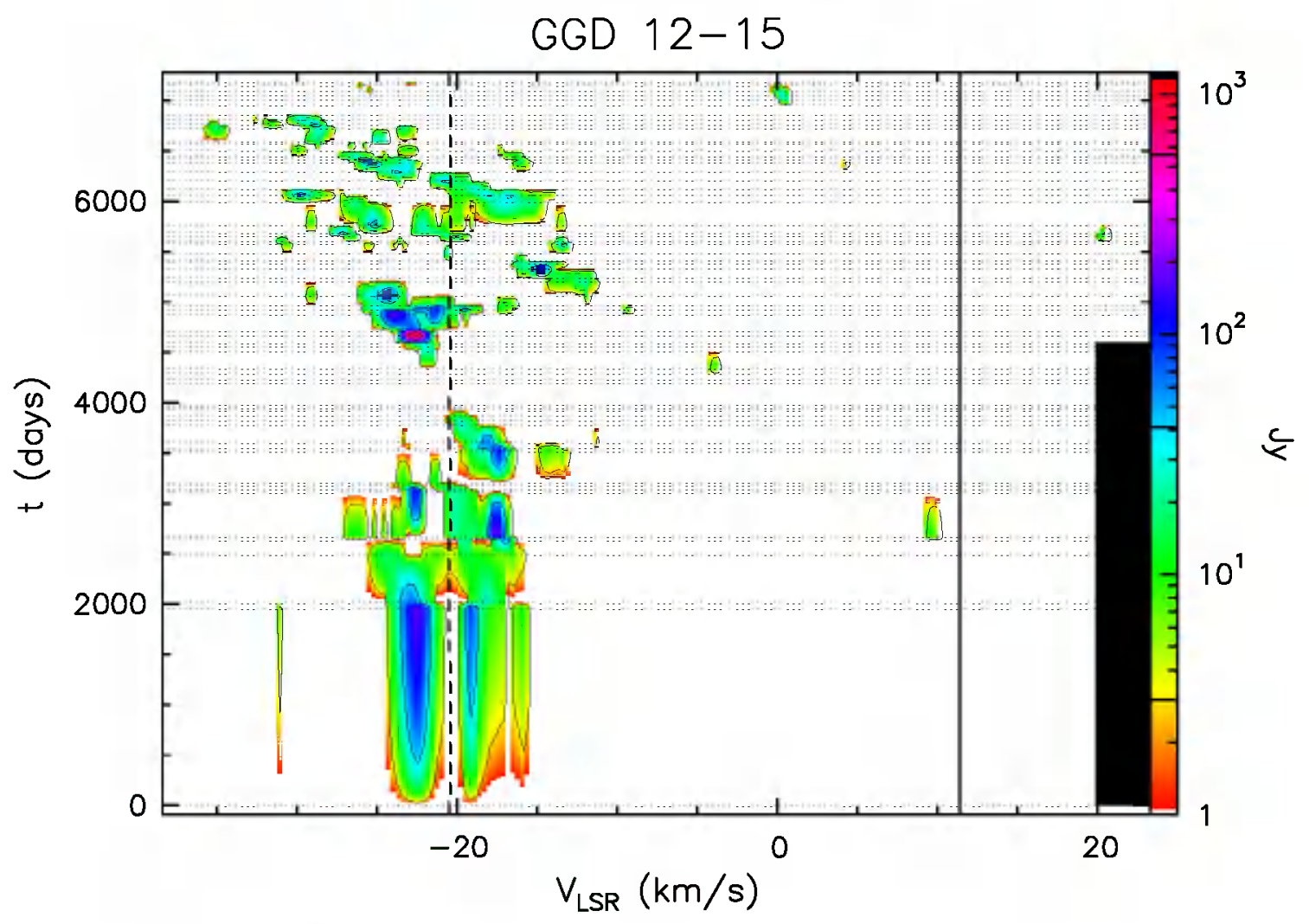

Fig. A.16. c Same as previous figure, but "zoomed" to velocity range over which emission has been detected. 


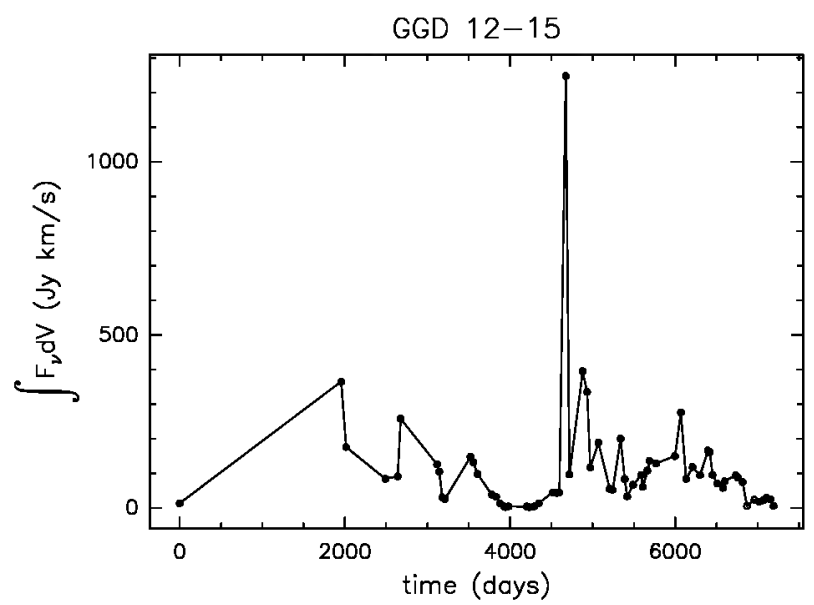

Fig. A.16. d Integral of the flux density over the observed velocity range as a function of time for source GGD 12-15.

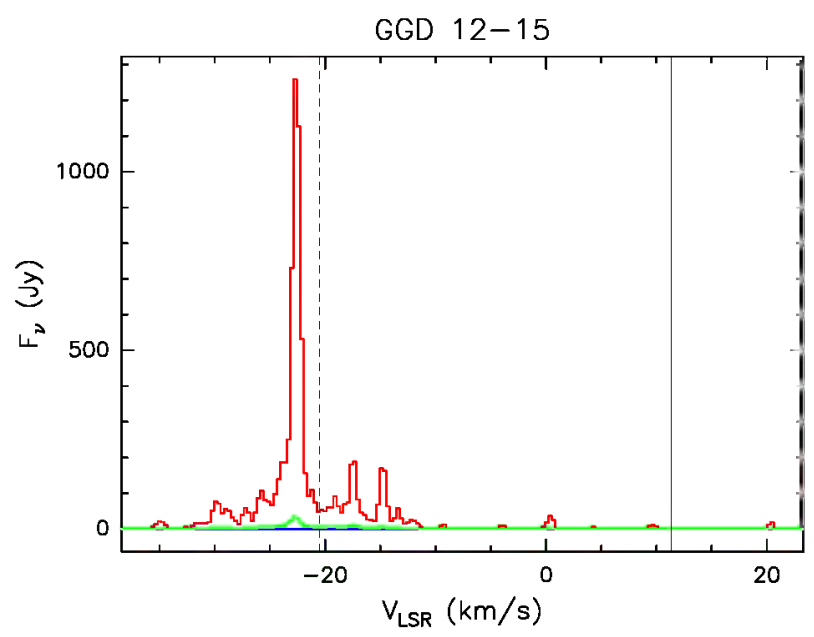

Fig. A.16. e Upper (red) and lower (blue) envelopes and mean spectrum (green) of source GGD 12-15 measured during our monitoring. The vertical solid line marks the velocity of the associated thermal molecular gas. The vertical dashed line marks the mean velocity derived from the histogram of the rate-of-occurrence.

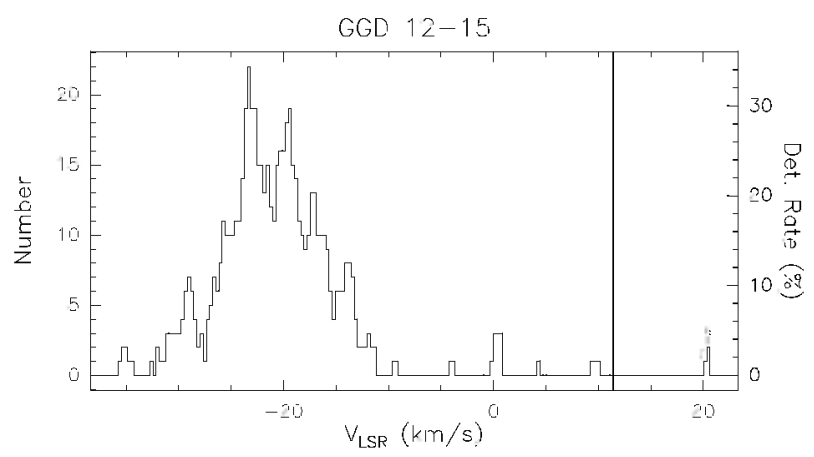

Fig. A.16. f Rate-of-occurrence plot for source GGD 12-15. The scale to the right refers to the dotted histogram, the scale to the left to the solid line histogram. The vertical solid line marks the velocity of the associated thermal molecular gas. 
$\operatorname{Sh~} 2-255 / 7$
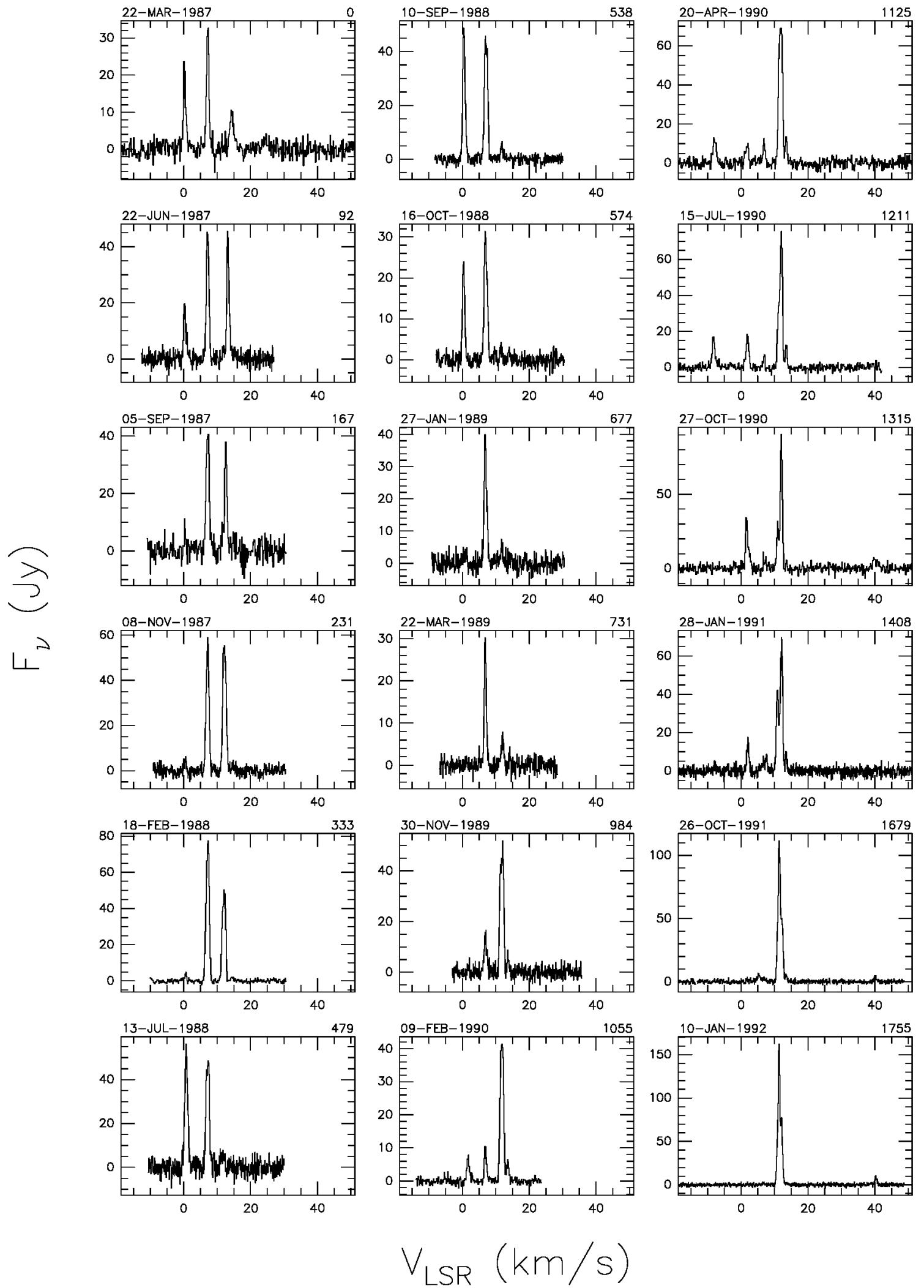

Fig. A.17. a Spectra of source Sh 2-255/7 with antoscaled flux density scale. The date of observation is shown above the top left corner of each spectrum and the number of days elapsed since the first observation is given above the top right corner. The velocity scale is the same for all spectra. 
Sh $2-255 / 7$
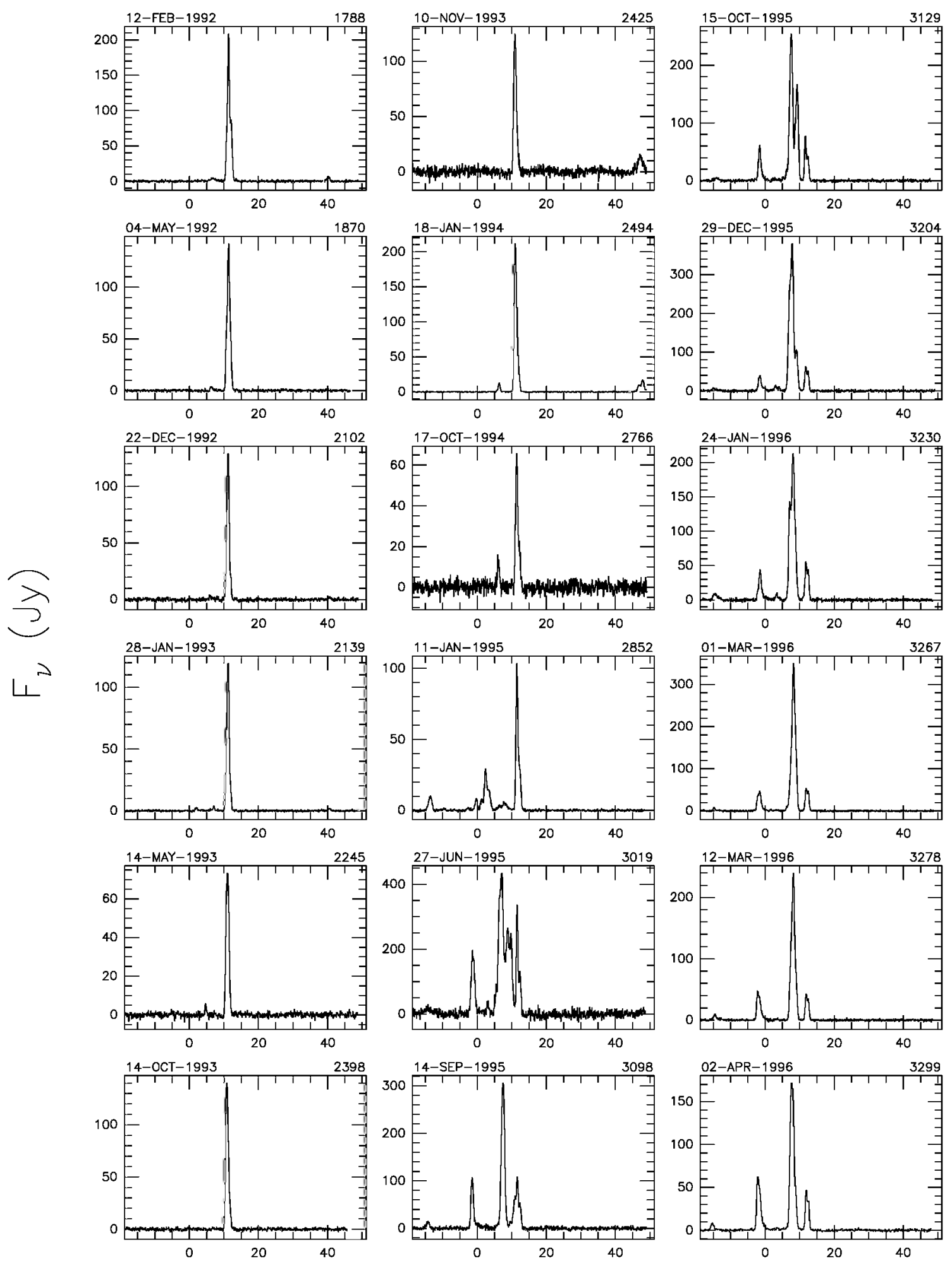

$$
V_{\text {LSR }}(\mathrm{km} / \mathrm{s})
$$

Fig. A.17. a continued. 
Sh $2-255 / 7$
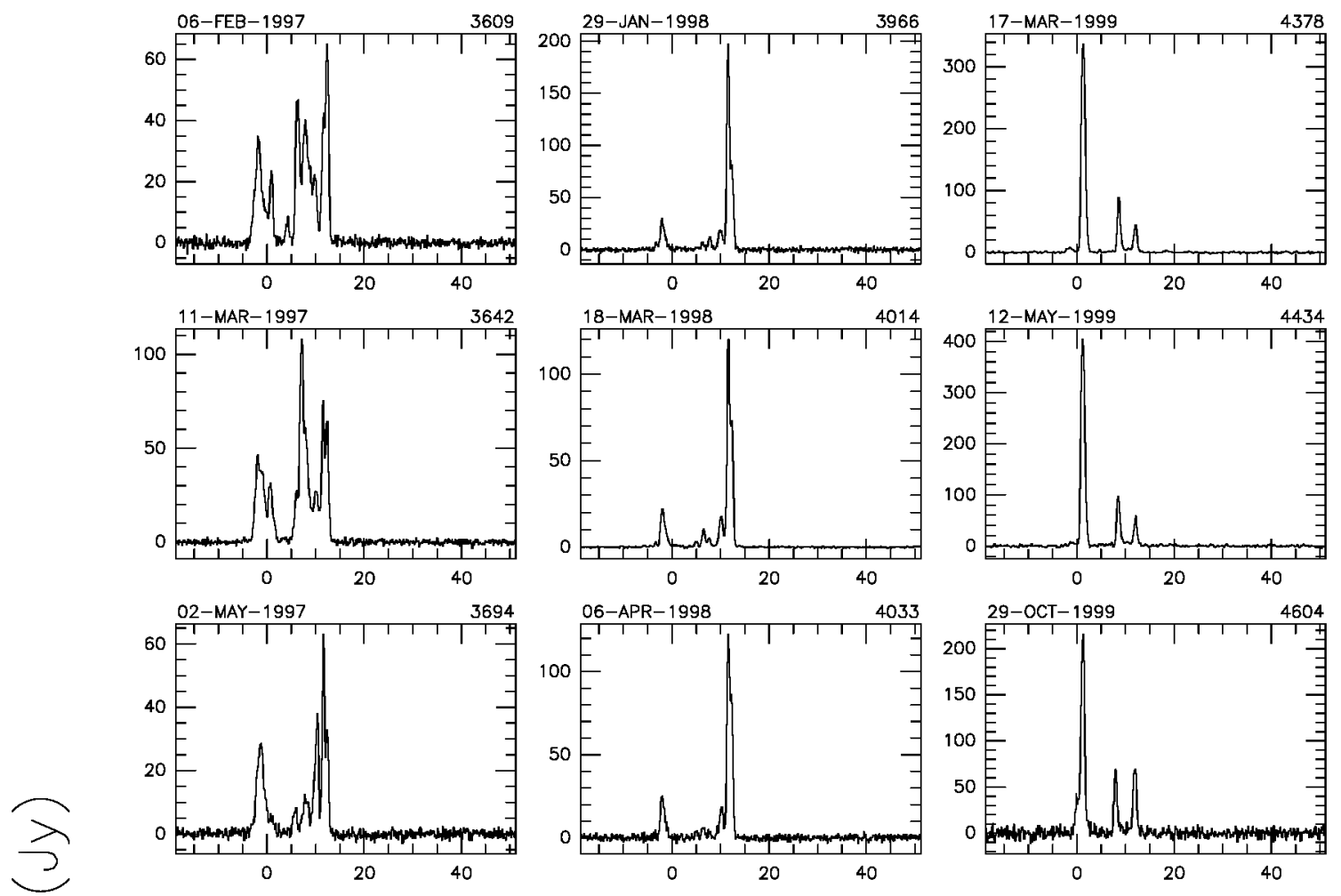

$\sqcup^{2}$
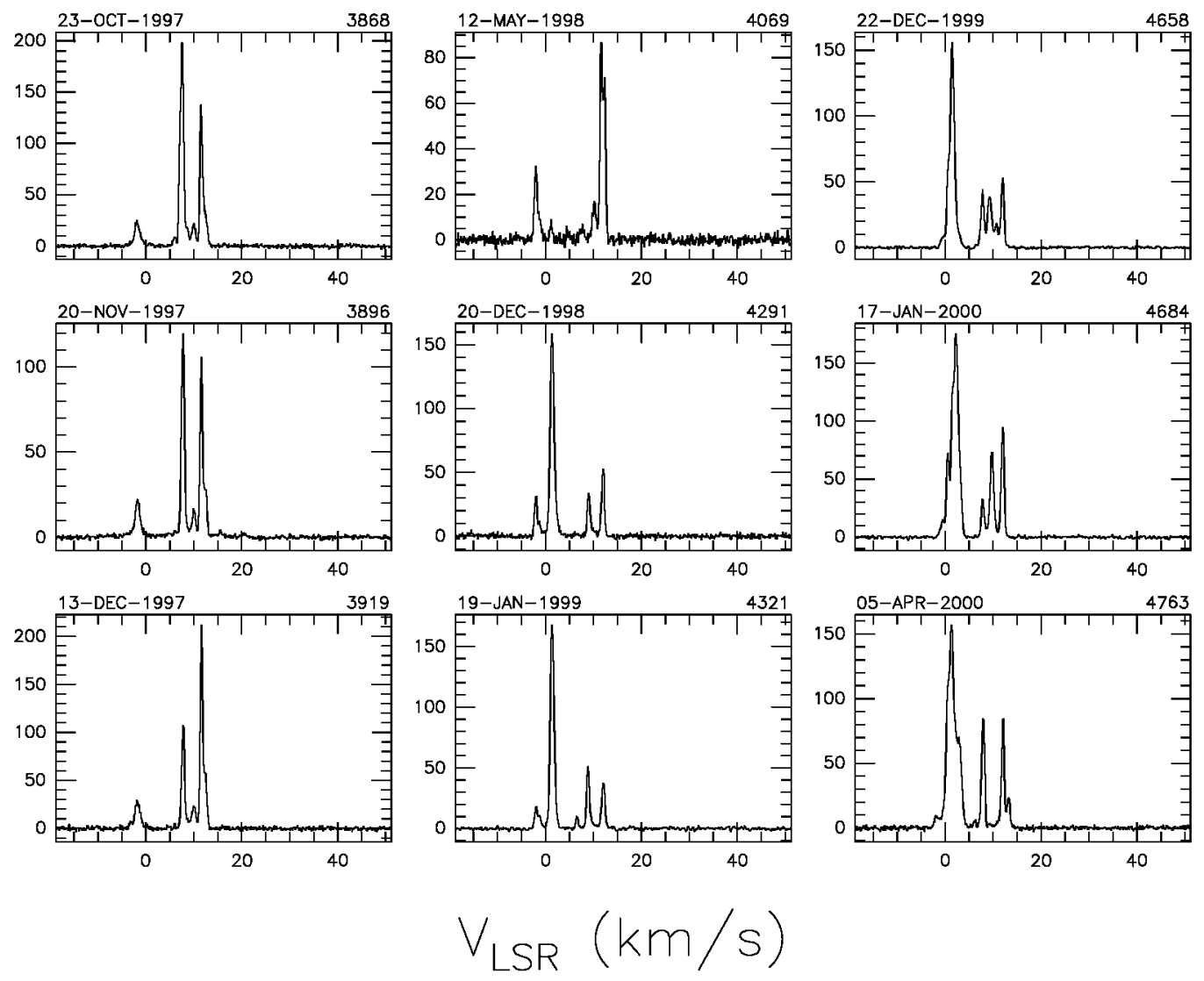

Fig. A.17. a continued. 

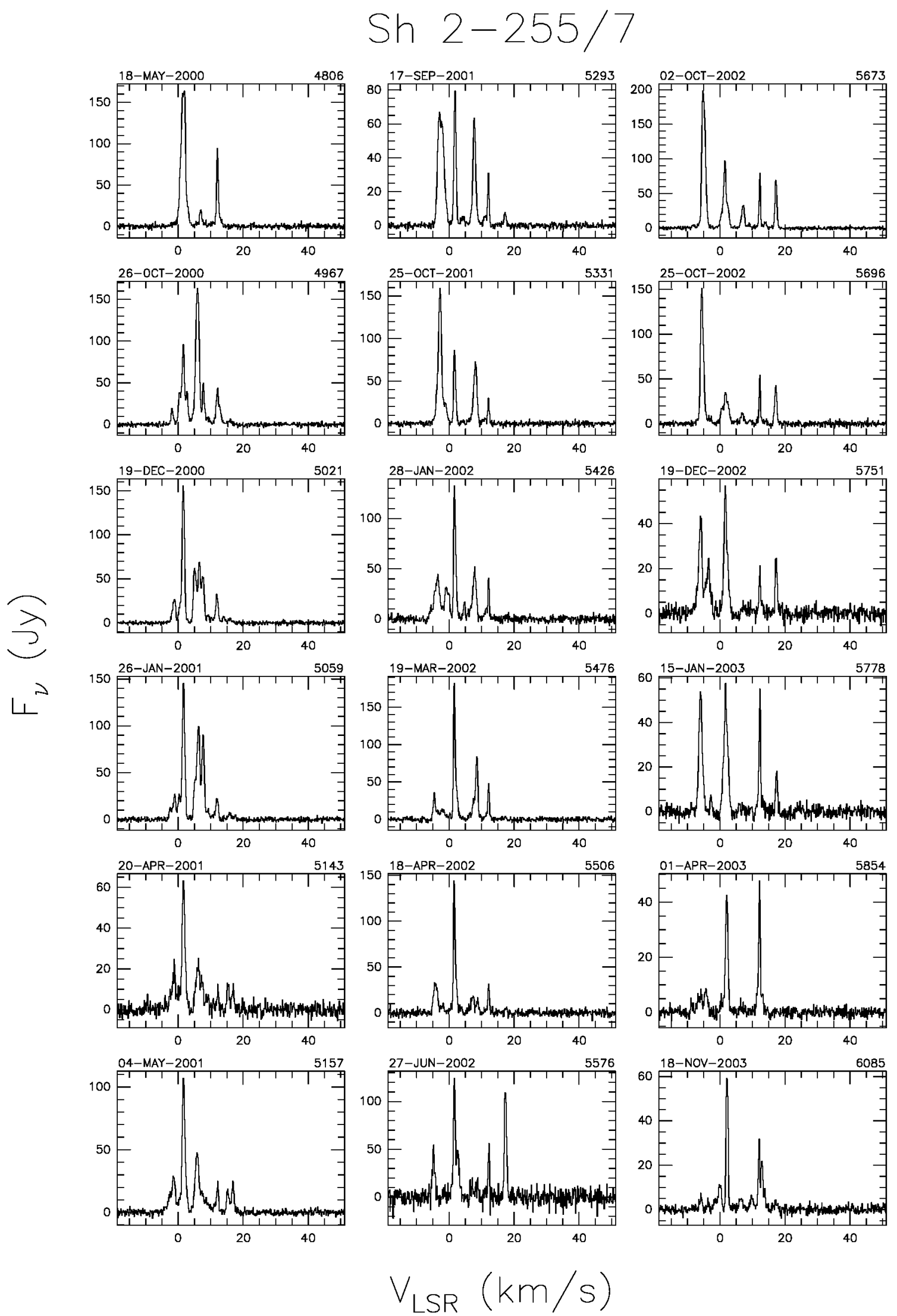

Fig. A.17. a continued. 
Sh $2-255 / 7$
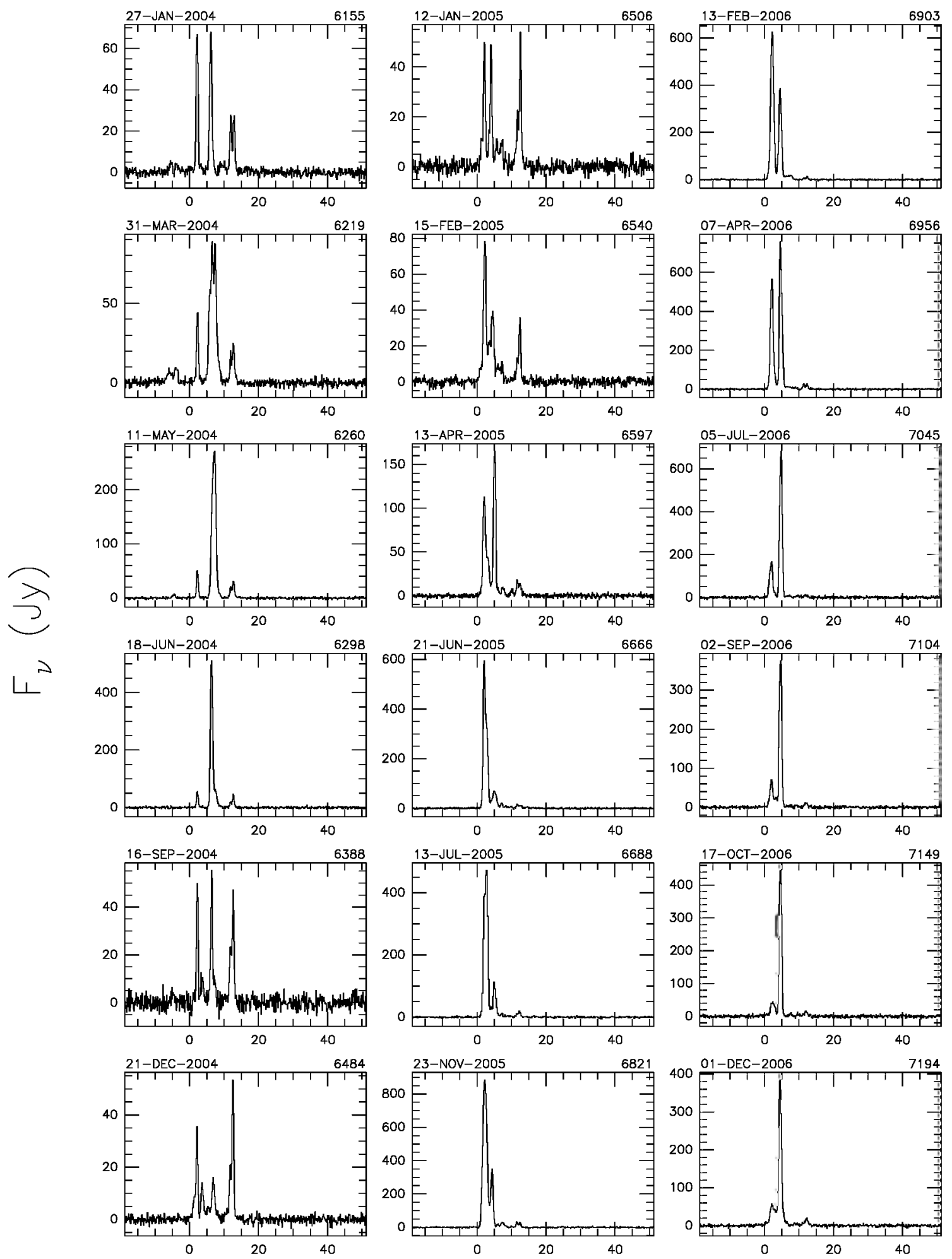

$$
V_{\text {LSR }}(\mathrm{km} / \mathrm{s})
$$

Fig. A.17. a continued. 
Sh $2-255 / 7$
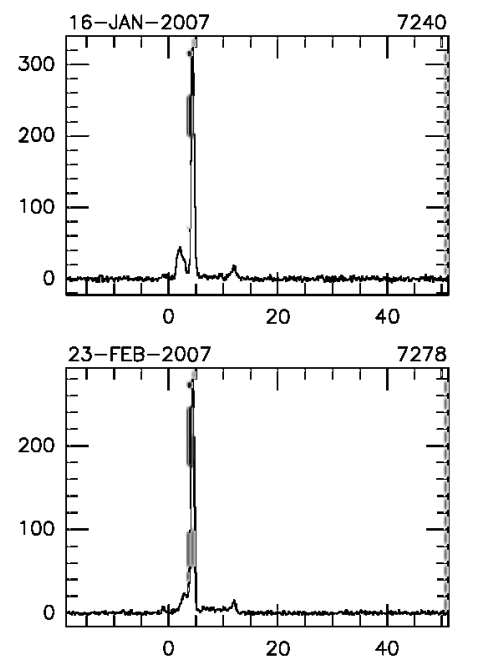

3

$L^{\lambda}$

$$
V_{\text {LSR }}(\mathrm{km} / \mathrm{s})
$$

Fig. A.17. a continued. 


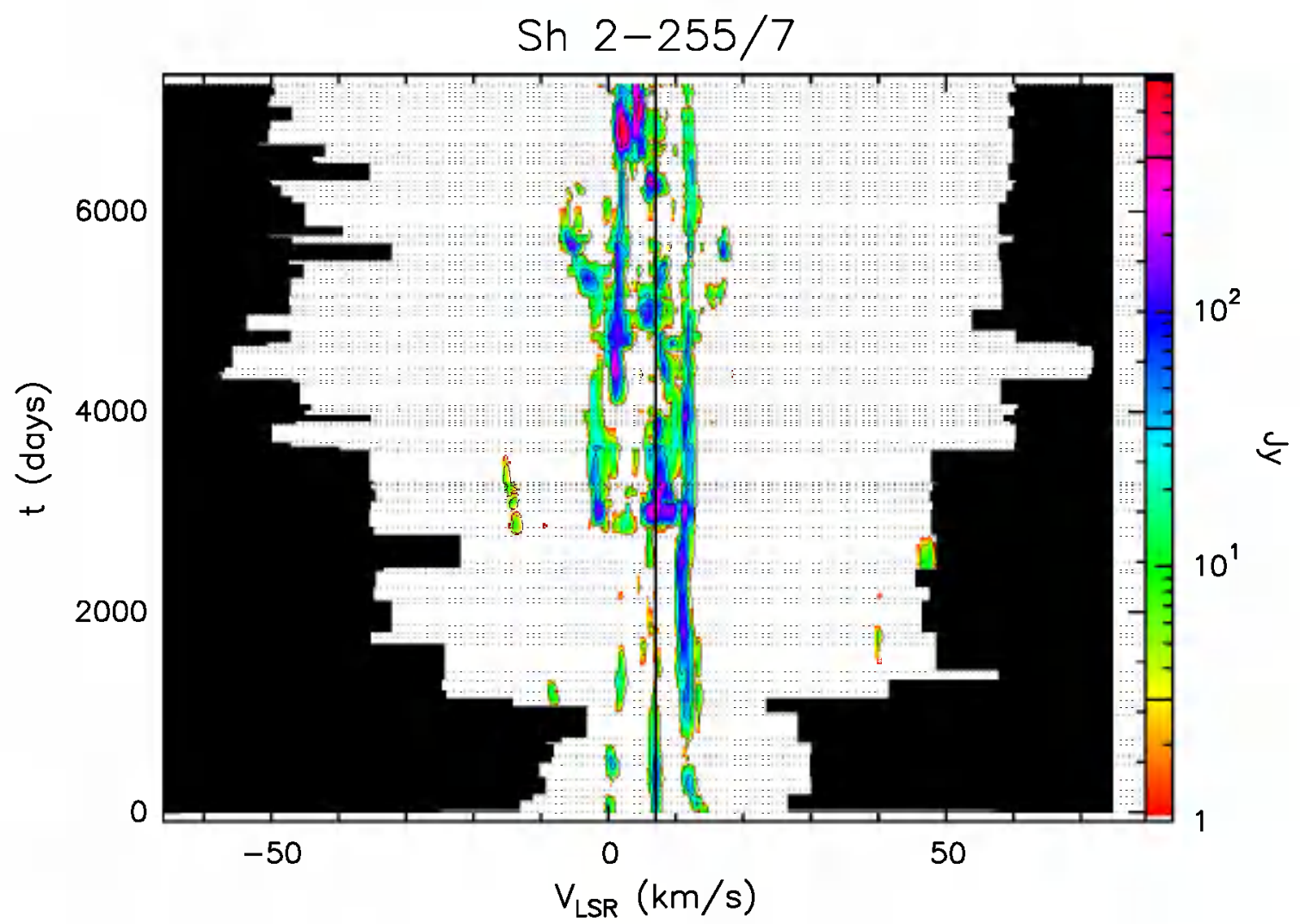

Fig. A.17. b Velocity-time-flux density full plot for source Sh $2-255 / 7$. The vertical solid line indicates the velocity of the associated thermal molecular gas. The flux density scale is shown by the bar on the right. In this bar the three lines give the flux density of the drawn contours.

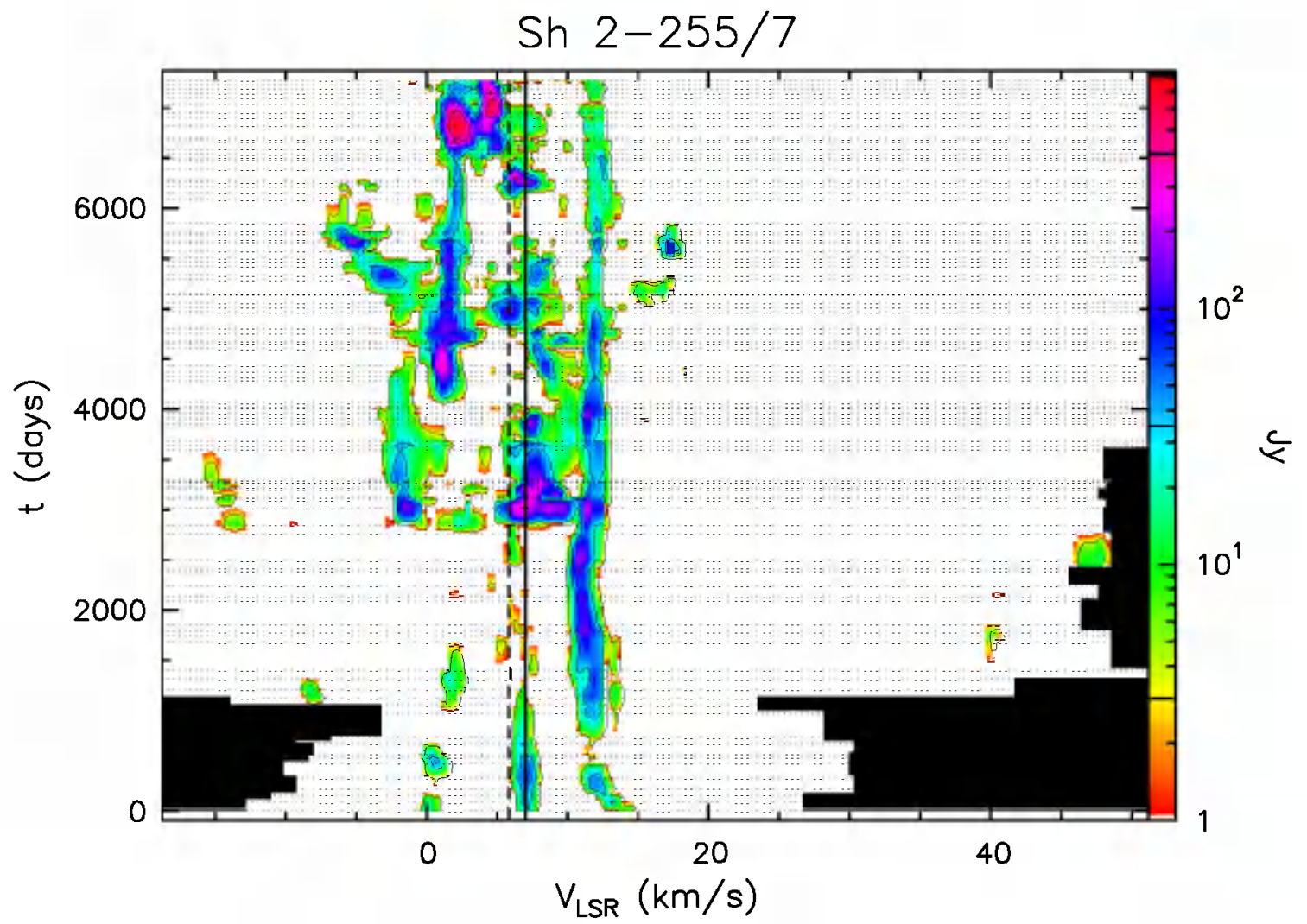

Fig. A.17. c Same as previous figure, but "zoomed" to velocity range over which emission has been detected. 


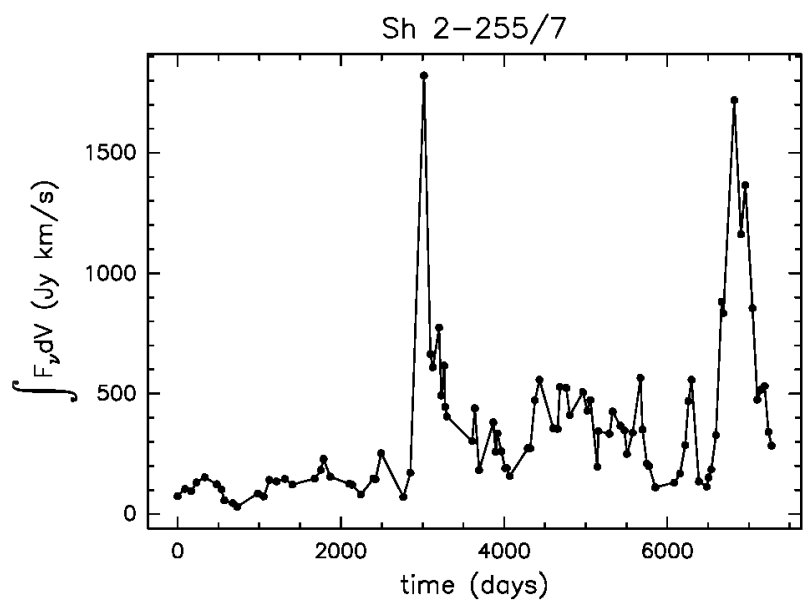

Fig. A.17. $d$ Integral of the flux density over the observed velocity range as a function of time for source Sh 2-255/7.

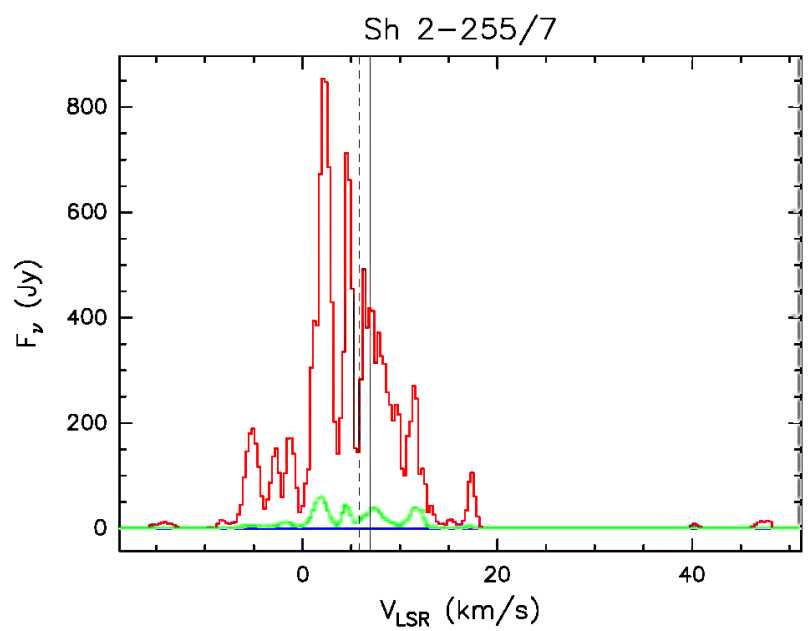

Fig. A.17. e Upper (red) and lower (blue) envelopes and mean spectrum (green) of source Sh 2-255/7 measured during our monitoring. The vertical solid line marks the velocity of the associated thermal molecular gas. The vertical dashed line marks the mean velocity derived from the histogram of the rate-of-occurrence.

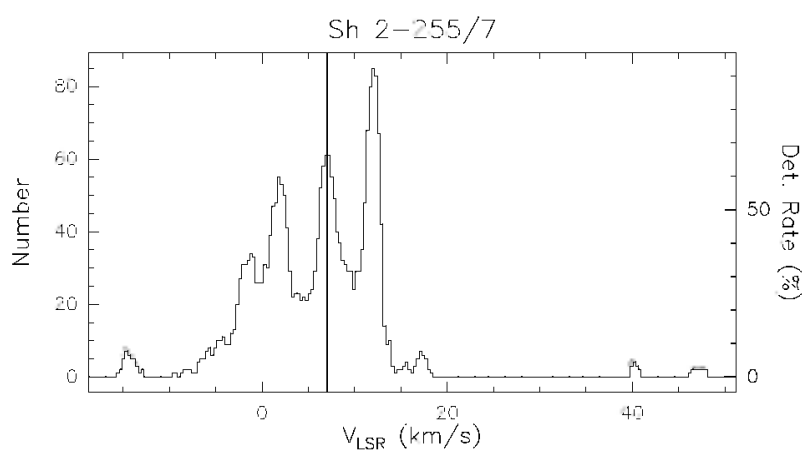

Fig. A.17. f Rate-of-occurrence plot for source Sh 2-255/7. The scale to the right refers to the dotted histogram, the scale to the left to the solid line histogram. The vertical solid line marks the velocity of the associated thermal molecular gas. 

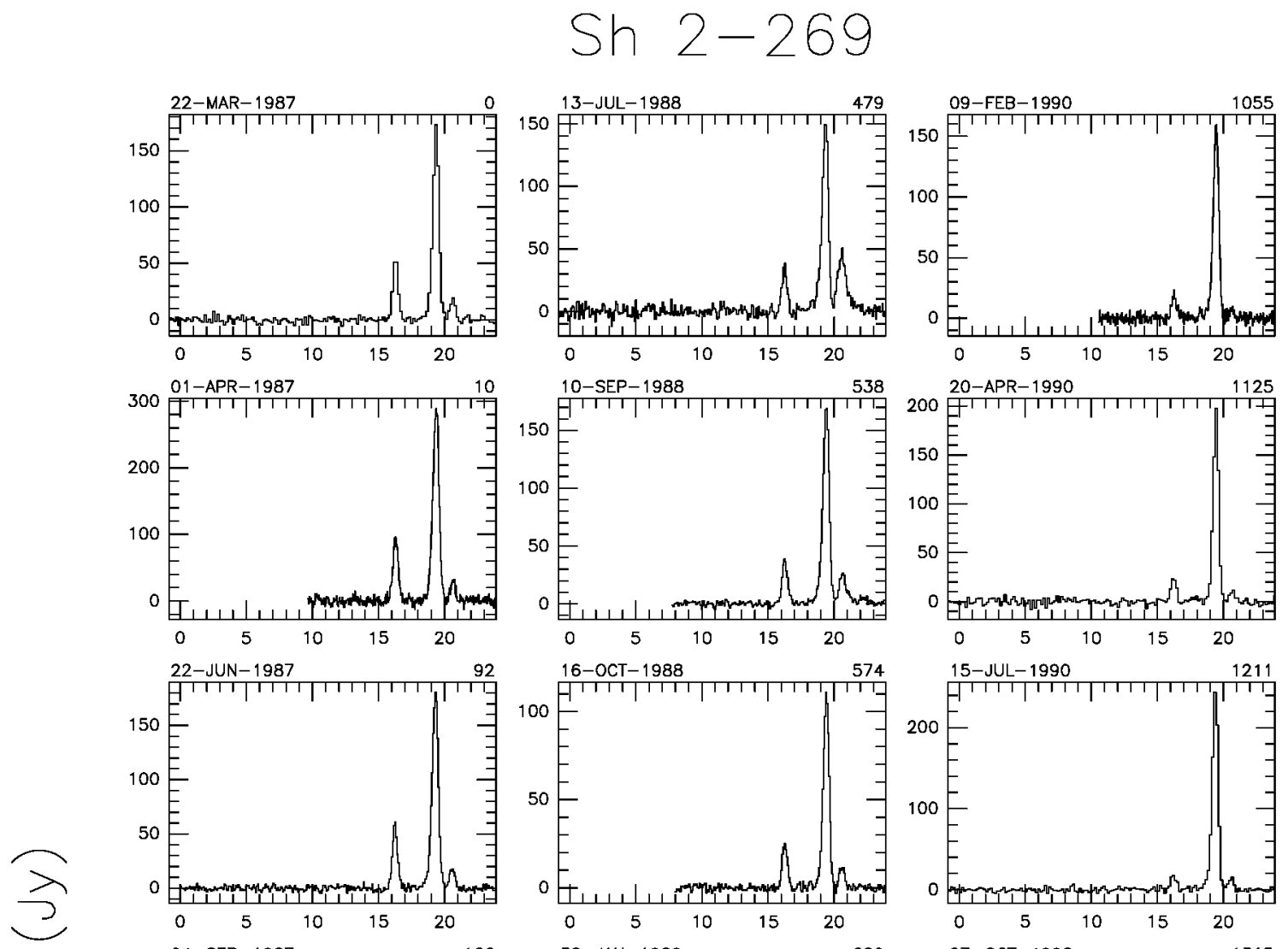

$L^{2}$
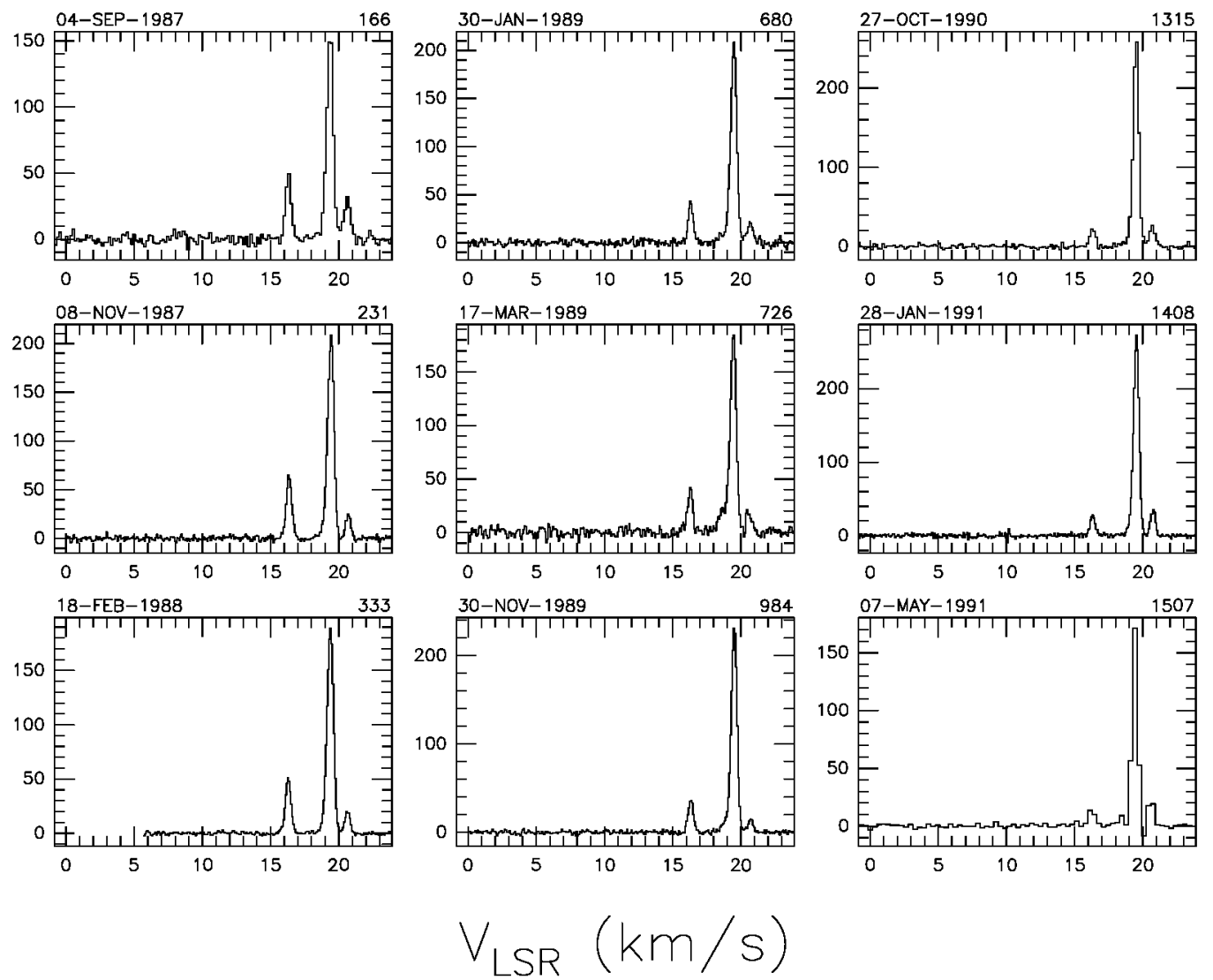

Fig. A.18. a Spectra of source Sh 2-269 with autoscaled flux density scale. The date of observation is shown above the top left corner of each spectrum and the number of days elapsed since the first observation is given above the top right corner. The velocity scale is the same for all spectra. 
Sh 2-269
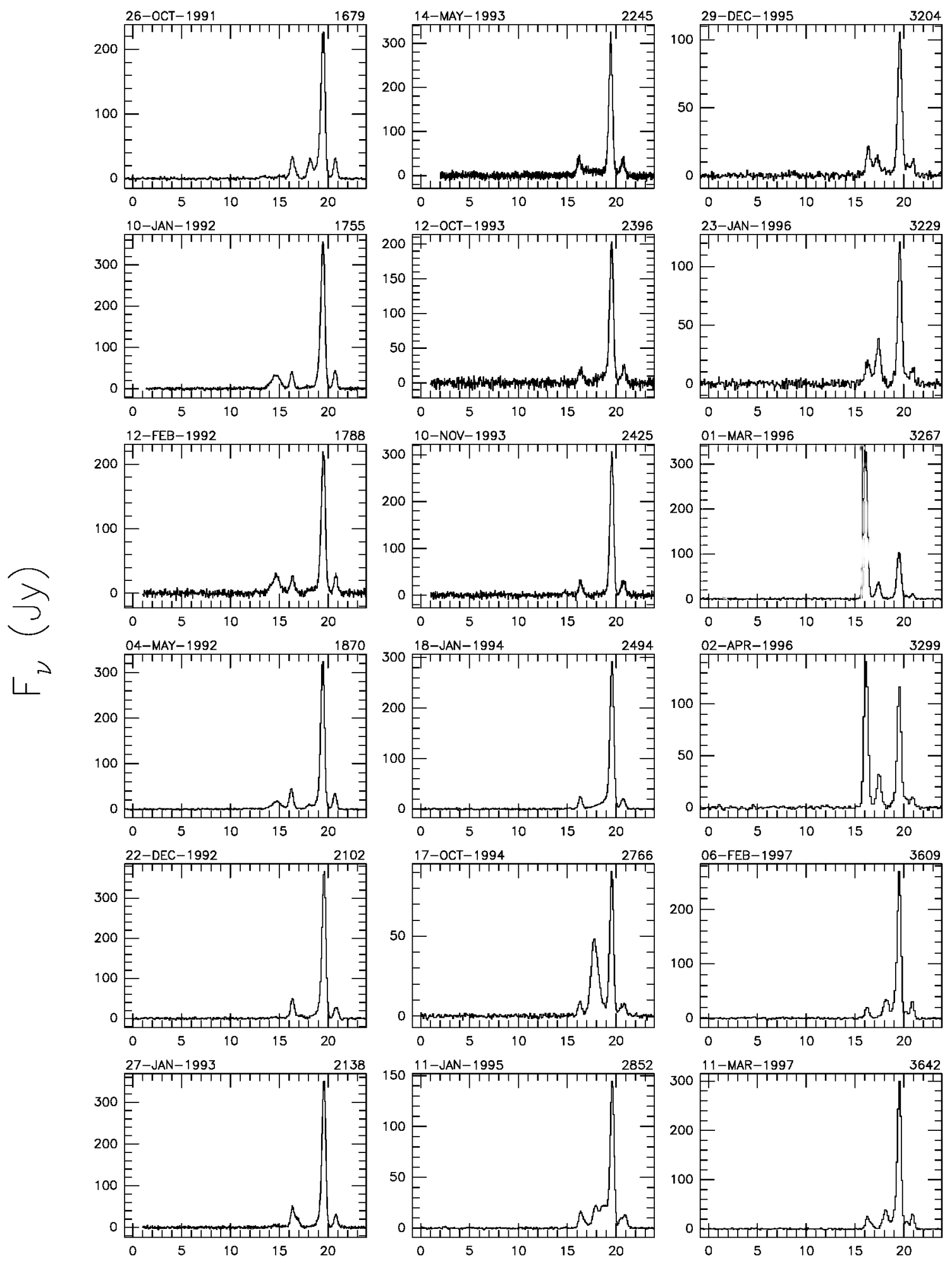

$$
V_{\text {LSR }}(\mathrm{km} / \mathrm{s})
$$

Fig. A.18. a continued. 
Sh 2-269
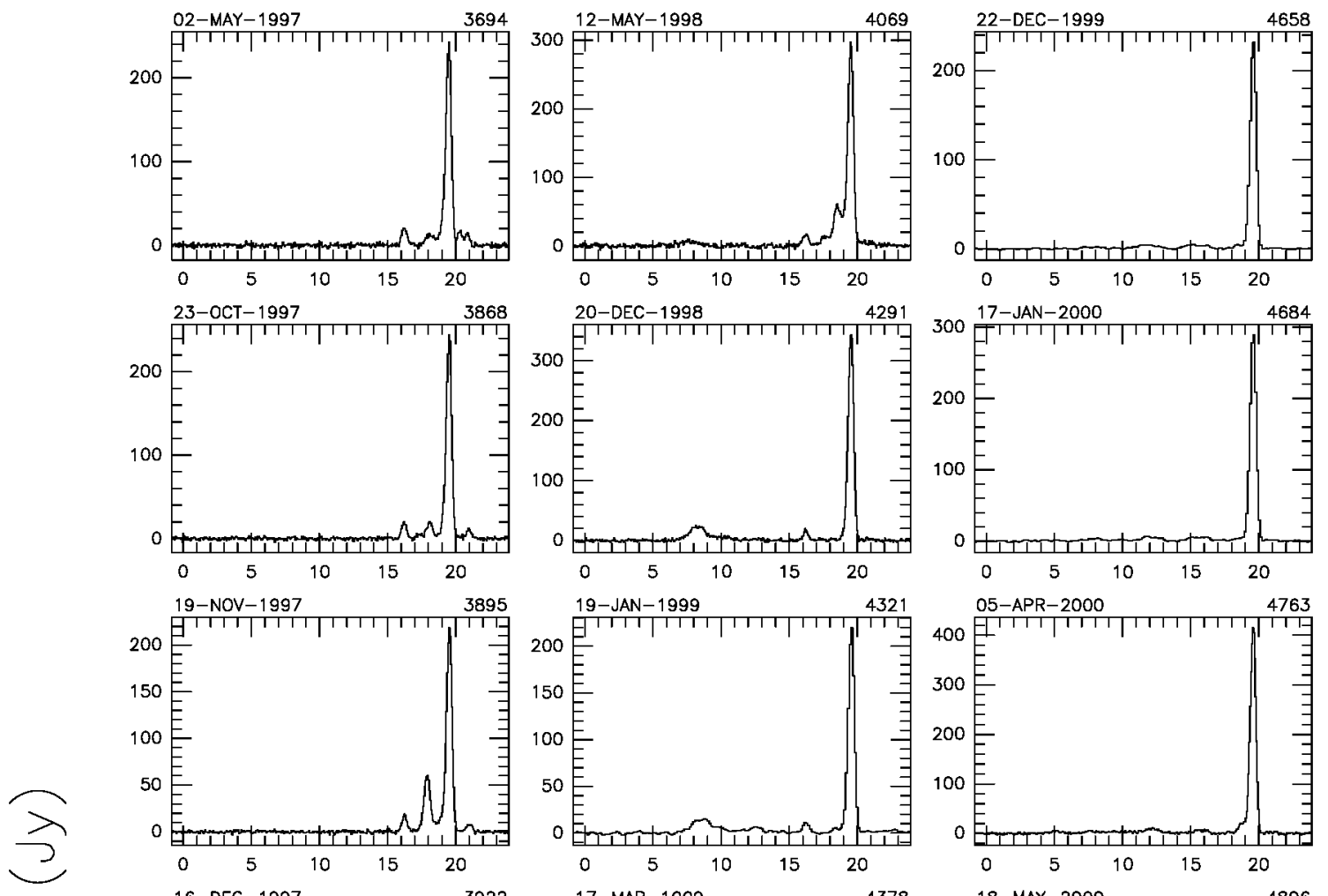

เ
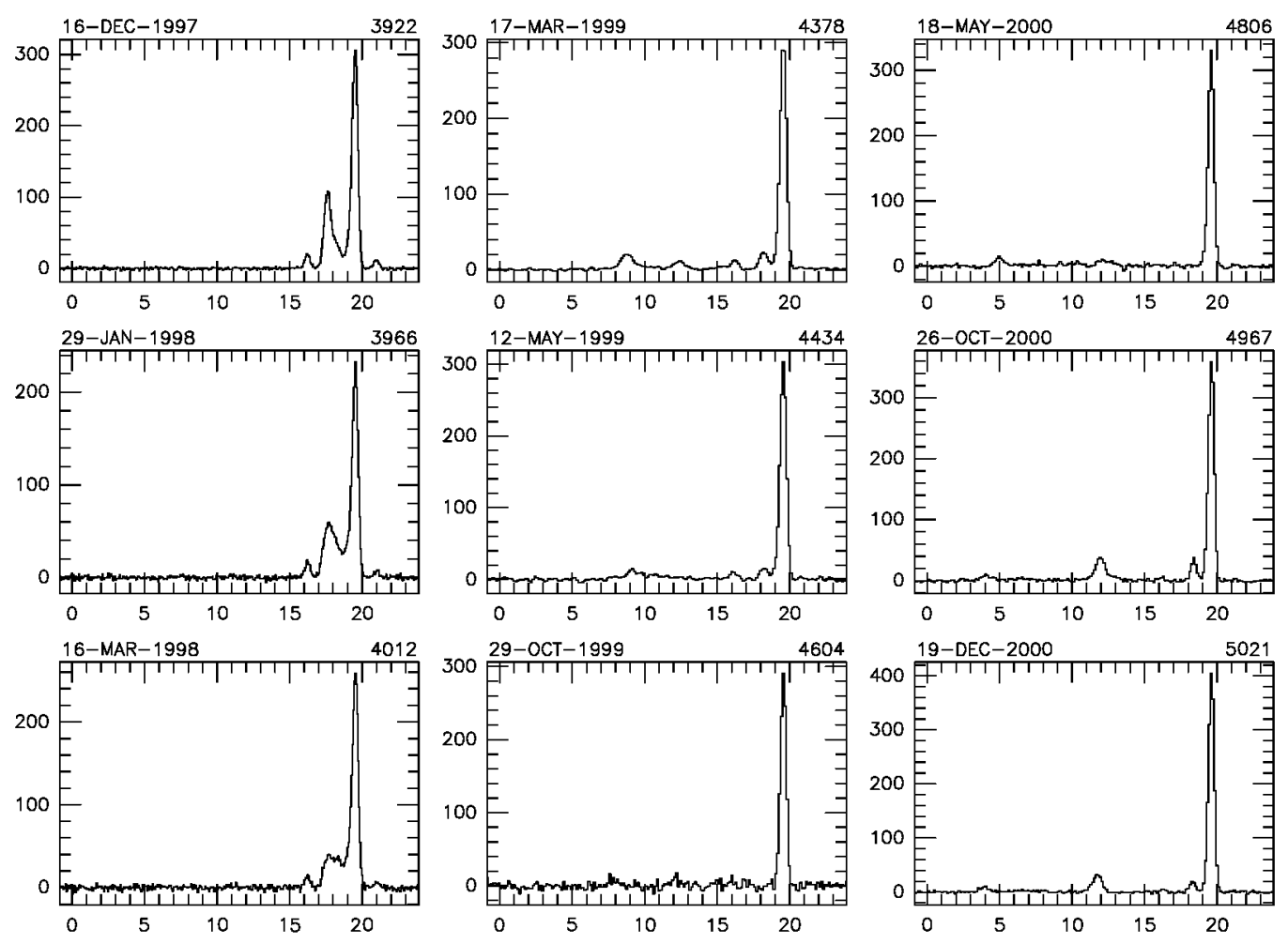

$$
V_{\text {LSR }}(\mathrm{km} / \mathrm{s})
$$

Fig. A.18. a continued. 
Sh 2-269
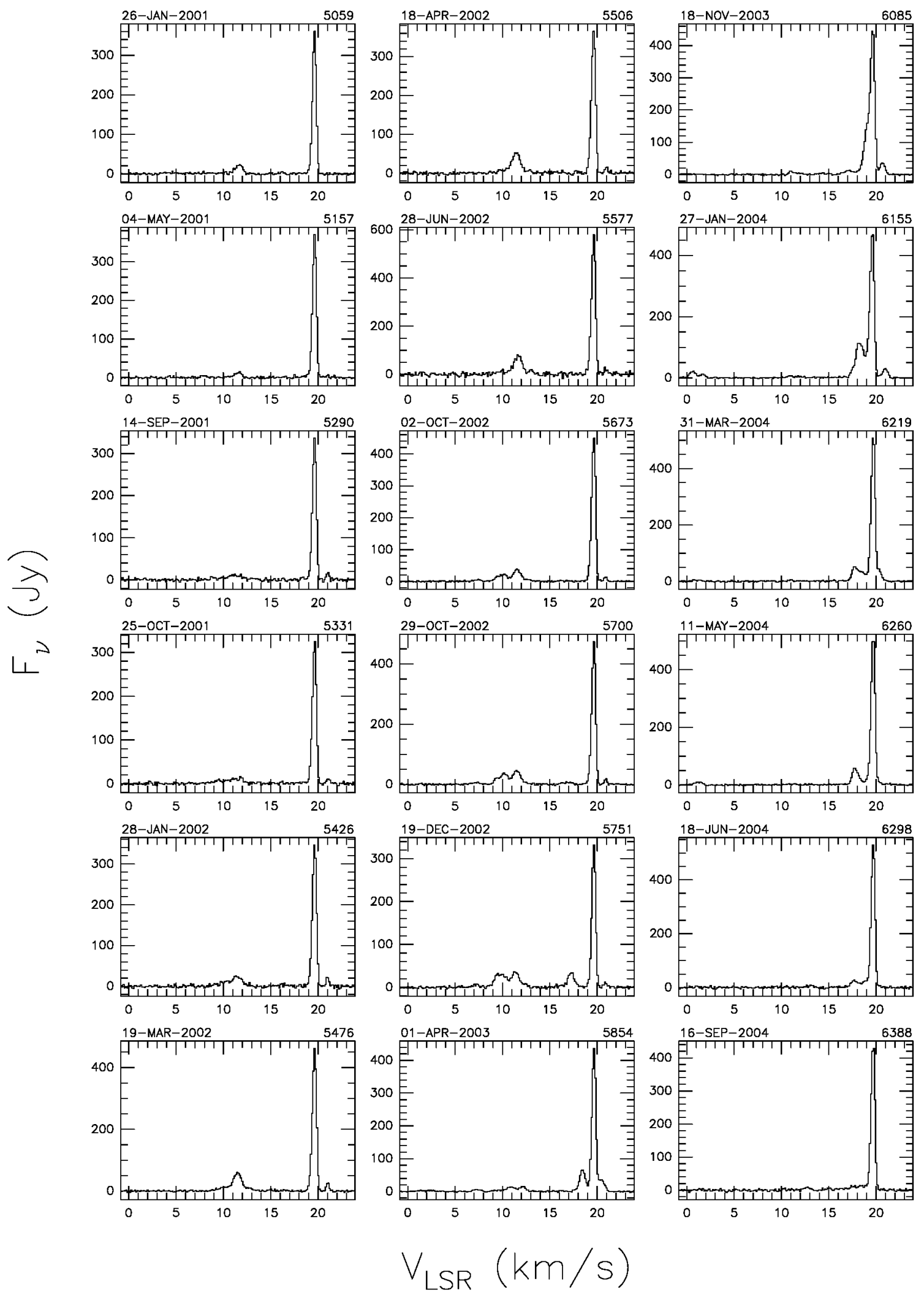

Fig. A.18. a continued. 
Sh 2-269
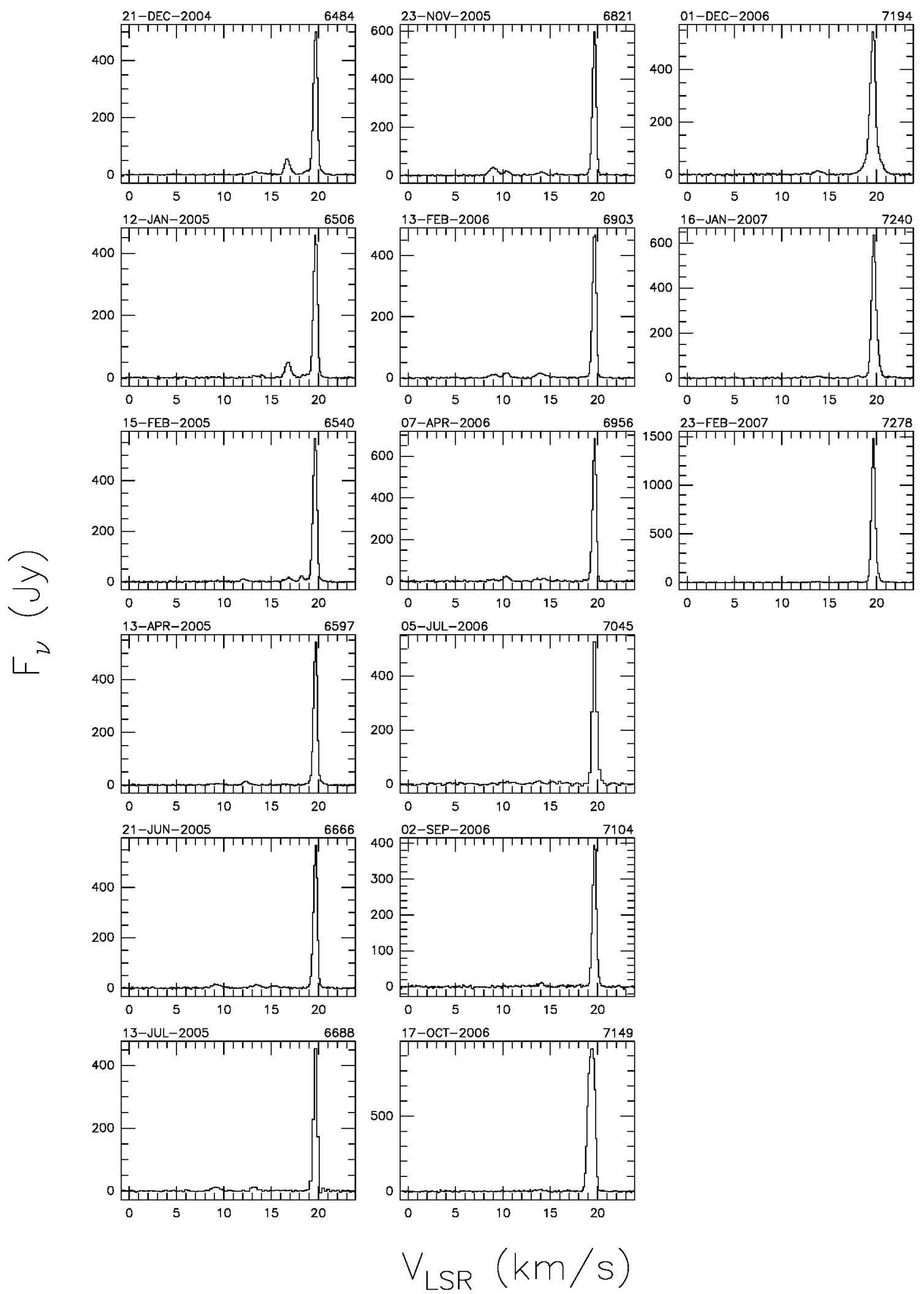

Fig. A.18. a continued. 


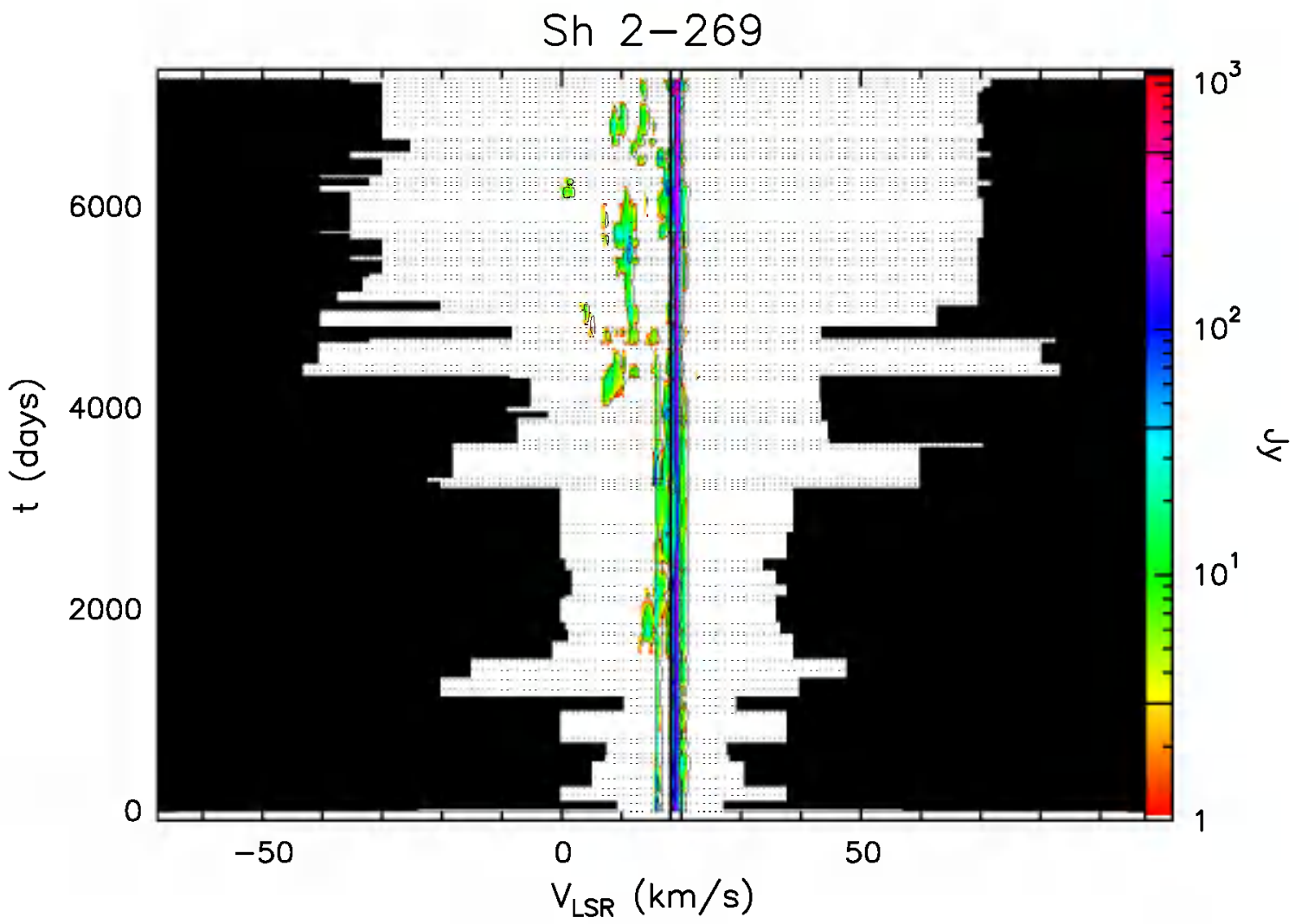

Fig. A.18. b Velocity-time-flux density full plot for source Sh 2-269. The vertical solid line indicates the velocity of the associated thermal molecular gas. The flux density scale is shown by the bar on the right. In this bar the three lines give the flux density of the drawn contours.

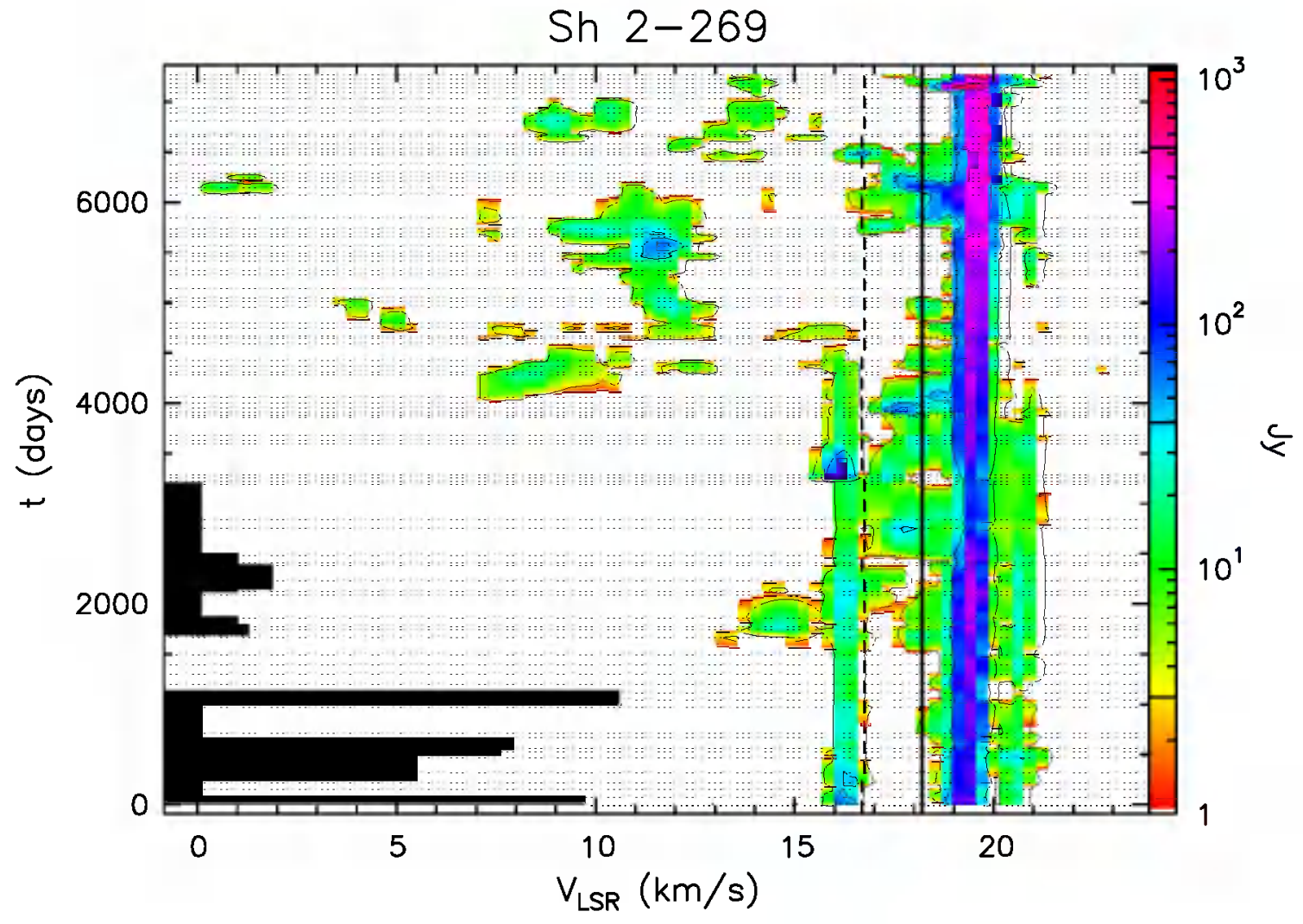

Fig. A.18. c Same as previous figure, but "zoomed" to velocity range over which emission has been detected. 


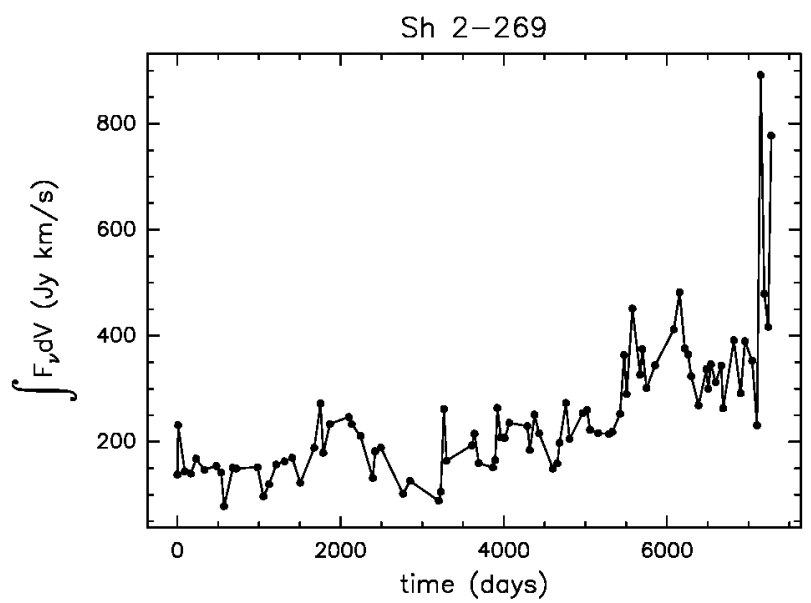

Fig. A.18. d Integral of the flux density over the observed velocity range as a function of time for source Sh 2-269.

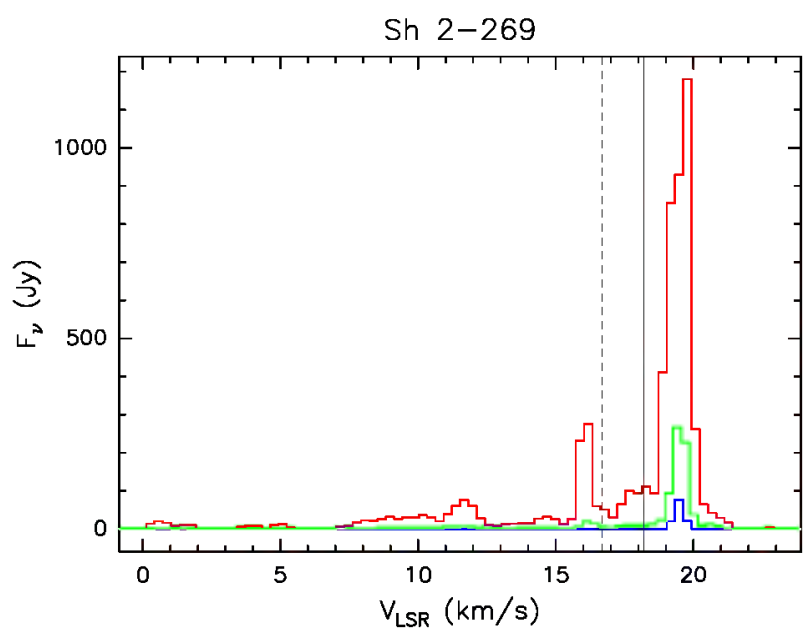

Fig. A.18. e Upper (red) and lower (blue) envelopes and mean spectrum (green) of source Sh 2-269 measured during our monitoring. The vertical solid line marks the velocity of the associated thermal molecular gas. The vertical dashed line marks the mean velocity derived from the histogram of the rate-of-occurrence.

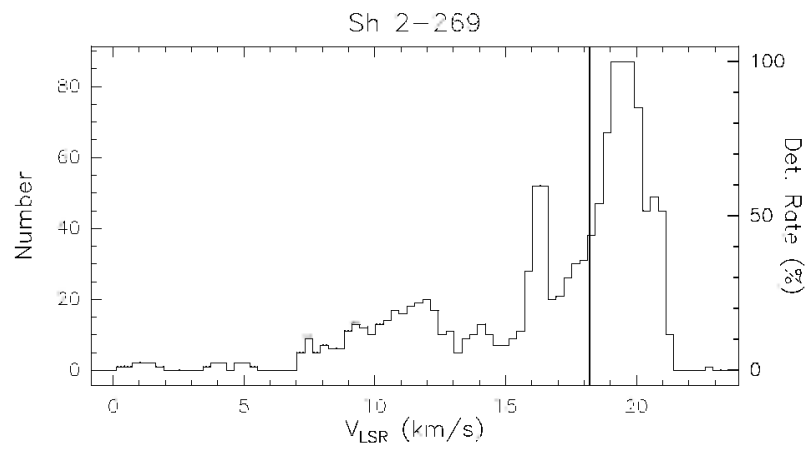

Fig. A.18. f Rate-of-occurrence plot for source Sh 2-269. The scale to the right refers to the dotted histogram. the scale to the left to the solid line histogram. The vertical solid line marks the velocity of the associated thermal molecular gas. 
NGC 2264
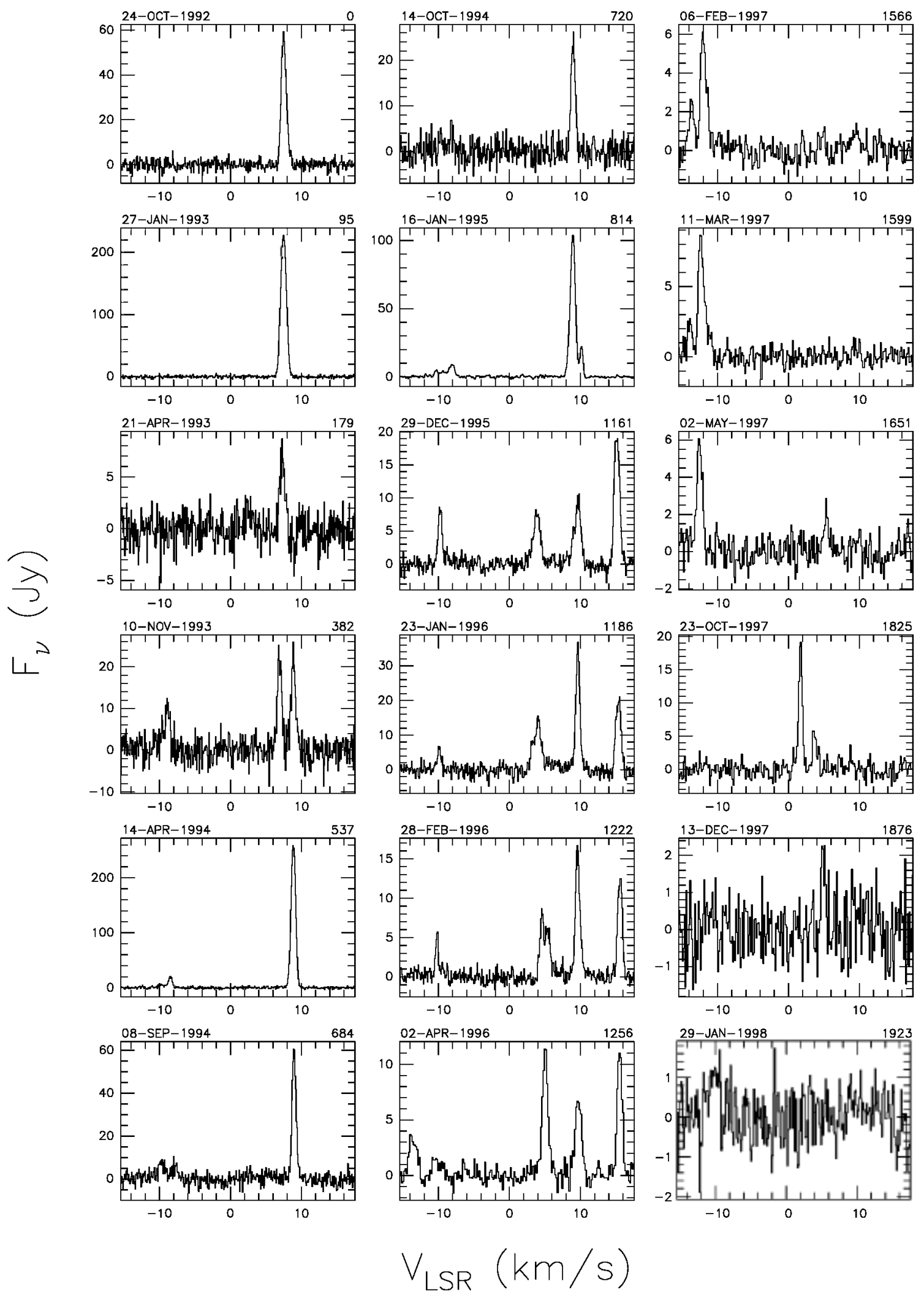

Fig. A.19. a Spectra of source NGC 2264 with autoscaled flux density scale. The date of observation is shown above the top left corner of each spectrum and the number of days elapsed since the first observation is given above the top right corner. The velocity scale is the same for all spectra. 
NGC 2264
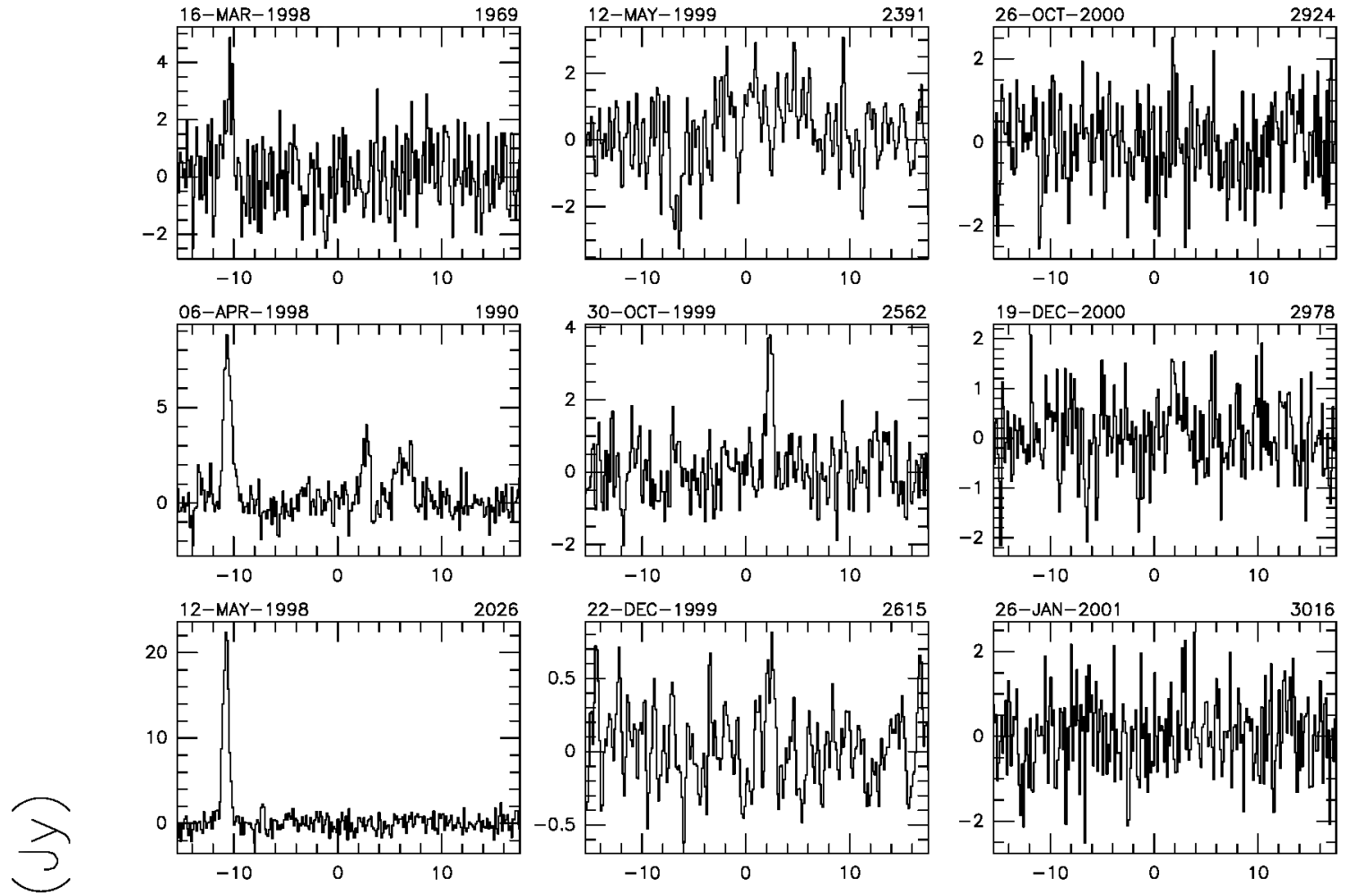

$4^{2}$
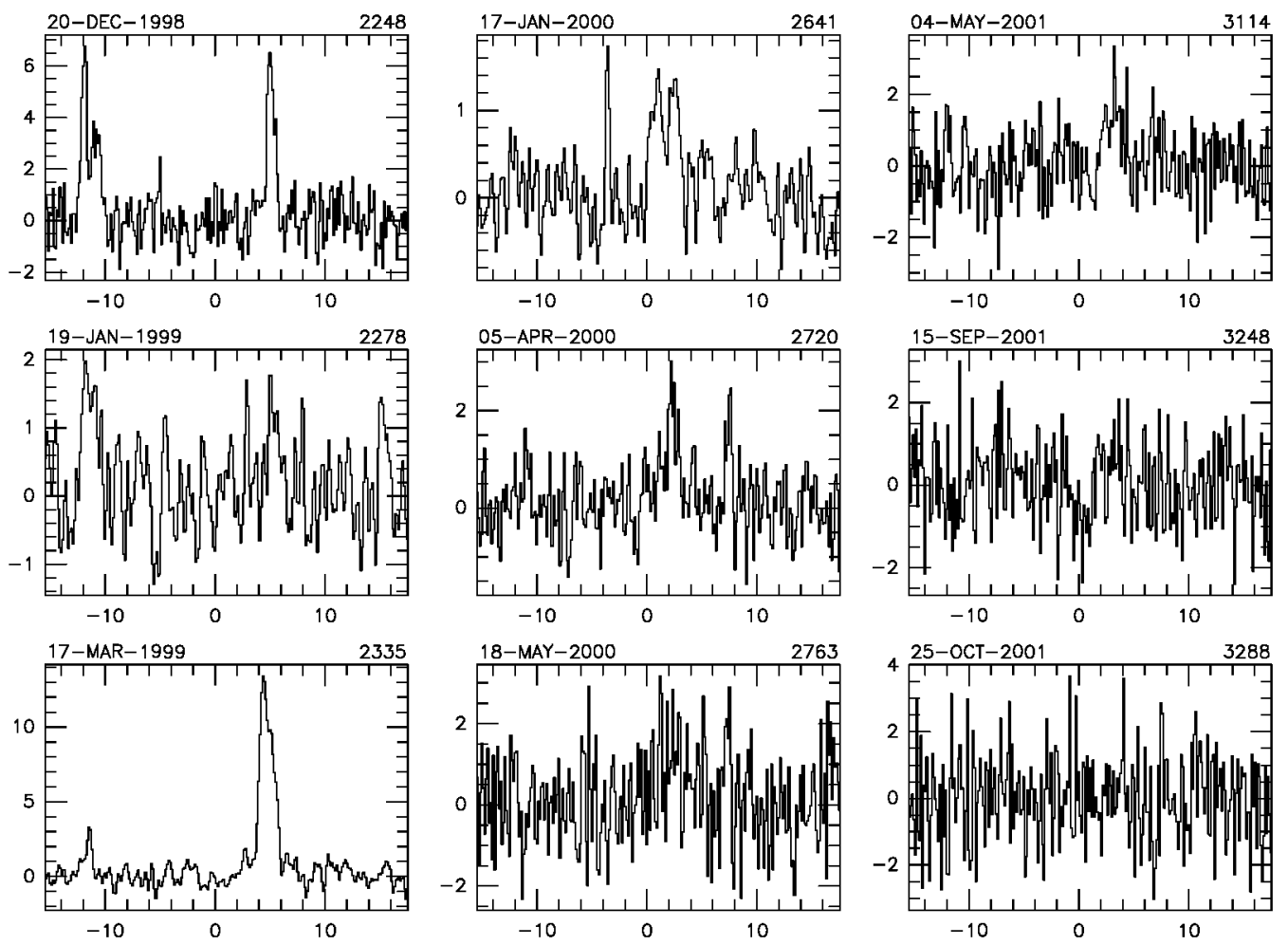

Fig. A.19. a continued.

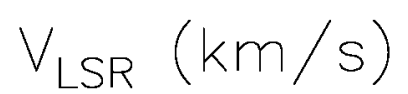


NGC 2264
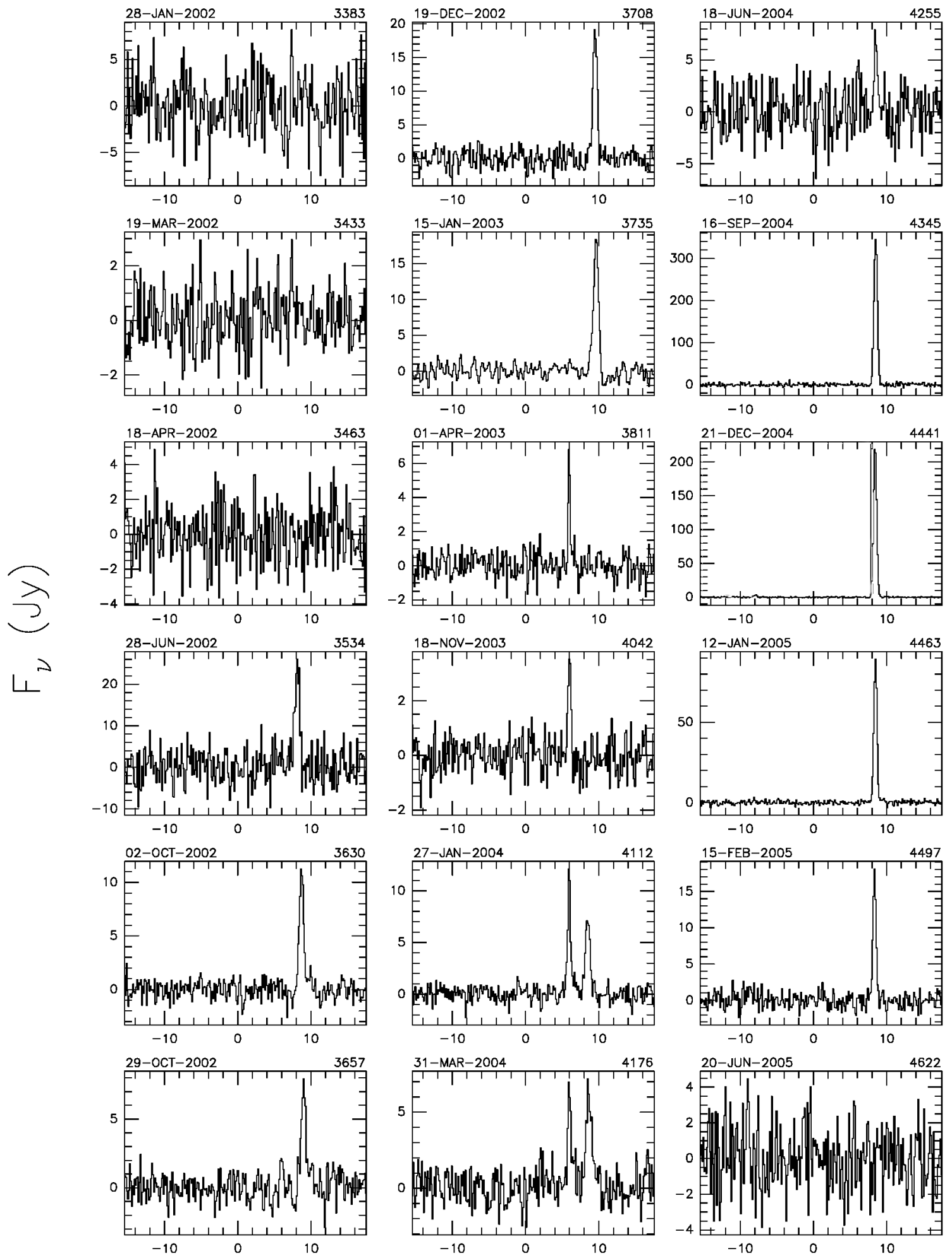

$$
V_{\text {LSR }}(\mathrm{km} / \mathrm{s})
$$

Fig. A.19. a continued. 


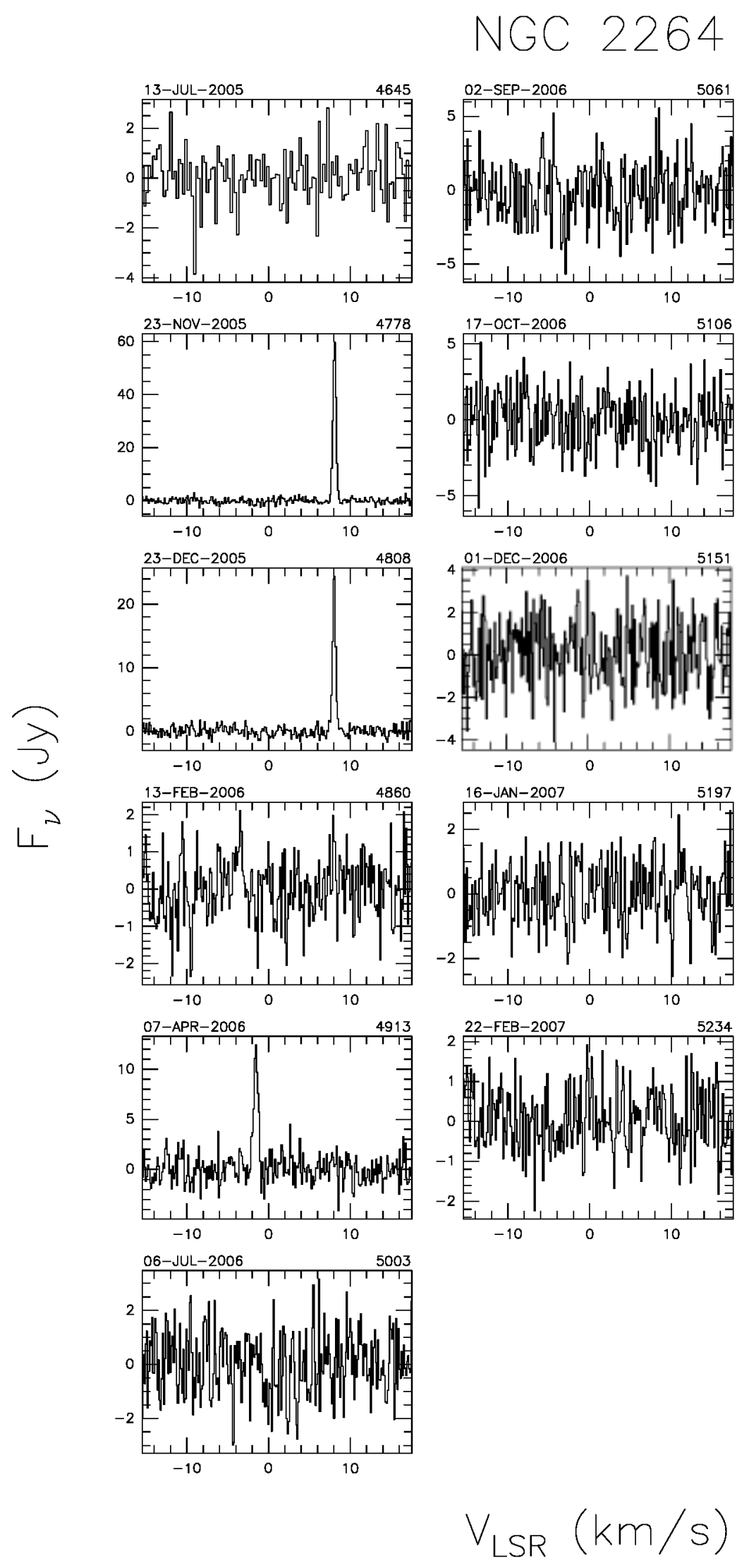

Fig. A.19. a continued. 


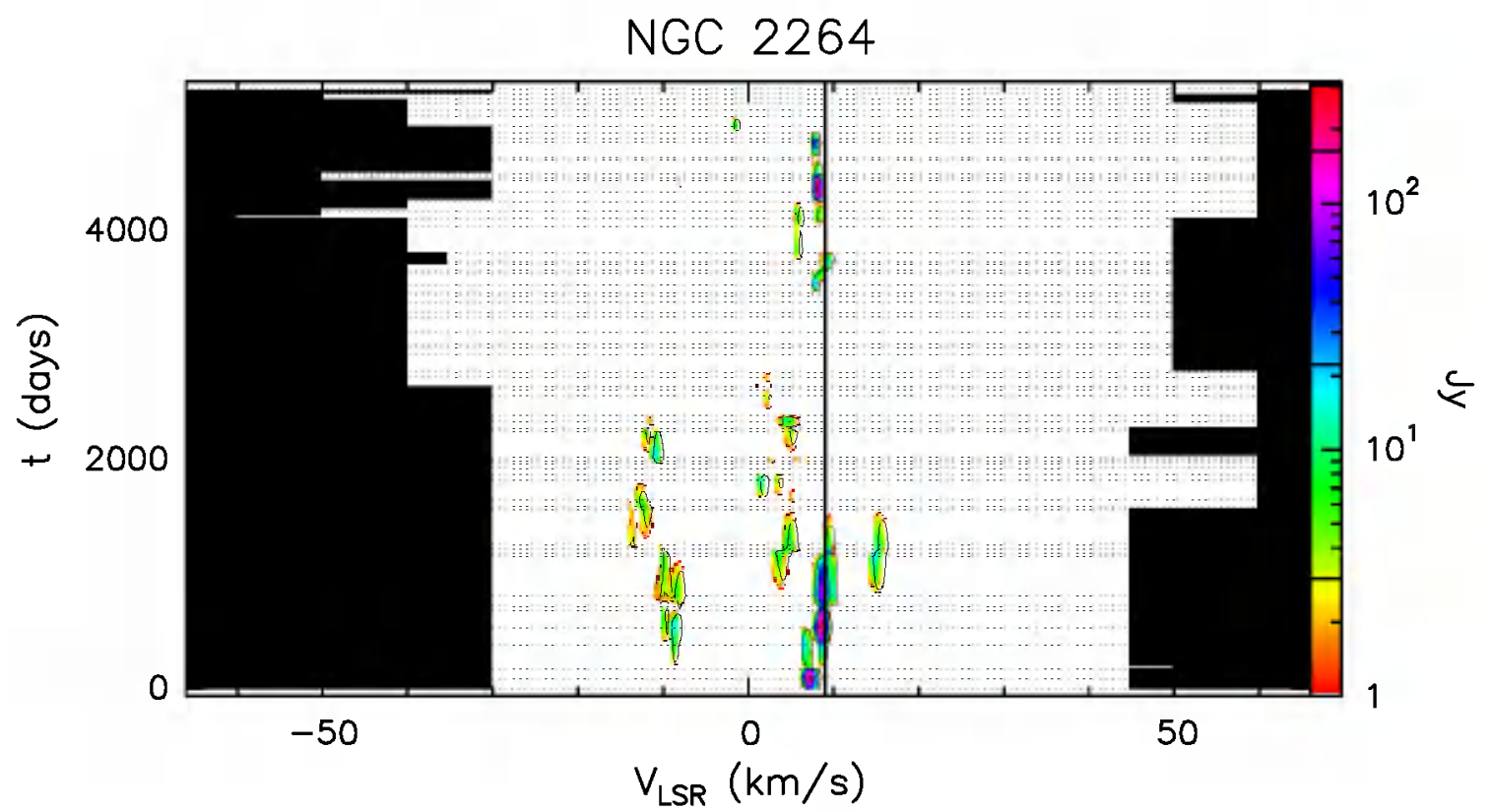

Fig. A.19. b Velocity-time-flux density full plot for source NGC 2264. The vertical solid line indicates the velocity of the associated thermal molecular gas. The flux density scale is shown by the bar on the right. In this bar the three lines give the flux density of the drawn contours.

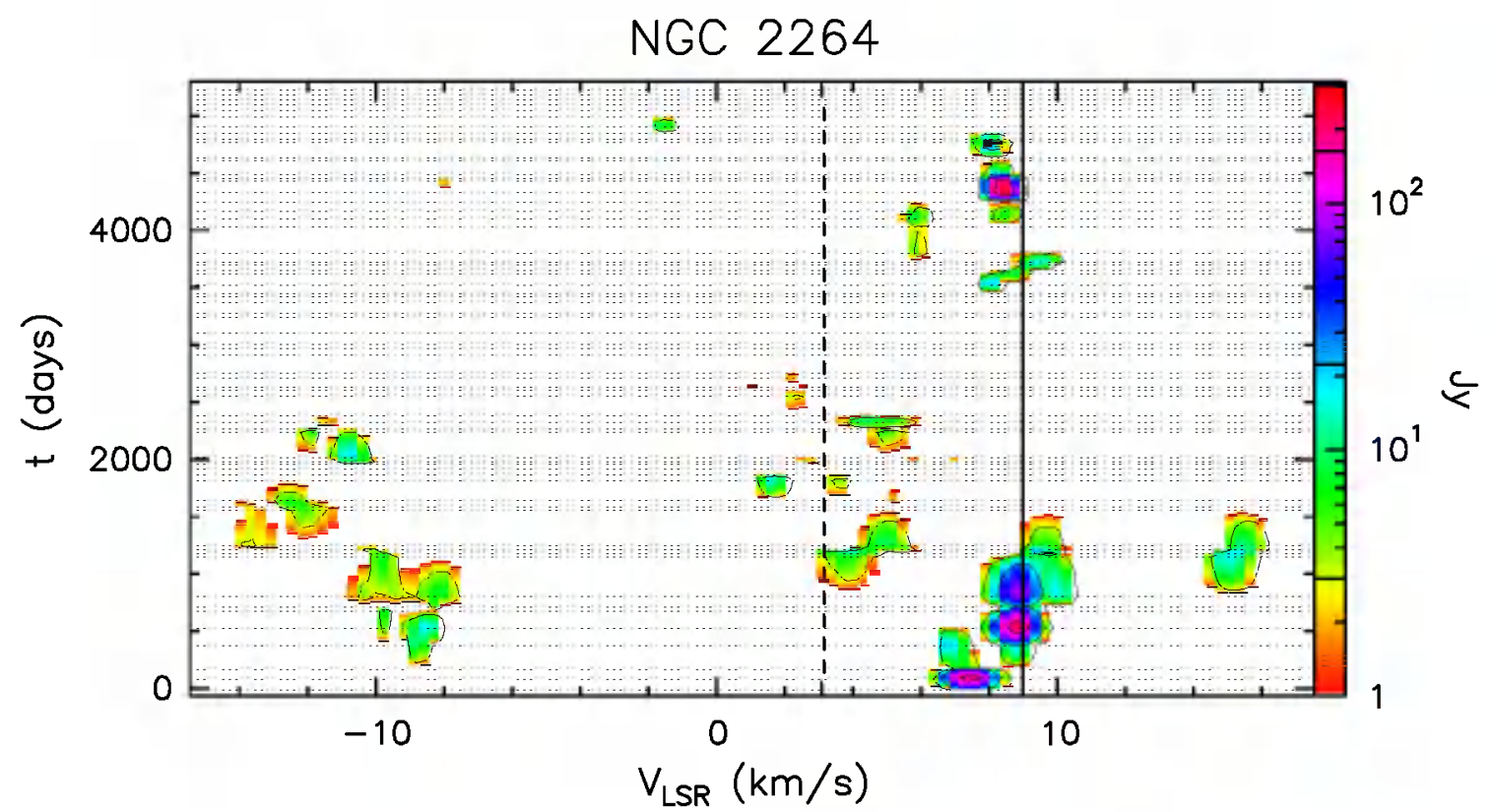

Fig. A.19. c Same as previous figure, but "zoomed" to velocity range over which emission has been detected. 


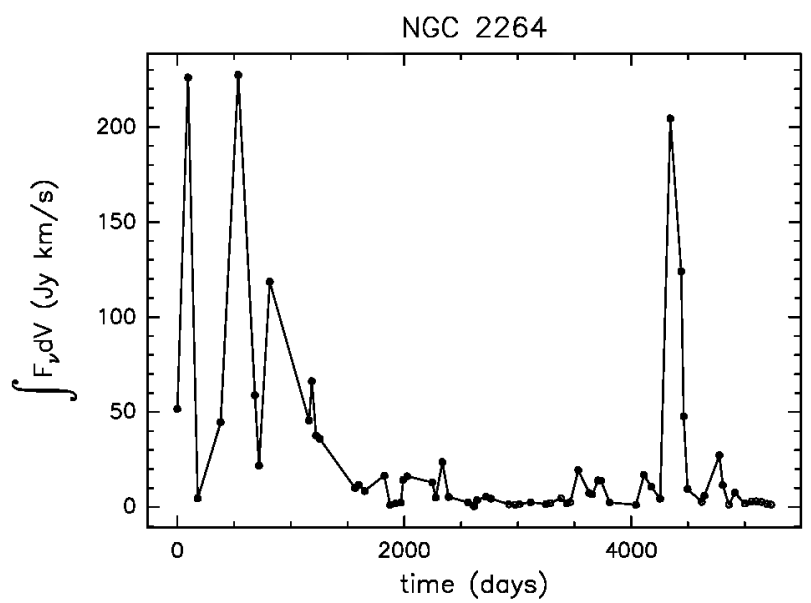

Fig. A.19. d Integral of the flux density over the observed velocity range as a function of time for source NGC 2264.

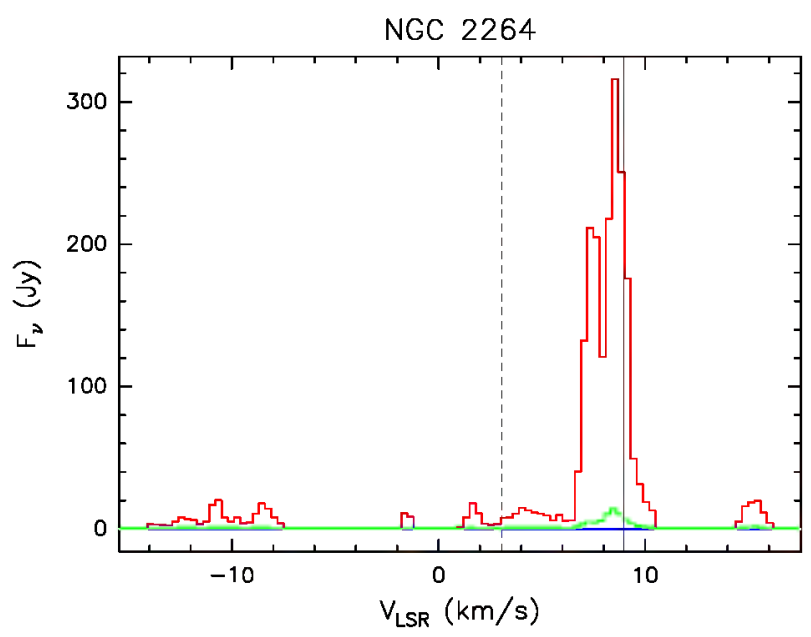

Fig. A.19. e Upper (red) and lower (blue) envelopes and mean spectrum (green) of source NGC 2264 measured during our monitoring. The vertical solid line marks the velocity of the associated thermal molecular gas. The vertical dashed line marks the mean velocity derived from the histogram of the rate-of-occurrence.

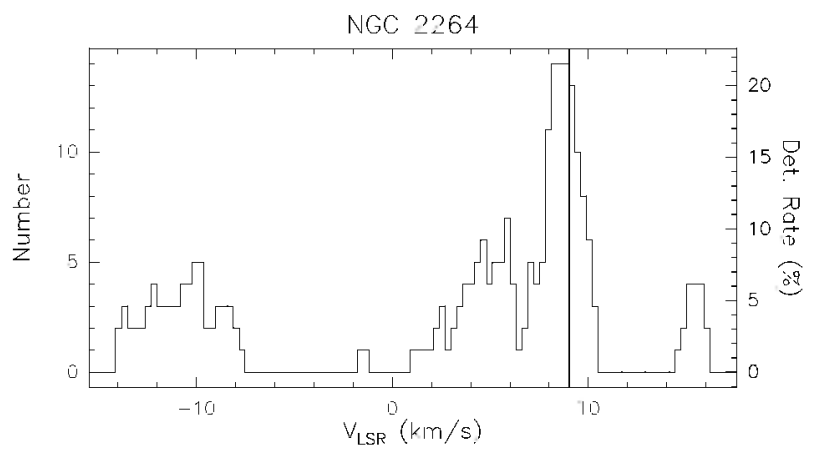

Fig. A.19. f Rate-of-occurrence plot for source NGC 2264. The scale to the right refers to the dotted histogram. the scale to the left to the solid line histogram. The vertical solid line marks the velocity of the associated thermal molecular gas. 


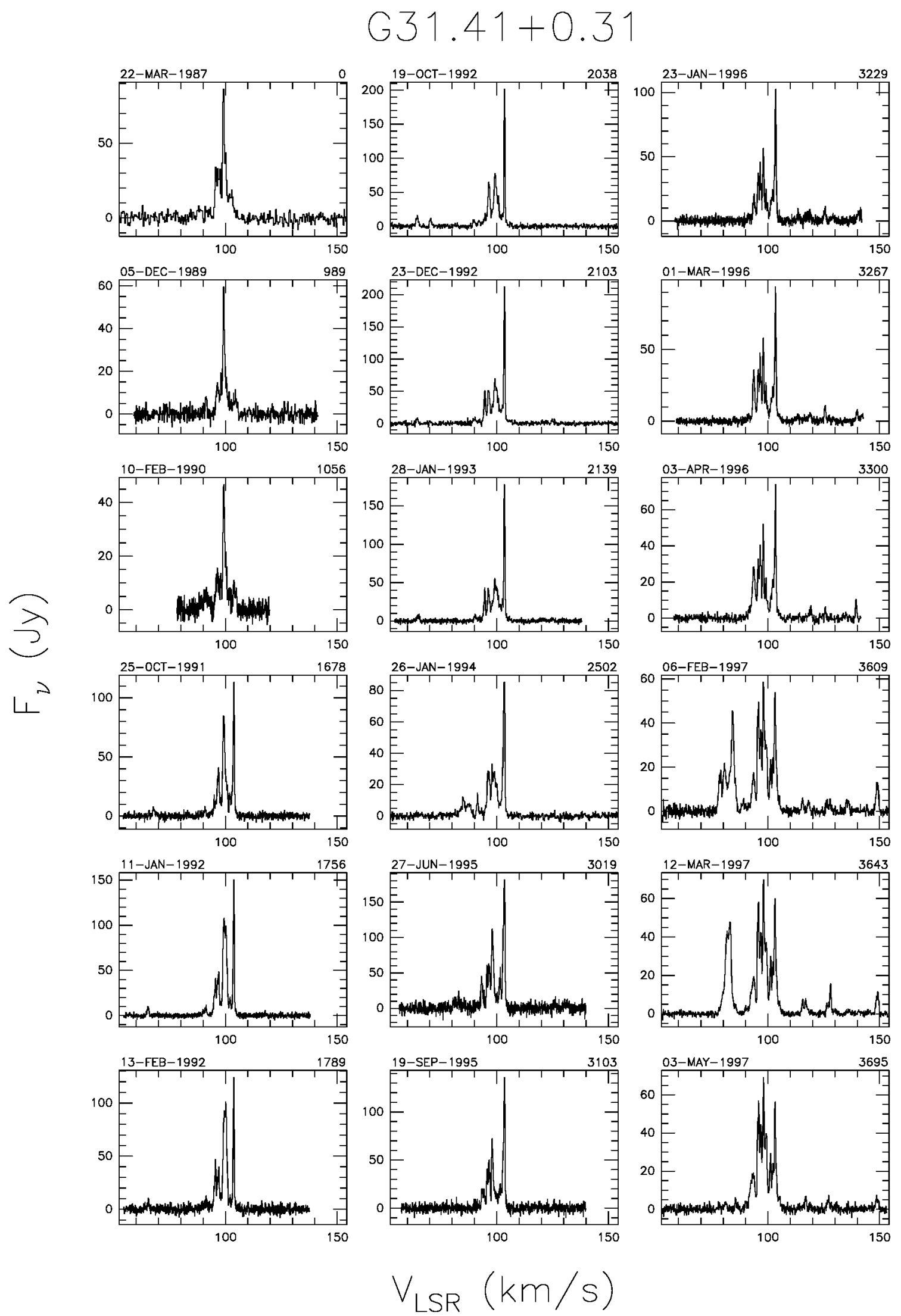

Fig. A.20. a Spectra of source G31.41+0.31 with autoscaled flux density scale. The date of observation is shown above the top left corner of each spectrum and the number of days elapsed since the first observation is given above the top right corner. The velocity scale is the same for all spectra. 


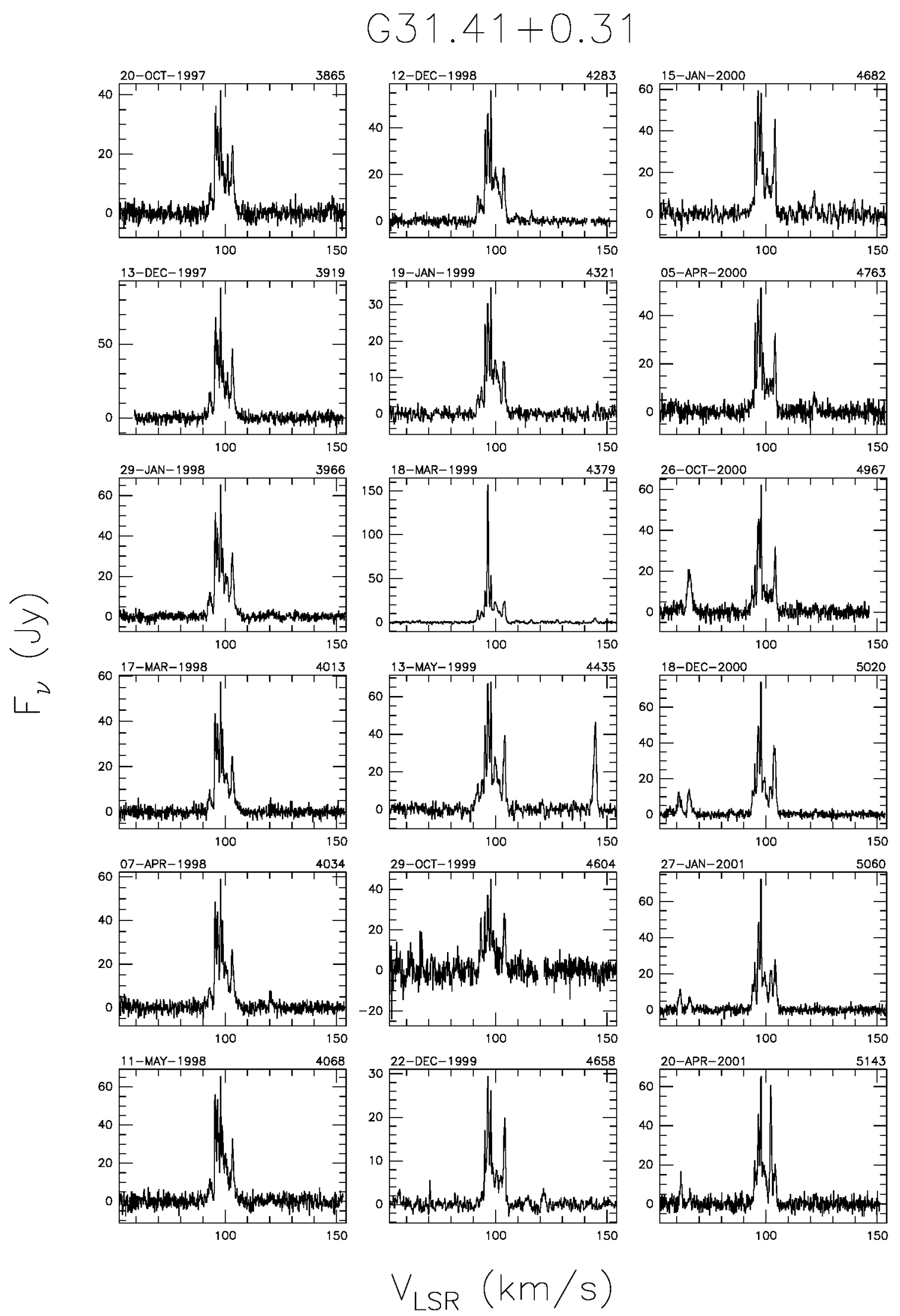

Fig. A.20. a continued. 


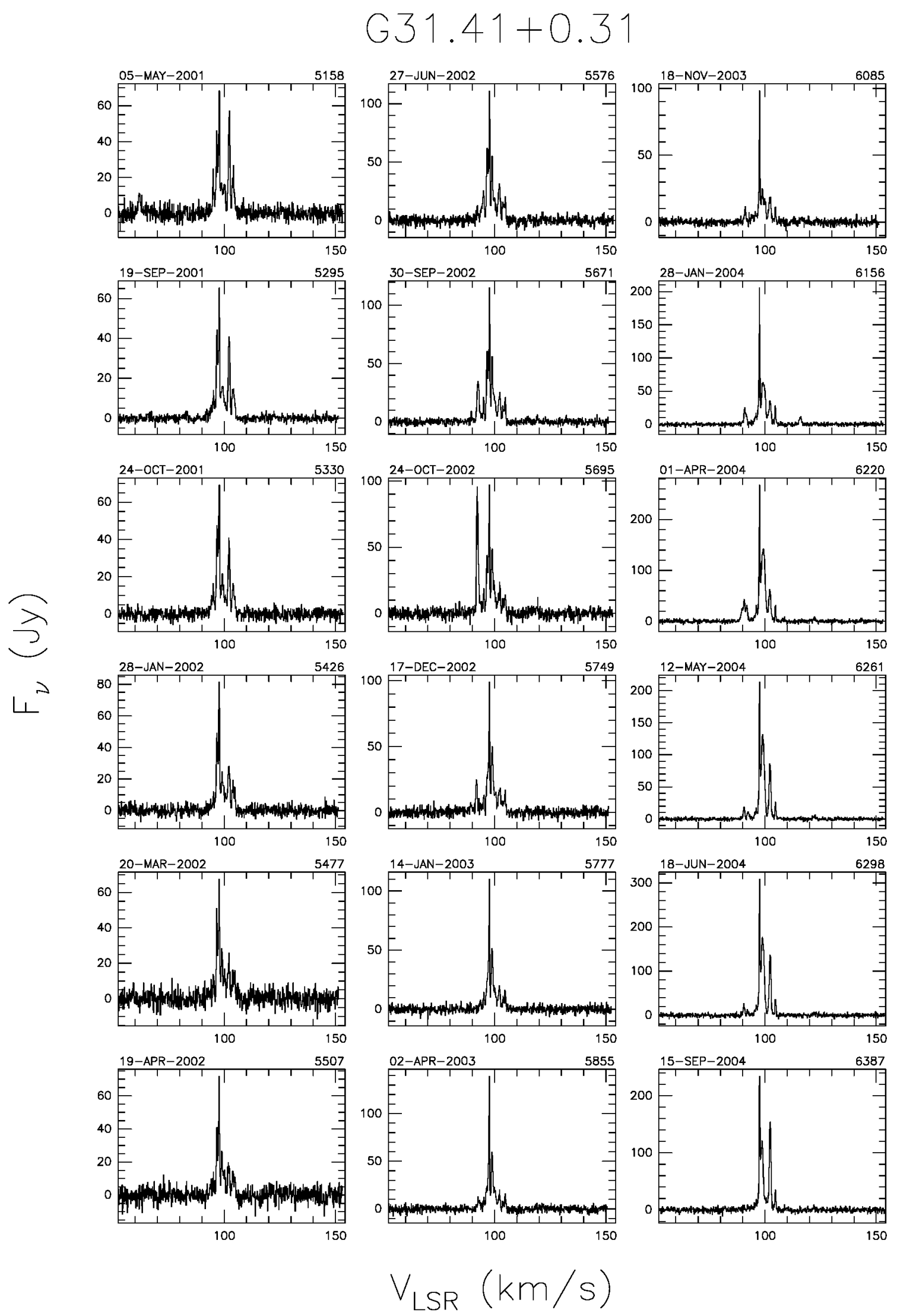

Fig. A.20. a continued. 


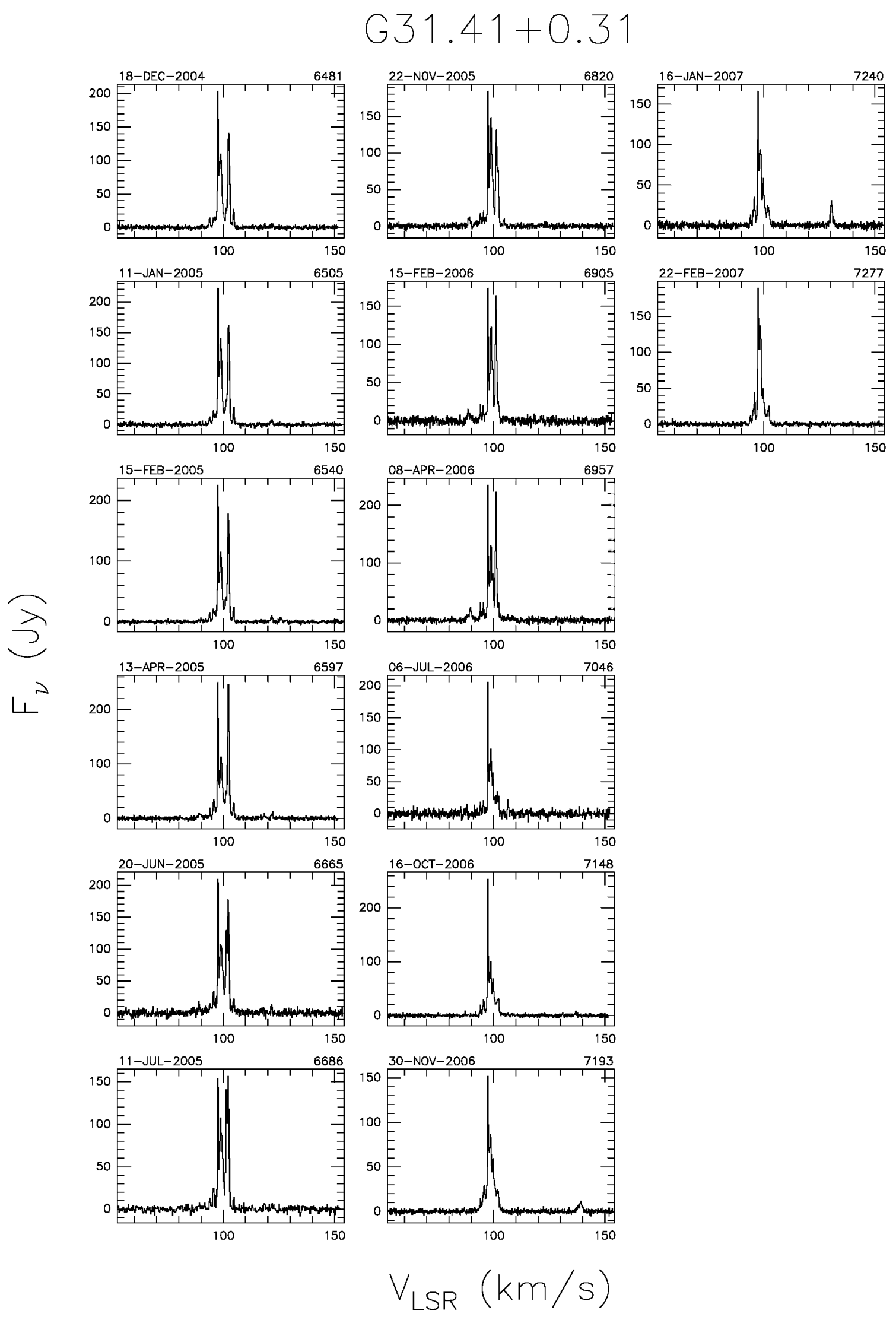

Fig. A.20. a continued. 


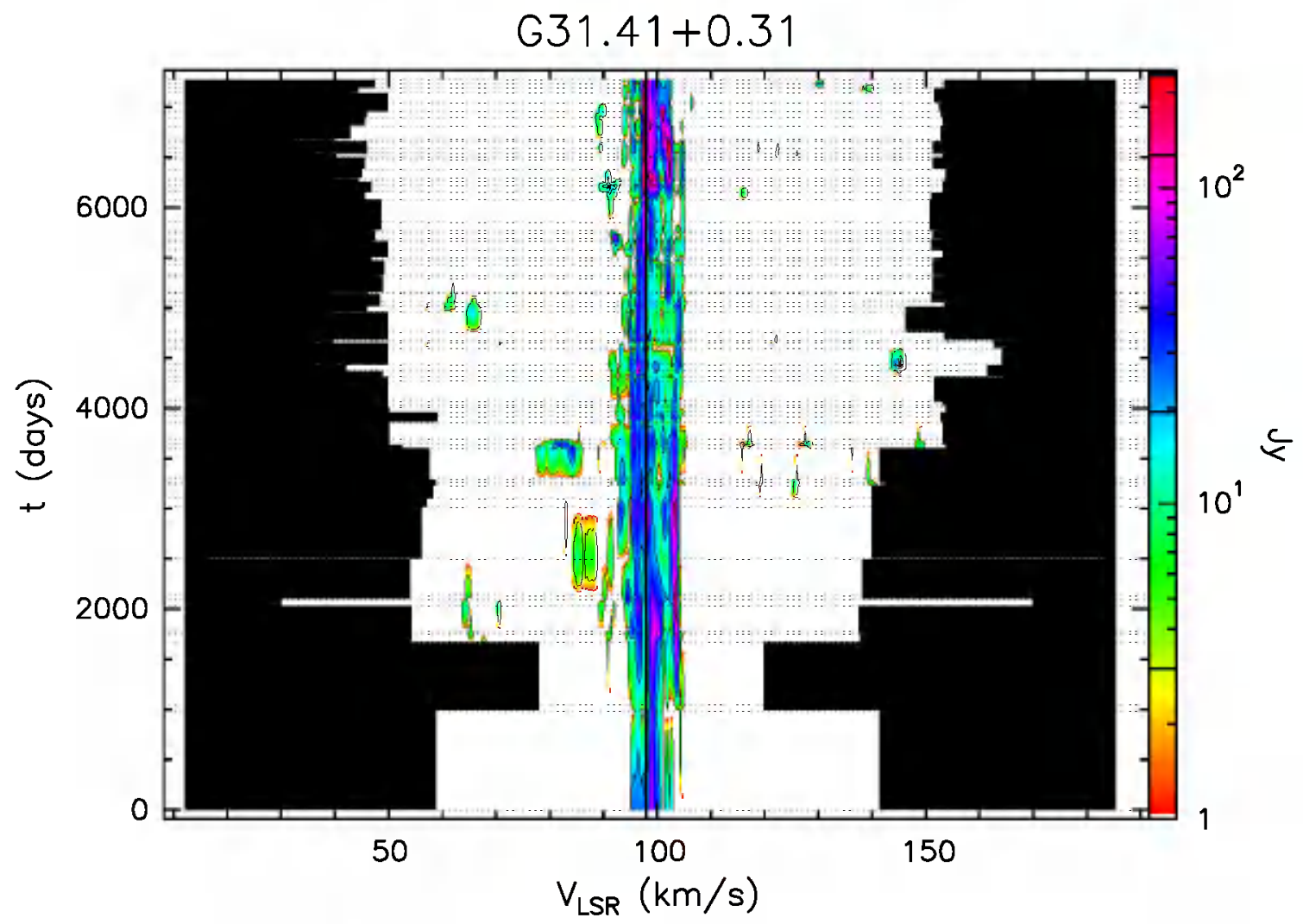

Fig. A.20. b Velocity-time-flux density full plot for source G31.41+0.31. The vertical solid line indicates the velocity of the associated thermal molecular gas. The flux density scale is shown by the bar on the right. In this bar the three lines give the flux density of the drawn contours.

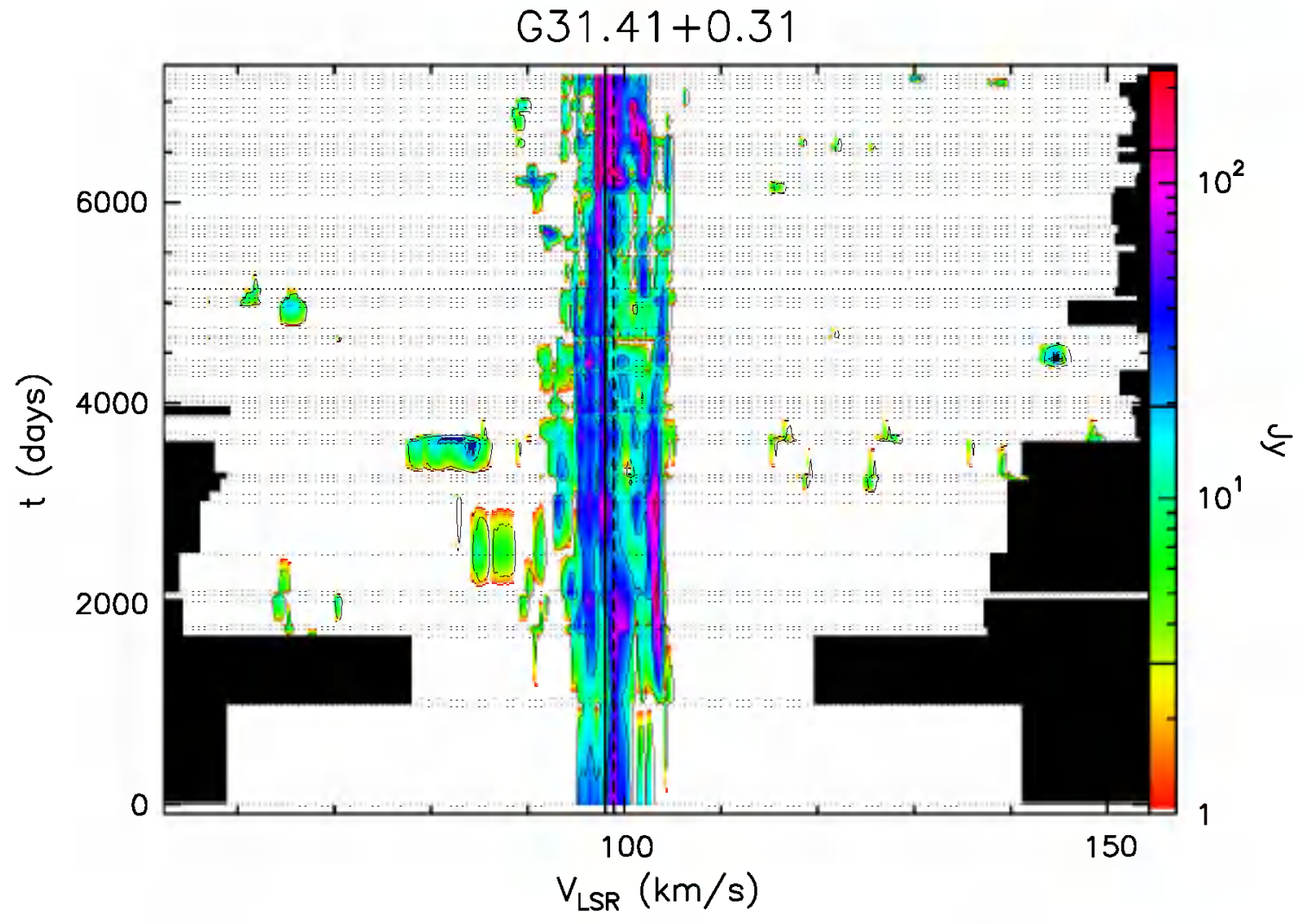

Fig. A.20. c Same as previous figure, but "zoomed" to velocity range over which emission has been detected. 


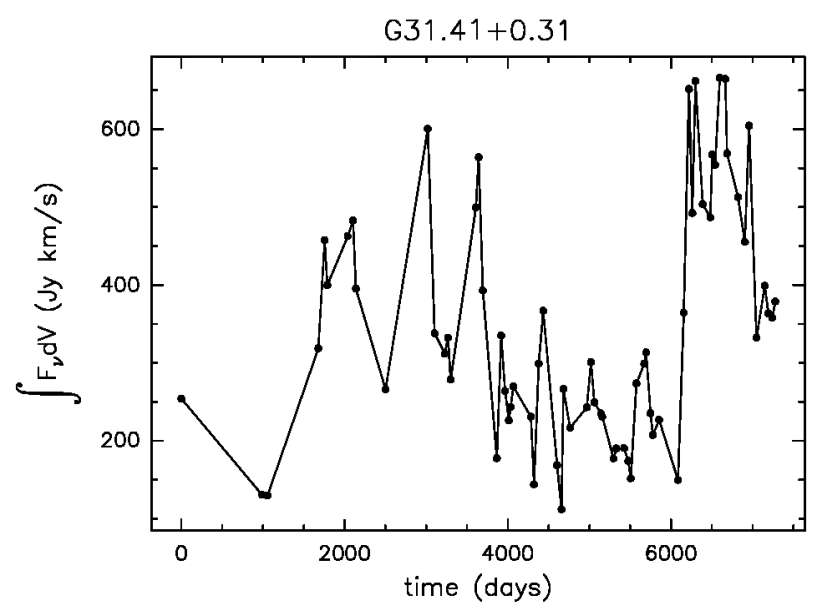

Fig. A.20. d Integral of the flux density over the observed velocity range as a function of time for source G31.41+0.31.

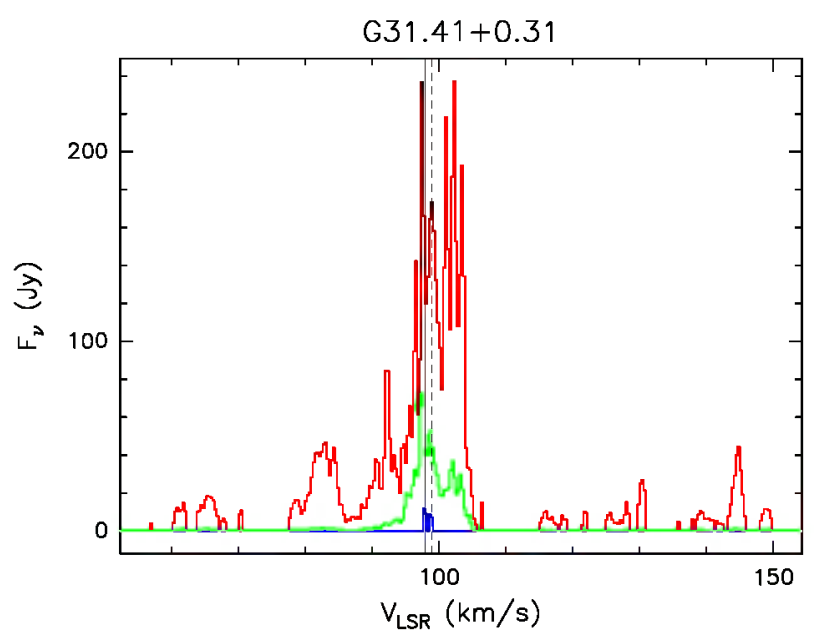

Fig. A.20. e Upper (red) and lower (blue) envelopes and mean spectrum (green) of source G31.41+0.31 measured during our monitoring. The vertical solid line marks the velocity of the associated thermal molecular gas. The vertical dashed line marks the mean velocity derived from the histogram of the rate-of-occurrence.

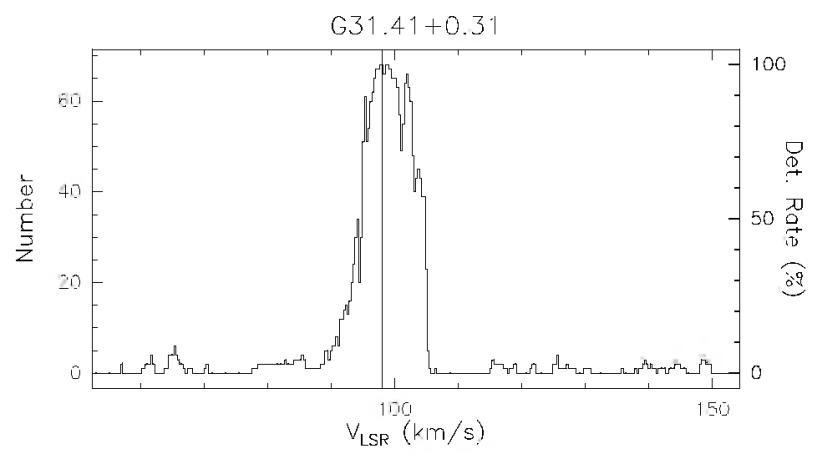

Fig. A.20. f Rate-of-occurrence plot for source G31.41+0.31. The scale to the right refers to the dotted histogram, the scale to the left to the solid line histogram. The vertical solid line marks the velocity of the associated thermal molecular gas. 


\section{W43 Main3}
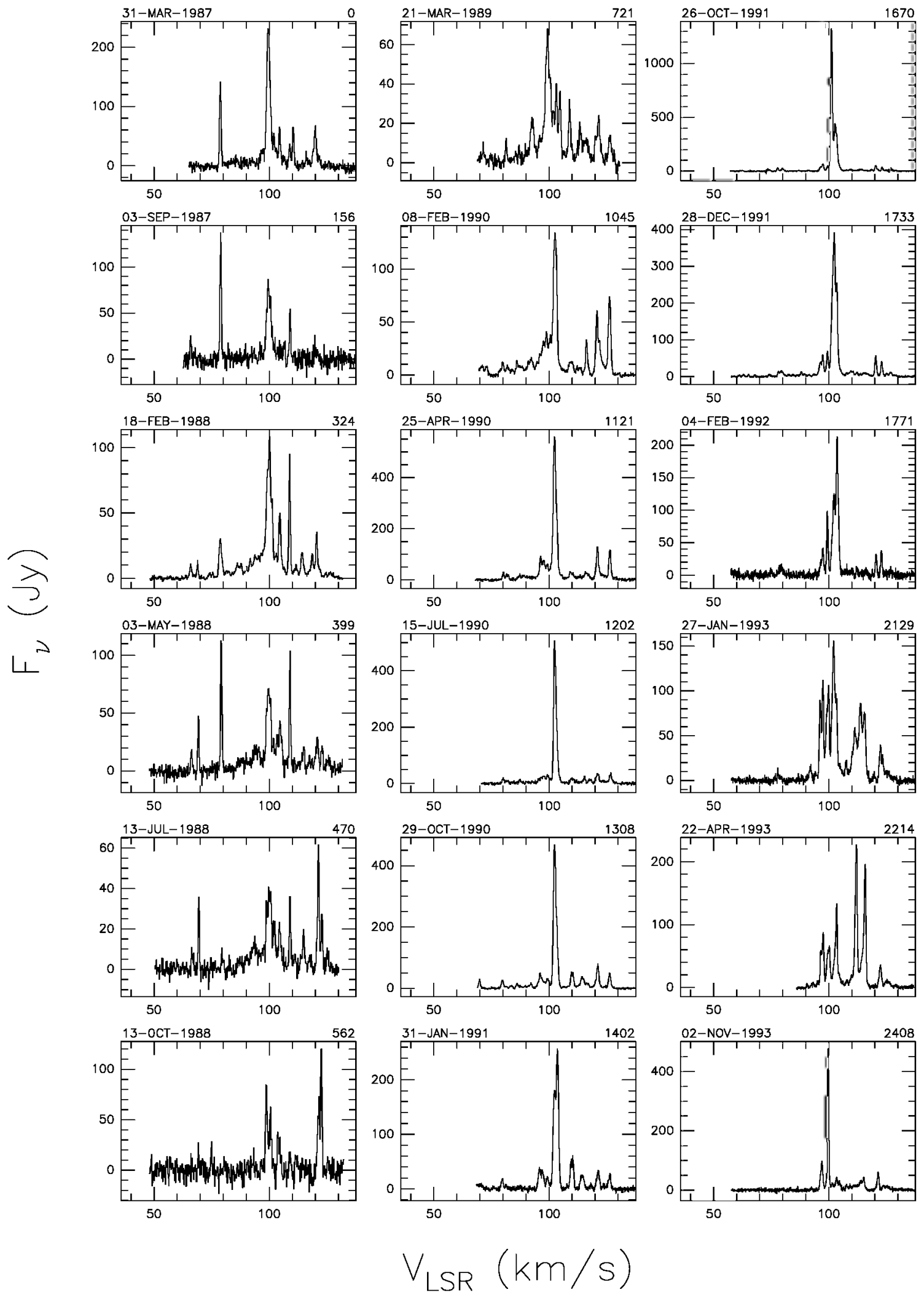

Fig. A.21. a Spectra of source W43 Main3 with autoscaled flux density scale. The date of observation is shown above the top left corner of each spectrum and the number of days elapsed since the first observation is given above the top right corner. The velocity scale is the same for all spectra. 


\section{W43 Main 3}
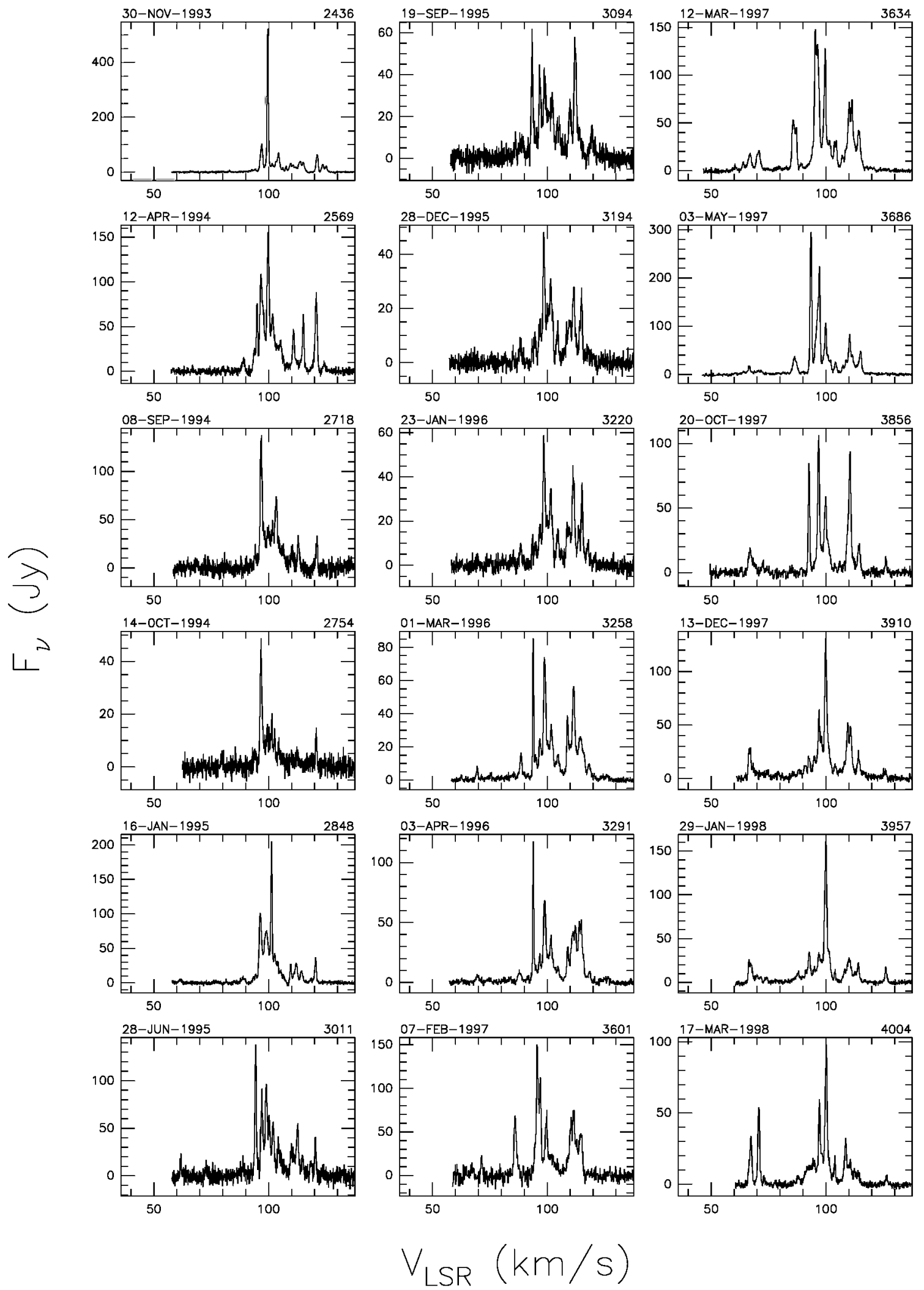

Fig. A.21. a continued. 


\section{W43 Main3}
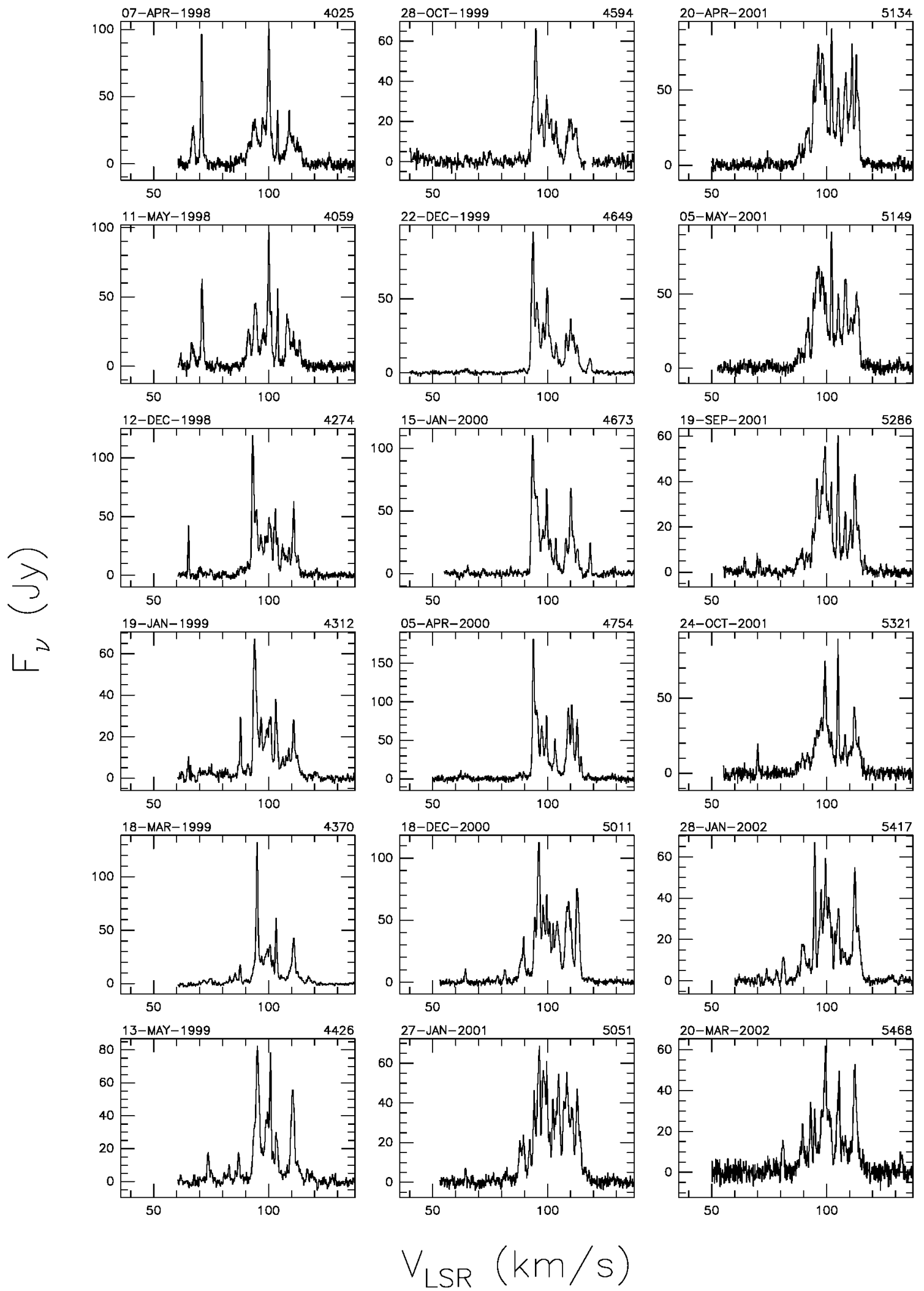

Fig. A.21. a continued. 


\section{W43 Main3}
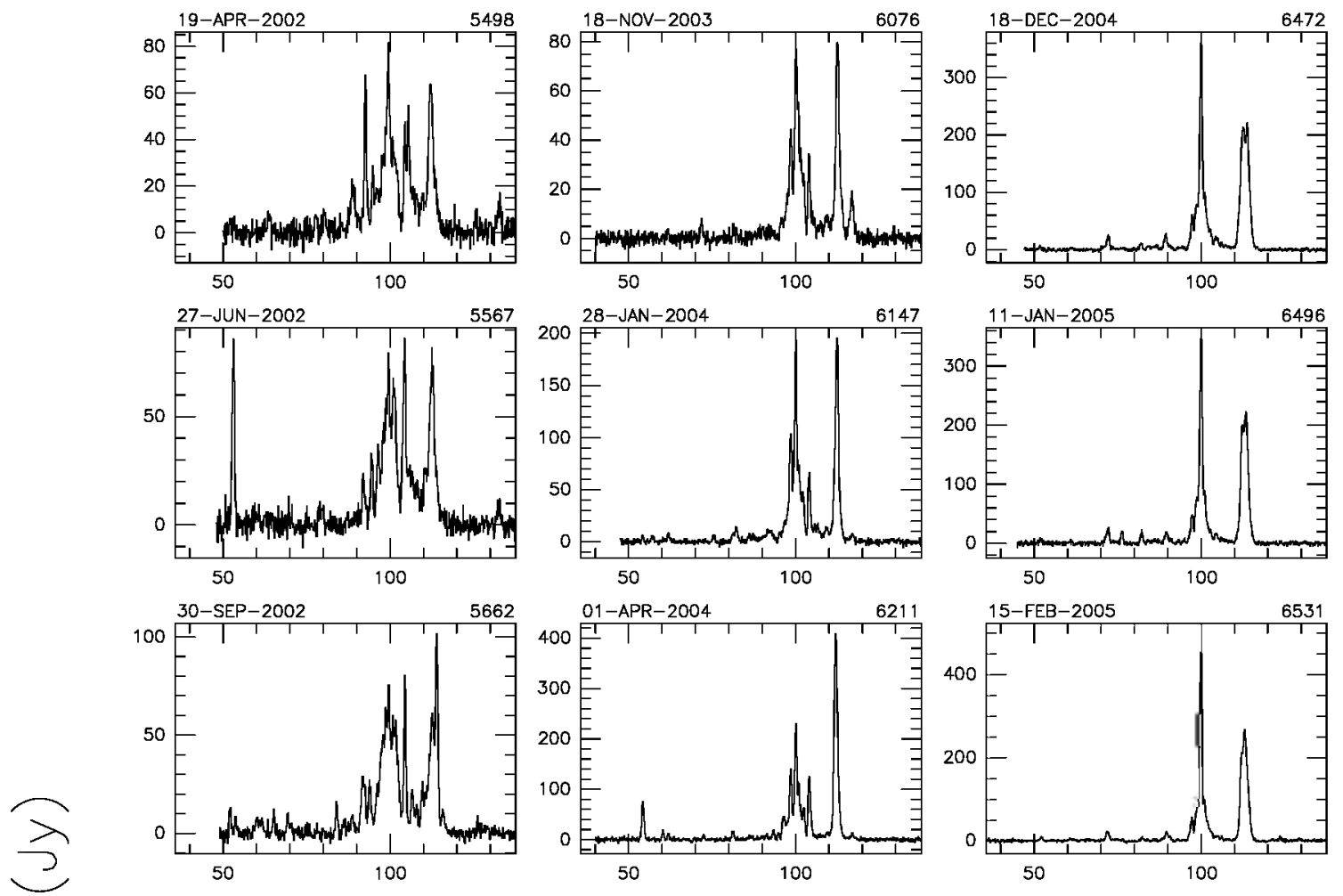

$L^{2}$
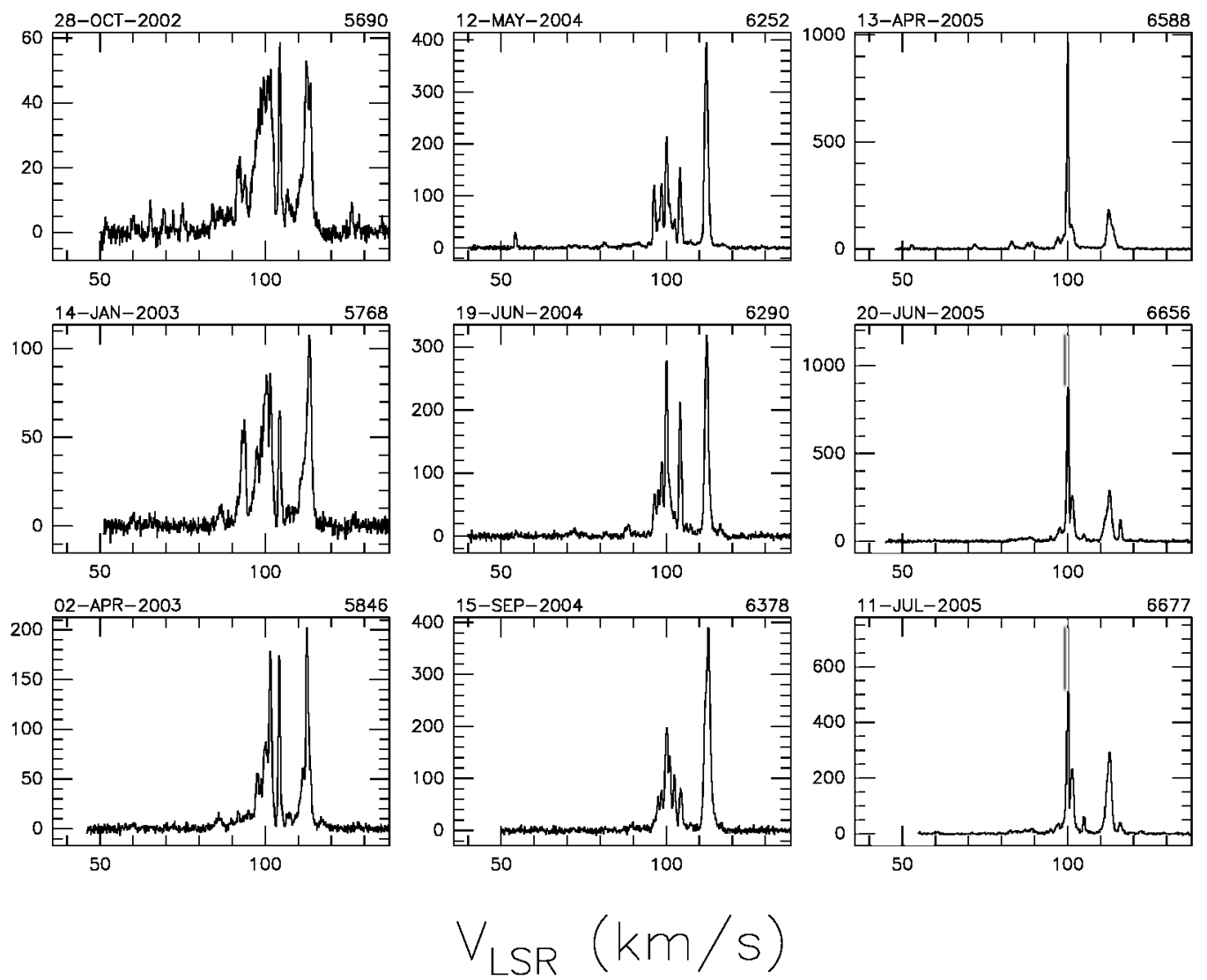

Fig. A.21. a continued. 


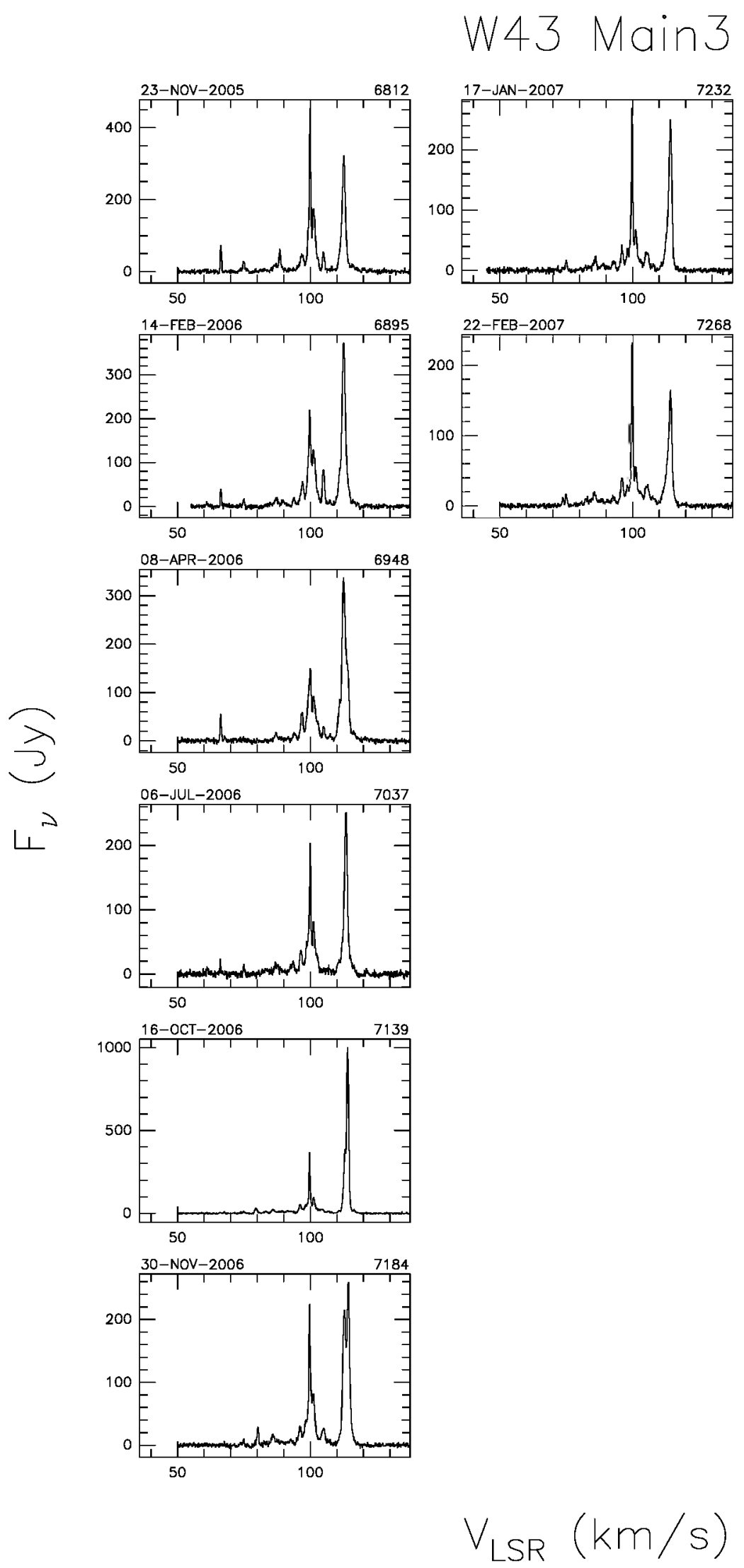

Fig. A.21. a continued. 


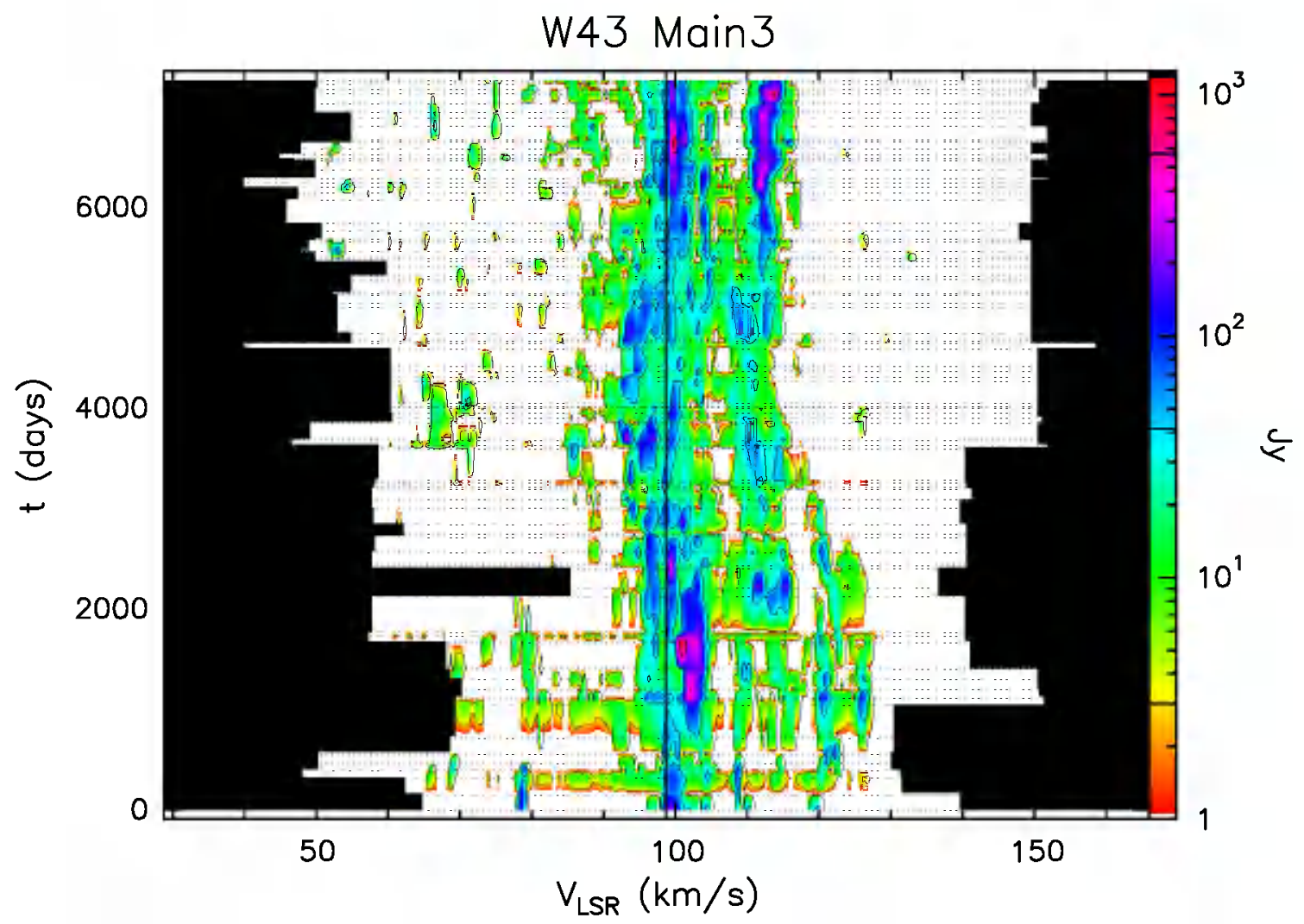

Fig. A.21. b Velocity-time-flux density full plot for source W43 Main3. The vertical solid line indicates the velocity of the associated thermal molecular gas. The flux density scale is shown by the bar on the right. In this bar the three lines give the flux density of the drawn contours.

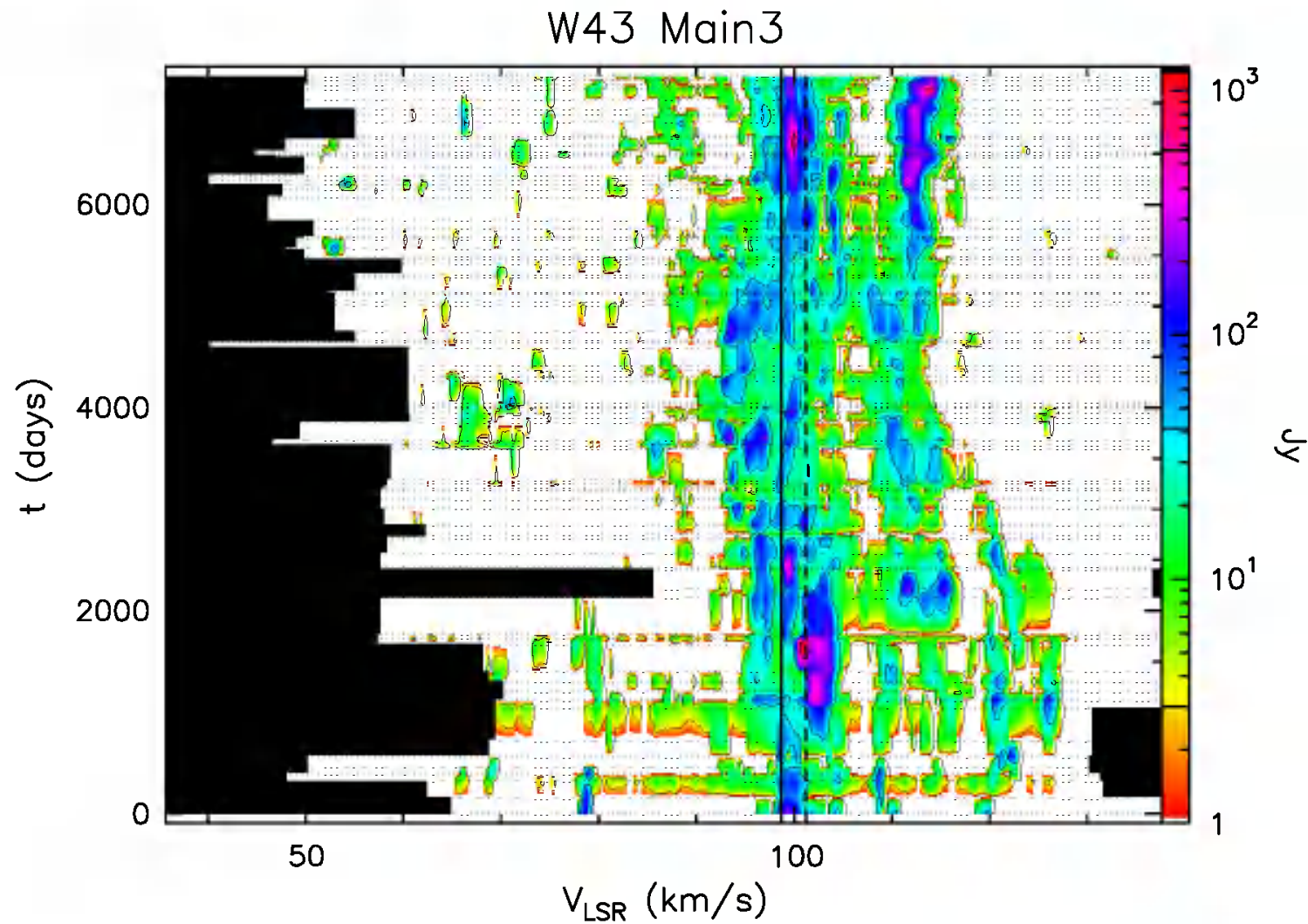

Fig. A.21. c Same as previous figure, but "zoomed" to velocity range over which emission has been detected. 


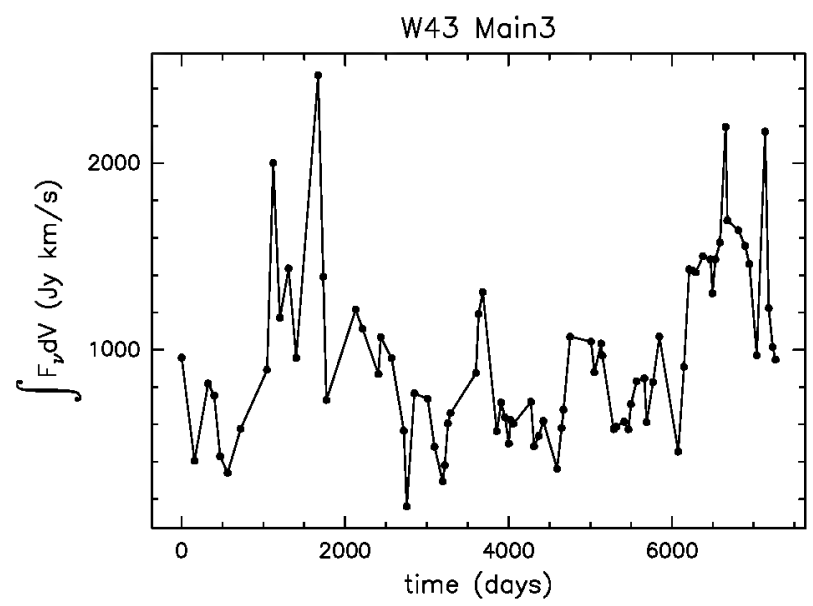

Fig. A.21. d Integral of the flux density over the observed velocity range as a function of time for source W43 Main3.

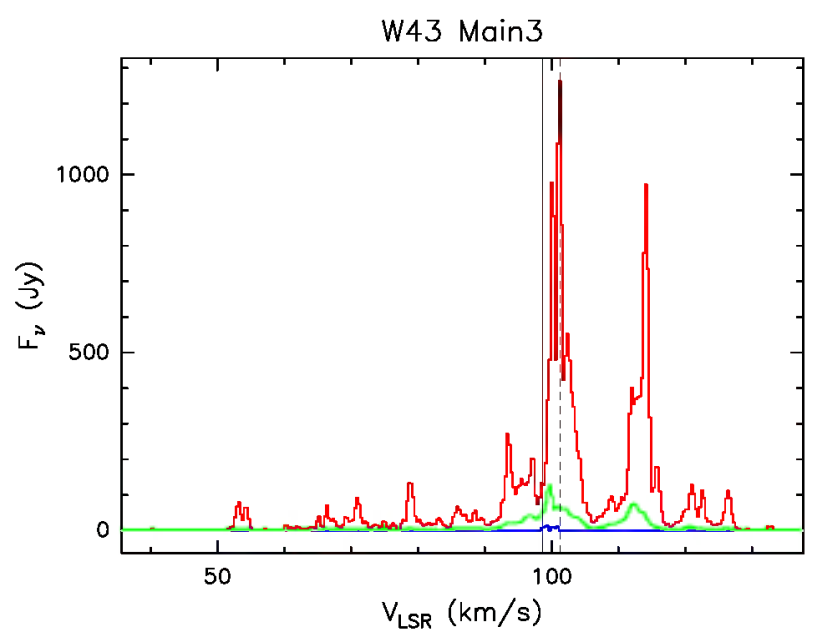

Fig. A.21. e Upper (red) and lower (blue) envelopes and mean spectrum (green) of source W43 Main3 measured during our monitoring. The vertical solid line marks the velocity of the associated thermal molecular gas. The vertical dashed line marks the mean velocity derived from the histogram of the rate-of-occurrence.

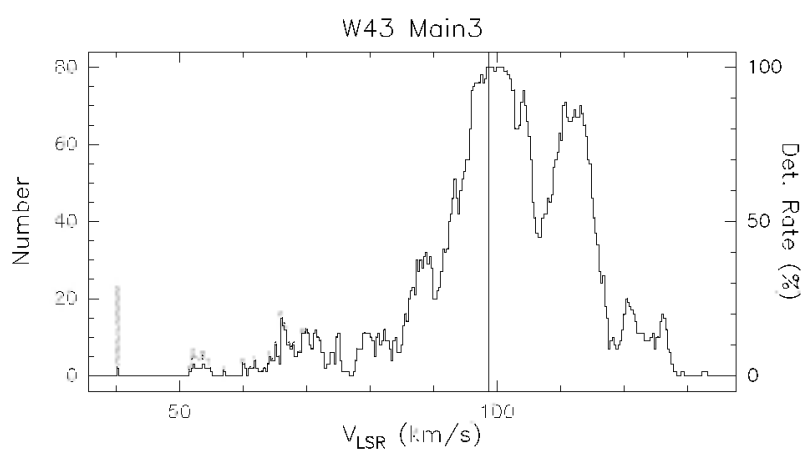

Fig. A.21. f Rate-of-occurrence plot for source W43 Main3. The scale to the right refer's to the dotted histogram, the scale to the left to the solid line histogram. The vertical solid line marks the velocity of the associated thermal molecular gas. 

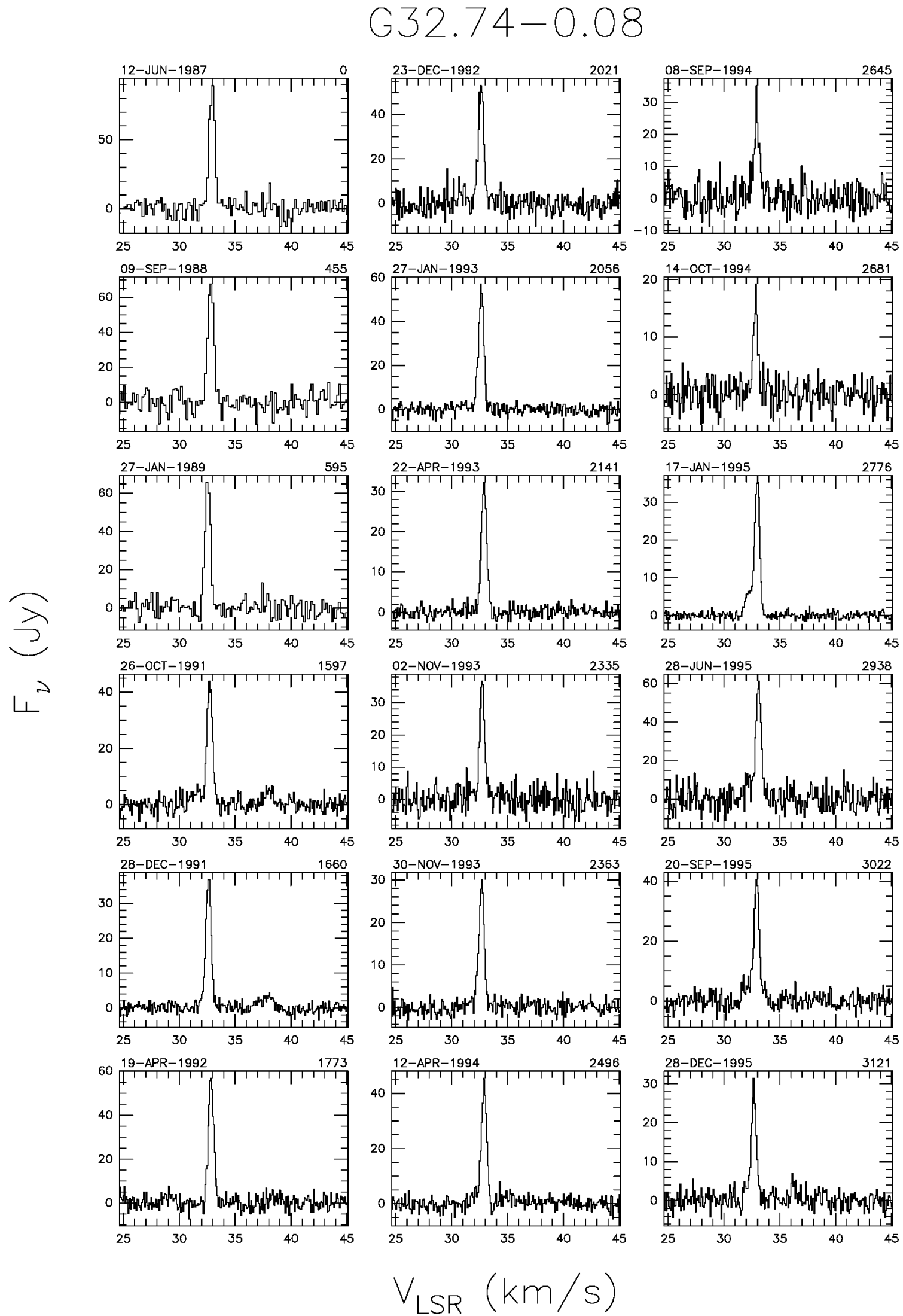

Fig. A.22. a Spectra of source G32.74-0.08 with autoscaled flux density scale. The date of observation is shown above the top left corner of each spectrum and the number of days elapsed since the first observation is given above the top right corner. The velocity scale is the same for all spectra. 

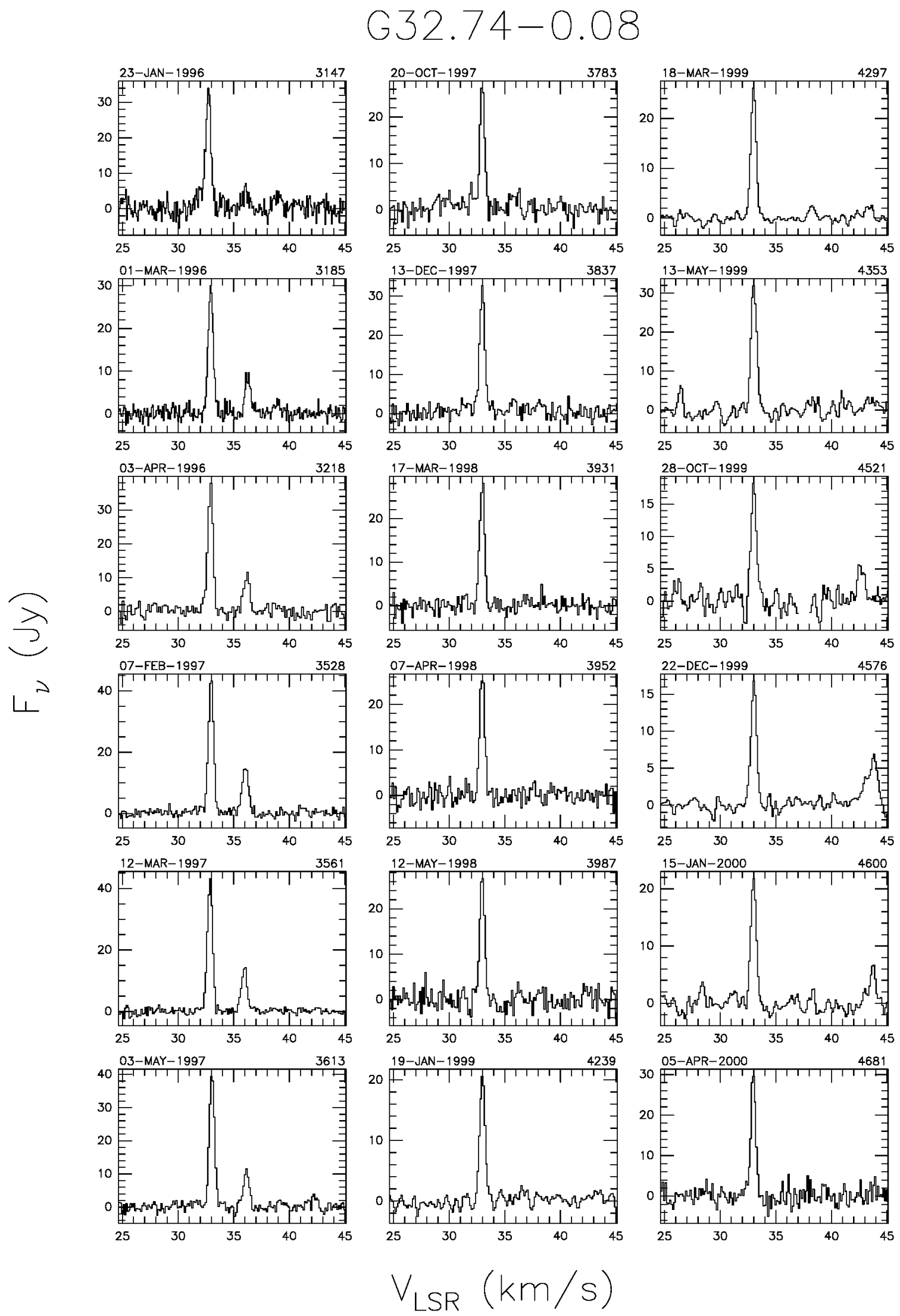

Fig. A.22. a continued. 
G32.74-0.08
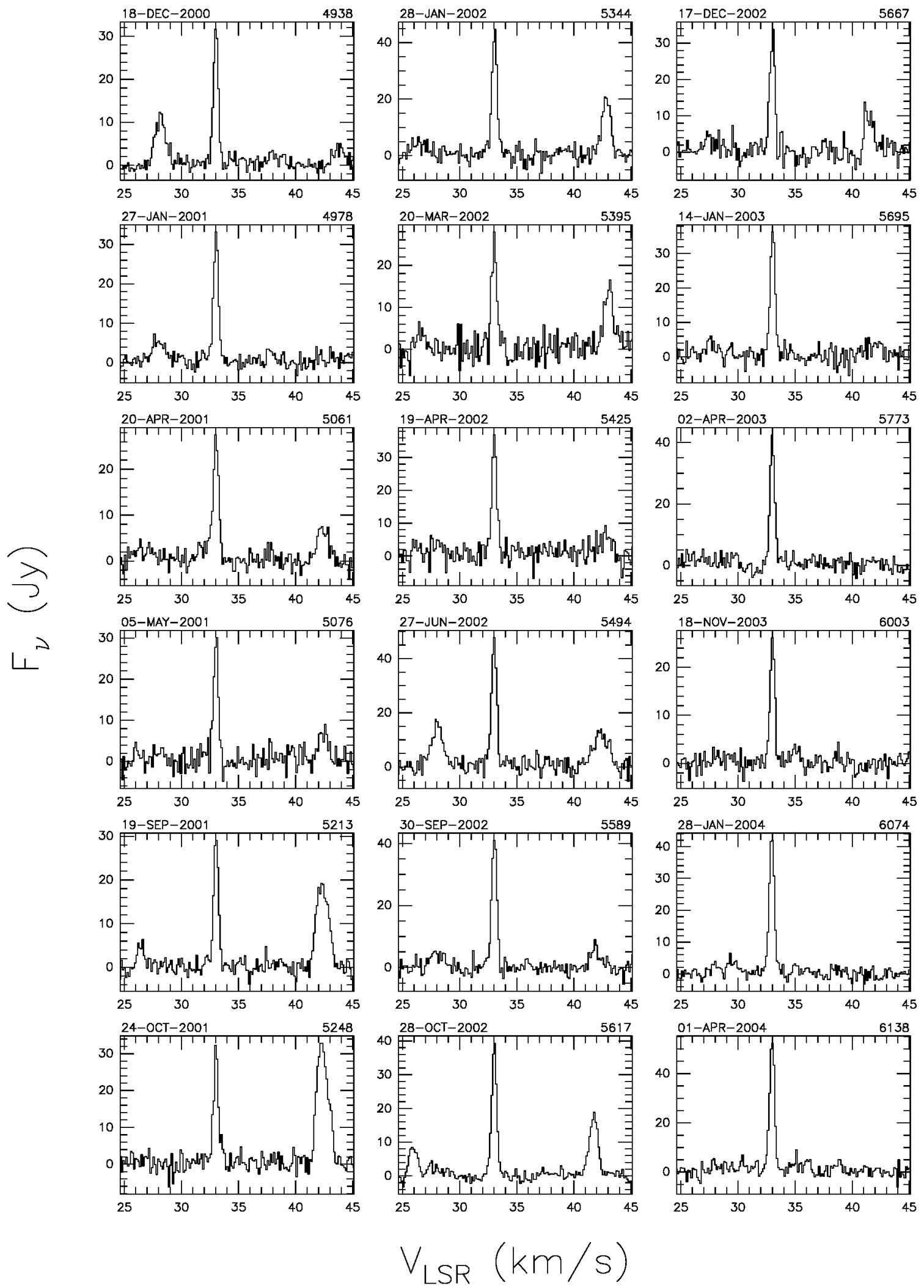

Fig. A.22. a continued. 


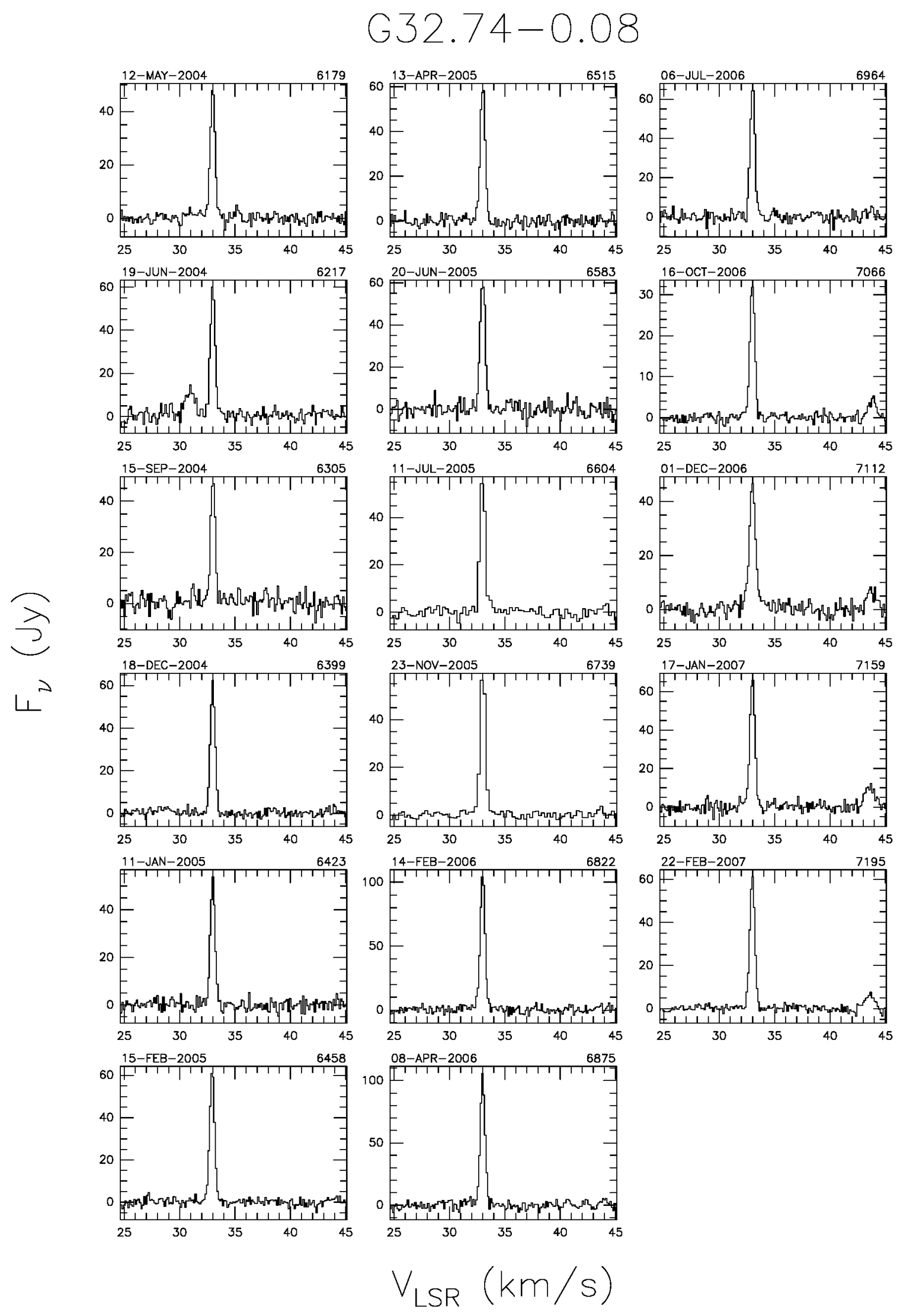

Fig. A.22. a continued. 


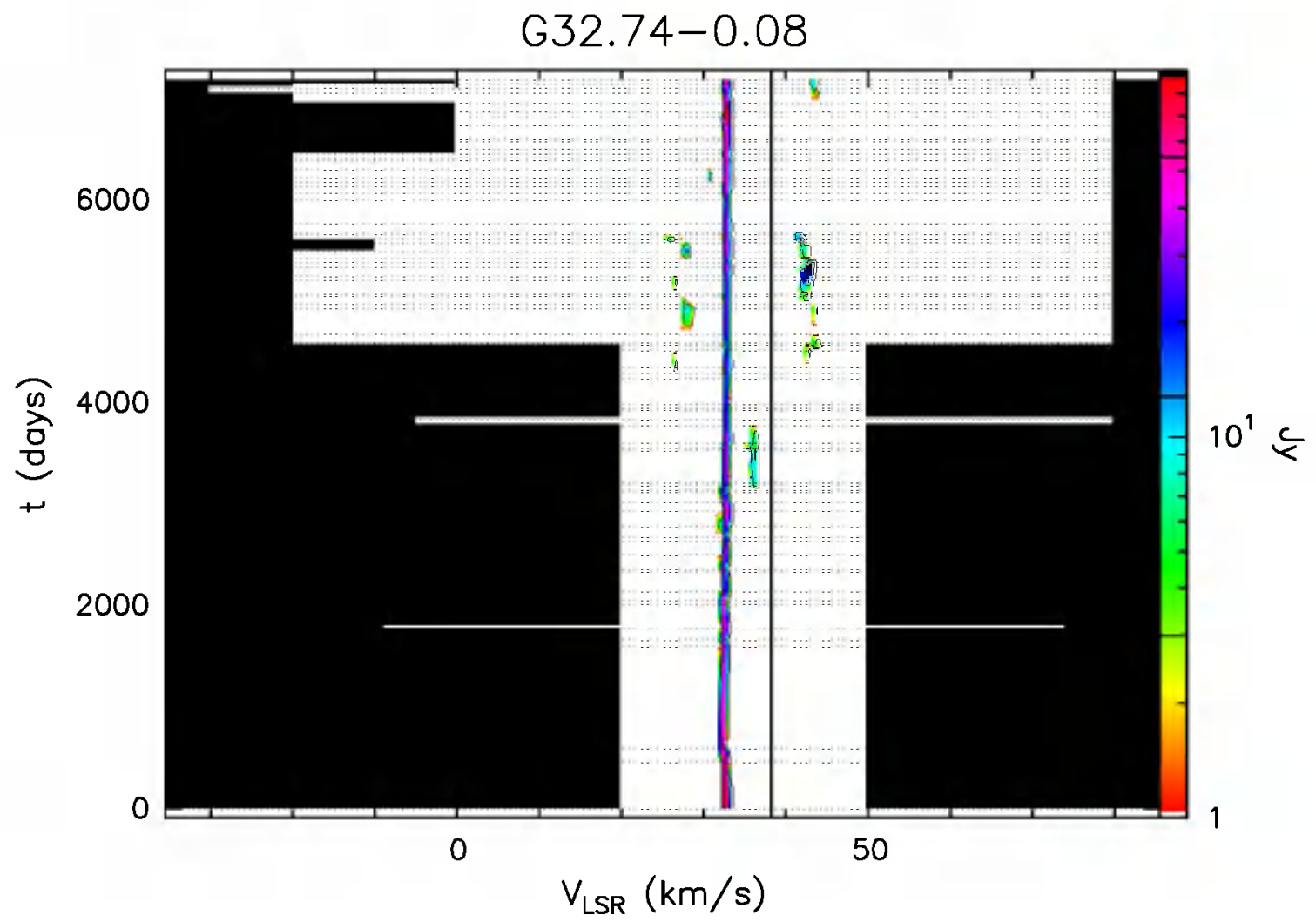

Fig. A.22. b Velocity-time-flux density full plot for source G32.74-0.08. The vertical solid line indicates the velocity of the associated thermal molecular gas. The flux density scale is shown by the bar on the right. In this bar the three lines give the flux density of the drawn contours.

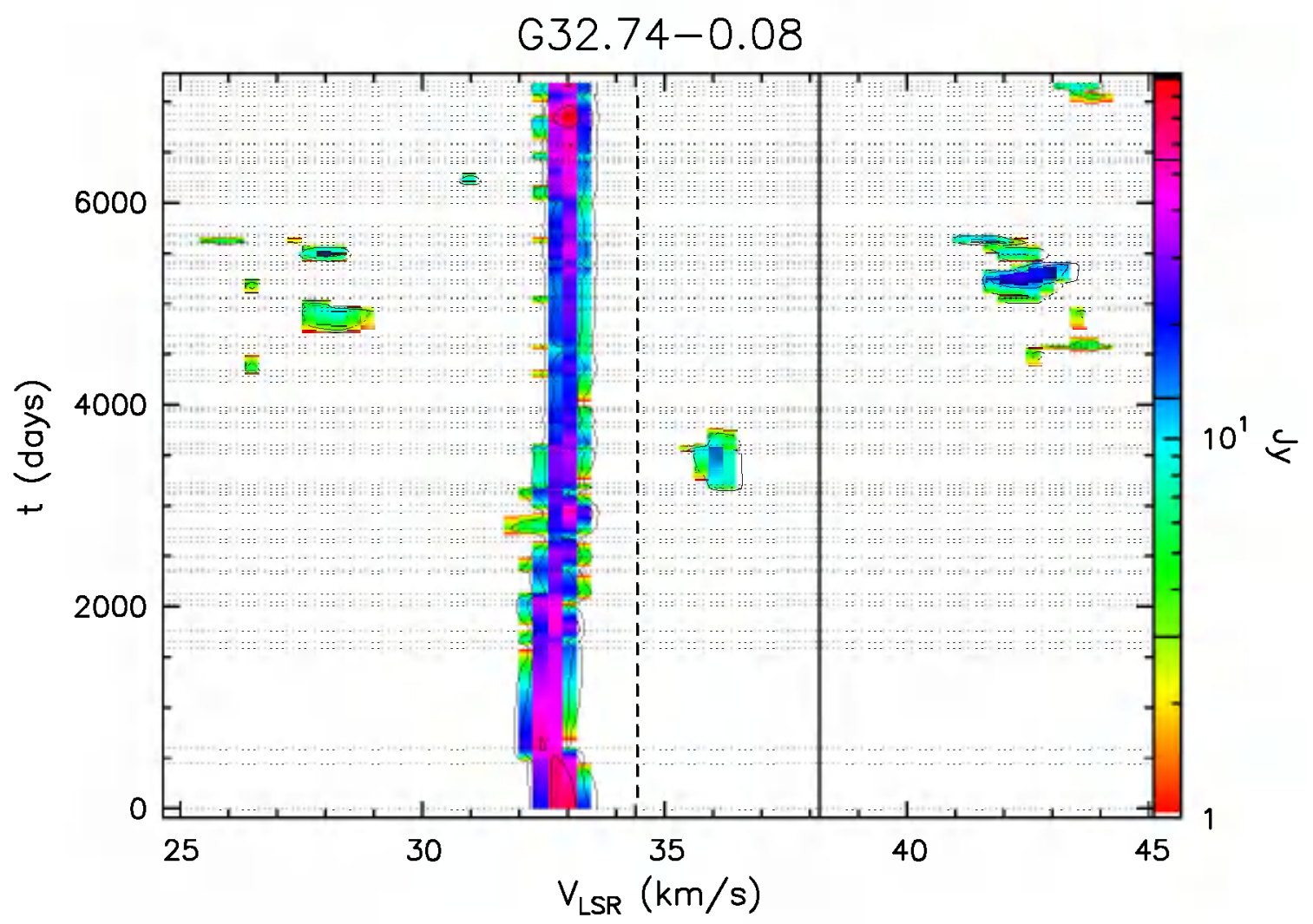

Fig. A.22. c Same as previous figure, but "zoomed" to velocity range over which emission has been detected. 


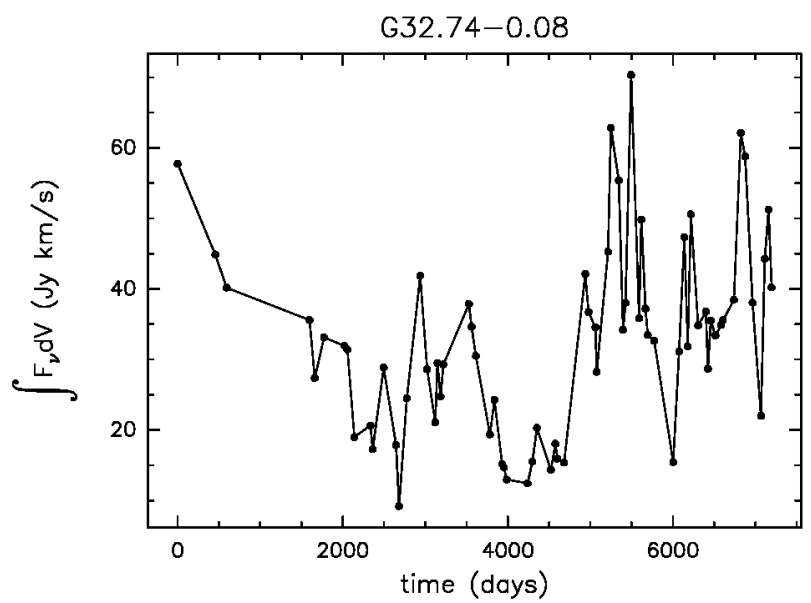

Fig. A.22. d Integral of the flux density over the observed velocity range as a function of time for source G32.74-0.08.

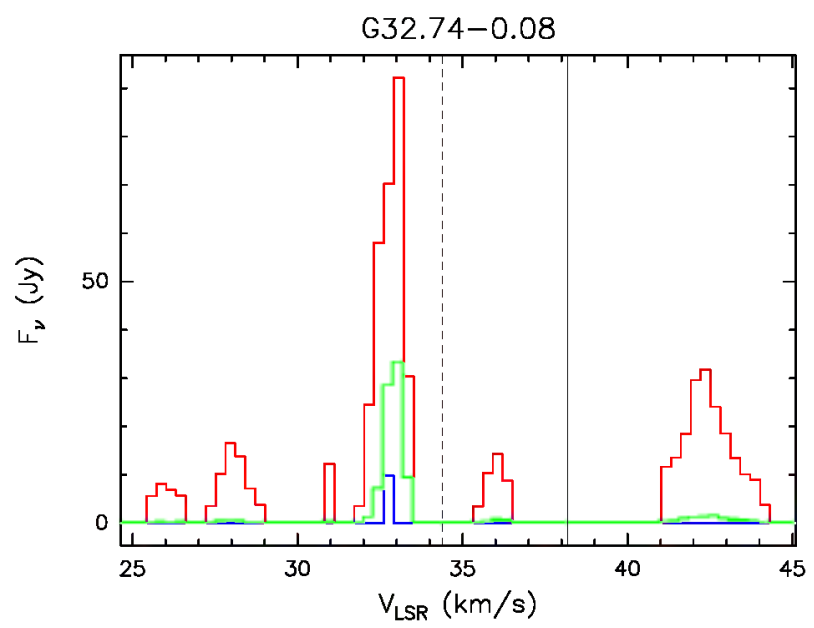

Fig. A.22. e Upper (red) and lower (blue) envelopes and mean spectrum (green) of source G32.74-0.08 measured during onr monitoring. The vertical solid line marks the velocity of the associated thermal molecular gas. The vertical dashed line marks the mean velocity derived from the histogram of the rate-of-occurrence.

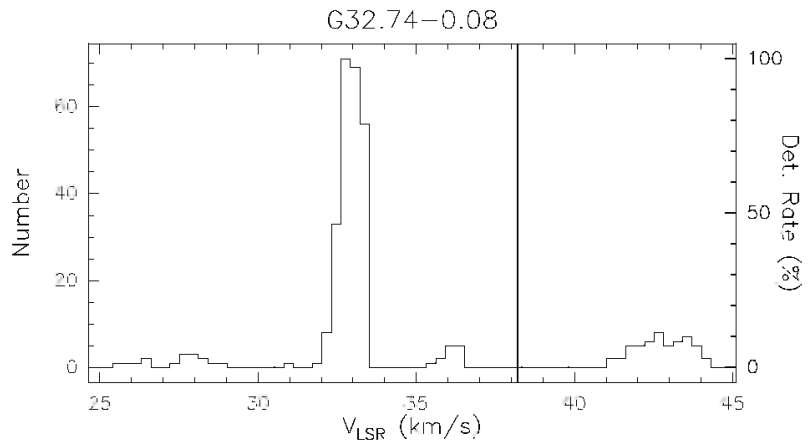

Fig. A.22. f Rate-of-occurrence plot for source G32.74-0.08. The scale to the right refer's to the dotted histogram, the scale to the left to the solid line histogram. The vertical solid line marks the velocity of the associated thermal molecular gas. 


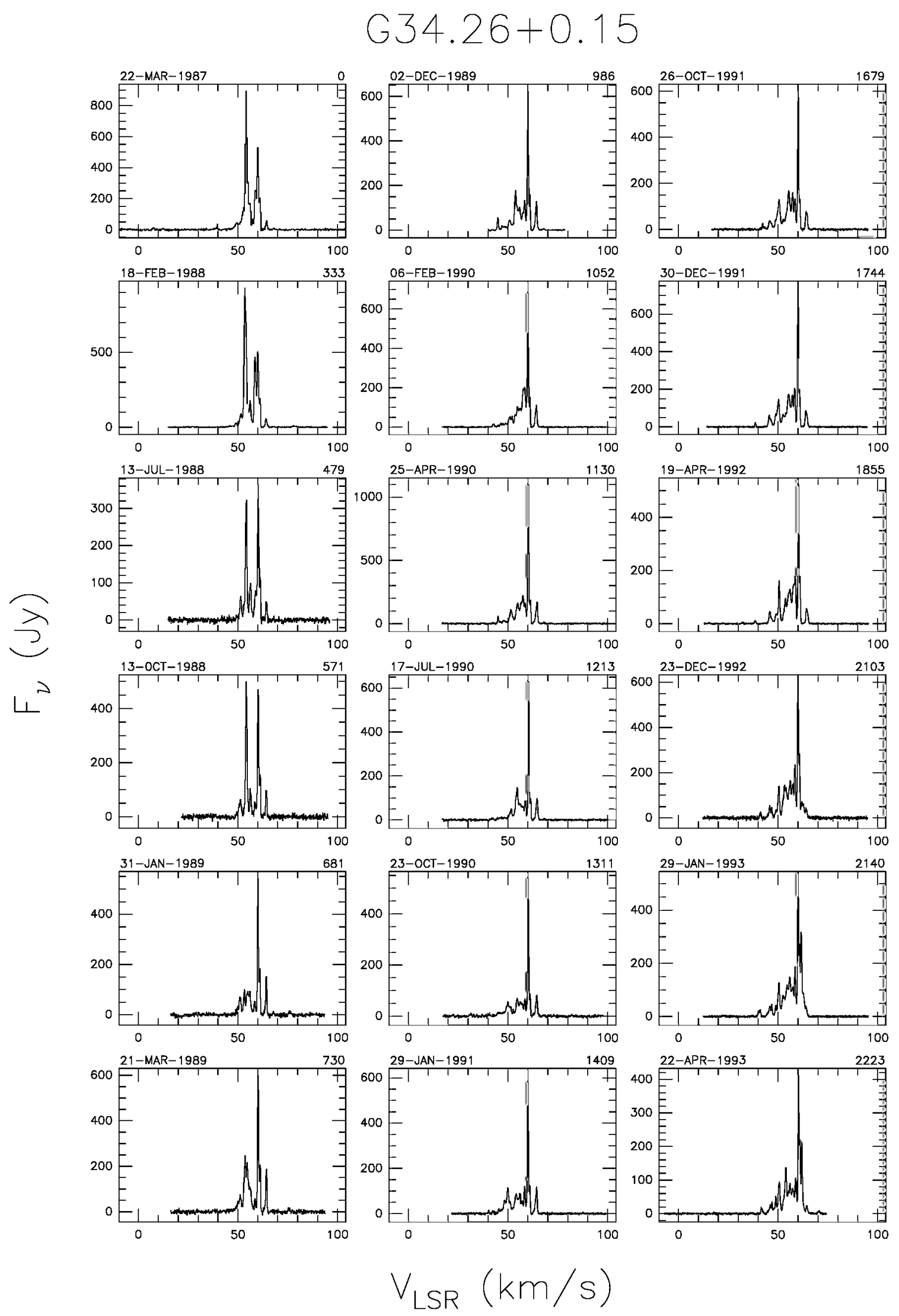

Fig. A.23. a Spectra of source G34.26+0.15 with autoscaled flux density scale. The date of observation is shown above the top left corner of each spectrum and the number of days elapsed since the first observation is given above the top right corner. The velocity scale is the same for all spectra. 


$$
634.26+0.15
$$
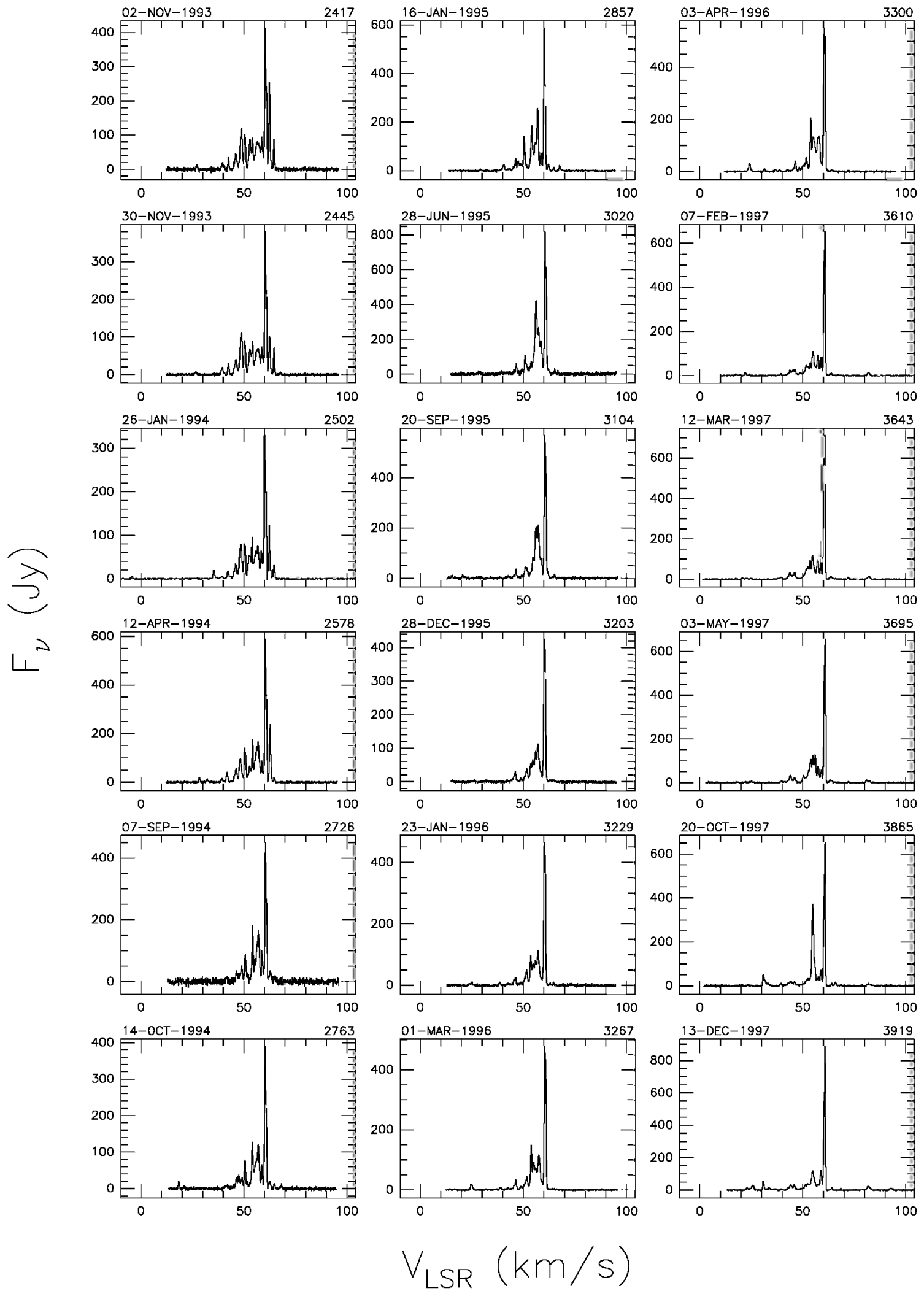

Fig. A.23. a continued. 


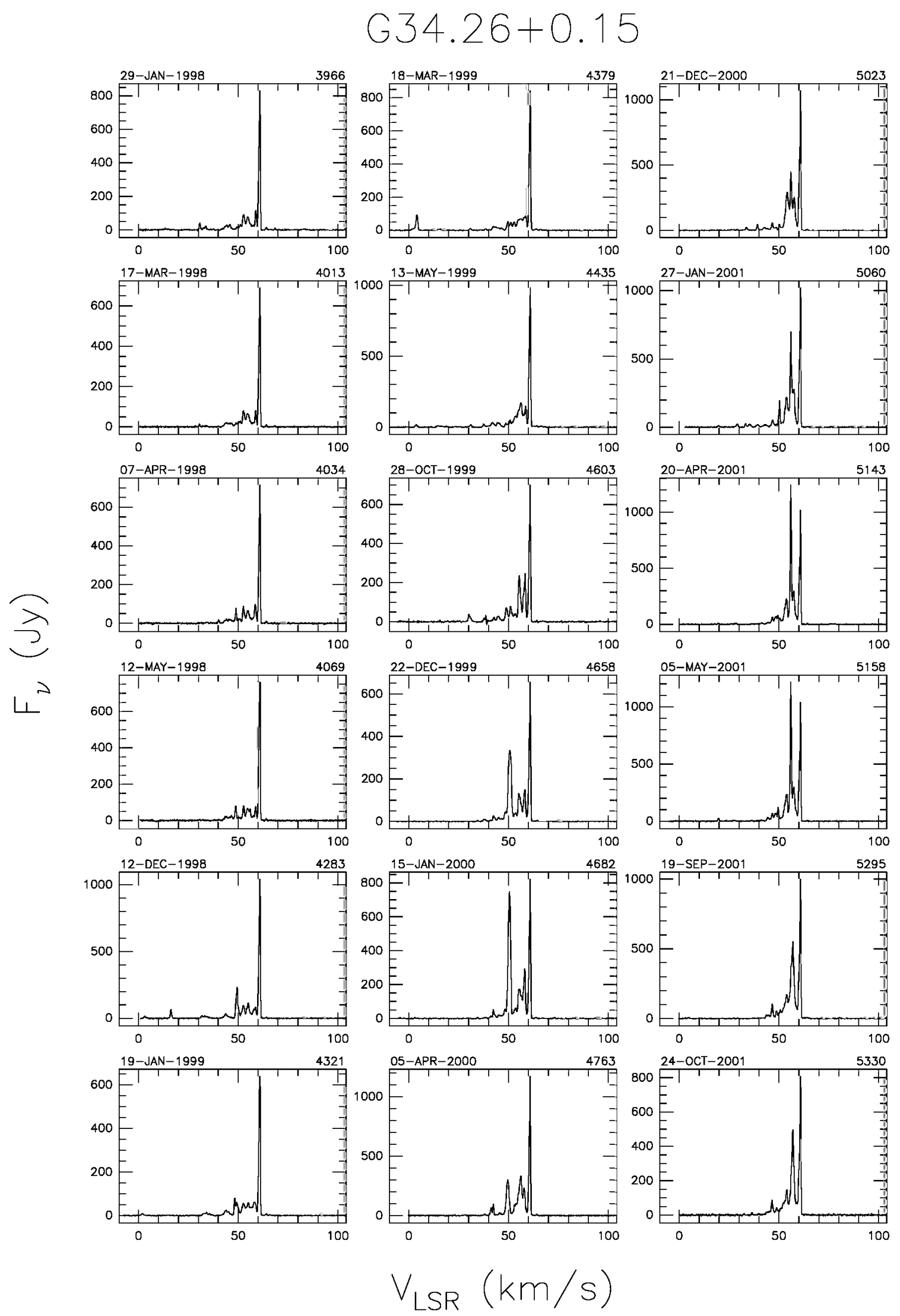

Fig. A.23. a continued. 

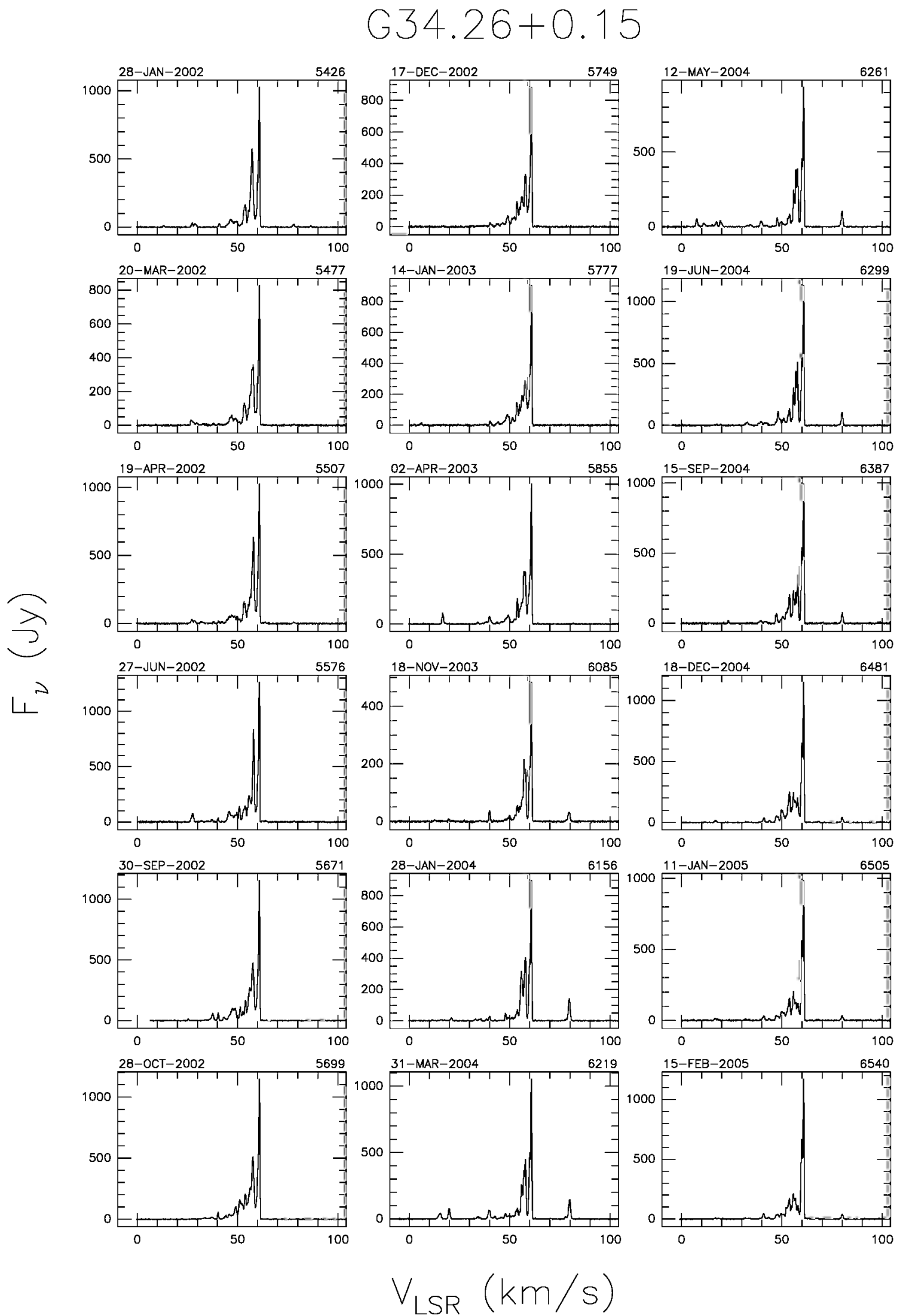

Fig. A.23. a continued. 


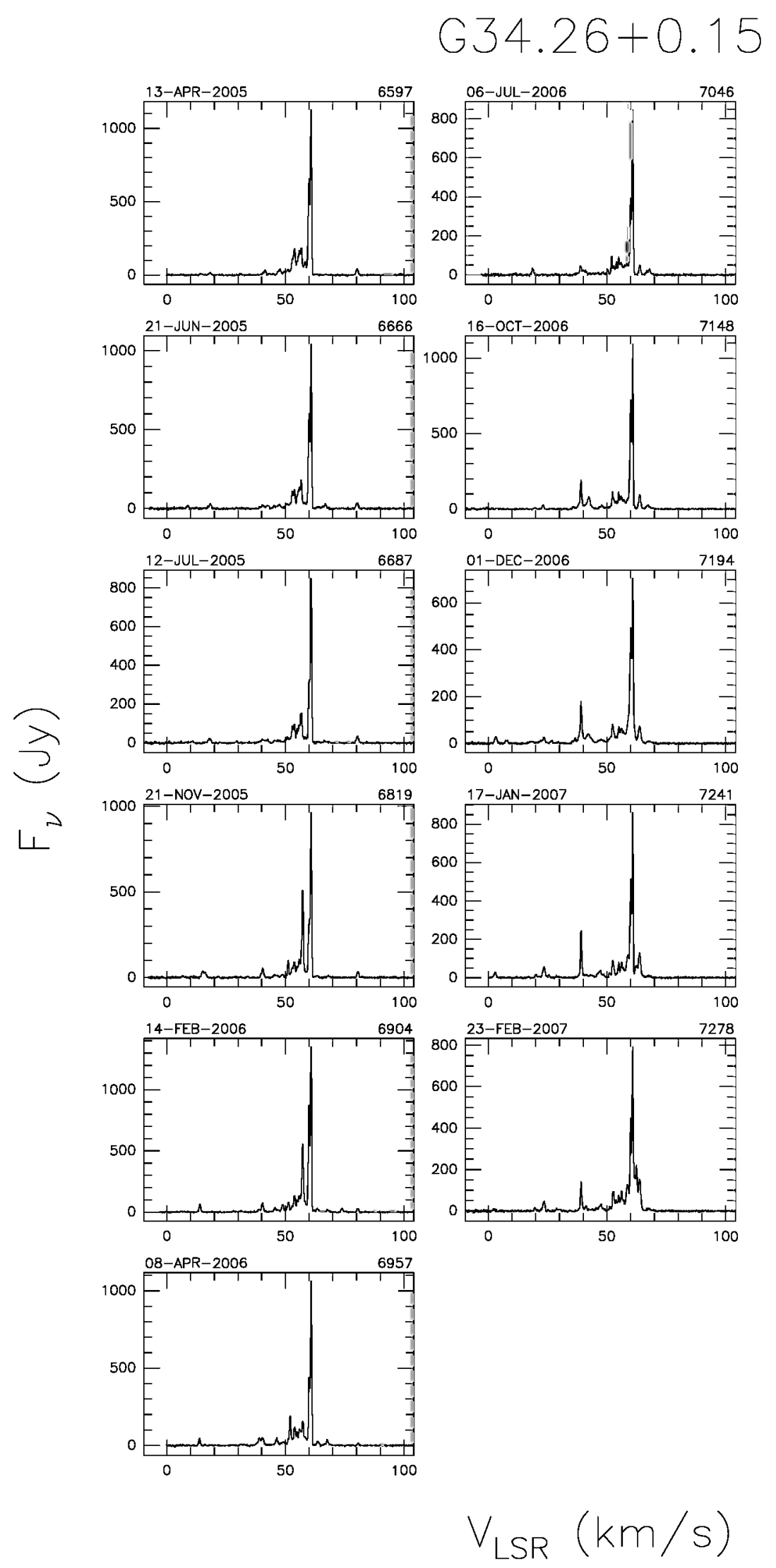

Fig. A.23. a continued. 


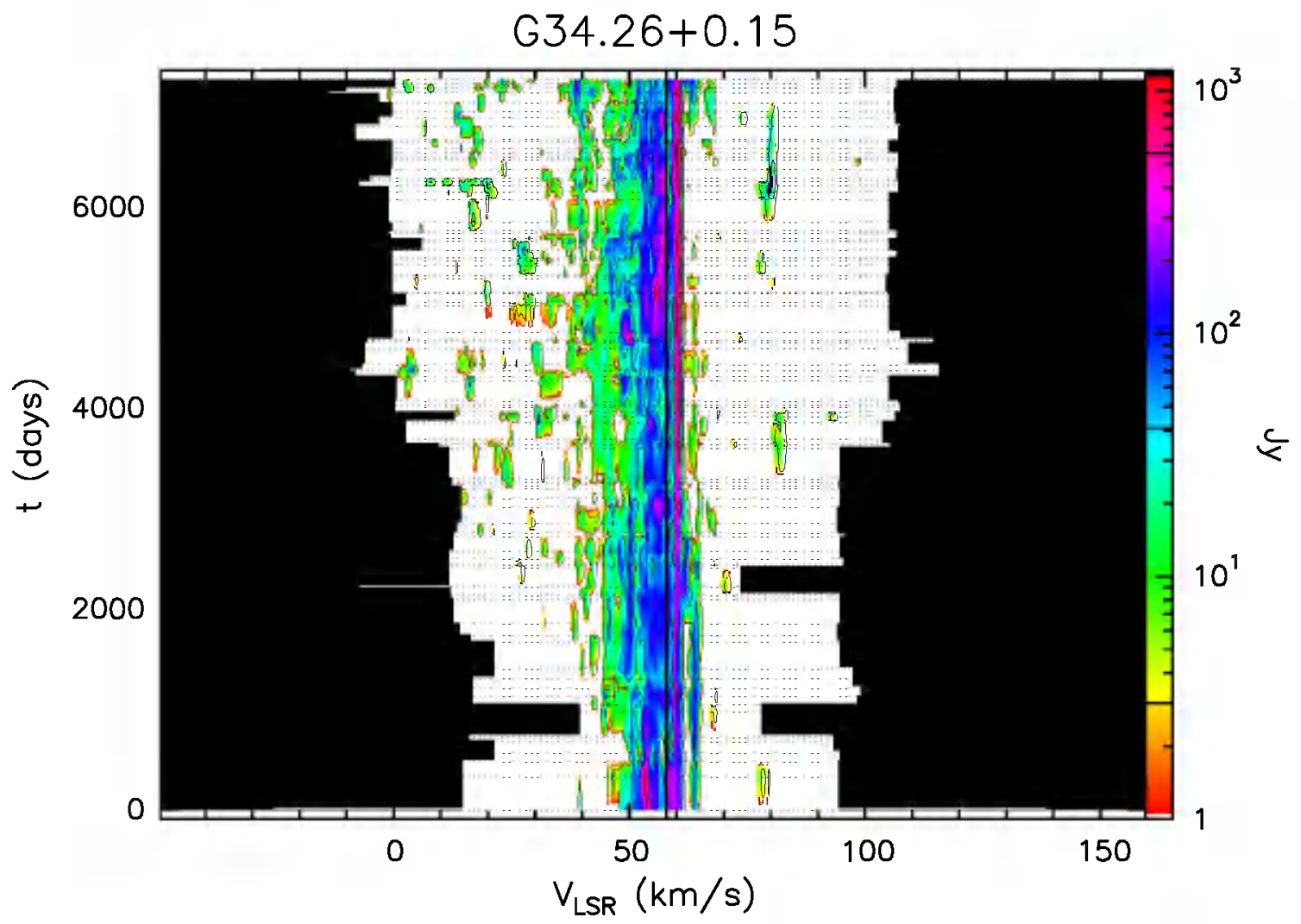

Fig. A.23. b Velocity-time-flux density full plot for source G34.26+0.15. The vertical solid line indicates the velocity of the associated thermal molecular gas. The flux density scale is shown by the bar on the right. In this bar the three lines give the flux density of the diawn contours.

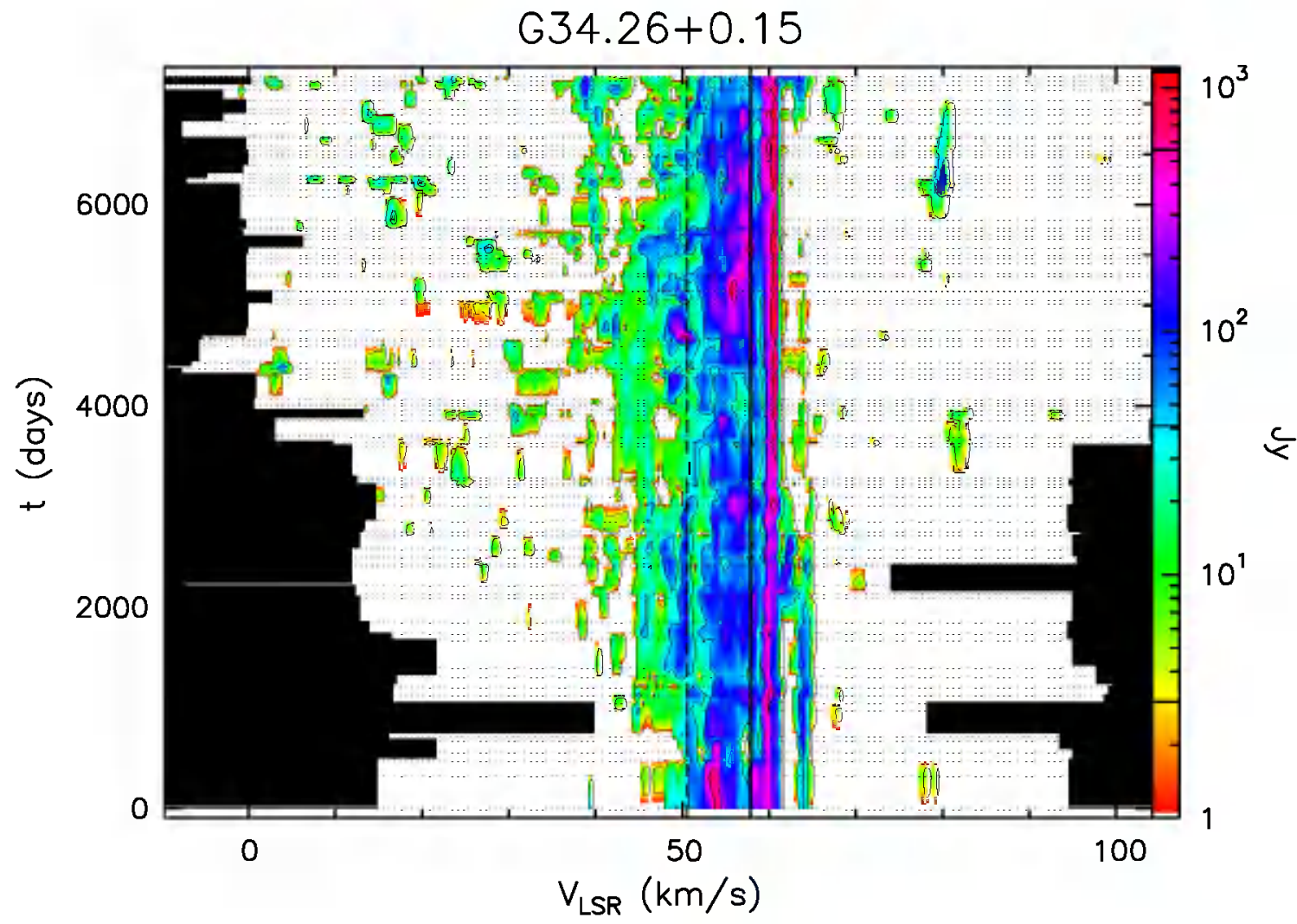

Fig. A.23. c Same as previous figure, but "zoomed" to velocity range over which emission has been detected. 


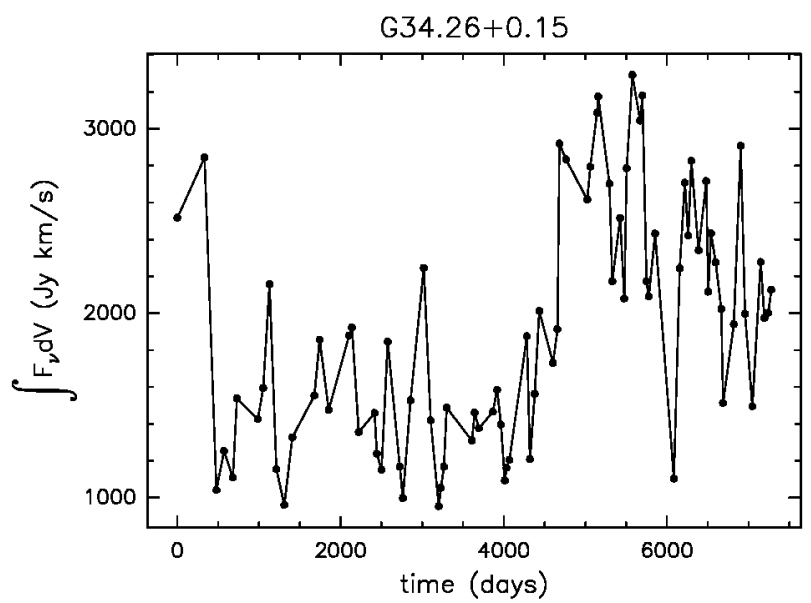

Fig. A.23. d Integral of the flux density over the observed velocity range as a function of time for source G34.26+0.15.

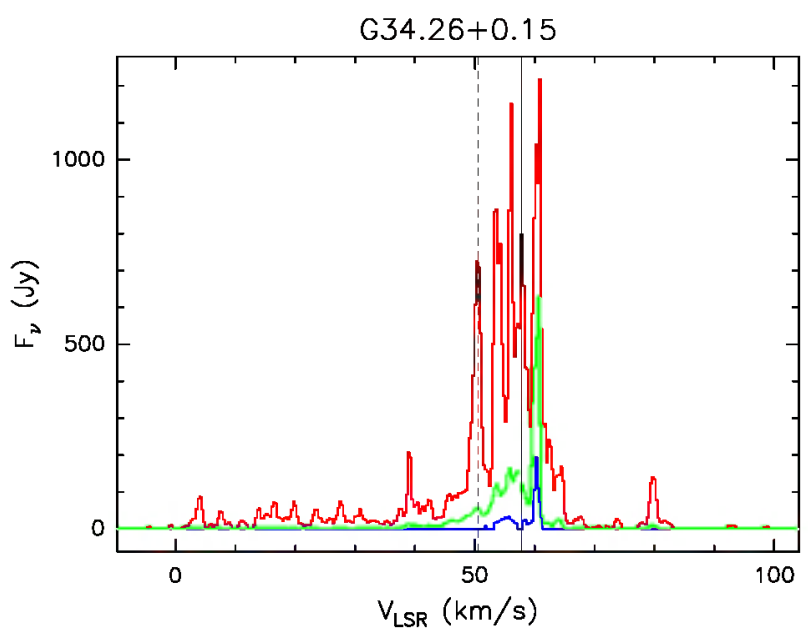

Fig. A.23. e Upper (red) and lower (blue) envelopes and mean spectrum (green) of source G34.26+0.15 measured during our monitoring. The vertical solid line marks the velocity of the associated thermal molecular gas. The vertical dashed line marks the mean velocity derived from the histogram of the rate-of-occurrence.

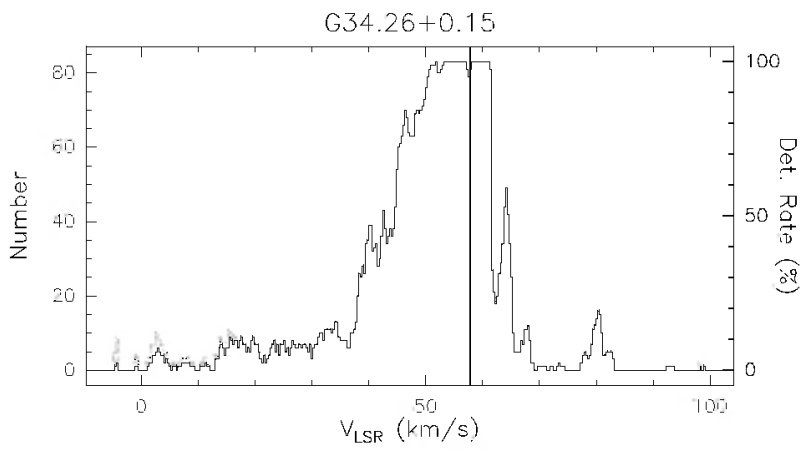

Fig. A.23. f Rate-of-occurrence plot for source G34.26+0.15. The scale to the right refers to the dotted histogram. the scale to the left to the solid line histogram. The vertical solid line marks the velocity of the associated thermal molecular gas. 


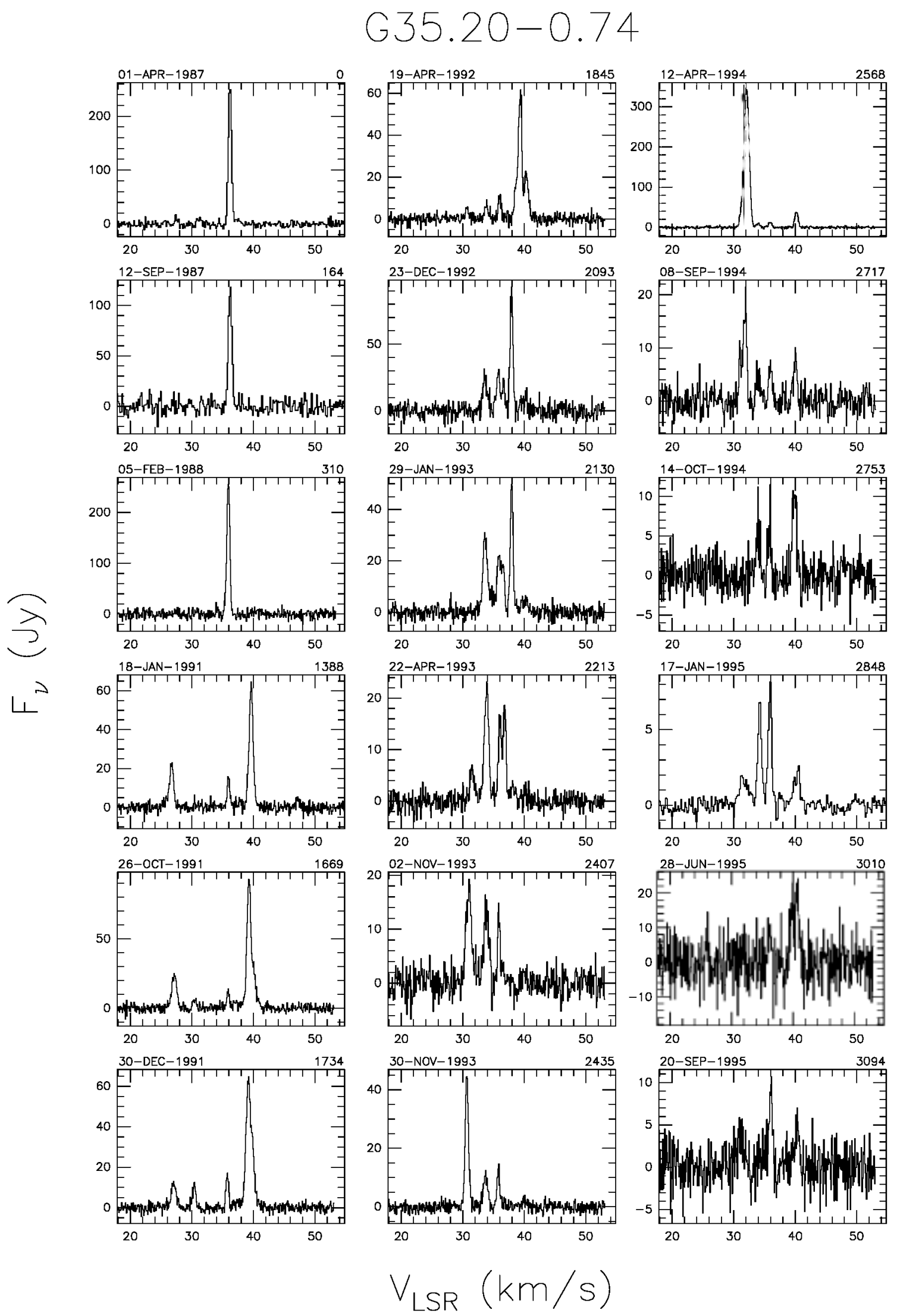

Fig. A.24. a Spectra of source G35.20-0.74 with autoscaled flux density scale. The date of observation is shown above the top left corner of each spectrum and the number of days elapsed since the first observation is given above the top right corner. The velocity scale is the same for all spectra. 
$635.20-0.74$
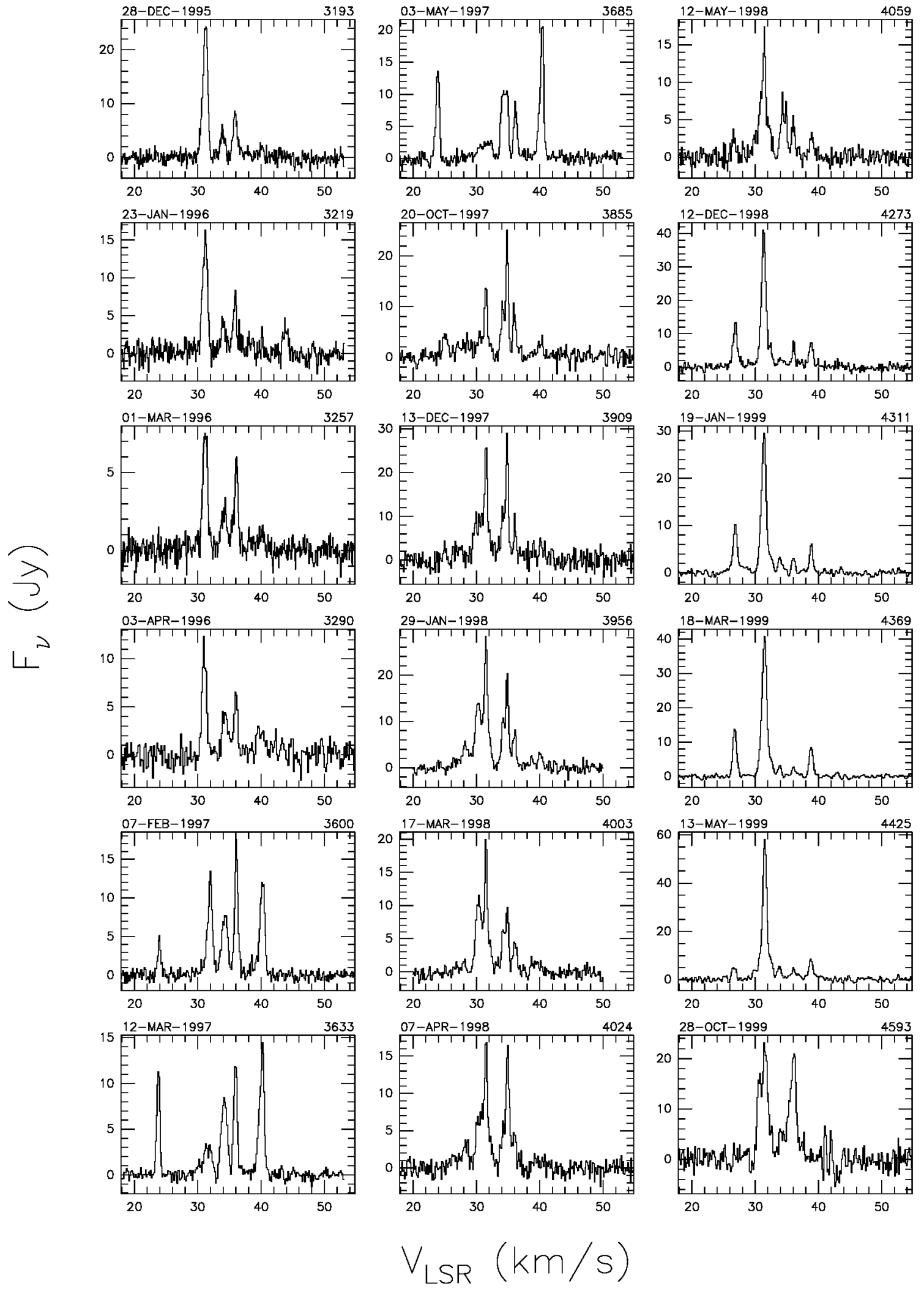

Fig. A.24. a continued. 


$$
635.20-0.74
$$
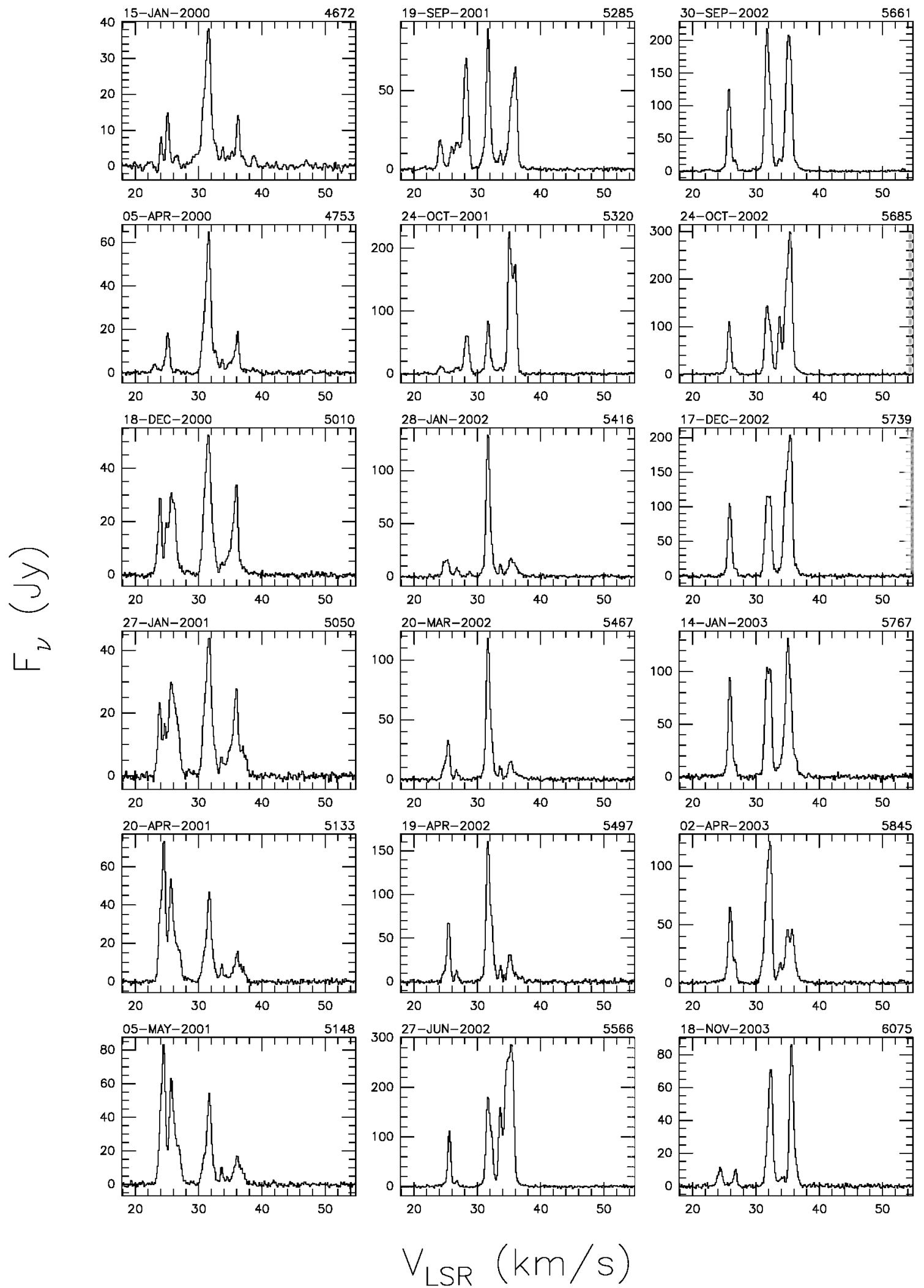

Fig. A.24. a continued. 


$$
\text { G35.20-0.74 }
$$
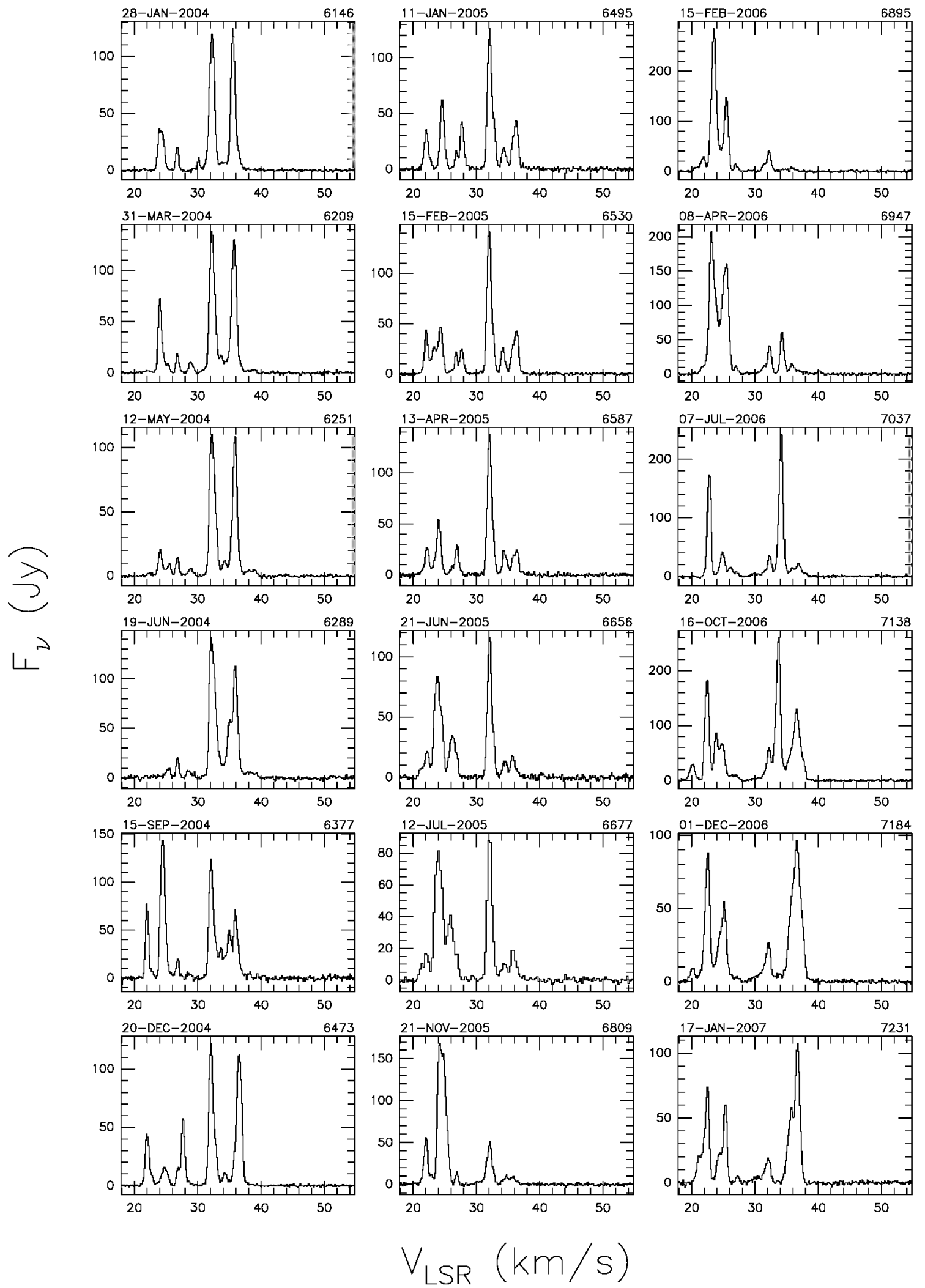

Fig. A.24. a continued. 


$$
\text { G35.20-0.74 }
$$

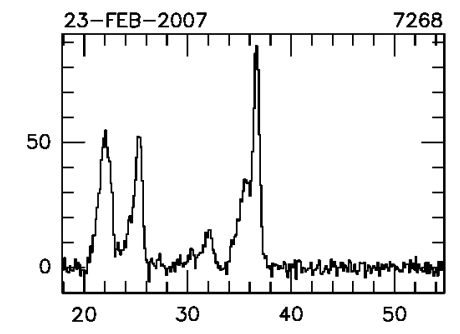

3

$L^{\lambda}$

$$
V_{\text {LSR }}(\mathrm{km} / \mathrm{s})
$$

Fig. A.24. a continued. 


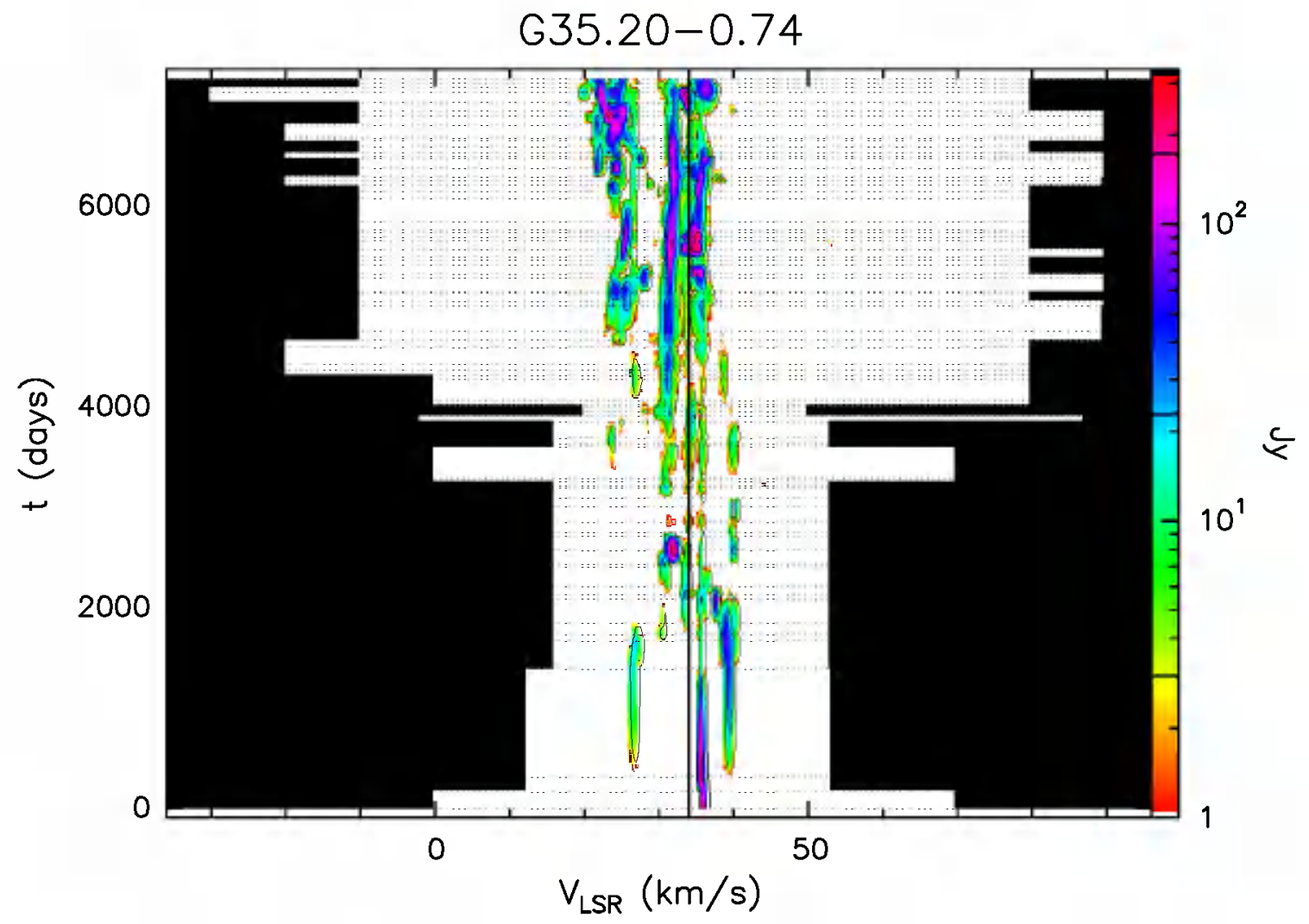

Fig. A.24. b Velocity-time-flux density full plot for source G35.20-0.74. The vertical solid line indicates the velocity of the associated thermal molecular gas. The flux density scale is shown by the bar on the right. In this bar the three lines give the flux density of the drawn contours.

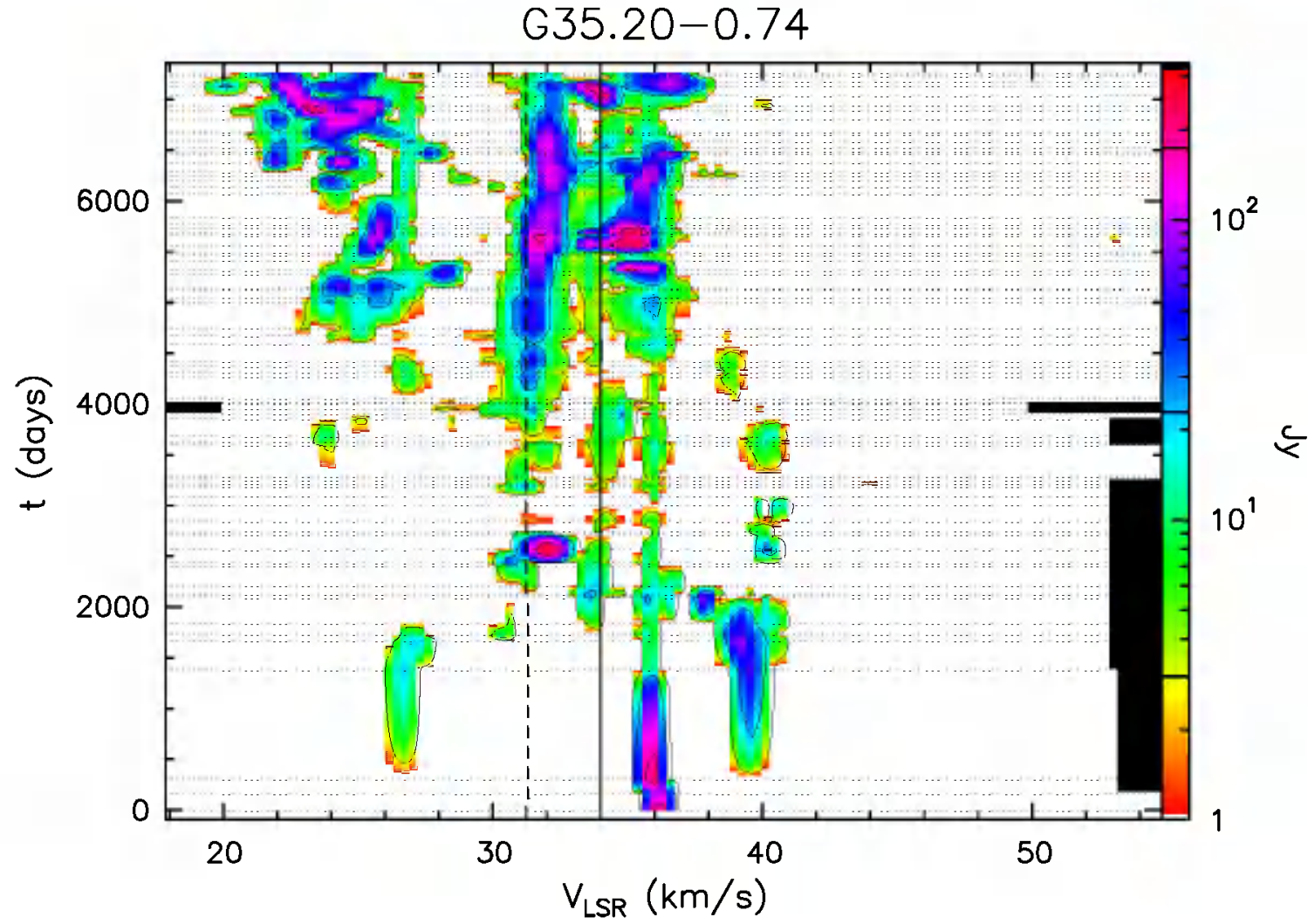

Fig. A.24. c Same as previous figure. but "zoomed" to velocity range over which emission has been detected. 


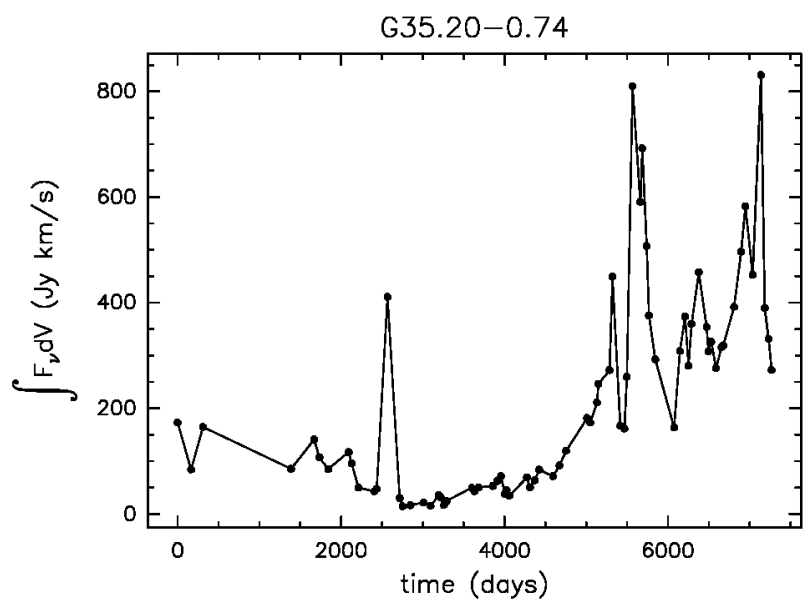

Fig. A.24. d Integral of the flux density over the observed velocity range as a function of time for source G35.20-0.74.

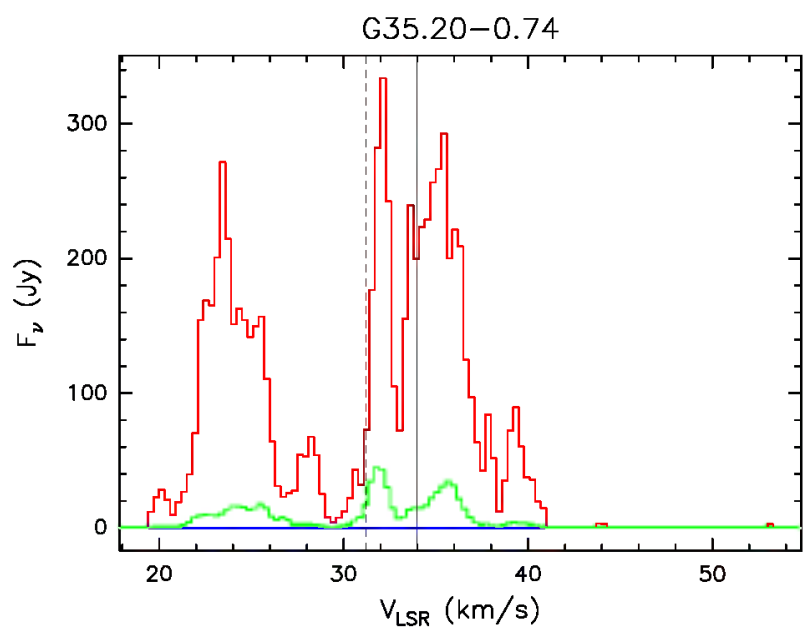

Fig. A.24. e Upper (red) and lower (blue) envelopes and mean spectrum (green) of source G35.20-0.74 measured during our monitoring. The vertical solid line marks the velocity of the associated thermal molectllar gas. The vertical dashed line marks the mean velocity derived from the histogram of the rate-of-occurrence.

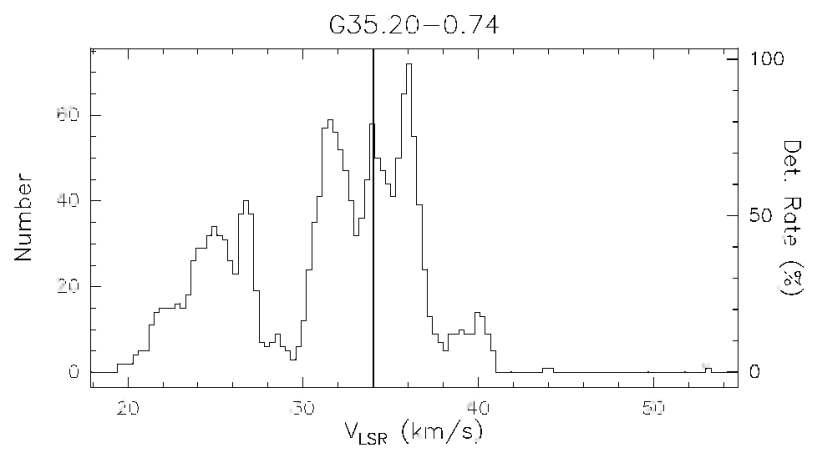

Fig. A.24. f Rate-of-occurrence plot for source G35.20-0.74. The scale to the right refers to the dotted histogram, the scale to the left to the solid line histogram. The vertical solid line marks the velocity of the associated thermal molecular gas. 


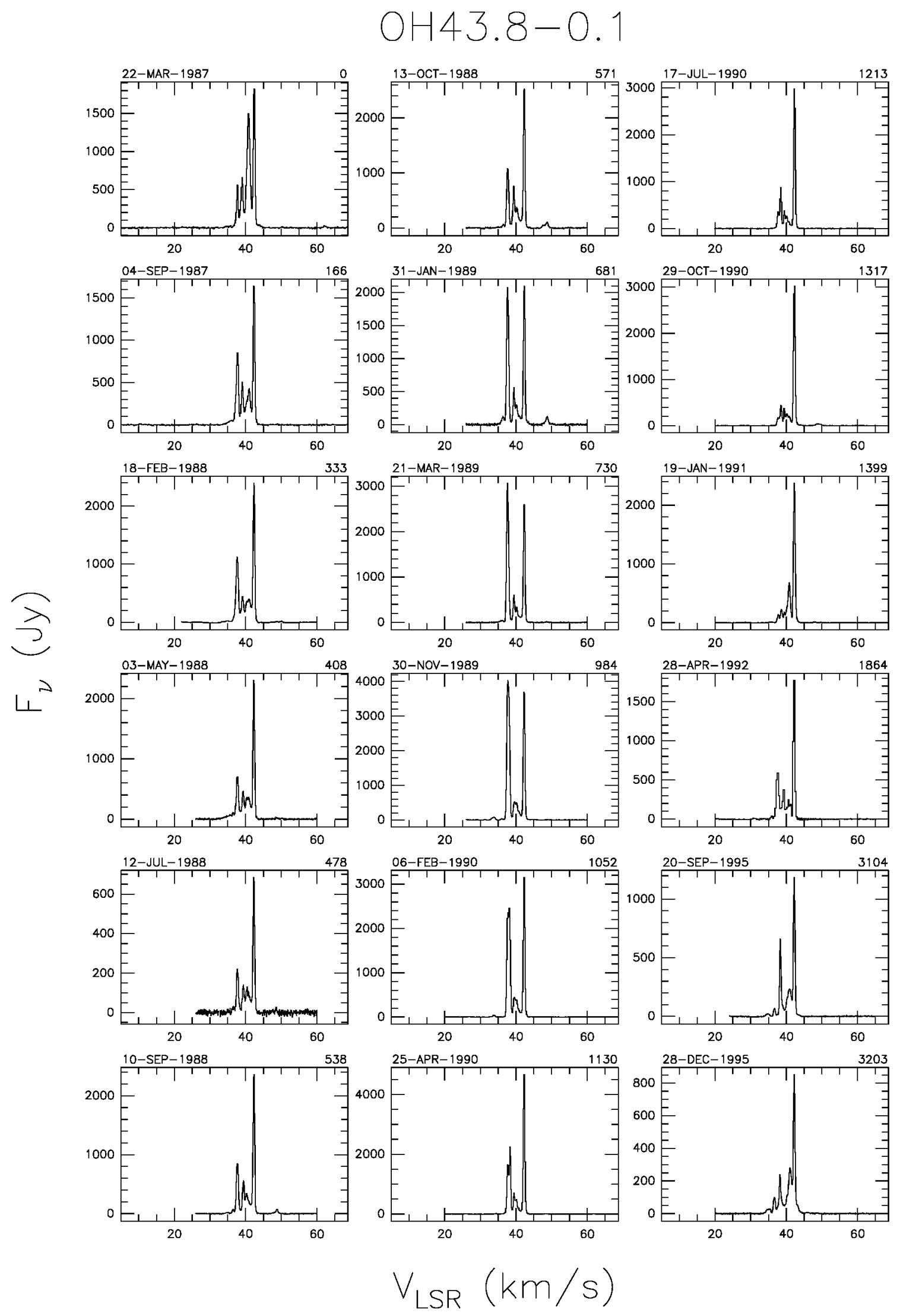

Fig. A.25. a Spectra of source OH43.8-0.1 with autoscaled flux density scale. The date of observation is shown above the top left corner of each spectrum and the number of days elapsed since the first observation is given above the top right corner. The velocity scale is the same for all spectia. 

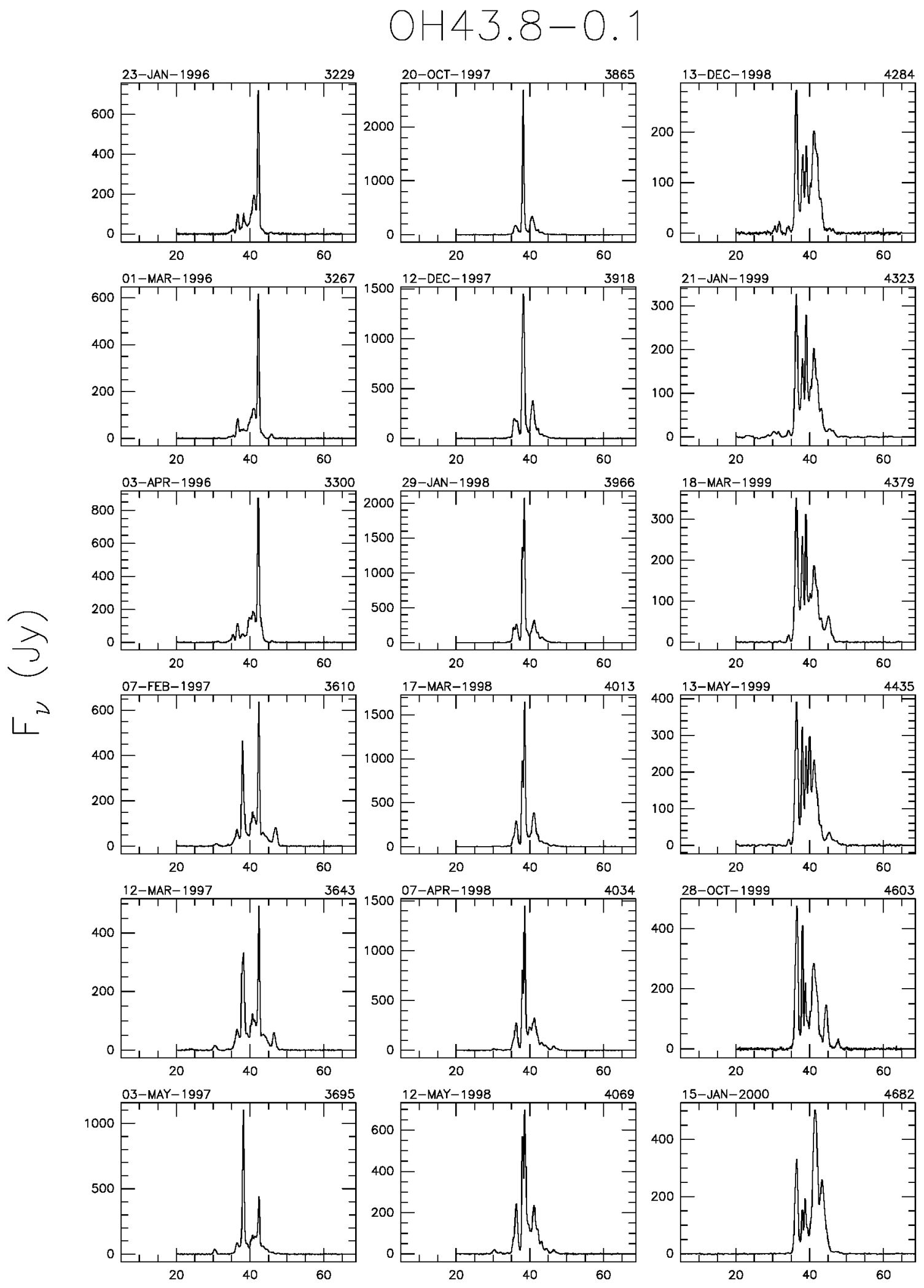

$$
V_{\text {LSR }}(\mathrm{km} / \mathrm{s})
$$

Fig. A.25. a continued. 


\section{$\mathrm{OH} 43.8-0.1$}
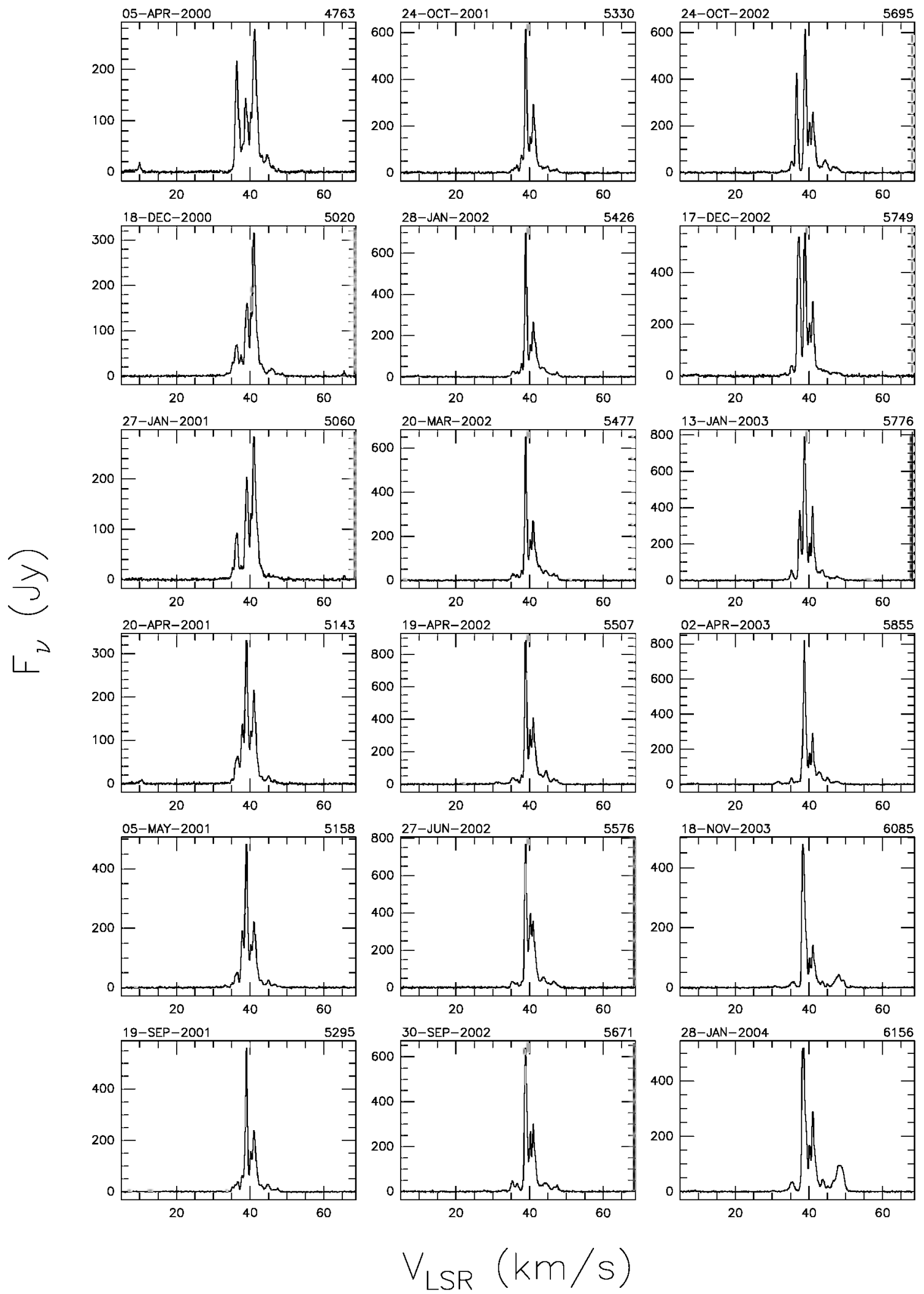

Fig. A.25. a continued. 


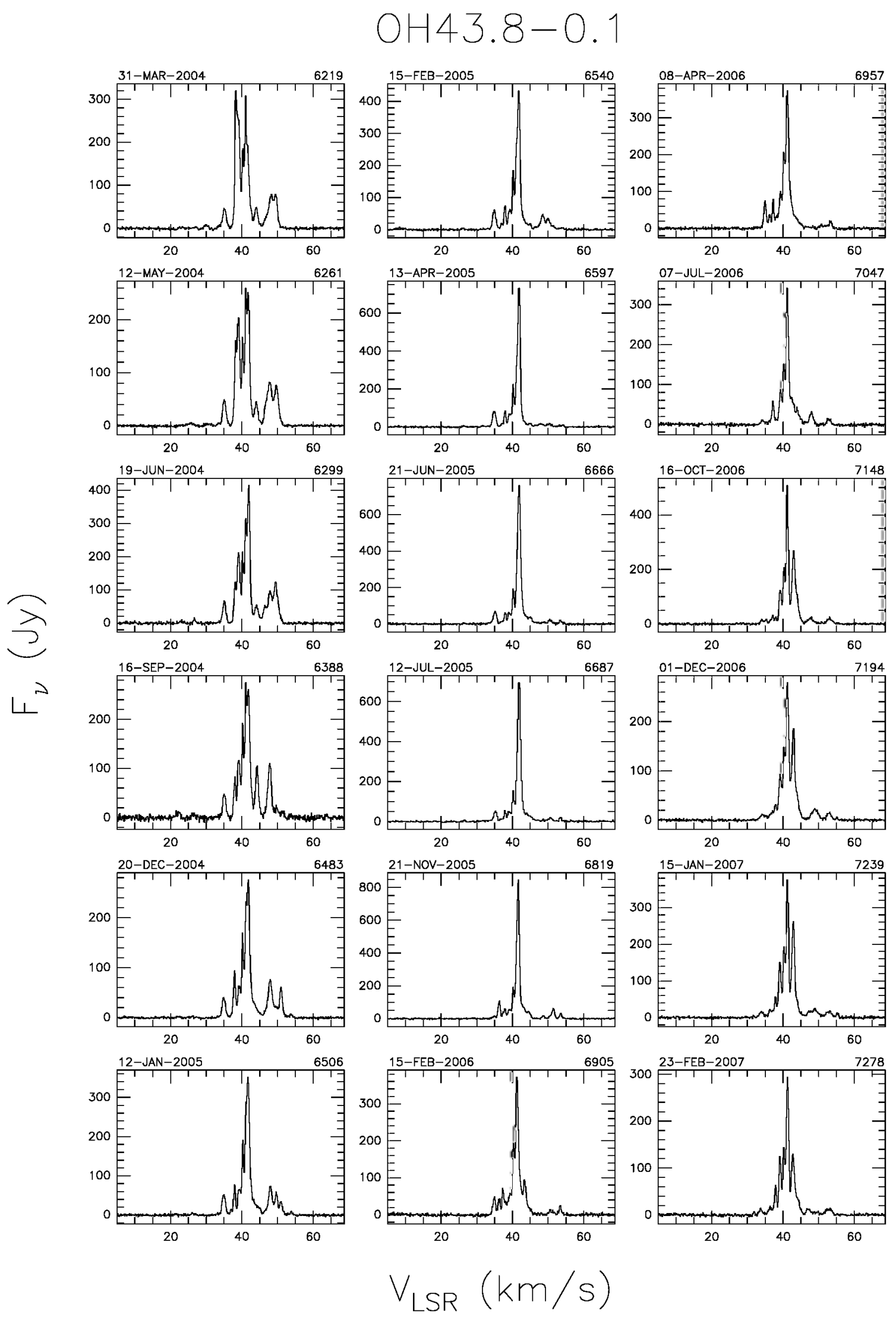

Fig. A.25. a continued. 


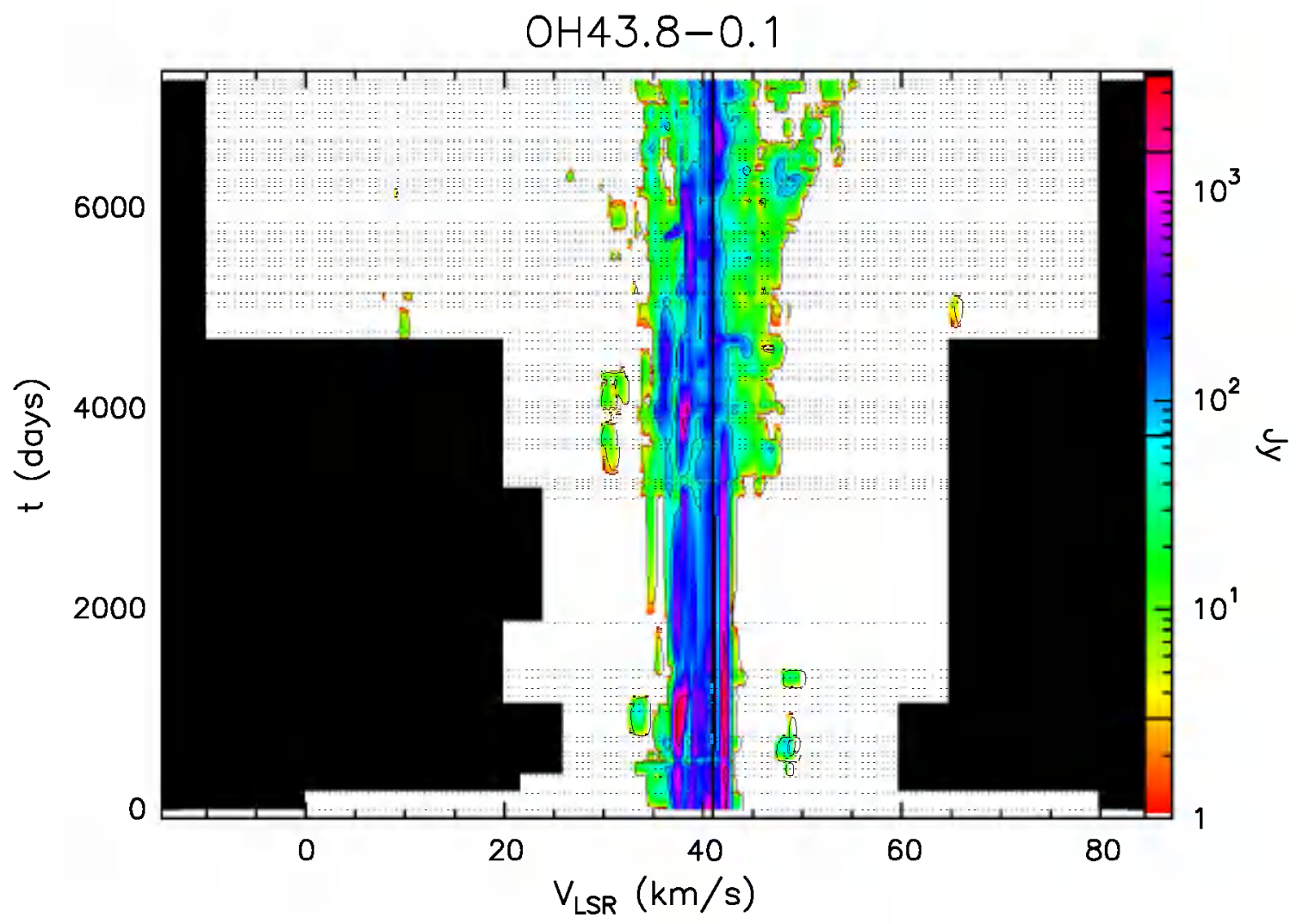

Fig. A.25. b Velocity-time-flux density full plot for source OH43.8-0.1. The vertical solid line indicates the velocity of the associated thermal molecular gas. The flux density scale is shown by the bar on the right. In this bar the three lines give the flux density of the drawn contours.

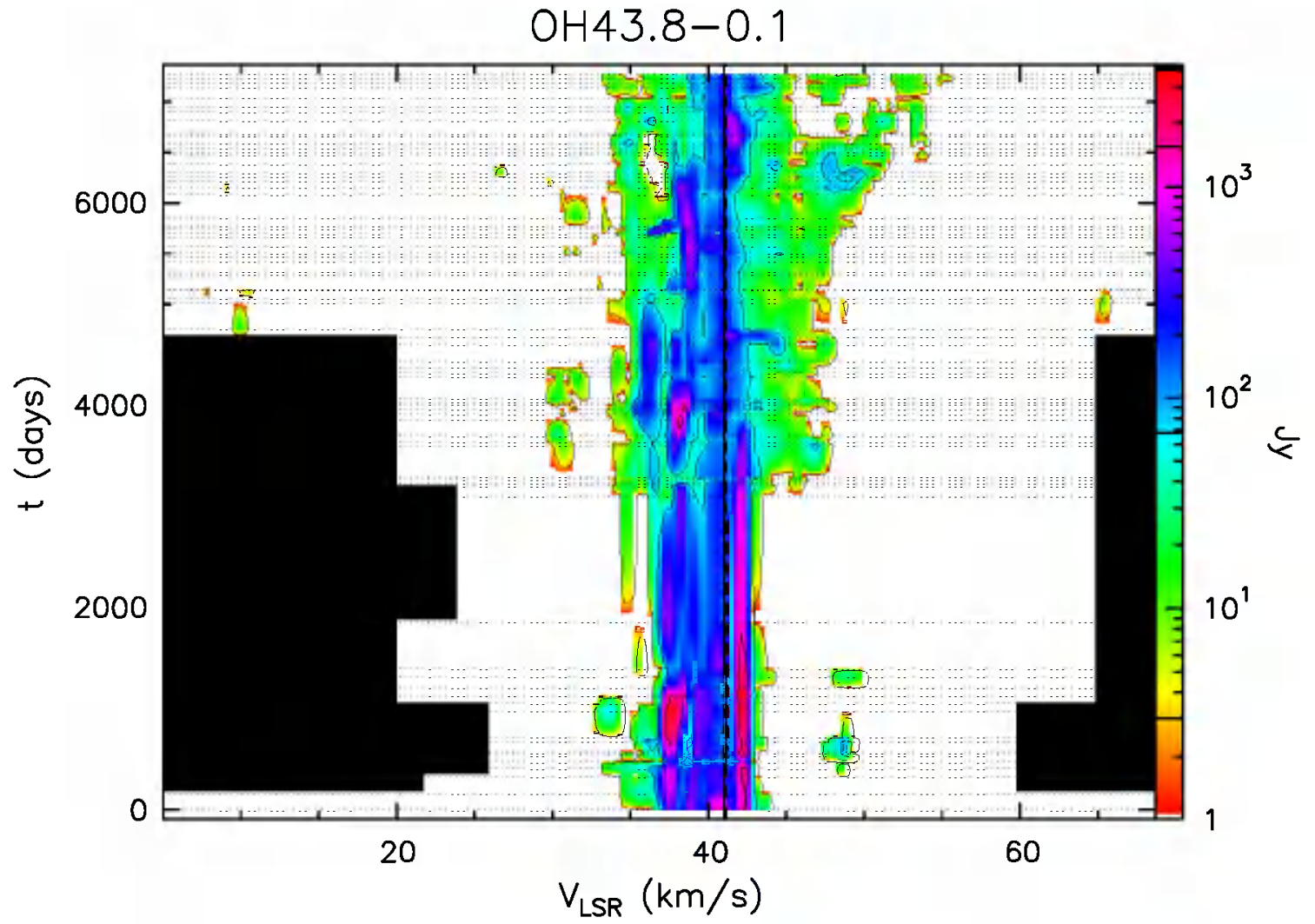

Fig. A.25. c Same as previous figure, but "zoomed" to velocity range over which emission has been detected. 


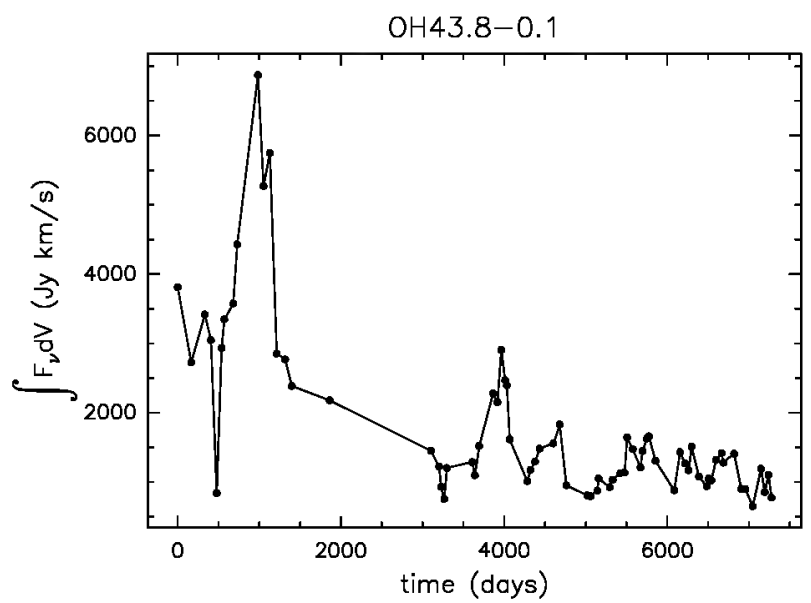

Fig. A.25. d Integral of the flux density over the observed velocity range as a function of time for source OH43.8-0.1.

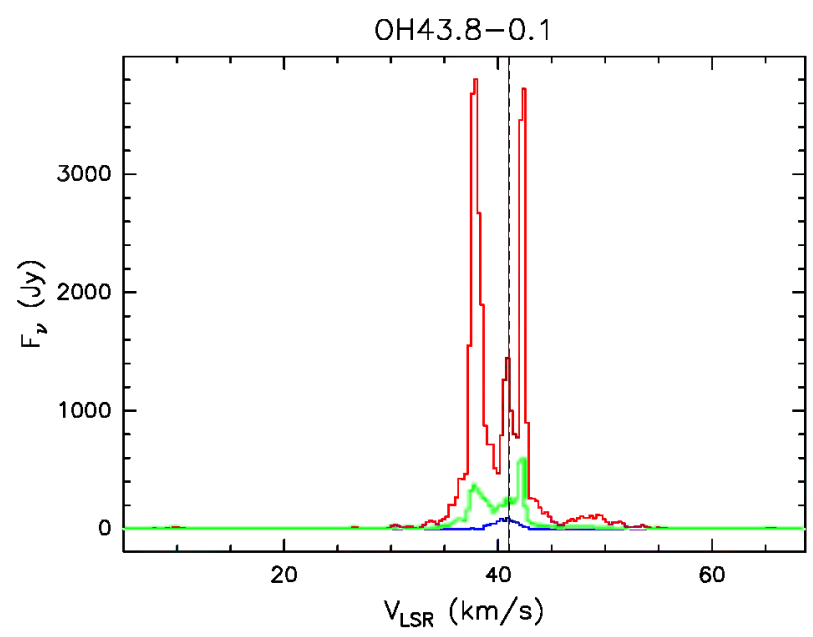

Fig. A.25. e Upper (red) and lower (blue) envelopes and mean spectrum (green) of source OH43.8-0.1 measured during our monitoring. The vertical solid line marks the velocity of the associated thermal molecular gas. The vertical dashed line marks the mean velocity derived from the histogram of the rate-of-occurrence.

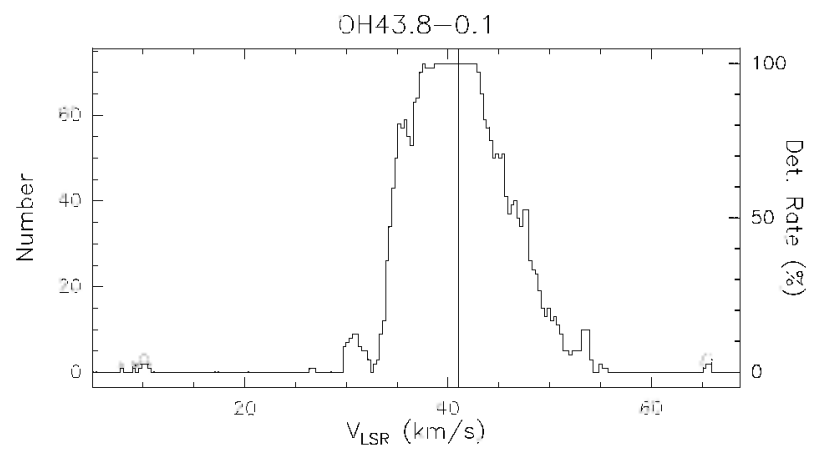

Fig. A.25. f Rate-of-occurrence plot for source OH43.8-0.1. The scale to the right refers to the dotted histogram, the scale to the left to the solid line histogram. The vertical solid line marks the velocity of the associated thermal molecular gas. 
$645.07+0.13$
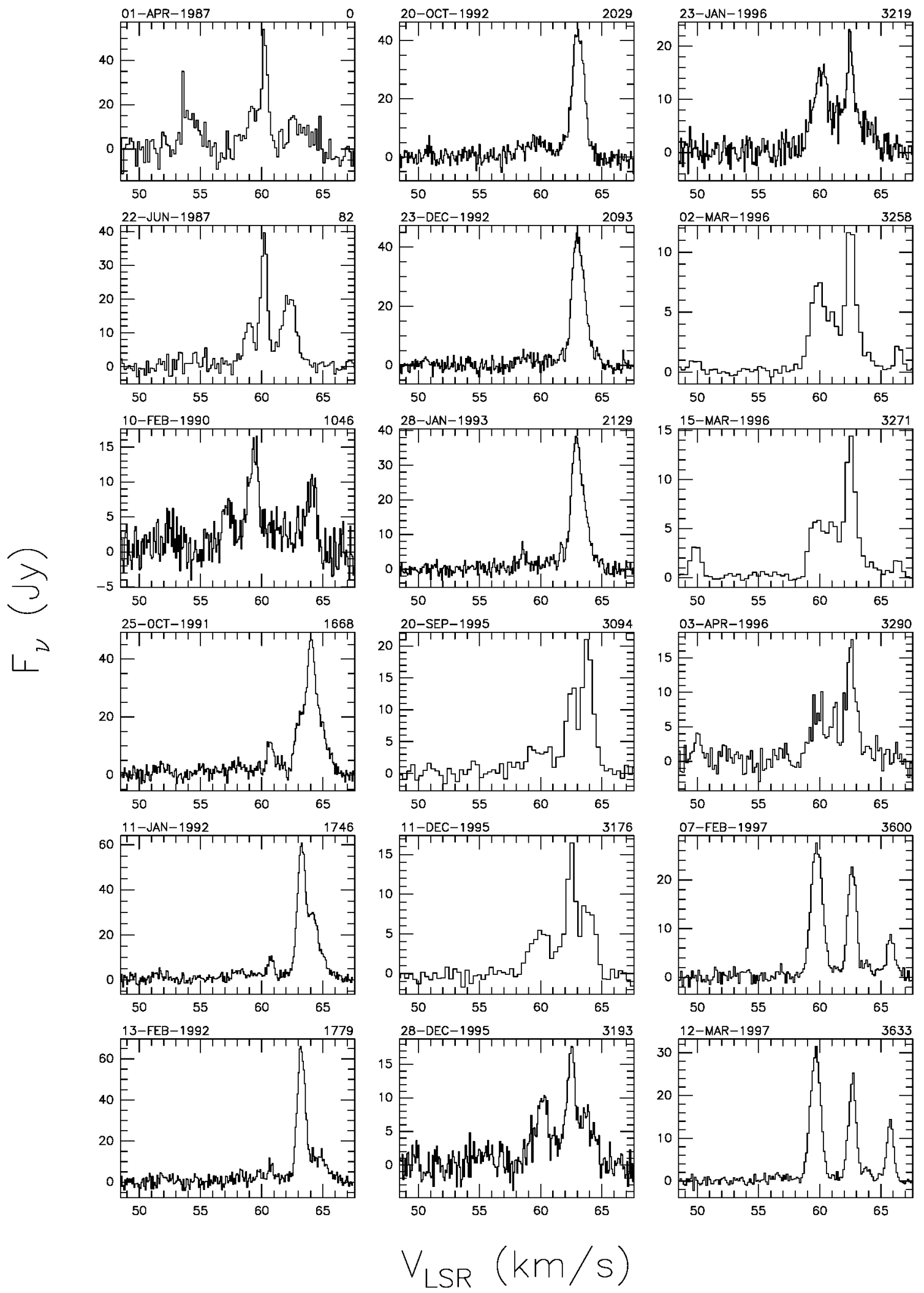

Fig. A.26. a Spectra of source G45.07+0.13 with autoscaled flux density scale. The date of observation is shown above the top left corner of each spectrum and the number of days elapsed since the first observation is given above the top right corner. The velocity scale is the same for all spectra. 


$$
645.07+0.13
$$
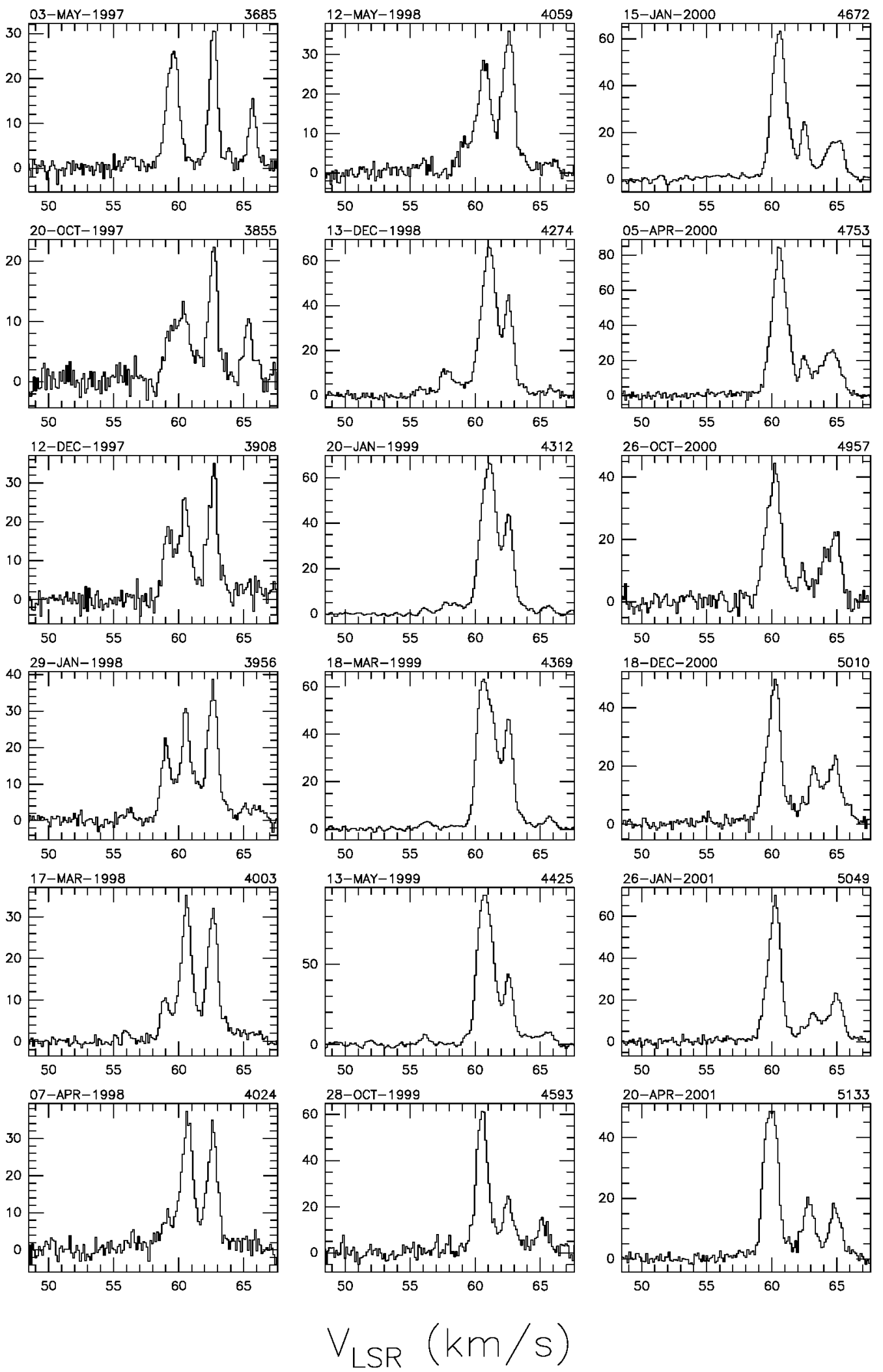

Fig. A.26. a continued. 
$645.07+0.13$
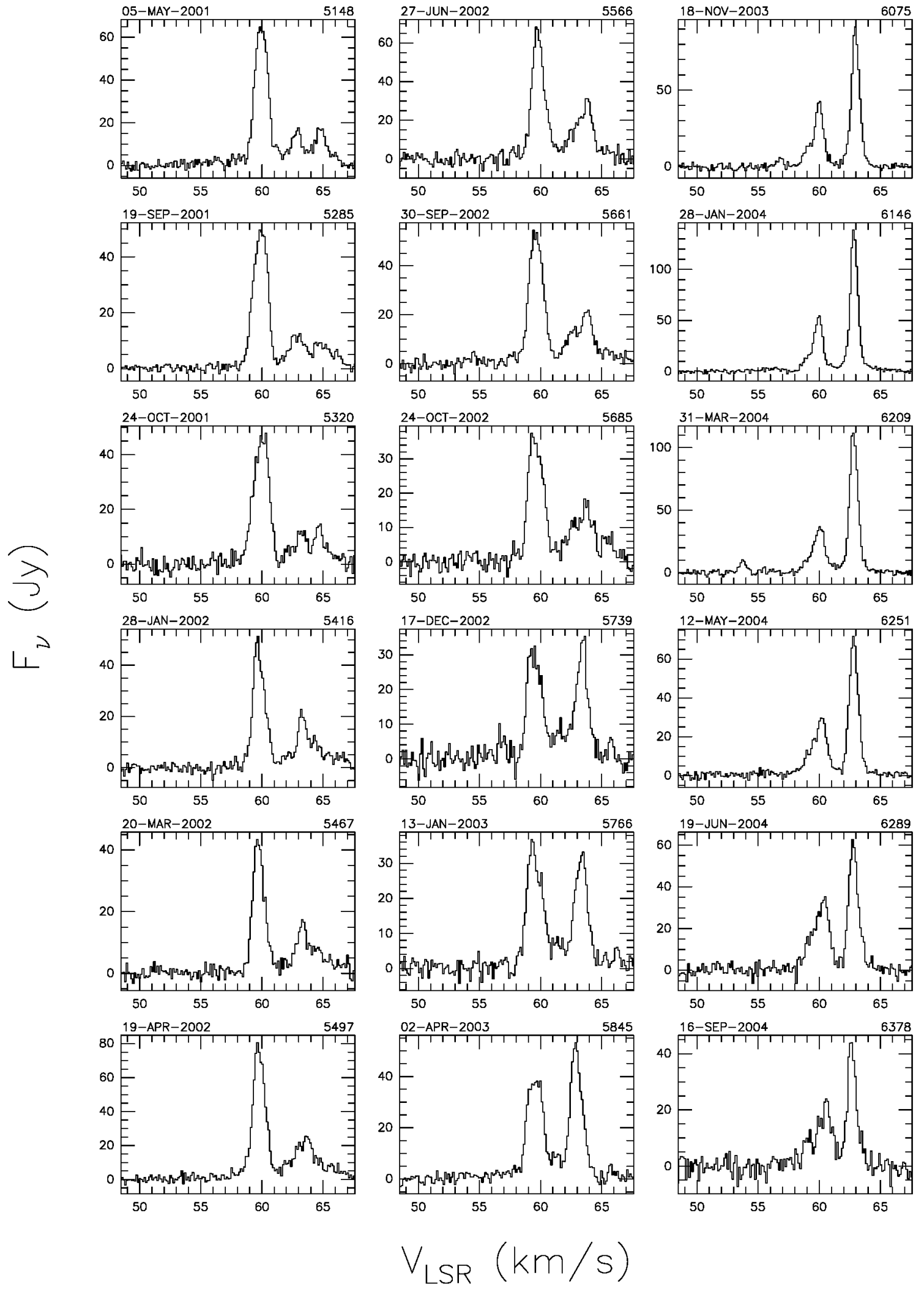

Fig. A.26. a continued. 
$G 45.07+0.13$
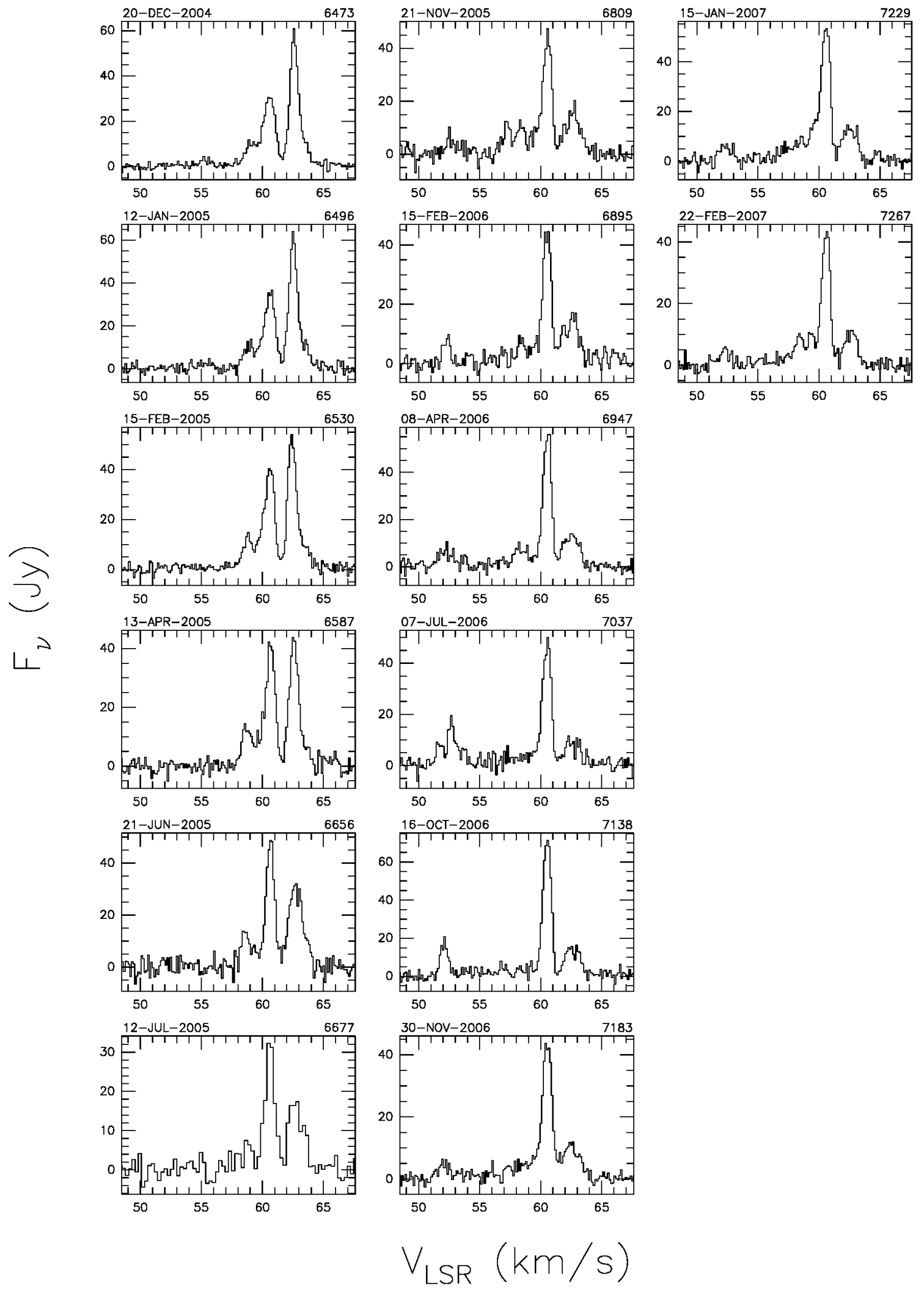

Fig. A.26. a continued. 


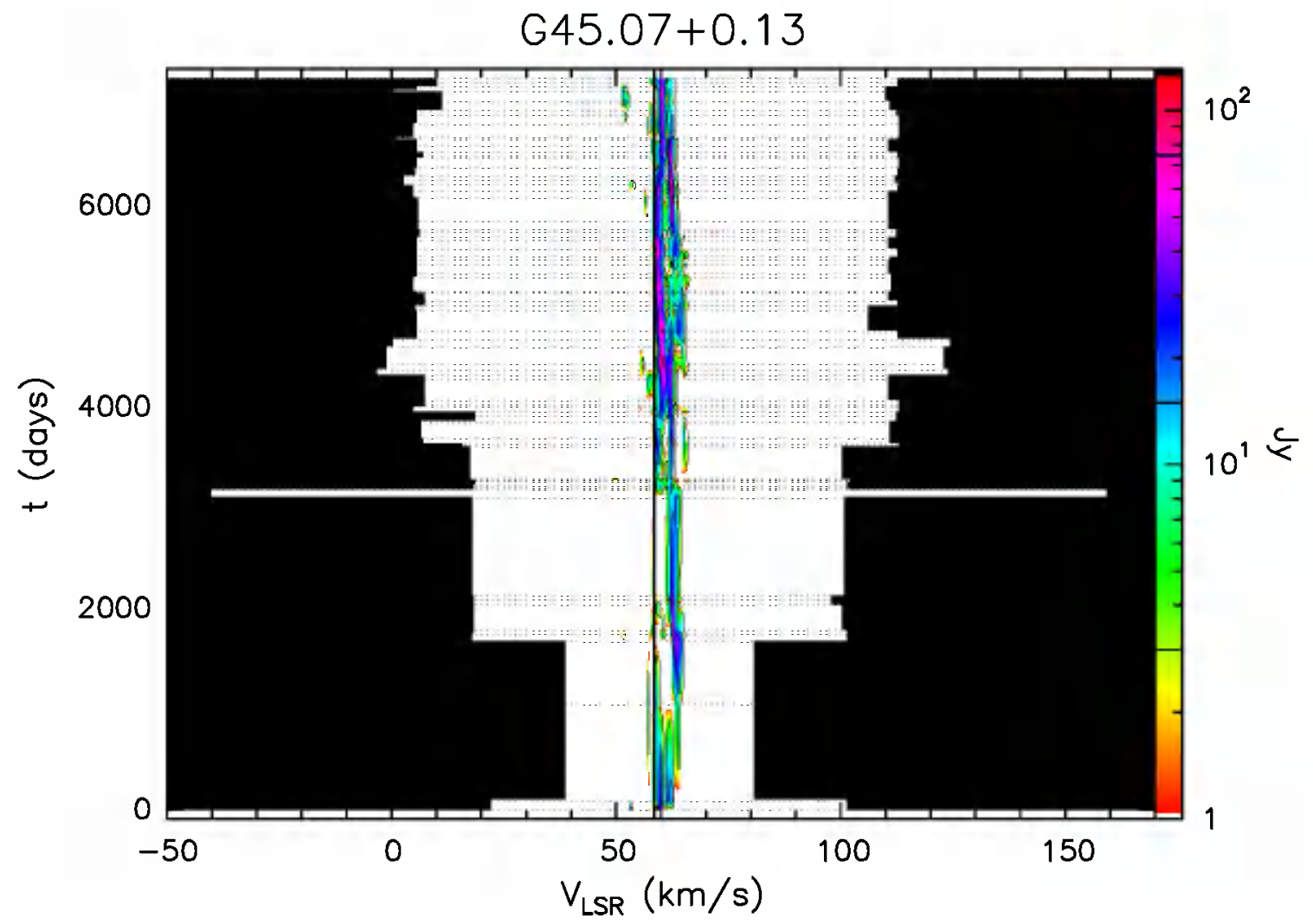

Fig. A.26. b Velocity-time-flux density full plot for source G45.07+0.13. The vertical solid line indicates the velocity of the associated thermal molecular gas. The flux density scale is shown by the bar on the right. In this bar the three lines give the flux density of the drawn contours.

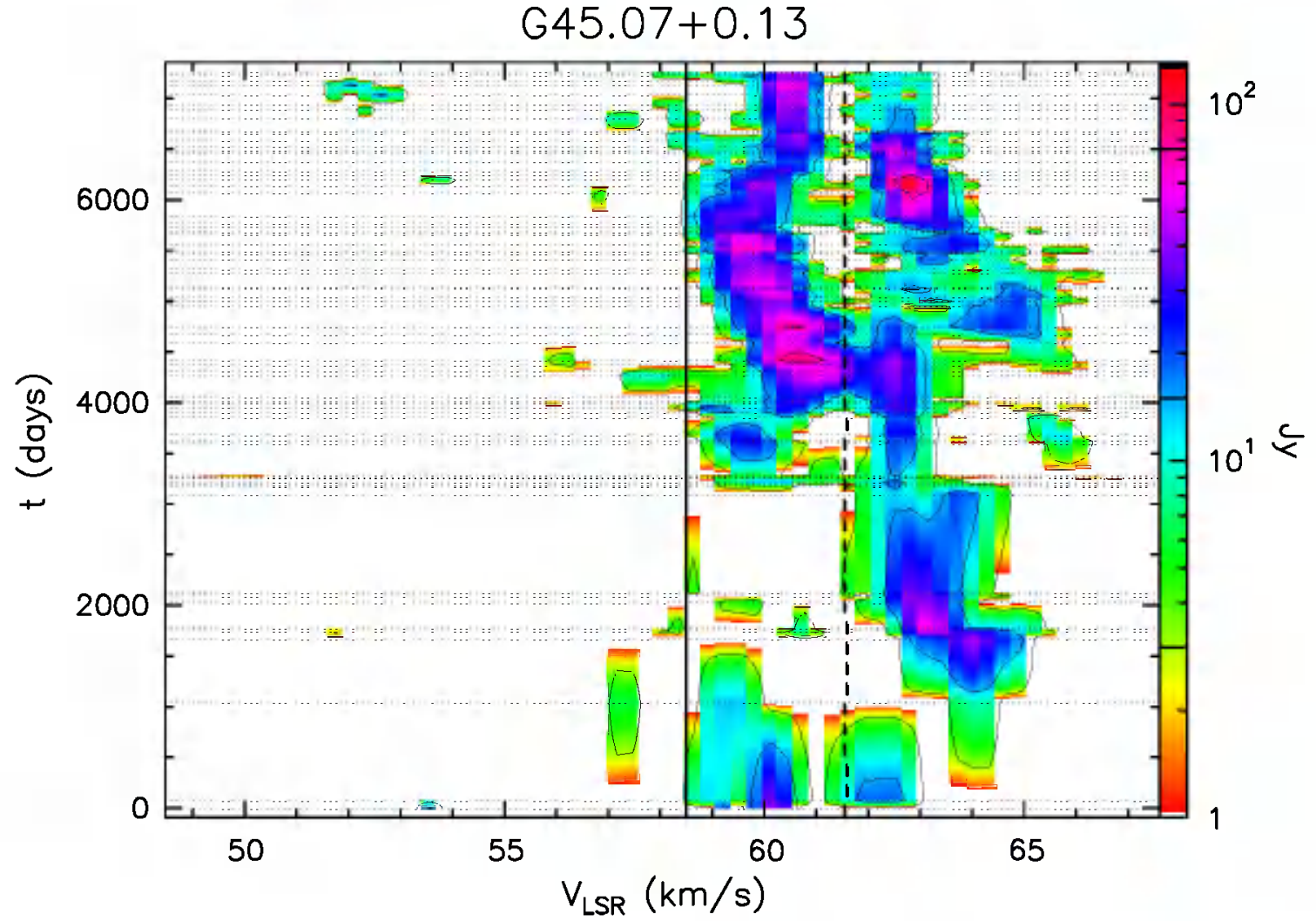

Fig. A.26. c Same as previous figure. but "zoomed" to velocity range over which emission has been detected. 


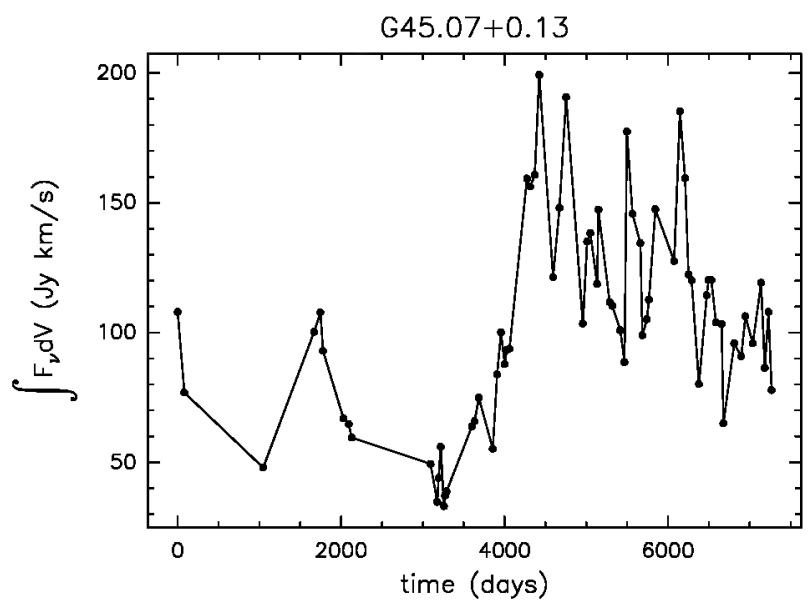

Fig. A.26. d Integral of the flux density over the observed velocity range as a function of time for source $\mathrm{G} 45 \cdot 07+0.13$.

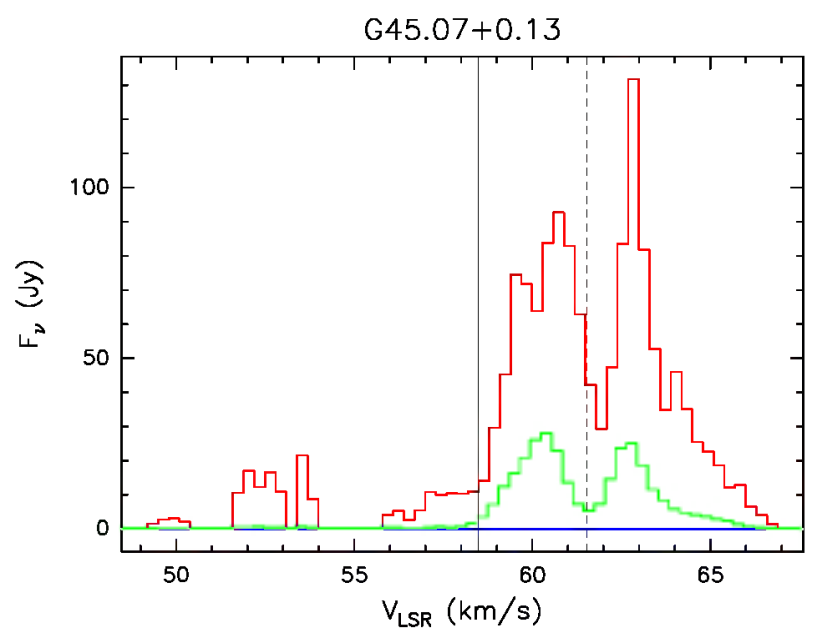

Fig. A.26. e Upper (red) and lower (blue) envelopes and mean spectrum (green) of source $\mathrm{G} 45.07+0.13$ measured during our monitoring. The vertical solid line marks the velocity of the associated thermal molectllar gas. The vertical dashed line marks the mean velocity derived from the histogram of the rate-of-occurrence.

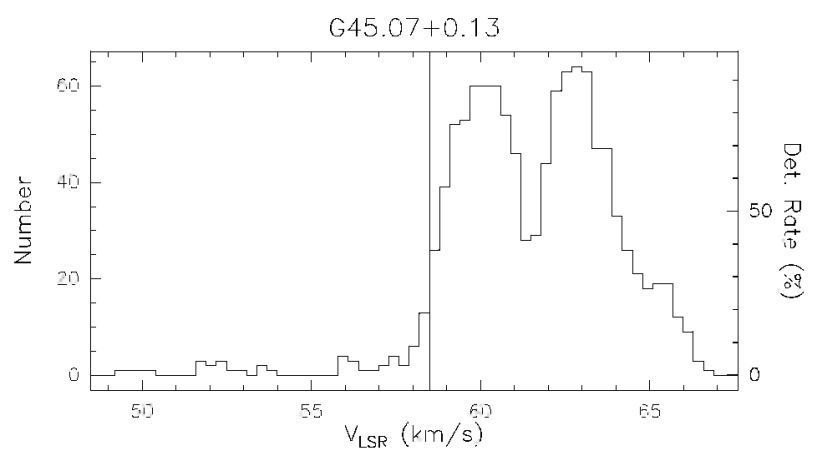

Fig. A.26. f Rate-of-occurrence plot for source G45.07+0.13. The scale to the right refers to the dotted histogram, the scale to the left to the solid line histogram. The vertical solid line marks the velocity of the associated thermal molecular gas. 
$659.78+0.06$
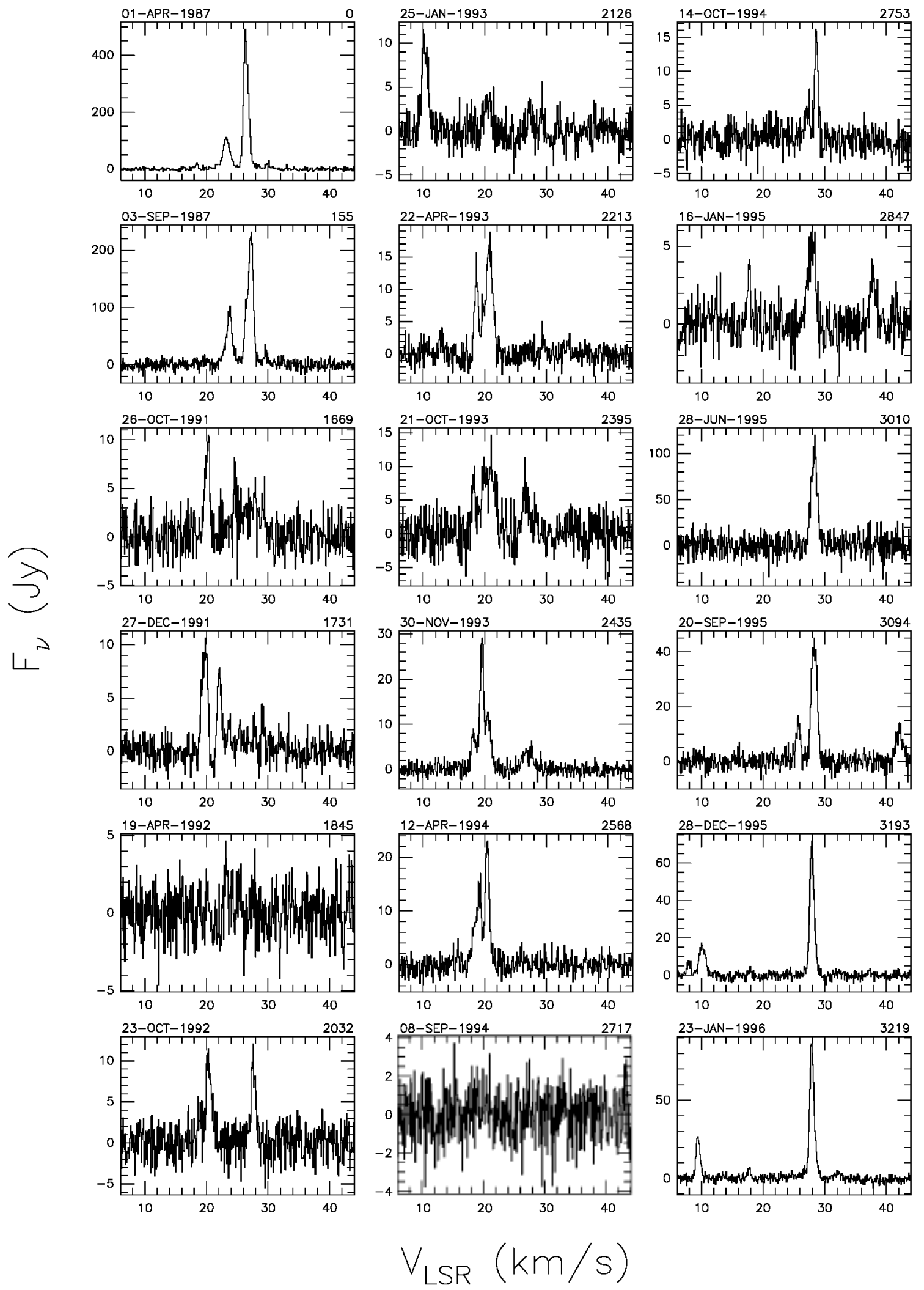

Fig. A.27. a Spectra of source G59.78+0.06 with autoscaled flux density scale. The date of observation is shown above the top left corner of each spectrum and the number of days elapsed since the first observation is given above the top right corner. The velocity scale is the same for all spectra. 

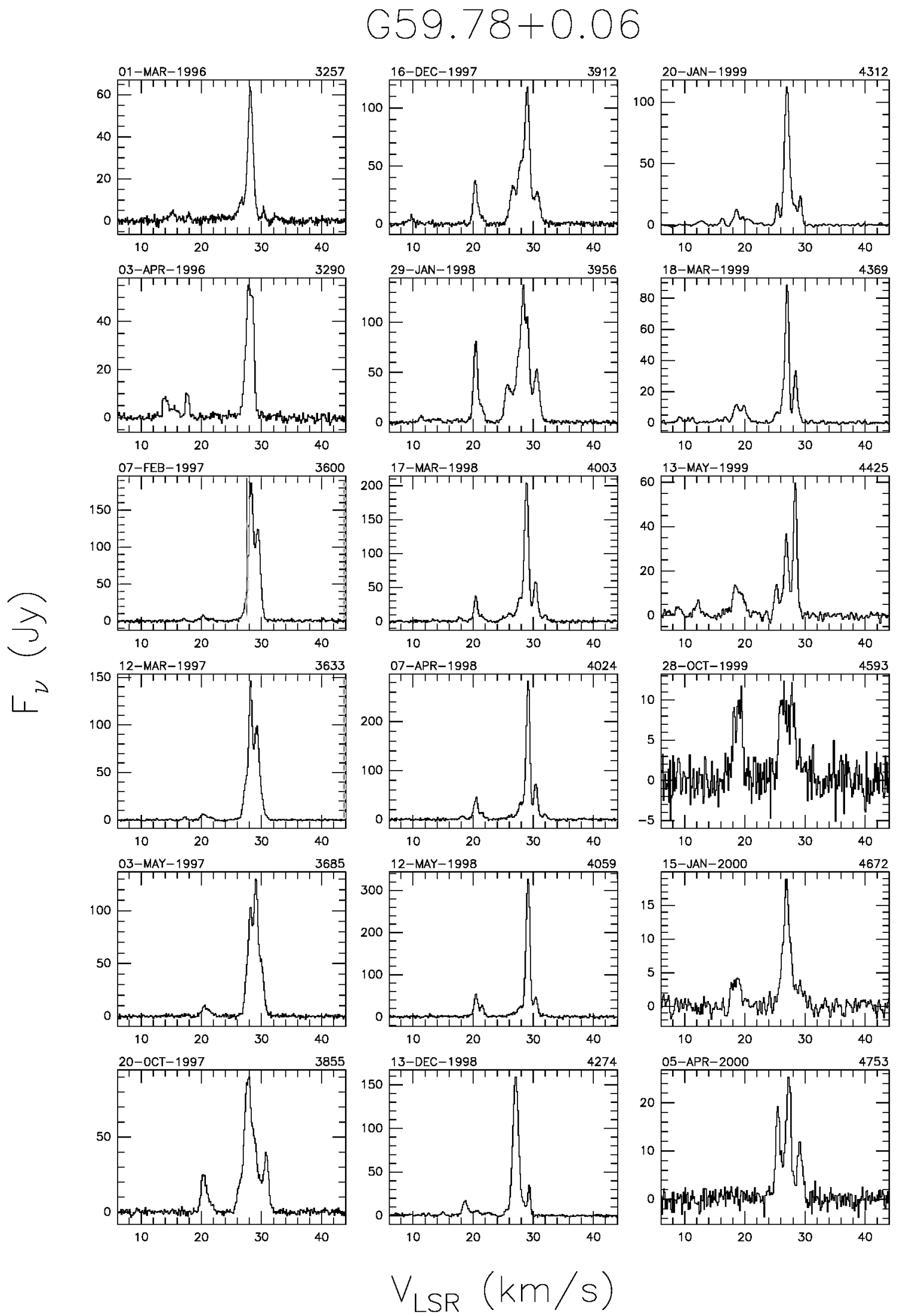

Fig. A.27. a continued. 
$\mathrm{G} 59.78+0.06$
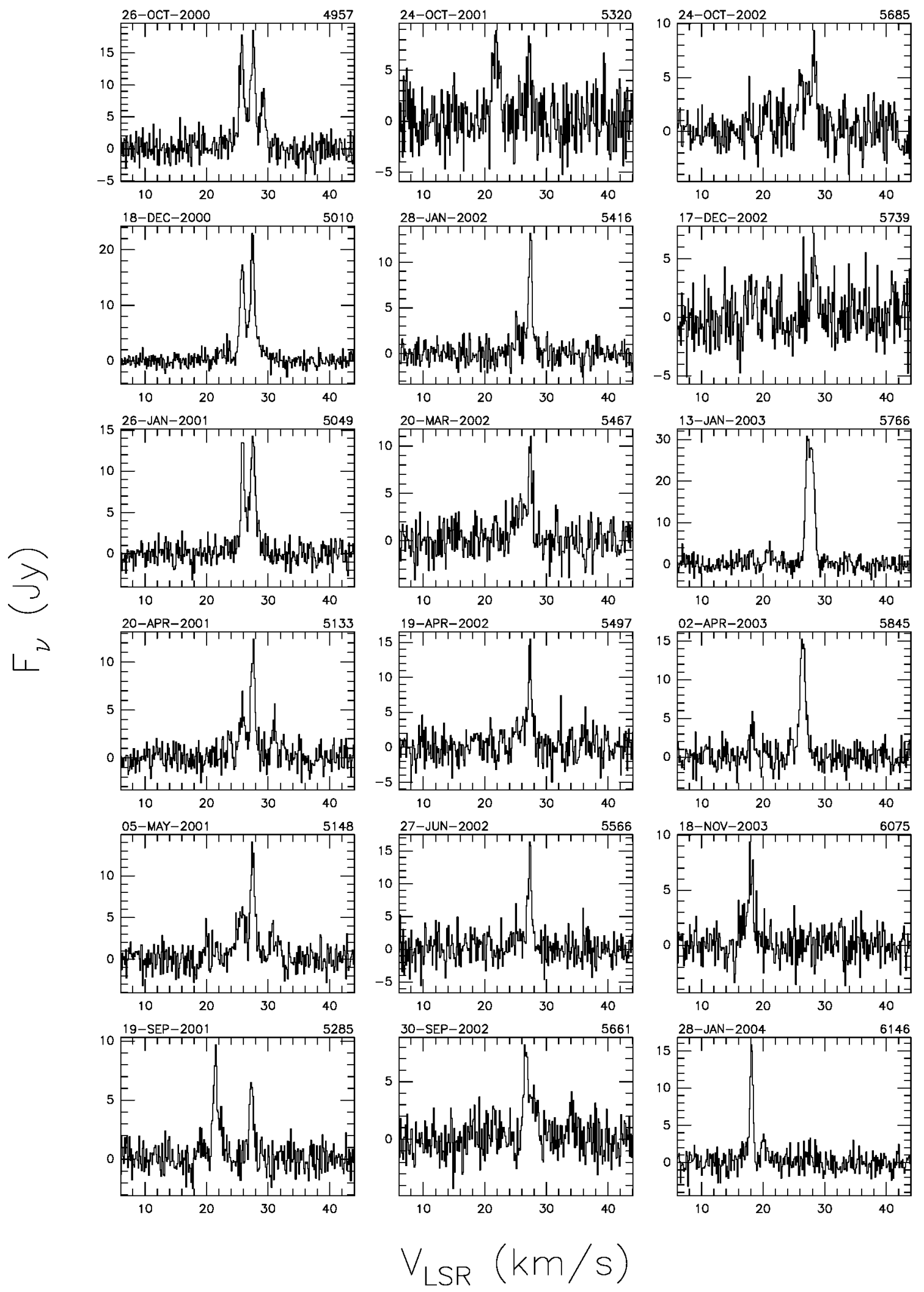

Fig. A.27. a continued. 
G59.78+0.06
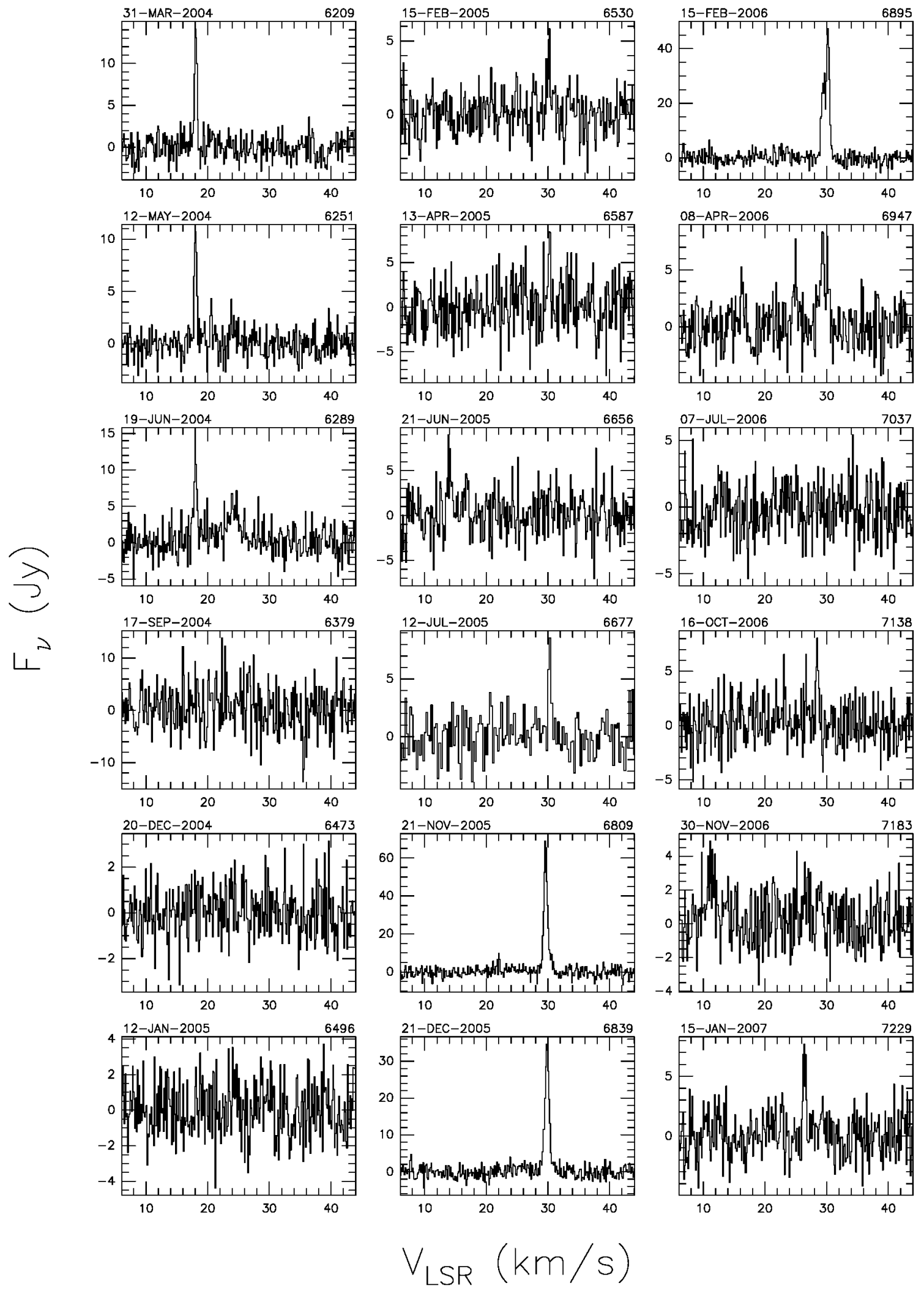

Fig. A.27. a continued. 
$\mathrm{G} 59.78+0.06$

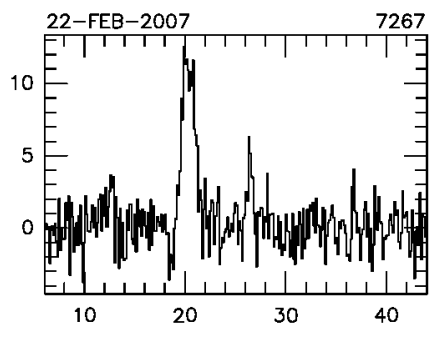

3

$\left\llcorner^{2}\right.$

$$
V_{\text {LSR }}(\mathrm{km} / \mathrm{s})
$$

Fig. A.27. a continued. 


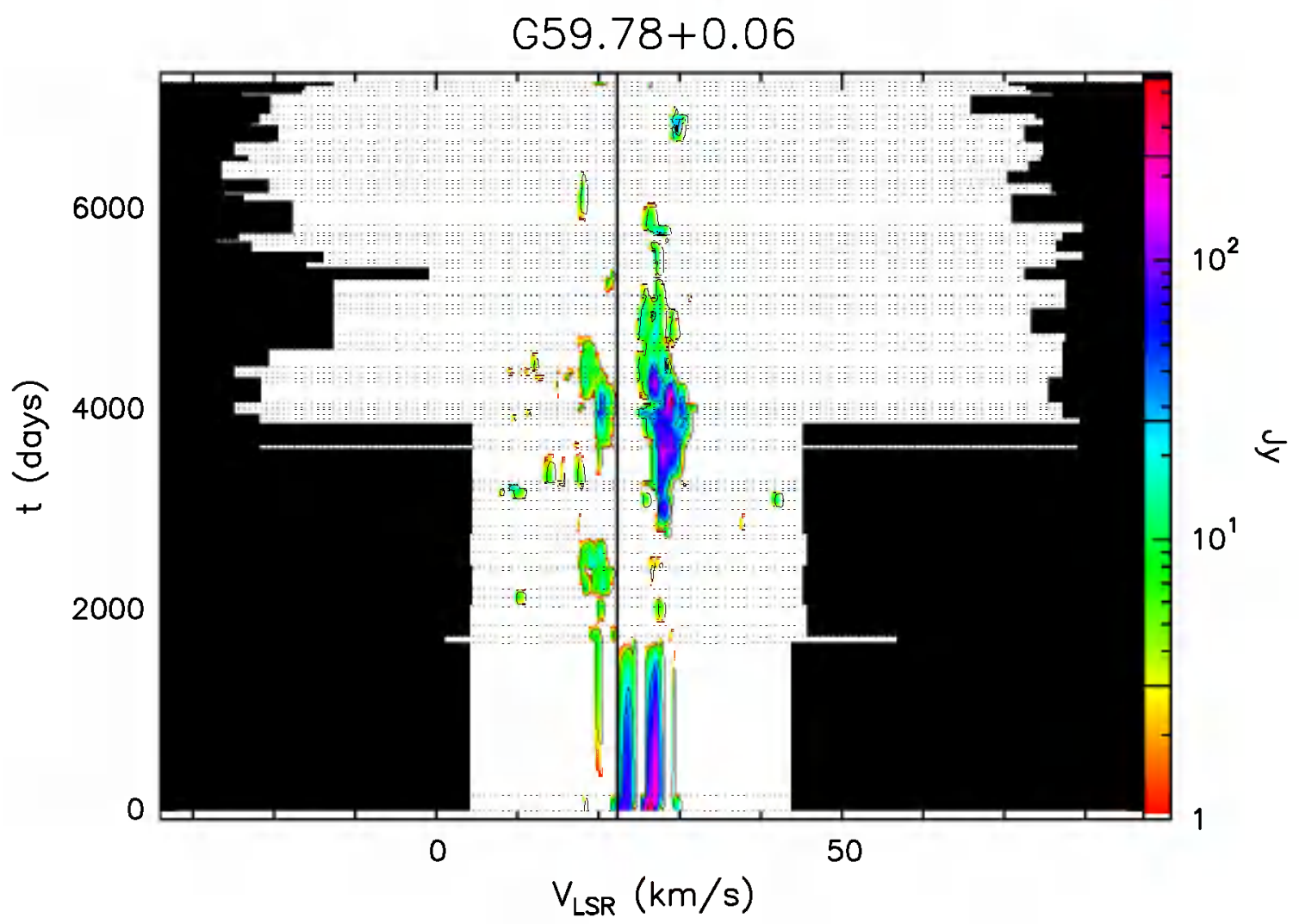

Fig. A.27. b Velocity-time-flux density full plot for source G59.78+0.06. The vertical solid line indicates the velocity of the associated thermal molecular gas. The flux density scale is shown by the bar on the right. In this bar the three lines give the flux density of the diawn contours.

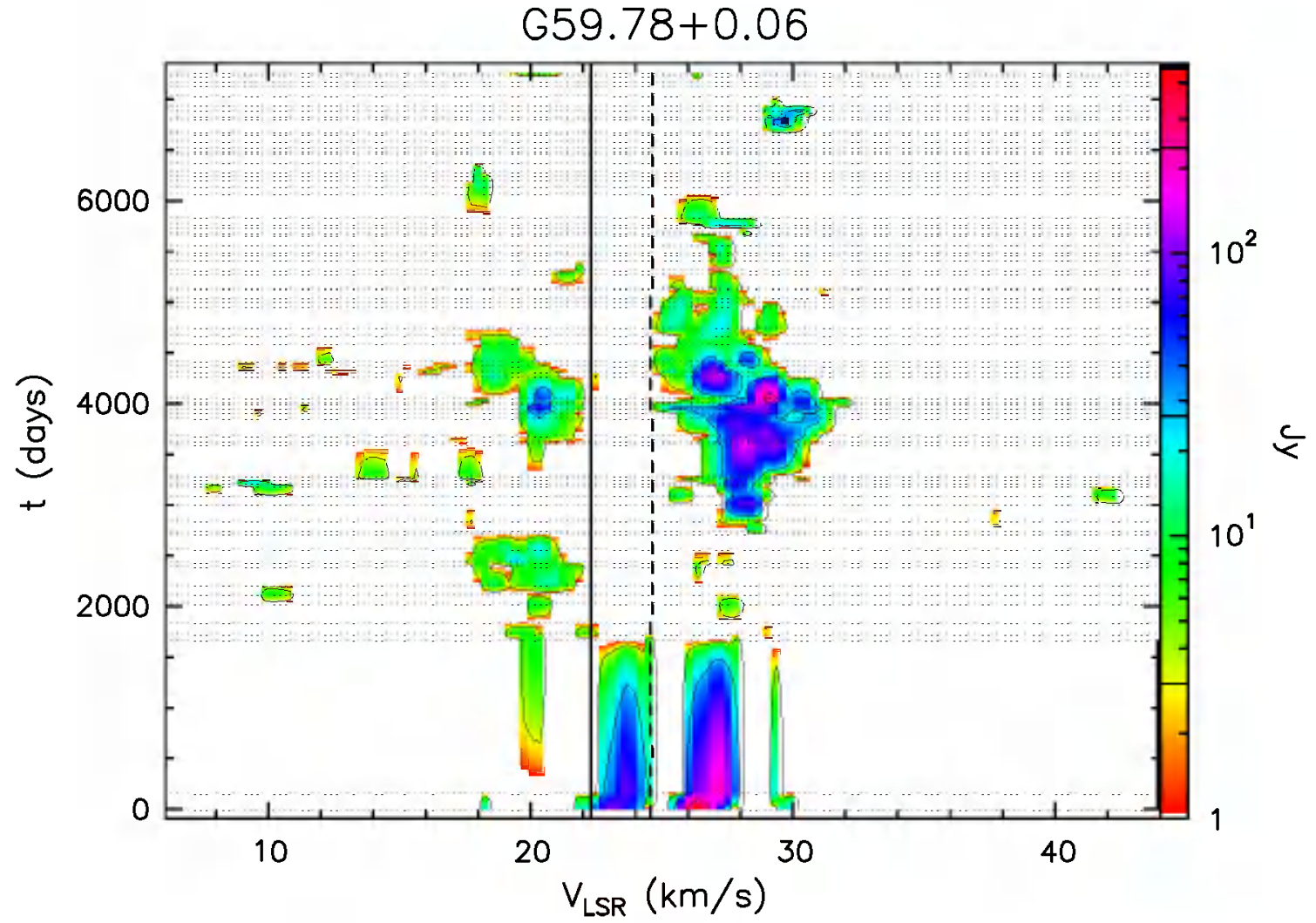

Fig. A.27. c Same as previous figure, but "zoomed" to velocity range over which emission has been detected. 


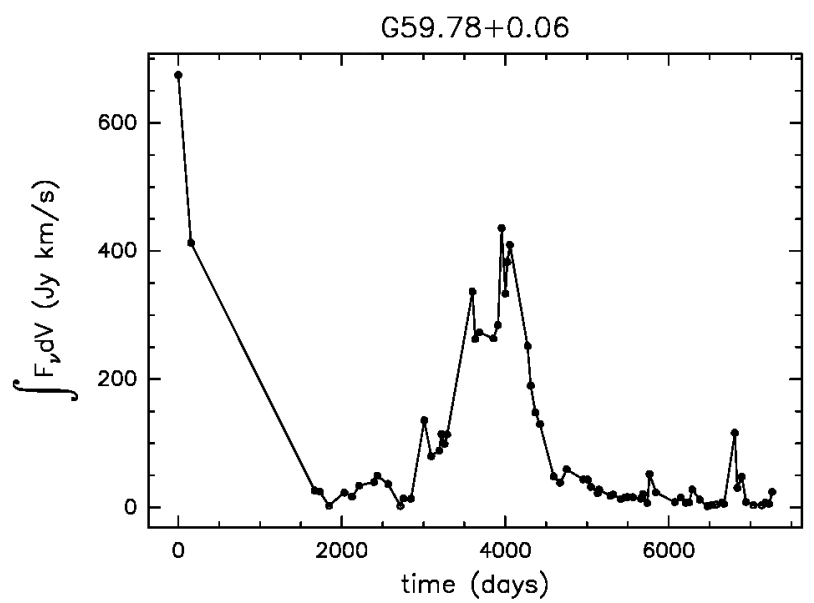

Fig. A.27. d Integral of the flux density over the observed velocity range as a function of time for source G59.78+0.06.

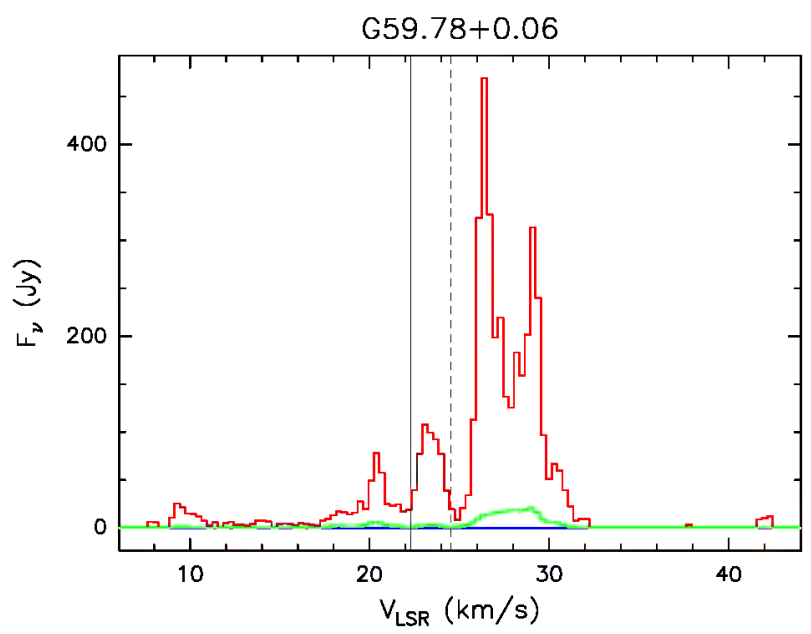

Fig. A.27. e Upper (red) and lower (blue) envelopes and mean spectrum (green) of source G59.78+0.06 measured during our monitoring. The vertical solid line marks the velocity of the associated thermal molecular gas. The vertical dashed line marks the mean velocity derived from the histogram of the rate-of-occurrence.

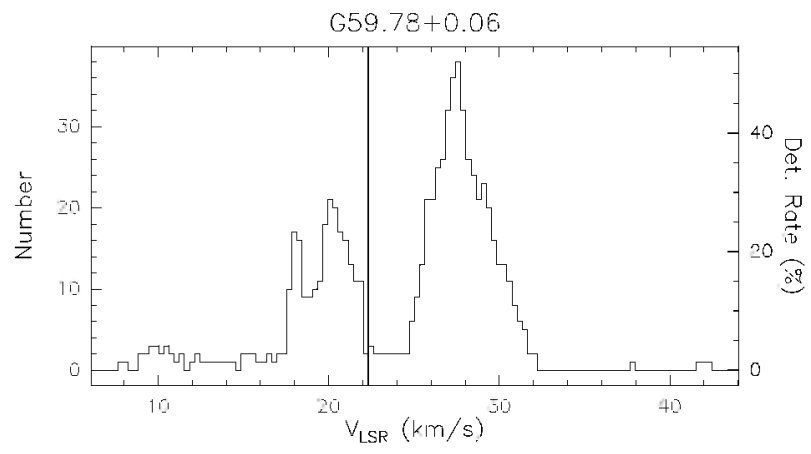

Fig. A.27. f Rate-of-occurrence plot for source G59.78+0.06. The scale to the right refers to the dotted histogram. the scale to the left to the solid line histogram. The vertical solid line marks the velocity of the associated thermal molecular gas. 


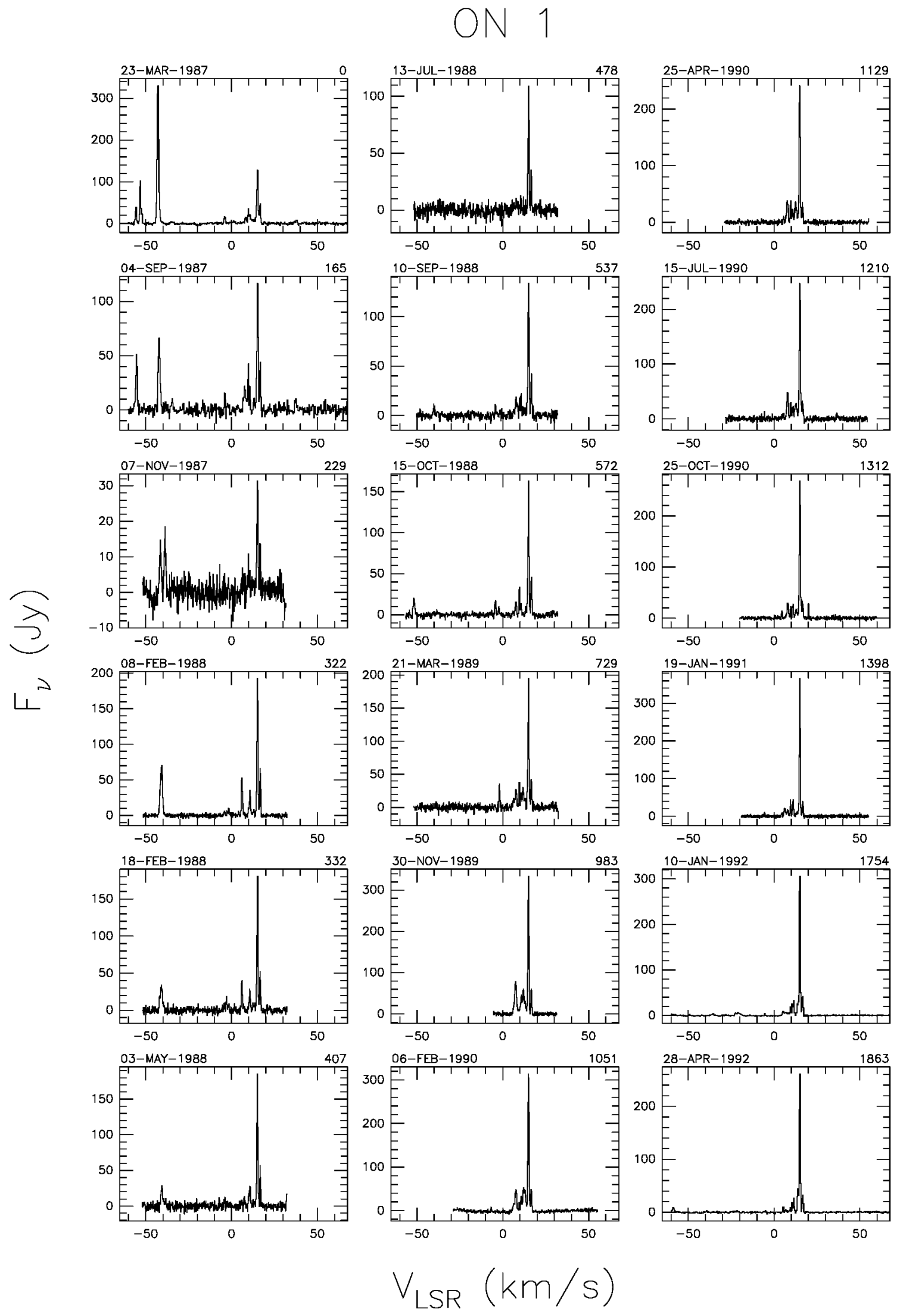

Fig. A.28. a Spectra of source ON 1 with autoscaled flux density scale. The date of observation is shown above the top left corner of each spectrum and the number of days elapsed since the first observation is given above the top right corner. The velocity scale is the same for all spectra. 


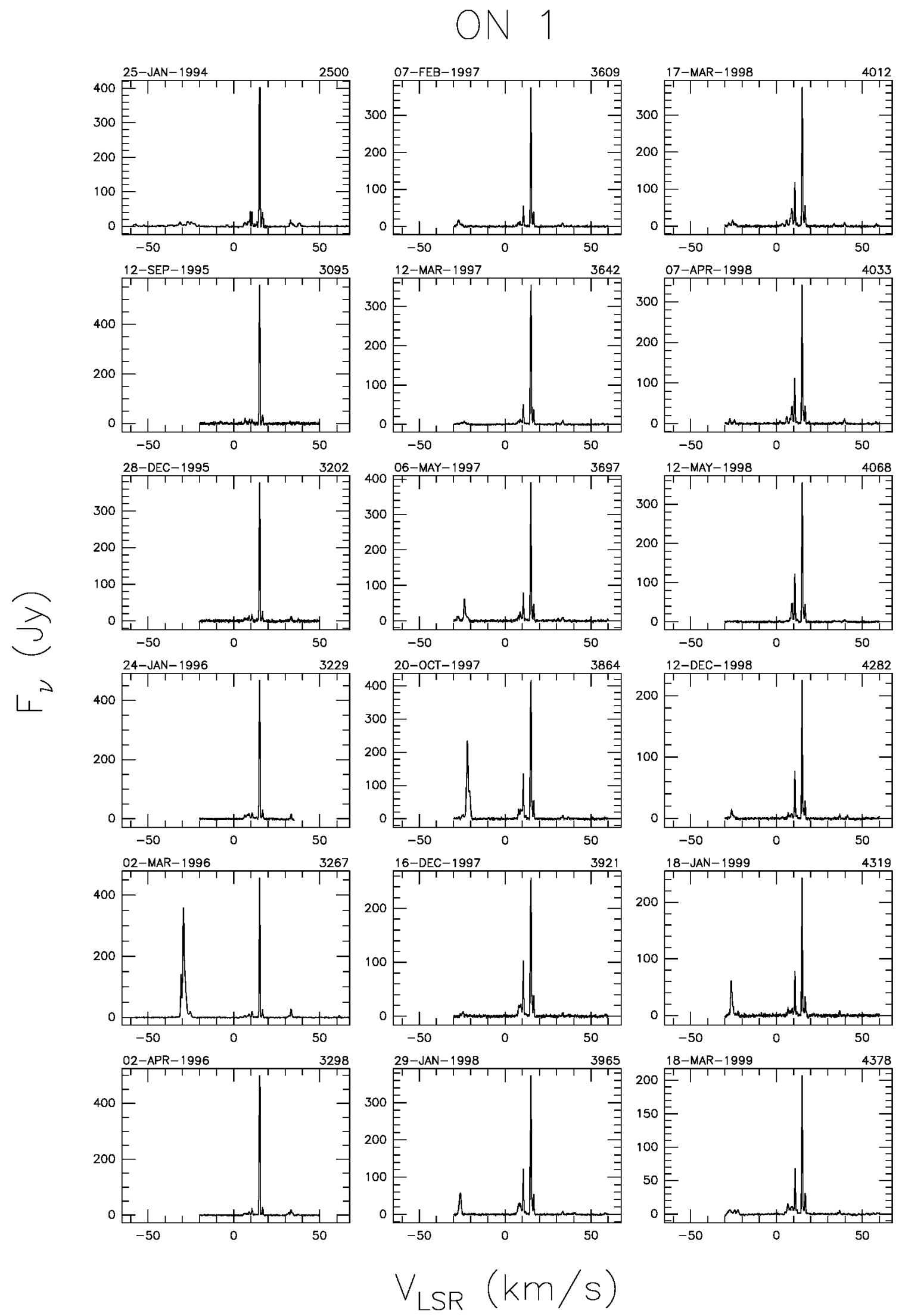

Fig. A.28. a continued. 


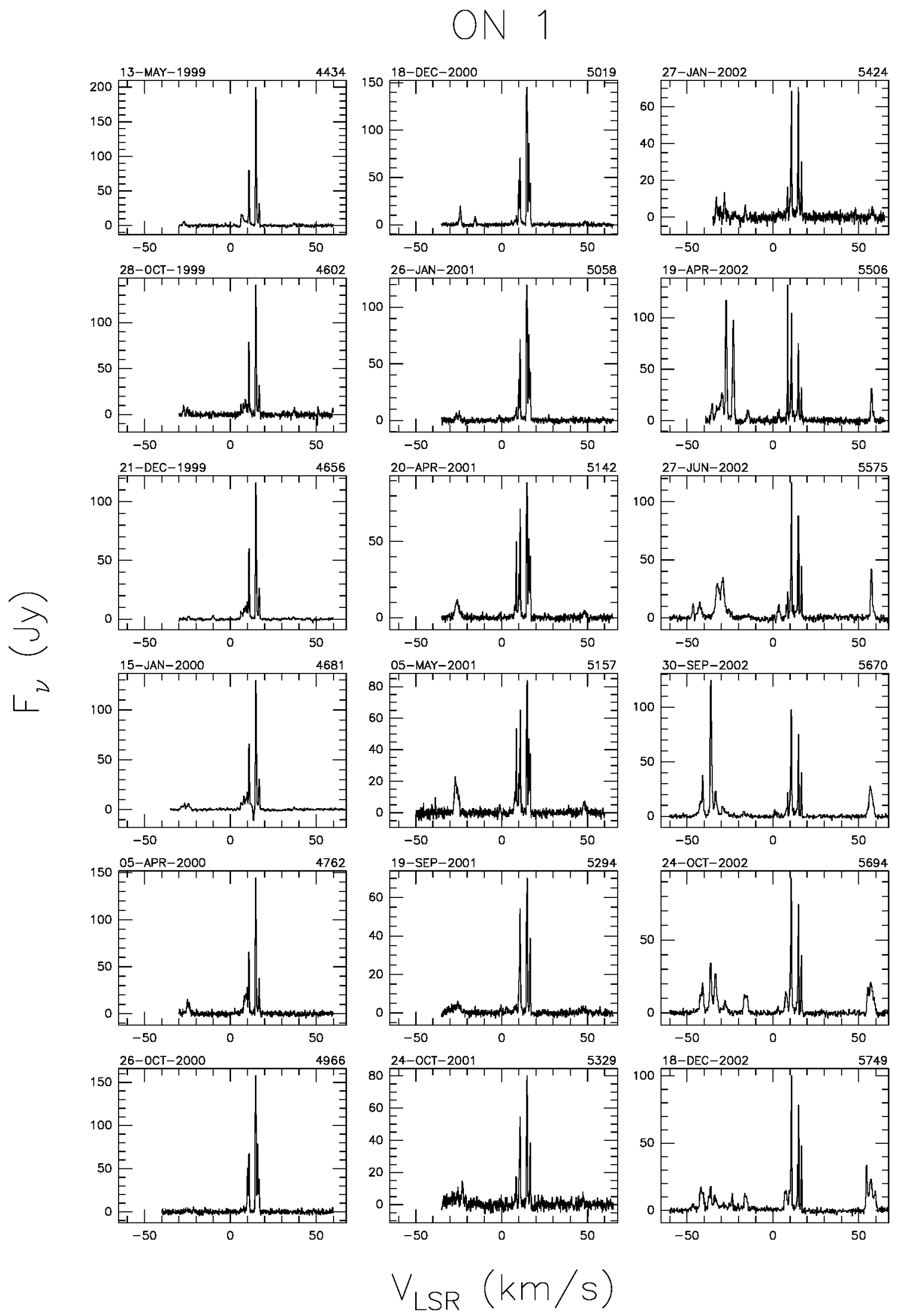

Fig. A.28. a continued. 


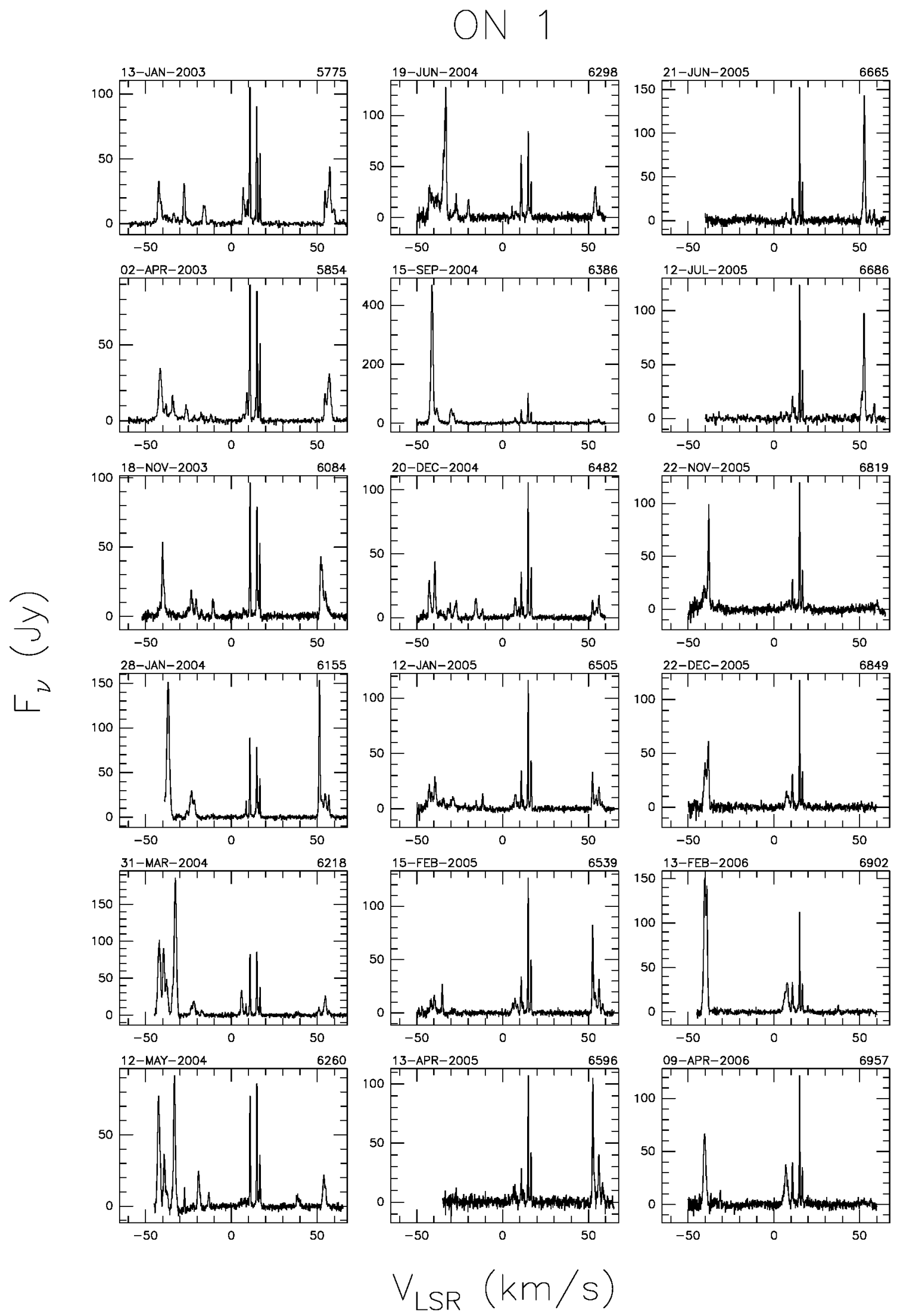

Fig. A.28. a continued. 
ON 1
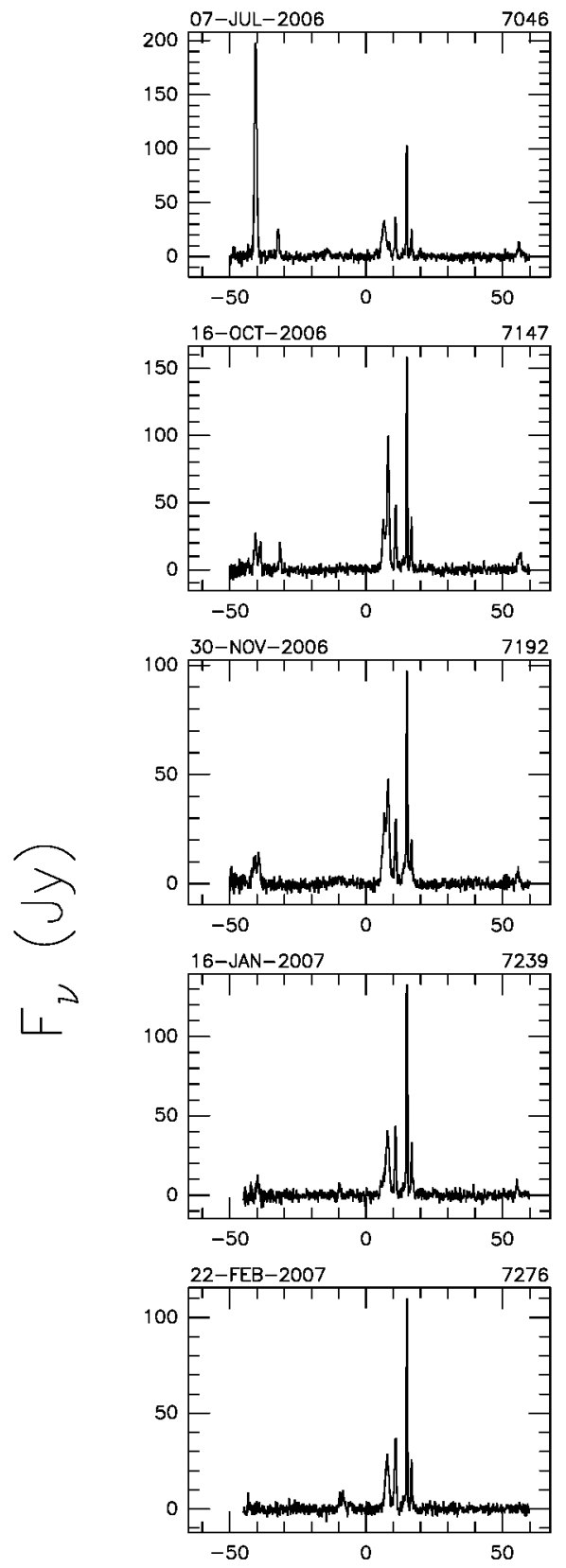

$V_{\text {LSR }}(\mathrm{km} / \mathrm{s})$

Fig. A.28. a continued. 


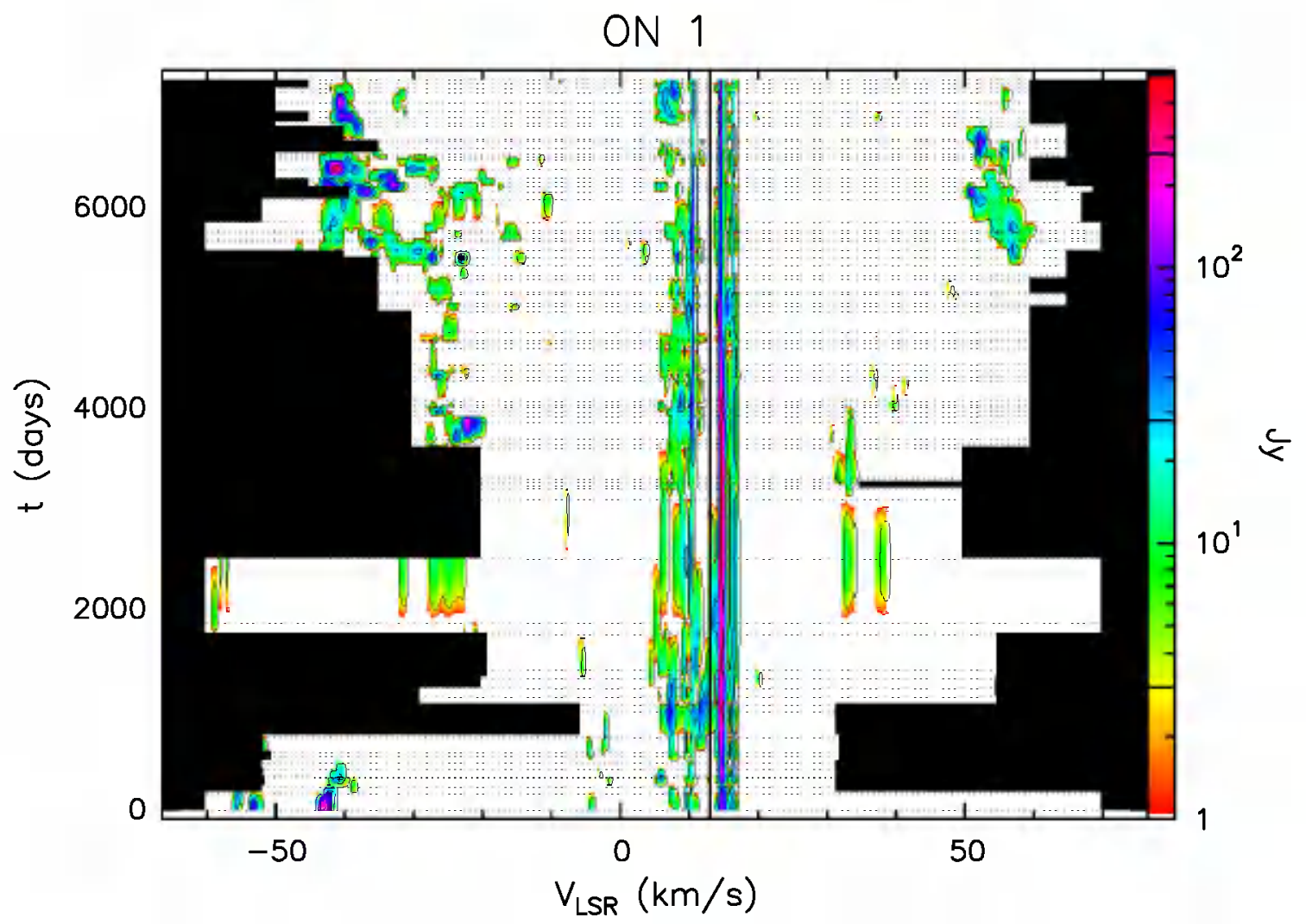

Fig. A.28. b Velocity-time-flux density full plot for source ON 1. The vertical solid line indicates the velocity of the associated thermal molecular gas. The flux density scale is shown by the bar on the right. In this bar the three lines give the flux density of the drawn contours.

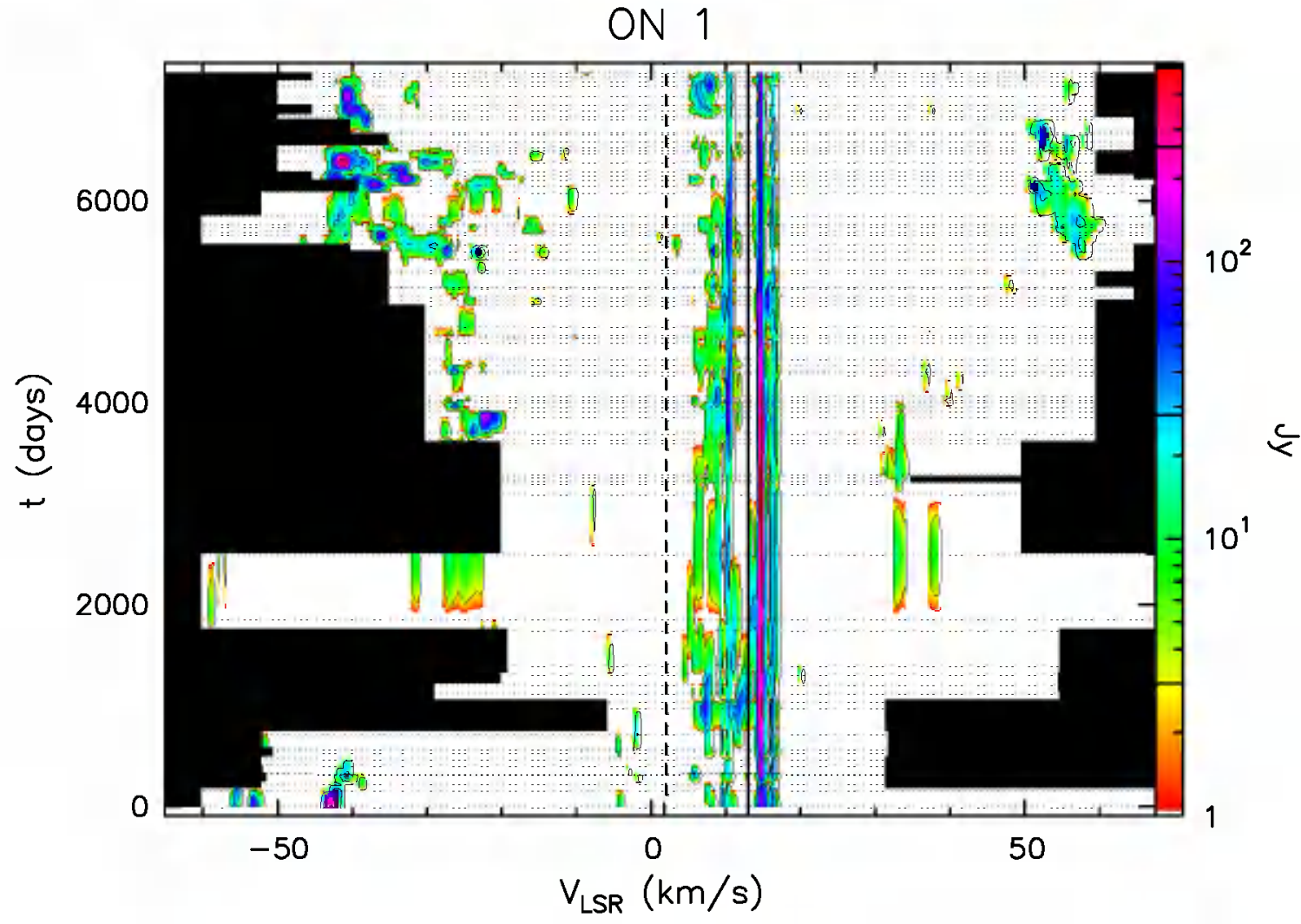

Fig. A.28. c Same as previous figure, but "zoomed" to velocity range over which emission has been detected. 


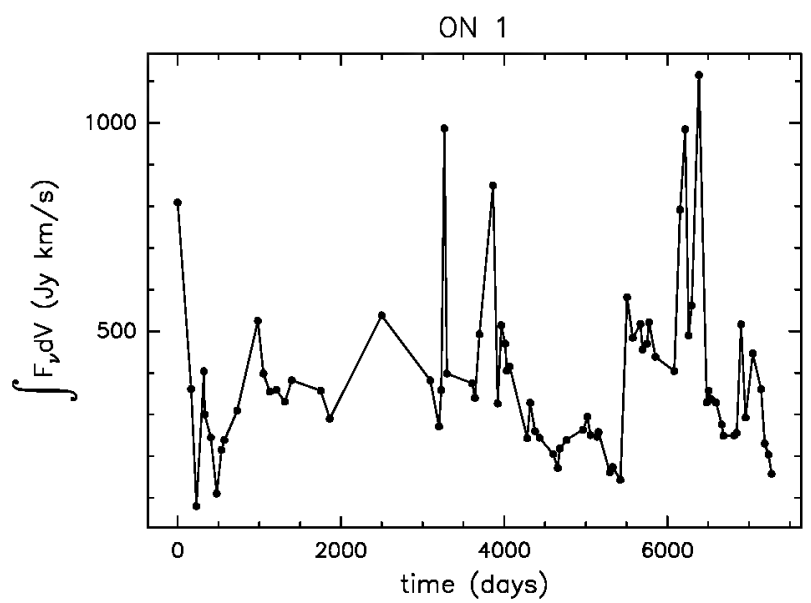

Fig. A.28. d Integral of the flux density over the observed velocity range as a function of time for source ON 1 .

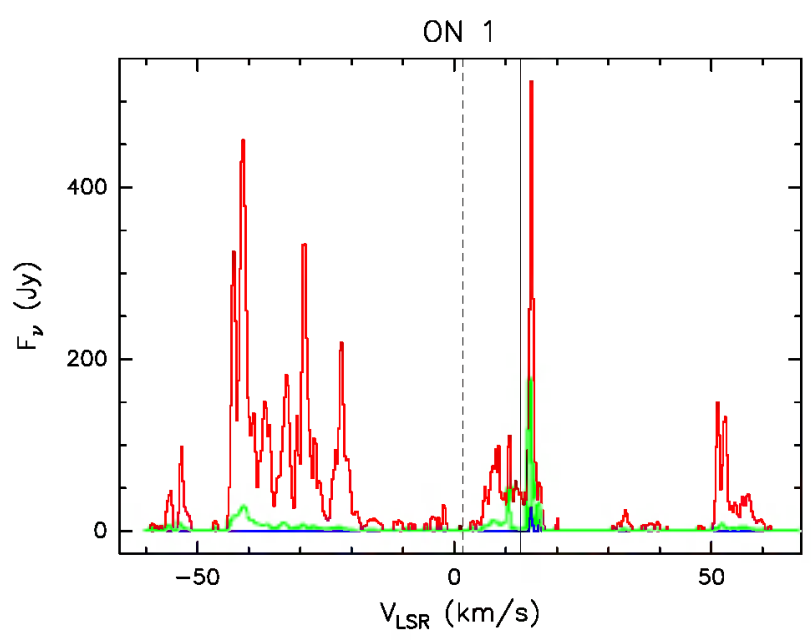

Fig. A.28. e Upper (red) and lower (blue) envelopes and mean spectrum (green) of source ON 1 measured during our monitoring. The vertical solid line marks the velocity of the associated theimal molecular gas. The vertical dashed line marks the mean velocity derived from the histogram of the rate-of-occurrence.

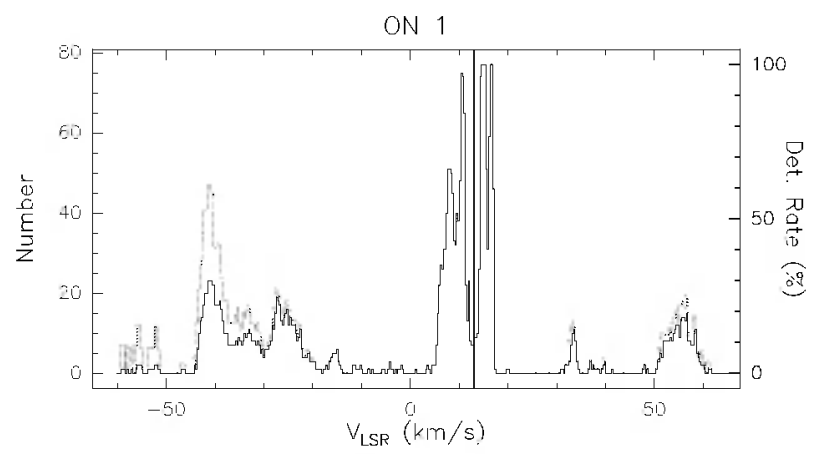

Fig. A.28. f Rate-of-occurrence plot for source ON 1. The scale to the right refers to the dotted histogram, the scale to the left to the solid line histogram. The vertical solid line marks the velocity of the associated thermal molecular gas. 


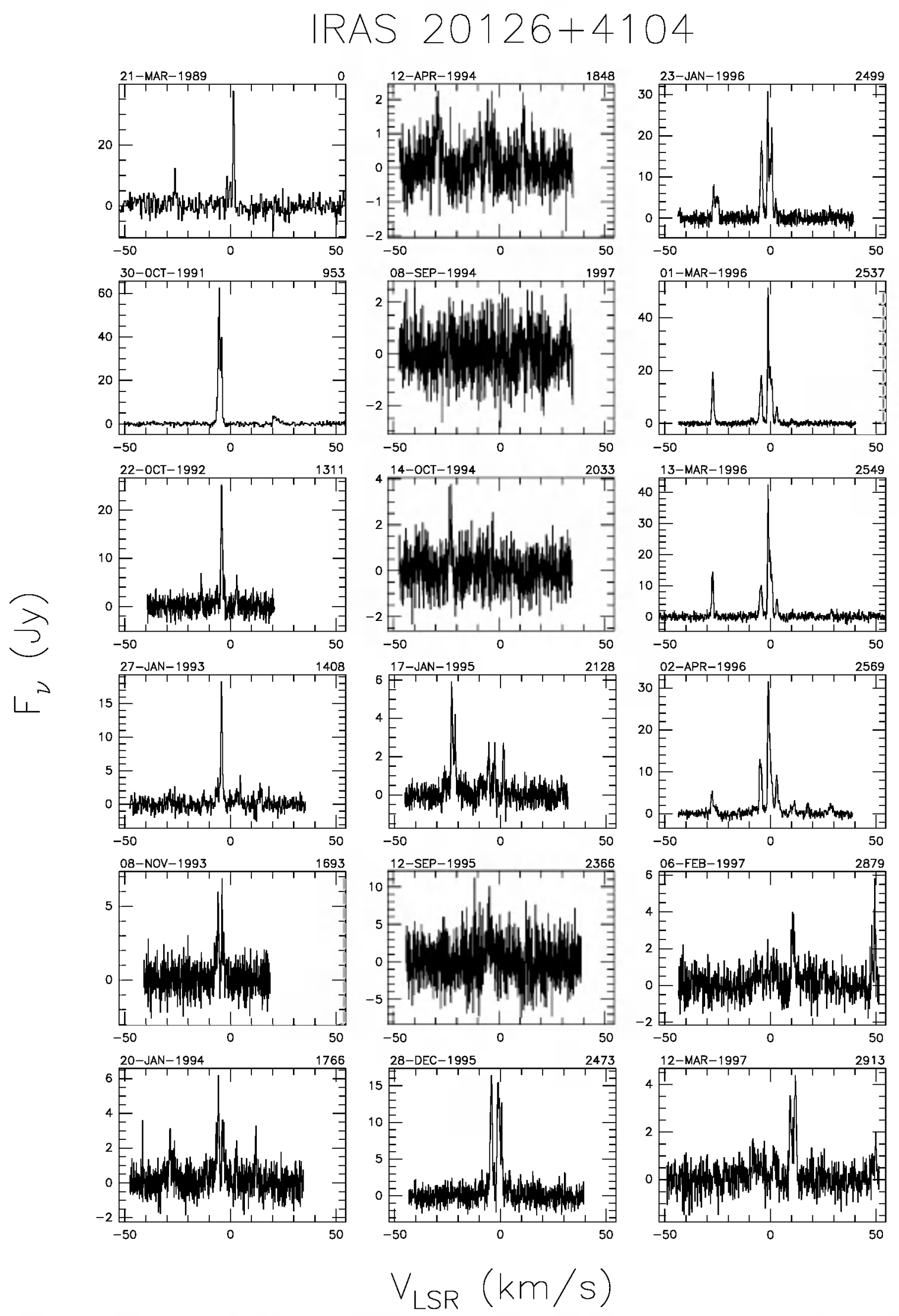

Fig. A.29. a Spectra of source IRAS 20126+4104 with autoscaled flux density scale. The date of observation is shown above the top left corner of each spectrum and the number of days elapsed since the first observation is given above the top right corner. The velocity scale is the same for all spectia. 


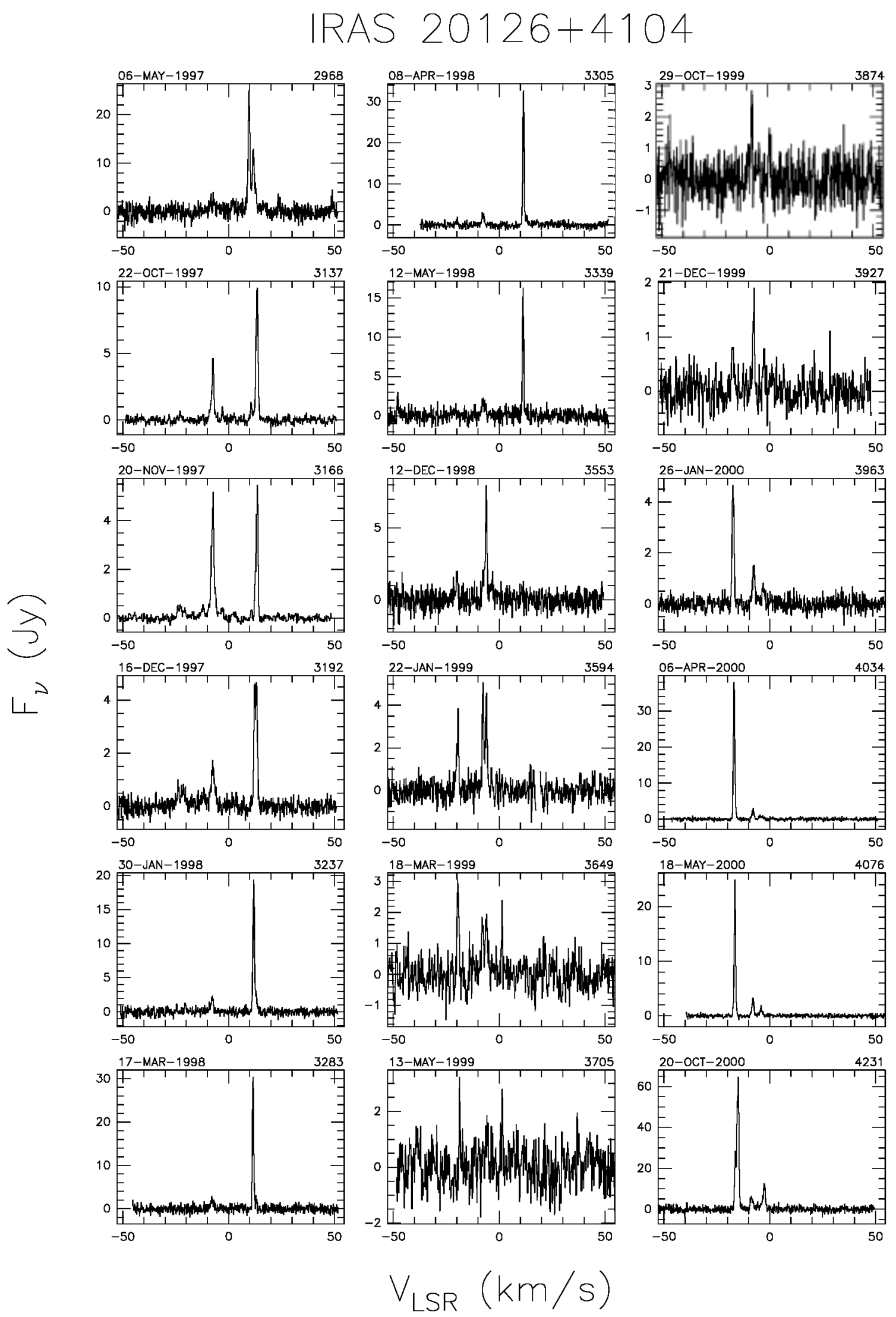

Fig. A.29. a continued. 
IRAS $20126+4104$
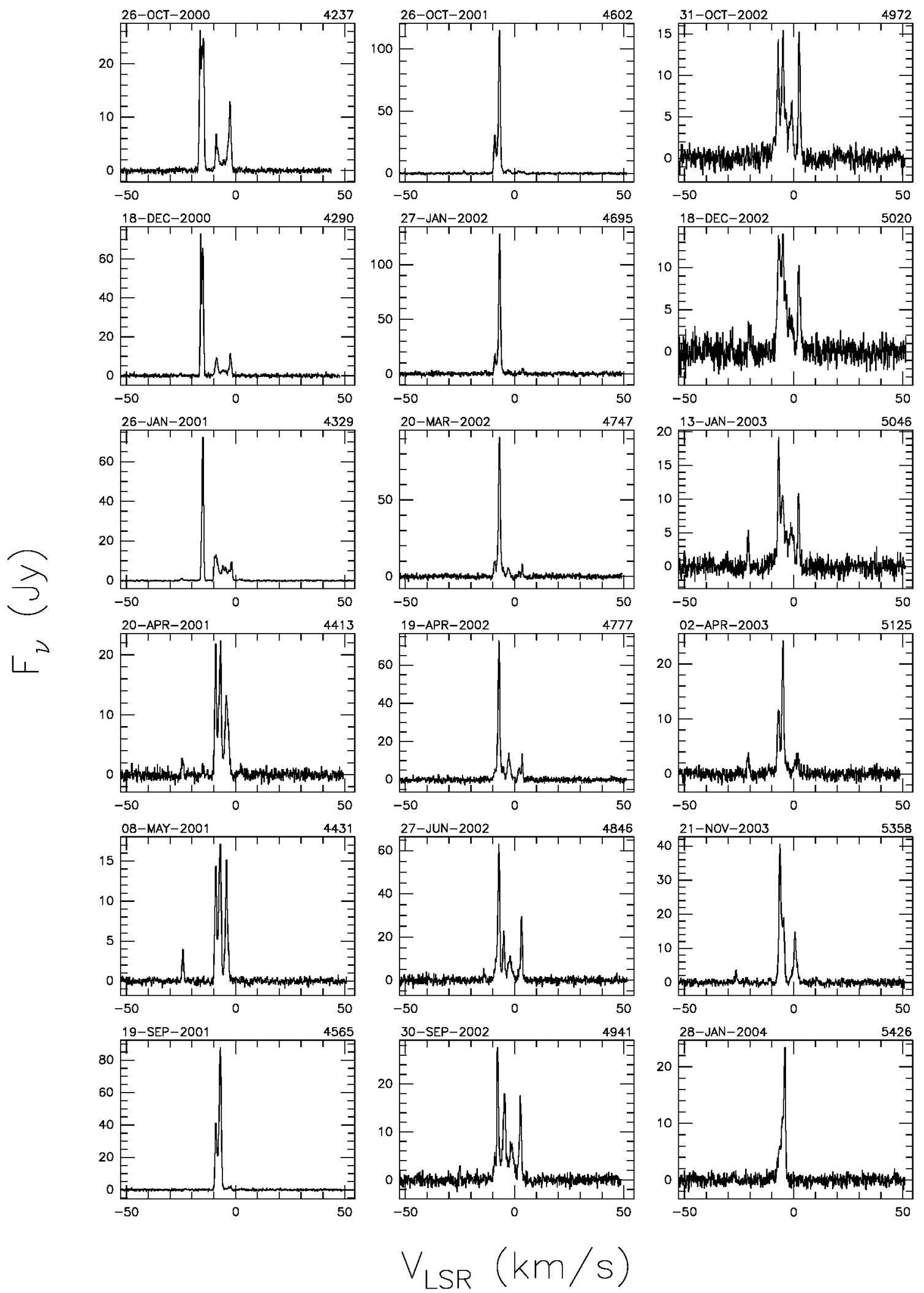

Fig. A.29. a continued. 


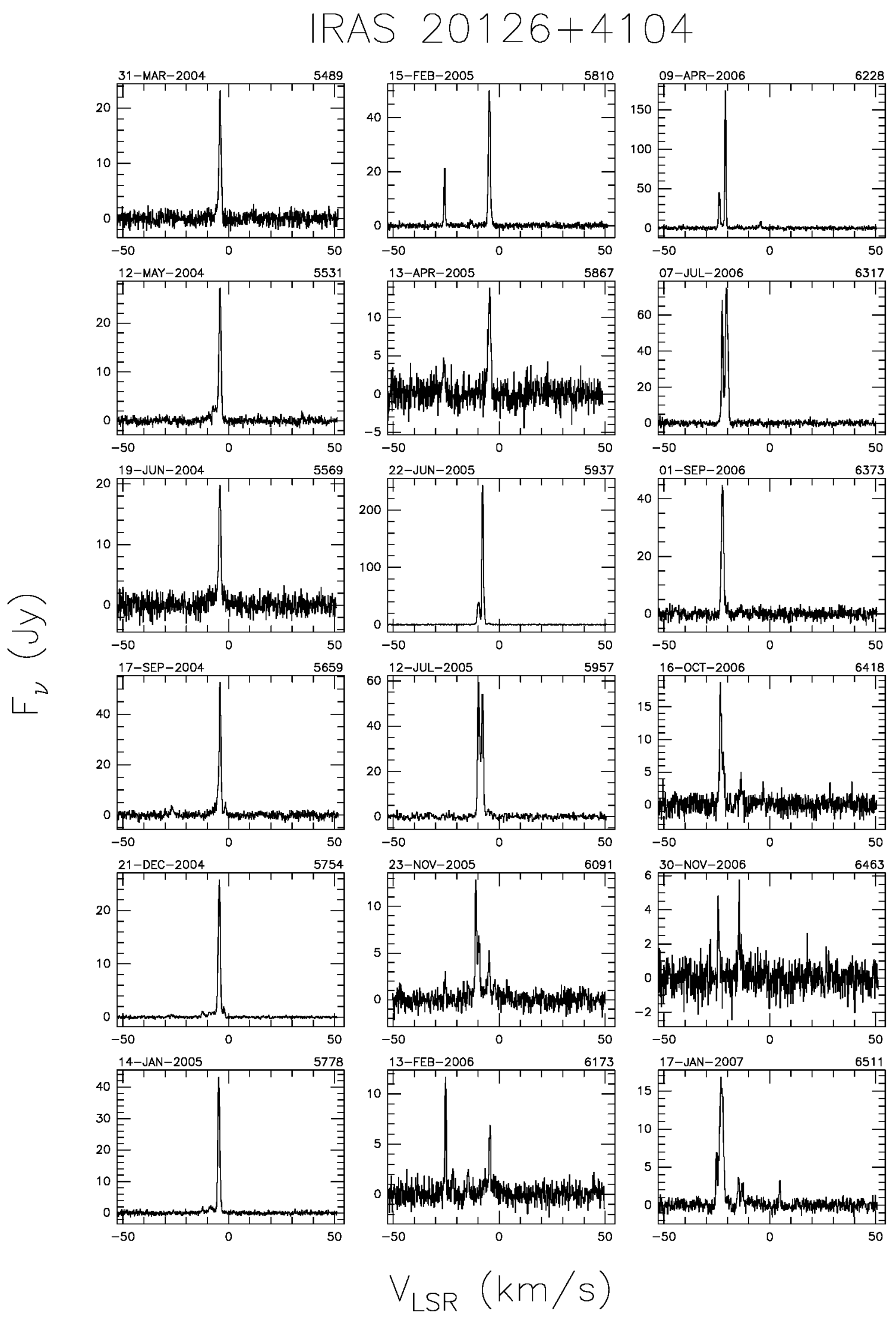

Fig. A.29. a continued. 


$$
\text { IRAS } 20126+4104
$$

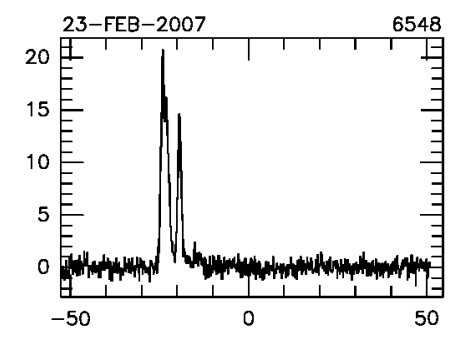

$\overbrace{1}^{\lambda}$

$$
V_{\text {LSR }}(k m / s)
$$

Fig. A.29. a continued. 


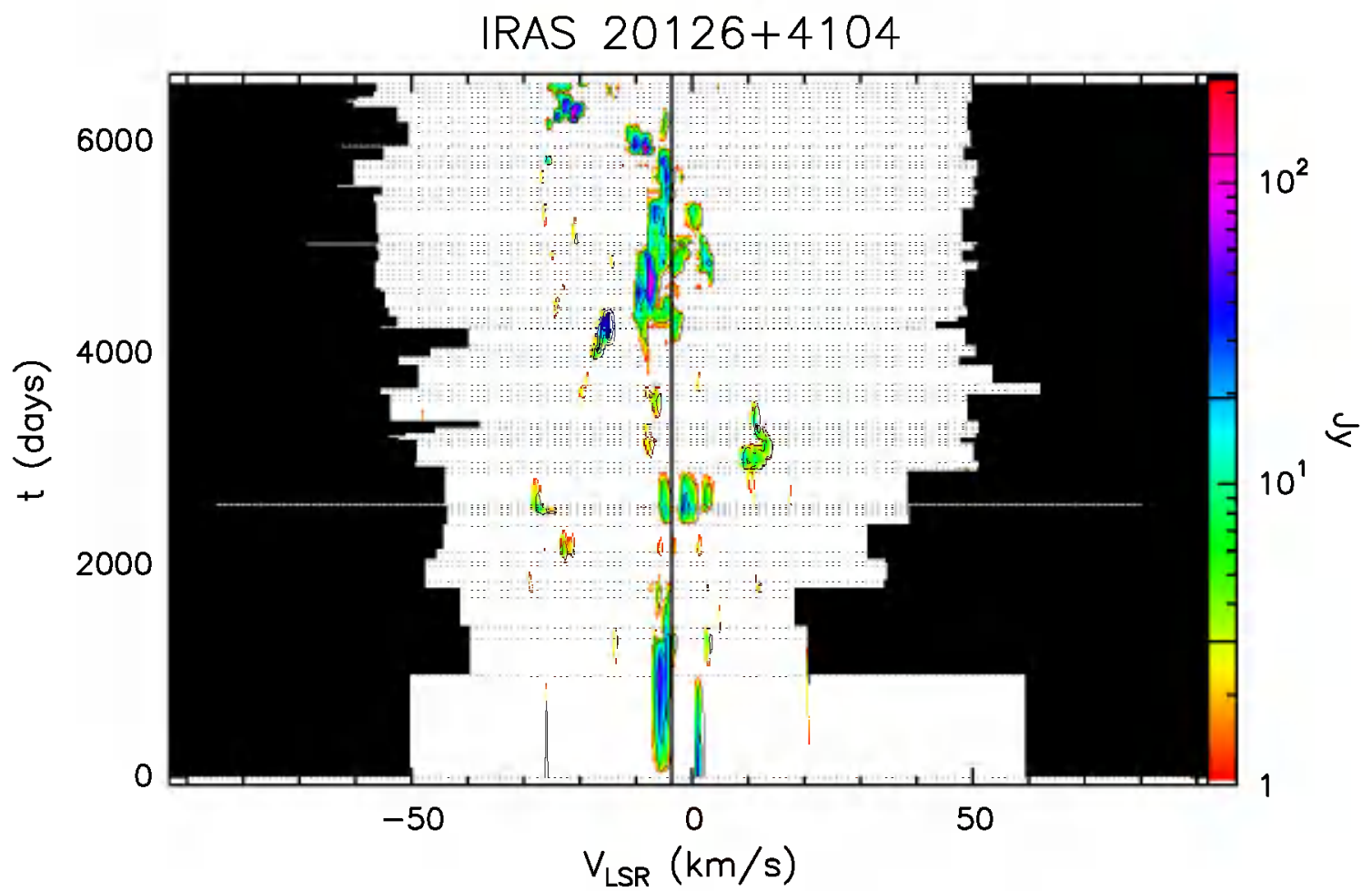

Fig. A.29. b Velocity-time-flux density full plot for source IRAS 20126+4104. The vertical solid line indicates the velocity of the associated thermal molecular gas. The flux density scale is shown by the bar on the right. In this bar the three lines give the flux density of the drawn contours.

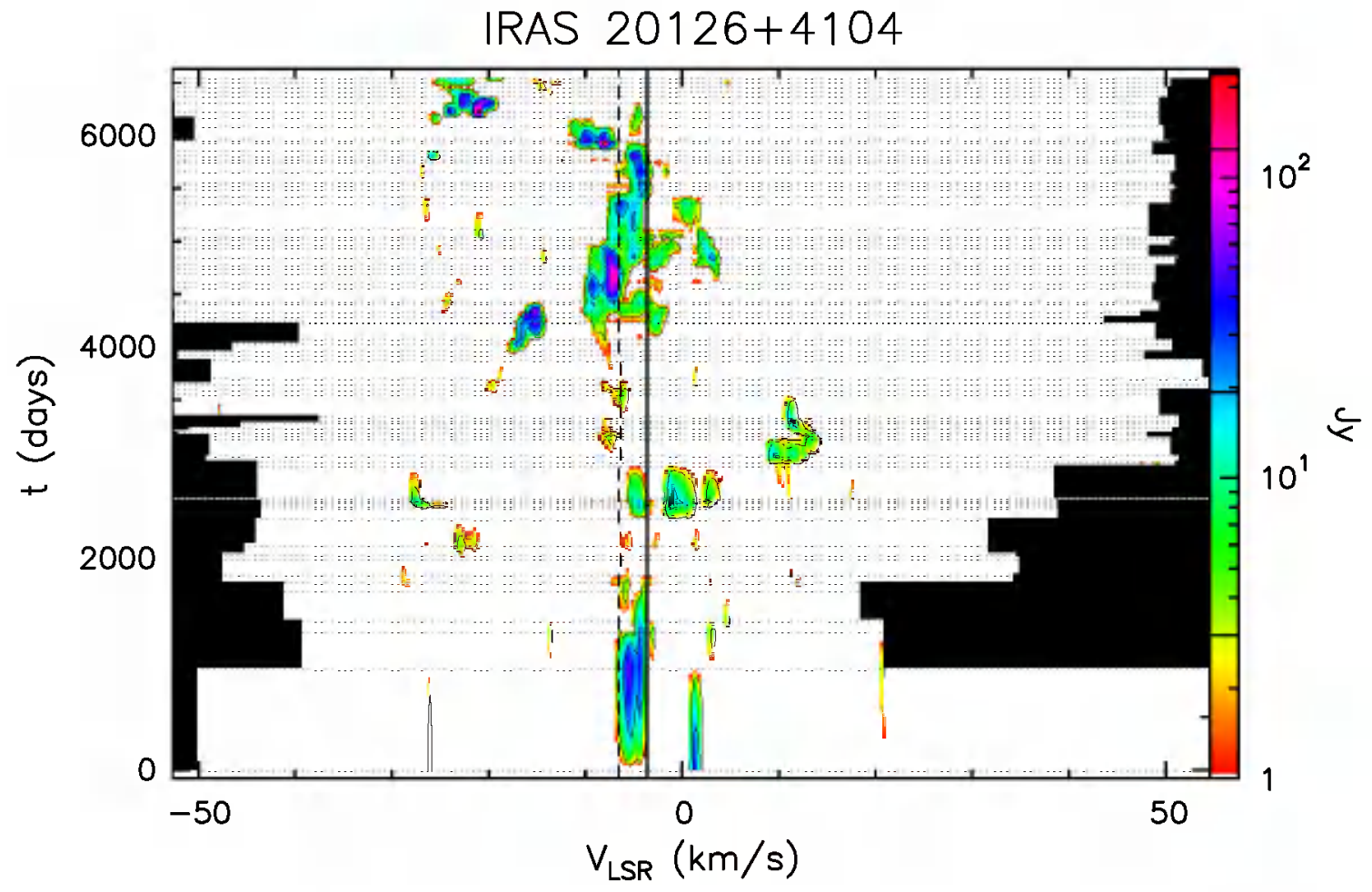

Fig. A.29. c Same as previous figure, but "zoomed" to velocity range over which emission has been detected. 


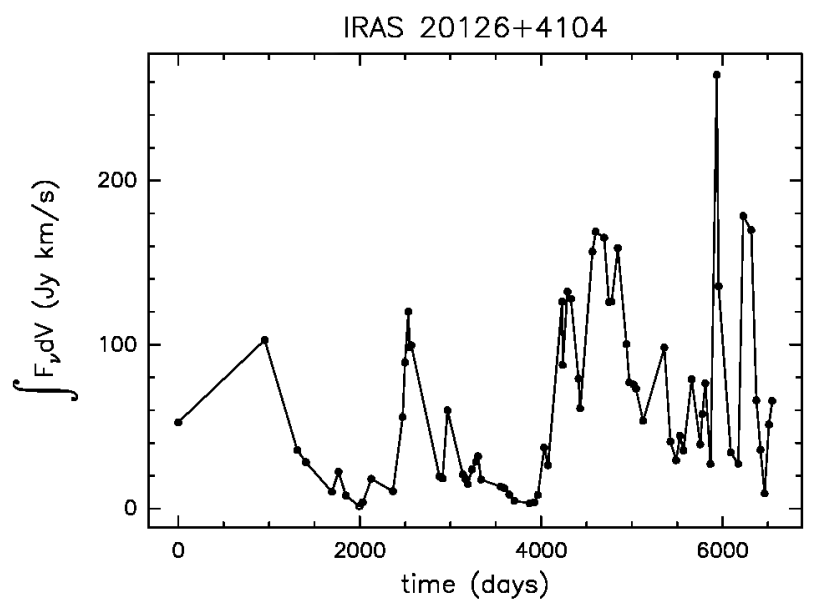

Fig. A.29. d Integral of the flux density over the observed velocity range as a function of time for source IRAS $20126+4104$.

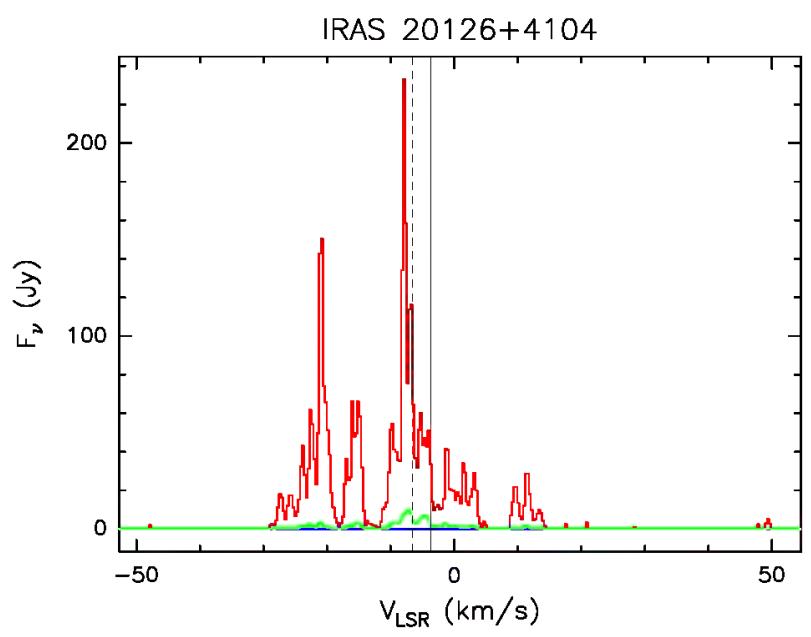

Fig. A.29. e Upper (red) and lower (blue) envelopes and mean spectrum (green) of source IRAS 20126+4104 measured during our monitoring. The vertical solid line marks the velocity of the associated thermal molecular gas. The vertical dashed line marks the mean velocity derived from the histogram of the rate-of-occurrence.

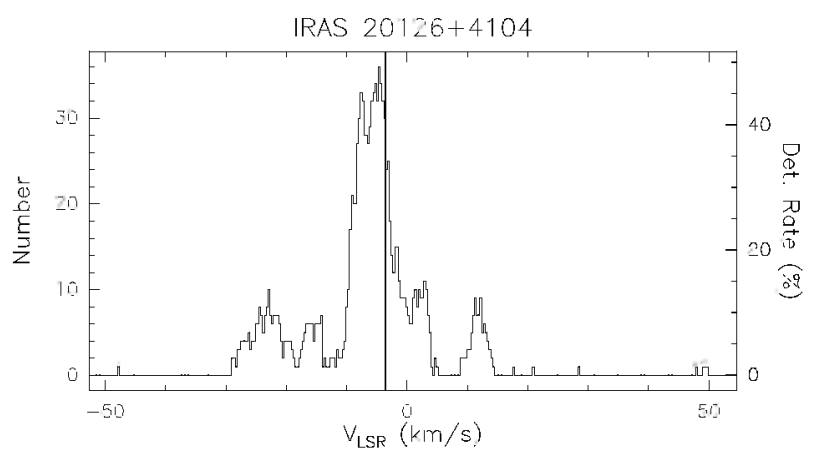

Fig. A.29. f Rate-of-occurrence plot for source IRAS 20126+4104. The scale to the right refers to the dotted histogram. the scale to the left to the solid line histogram. The vertical solid line marks the velocity of the associated thermal molecular gas. 


\section{AFGL 2591}
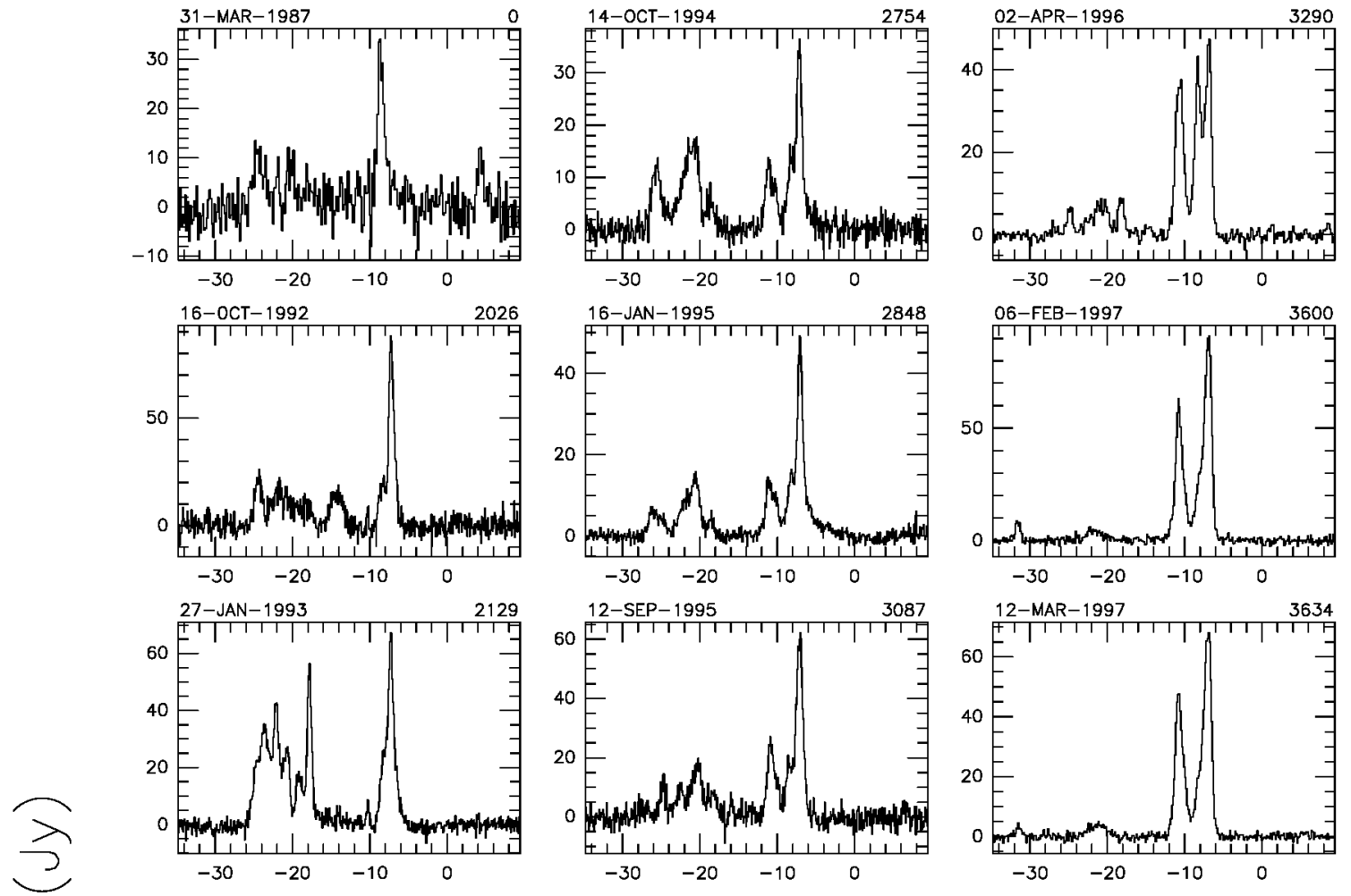

$\left\llcorner^{\lambda}\right.$
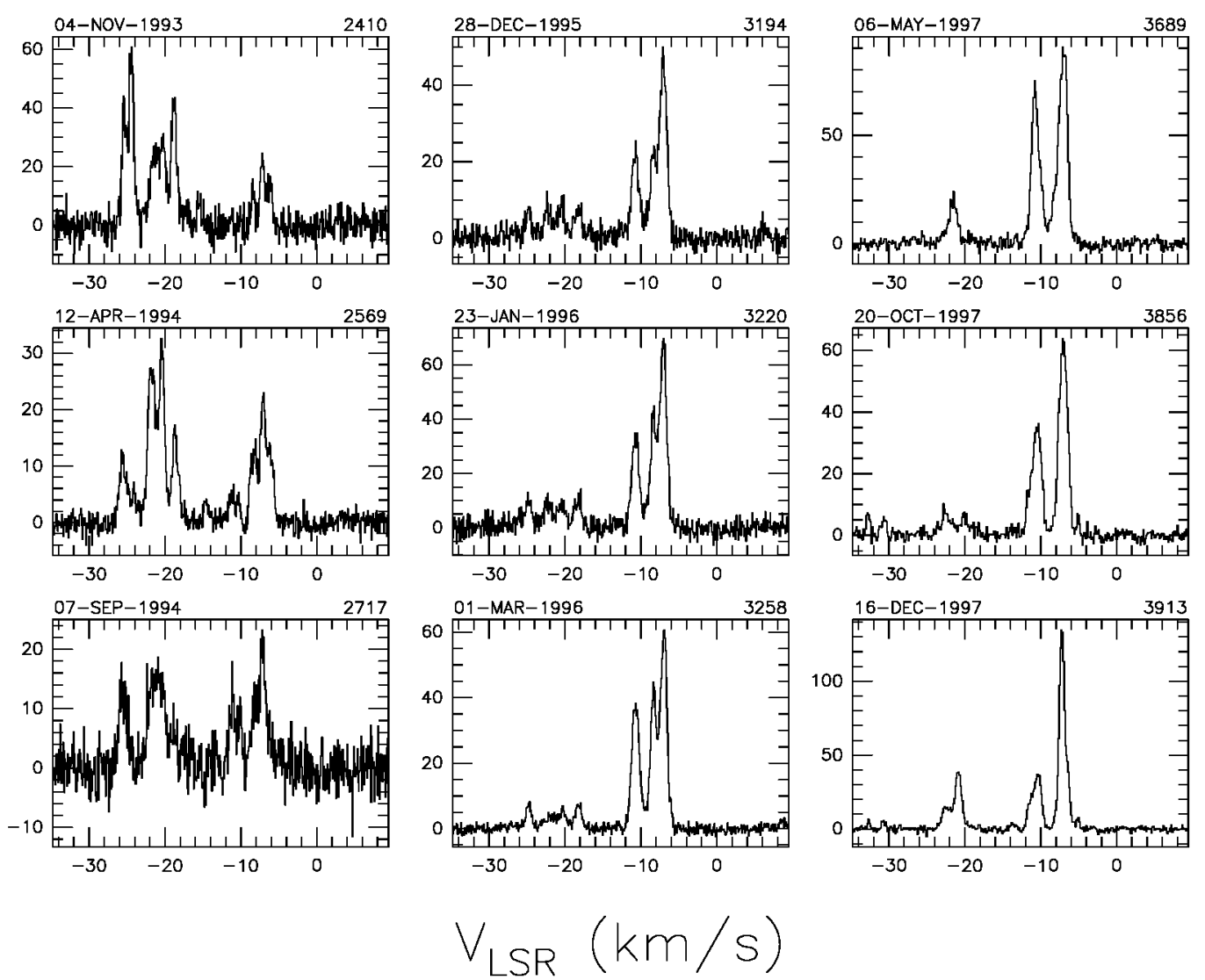

Fig. A.30. a Spectra of source AFGL 2591 with autoscaled flux density scale. The date of observation is shown above the top left corner of each spectrum and the number of days elapsed since the first observation is given above the top right corner. The velocity scale is the same for all spectra. 


\section{AFGL 2591}
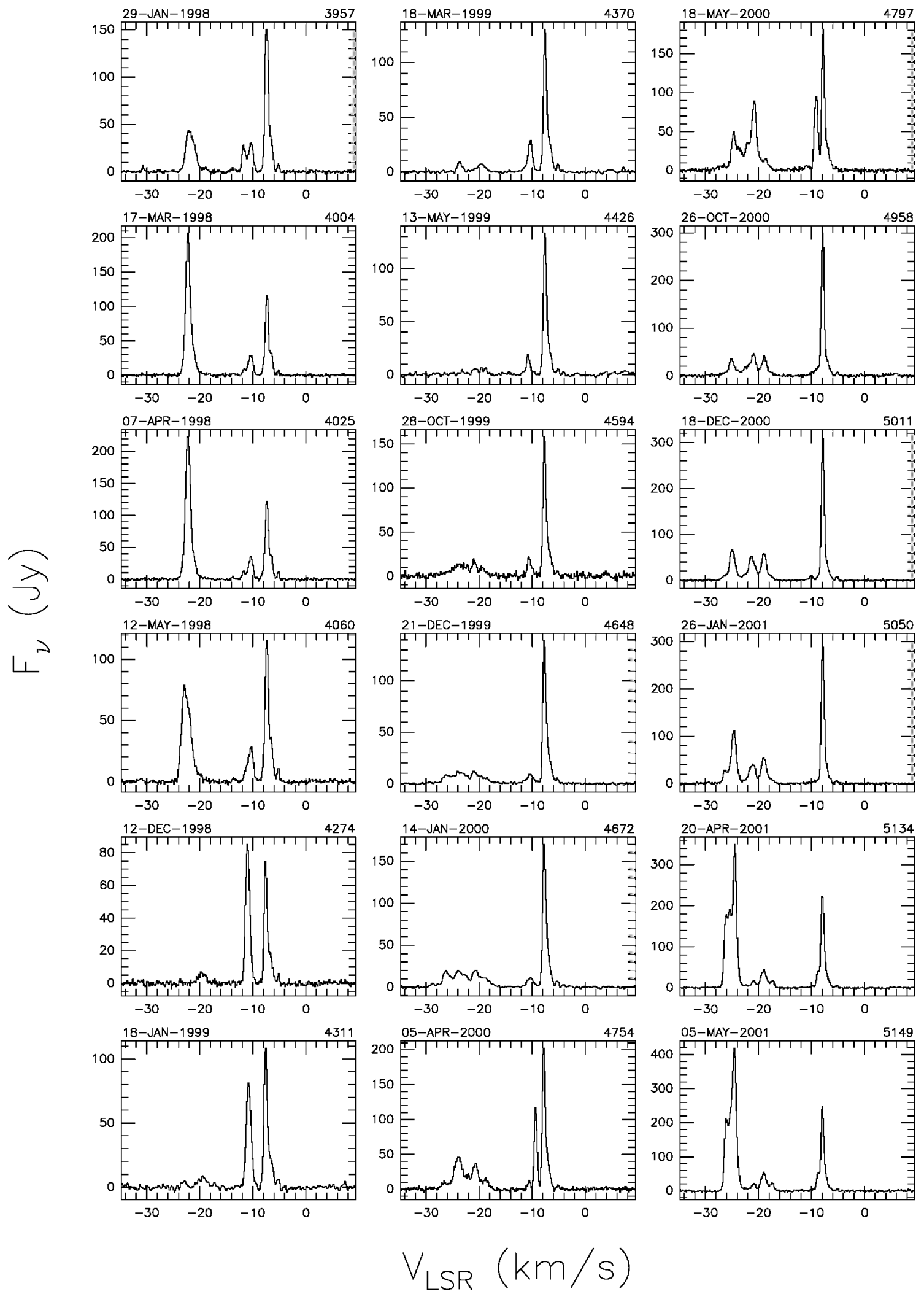

Fig. A.30. a continued. 


\section{AFGL 2591}
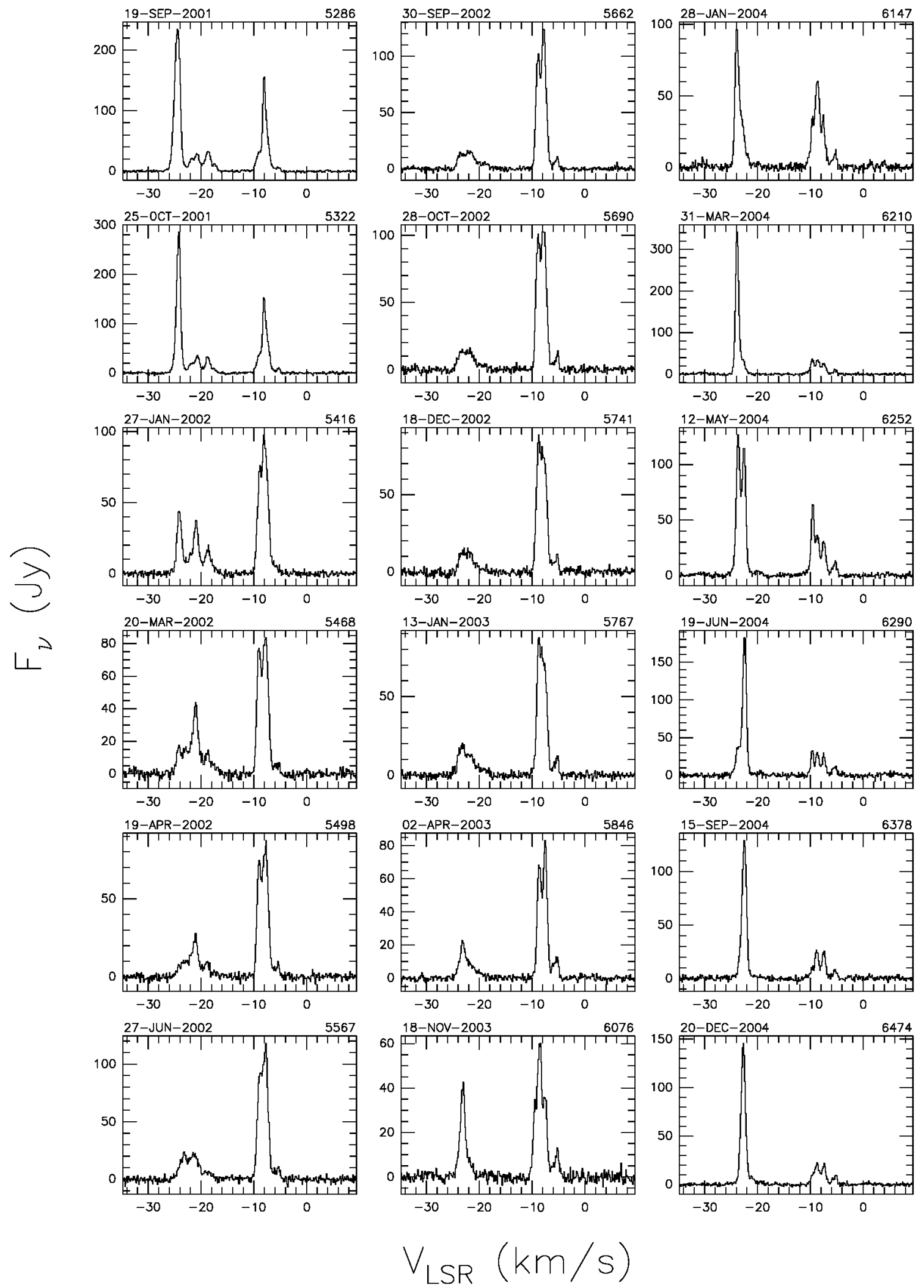

Fig. A.30. a continued. 


\section{AFGL 2591}
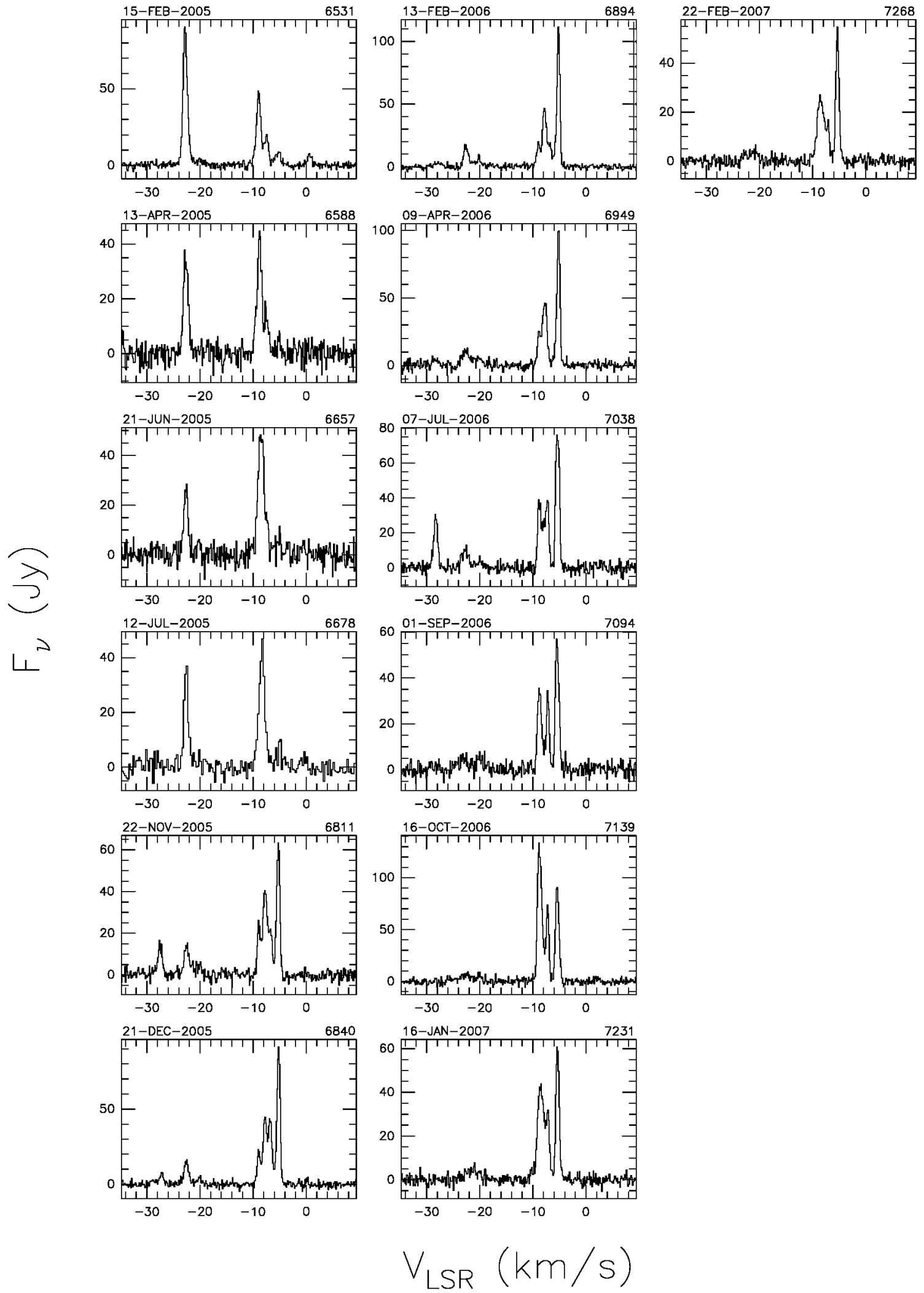

Fig. A.30. a continued. 


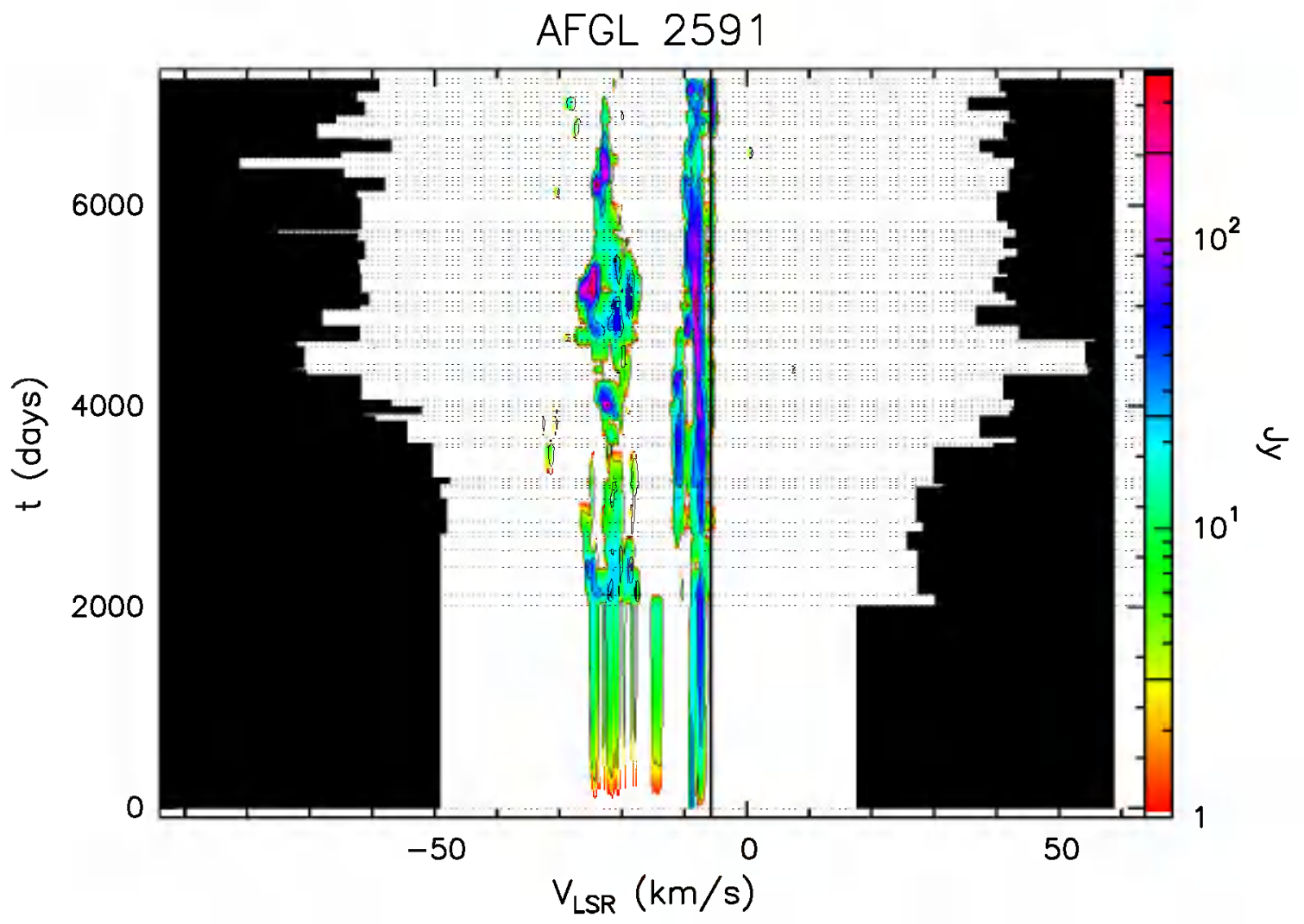

Fig. A.30. b Velocity-time-flux density full plot for source AFGL 2591. The vertical solid line indicates the velocity of the associated thermal molecular gas. The flux density scale is shown by the bar on the right. In this bar the three lines give the flux density of the drawn contours.

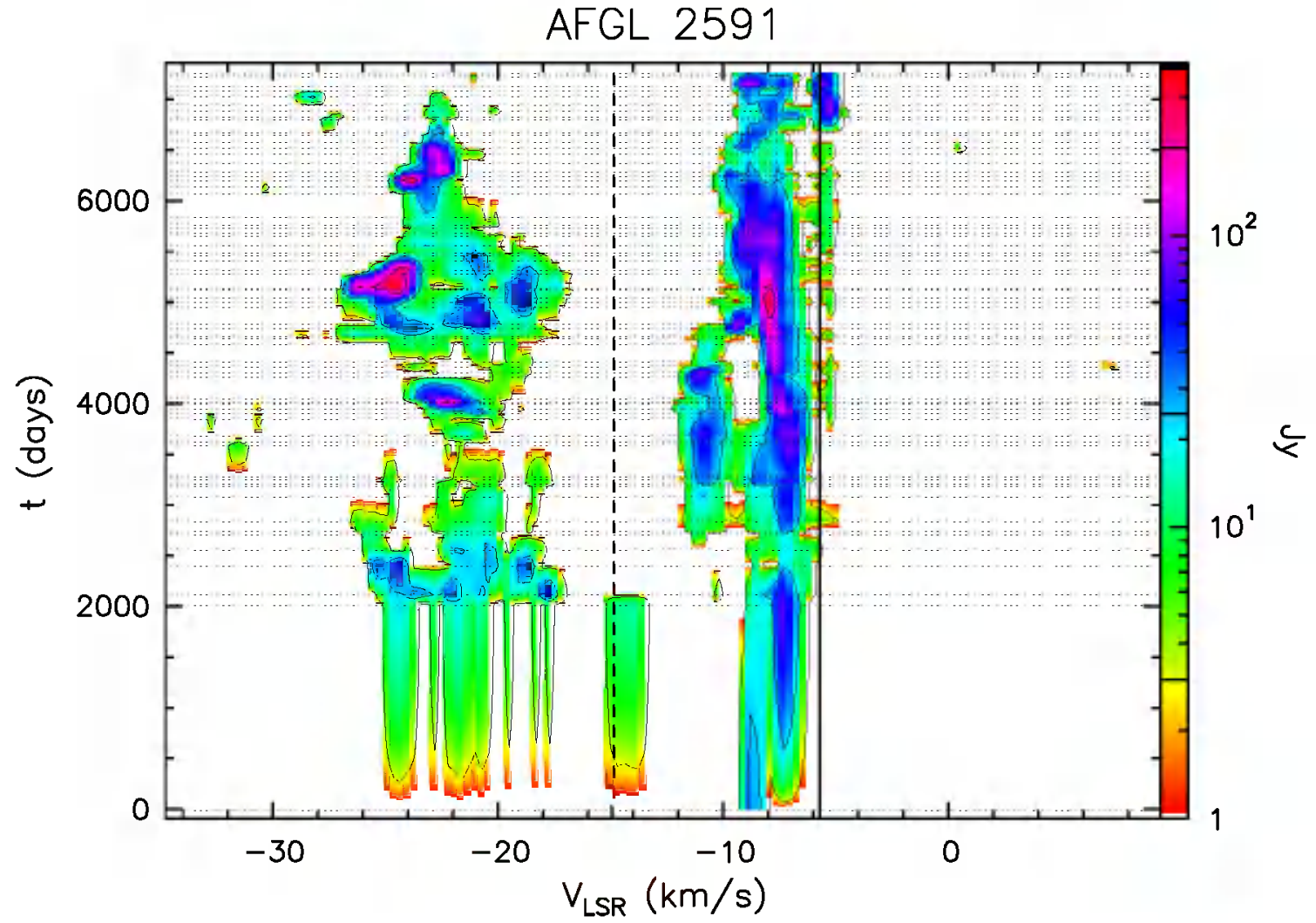

Fig. A.30. c Same as previous figure, but "zoomed" to velocity range over which emission has been detected. 


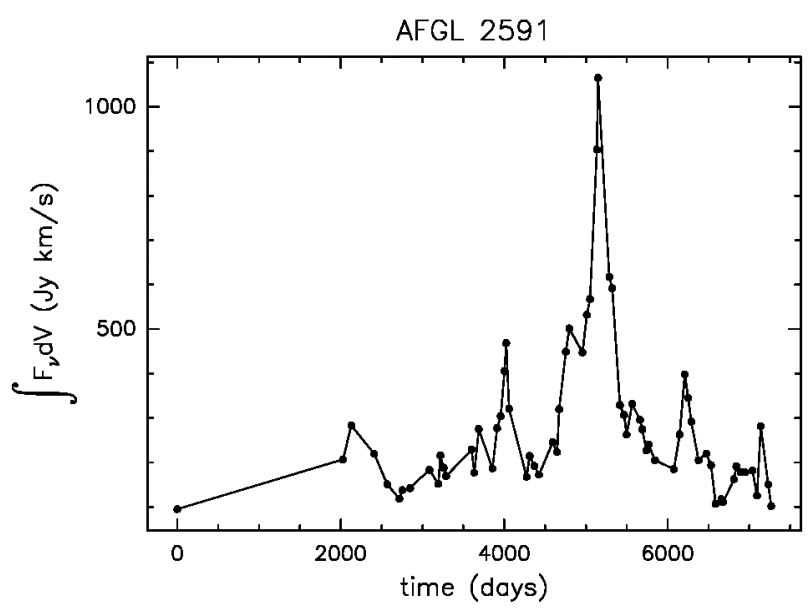

Fig. A.30. d Integral of the flux density over the observed velocity range as a function of time for source AFGL 2591.

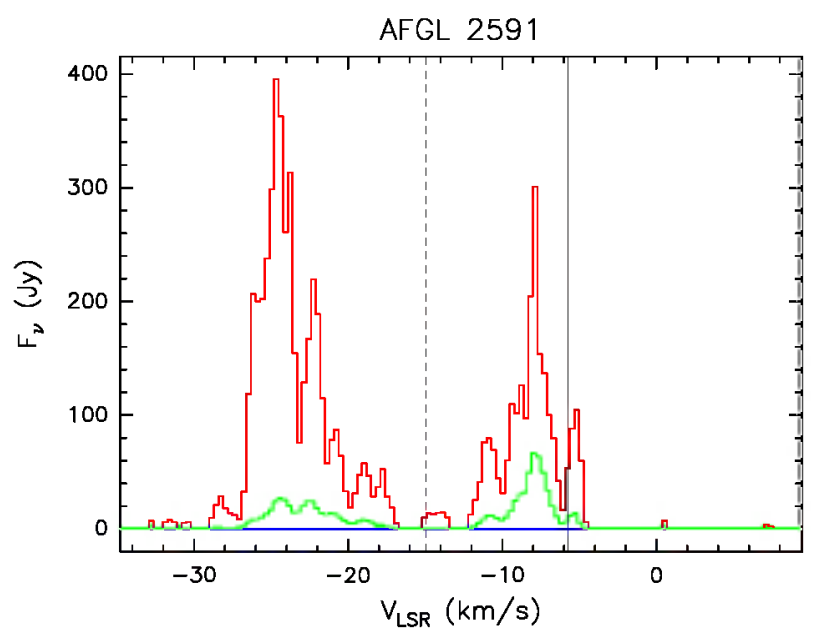

Fig. A.30. e Upper (red) and lower (blue) envelopes and mean spectrum (green) of source AFGL 2591 measured during our monitoring. The vertical solid line marks the velocity of the associated thermal molecular gas. The vertical dashed line marks the mean velocity derived from the histogram of the rate-of-occurrence.

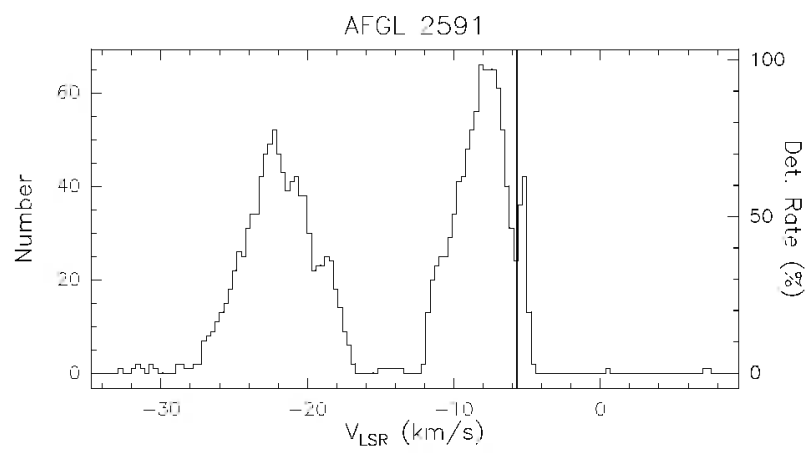

Fig. A.30. f Rate-of-occurrence plot for source AFGL 2591. The scale to the right refers to the dotted histogram. the scale to the left to the solid line histogram. The vertical solid line marks the velocity of the associated thermal molecular gas. 


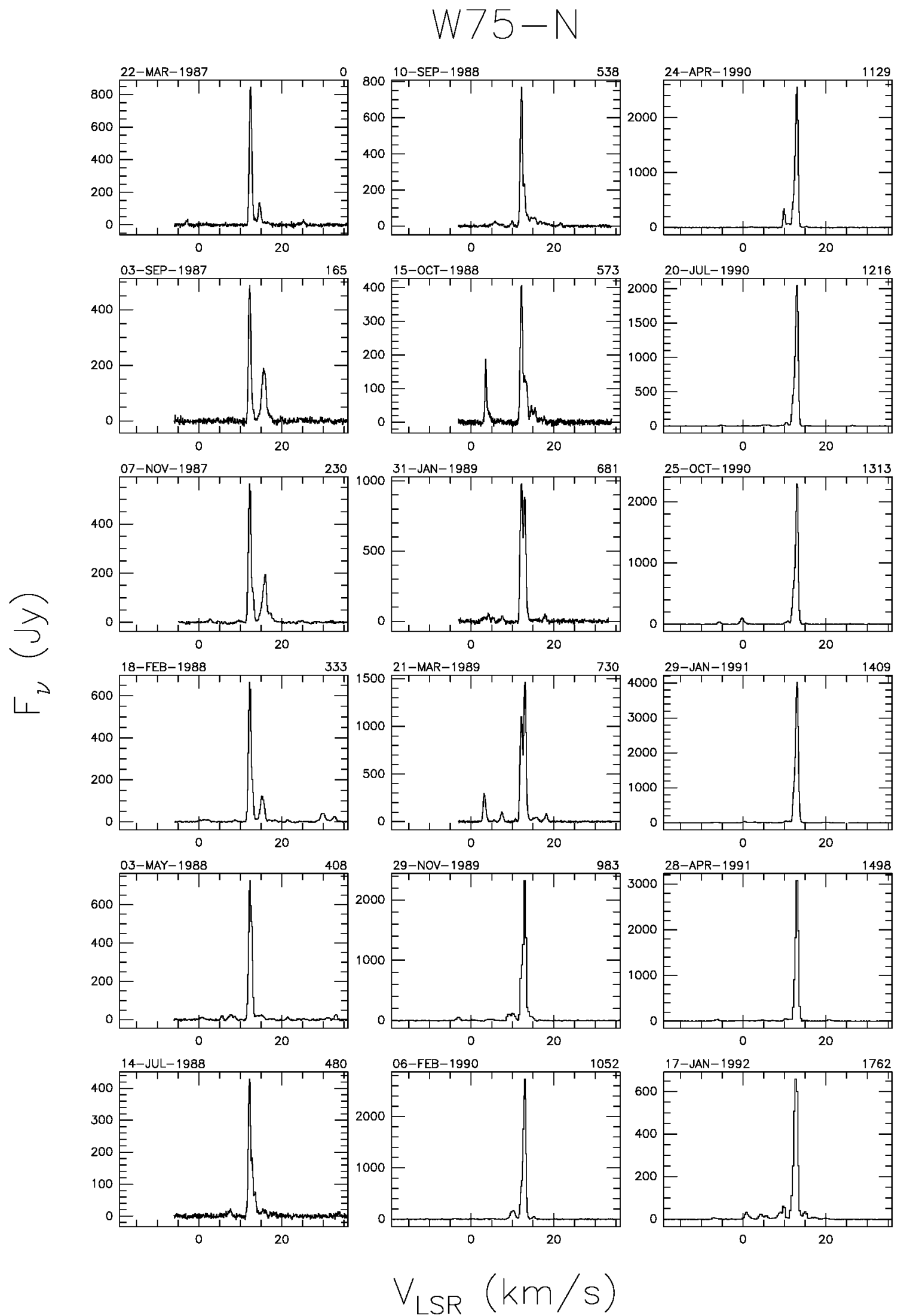

Fig. A.31. a Spectra of source W75-N with autoscaled flux density scale. The date of observation is shown above the top left corner of each spectrum and the number of days elapsed since the first observation is given above the top right corner. The velocity scale is the same for all spectra. 


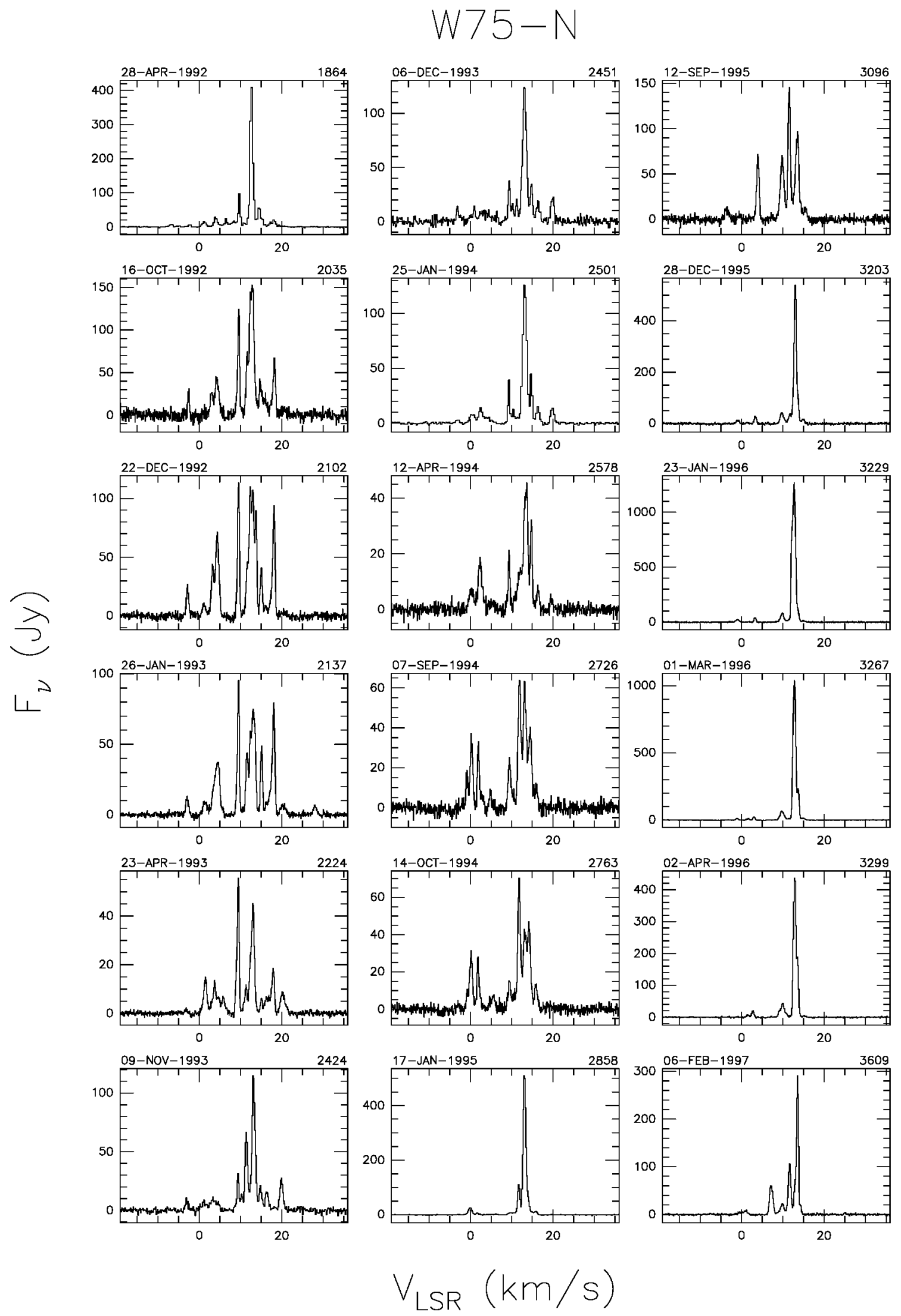

Fig. A.31. a continued. 


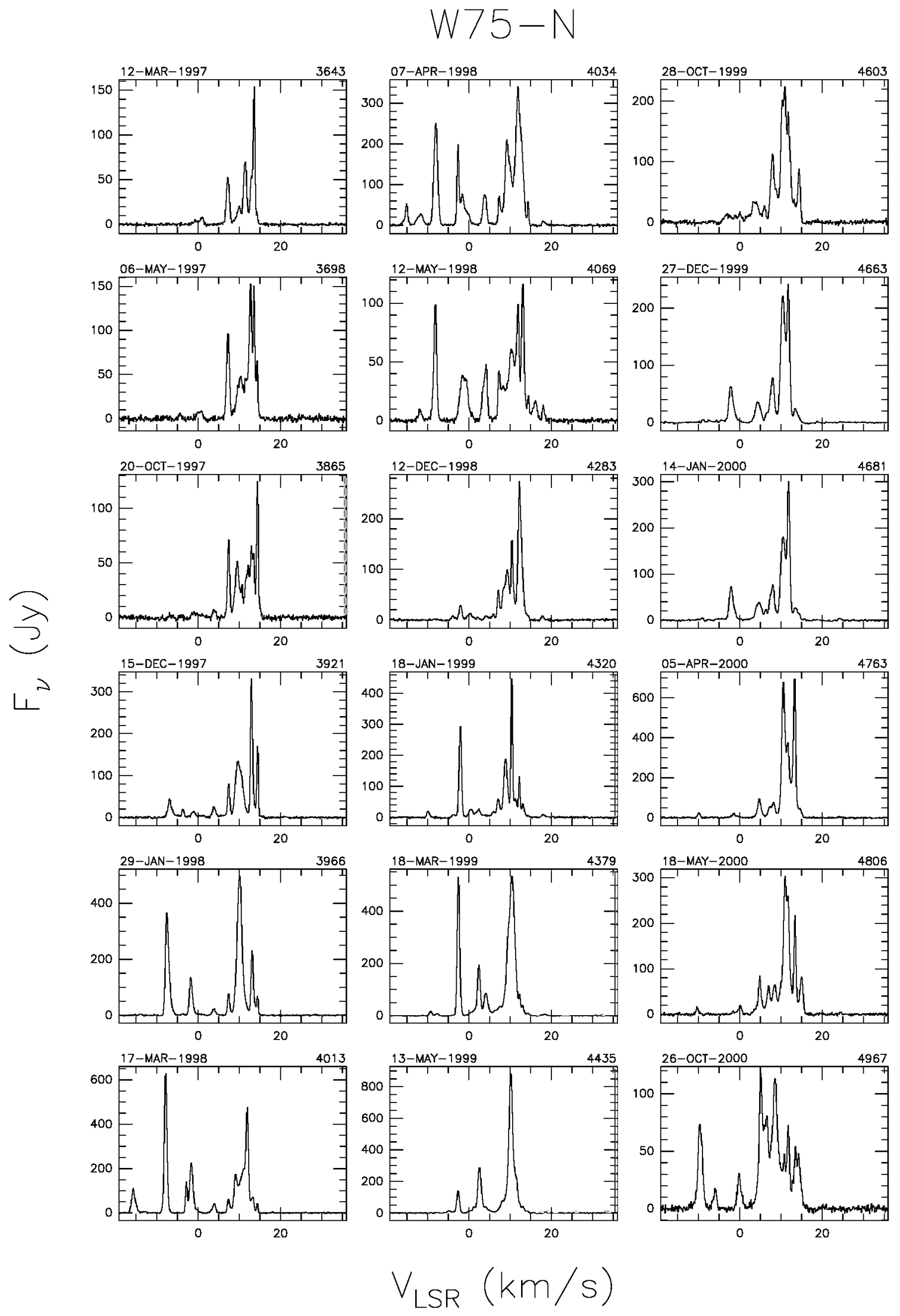

Fig. A.31. a continued. 


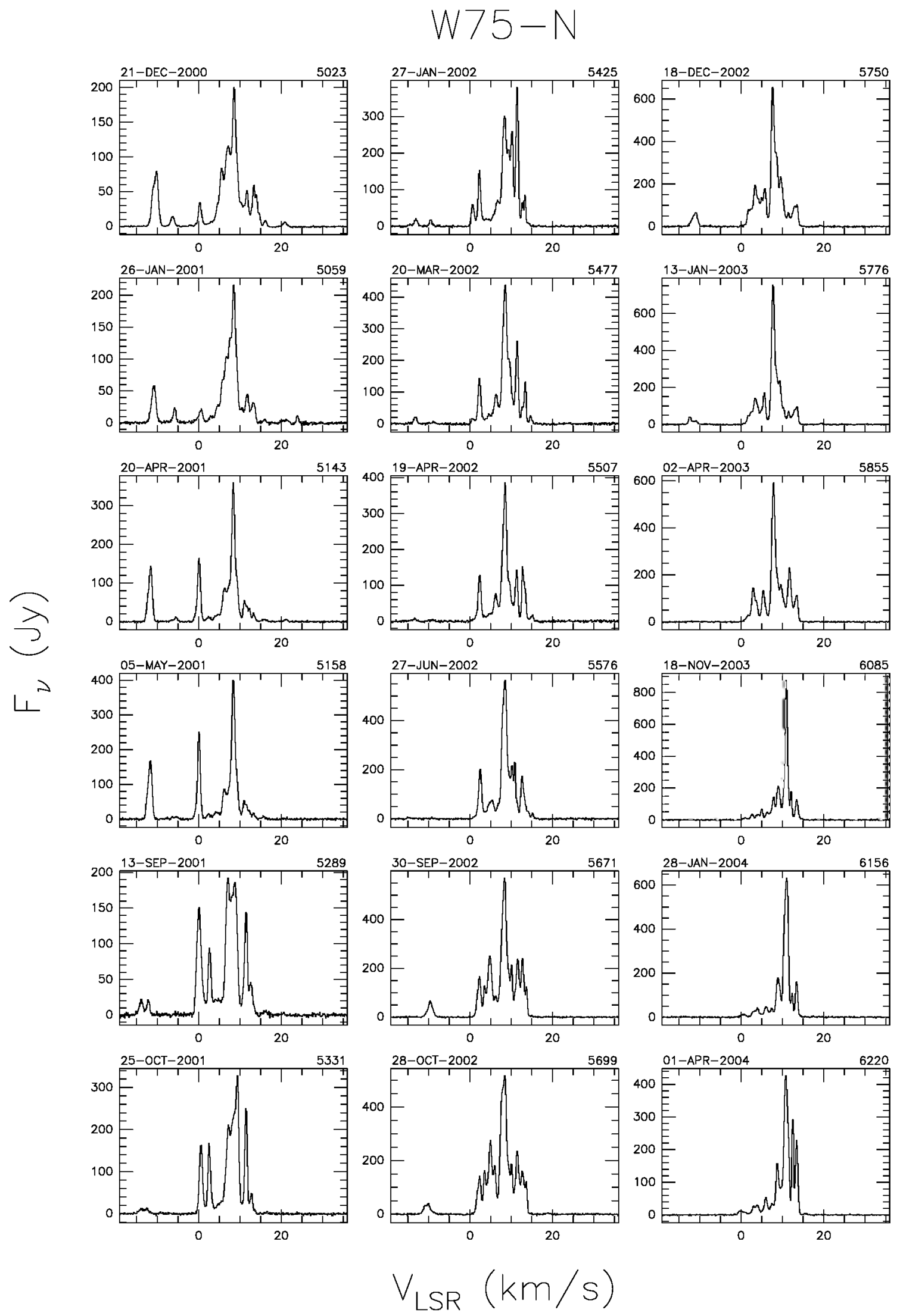

Fig. A.31. a continued. 


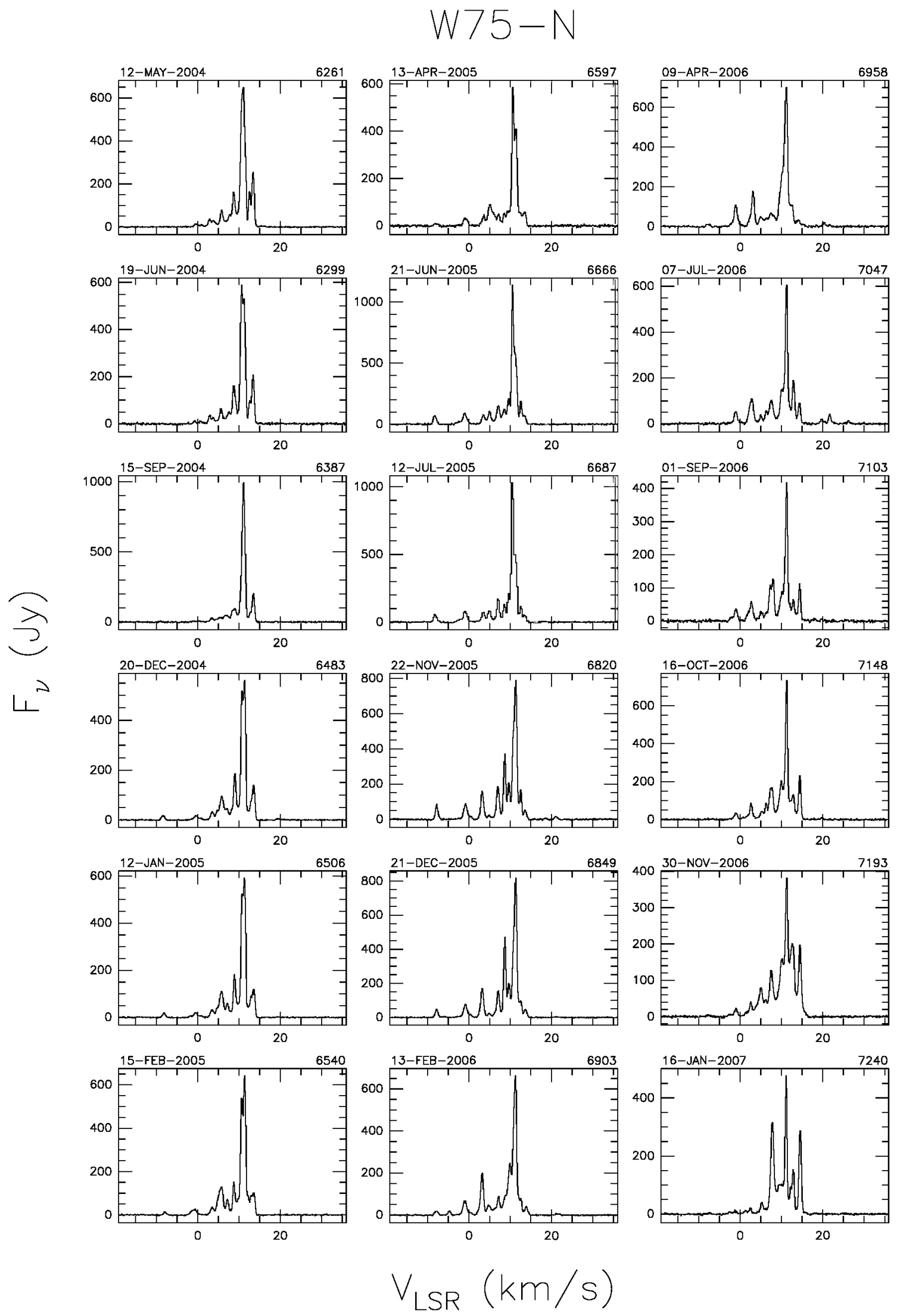

Fig. A.31. a continued. 


$$
\text { W75-N }
$$

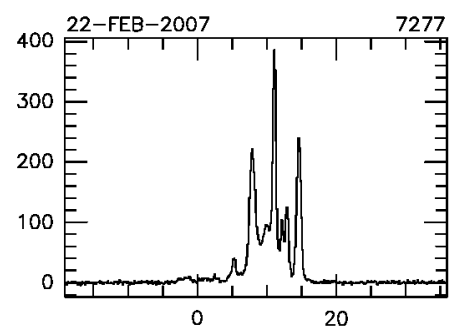

3

$L^{2}$

$$
V_{\text {LSR }}(\mathrm{km} / \mathrm{s})
$$

Fig. A.31. a continued. 


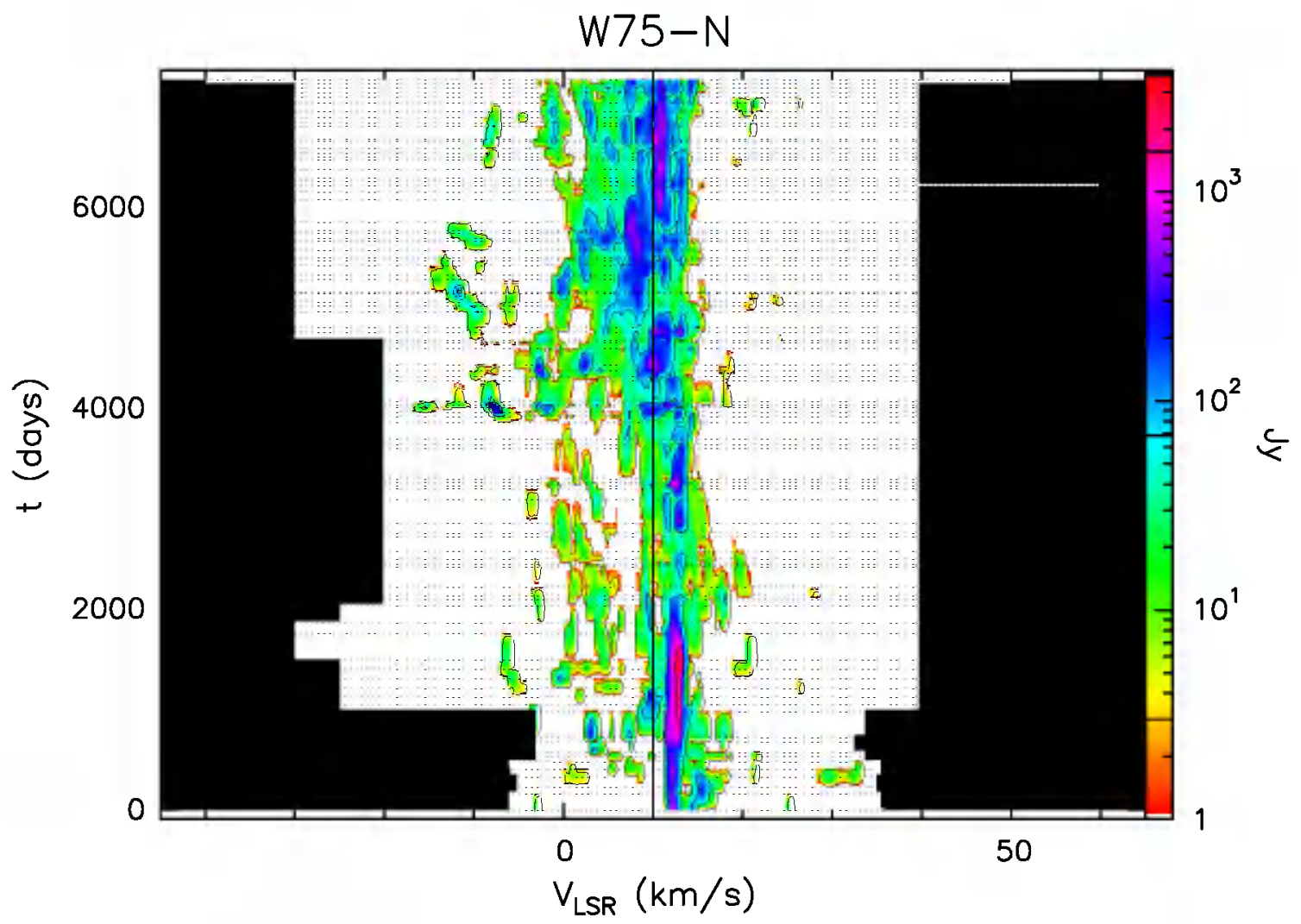

Fig. A.31. b Velocity-time-flux density full plot for source W75-N. The vertical solid line indicates the velocity of the associated thermal molecular gas. The flux density scale is shown by the bar on the right. In this bar the three lines give the flux density of the drawn contours.

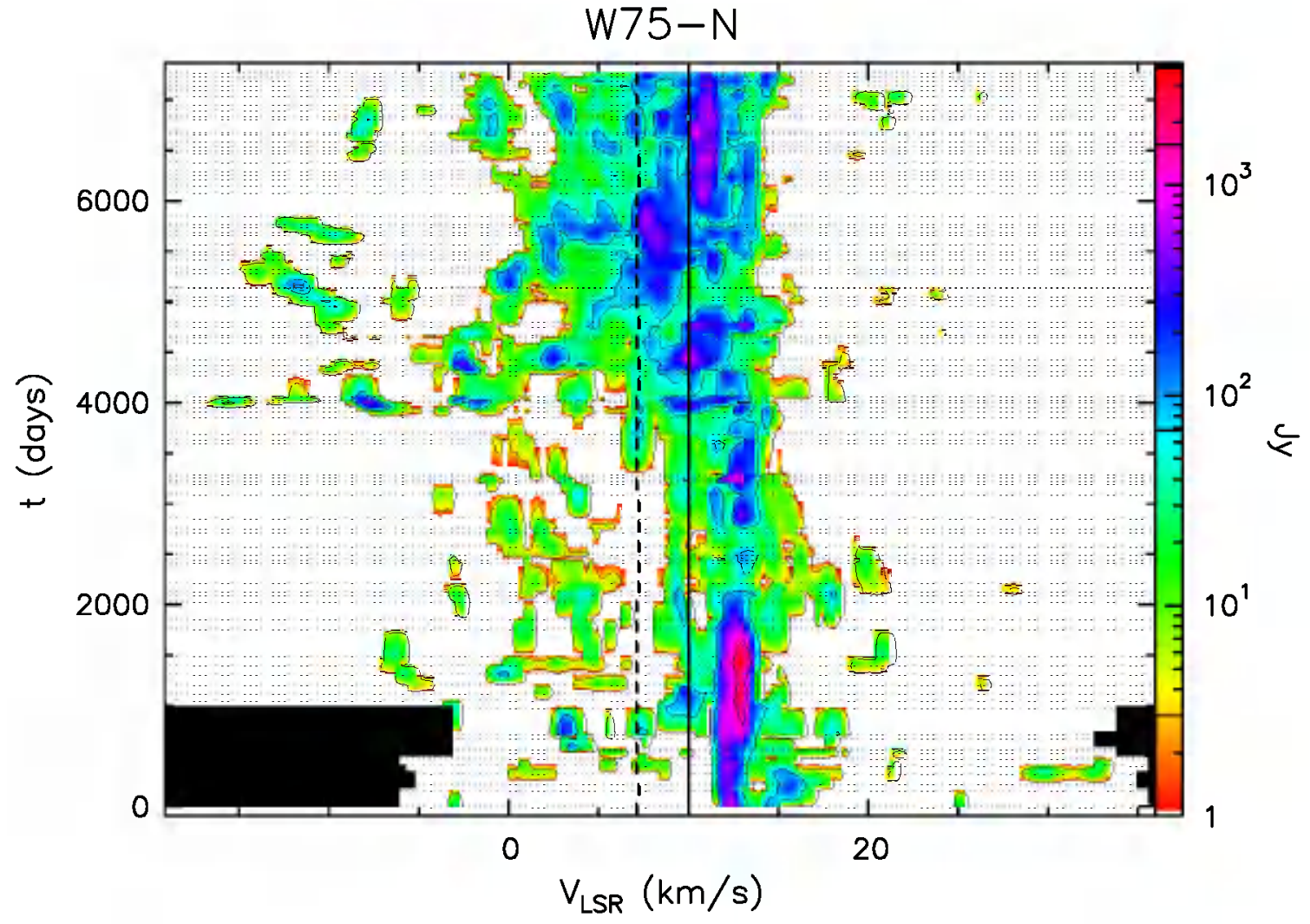

Fig. A.31. c Same as previous figure, but "zoomed" to velocity range over which emission has been detected. 


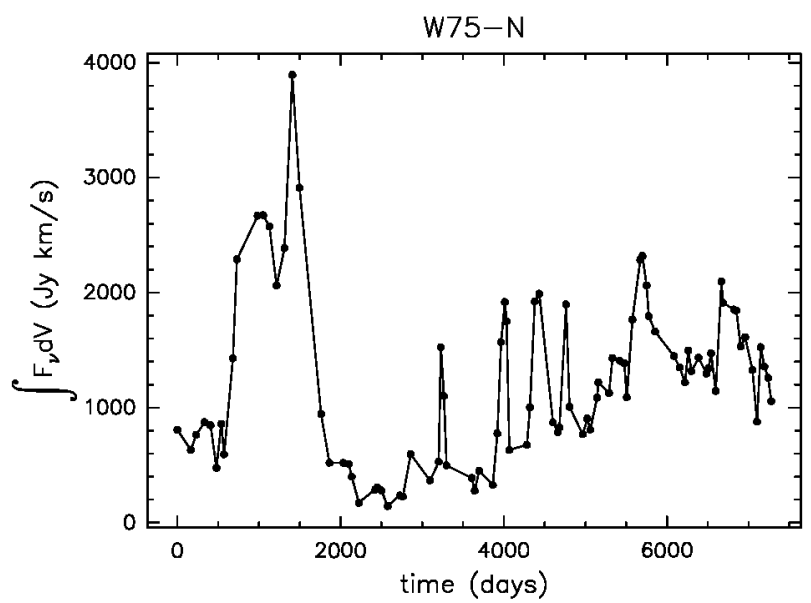

Fig. A.31. d Integral of the flux density over the observed velocity range as a function of time for source W75-N.

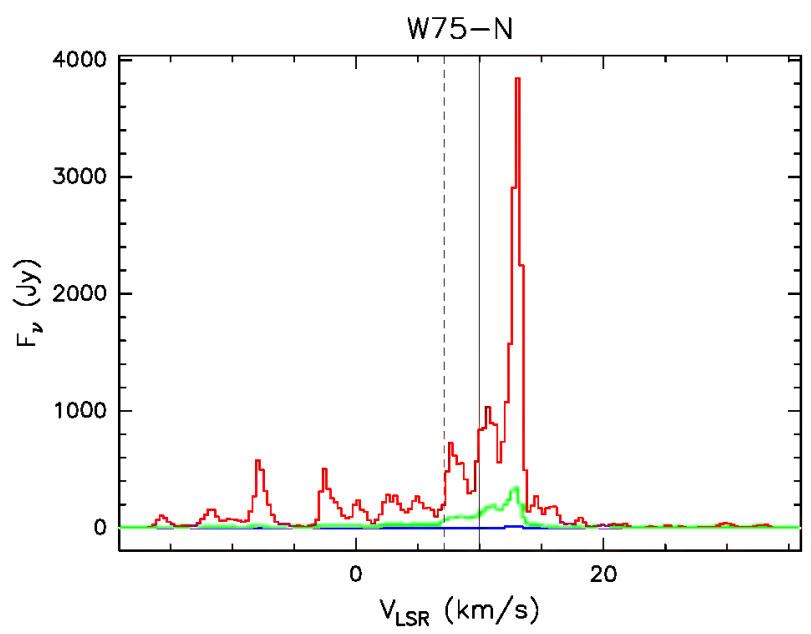

Fig. A.31. e Upper (red) and lower (blue) envelopes and mean spectrum (green) of source W75-N measured during our monitoring. The vertical solid line marks the velocity of the associated thermal molecular gas. The vertical dashed line marks the mean velocity derived from the histogram of the rate-of-occurrence.

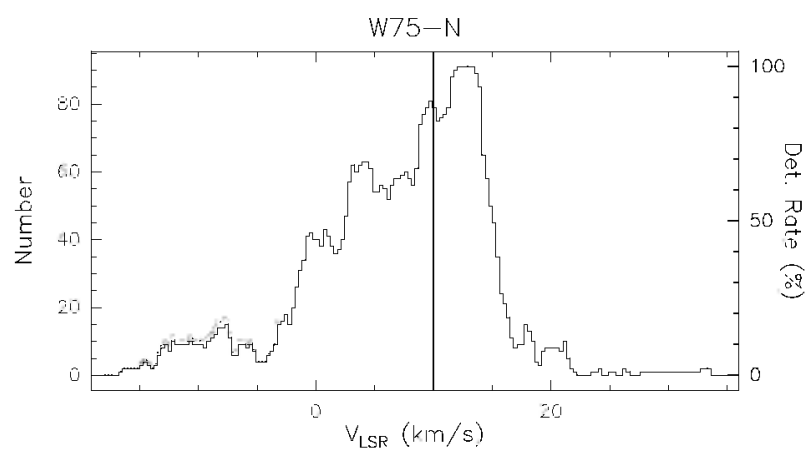

Fig. A.31. f Rate-of-occurrence plot for source W75-N. The scale to the right refers to the dotted histogram. the scale to the left to the solid line histogram. The vertical solid line marks the velocity of the associated thermal molecular gas. 

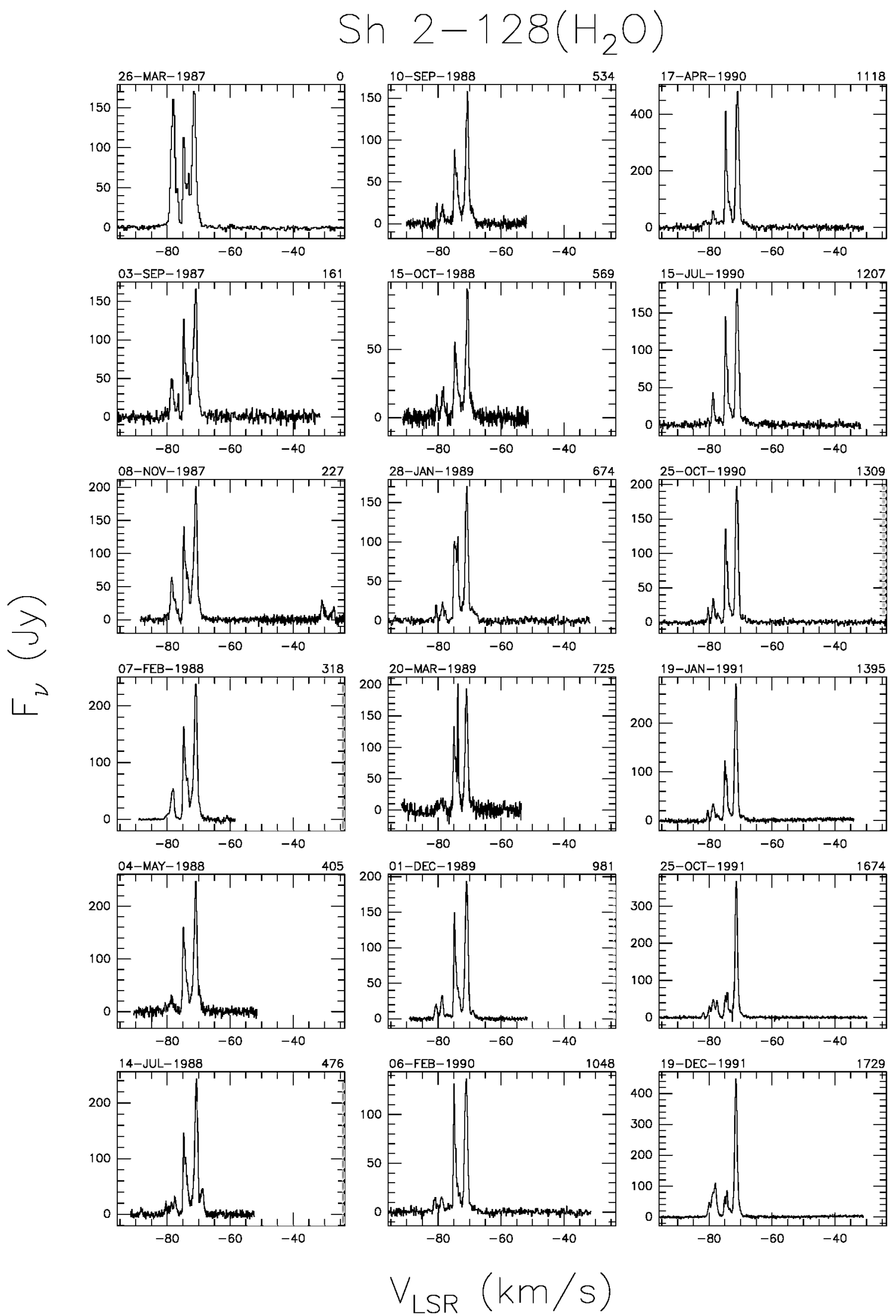

Fig. A.32. a Spectra of source $\mathrm{Sh} 2-128\left(\mathrm{H}_{2} \mathrm{O}\right)$ with antoscaled flux density scale. The date of observation is shown above the top left corner of each spectrum and the number of days elapsed since the first observation is given above the top right corner. The velocity scale is the same for all spectra. 


$$
\text { Sh } 2-128\left(\mathrm{H}_{2} \mathrm{O}\right)
$$
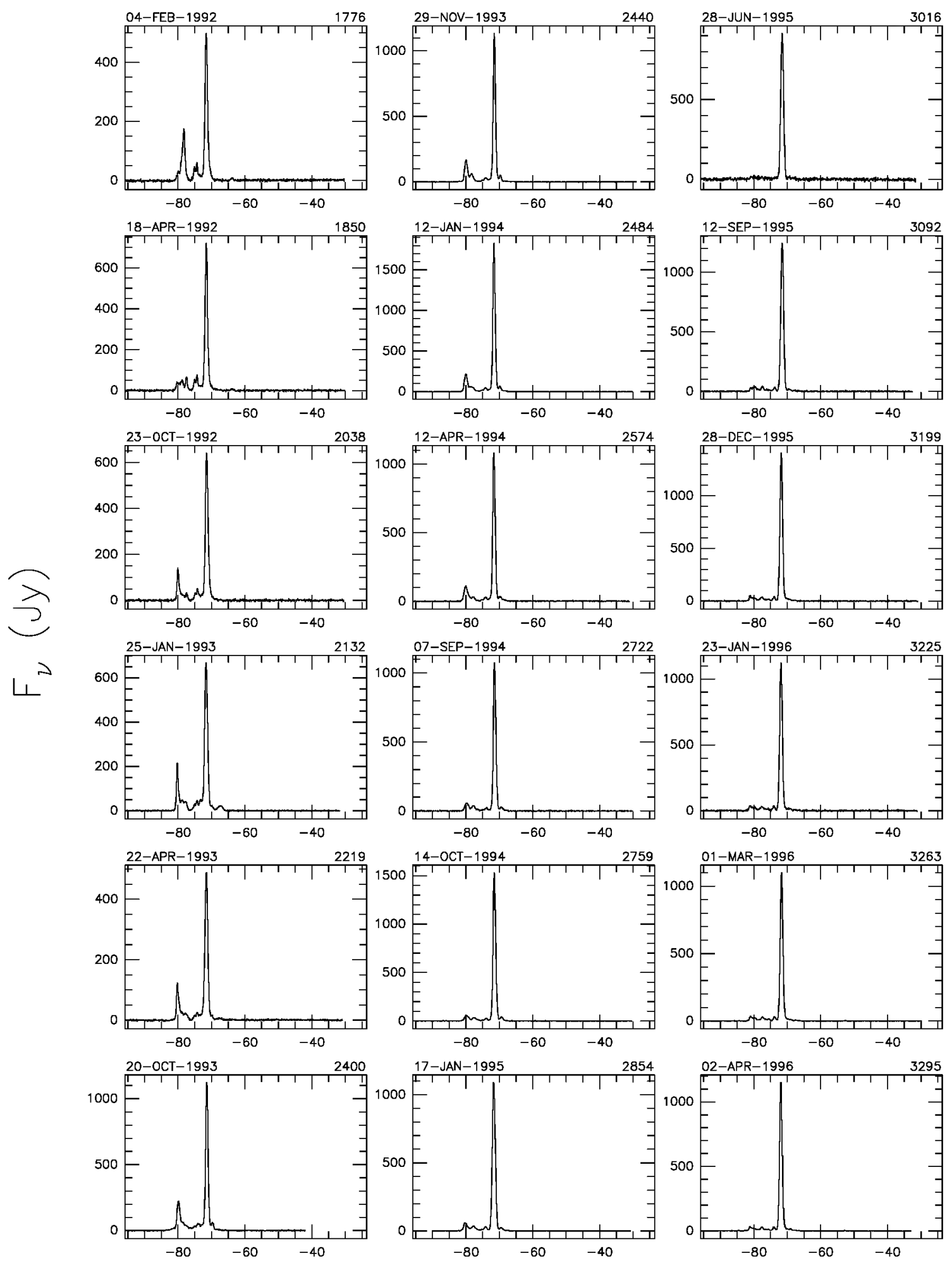

$$
V_{\text {LSR }}(\mathrm{km} / \mathrm{s})
$$

Fig. A.32. a continued. 


$$
\text { Sh } 2-128\left(\mathrm{H}_{2} \mathrm{O}\right)
$$
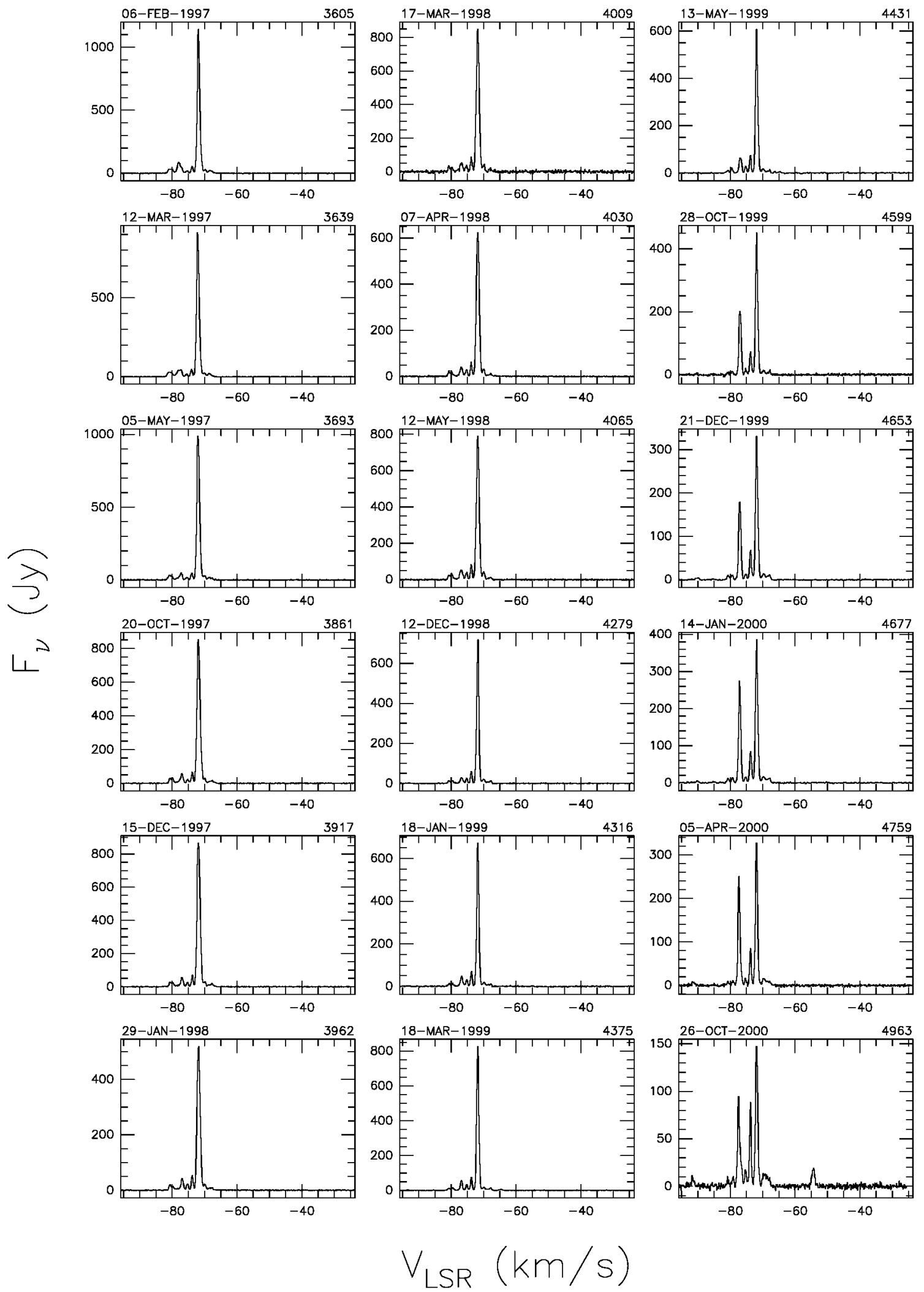

Fig. A.32. a continued. 


$$
\text { Sh } 2-128\left(\mathrm{H}_{2} \mathrm{O}\right)
$$
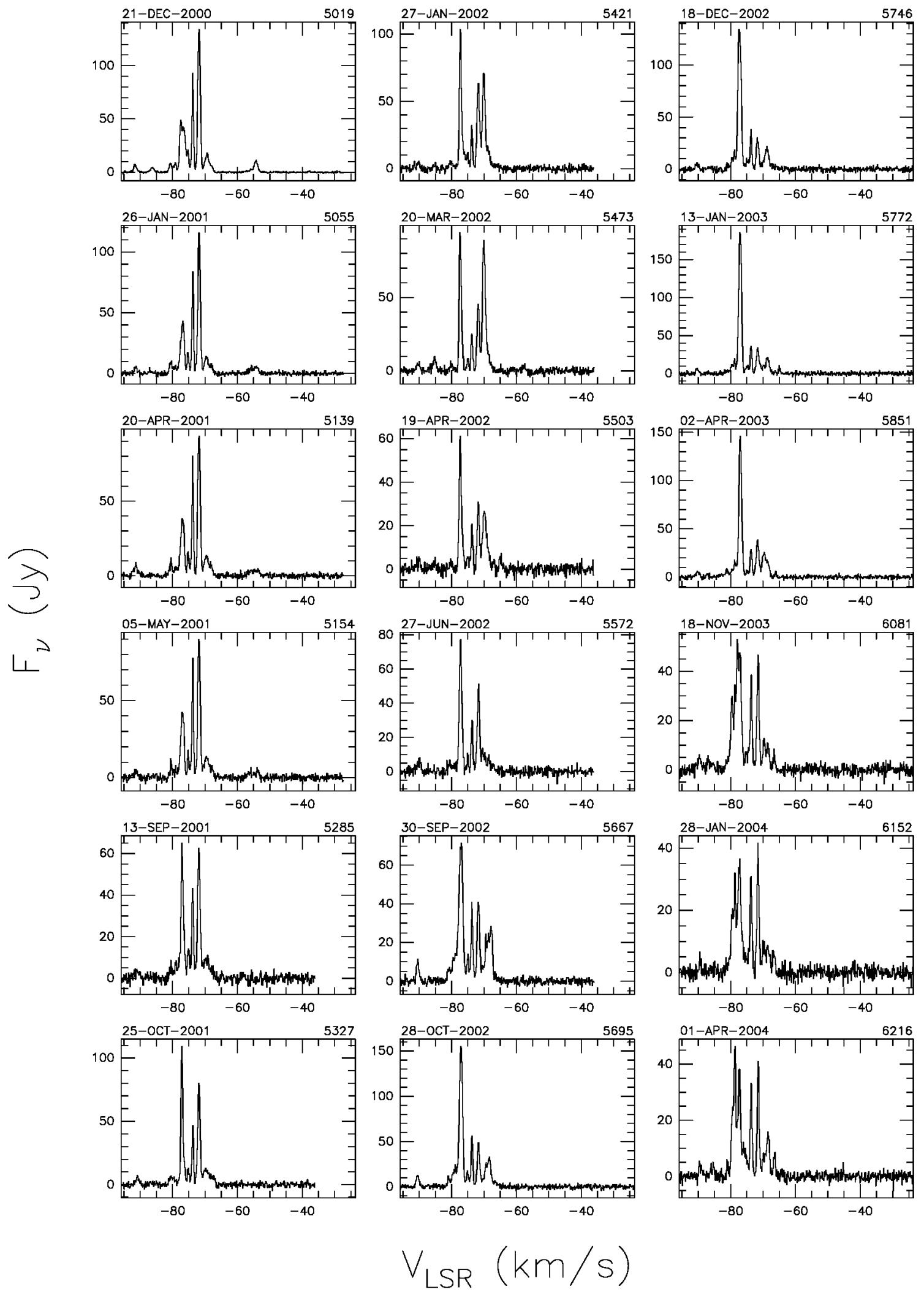

Fig. A.32. a continued. 


$$
\text { Sh } 2-128\left(\mathrm{H}_{2} \mathrm{O}\right)
$$
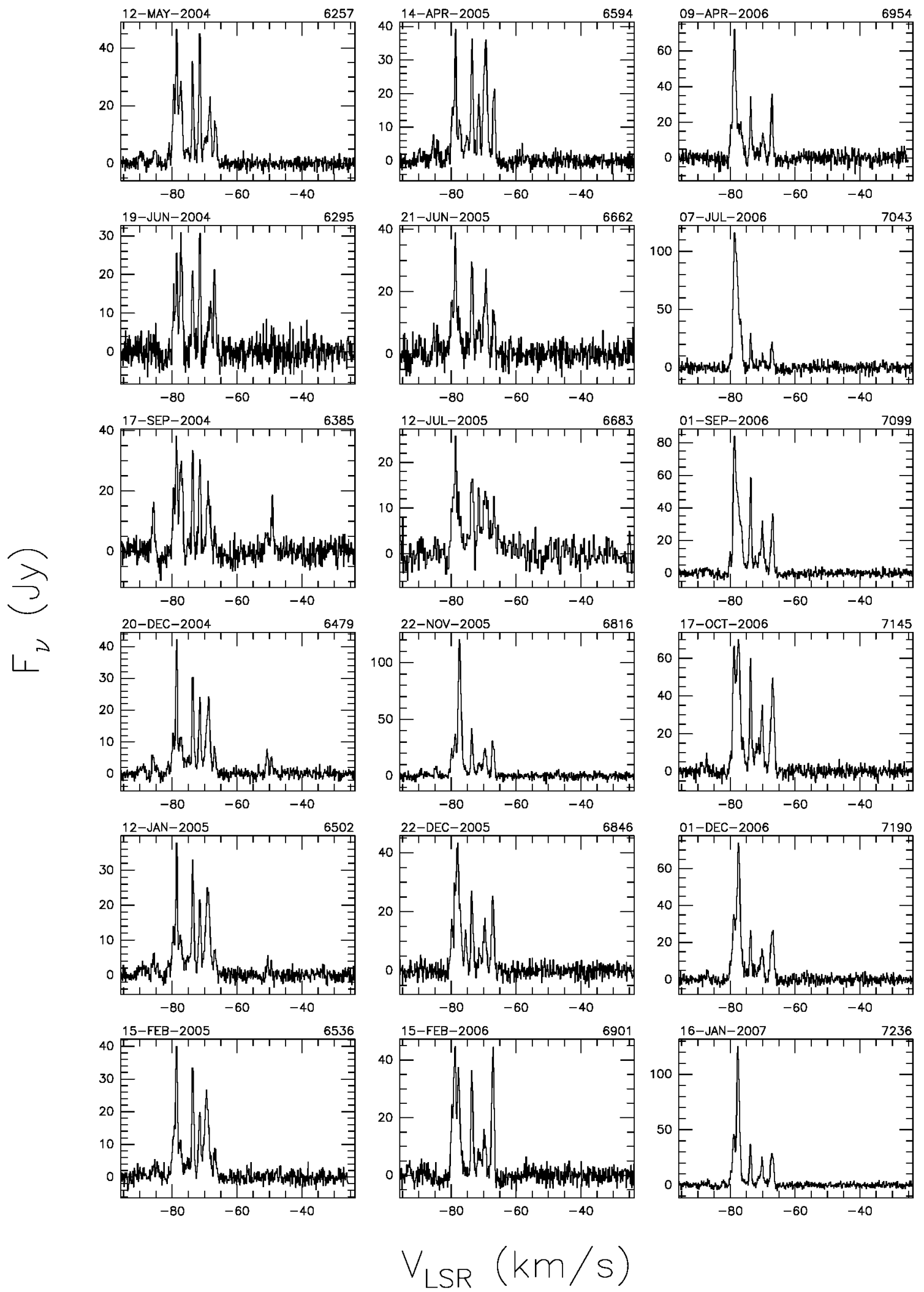

Fig. A.32. a continued. 
Sh $2-128\left(\mathrm{H}_{2} \mathrm{O}\right)$

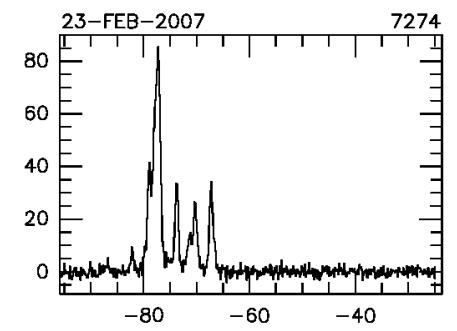

3

$\left\llcorner^{\curvearrowright}\right.$

$$
V_{\text {LSR }}(k m / s)
$$

Fig. A.32. a continued. 


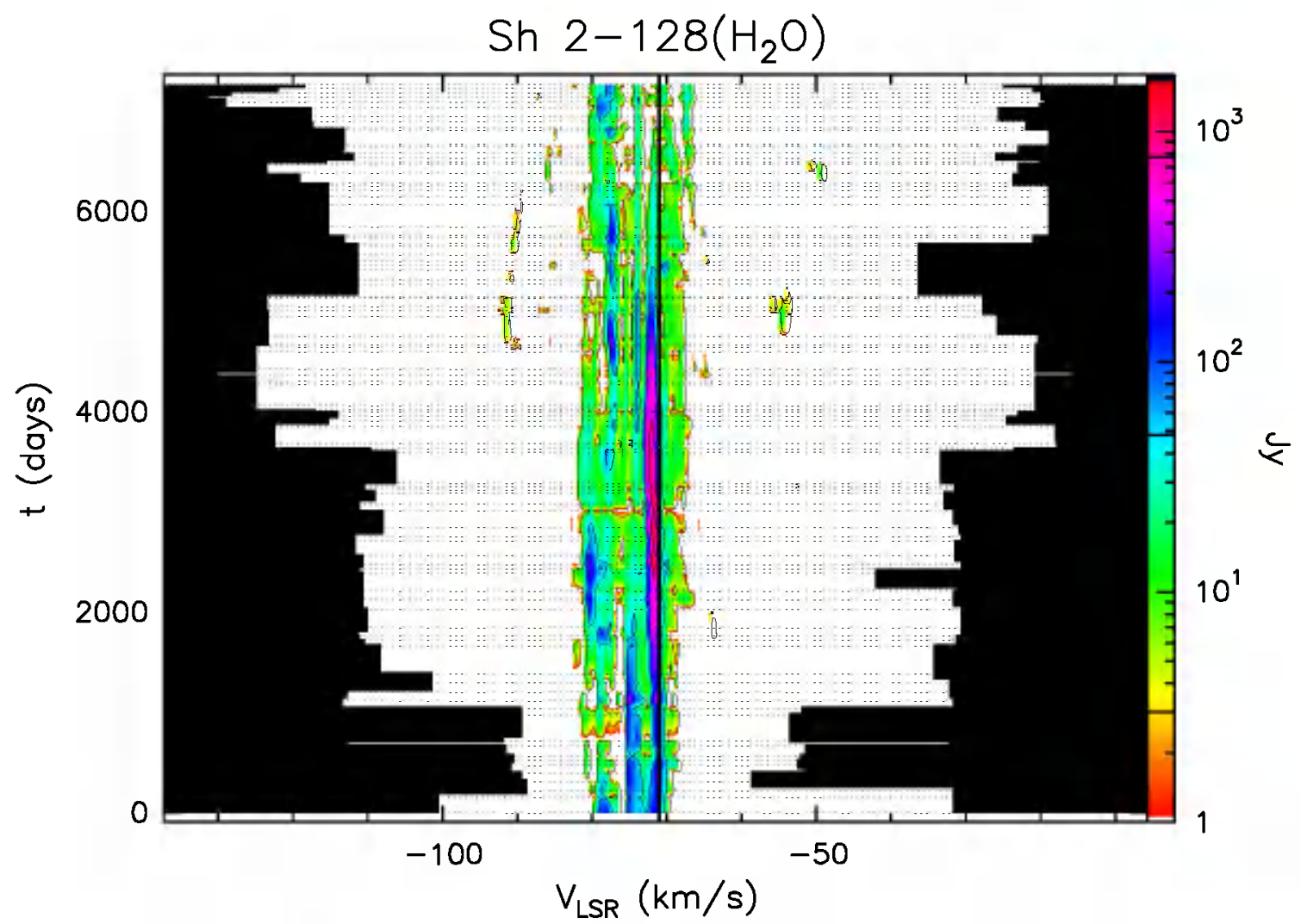

Fig. A.32. b Velocity-time-flux density full plot for source Sh $2-128\left(\mathrm{H}_{2} \mathrm{O}\right)$. The vertical solid line indicates the velocity of the associated thermal molecular gas. The flux density scale is shown by the bar on the right. In this bar the three lines give the flux density of the drawn contours.

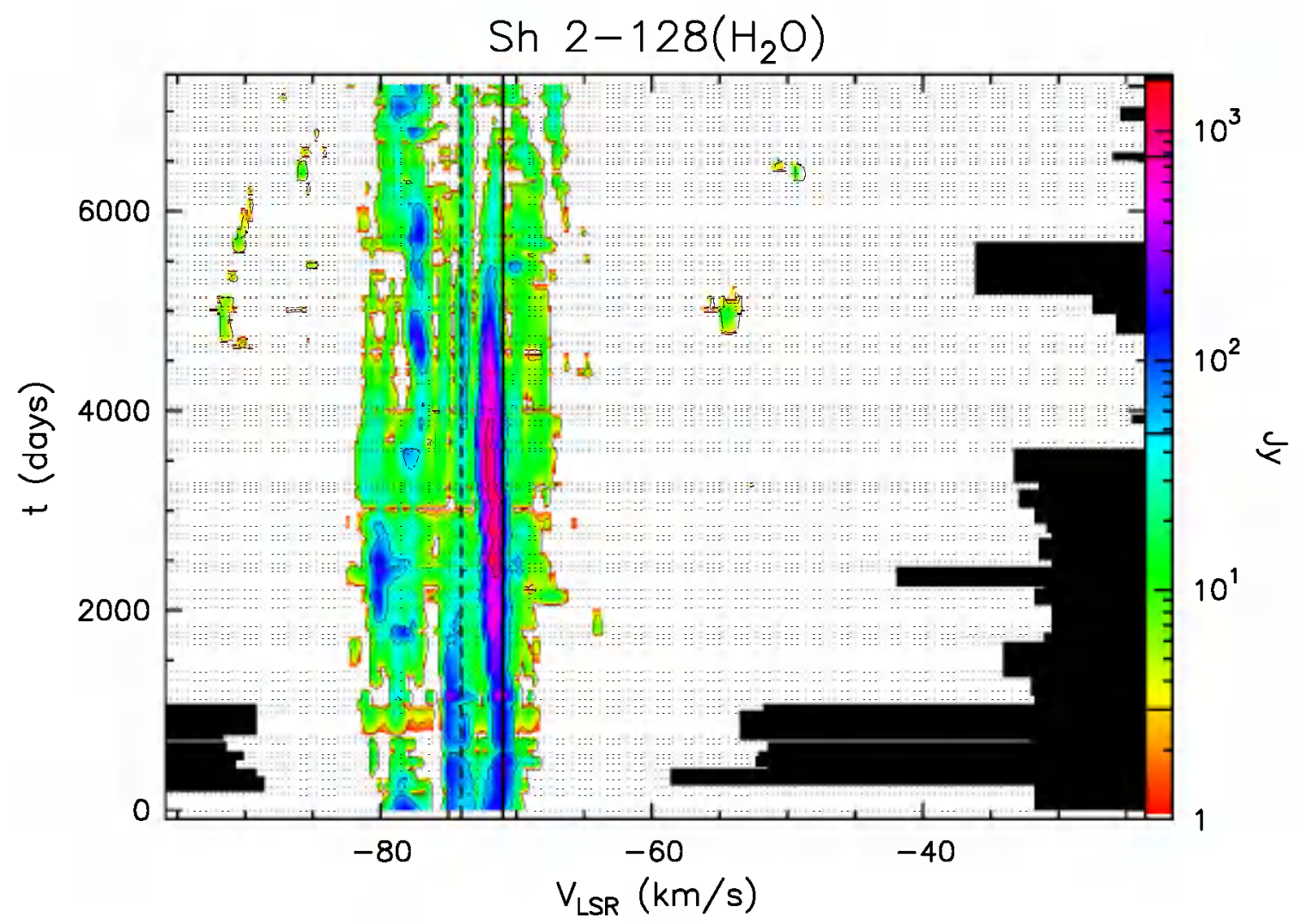

Fig. A.32. c Same as previous figure, but "zoomed" to velocity range over which emission has been detected. 


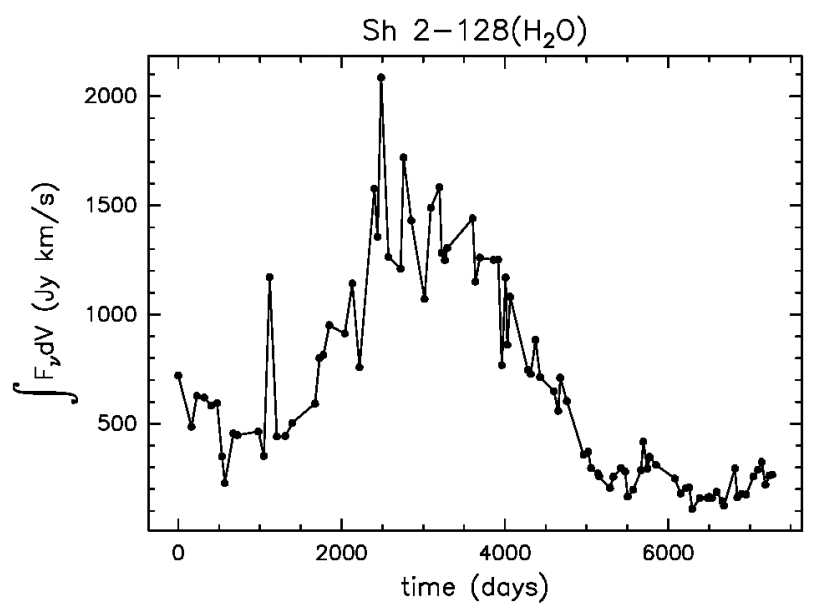

Fig. A.32. d Integral of the flux density over the observed velocity range as a function of time for source $\mathrm{Sh} 2-128\left(\mathrm{H}_{2} \mathrm{O}\right)$.

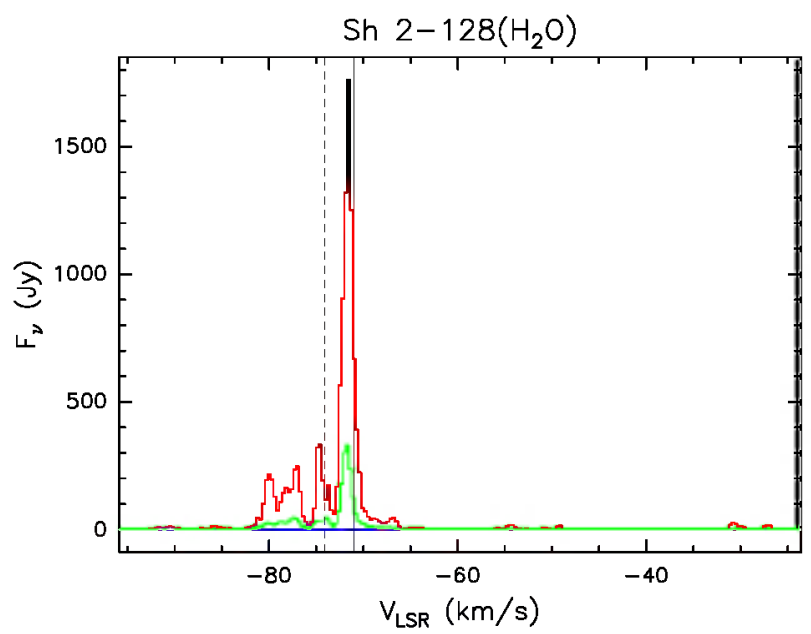

Fig. A.32. e Upper (red) and lower (blue) envelopes and mean spectrum (green) of source $\mathrm{Sh} 2-128\left(\mathrm{H}_{2} \mathrm{O}\right)$ measured during our monitoring. The vertical solid line marks the velocity of the associated thermal molecular gas. The vertical dashed line marks the mean velocity derived from the histogram of the rate-of-occurrence.

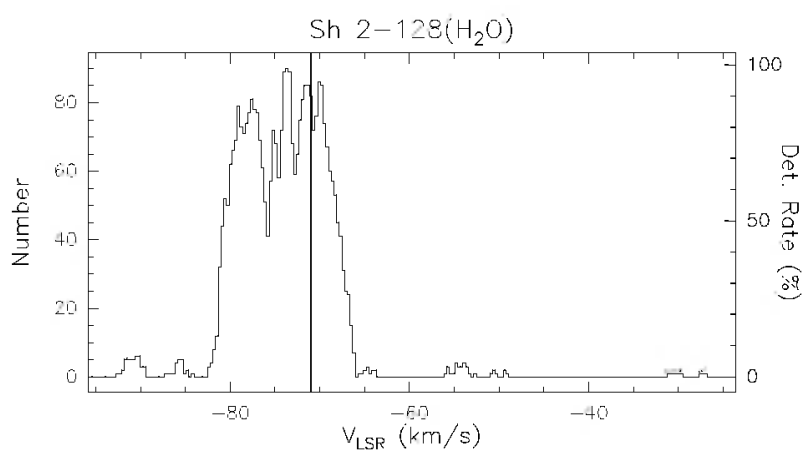

Fig. A.32. f Rate-of-occurrence plot for source $\mathrm{Sh} 2-128\left(\mathrm{H}_{2} \mathrm{O}\right)$. The scale to the right refers to the dotted histogram. the scale to the left to the solid line histogram. The vertical solid line marks the velocity of the associated thermal molecular gas. 


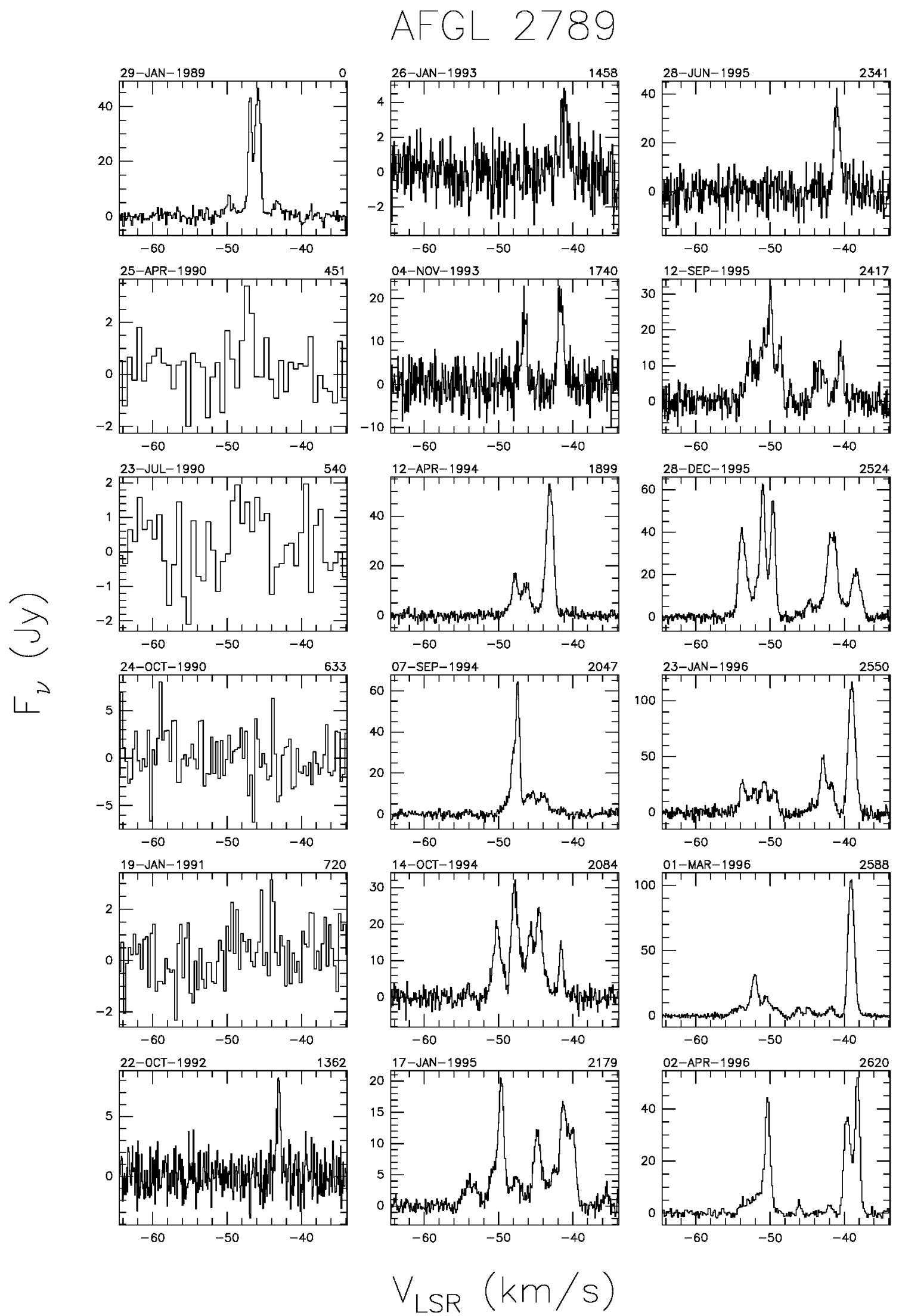

Fig. A.33. a Spectra of source AFGL 2789 with autoscaled flux density scale. The date of observation is shown above the top left corner of each spectrum and the number of days elapsed since the first observation is given above the top right corner. The velocity scale is the same for all spectra. 


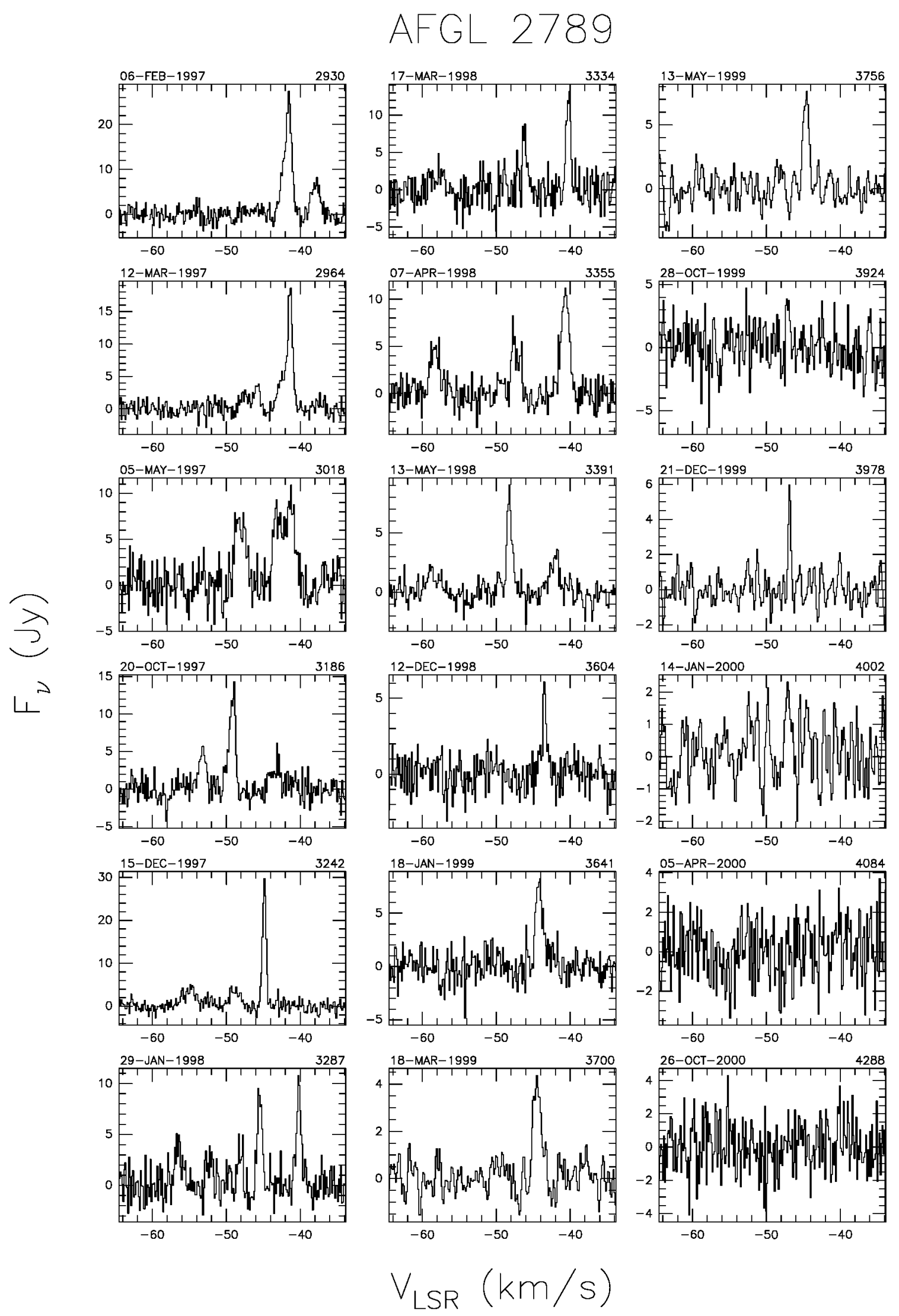

Fig. A.33. a continued. 


\section{AFGL 2789}
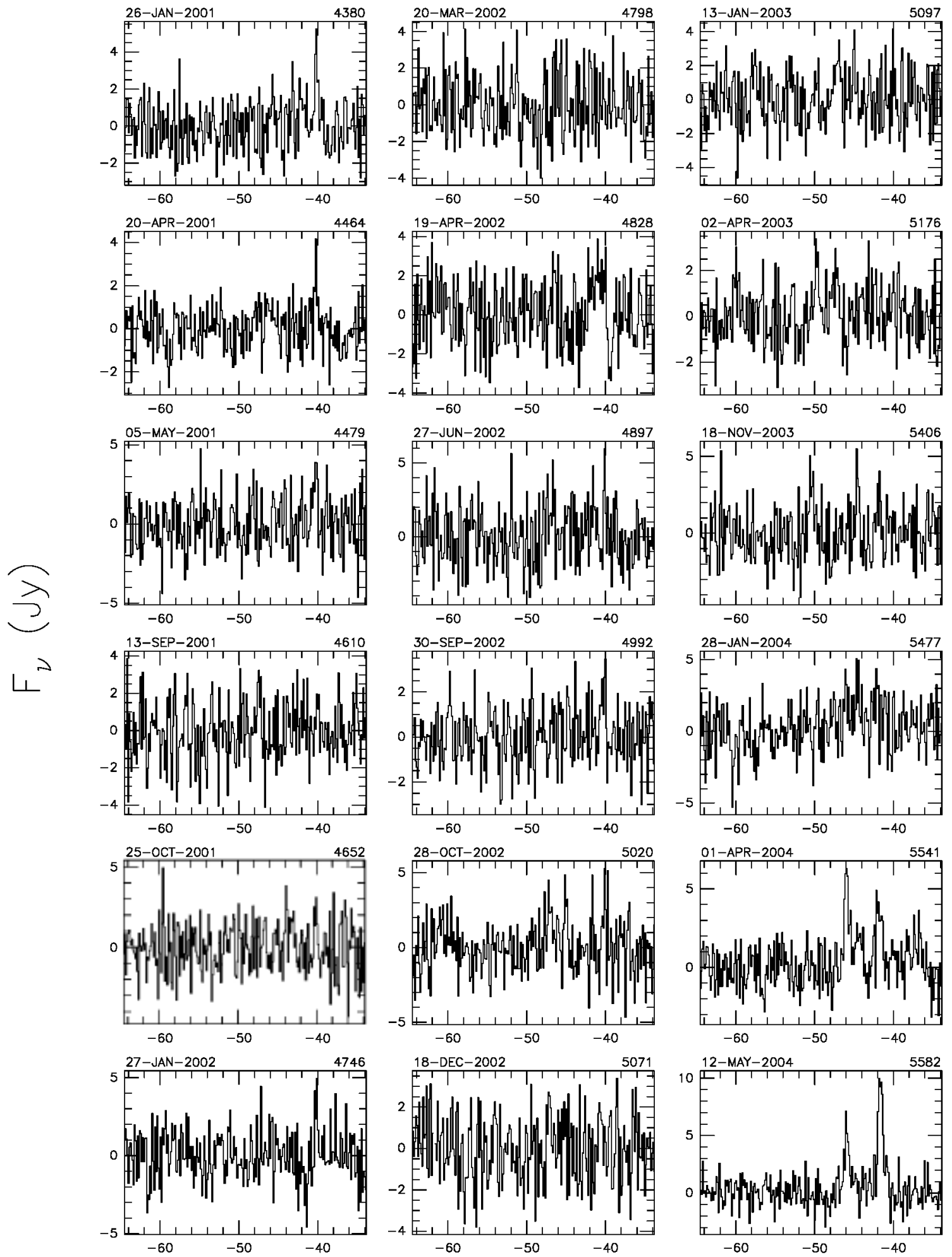

$$
V_{\text {LSR }}(\mathrm{km} / \mathrm{s})
$$

Fig. A.33. a continued. 

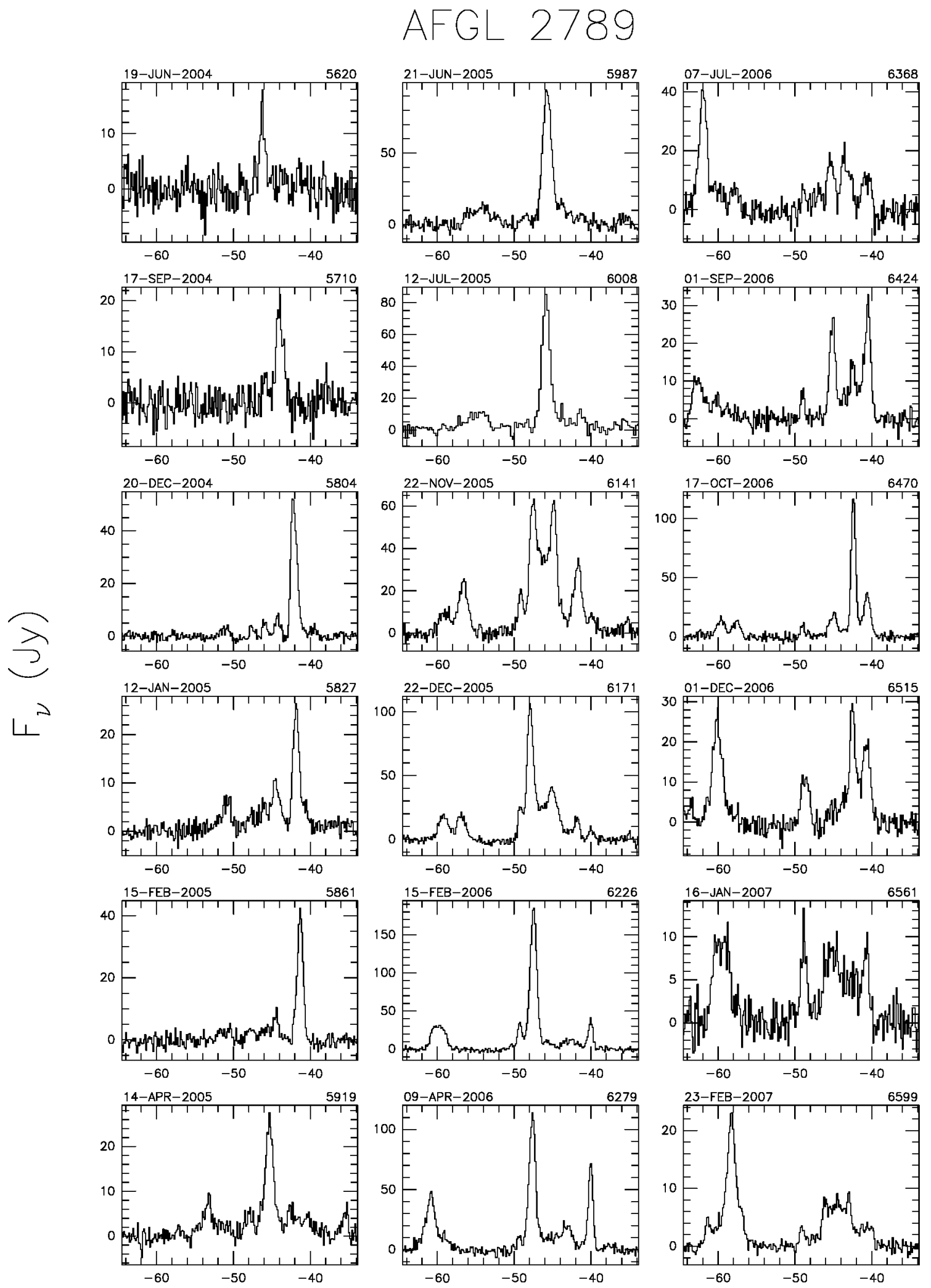

$$
V_{\text {LSR }}(\mathrm{km} / \mathrm{s})
$$

Fig. A.33. a continued. 


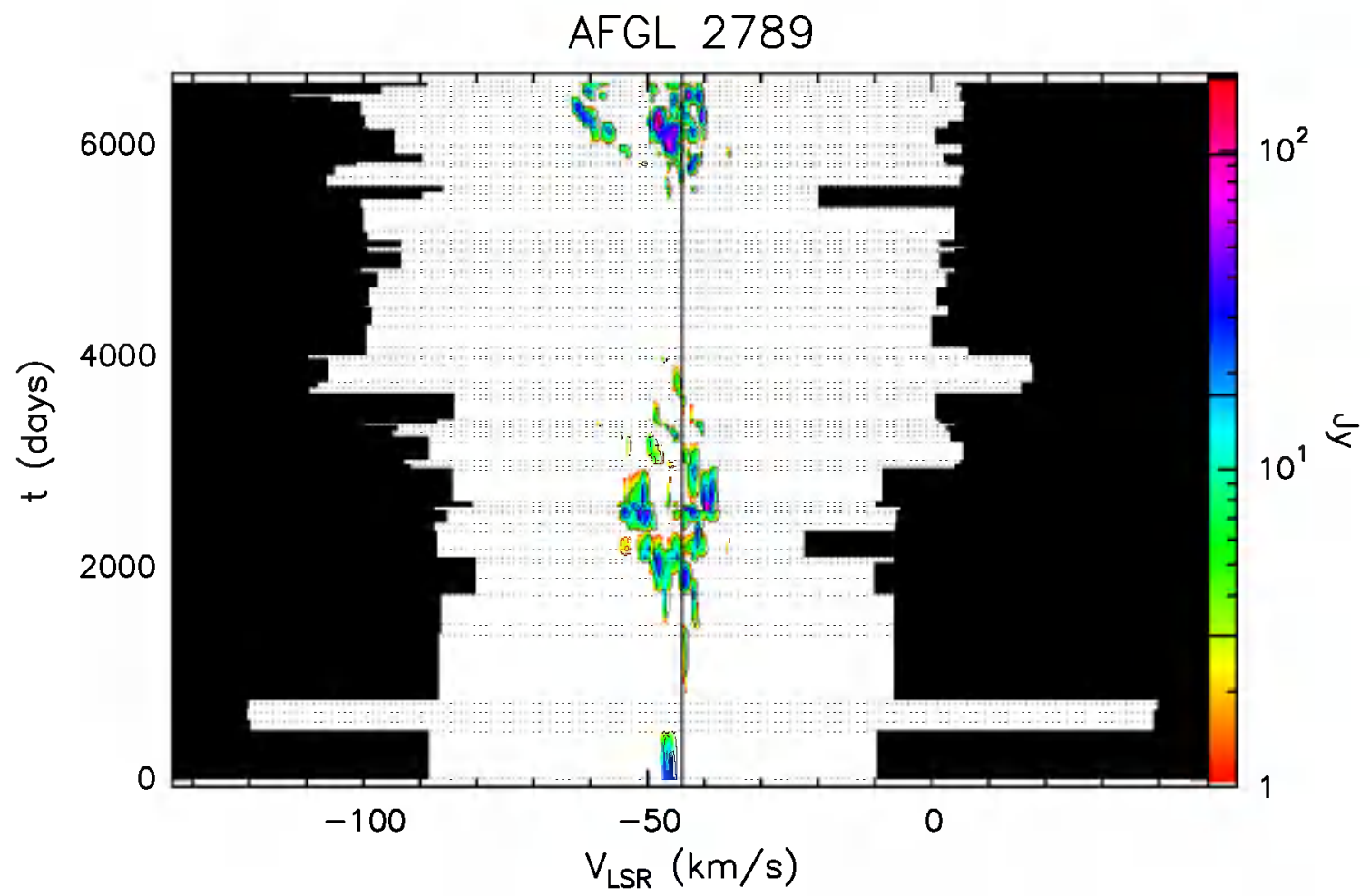

Fig. A.33. b Velocity-time-flux density full plot for source AFGL 2789. The vertical solid line indicates the velocity of the associated thermal molecular gas. The flux density scale is shown by the bar on the right. In this bar the three lines give the flux density of the drawn contours.

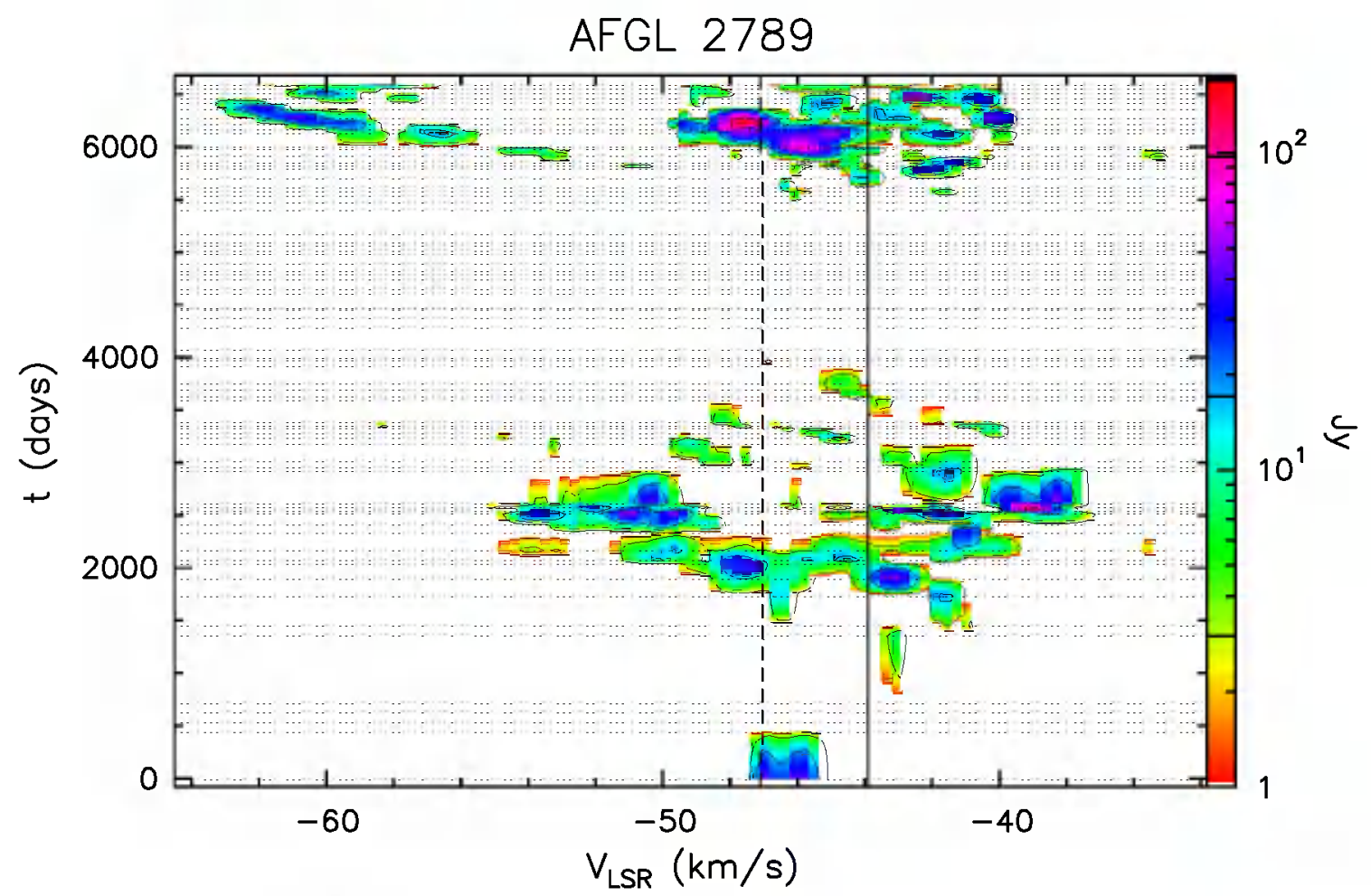

Fig. A.33. c Same as previous figure, but "zoomed" to velocity range over which emission has been detected. 


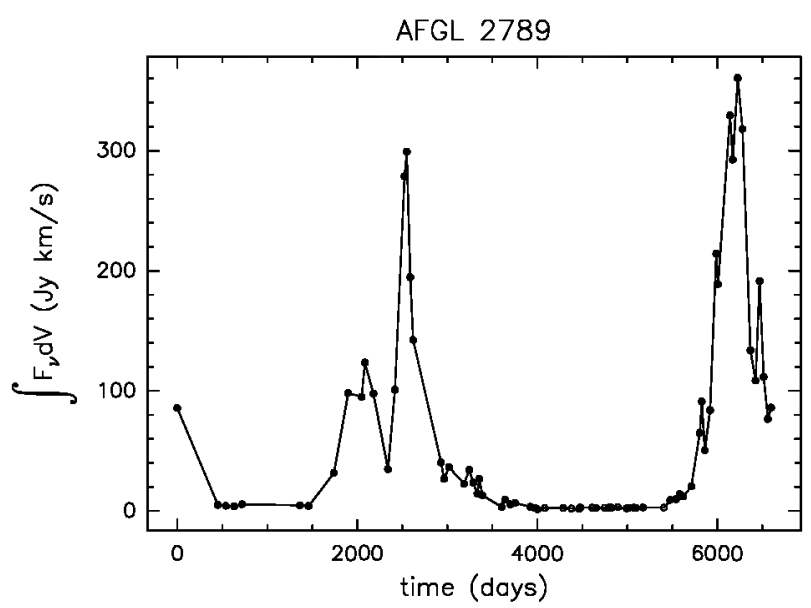

Fig. A.33. d Integral of the flux density over the observed velocity range as a function of time for source AFGL 2789.

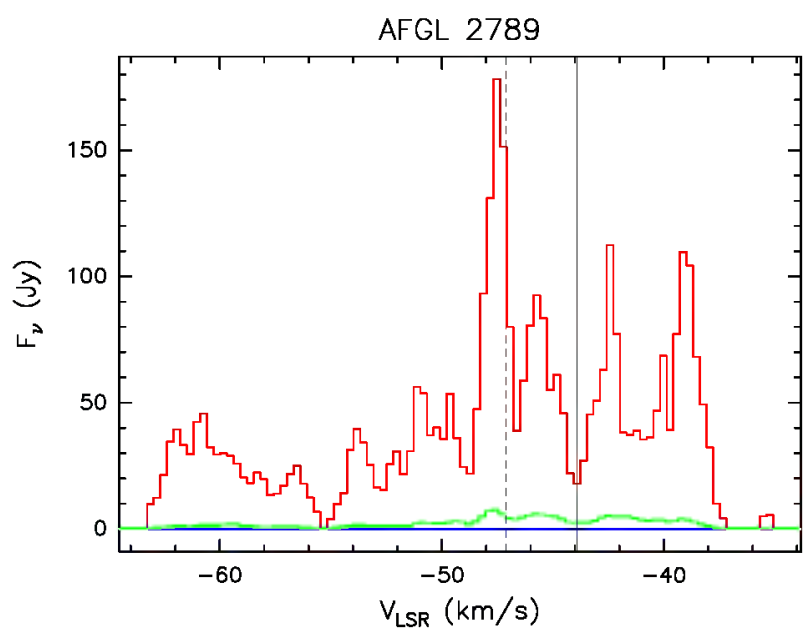

Fig. A.33. e Upper (red) and lower (blue) envelopes and mean spectrum (green) of source AFGL 2789 measured during our monitoring. The vertical solid line marks the velocity of the associated thermal molecular gas. The vertical dashed line marks the mean velocity derived from the histogram of the rate-of-occurrence.

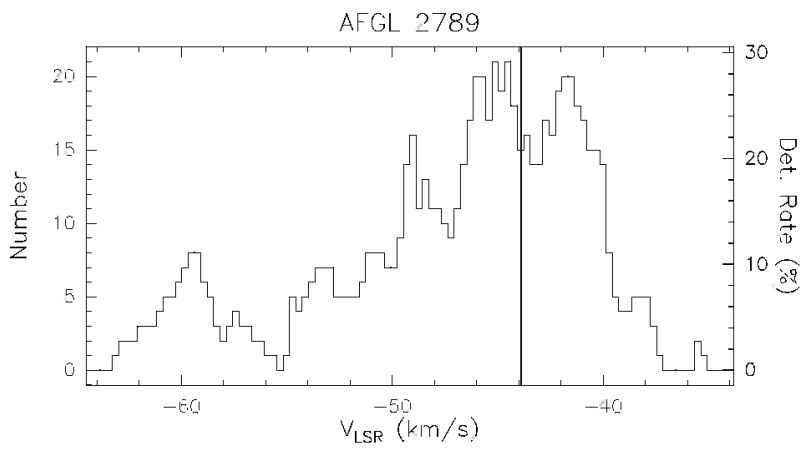

Fig. A.33. f Rate-of-occurrence plot for source AFGL 2789. The scale to the right refers to the dotted histogram, the scale to the left to the solid line histogram. The vertical solid line marks the velocity of the associated thermal molecular gas. 


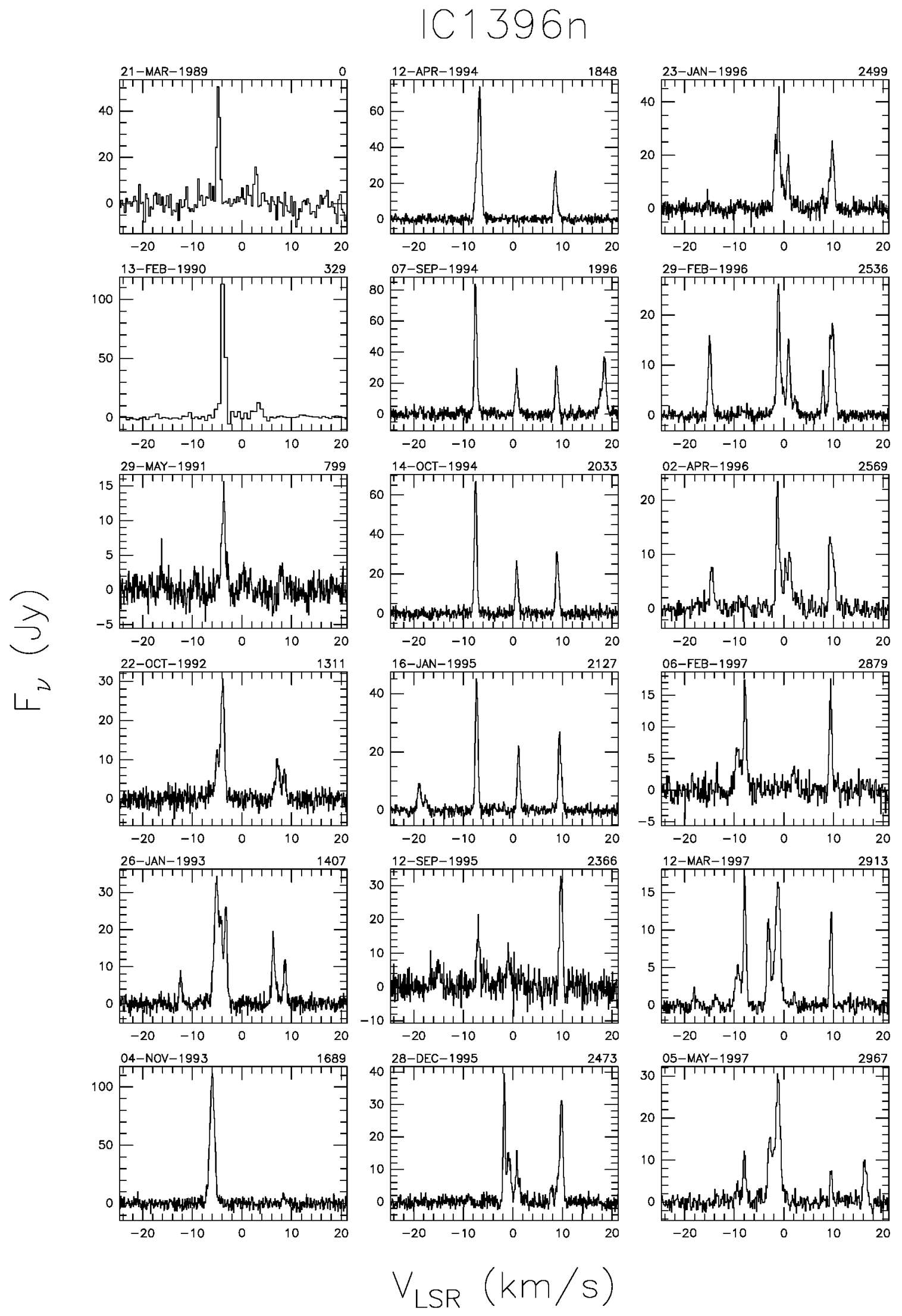

Fig. A.34. a Spectra of source IC 1396n with autoscaled flux density scale. The date of observation is shown above the top left corner of each spectrum and the number of days elapsed since the first observation is given above the top right corner. The velocity scale is the same for all spectra. 


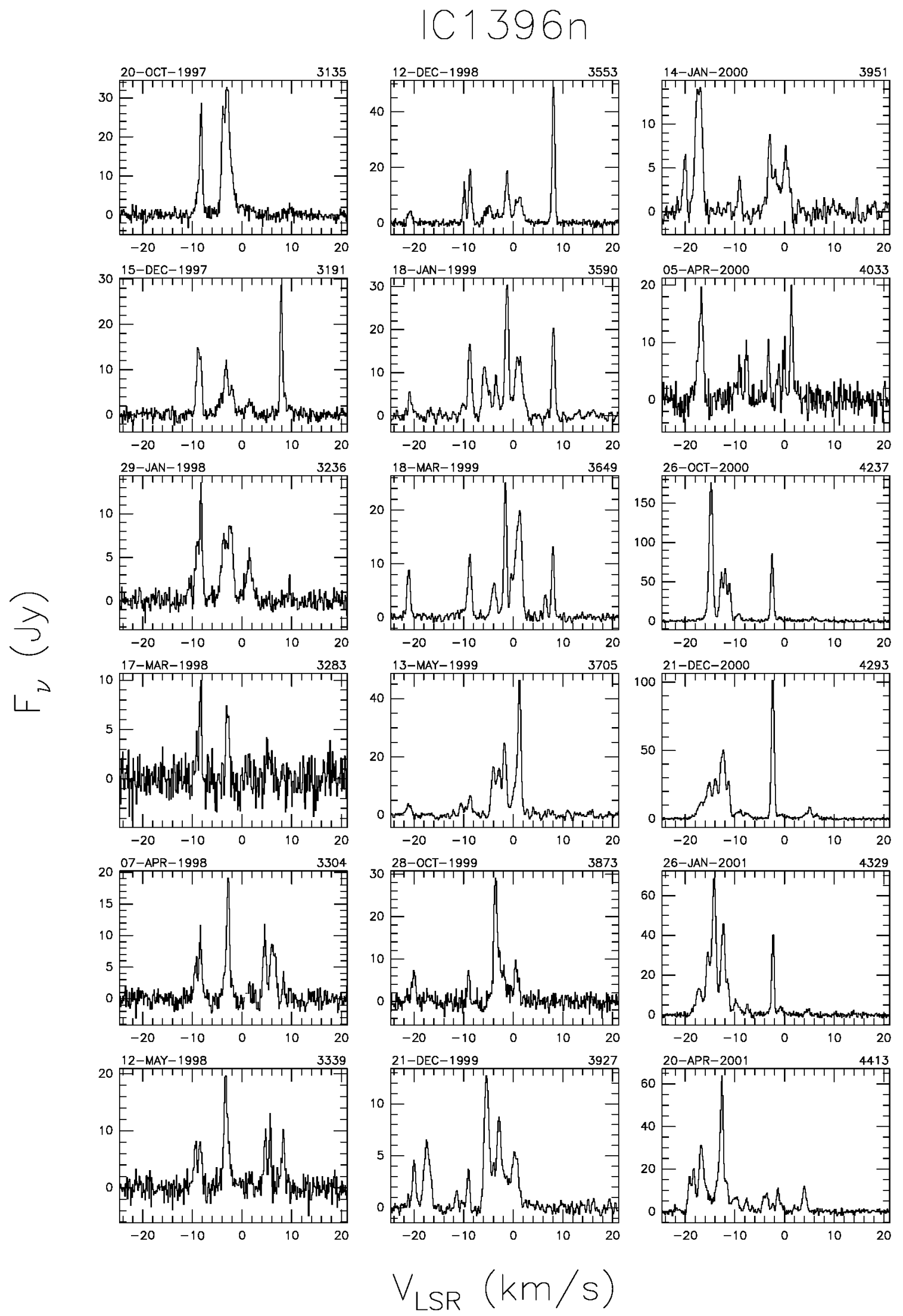

Fig. A.34. a continued. 


\section{IC1396n}
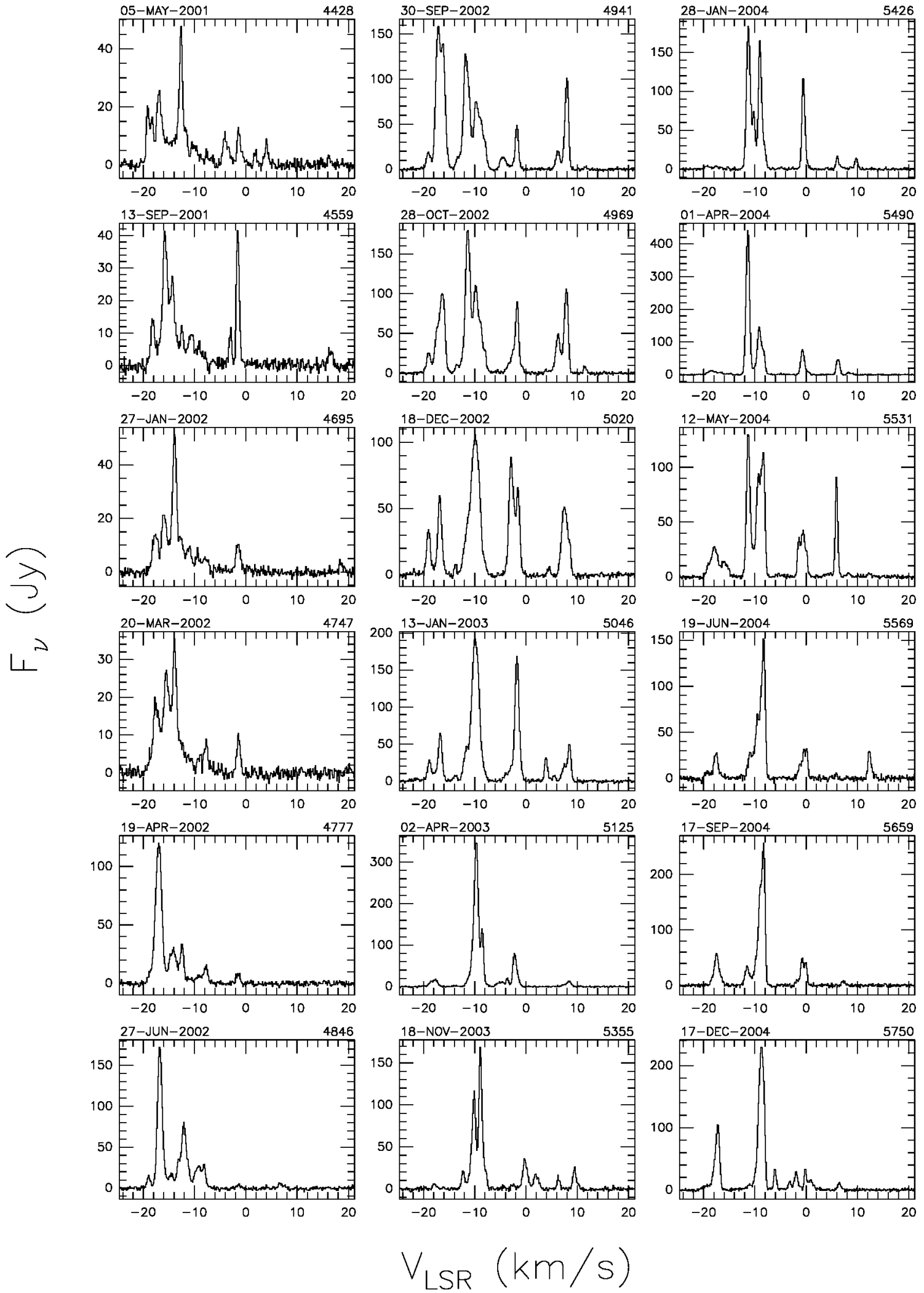

Fig. A.34. a continued. 


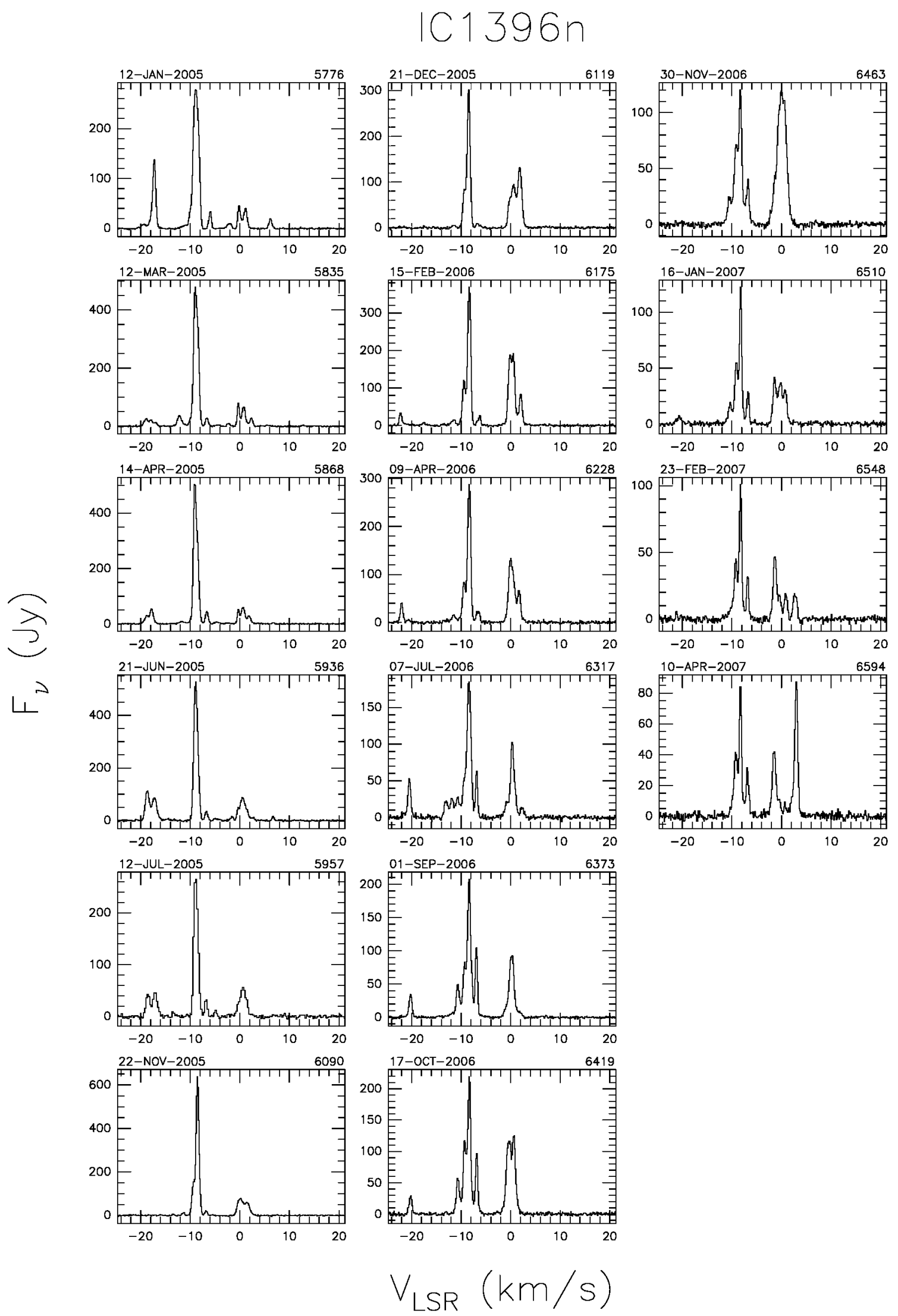

Fig. A.34. a continued. 


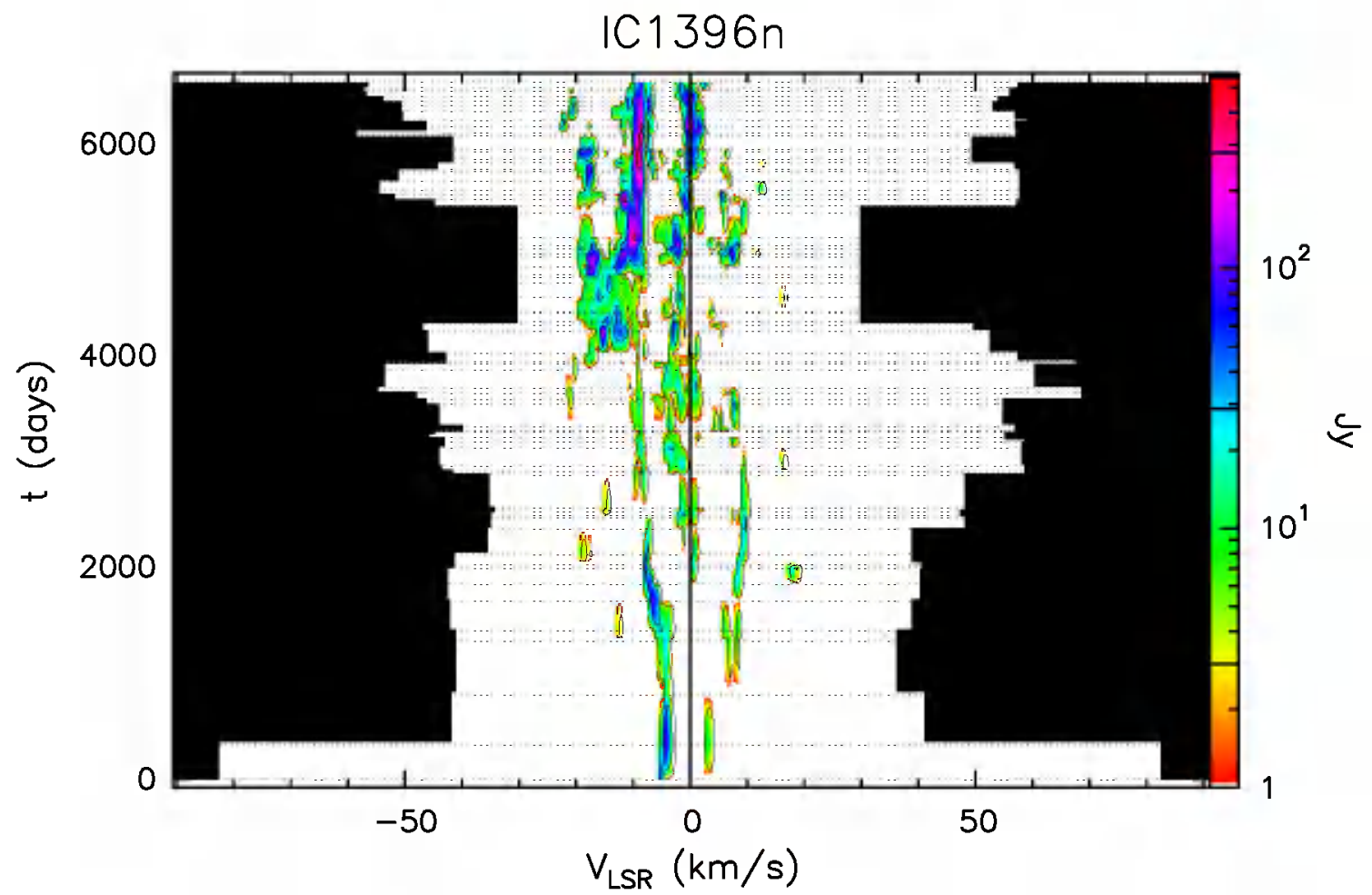

Fig. A.34. b Velocity-time-flux density full plot for source IC1396n. The vertical solid line indicates the velocity of the associated thermal molecular gas. The flux density scale is shown by the bar on the right. In this bar the three lines give the flux density of the drawn contours.

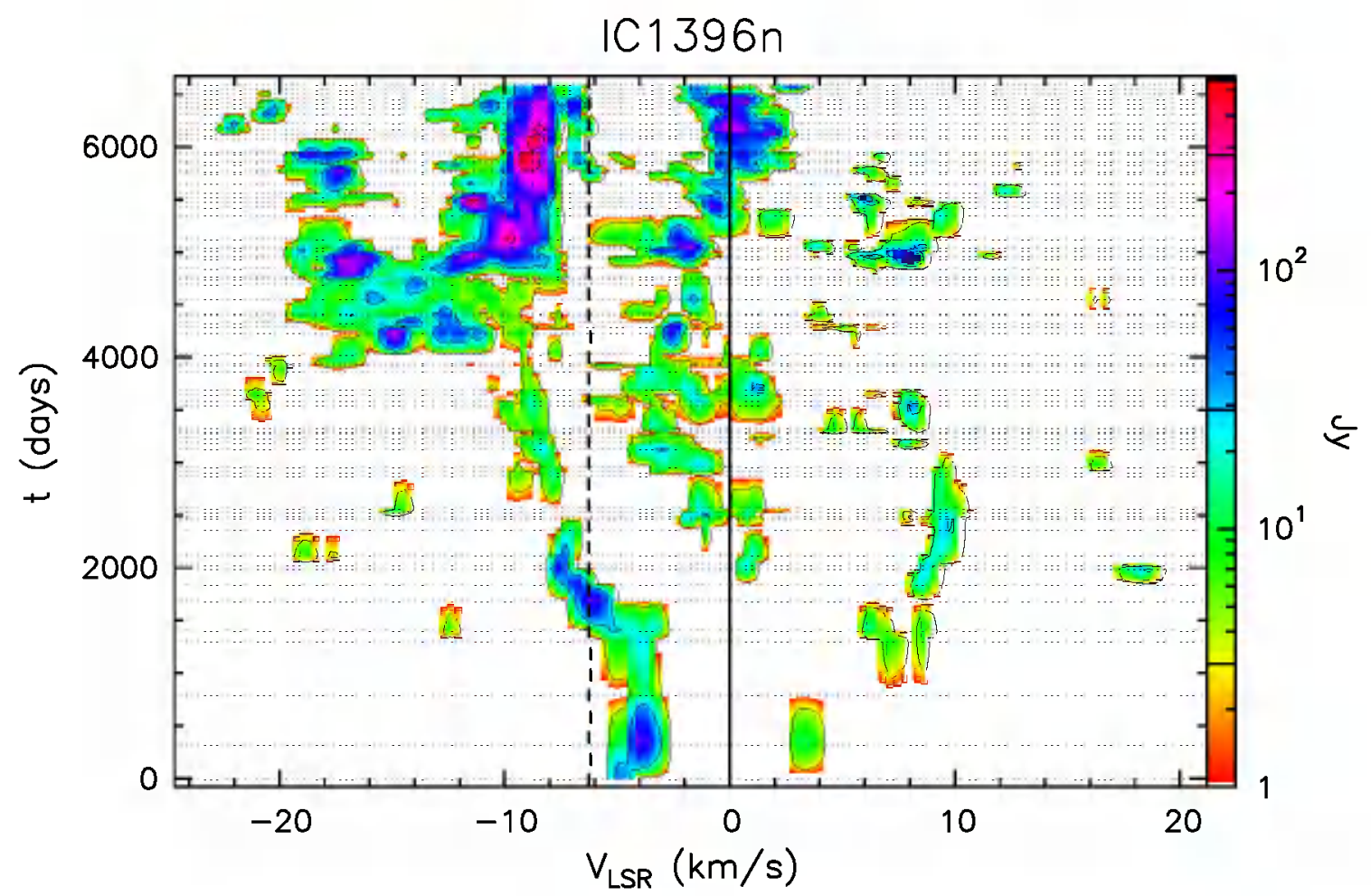

Fig. A.34. c Same as previous figure, but "zoomed" to velocity range over which emission has been detected. 


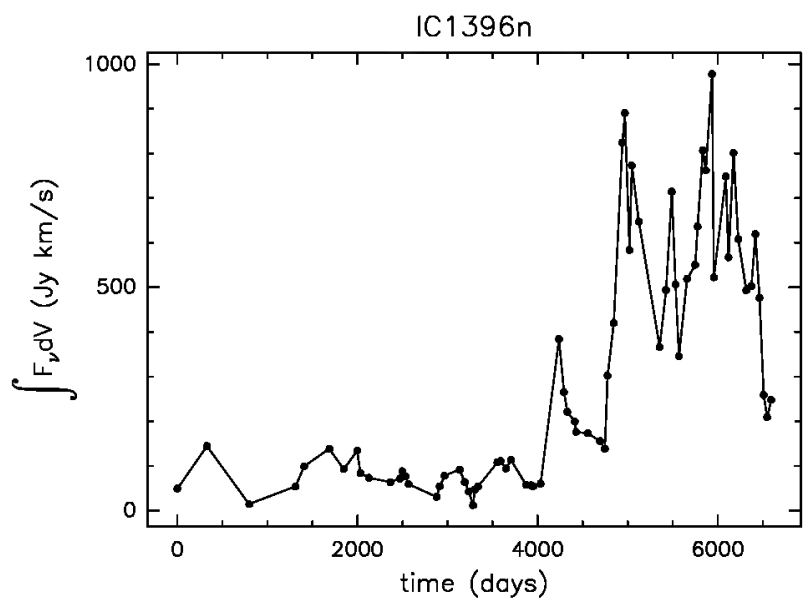

Fig. A.34. d Integral of the flux density over the observed velocity range as a function of time for source IC1396n.

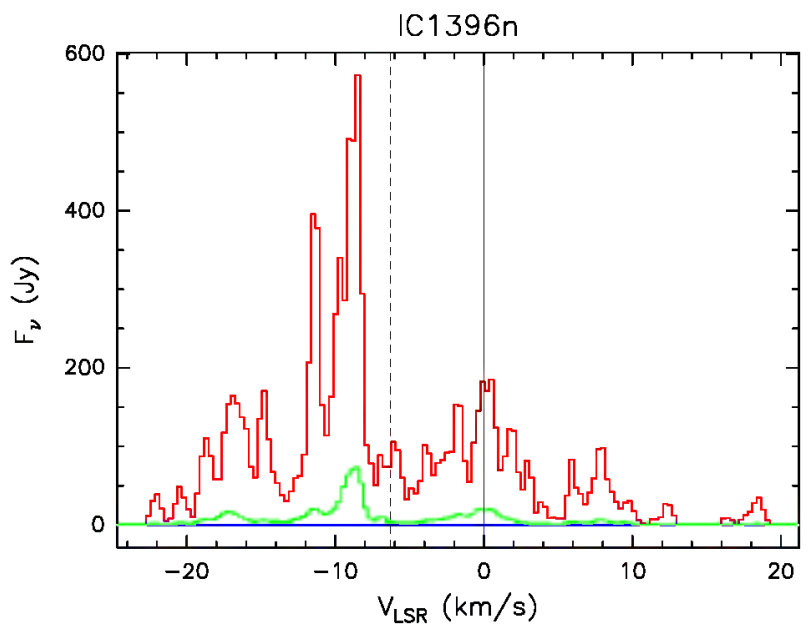

Fig. A.34. e Upper (red) and lower (blue) envelopes and mean spectrum (green) of source IC1396n measured during our monitoring. The vertical solid line marks the velocity of the associated thermal molecular gas. The vertical dashed line marks the mean velocity derived from the histogram of the rate-of-occurrence.

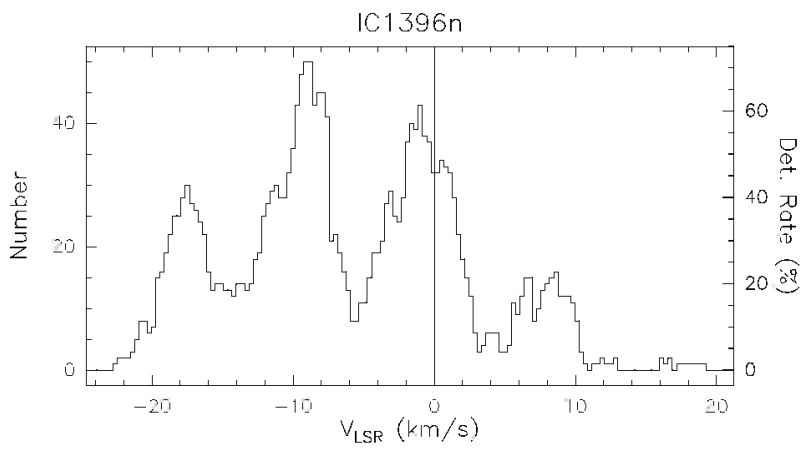

Fig. A.34. f Rate-of-occurrence plot for source IC1396n. The scale to the right refers to the dotted histogram, the scale to the left to the solid line histogram. The vertical solid line marks the velocity of the associated thermal molecular gas. 
NGC 7129 FIRS2
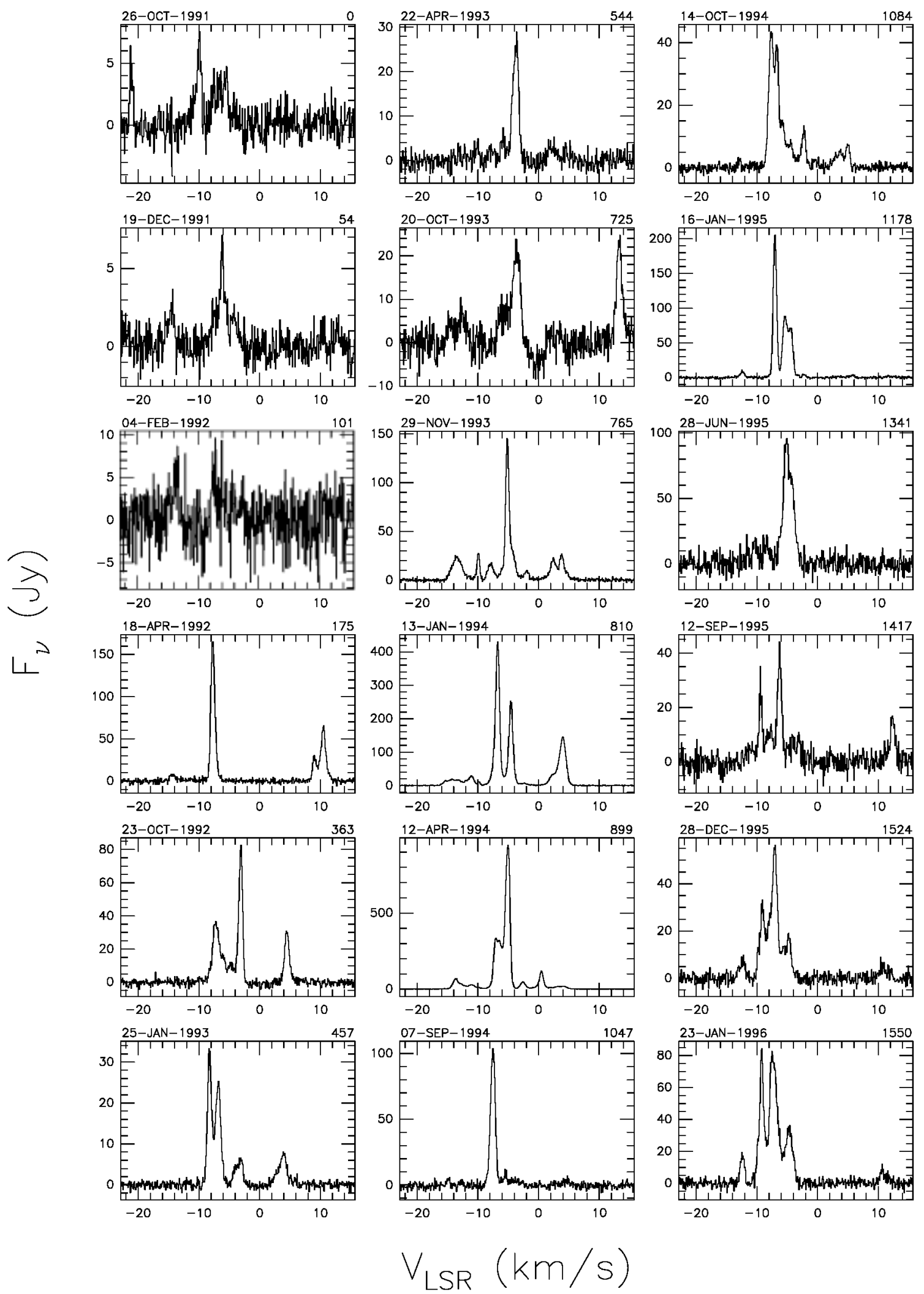

Fig. A.35. a Spectra of source NGC 7129 FIRS2 with autoscaled flux density scale. The date of observation is shown above the top left corner of each spectrum and the number of days elapsed since the first observation is given above the top right corner. The velocity scale is the same for all spectra. 
NGC 7129 FIRS2
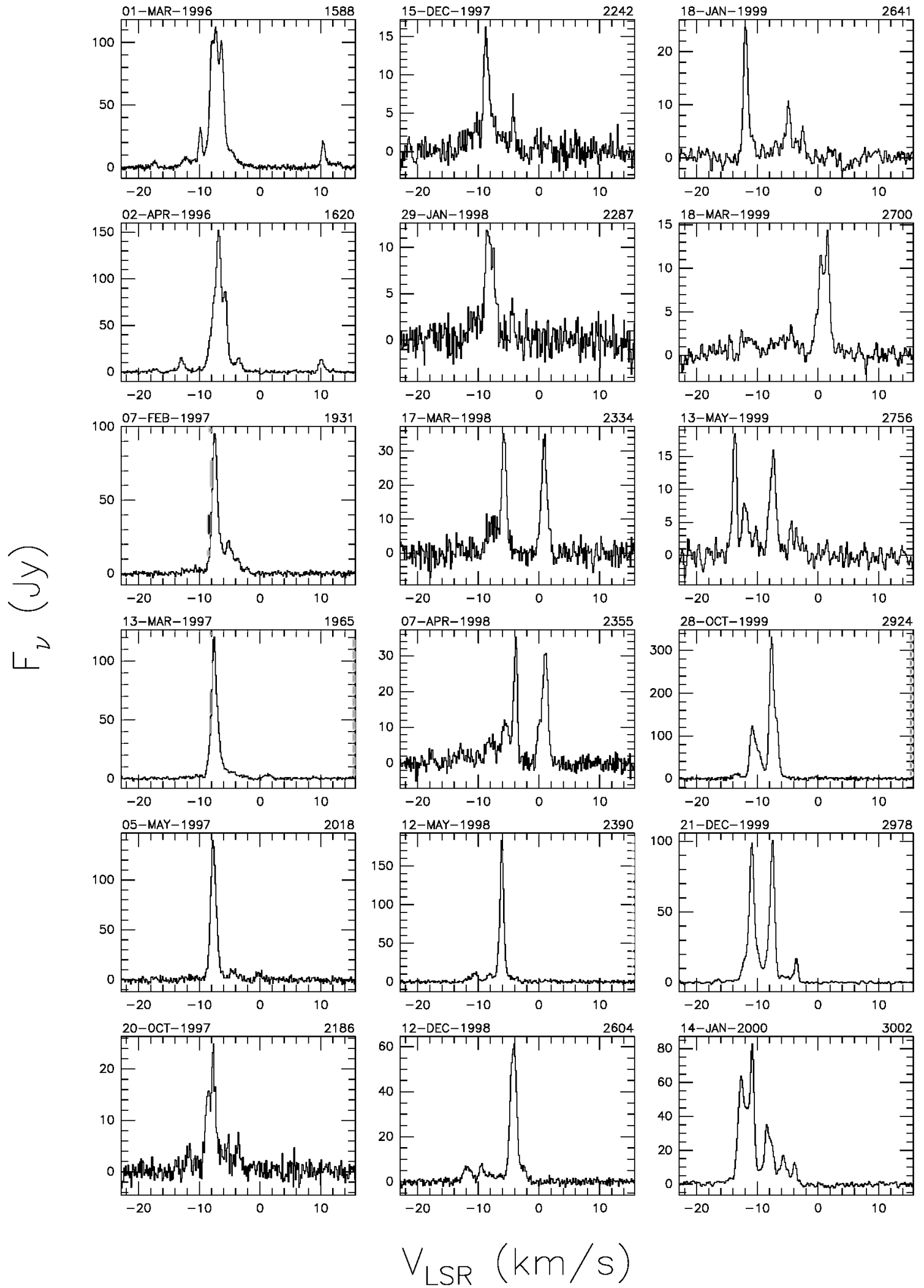

Fig. A.35. a continued. 
NGC 7129 FIRS2
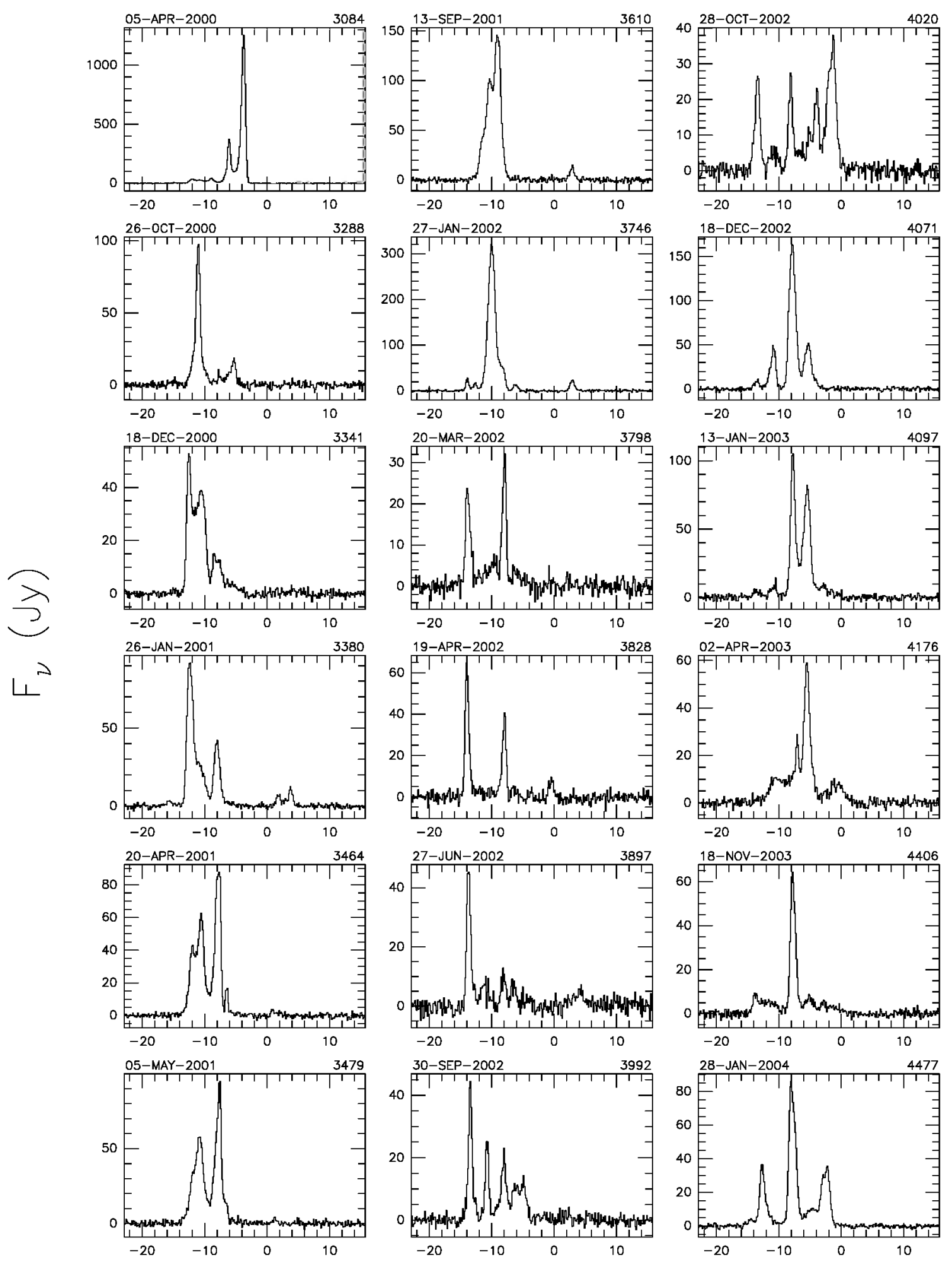

$$
V_{\text {LSR }}(\mathrm{km} / \mathrm{s})
$$

Fig. A.35. a continued. 
NGC 7129 FIRS2

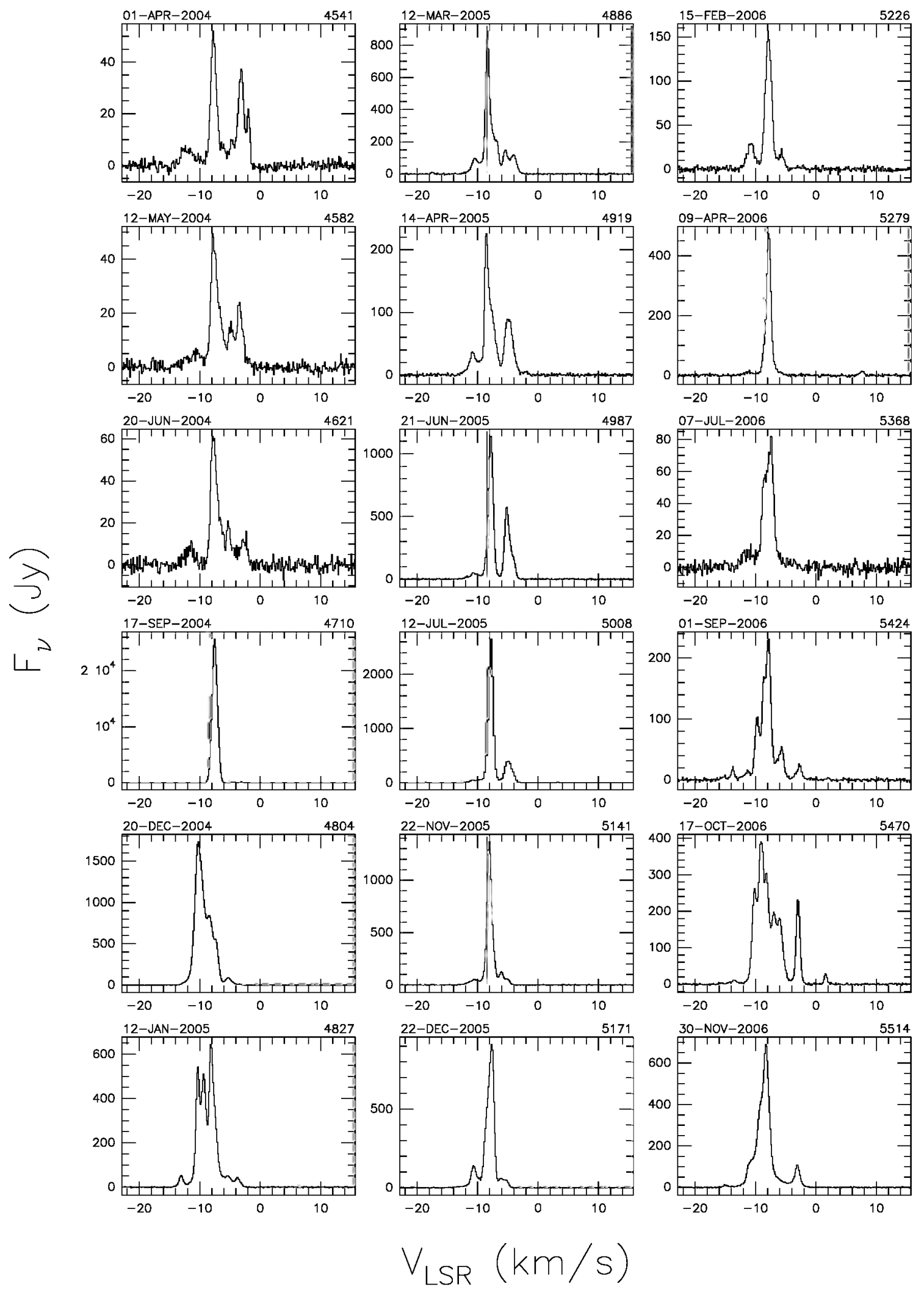

Fig. A.35. a continued. 
NGC 7129 FIRS2
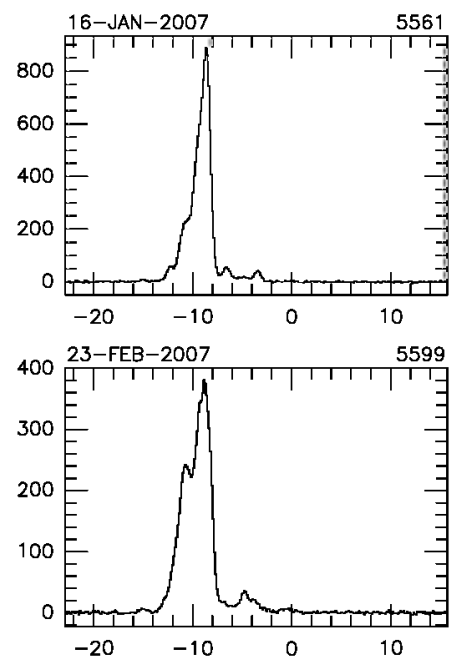

3

$L^{\lambda}$

$$
V_{\text {LSR }}(\mathrm{km} / \mathrm{s})
$$

Fig. A.35. a continued. 


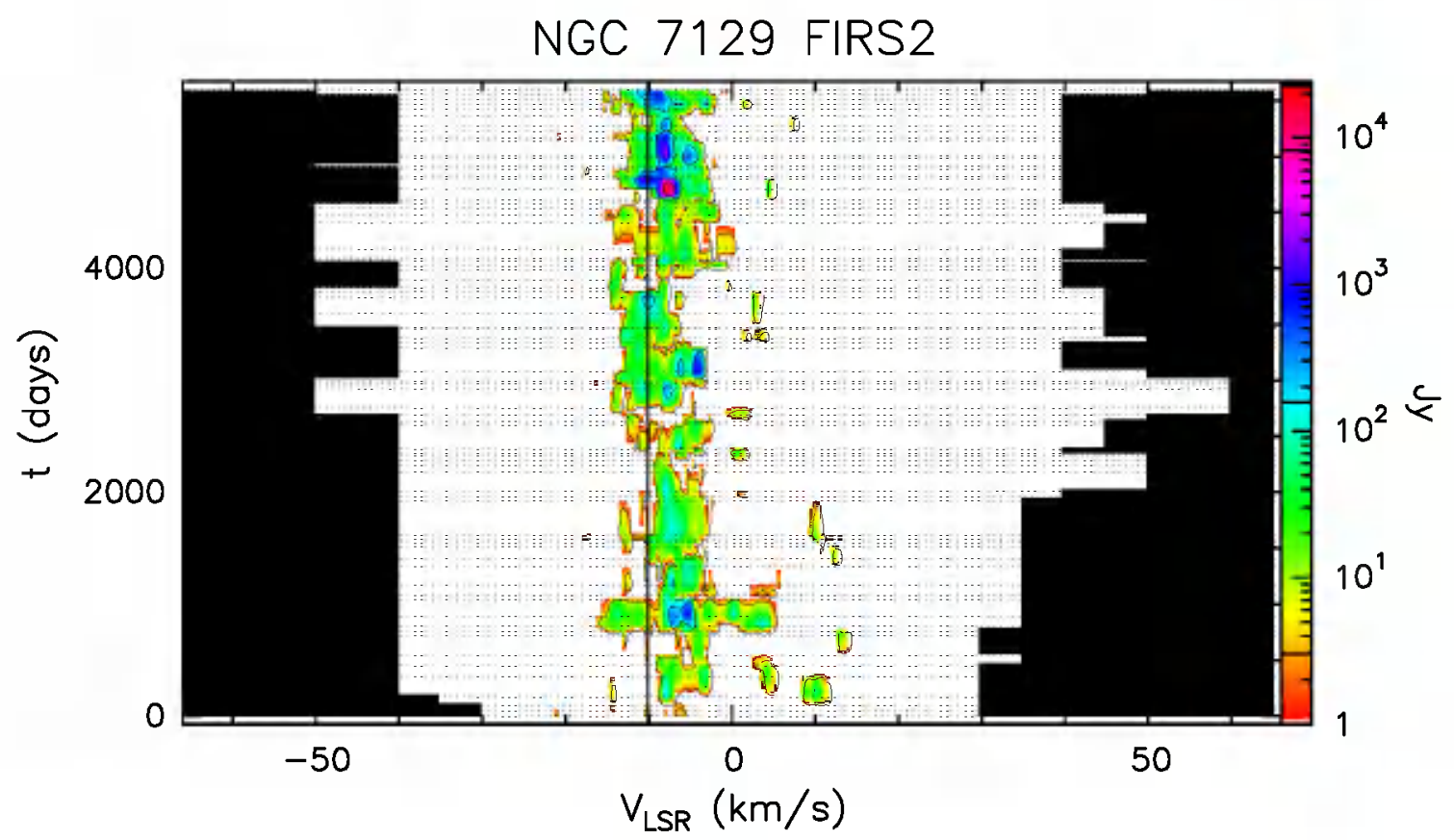

Fig. A.35. b Velocity-time-flux density full plot for source NGC 7129 FIRS2. The vertical solid line indicates the velocity of the associated thermal molecular gas. The flux density scale is shown by the bar on the right. In this bar the three lines give the flux density of the drawn contours.

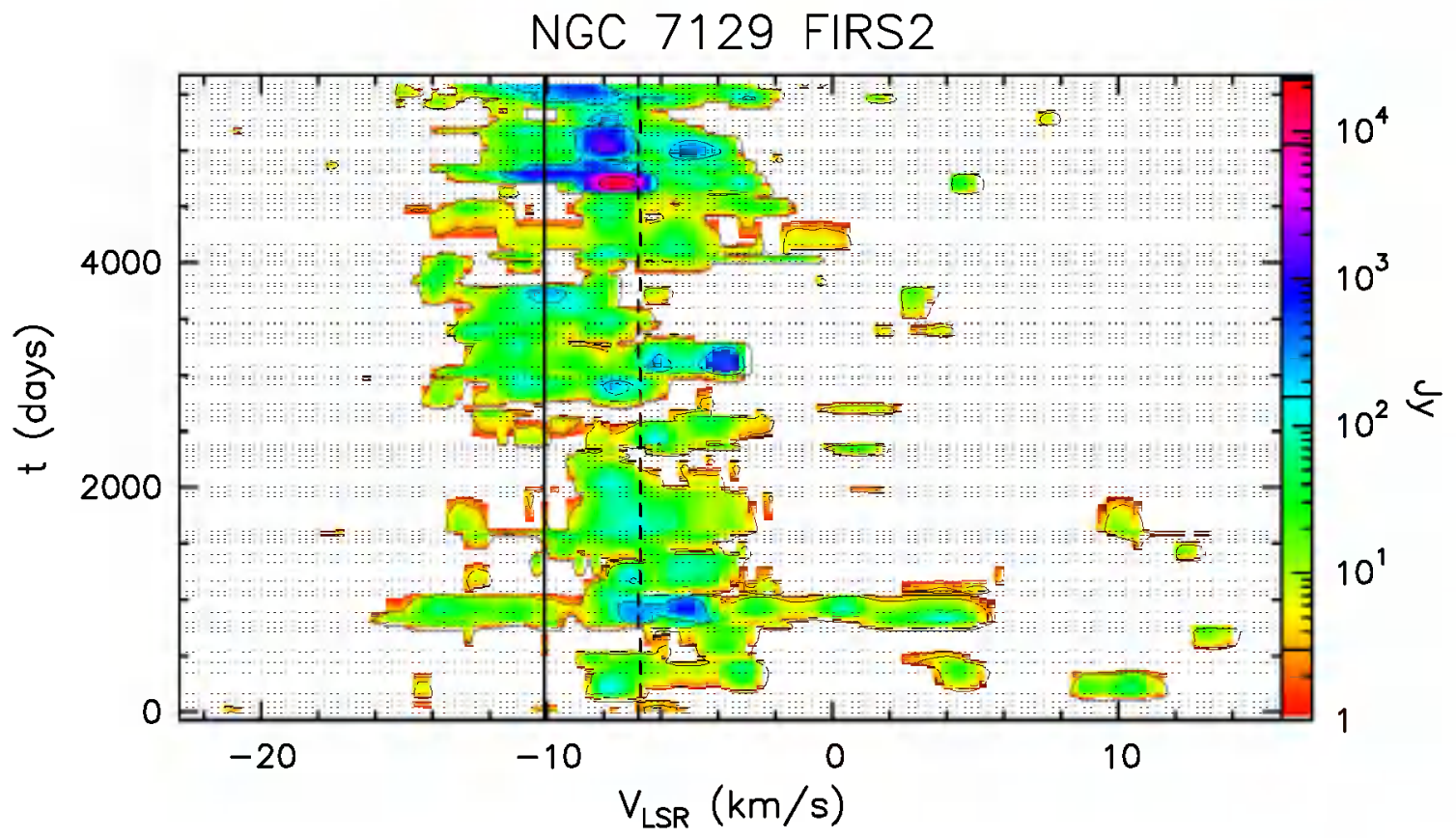

Fig. A.35. c Same as previous figure, but "zoomed" to velocity range over which emission has been detected. 


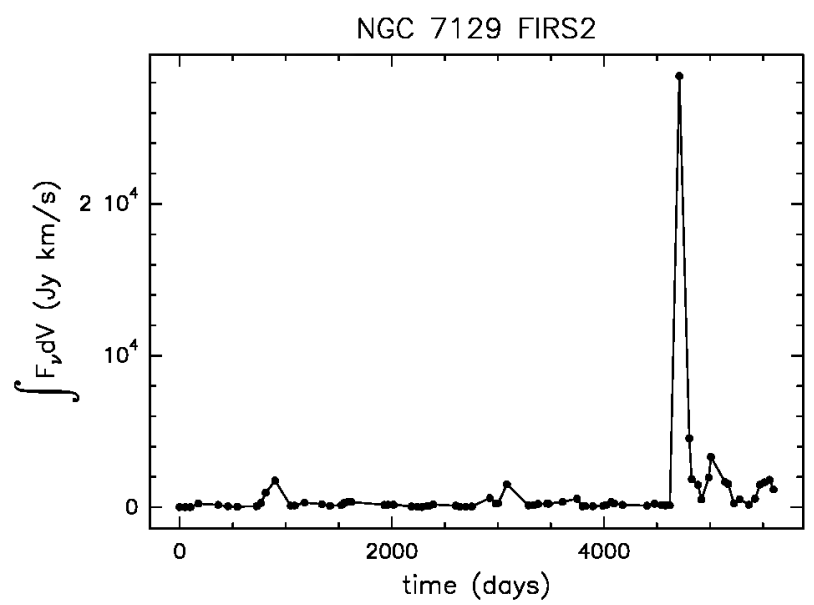

Fig. A.35. d Integral of the flux density over the observed velocity range as a function of time for source NGC 7129 FIRS2.

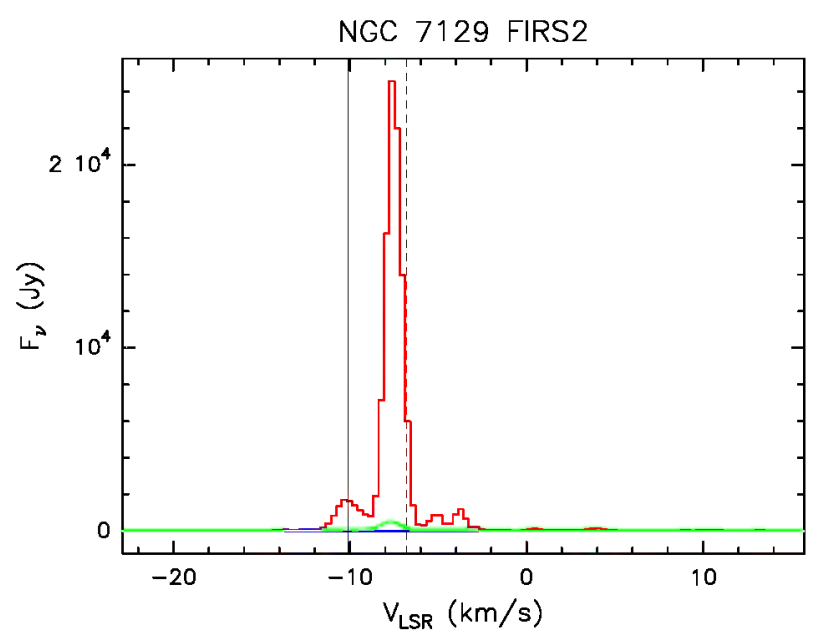

Fig. A.35. e Upper (red) and lower (blue) envelopes and mean spectrum (green) of source NGC 7129 FIRS2 measured during our monitoring. The vertical solid line marks the velocity of the associated thermal molecular gas. The vertical dashed line marks the mean velocity derived from the histogram of the rate-of-occurrence.

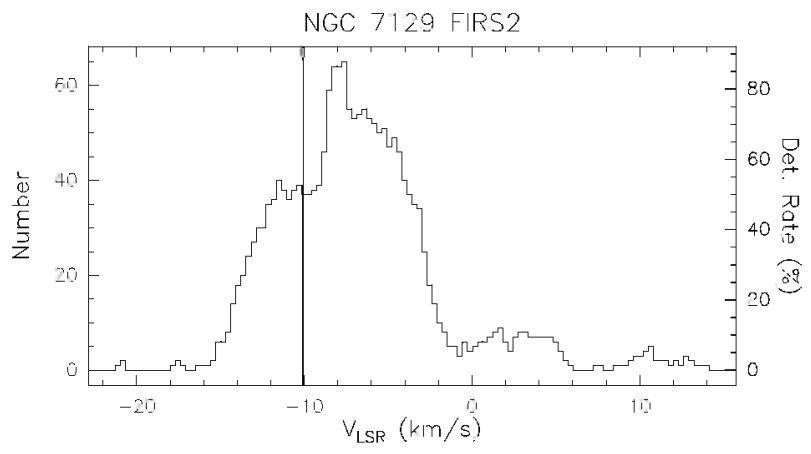

Fig. A.35. f Rate-of-occurrence plot for source NGC 7129 FIRS2. The scale to the right refers to the dotted histogram, the scale to the left to the solid line histogram. The vertical solid line marks the velocity of the associated thermal molecular gas. 


\section{Sh $2-140$ IRS 1}
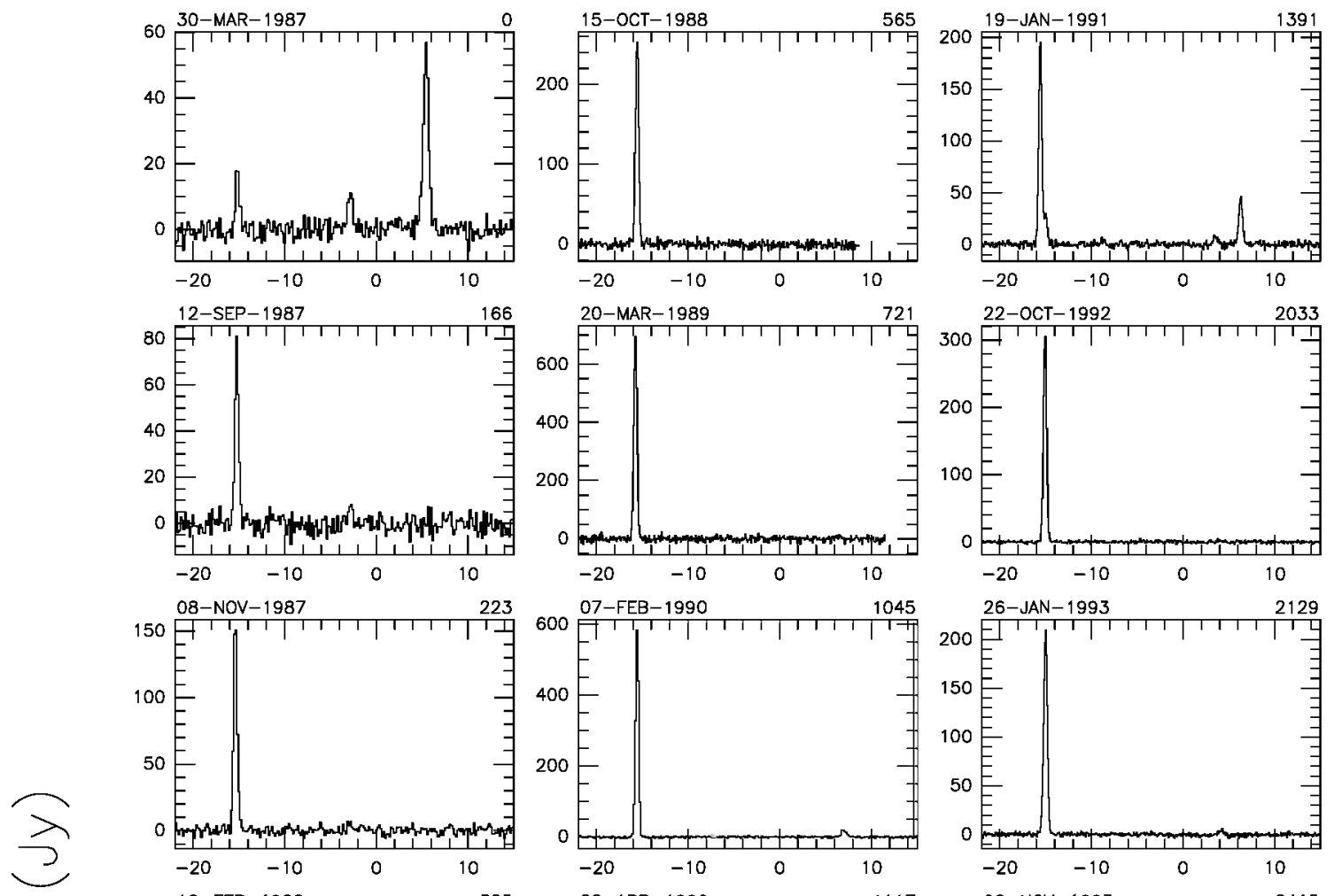

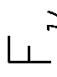
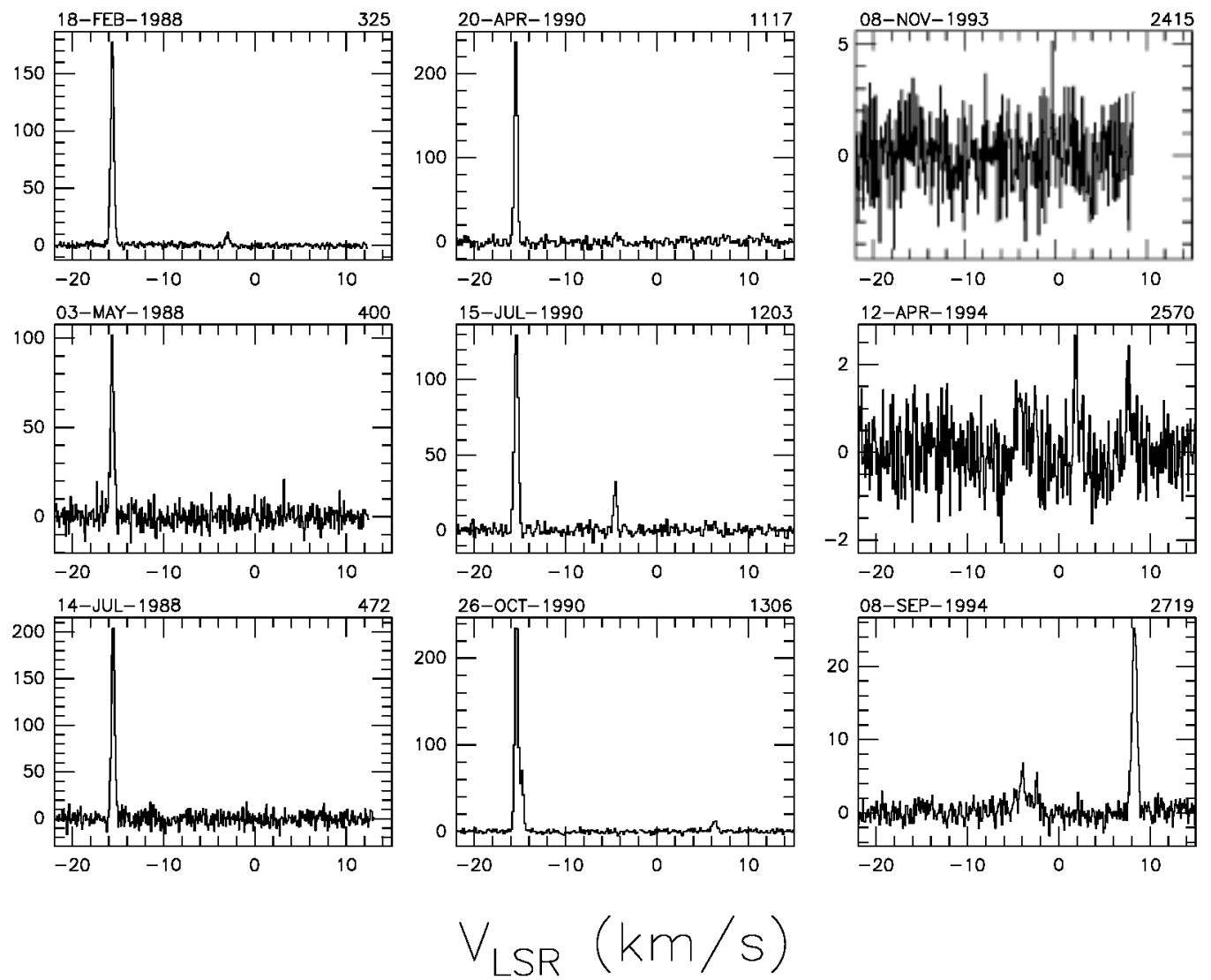

Fig. A.36. a Spectra of source Sh 2-140 IRS 1 with autoscaled flux density scale. The date of observation is shown above the top left corner of each spectrum and the number of days elapsed since the first observation is given above the top right corner. The velocity scale is the same for all spectra. 
Sh 2-140 |RS1
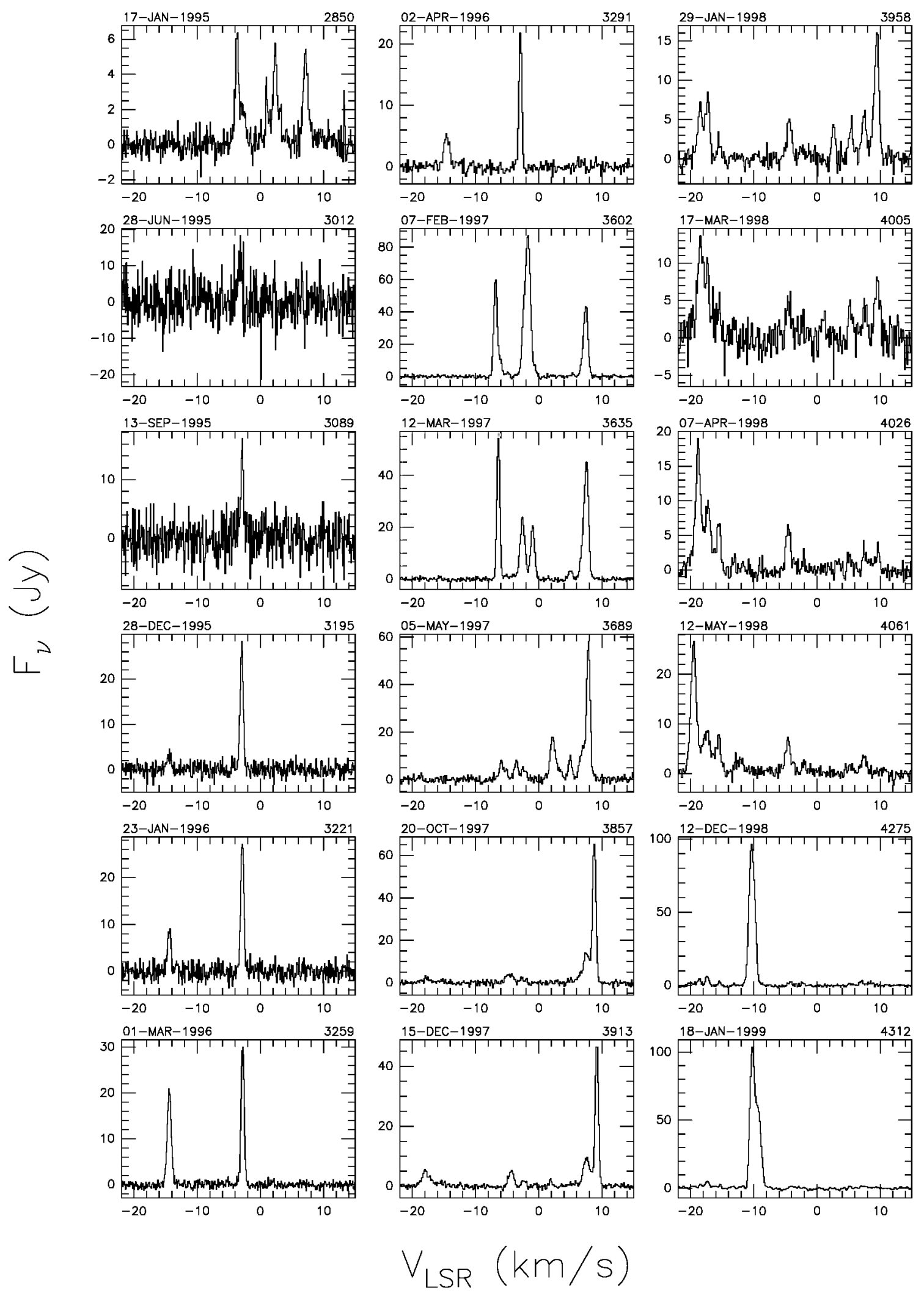

Fig. A.36. a continued. 
Sh 2-140 IRS1
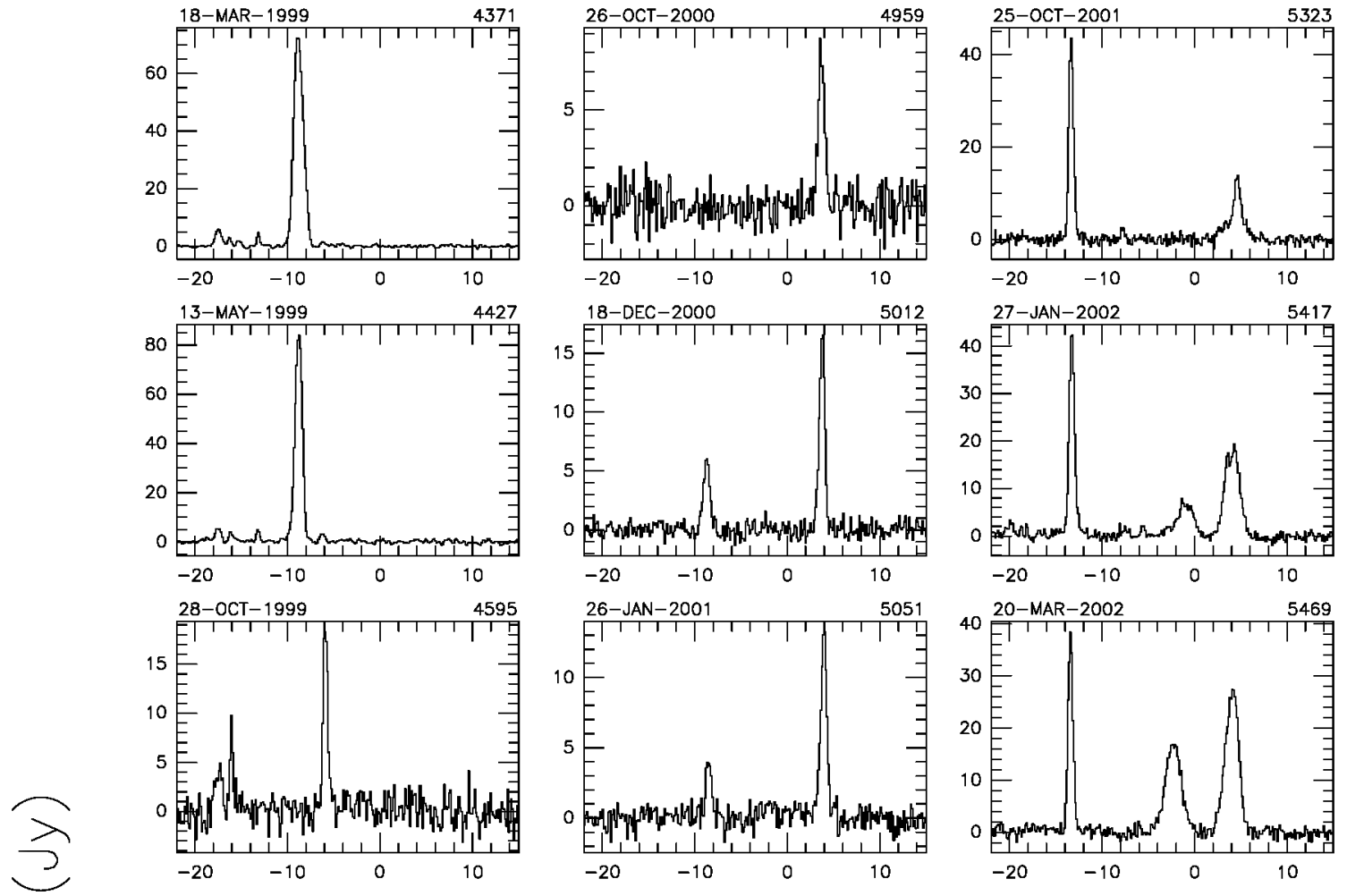

$L^{2}$
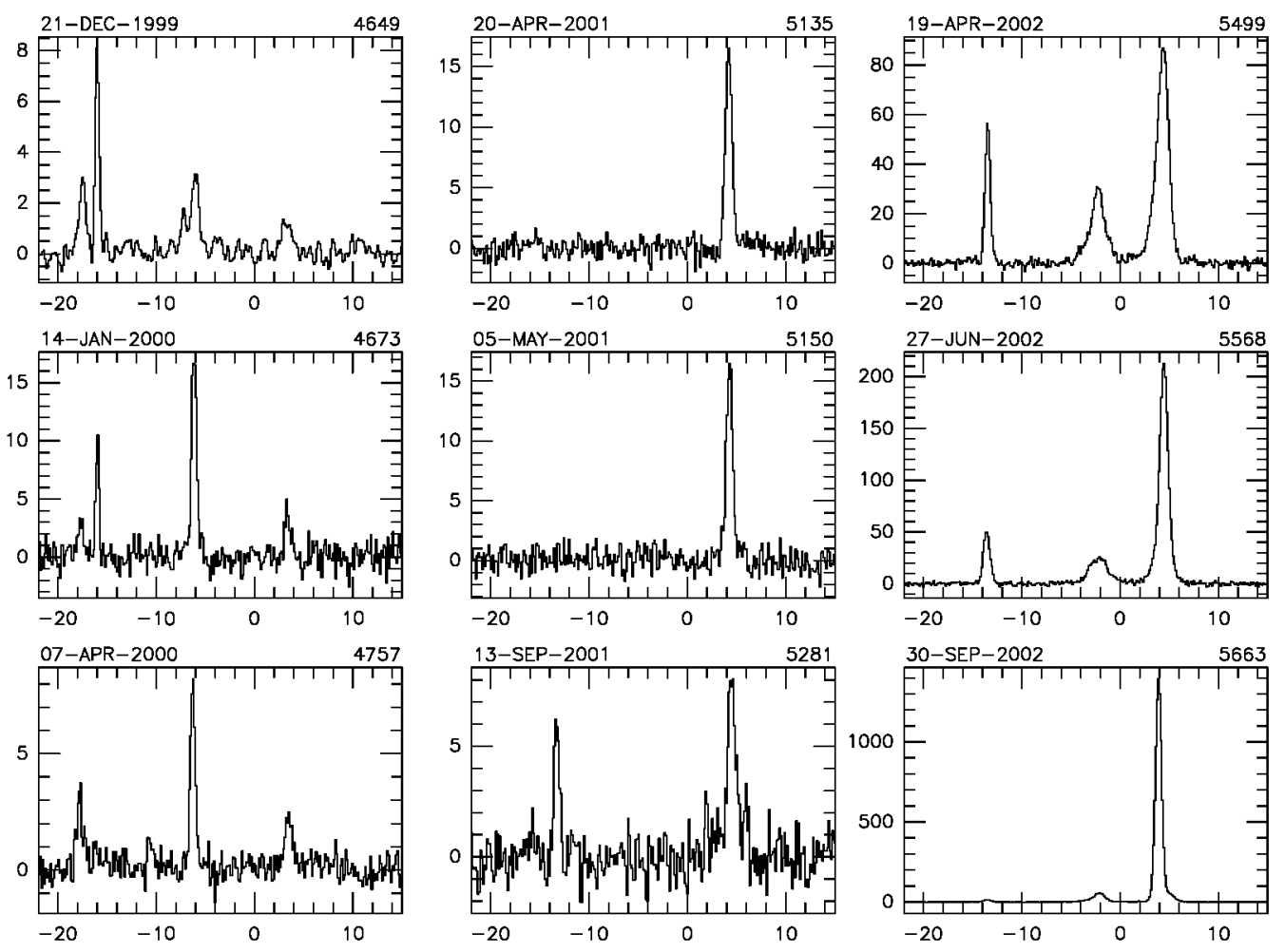

$$
V_{\text {LSR }}(\mathrm{km} / \mathrm{s})
$$

Fig. A.36. a continued. 

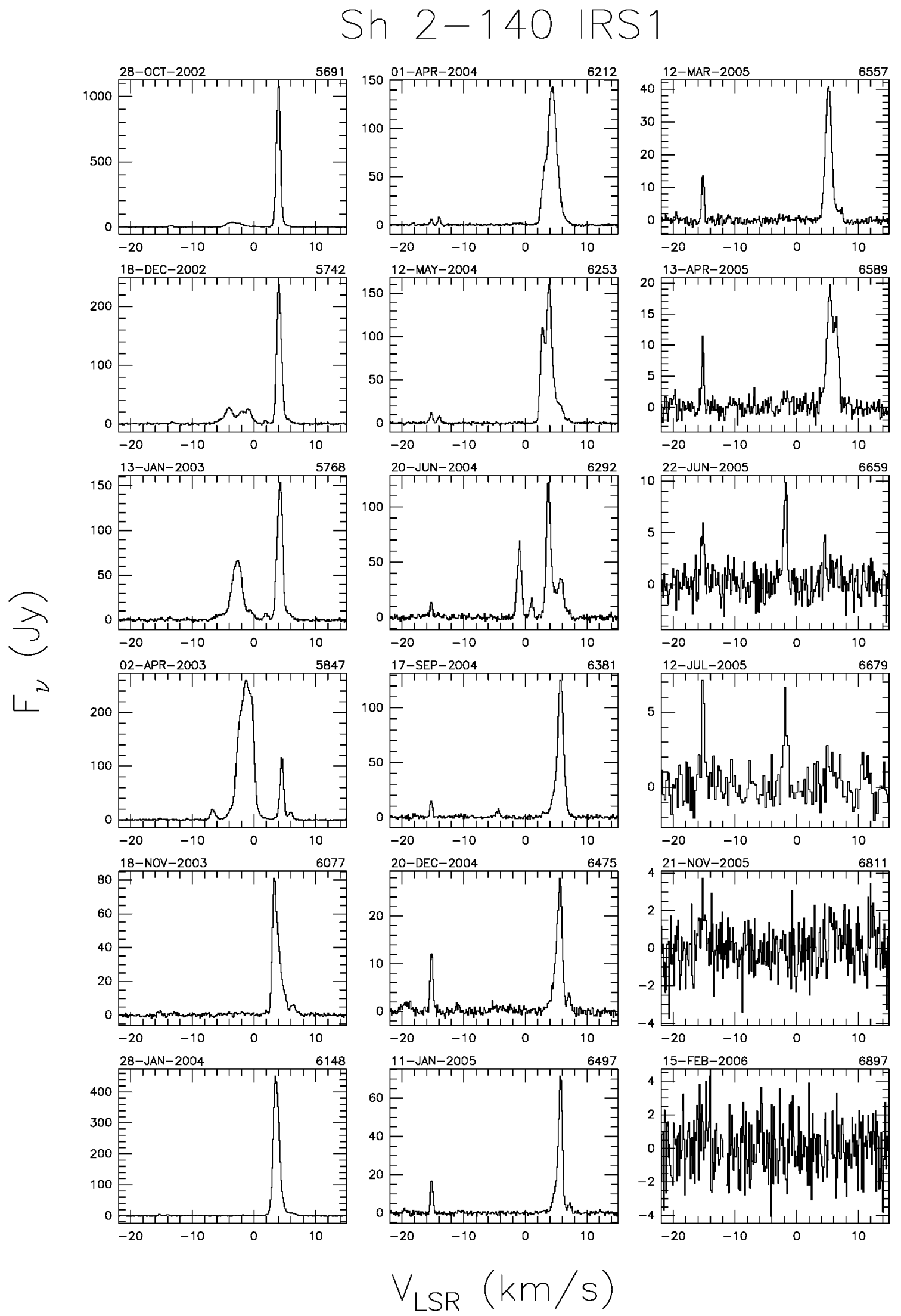

Fig. A.36. a continued. 
Sh 2-140 IRS1
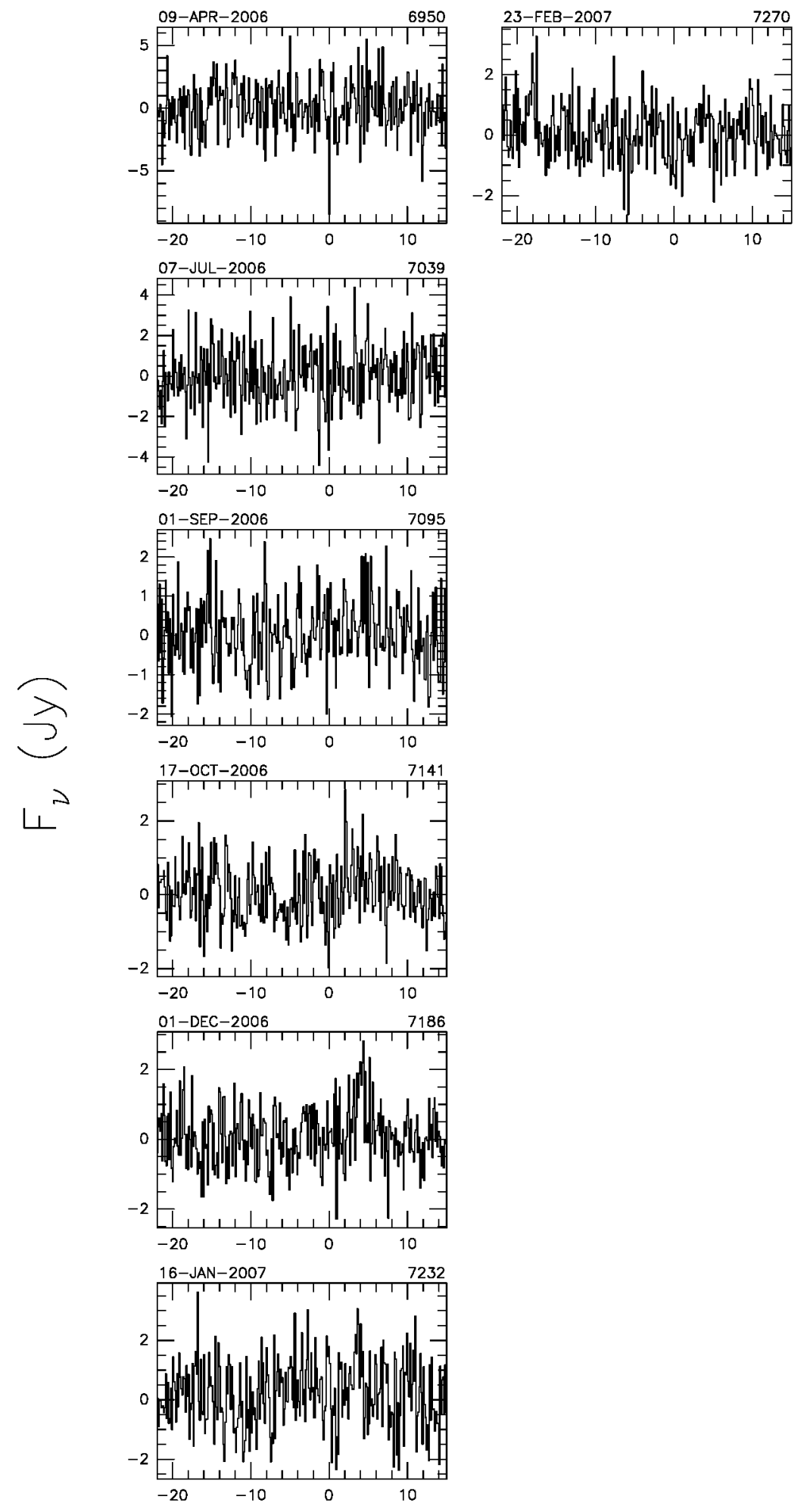

$$
V_{\text {LSR }}(\mathrm{km} / \mathrm{s})
$$

Fig. A.36. a continued. 


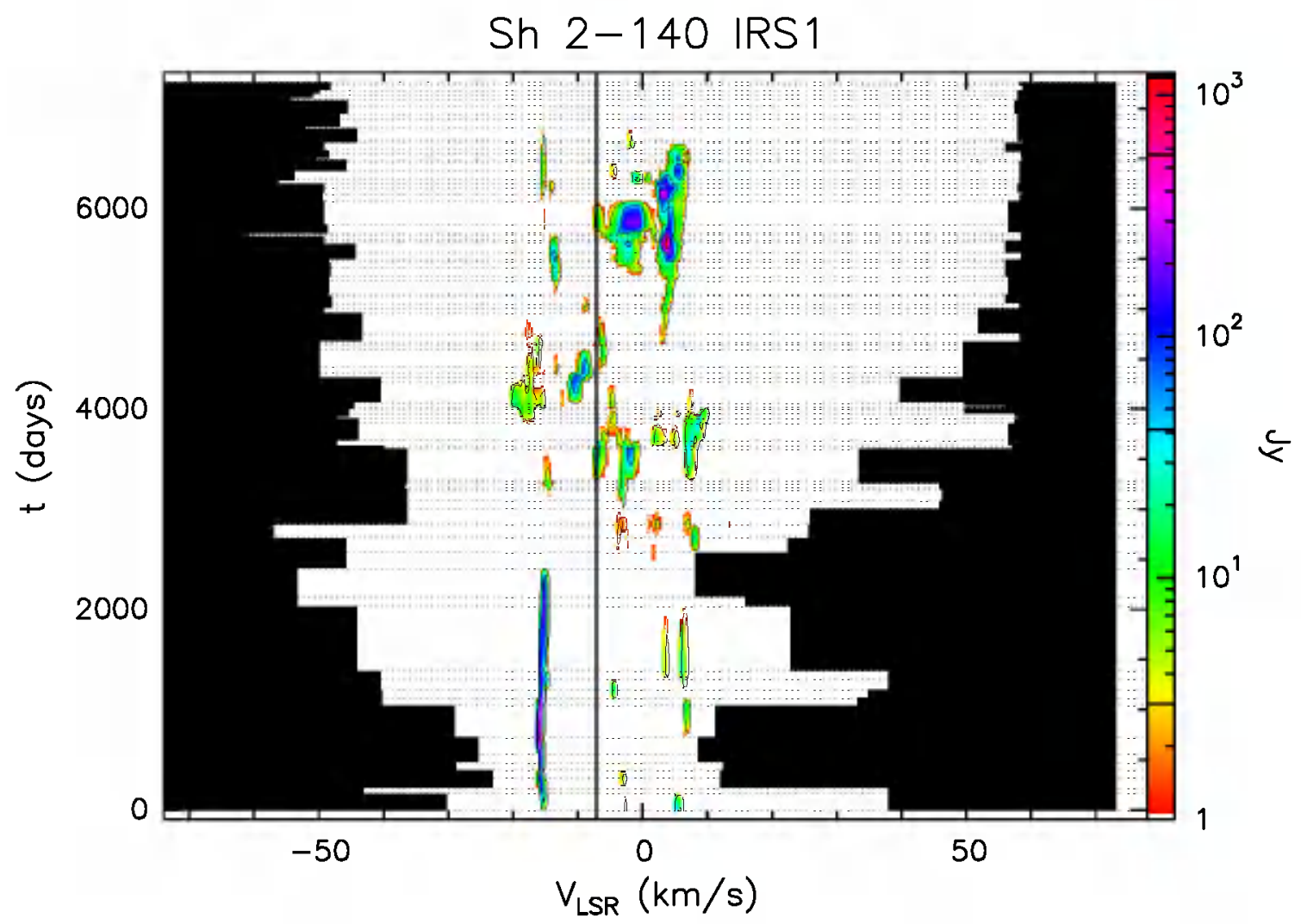

Fig. A.36. b Velocity-time-flux density full plot for source Sh 2-140 IRS I. The vertical solid line indicates the velocity of the associated thermal molecular gas. The flux density scale is shown by the bar on the right. In this bar the three lines give the flux density of the drawn contours.

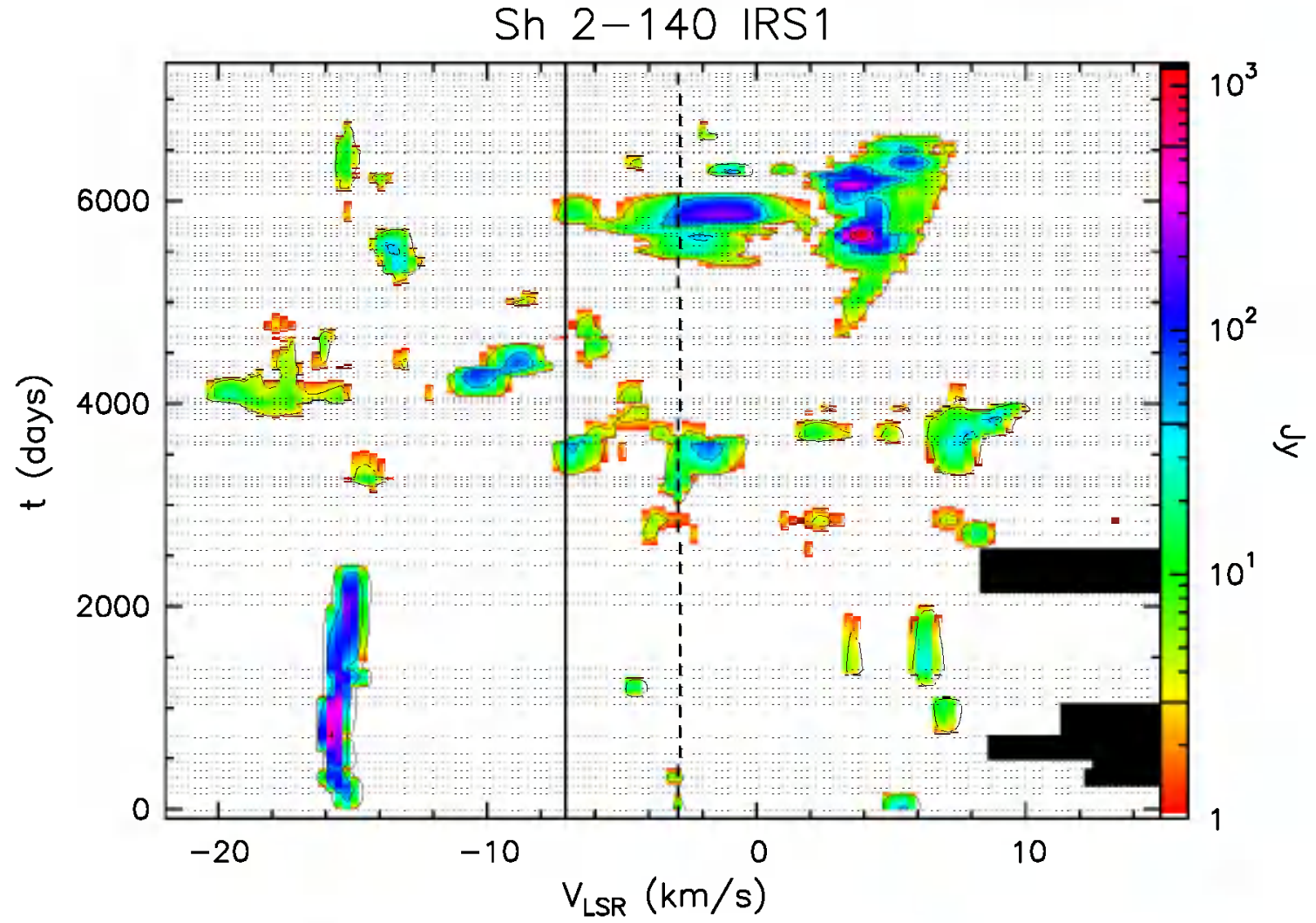

Fig. A.36. c Same as previous figure. but "zoomed" to velocity range over which emission has been detected. 


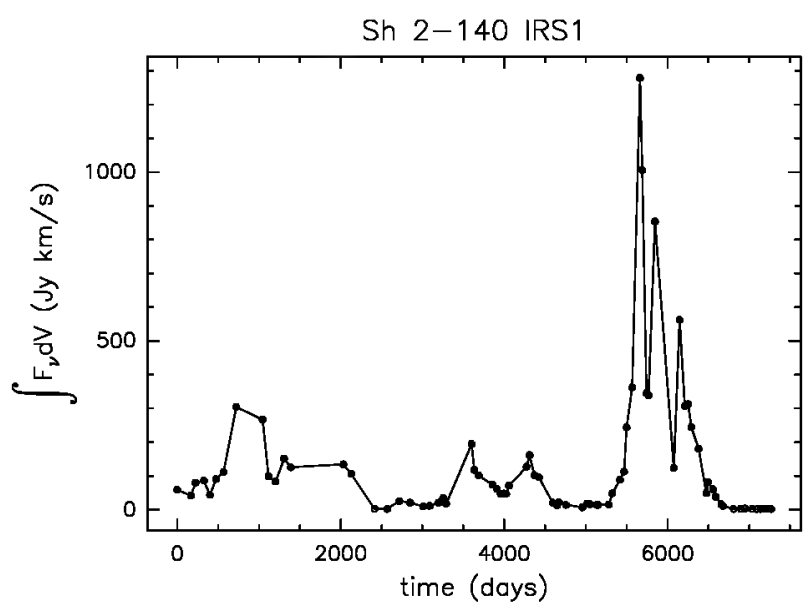

Fig. A.36. d Integral of the flux density over the observed velocity range as a function of time for source Sh 2-140 IRS1.

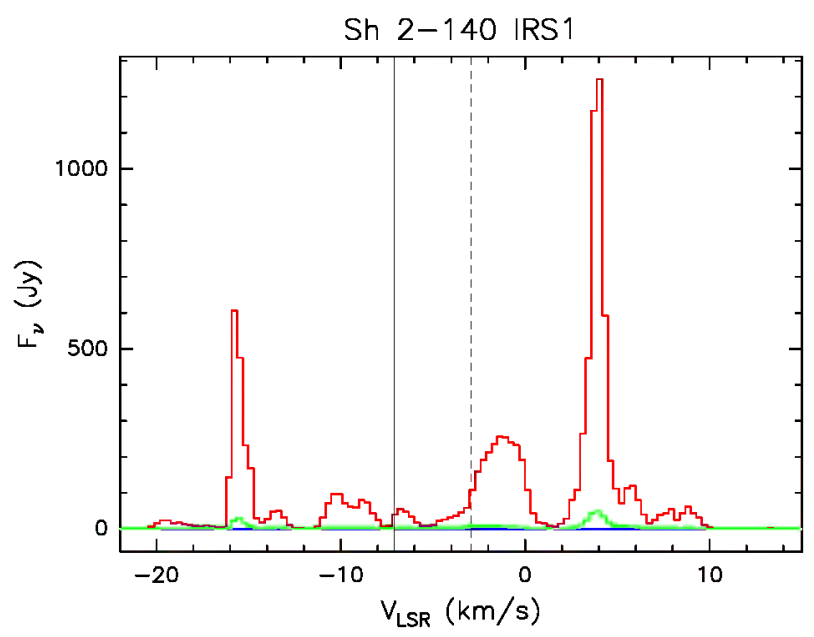

Fig. A.36. e Upper (red) and lower (blue) envelopes and mean spectrum (green) of source Sh 2-140 IRS1 measured during our monitoring. The vertical solid line marks the velocity of the associated thermal molecular gas. The vertical dashed line marks the mean velocity derived from the histogram of the rate-of-occurrence.

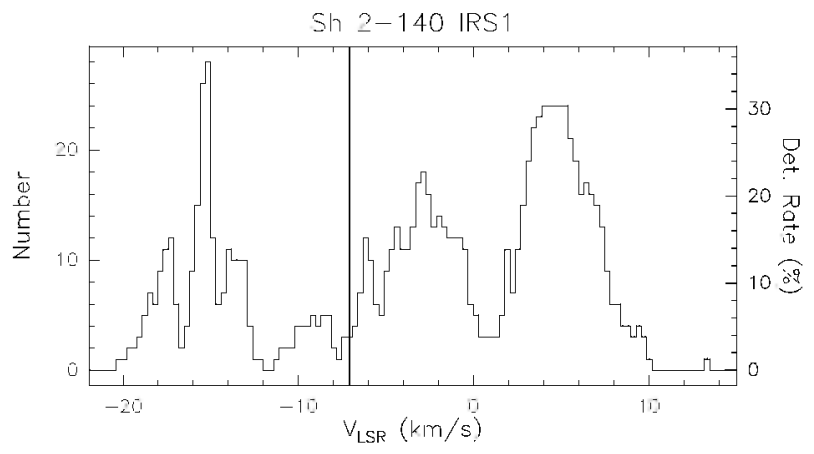

Fig. A.36. f Rate-of-occurrence plot for source Sh 2-140 IRS1. The scale to the right refers to the dotted histogram, the scale to the left to the solid line histogram. The vertical solid line marks the velocity of the associated thermal molecular gas. 


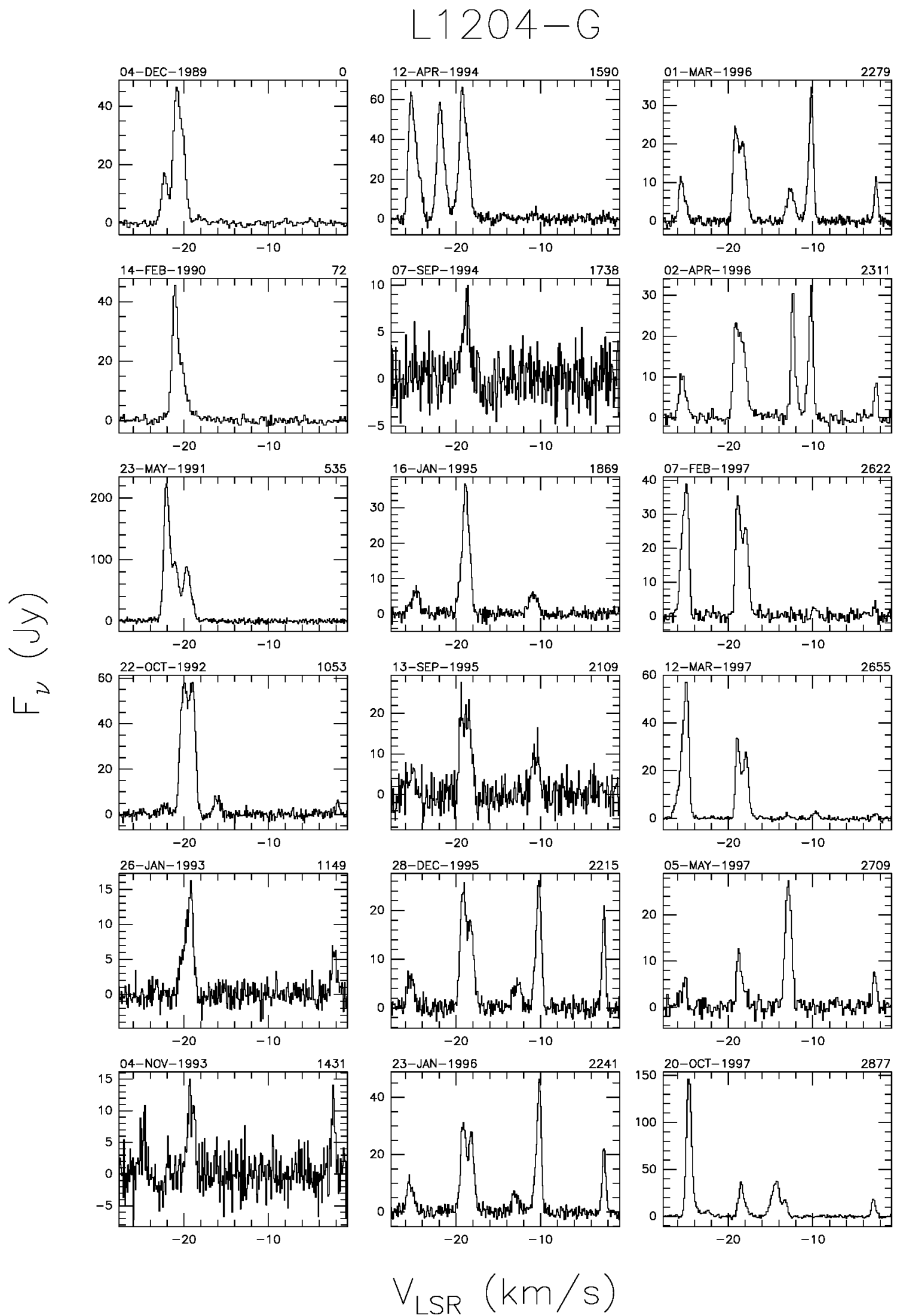

Fig. A.37. a Spectra of source L1204-G with autoscaled flux density scale. The date of observation is shown above the top left corner of each spectrum and the number of days elapsed since the first observation is given above the top right corner. The velocity scale is the same for all spectra. 


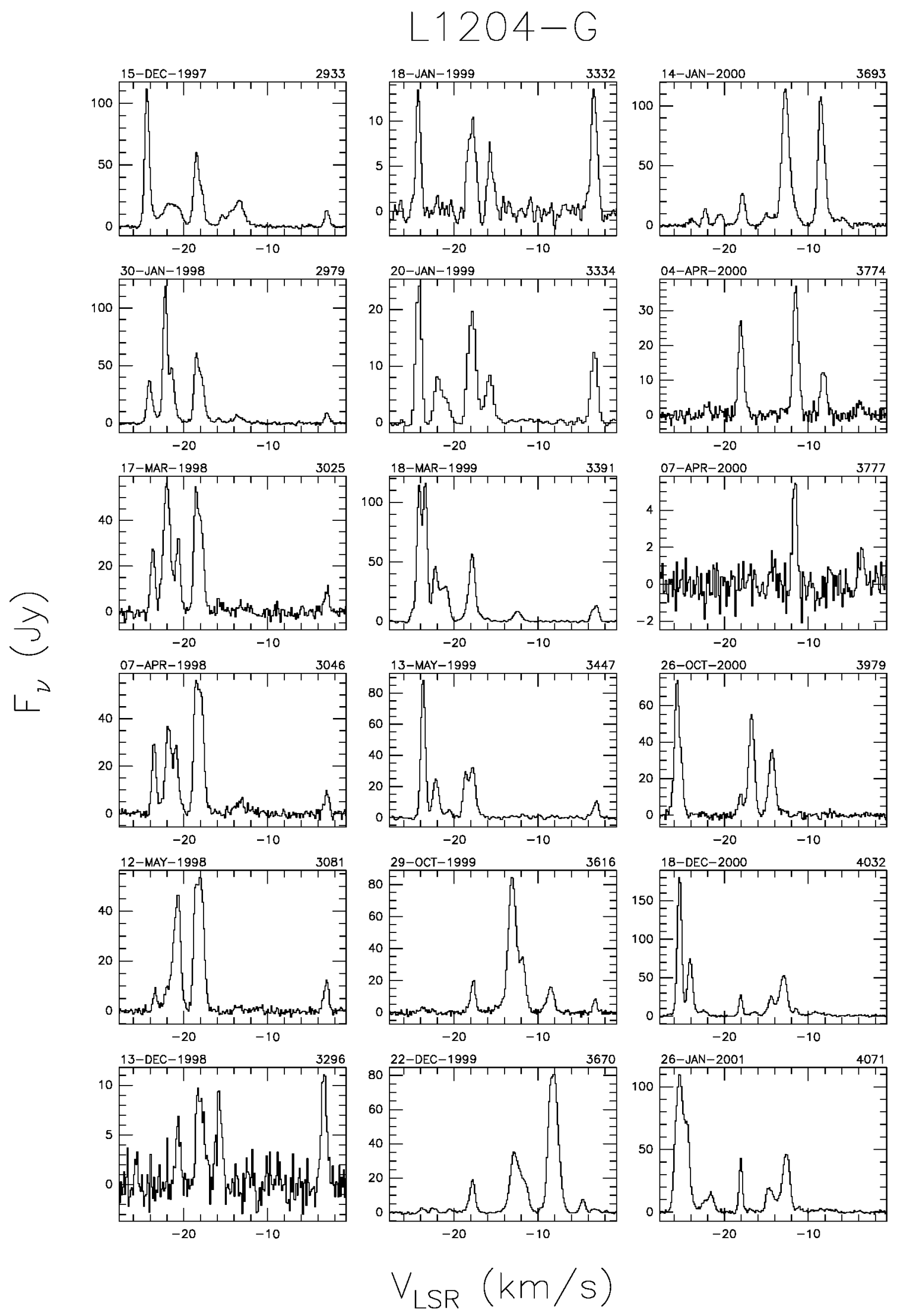

Fig. A.37. a continued. 


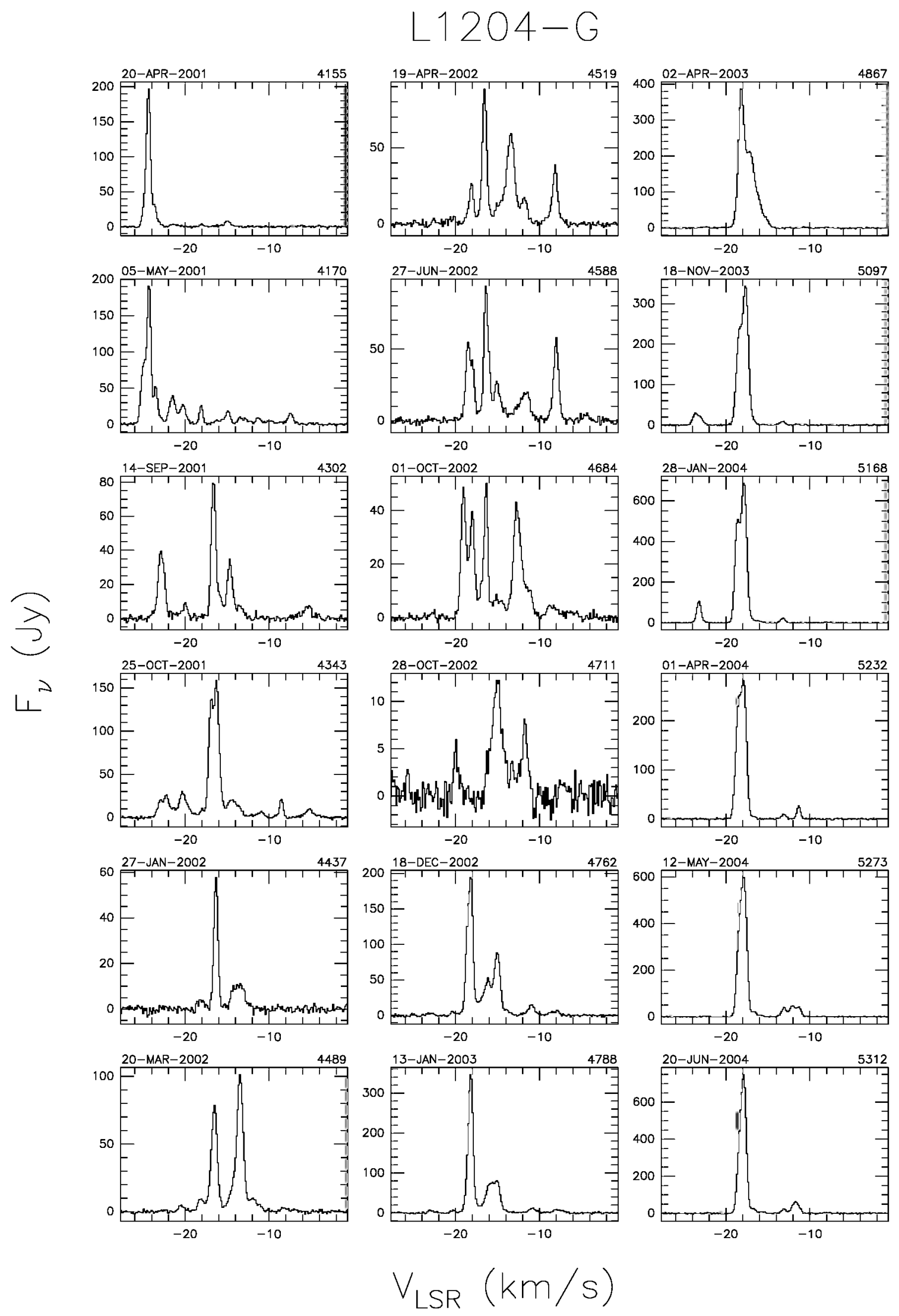

Fig. A.37. a continued. 


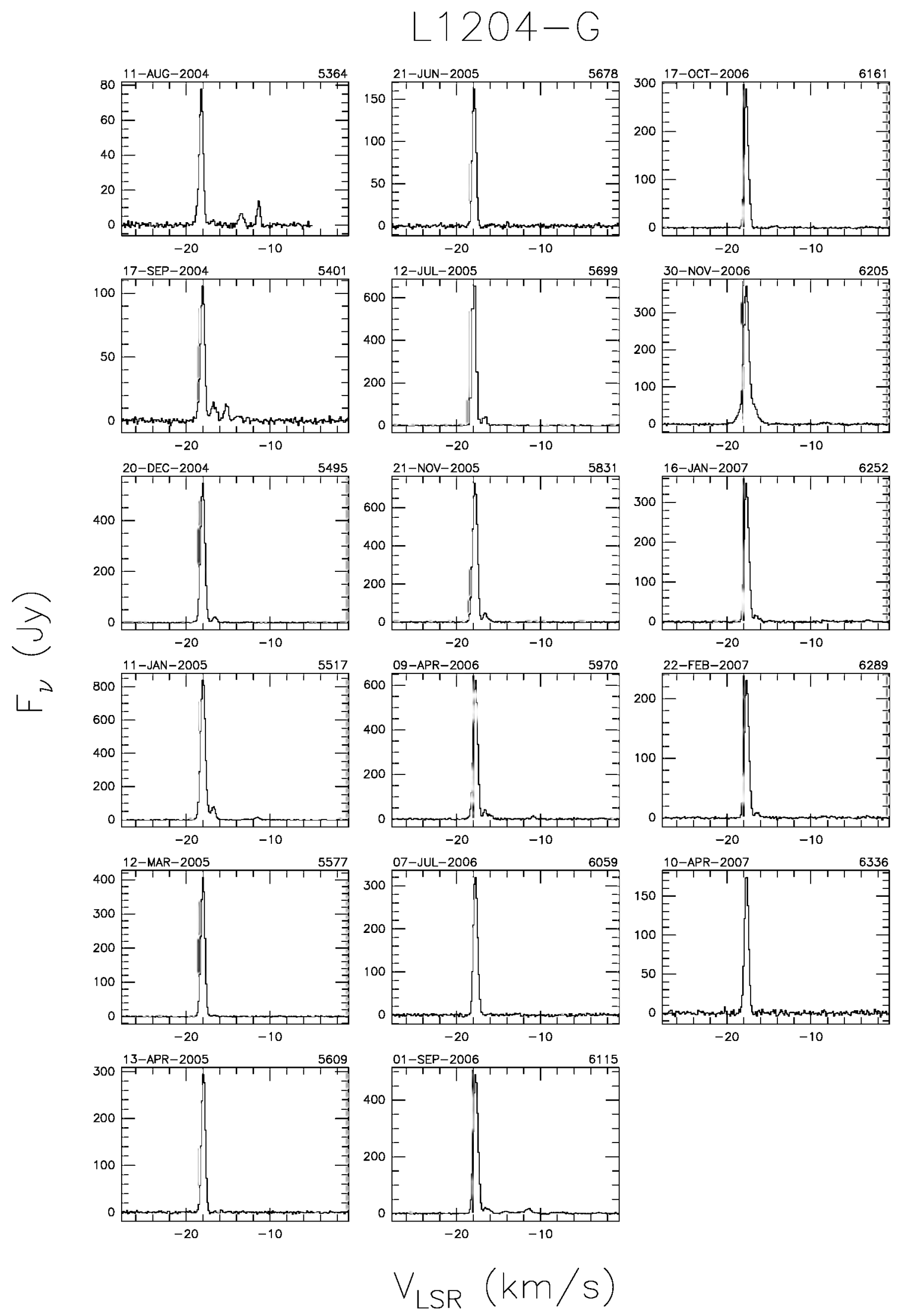

Fig. A.37. a continued. 


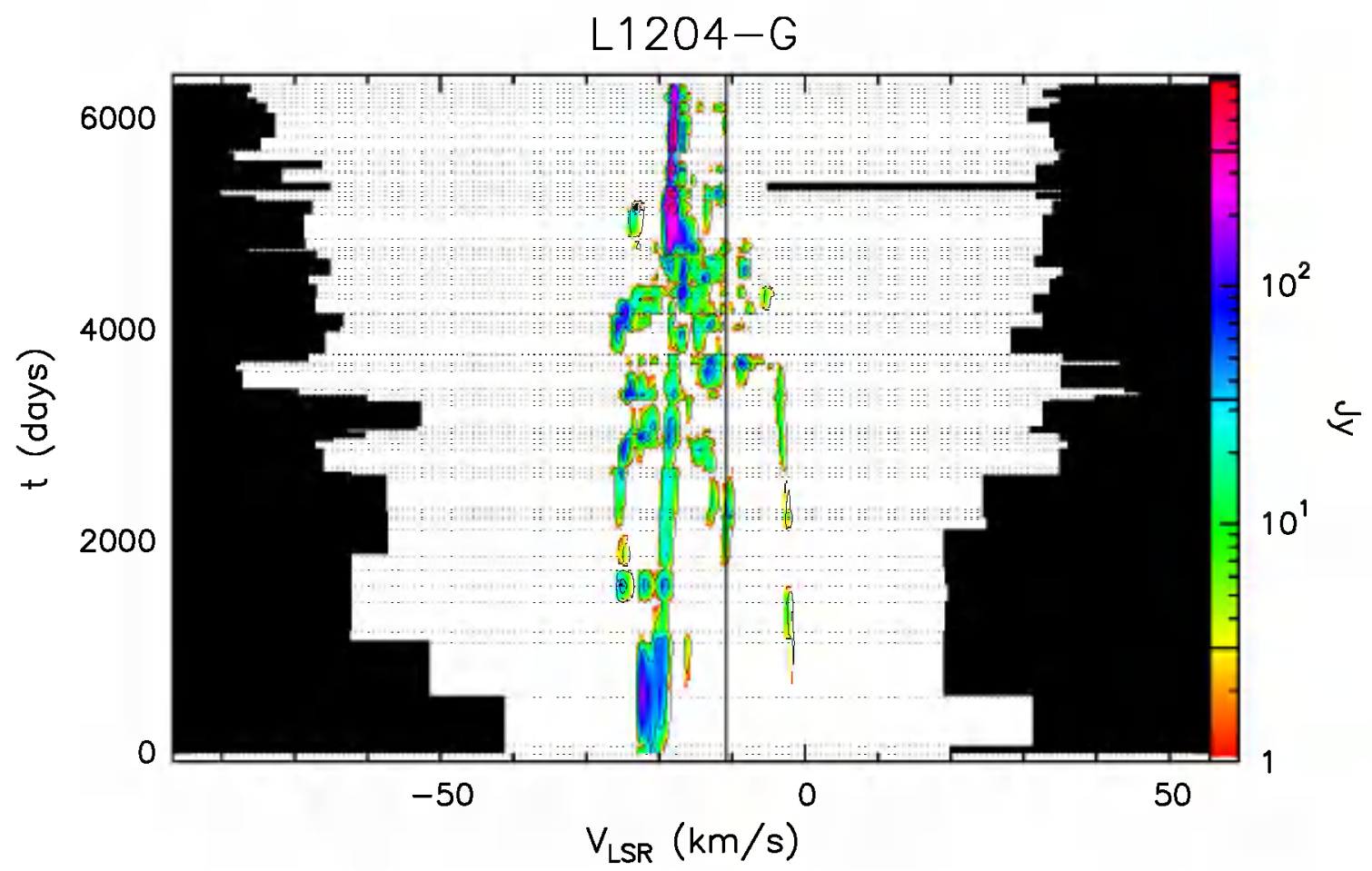

Fig. A.37. b Velocity-time-flux density full plot for source L1204-G. The vertical solid line indicates the velocity of the associated thermal molecular gas. The flux density scale is shown by the bar on the right. In this bar' the three lines give the flux density of the drawn contours.

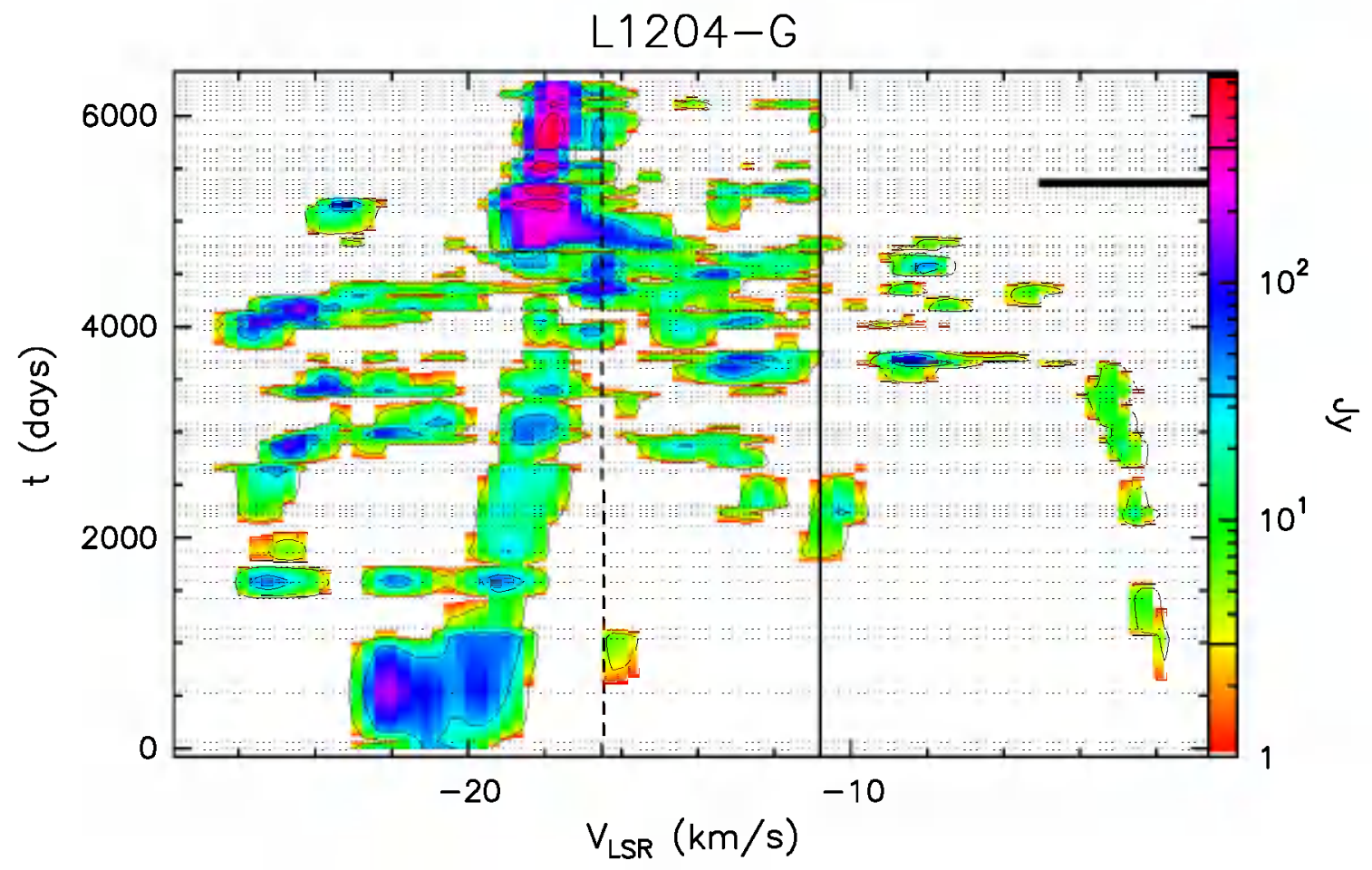

Fig. A.37. c Same as previous figure, but "zoomed" to velocity range over which emission has been detected. 


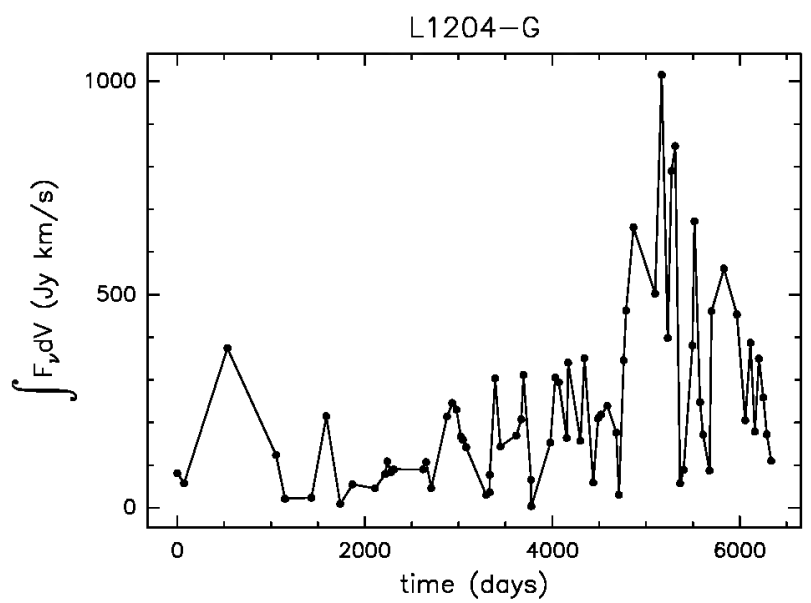

Fig. A.37. d Integral of the flux density over the observed velocity range as a function of time for source L1204-G.

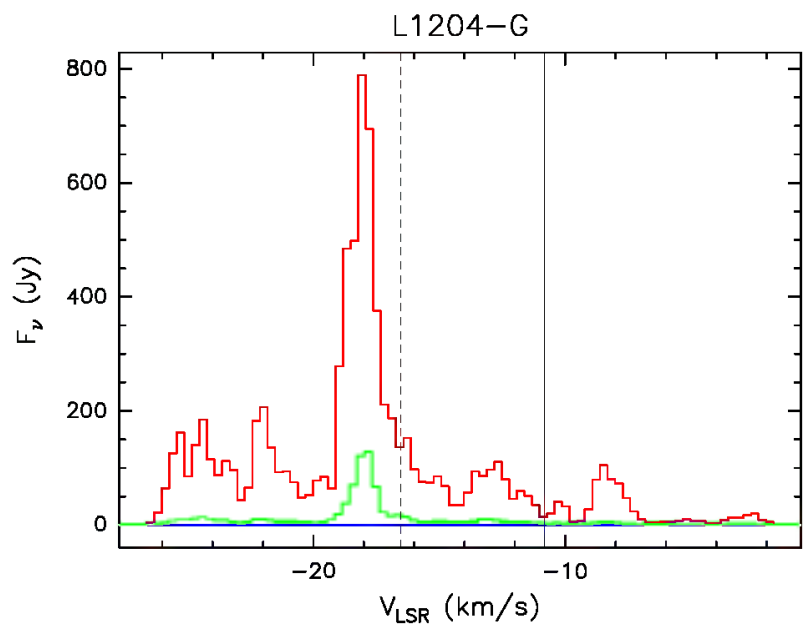

Fig. A.37. e Upper (red) and lower (blue) envelopes and mean spectrum (green) of source L1204-G measured during our monitoring. The vertical solid line marks the velocity of the associated thermal molecular gas. The vertical dashed line marks the mean velocity derived from the histogram of the rate-of-occurrence.

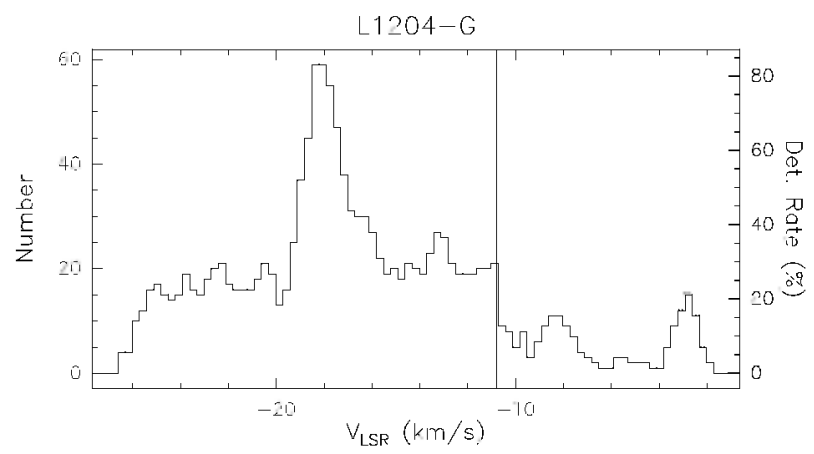

Fig. A.37. f Rate-of-occurrence plot for source L1204-G. The scale to the right refers to the dotted histogram, the scale to the left to the solid line histogram. The vertical solid line marks the velocity of the associated thermal molecular gas. 


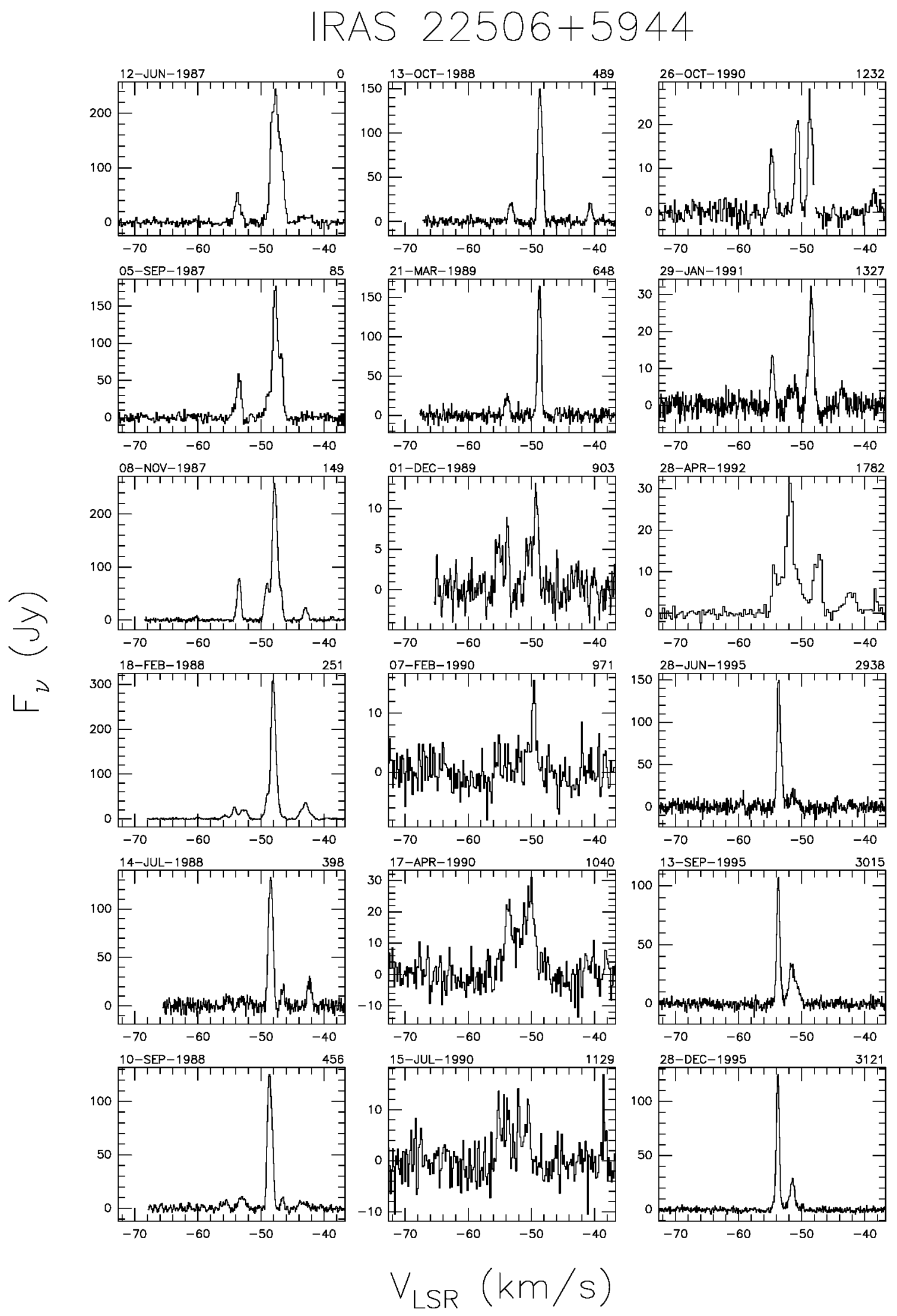

Fig. A.38. a Spectra of source IRAS $22506+5944$ with autoscaled flux density scale. The date of observation is shown above the top left corner of each spectrum and the number of days elapsed since the first observation is given above the top right corner. The velocity scale is the same for all spectra. 
IRAS $22506+5944$
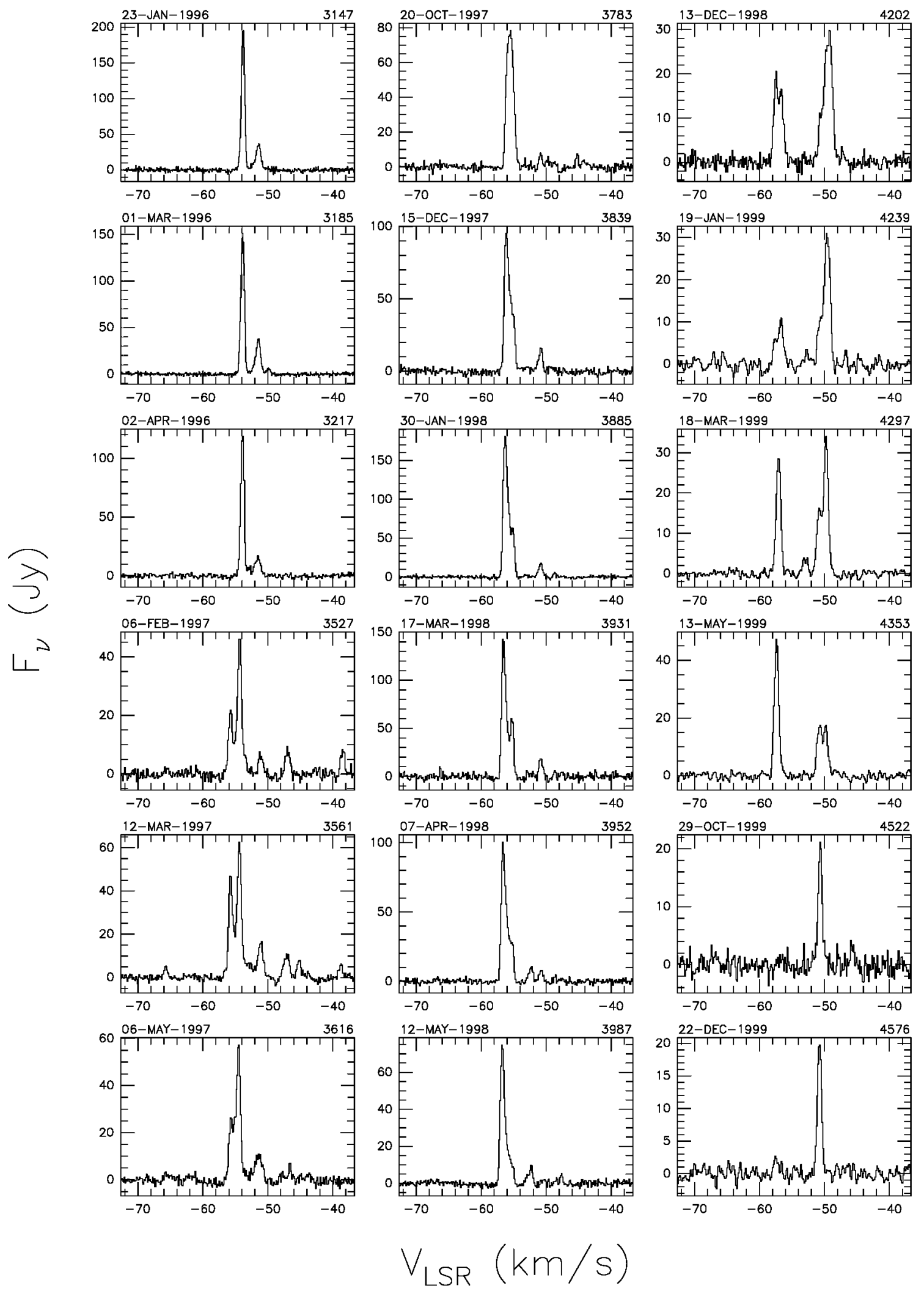

Fig. A.38. a continued. 
IRAS $22506+5944$
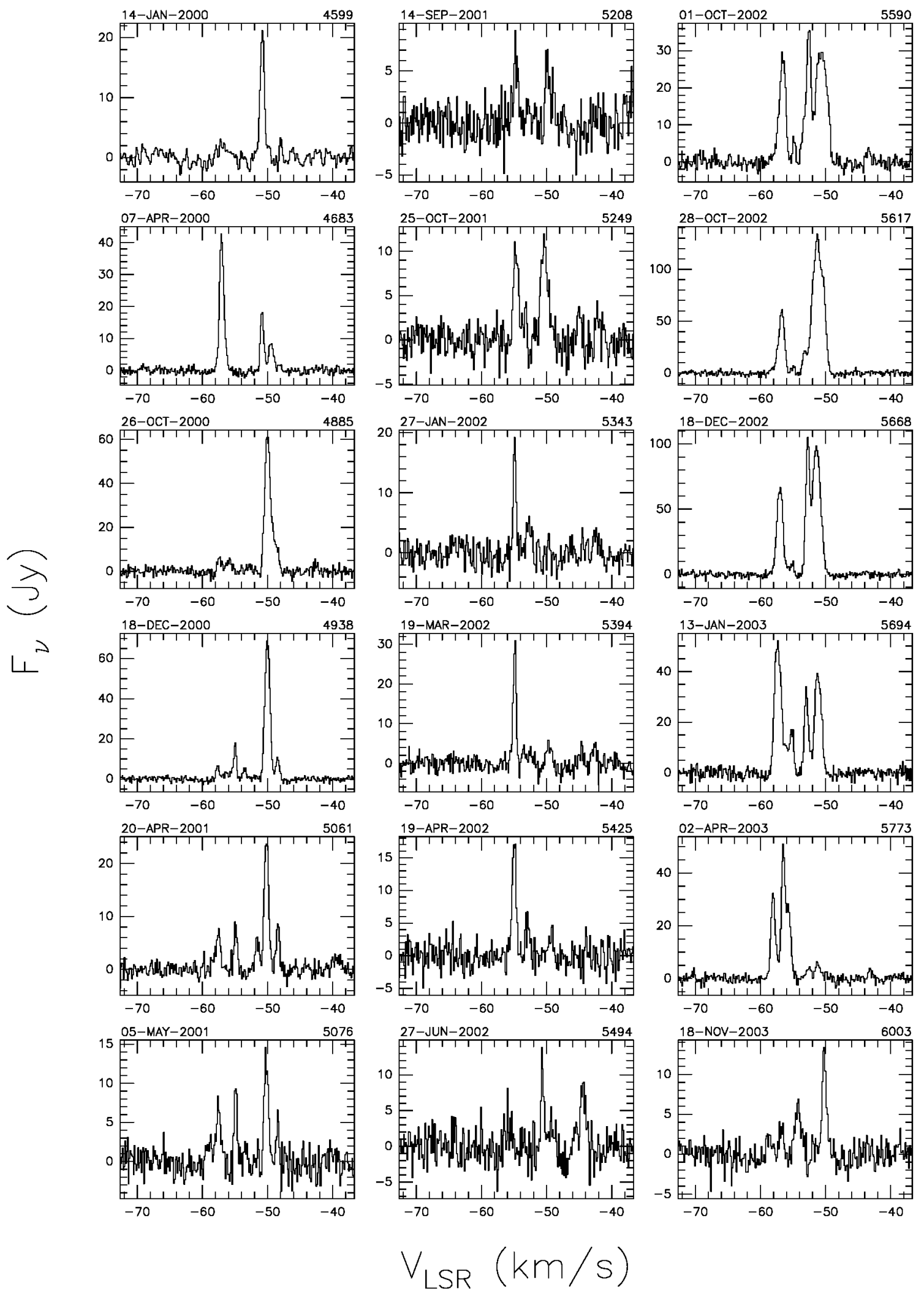

Fig. A.38. a continued. 
IRAS $22506+5944$
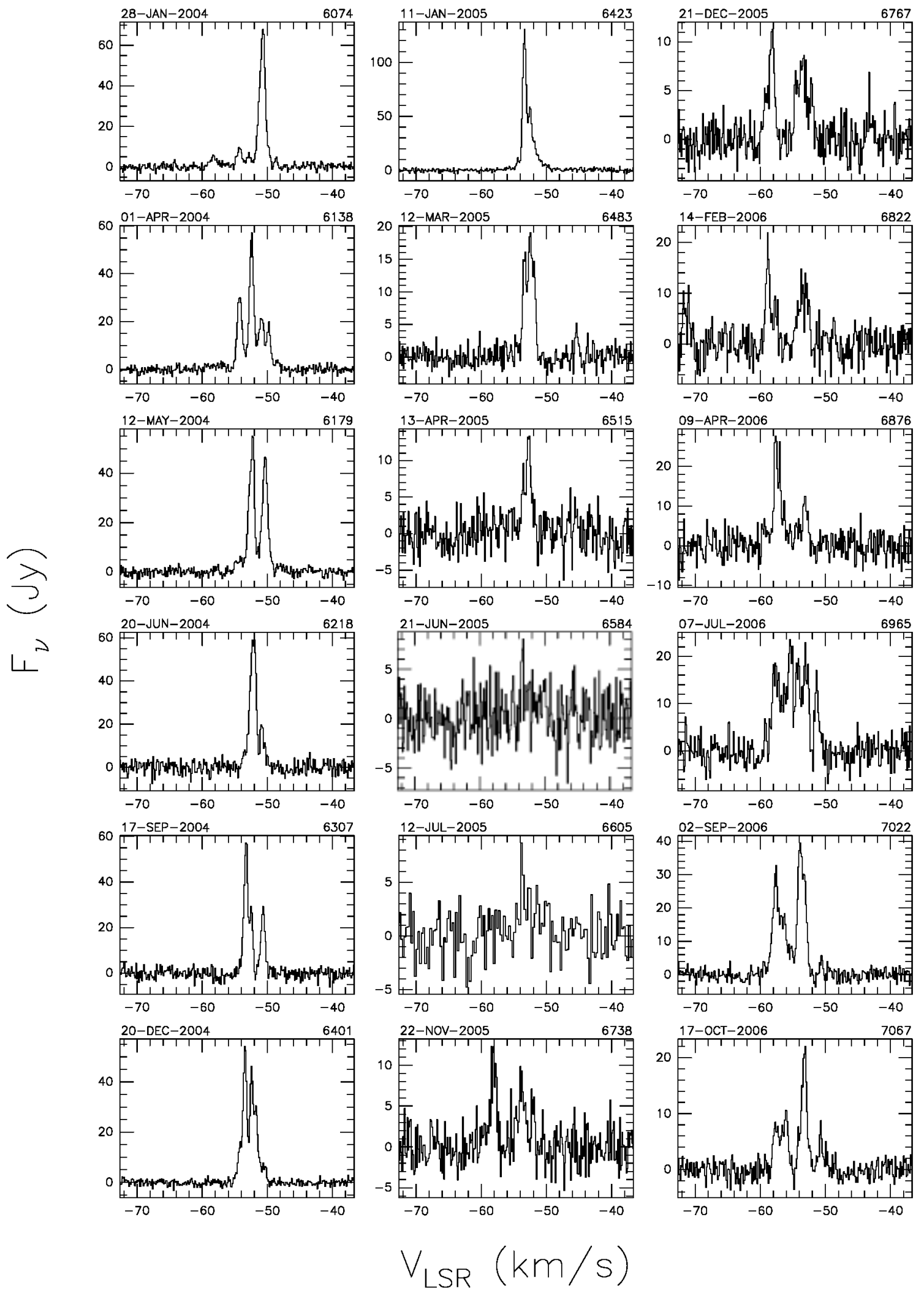

Fig. A.38. a continued. 


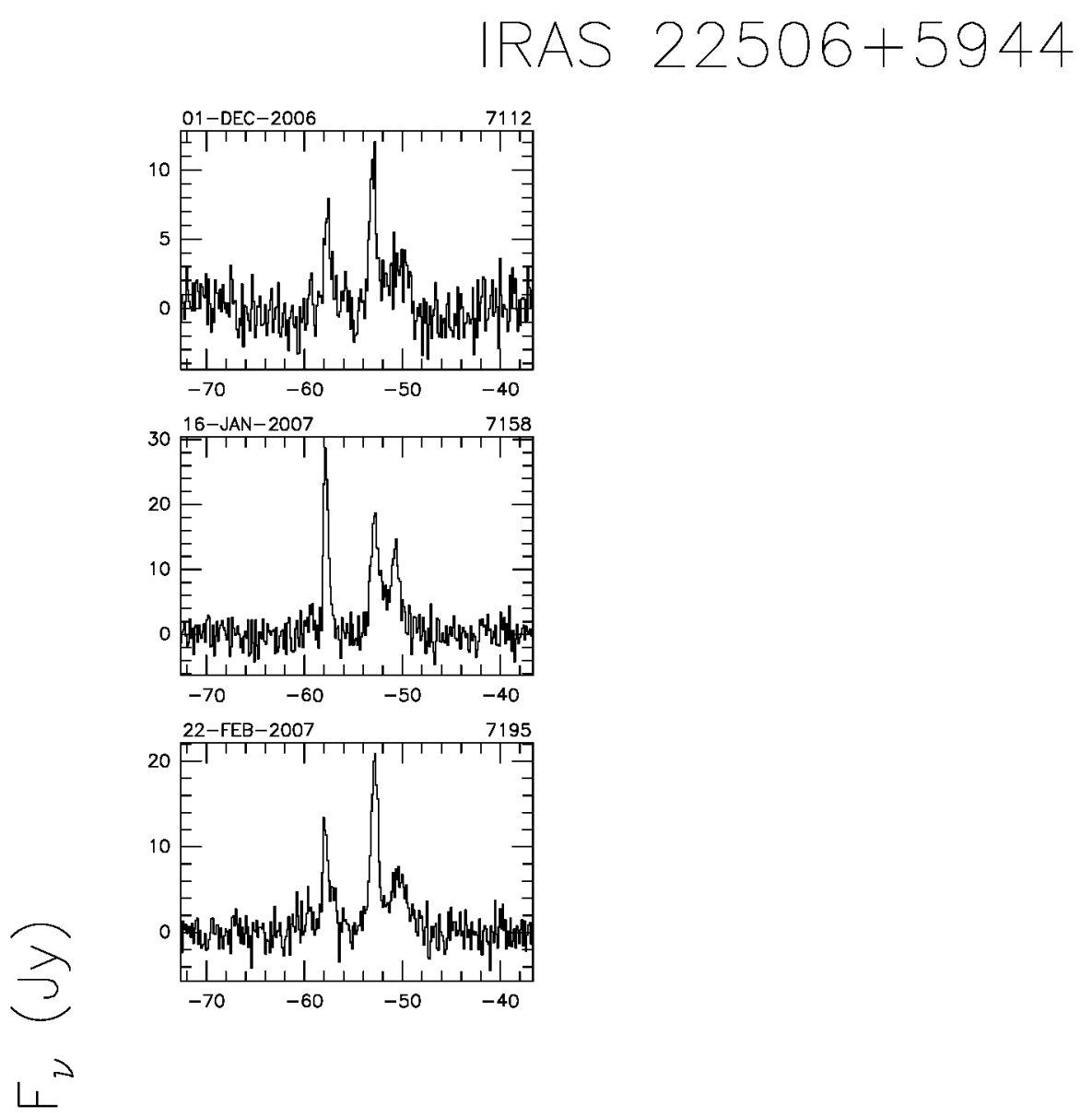

$V_{\text {LSR }}(\mathrm{km} / \mathrm{s})$

Fig. A.38. a continued. 


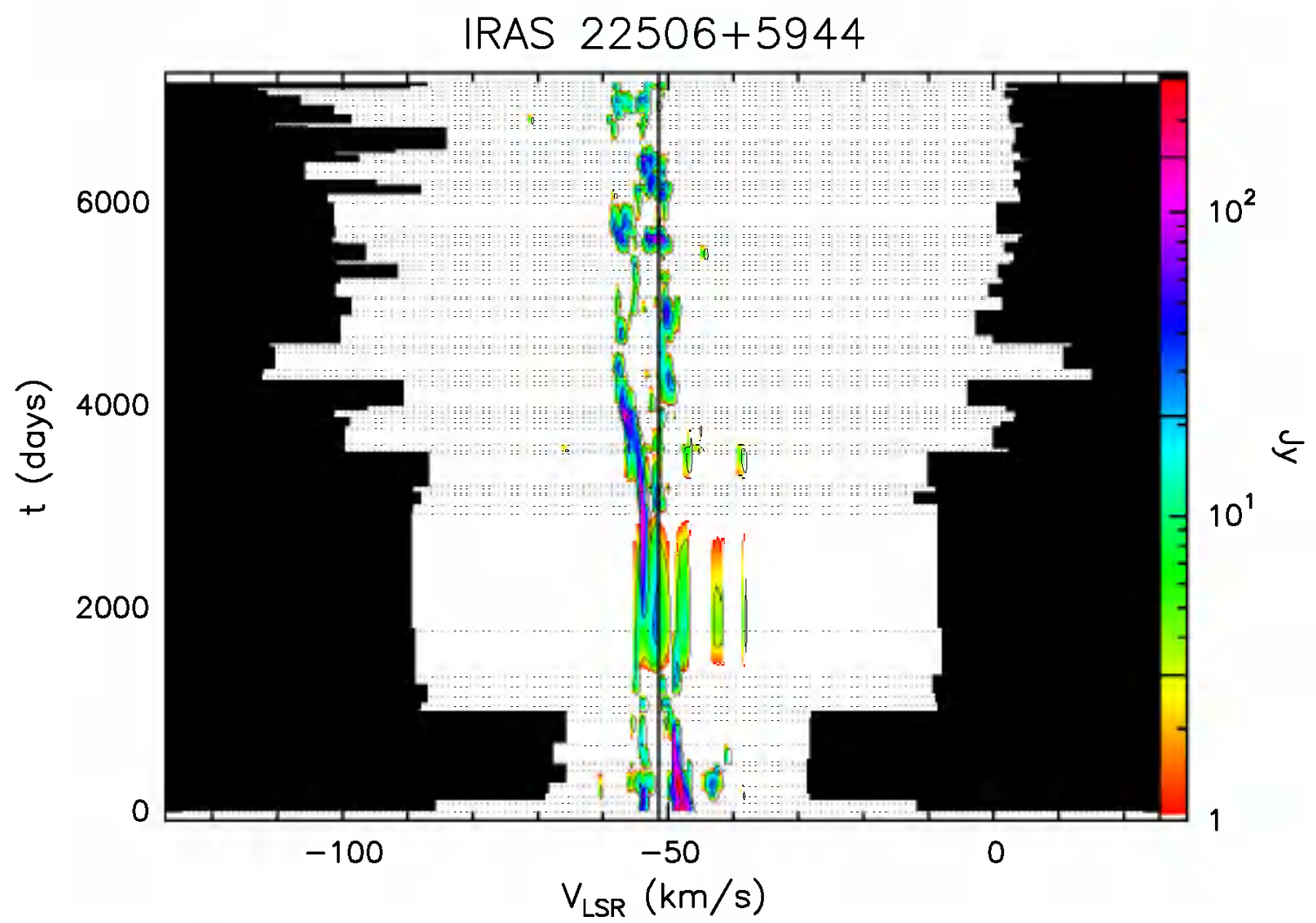

Fig. A.38. b Velocity-time-flux density full plot for source IRAS $22506+5944$. The vertical solid line indicates the velocity of the associated thermal molecular gas. The flux density scale is shown by the bar on the right. In this bar the three lines give the flux density of the drawn contours.

IRAS $22506+5944$

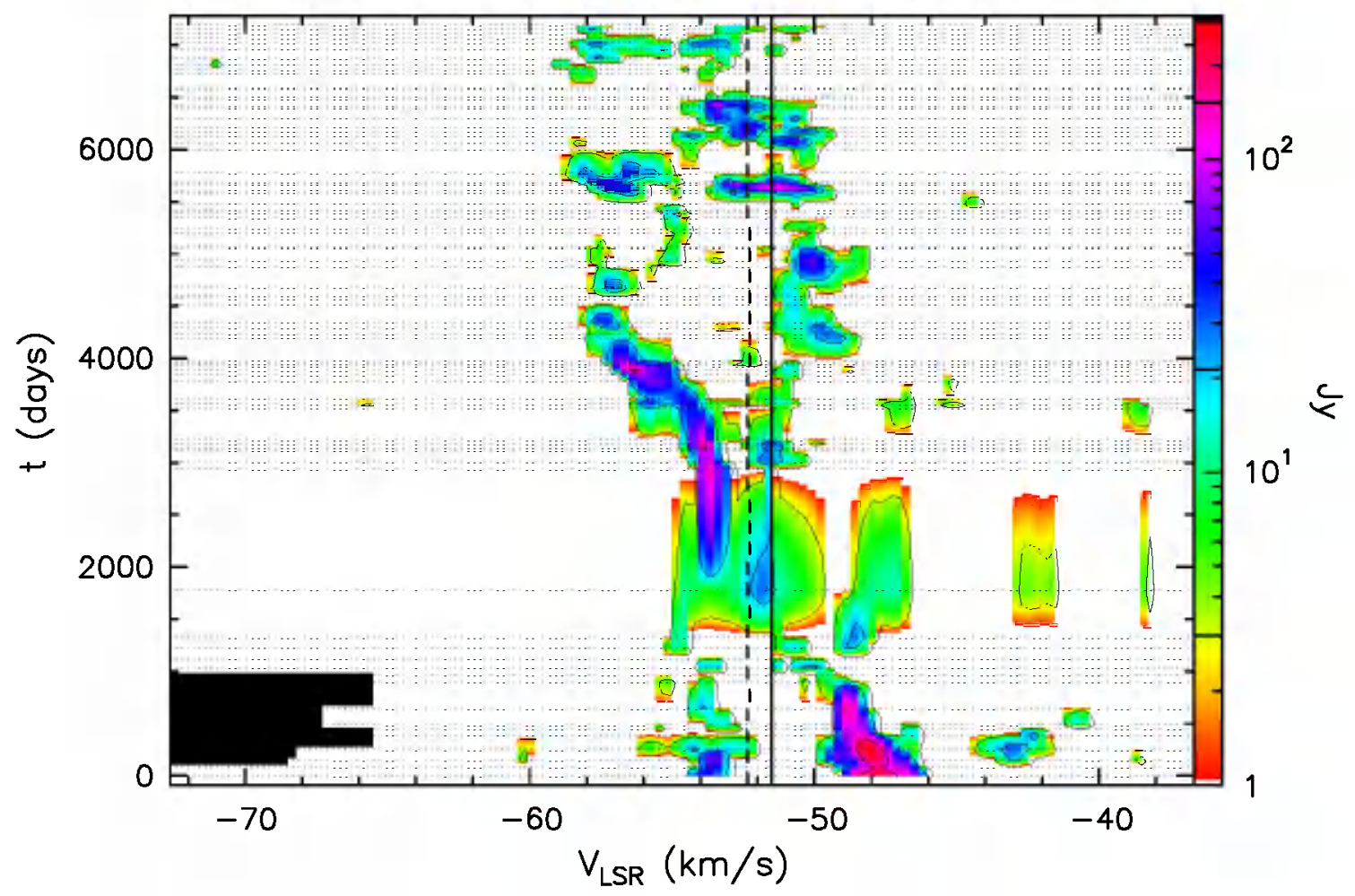

Fig. A.38. c Same as previous figure, but "zoomed" to velocity range over which emission has been detected. 


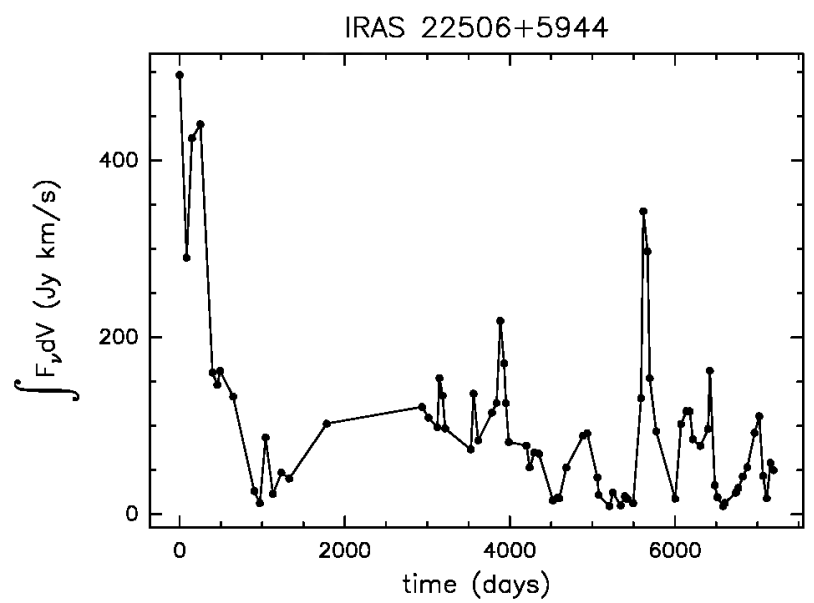

Fig. A.38. d Integral of the flux density over the observed velocity range as a function of time for source IRAS $22506+5944$.

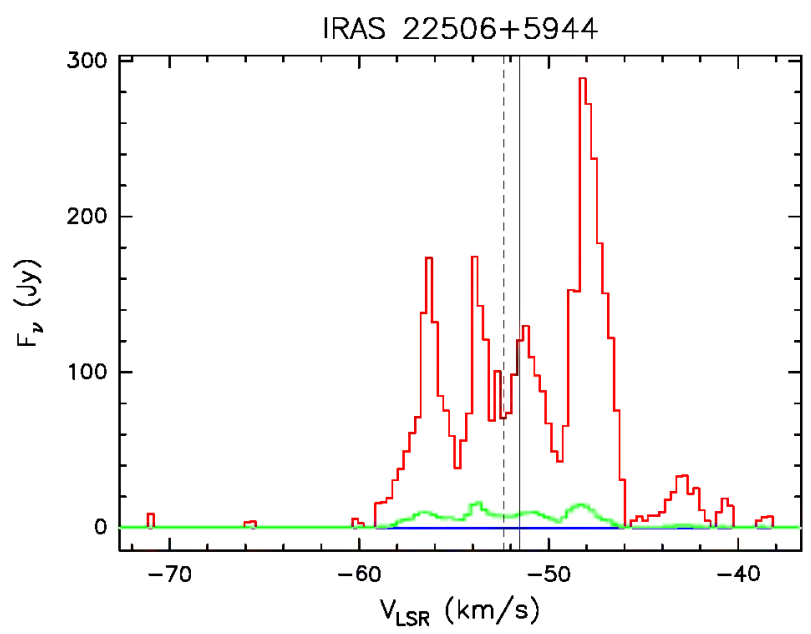

Fig. A.38. e Upper (red) and lower (blue) envelopes and mean spectrum (green) of source IRAS $22506+5944$ measured during our monitoring. The vertical solid line marks the velocity of the associated thermal molecular gas. The vertical dashed line marks the mean velocity derived from the histogram of the rate-of-occurrence.

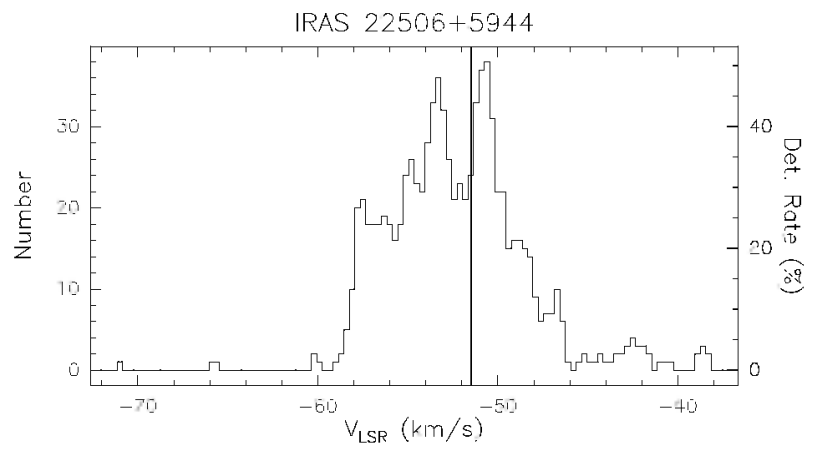

Fig. A.38. f Rate-of-occurrence plot for source IRAS 22506+5944. The scale to the right refers to the dotted histogram, the scale to the left to the solid line histogram. The vertical solid line marks the velocity of the associated thermal molecular gas. 


\section{Cepheus A}
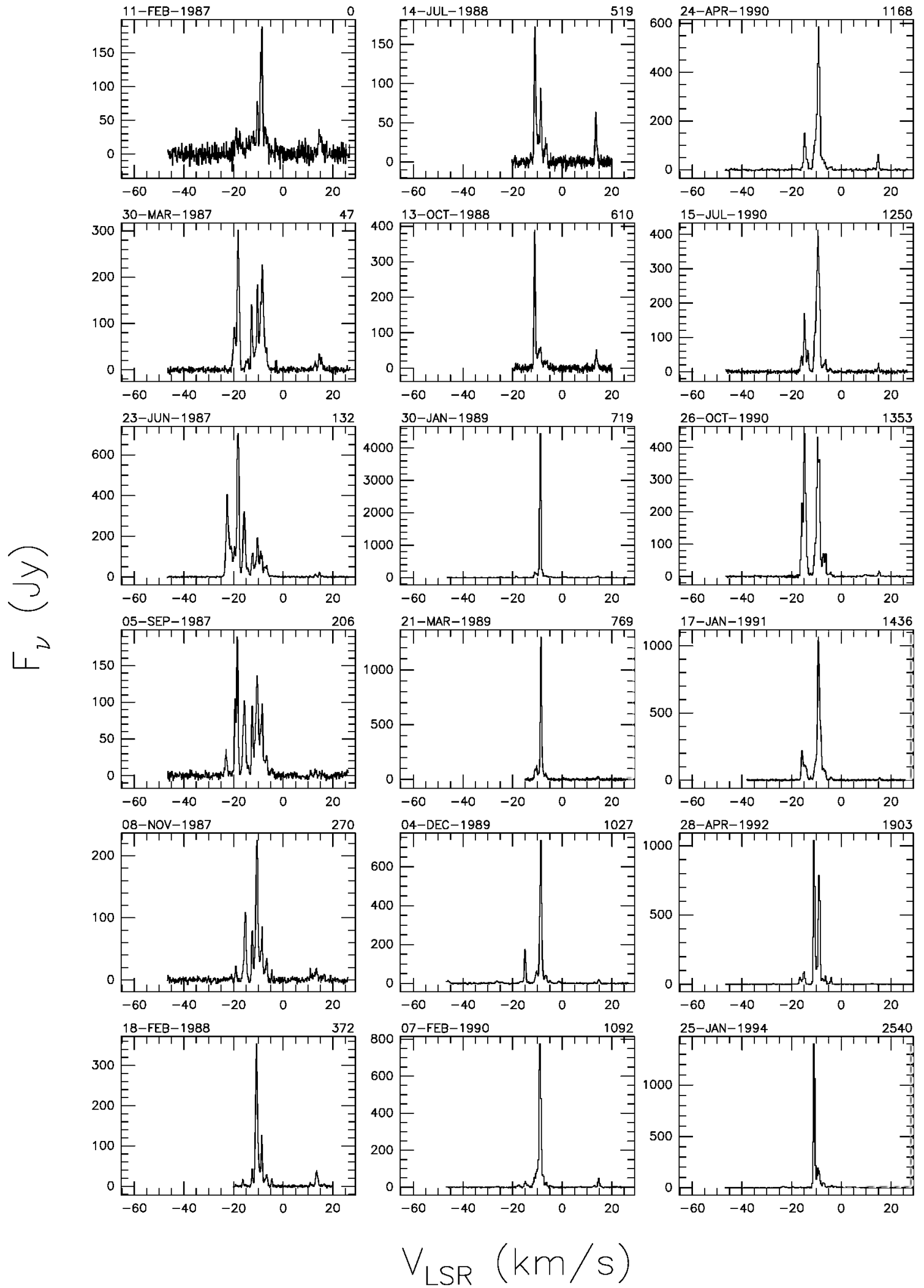

Fig. A.39. a Spectra of source Cepheus A with antoscaled flux density scale. The date of observation is shown above the top left corner of each spectrum and the number of days elapsed since the first observation is given above the top right corner. The velocity scale is the same for all spectra. 


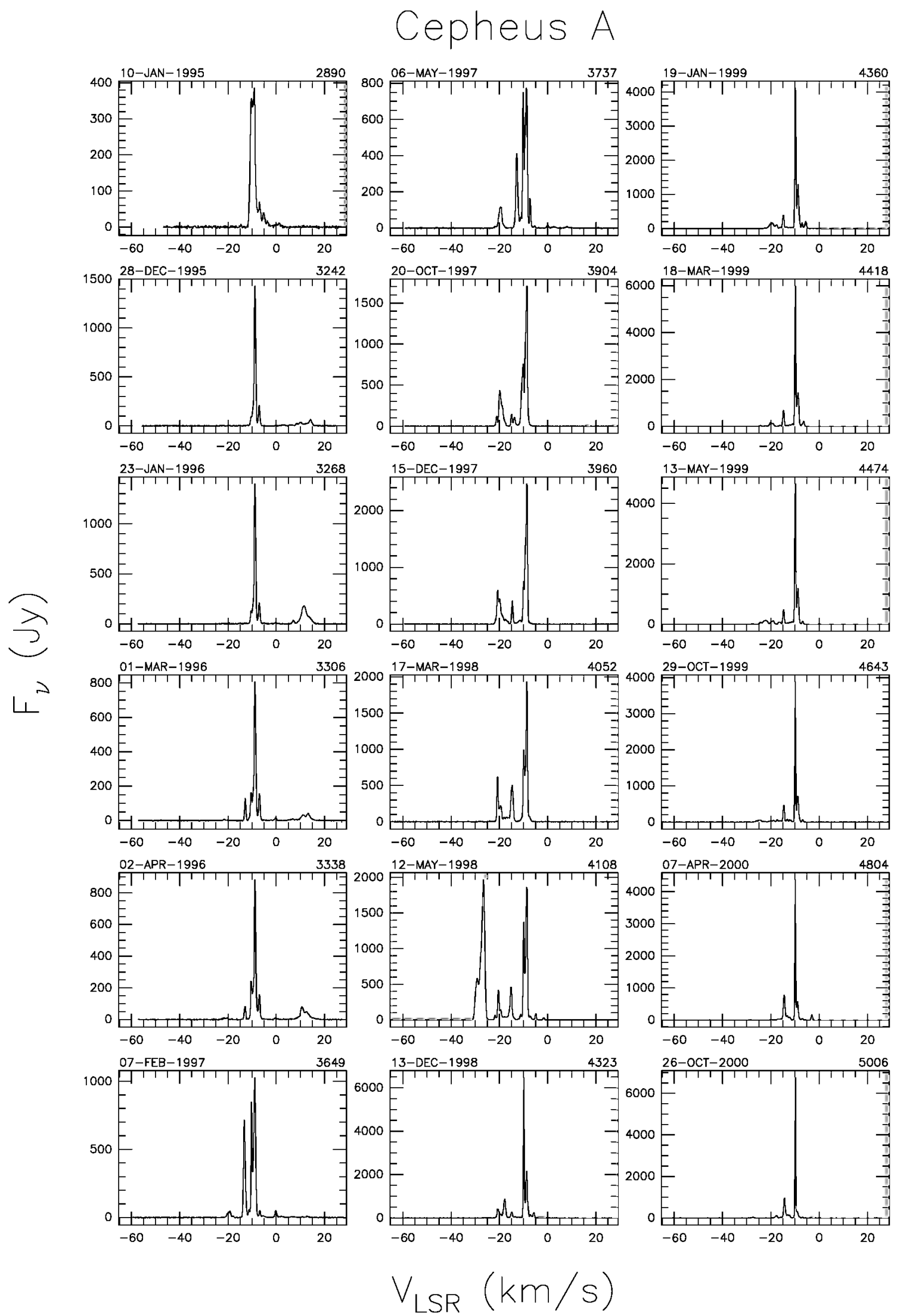

Fig. A.39. a continued. 


\section{Cepheus A}
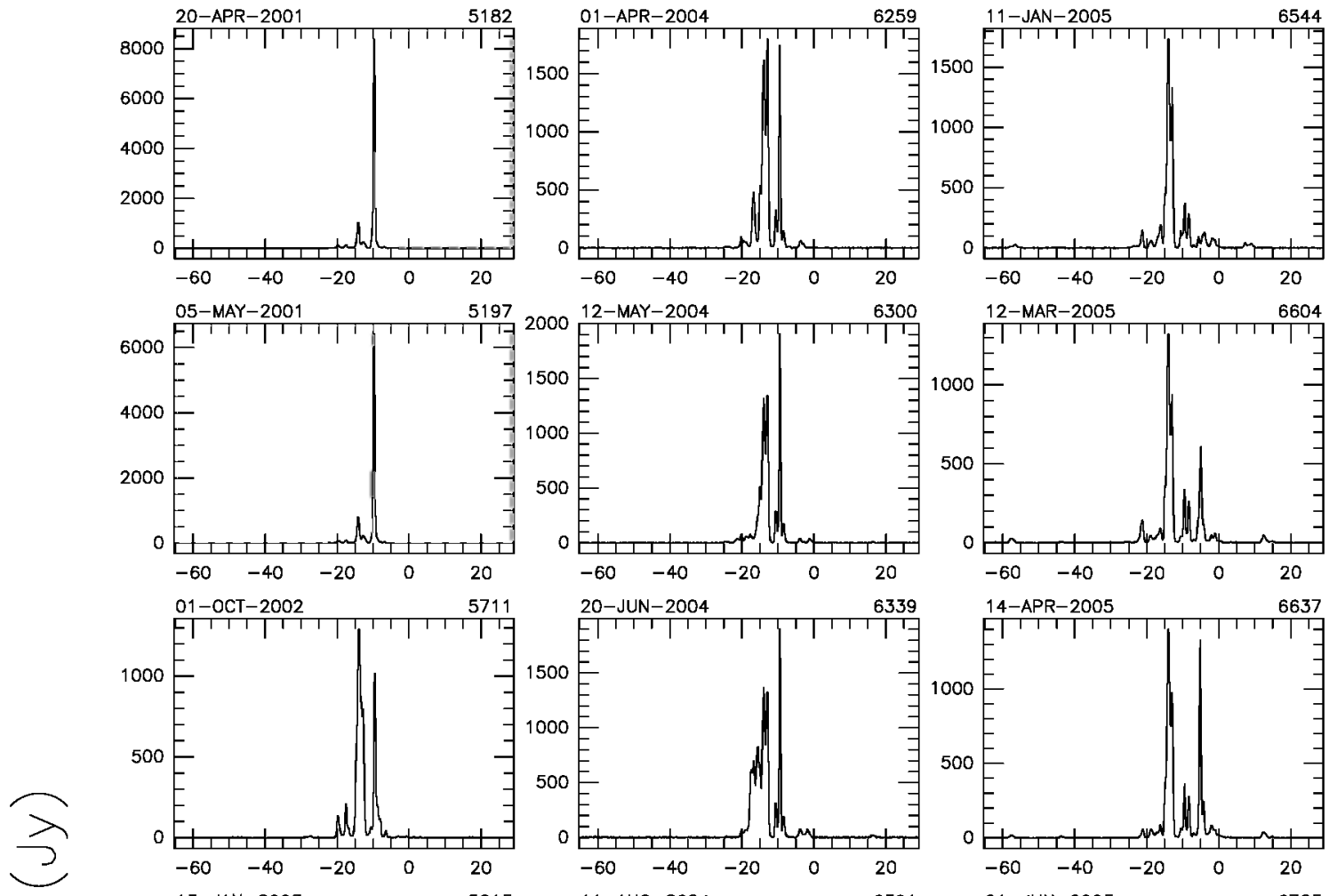

ᄂ
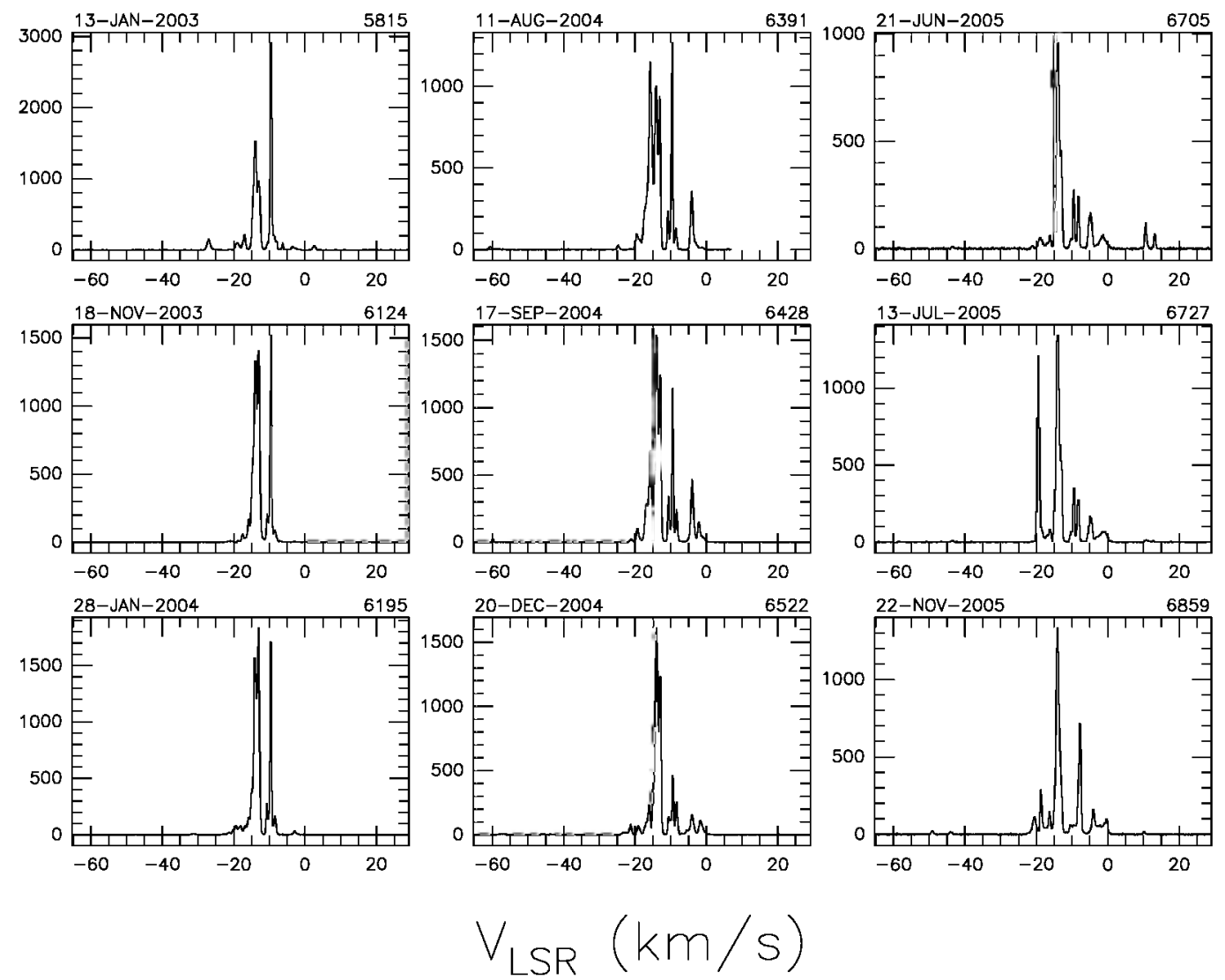

Fig. A.39. a continued. 
638

M. Felli et al.: Variability of $\mathrm{H}_{2} \mathrm{O}$ masers in SFR

Cepheus A
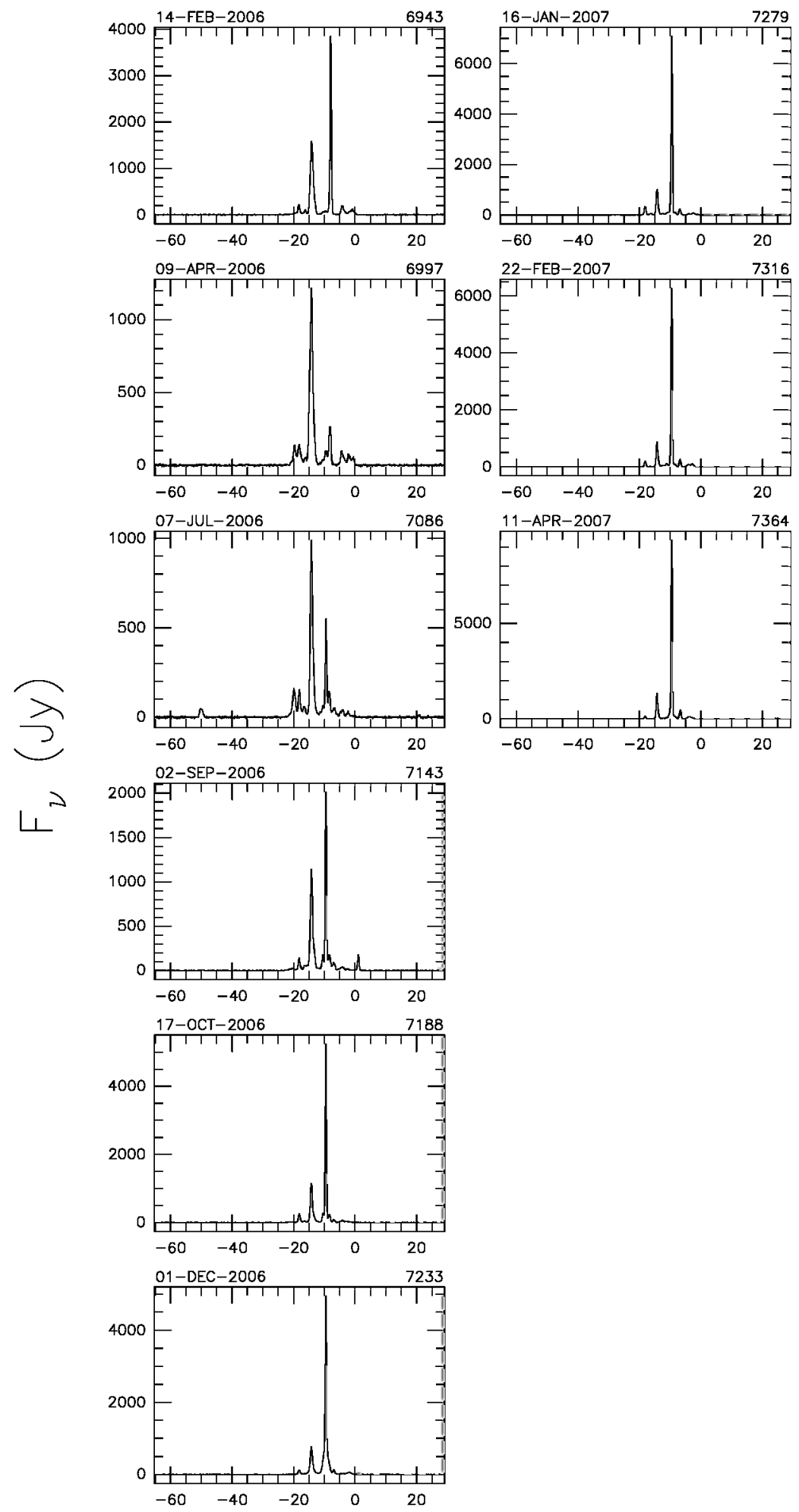

$$
V_{\text {LR }}(\mathrm{km} / \mathrm{s})
$$

Fig. A.39. a continued. 


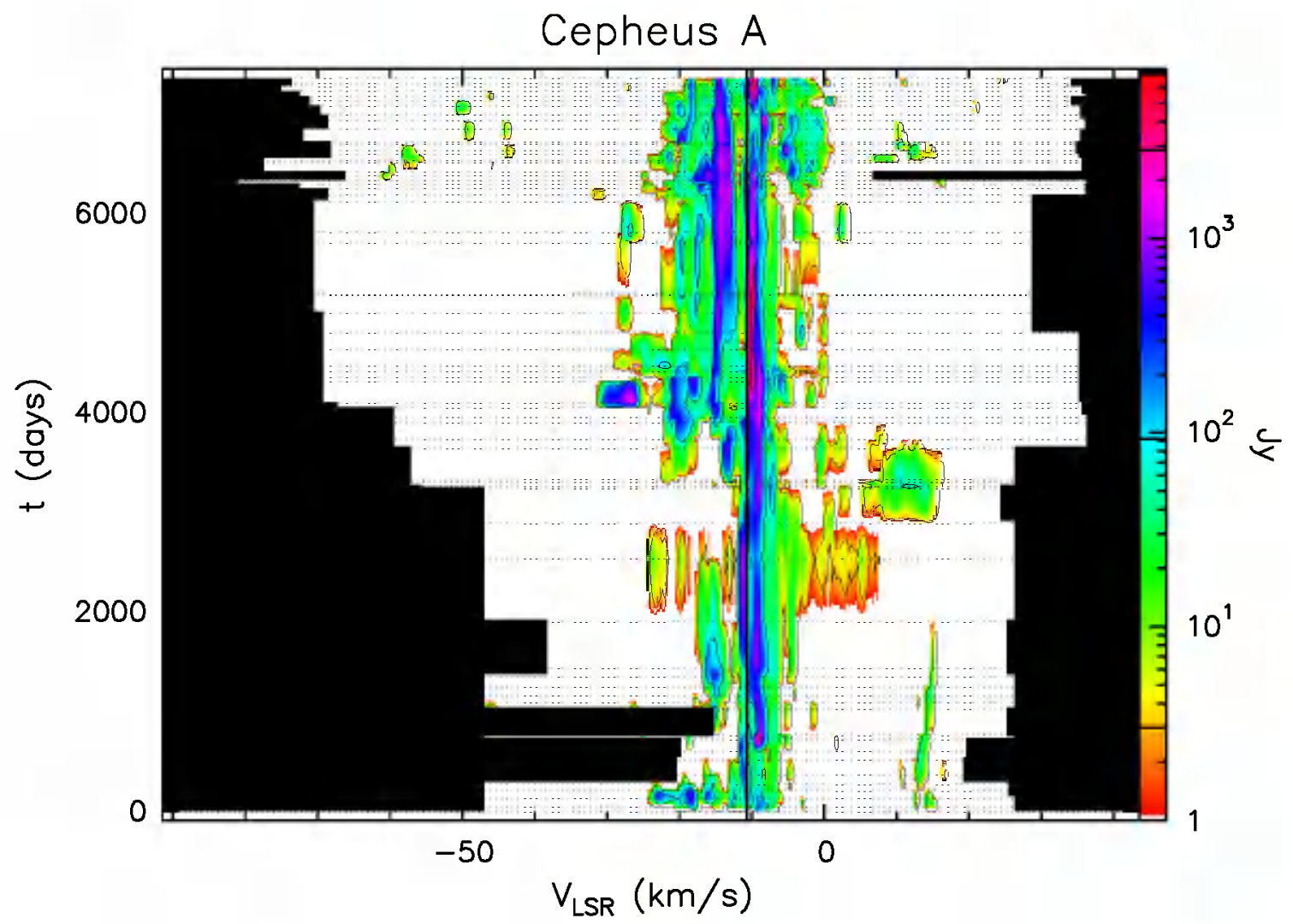

Fig. A.39. b Velocity-time-flux density full plot for source Cepheus A. The vertical solid line indicates the velocity of the associated thermal molecular gas. The flux density scale is shown by the bar on the right. In this bar the three lines give the flux density of the drawn contours.

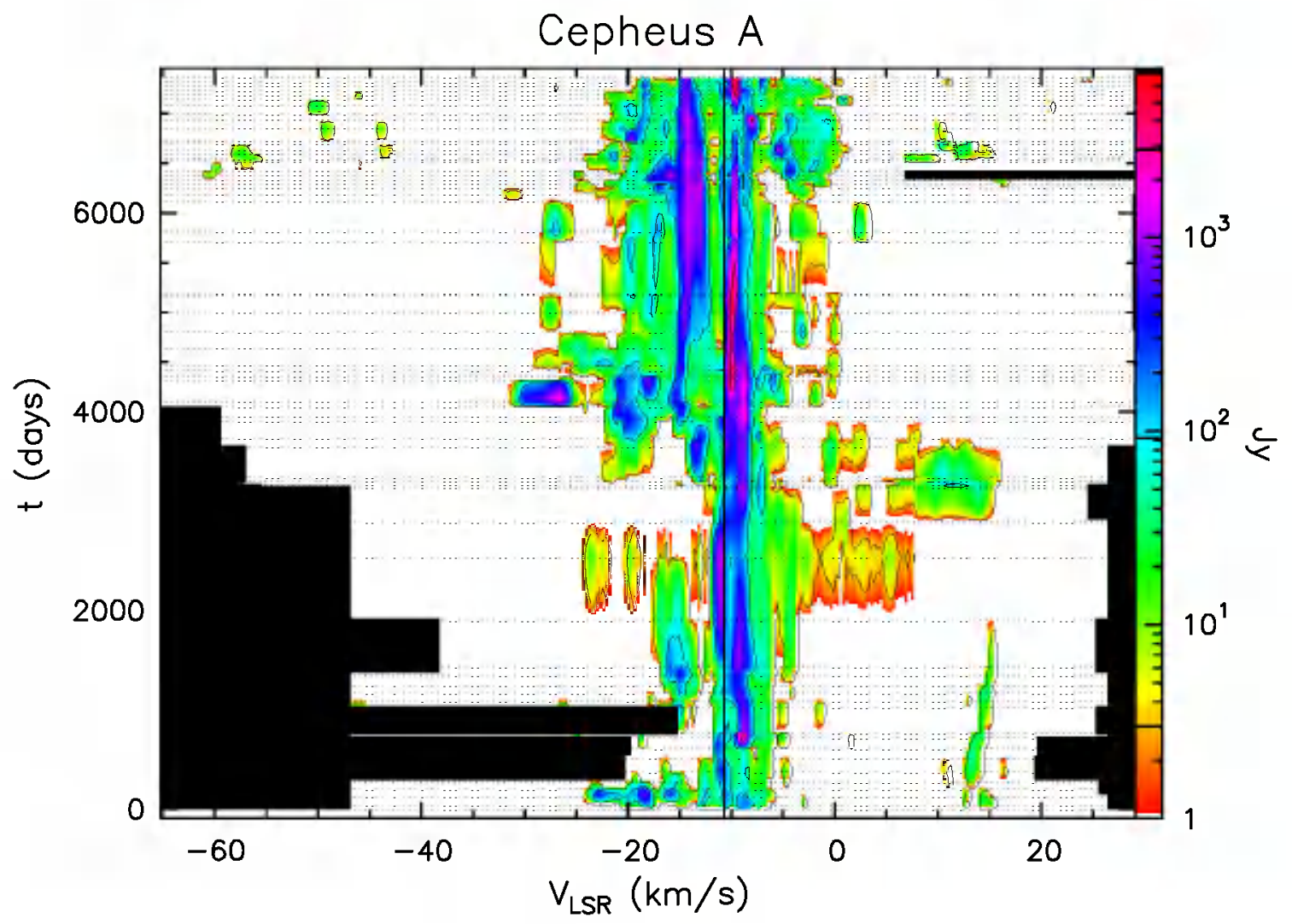

Fig. A.39. c Same as previous figure, but "zoomed" to velocity range over which emission has been detected. 


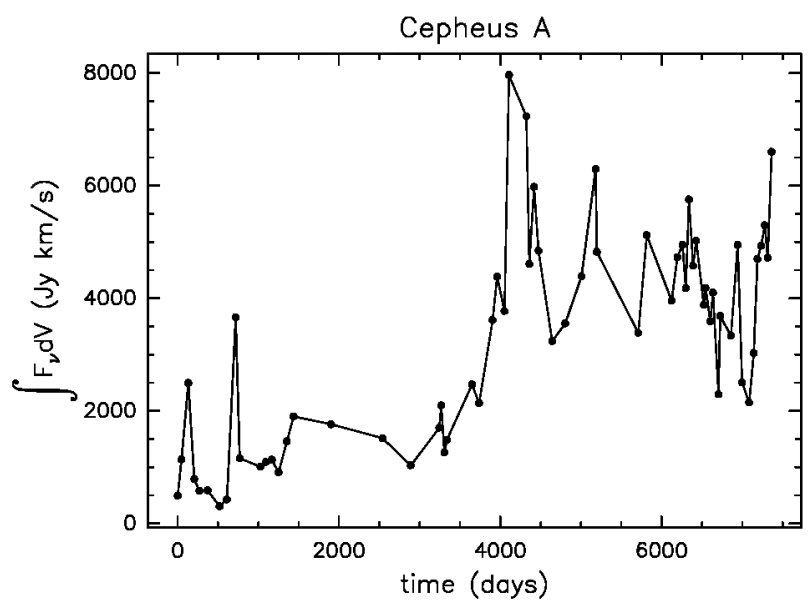

Fig. A.39. d Integral of the flux density over the observed velocity range as a function of time for source Cepheus A.

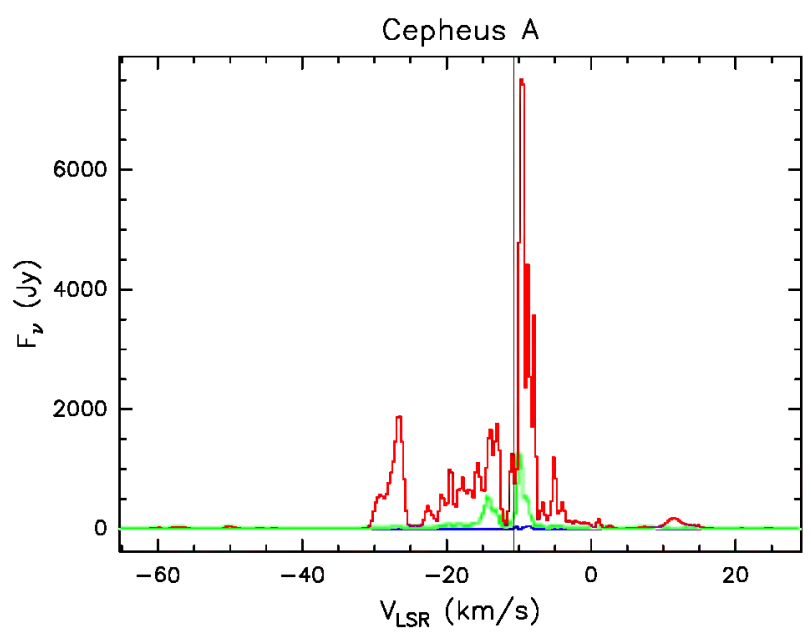

Fig. A.39. e Upper (red) and lower (blue) envelopes and mean spectrum (green) of source Cepheus A measured during our monitoring. The vertical solid line marks the velocity of the associated thermal molecular gas. The vertical dashed line marks the mean velocity derived from the histogram of the rate-of-occurrence.

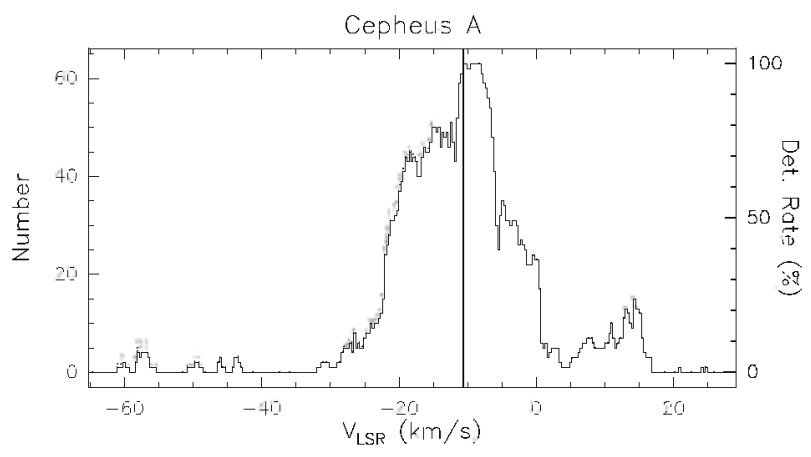

Fig. A.39. f Rate-of-occurrence plot for source Cepheus A. The scale to the right refers to the dotted histogram, the scale to the left to the solid line histogram. The vertical solid line marks the velocity of the associated thermal molecular gas. 


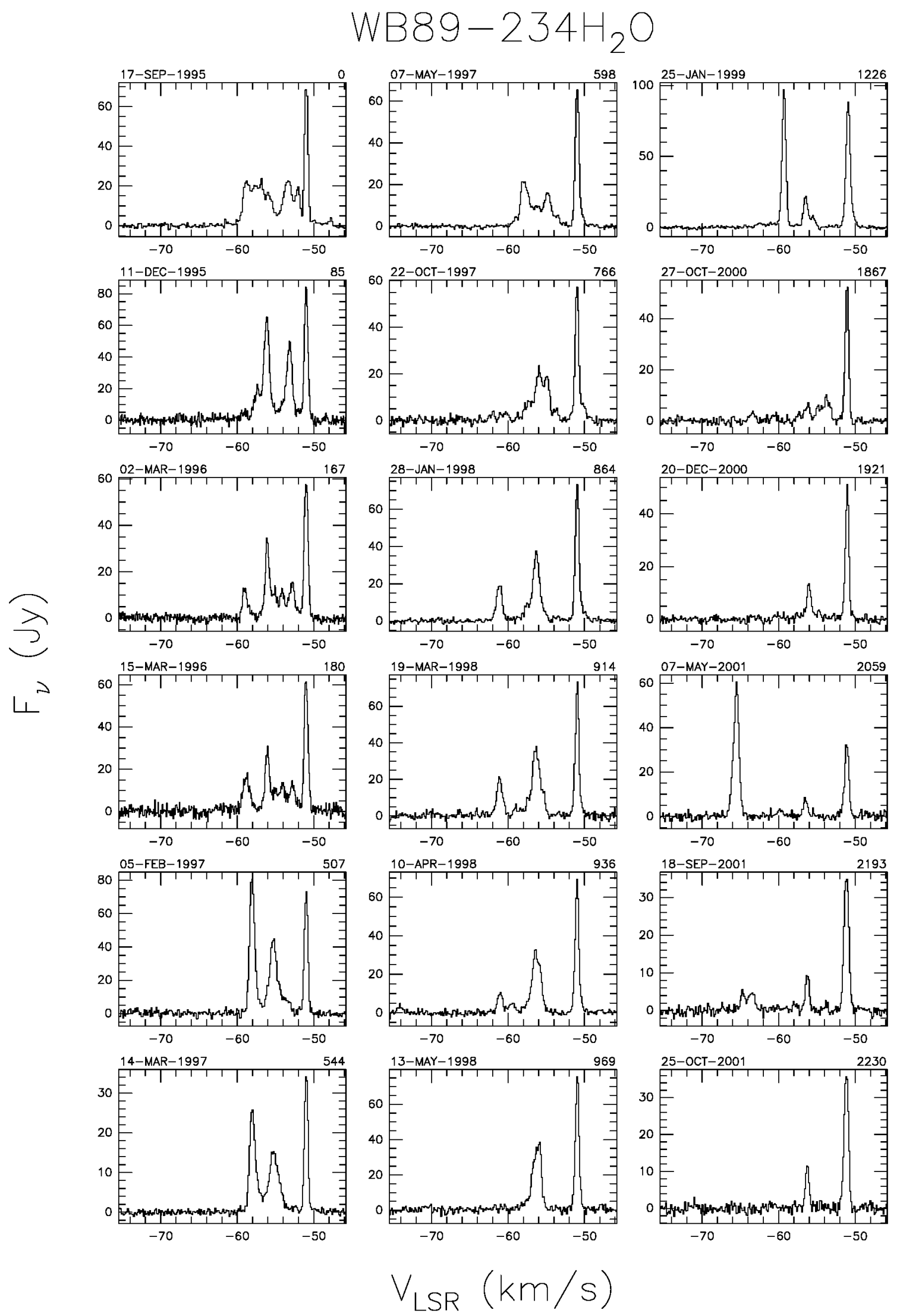

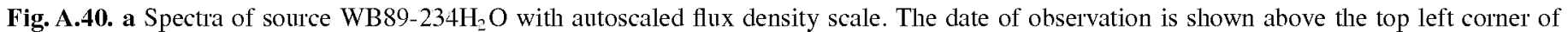
each spectrum and the number of days elapsed since the first observation is given above the top right corner. The velocity scale is the same for all spectra. 


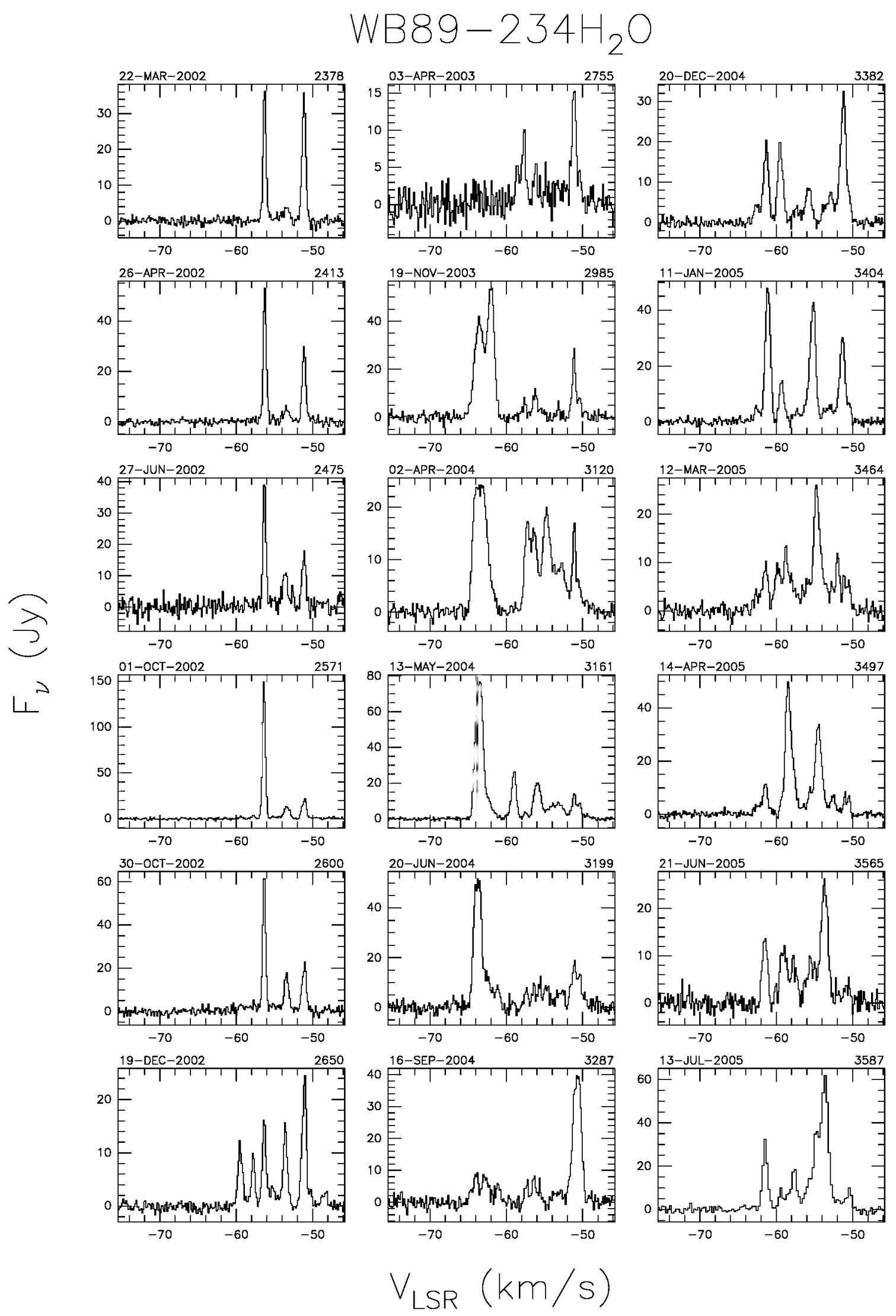

Fig. A.40. a continued. 
WB89-234 $\mathrm{H}_{2} \mathrm{O}$
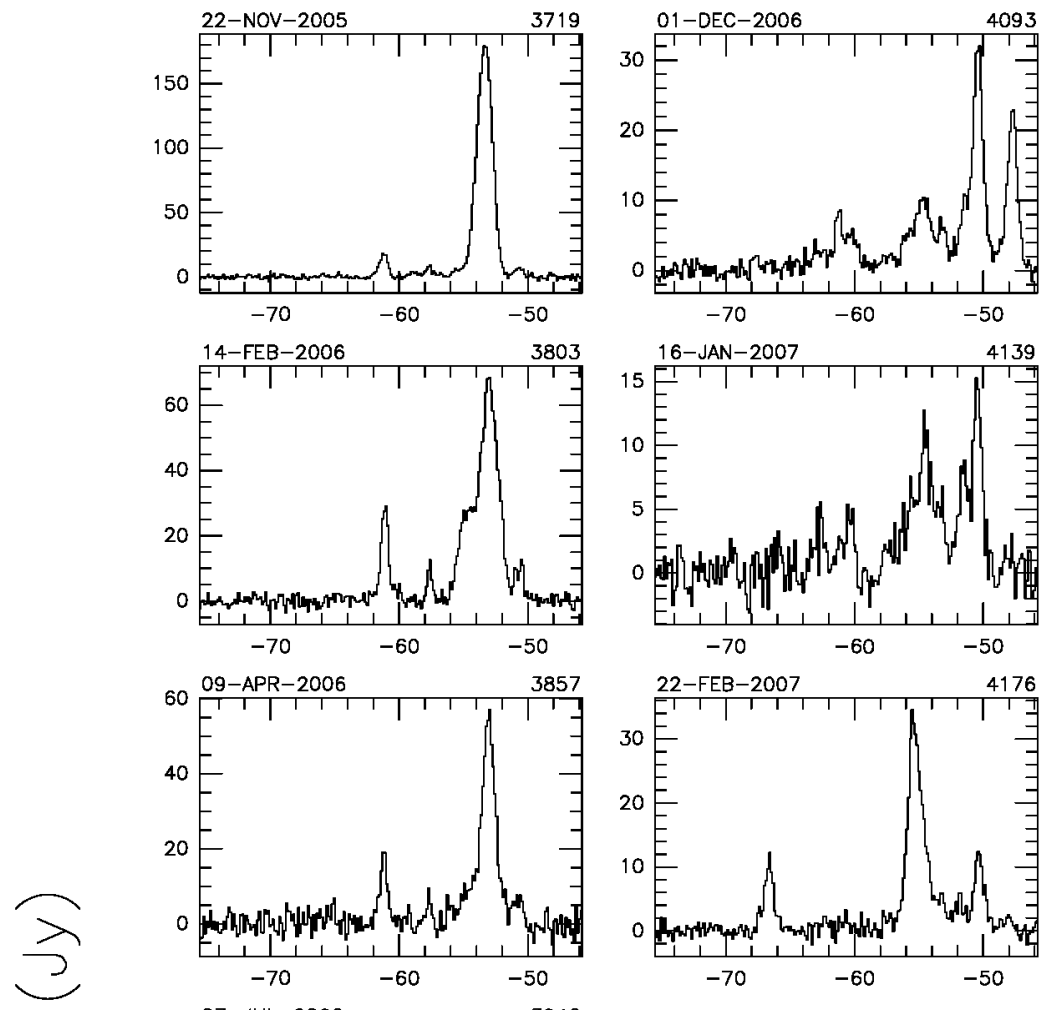

เ
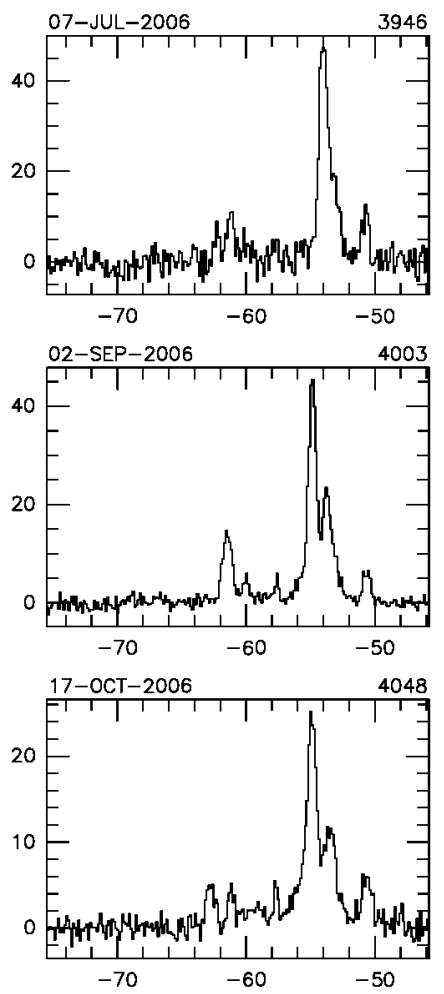

$V_{\text {LSR }}(k m / s)$

Fig. A.40. a continued. 


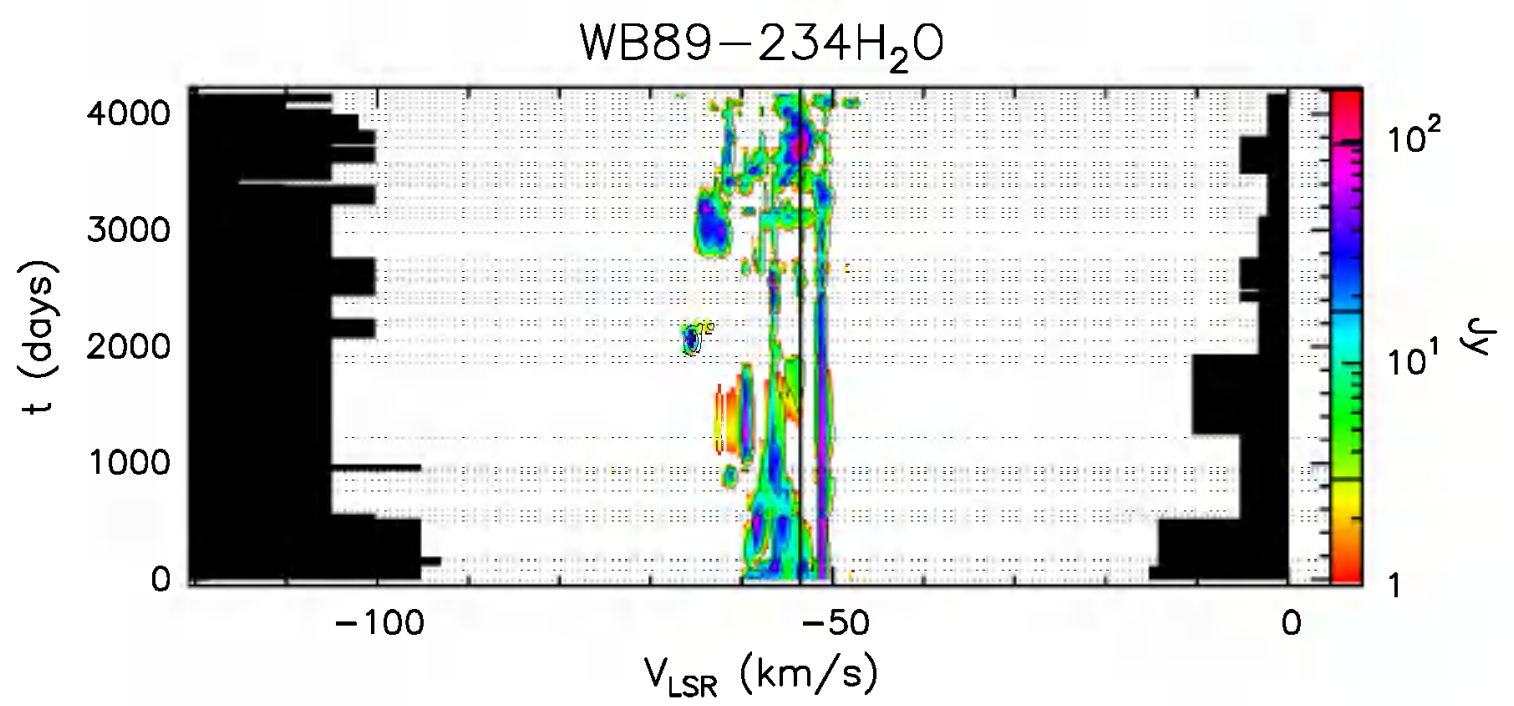

Fig. A.40. b Velocity-time-flux density full plot for source WB89-234 $\mathrm{H}_{2} \mathrm{O}$. The vertical solid line indicates the velocity of the associated thermal molecular gas. The flux density scale is shown by the bar on the right. In this bar the three lines give the flux density of the drawn contours.

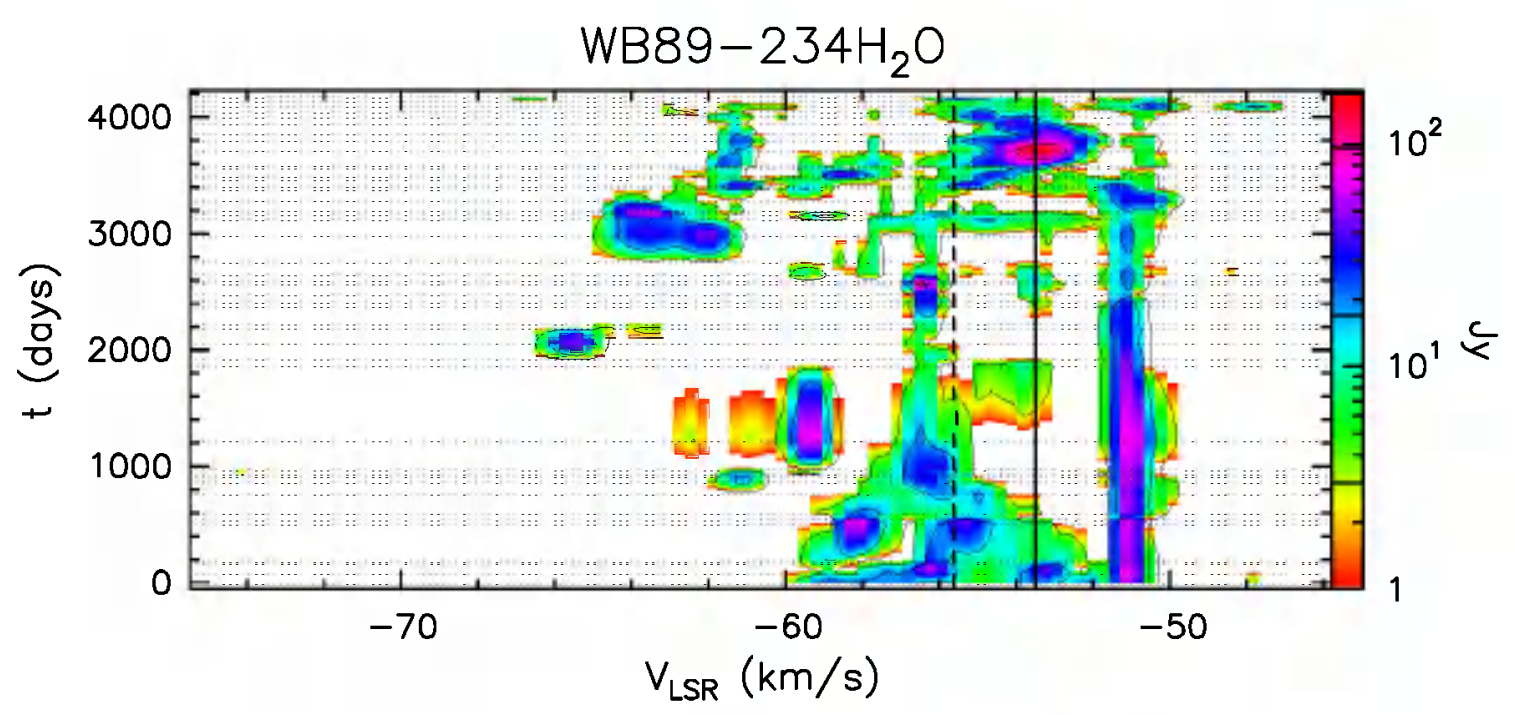

Fig. A.40. c Same as previous figure, but "zoomed" to velocity range over which emission has been detected. 


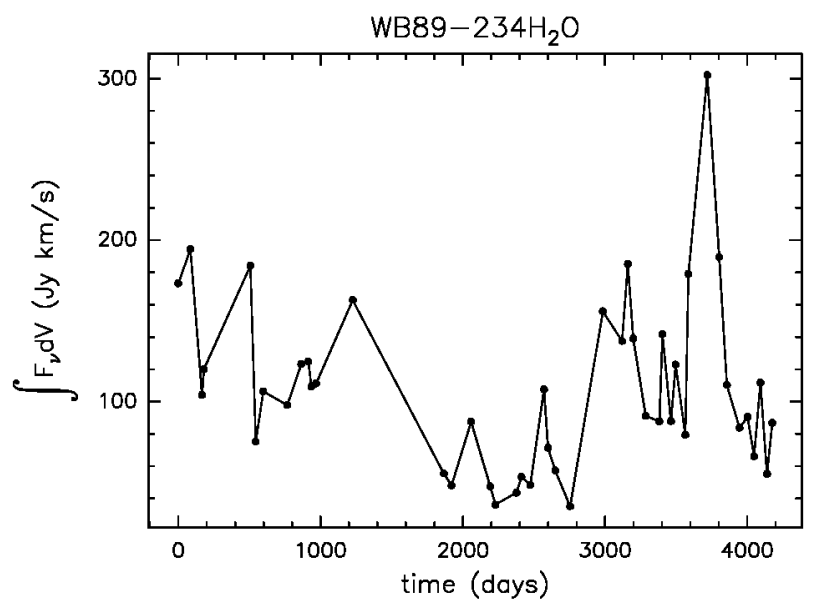

Fig. A.40. d Integral of the flux density over the observed velocity range as a function of time for source WB89-234 $\mathrm{H}_{2} \mathrm{O}$.

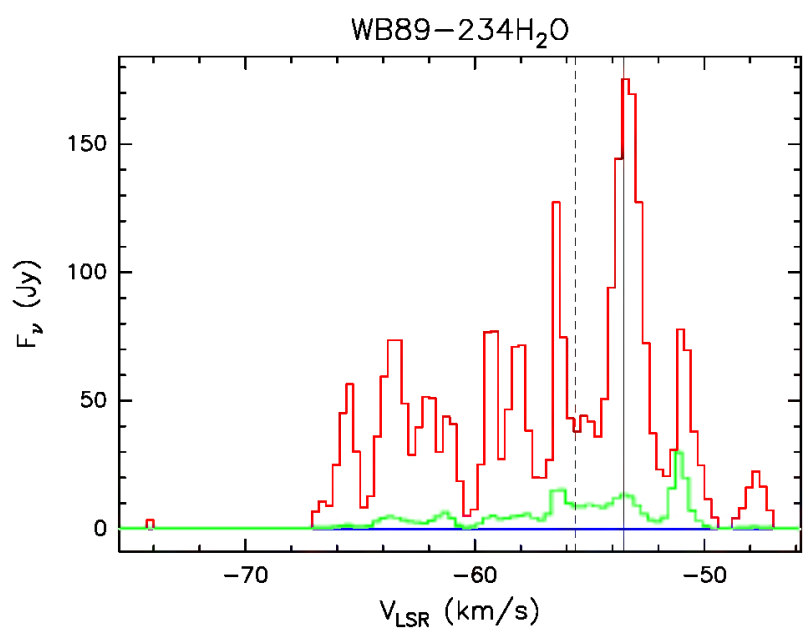

Fig. A.40. e Upper (red) and lower (blue) envelopes and mean spectrum (green) of source WB89-234 $\mathrm{H}_{2} \mathrm{O}$ measured during our monitoring. The vertical solid line marks the velocity of the associated thermal molecular gas. The vertical dashed line marks the mean velocity derived from the histogram of the rate-of-occurrence.

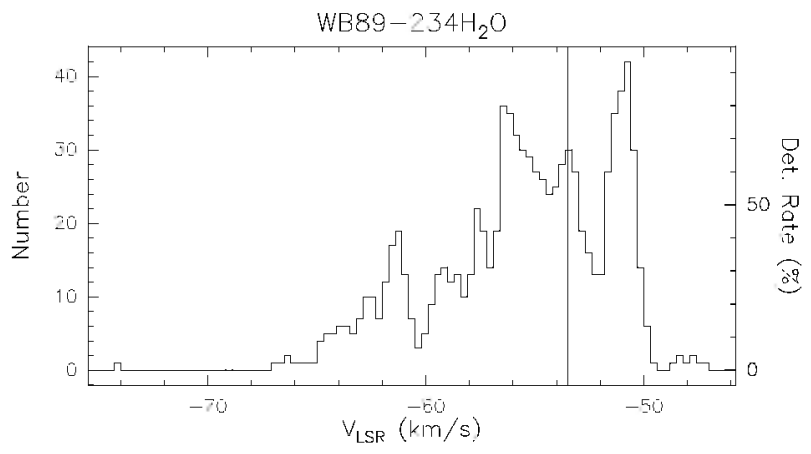

Fig. A.40. f Rate-of-occurrence plot for source WB89-234 $\mathrm{H}_{2} \mathrm{O}$. The scale to the right refers to the dotted histogram, the scale to the left to the solid line histogram. The vertical solid line marks the velocity of the associated thermal molecular gas. 


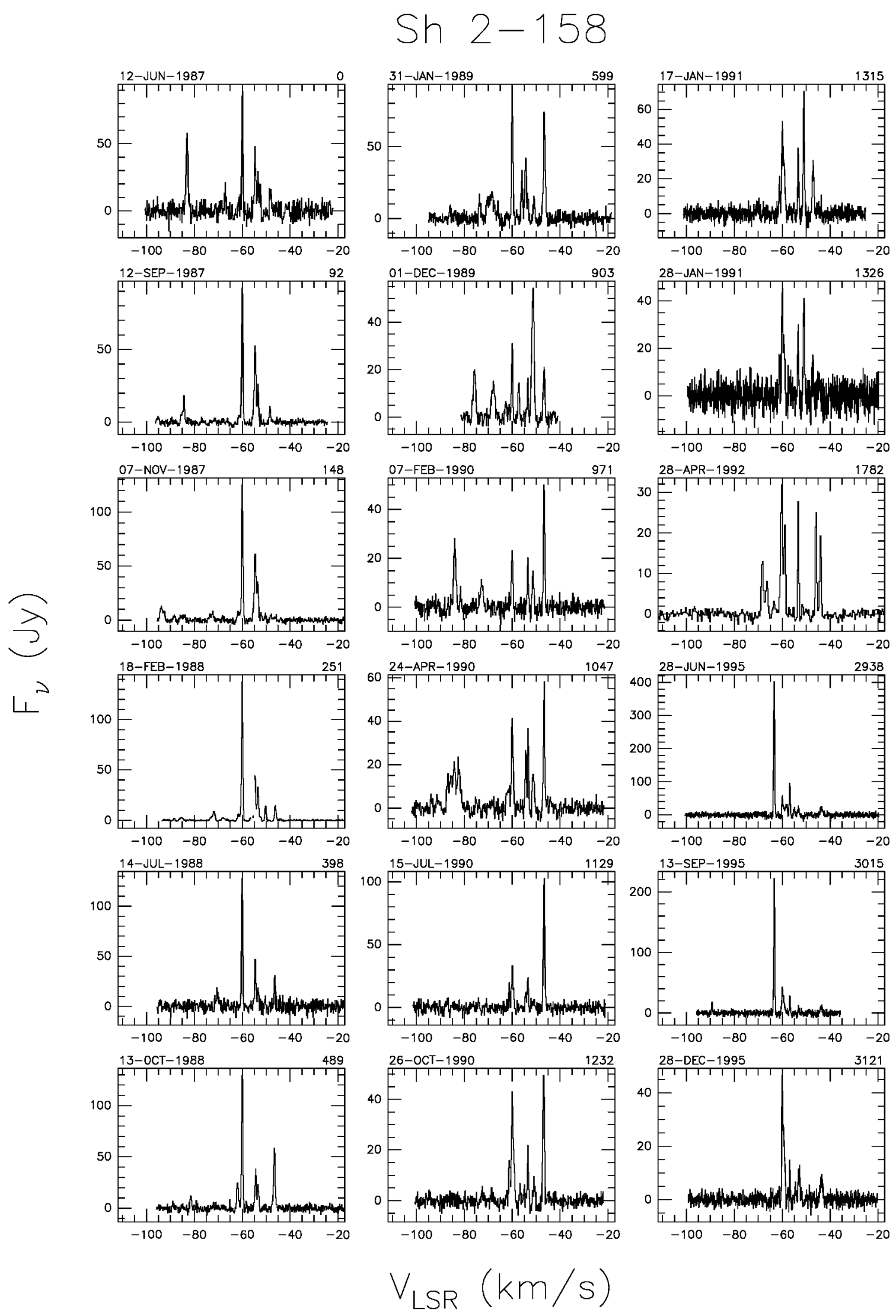

Fig. A.41. a Spectra of source Sh 2-158 with autoscaled flux density scale. The date of observation is shown above the top left corner of each spectrum and the number of days elapsed since the first observation is given above the top right corner. The velocity scale is the same for all spectra. 
Sh 2-158
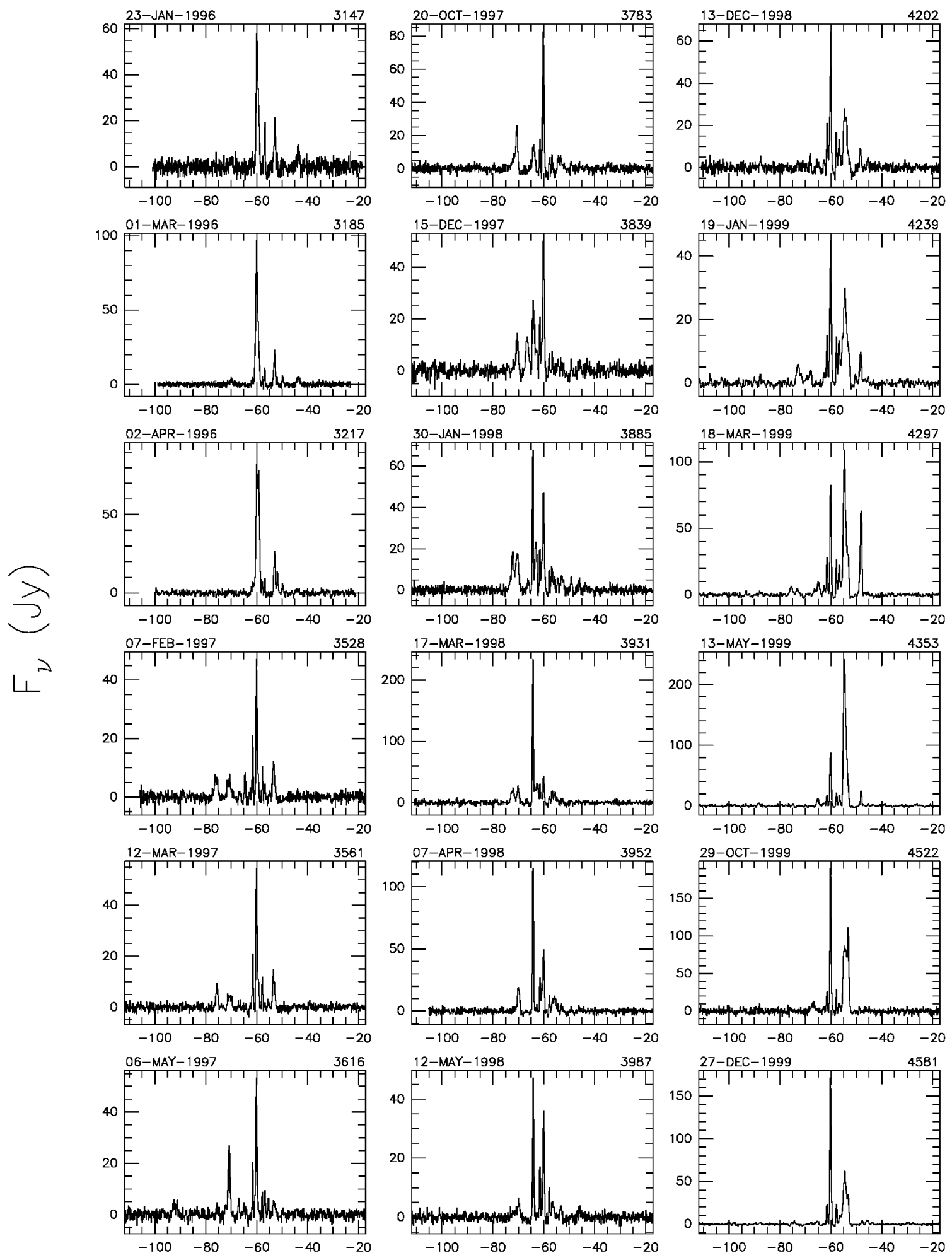

$$
V_{\text {LSR }}(\mathrm{km} / \mathrm{s})
$$

Fig. A.41. a continued. 

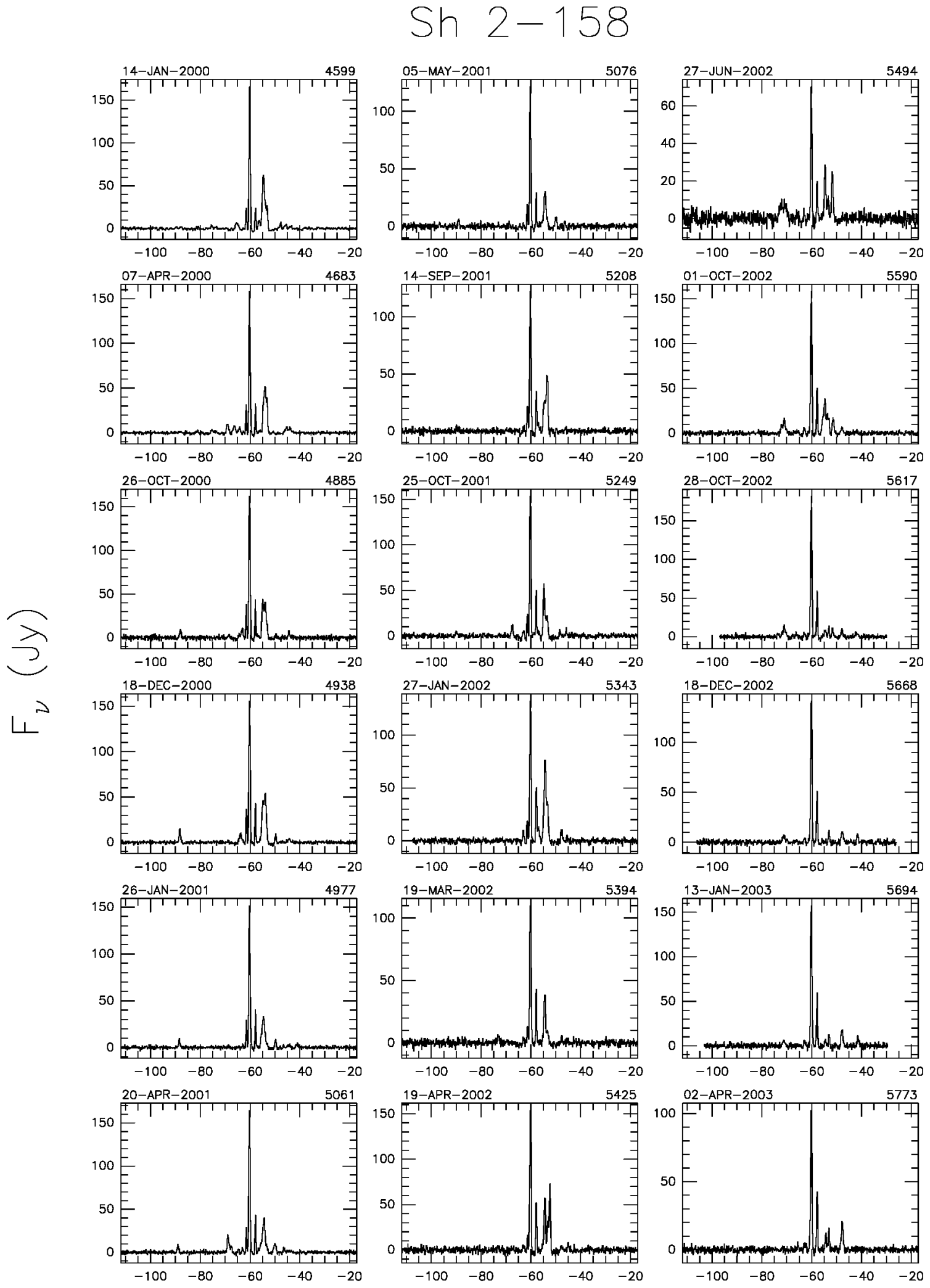

$$
V_{\text {LSR }}(\mathrm{km} / \mathrm{s})
$$

Fig. A.41. a continued. 
Sh 2-158
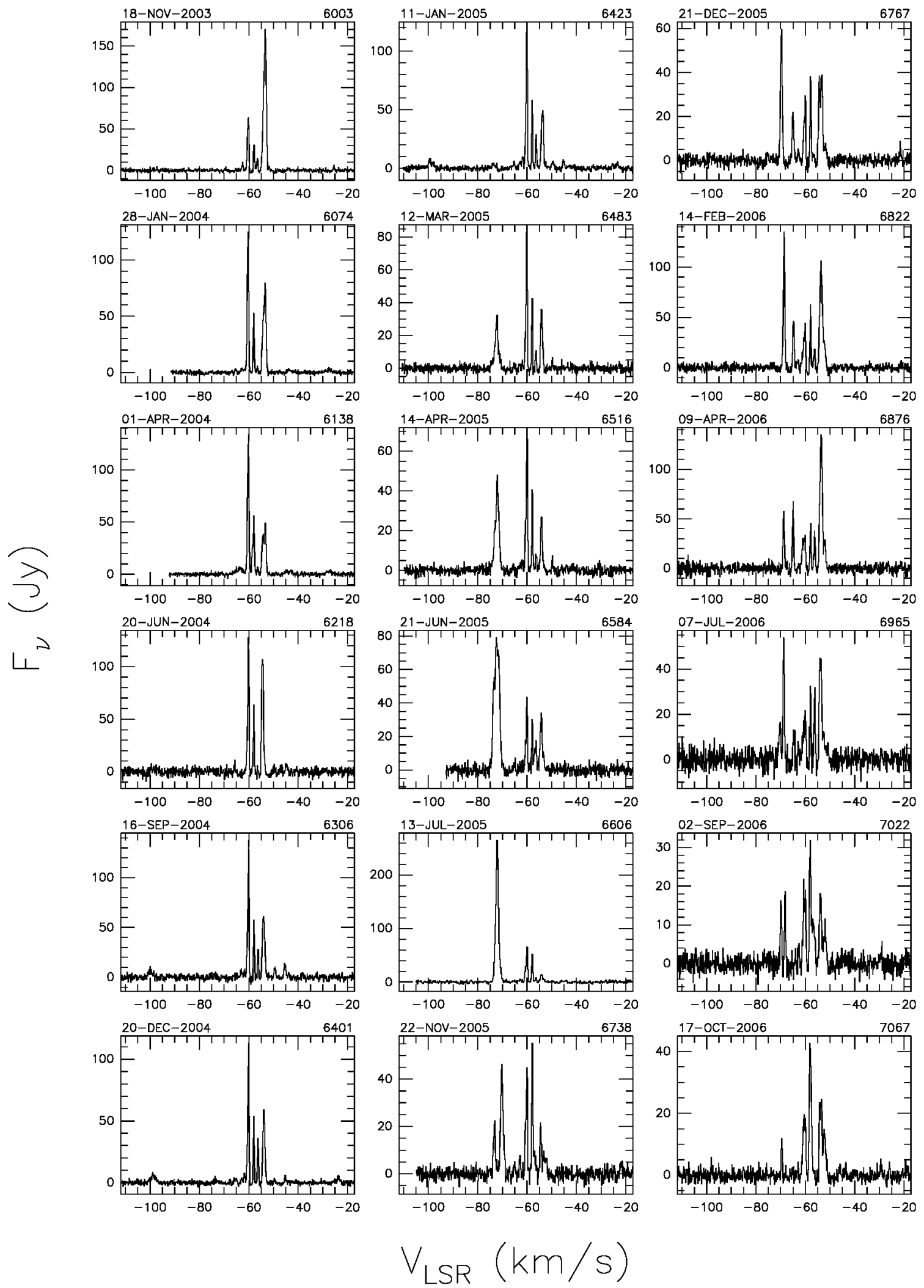

Fig. A.41. a continued. 
Sh $2-158$
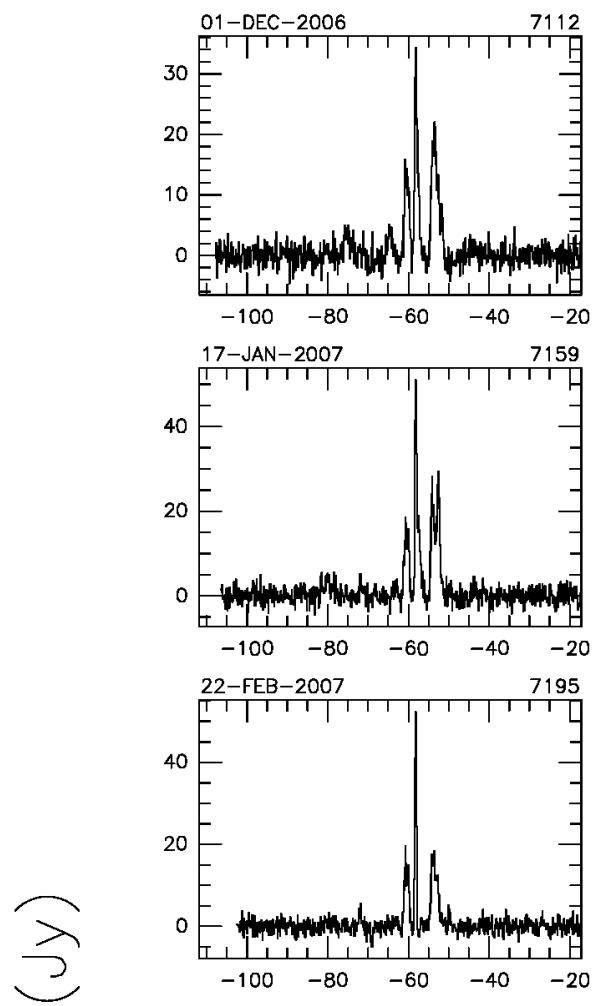

$\lambda$

$V_{\text {LSR }}(\mathrm{km} / \mathrm{s})$

Fig. A.41. a continued. 


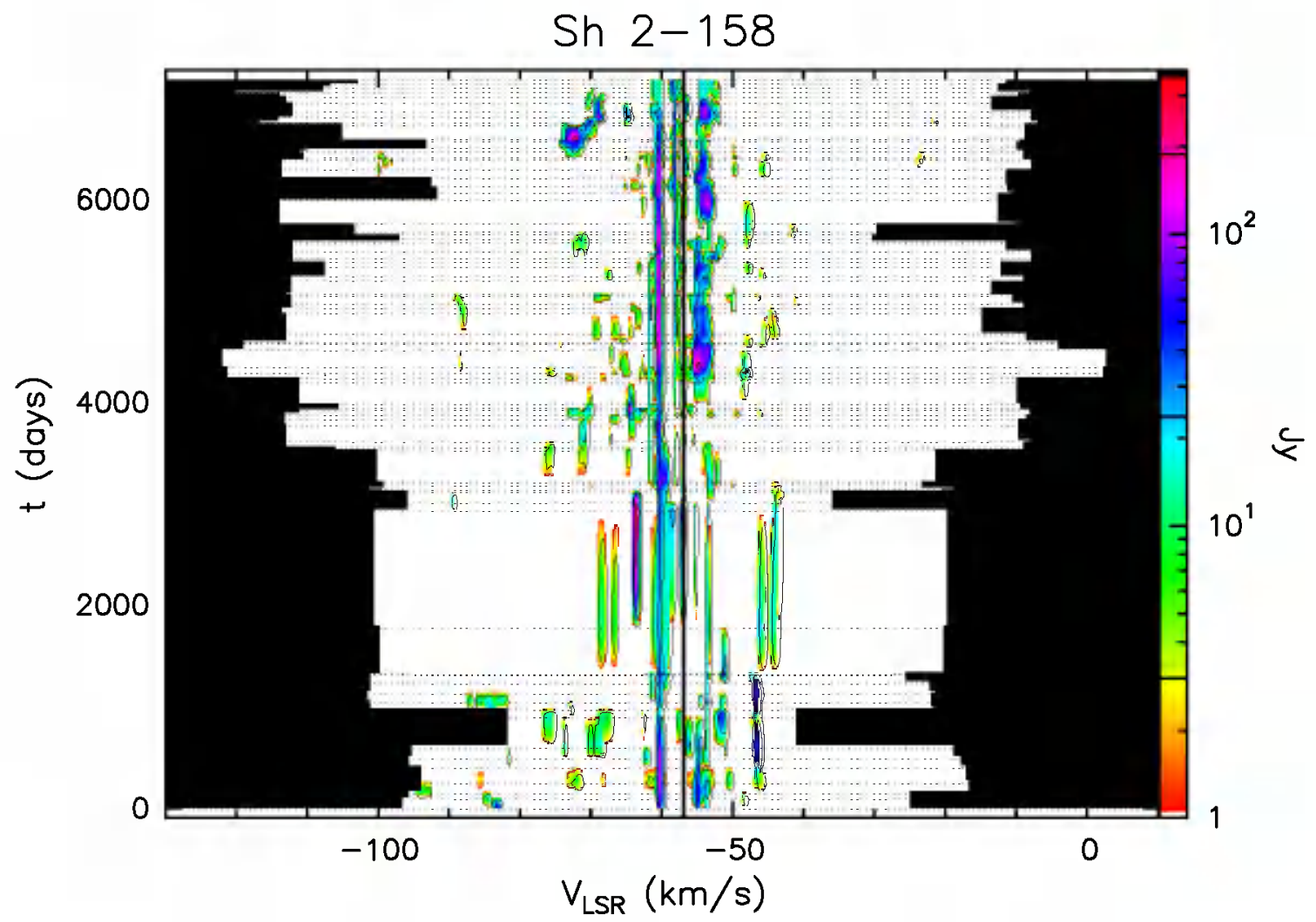

Fig. A.41. b Velocity-time-flux density full plot for source Sh 2-158. The vertical solid line indicates the velocity of the associated thermal molecular gas. The flux density scale is shown by the bar on the right. In this bar the three lines give the flux density of the drawn contours.

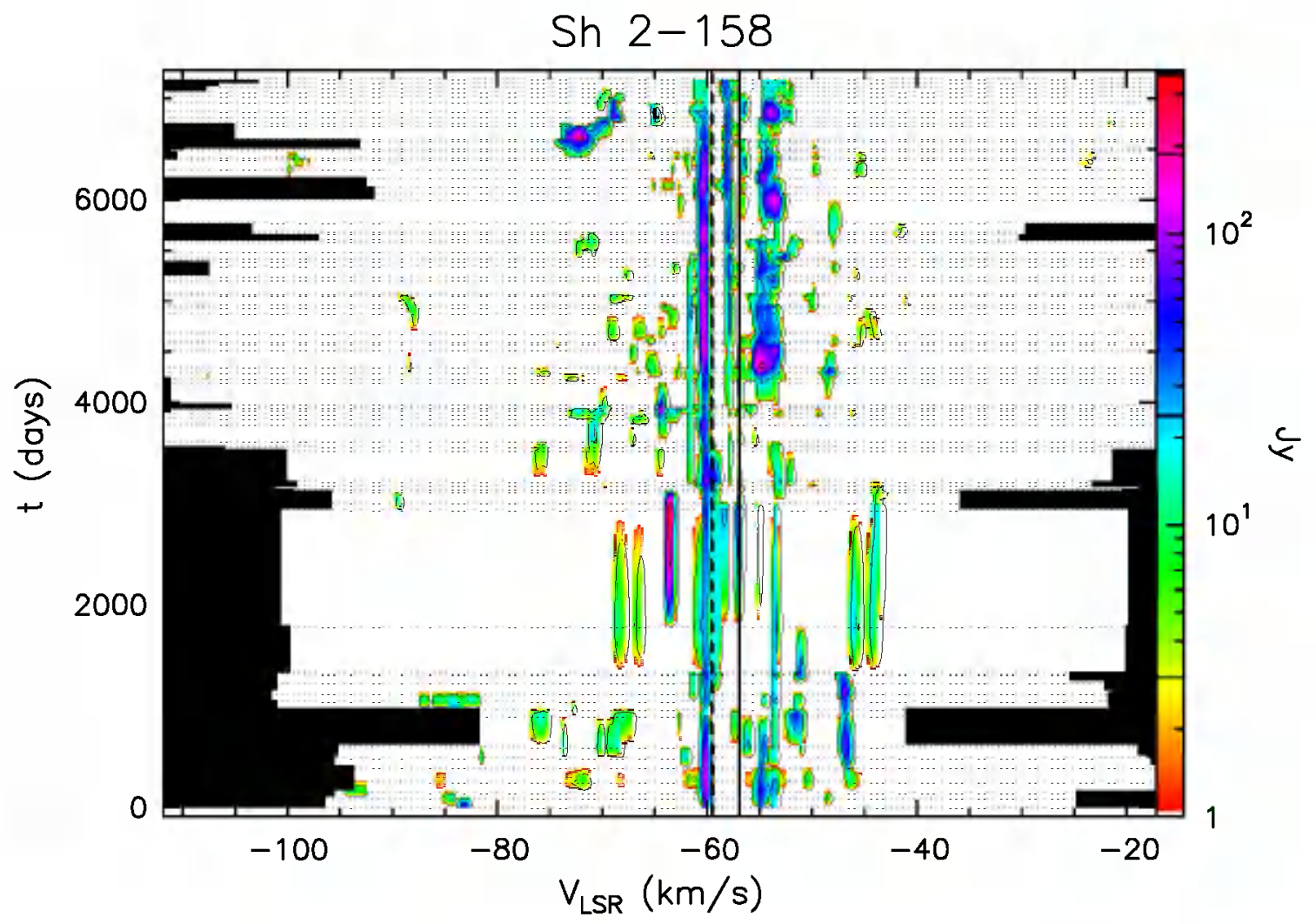

Fig. A.41. c Same as previous figure, but "zoomed" to velocity range over which emission has been detected. 


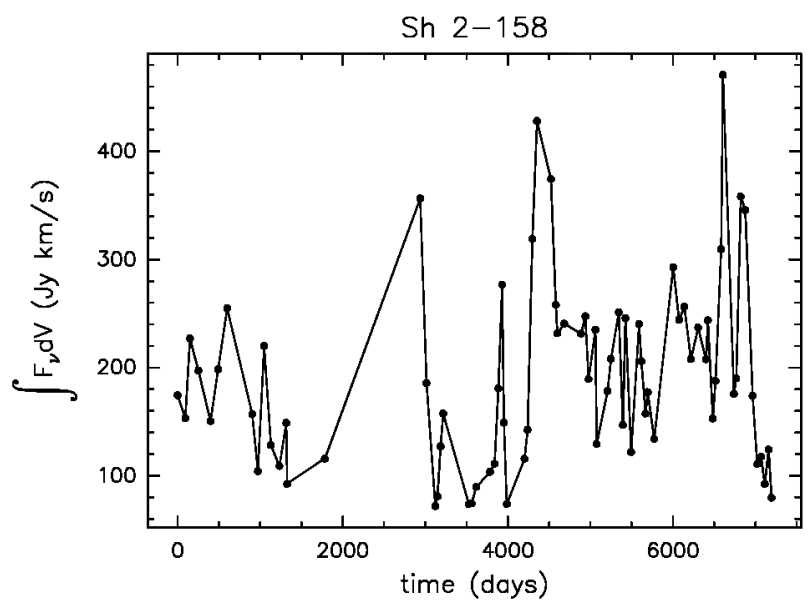

Fig. A.41. d Integral of the flux density over the observed velocity range as a function of time for source Sh 2-158.

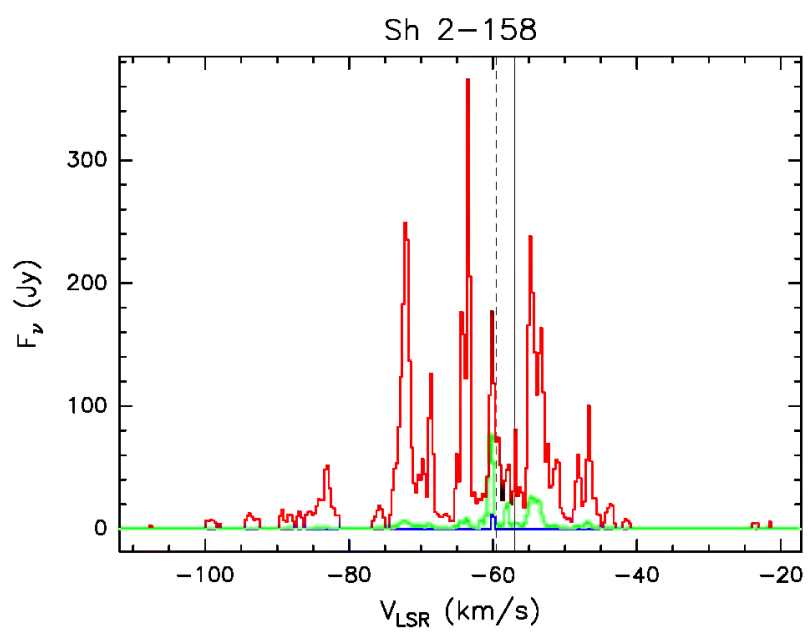

Fig. A.41. e Upper (red) and lower (blue) envelopes and mean spectrum (green) of source Sh 2-158 measured during our monitoring. The vertical solid line marks the velocity of the associated thermal molecular gas. The vertical dashed line marks the mean velocity derived from the histogram of the rate-of-occurrence.

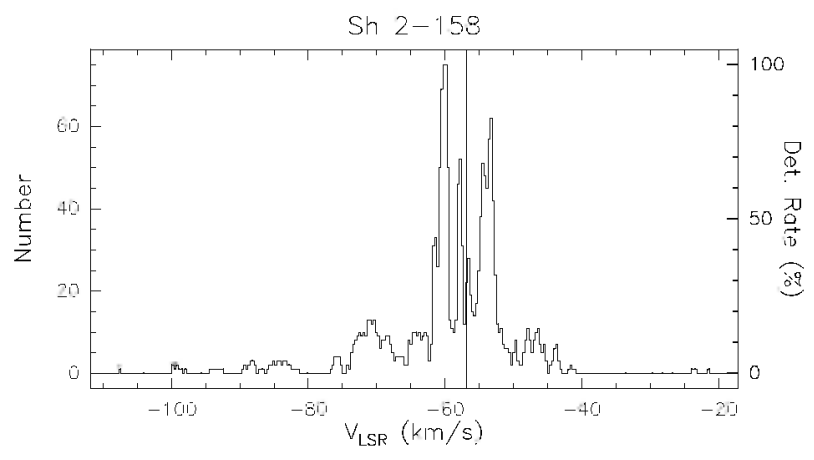

Fig. A.41. f Rate-of-occurrence plot for source Sh 2-158. The scale to the right refers to the dotted histogram, the scale to the left to the solid line histogram. The vertical solid line marks the velocity of the associated thermal molecular gas. 


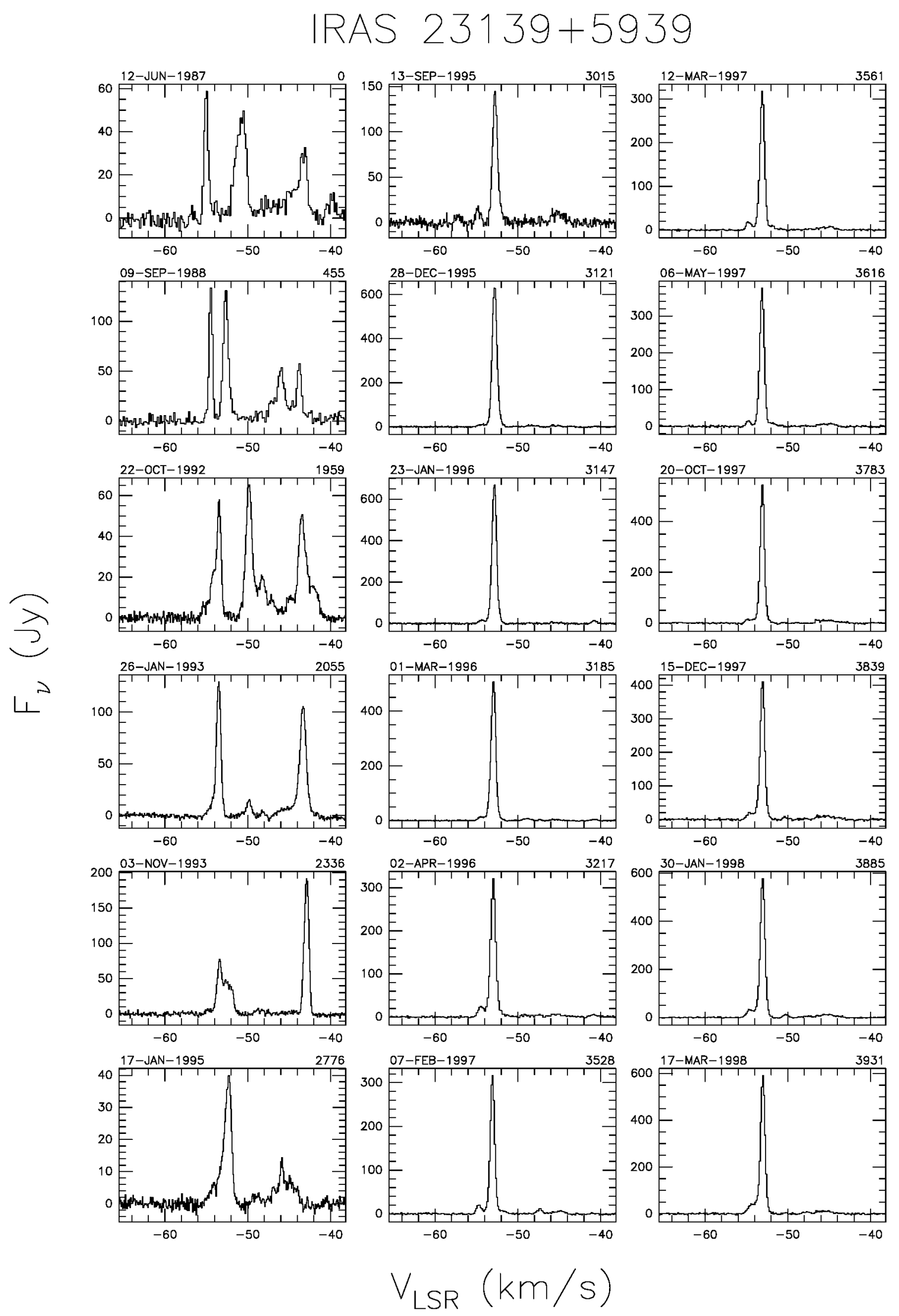

Fig. A.42. a Spectra of source IRAS $23139+5939$ with autoscaled flux density scale. The date of observation is shown above the top left corner of each spectrum and the number of days elapsed since the first observation is given above the top right corner. The velocity scale is the same for all spectia. 
IRAS $23139+5939$
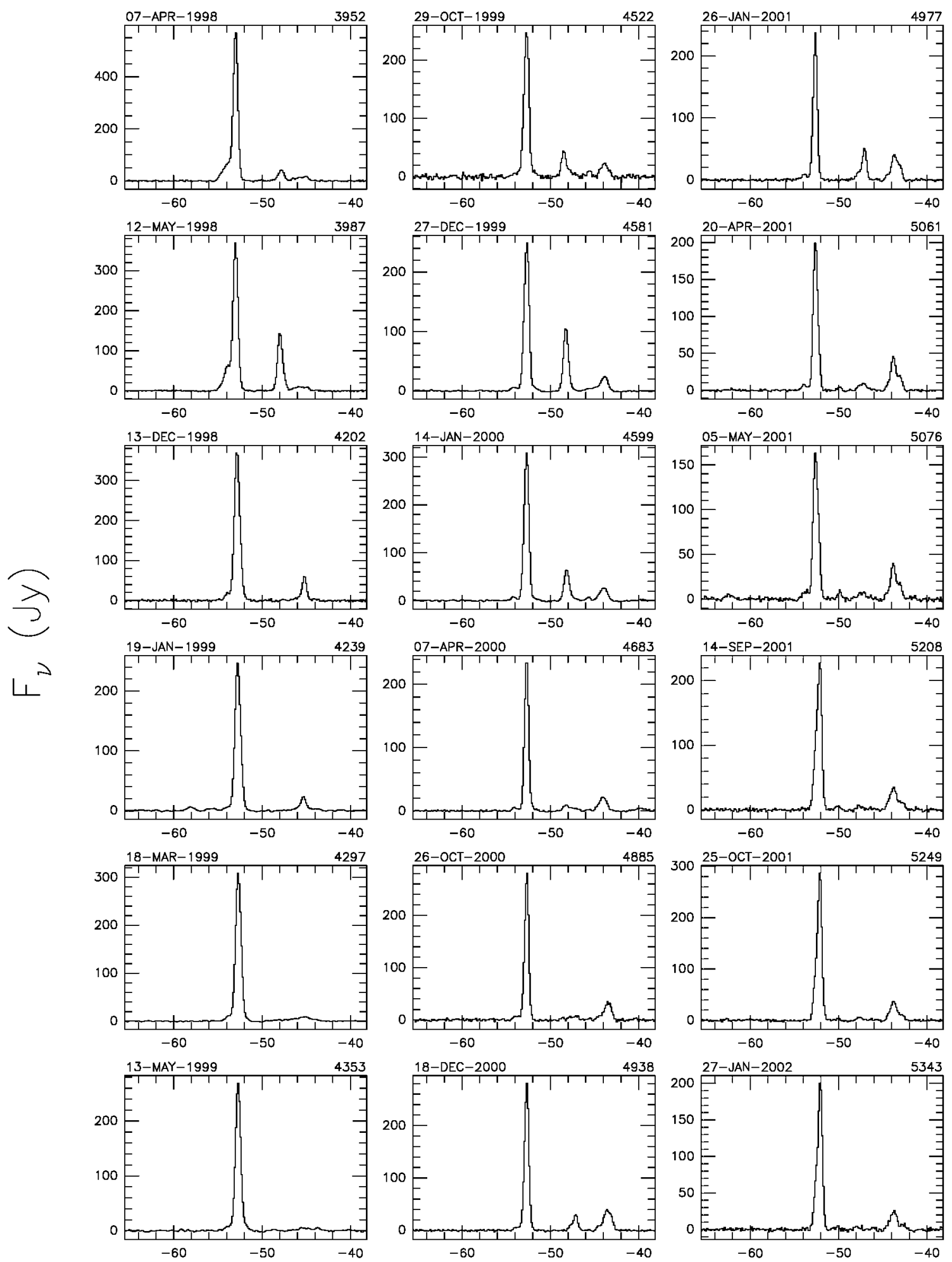

$$
V_{\text {LSR }}(\mathrm{km} / \mathrm{s})
$$

Fig. A.42. a continued. 


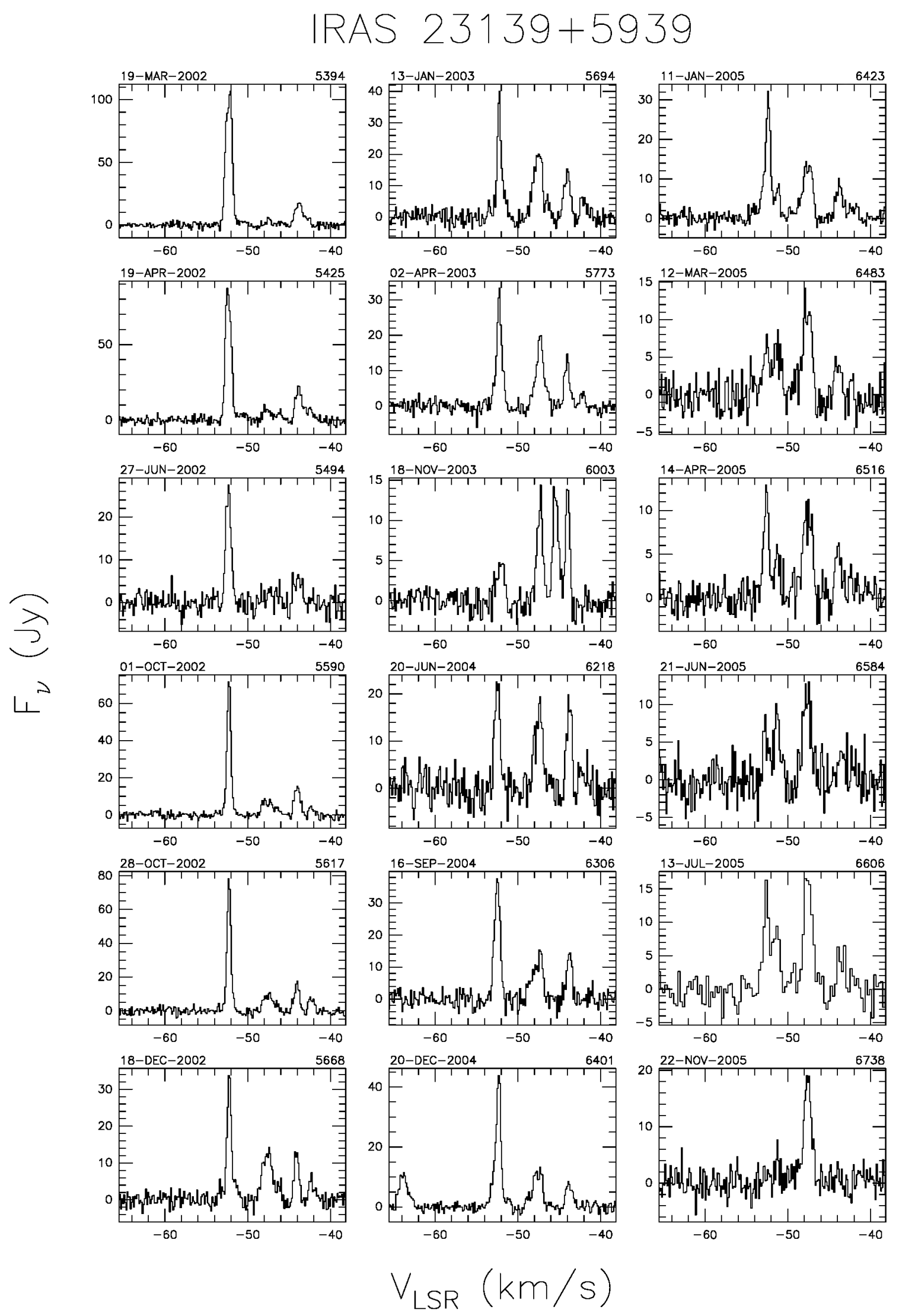

Fig. A.42. a continued. 


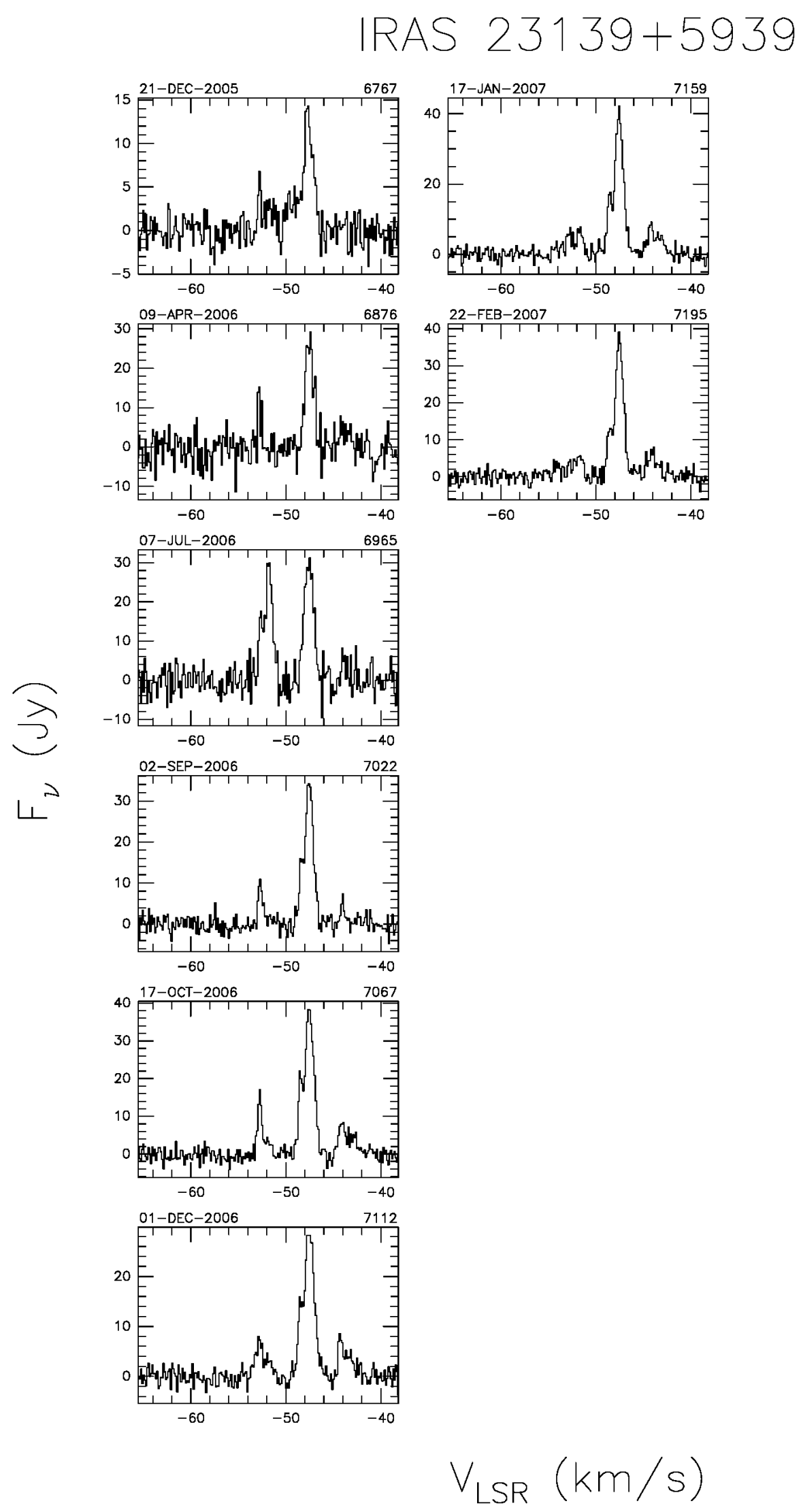

Fig. A.42. a continued. 


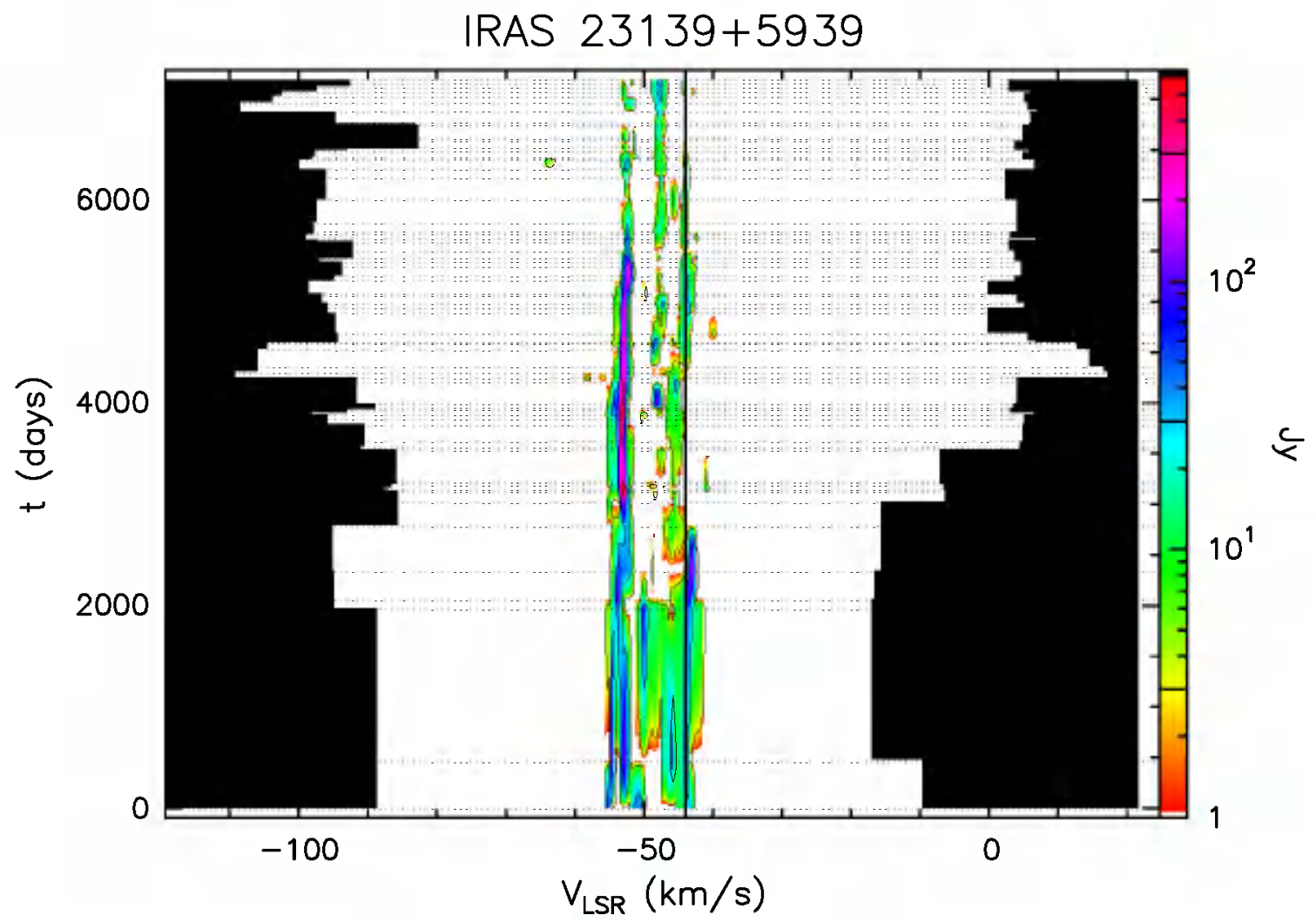

Fig. A.42. b Velocity-time-flux density full plot for source IRAS $23139+5939$. The vertical solid line indicates the velocity of the associated thermal molecular gas. The flux density scale is shown by the bar on the right. In this bar the three lines give the flux density of the drawn contours.

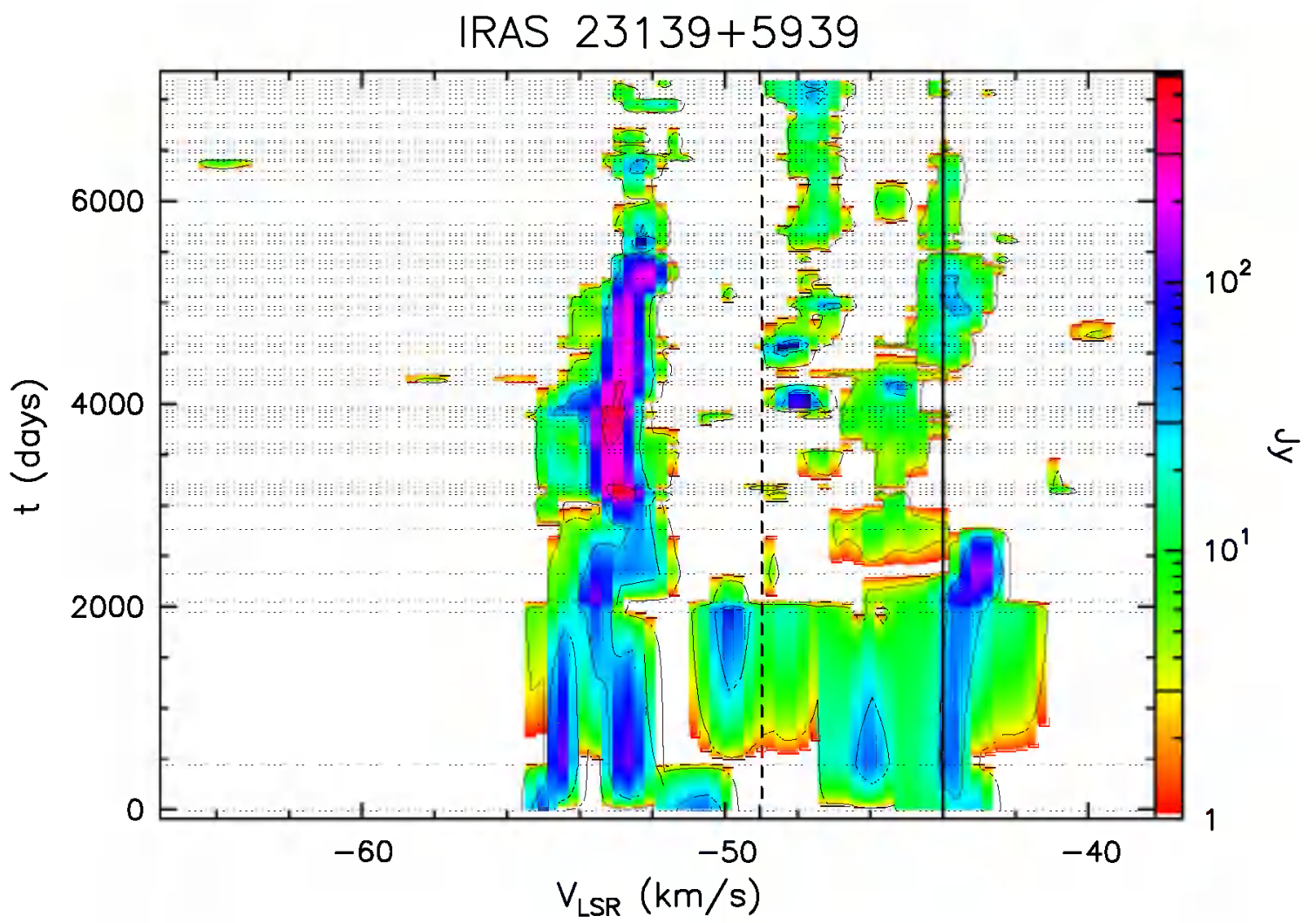

Fig. A.42. c Same as previous figure, but "zoomed" to velocity range over which emission has been detected. 


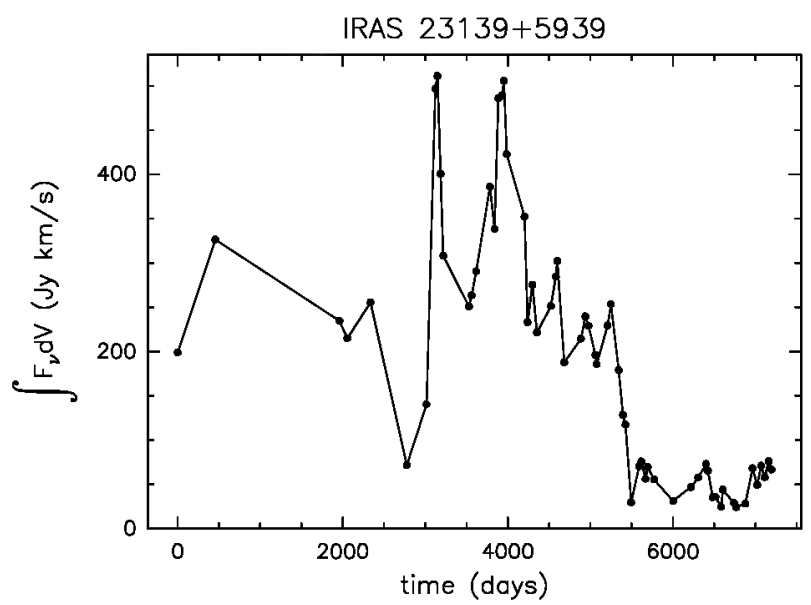

Fig. A.42. d Integral of the flux density over the observed velocity range as a function of time for source IRAS $23139+5939$.

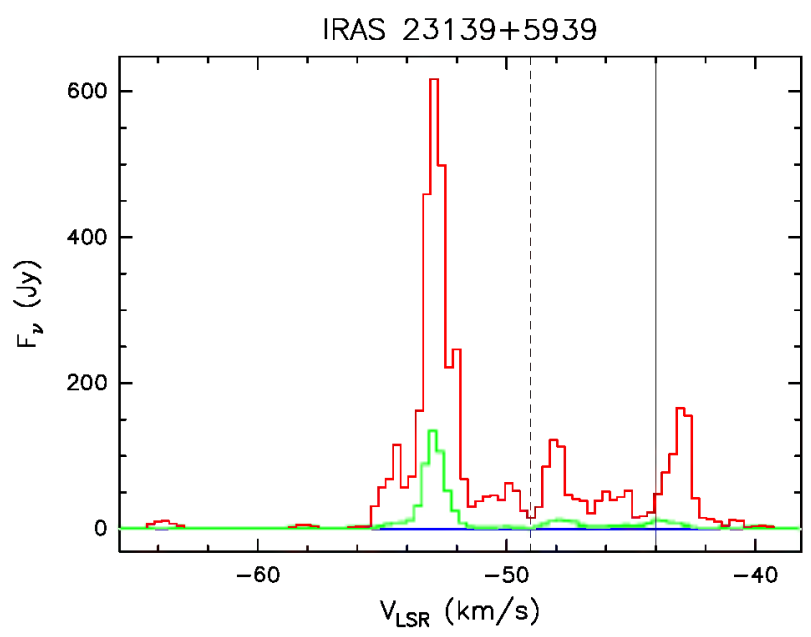

Fig. A.42. e Upper (red) and lower (blue) envelopes and mean spectrum (green) of source IRAS $23139+5939$ measured during our monitoring. The vertical solid line marks the velocity of the associated thermal molecular gas. The vertical dashed line marks the mean velocity derived from the histogram of the rate-of-occurrence.

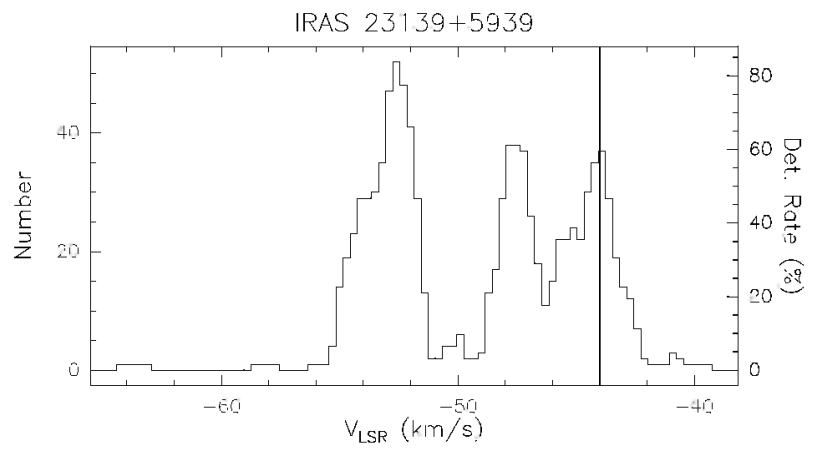

Fig. A.42. f Rate-of-occurrence plot for source IRAS $23139+5939$. The scale to the right refers to the dotted histogram, the scale to the left to the solid line histogram. The vertical solid line marks the velocity of the associated thermal molecular gas. 


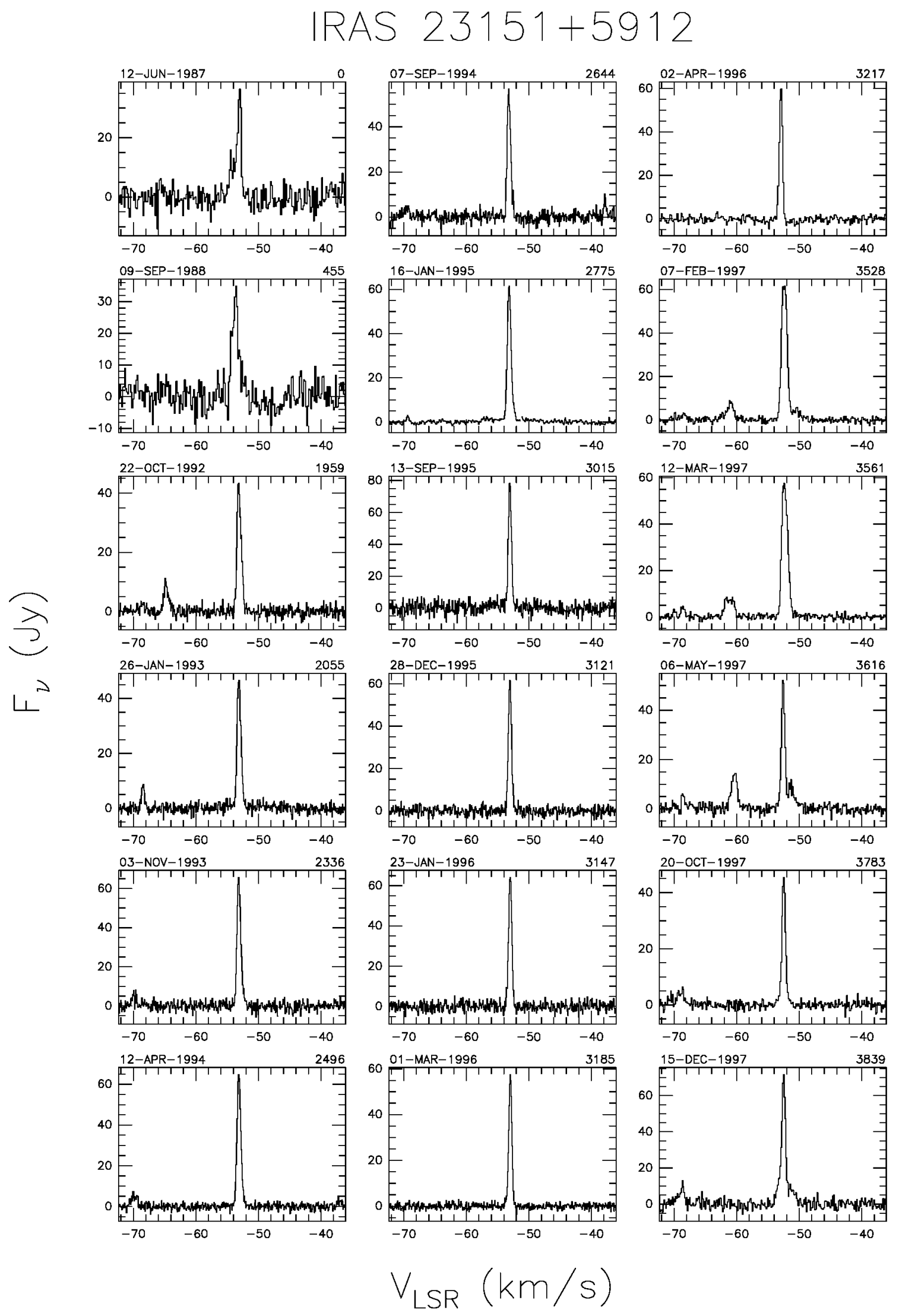

Fig. A.43. a Spectra of source IRAS $23151+5912$ with autoscaled flux density scale. The date of observation is shown above the top left corner of each spectrum and the number of days elapsed since the first observation is given above the top right corner. The velocity scale is the same for all spectia. 
IRAS $23151+5912$
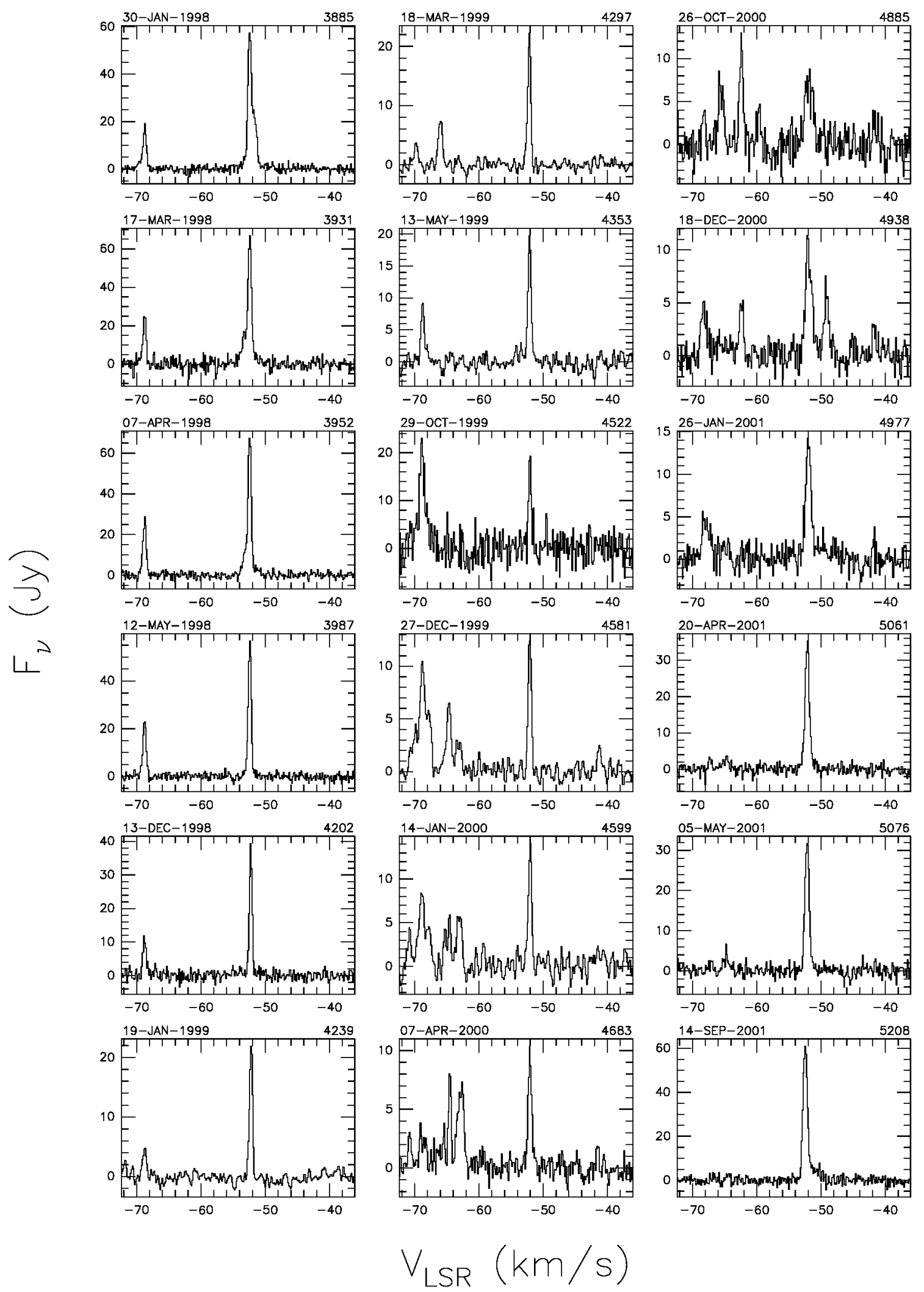

Fig. A.43. a continued. 
IRAS $23151+5912$
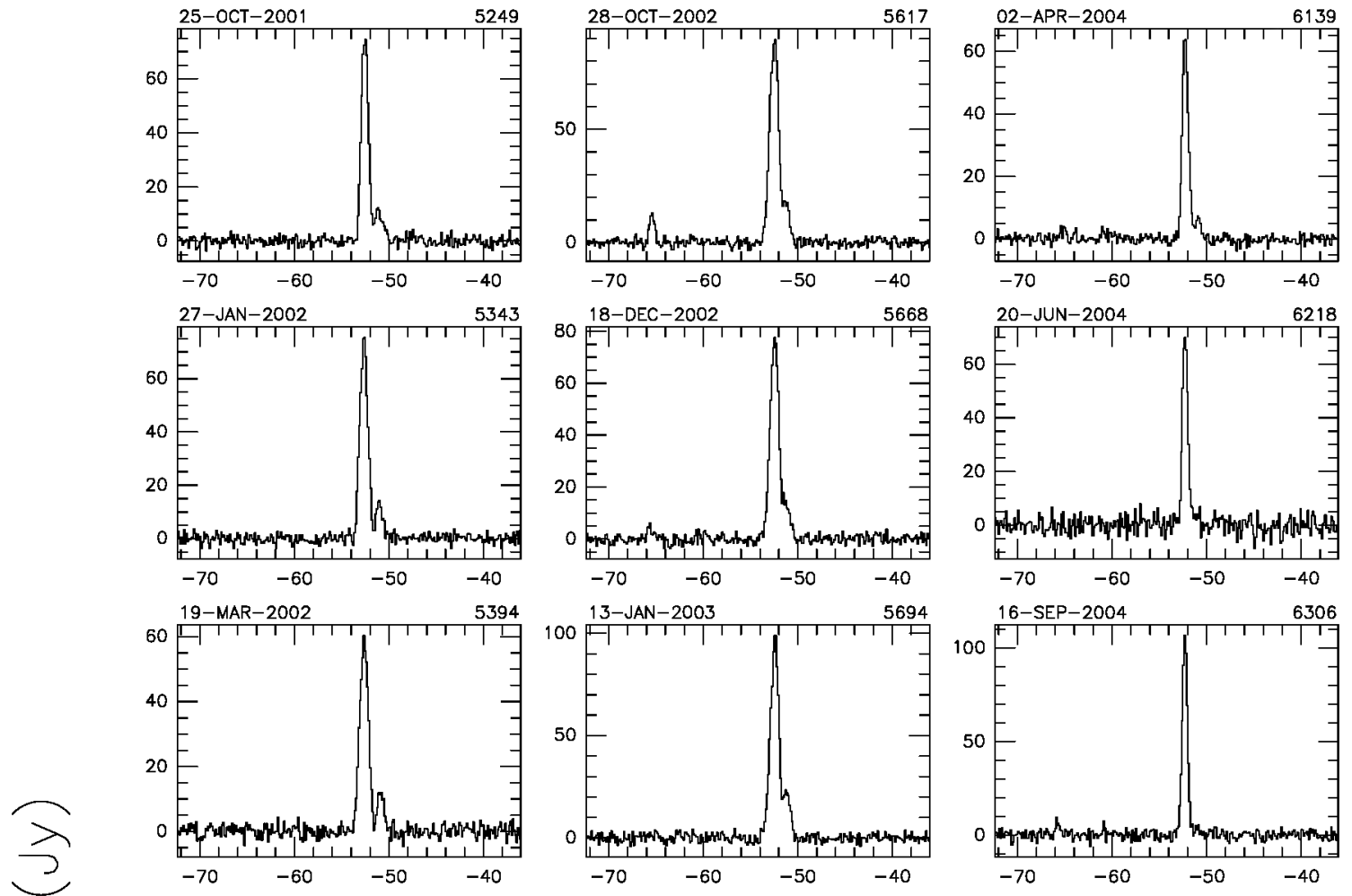

L
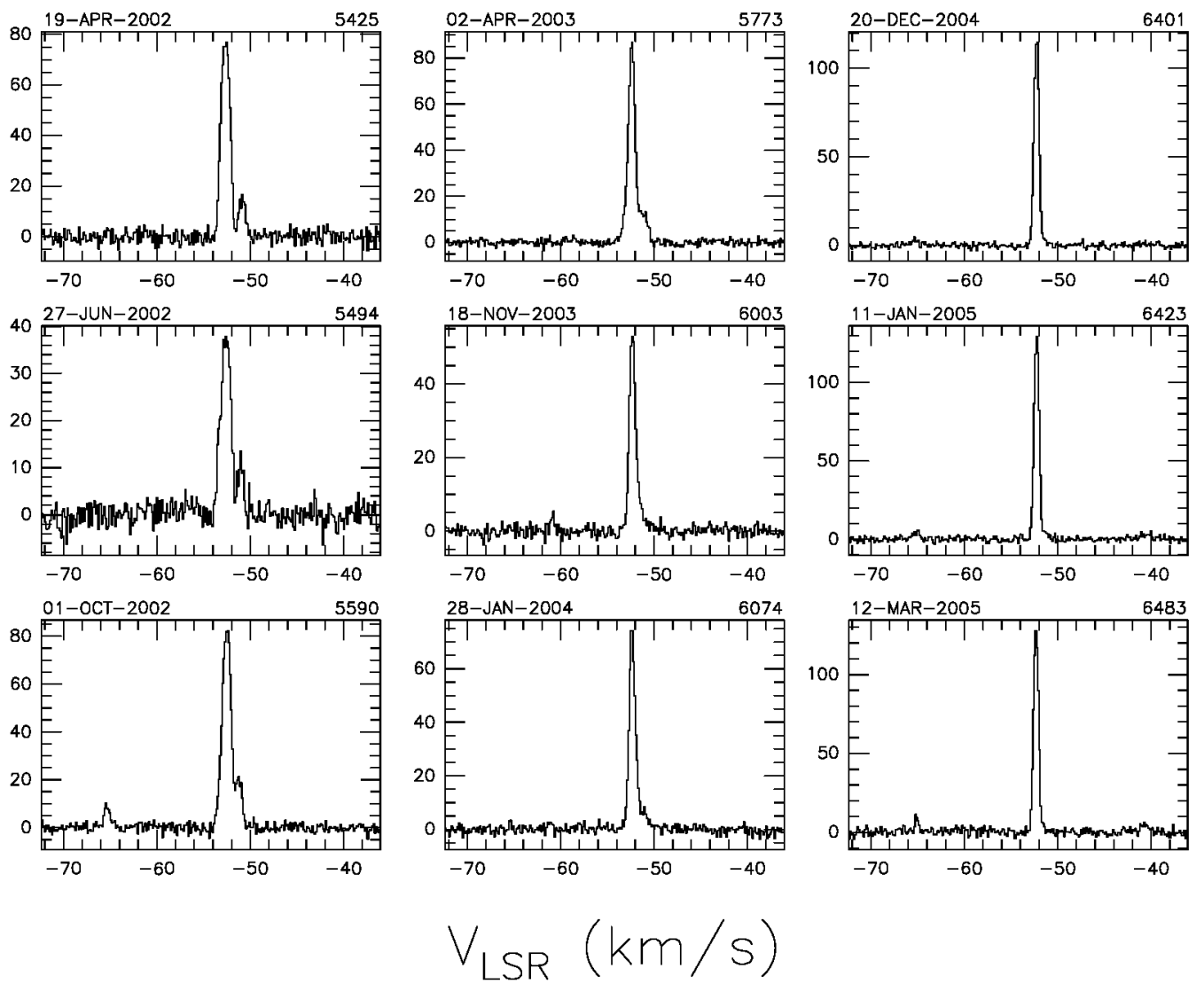

Fig. A.43. a continued. 


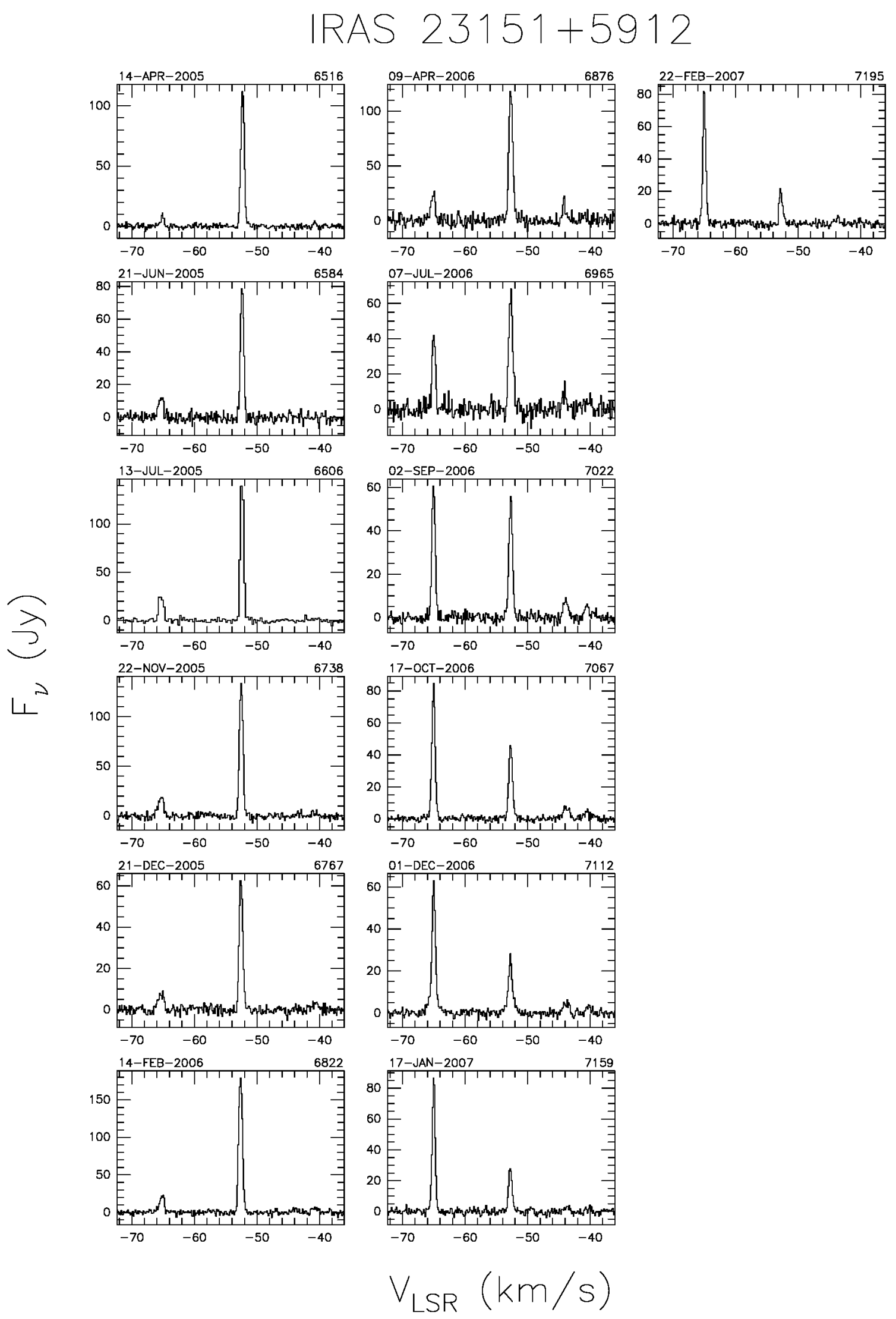

Fig. A.43. a continued. 


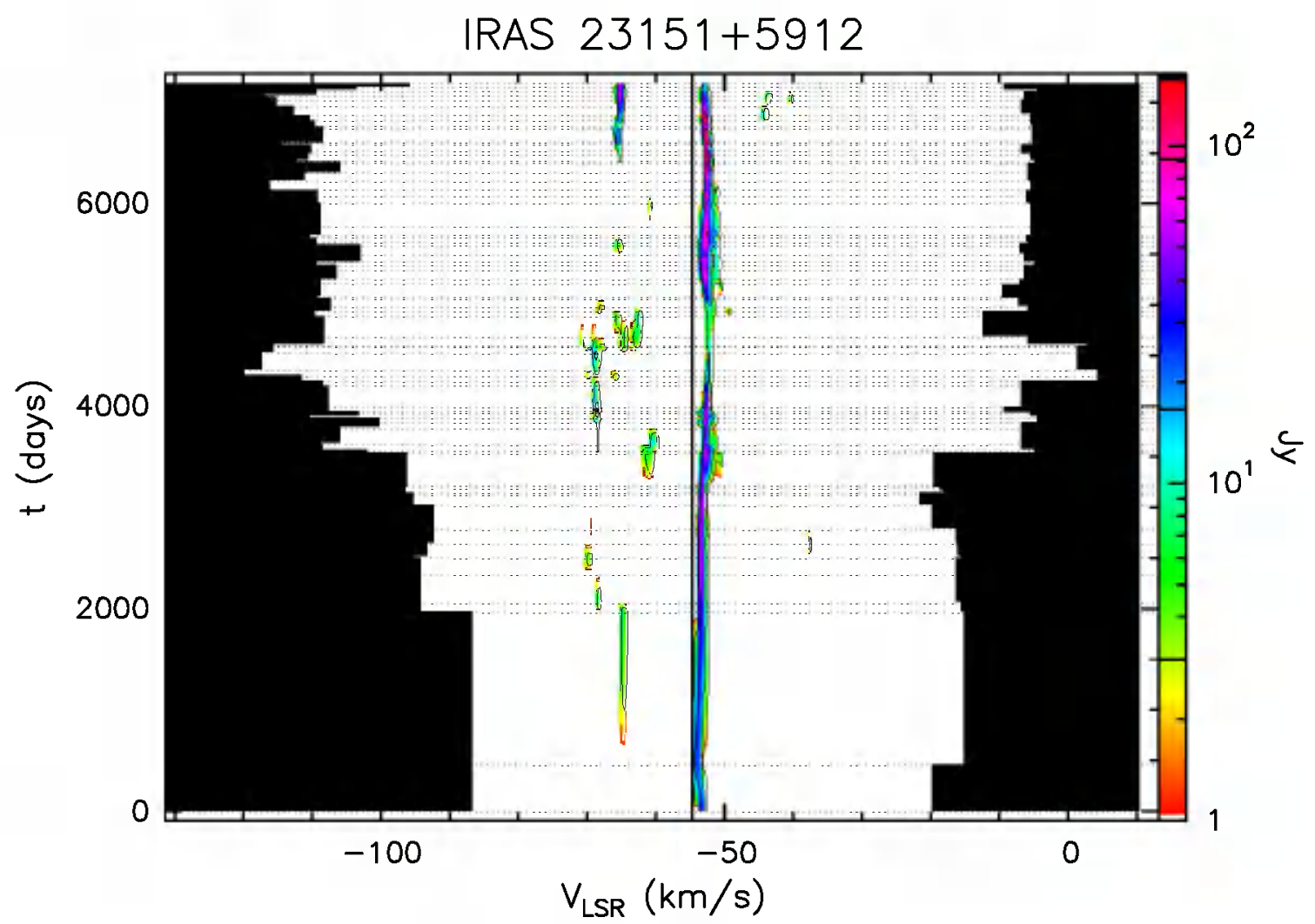

Fig. A.43. b Velocity-time-flux density full plot for source IRAS $23151+5912$. The vertical solid line indicates the velocity of the associated thermal molecular gas. The flux density scale is shown by the bar on the right. In this bar the three lines give the flux density of the drawn contours.

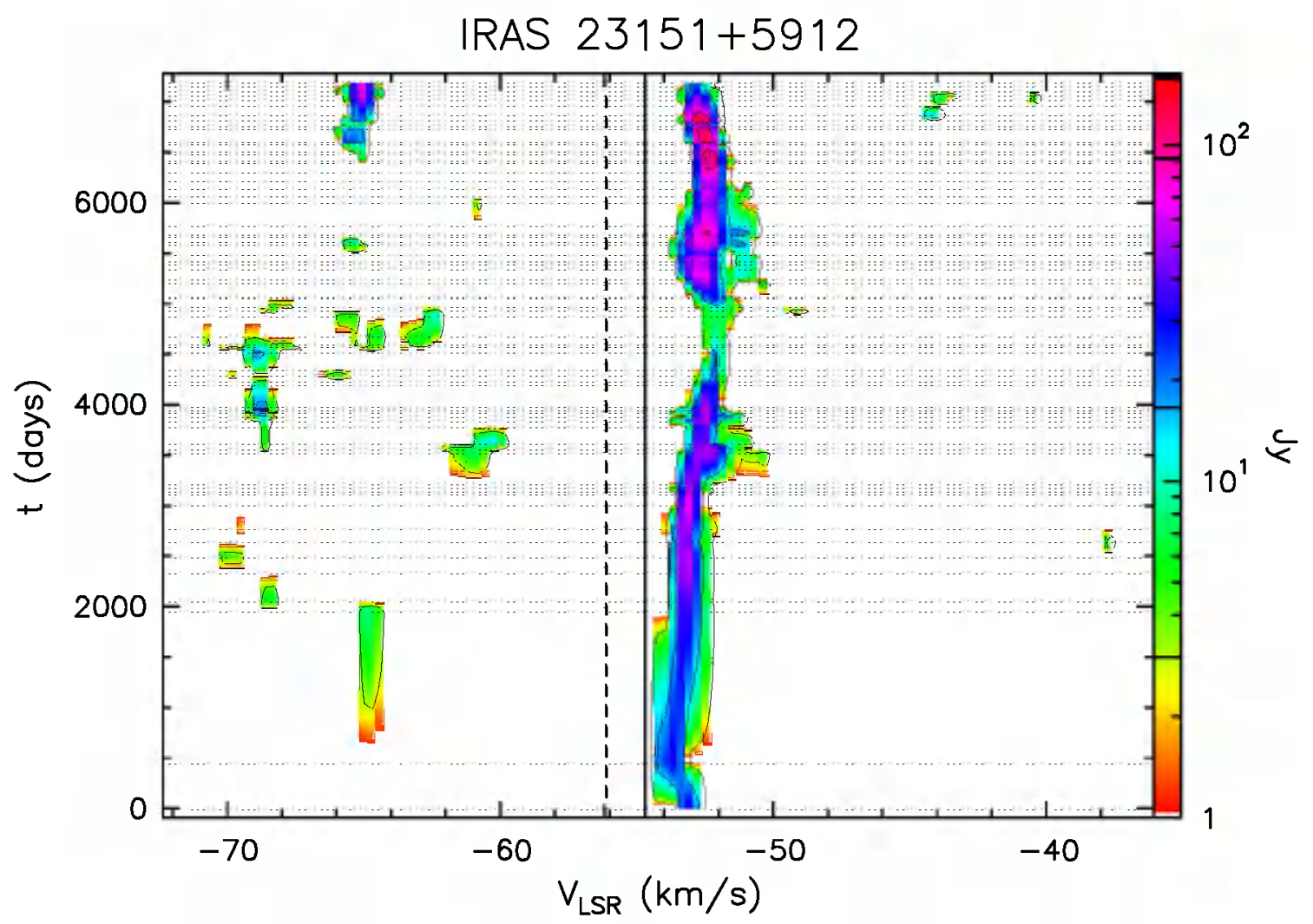

Fig. A.43. c Same as previous figure, but "zoomed" to velocity range over which emission has been detected. 


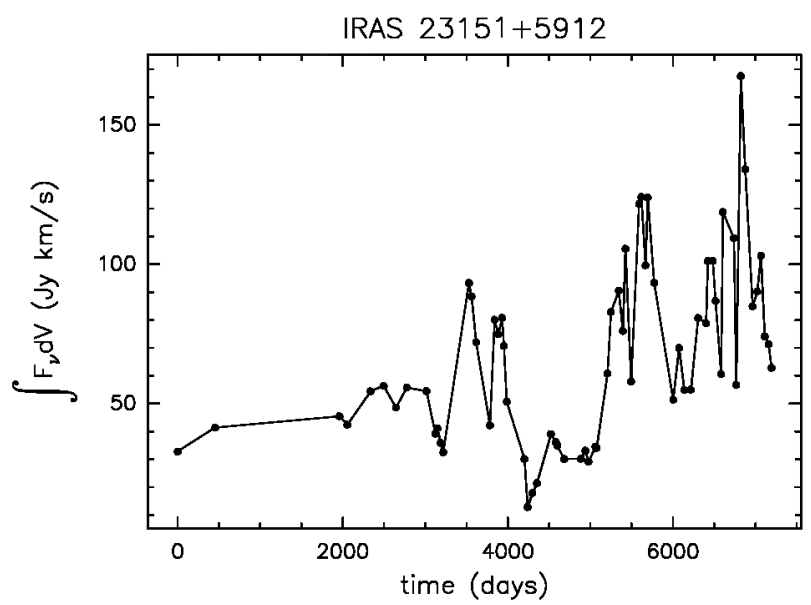

Fig. A.43. d Integral of the flux density over the observed velocity range as a function of time for source IRAS $23151+5912$.

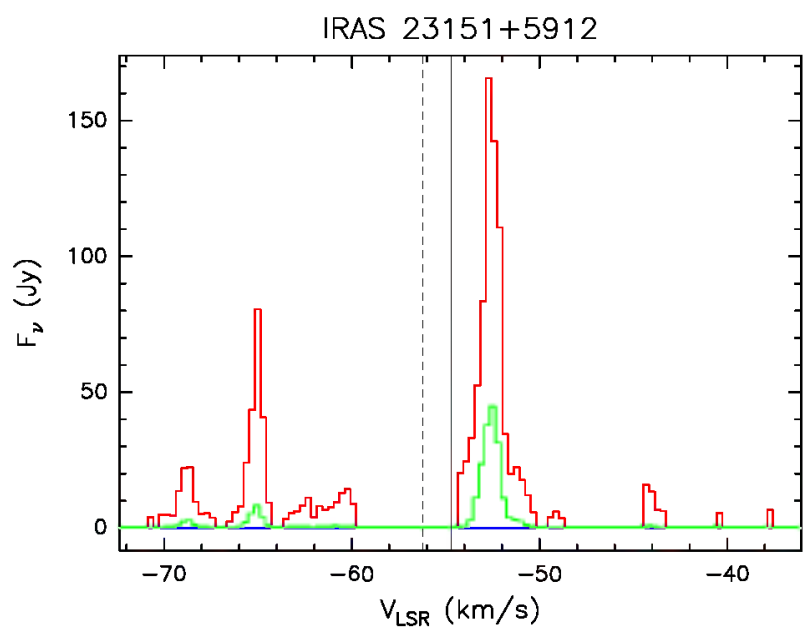

Fig. A.43. e Upper (red) and lower (blue) envelopes and mean spectrum (green) of source IRAS $23151+5912$ measured during our monitoring. The vertical solid line marks the velocity of the associated thermal molecular gas. The vertical dashed line marks the mean velocity derived from the histogram of the rate-of-occurrence.

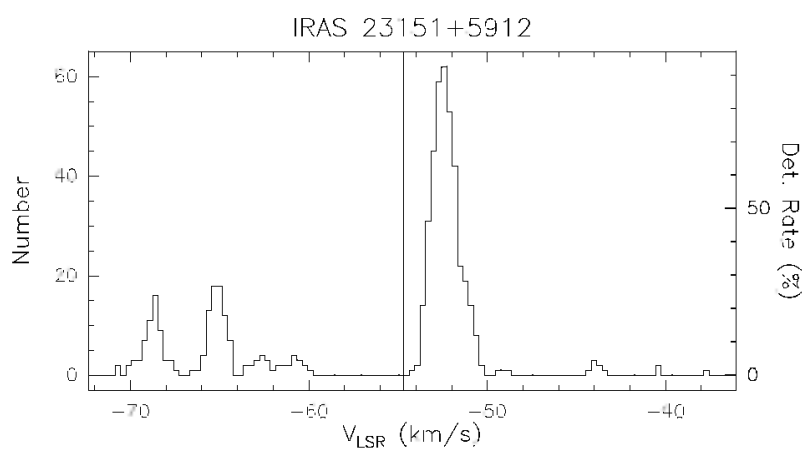

Fig. A.43. f Rate-of-occurrence plot for source IRAS 23151+5912. The scale to the right refers to the dotted histogram, the scale to the left to the solid line histogram. The vertical solid line marks the velocity of the associated thermal molecular gas. 